\title{
Beyond Regular Semigroups
}

\author{
Yan Hui Wang
}

$\mathrm{PhD}$

\author{
University of York \\ Department of Mathematics
}

March 2012 


\section{Abstract}

The topic of this thesis is the class of weakly $U$-abundant semigroups. This class is very wide, containing inverse, orthodox, regular, ample, adequate, quasiadequate, concordant, abundant, restriction, Ehresmann and weakly abundant semigroups. A semigroup $S$ with subset of idempotents $U$ is weakly $U$-abundant if every $\widetilde{\mathcal{R}}_{U}$-class and every $\widetilde{\mathcal{L}}_{U}$-class contains an idempotent of $U$, where $\widetilde{\mathcal{R}}_{U}$ and $\widetilde{\mathcal{L}}_{U}$ are relations extending the well known Green's relations $\mathcal{R}$ and $\mathcal{L}$. We assume throughout that our semigroups satisfy a condition known as the Congruence Condition $(\mathrm{C})$.

We take several approaches to weakly $U$-abundant semigroups. Our first results describe those that are analogous to completely simple semigroups. Together with an existing result of Ren this determines the structure of those weakly $U$ abundant semigroups that are analogues of completely regular semigroups, that is, they are superabundant. Our description is in terms of a semilattice of rectangular bands of monoids.

The second strand is to aim for an extension of the Hall-Yamada theorem for orthodox semigroups as spined products of inverse semigroups and fundamental orthodox semigroups. To this end we consider weakly $B$-orthodox semigroups, where $B$ is a band. We note that if $B$ is a semilattice then a weakly $B$-orthodox semigroup is exactly an Ehresmann semigroup. We provide a description of a weakly $B$-orthodox semigroup $S$ as a spined product of a fundamental weakly $\bar{B}$ orthodox semigroup $S_{B}$ (depending only on $B$ ) and $S / \gamma_{B}$, where $\bar{B}$ is isomorphic to $B$ and $\gamma_{B}$ is the analogue of the least inverse congruence on an orthodox semigroup. This result is an analogue of the Hall-Yamada theorem for orthodox semigroups. In the case that $B$ is a normal band, or $S$ is weakly $B$-superabundant, we find a closed form $\delta_{B}$ for $\gamma_{B}$, which simplifies our result to a straightforward form. 
For the above to work smoothly in the case $S$ is weakly $B$-superabundant, we need to find a canonical fundamental weakly $B$-superabundant subsemigroup of $S_{B}$. This we do, and give the corresponding answers in the case of the Hall semigroup $W_{B}$ and a number of intervening semigroups.

We then change our direction. A celebrated result of Nambooripad shows that regular semigroups are determined by ordered groupoids built over a regular biordered set. Our aim, achieved at the end of the thesis, is to extend Nambooripad's work to weakly $U$-regular semigroups, that is, weakly $U$-abundant semigroups with (C) and $U$ generating a regular subsemigroup whose set of idempotents is $U$.

As an intervening step we consider weakly $B$-orthodox semigroups in this light. We take two approaches. The first is via a new construction of an inductive generalised category over a band. In doing so we produce a new approach to characterising orthodox semigroups, by using inductive generalised groupoids. We show that the category of weakly $B$-orthodox semigroups is isomorphic to the category of inductive generalised categories over bands. Our approach is influenced by that of Nambooripad, however, there are significant differences in strategy, the first being the introduction of generalised categories and the second being that it is more convenient to consider (generalised) categories equipped with pre-orders, rather than with partial orders. Our work may be regarded as extending a result of Lawson for Ehresmann semigroups. We also examine the trace of a weakly $B$-orthodox semigroup, which is a primitive weakly $B$-orthodox semigroup.

We then take a more 'traditional' approach to weakly $B$-orthodox semigroups via band categories and weakly orthodox categories over a band, equipped with two pre-orders. We show that the category of weakly $B$-orthodox semigroups is equivalent to the category of weakly orthodox categories over bands. To do so we must substantially adjust Armstrong's method for concordant semigroups.

Finally, we consider the most general case of weakly $U$-regular semigroups. Following Nambooripad's theorem, which establishes a correspondence between algebraic structures (inverse semigroups) and ordered structures (inductive groupoids), we build a correspondence between the category of weakly $U$-regular semigroups and the category of weakly regular categories over regular biordered sets, equipped with two pre-orders. 


\section{Contents}

Abstract

Contents $\quad$ vi

List of Figures vii

Preface viii

Acknowledgments $\quad$ xiii

Author's Declaration $\quad$ Xv

1 Basic Theory I 1

1.1 Relations....................... . . . 1

1.2 Ordered sets . . . . . . . . . . . . . . . . . . . . 4

1.3 Semigroups and Green's relations . . . . . . . . . . . . . . 5

1.3.1 Basic definitions . . . . . . . . . . . . . 5 5

1.3.2 Green's relations . . . . . . . . . . . . . . . 8

1.3.3 Regular semigroups . . . . . . . . . . . . . . . . 11

1.3.4 Inverse semigroups . . . . . . . . . . . . . . . . . 13

1.3.5 Orthodox semigroups . . . . . . . . . . . . . . 14

1.3.6 Completely regular semigroups . . . . . . . . . . 15

1.4 Biordered sets . . . . . . . . . . . . . . . . . . . 17

1.5 Categories . . . . . . . . . . . . . . . . 26

2 Basic Theory II 32

2.1 Abundant semigroups . . . . . . . . . . . . . . . . . . . . 32

2.1.1 Relations $\mathcal{L}^{*}, \mathcal{R}^{*} \ldots \ldots . \ldots . \ldots . \ldots . . \ldots 32$ 
2.1.2 Abundant semigroups . . . . . . . . . . . . . . . 34

2.2 Weakly $U$-abundant semigroups . . . . . . . . . . . . . 35

2.2 .1 Relations $\widetilde{\mathcal{L}}_{U}, \widetilde{\mathcal{R}}_{U} \ldots \ldots \ldots \ldots \ldots \ldots \ldots$

2.2.2 Weakly $U$-abundant semigroups . . . . . . . . . . . 36

2.2 .3 Weakly $U$-regular semigroups _ . . . . . . . . . . . . 39

2.2.4 Weakly $B$-orthodox semigroups . . . . . . . . . . . 41

2.2 .5 Ehresmann semigroups . . . . . . . . . . . . . . . . 41

2.3 The idempotent connected condition . . . . . . . . . . . . 42

2.3 .1 (WIC), (IC) and (PIC) . . . . . . . . . . . . 43

2.3 .2 Special cases . . . . . . . . . . . . . . . . . 46

2.4 An analogue of the least inverse congruence . . . . . . . . 50

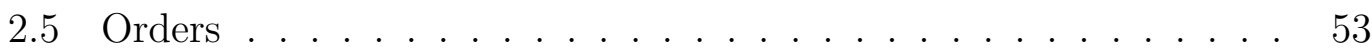

2.5.1 The weakly $U$-abundant case $\ldots \ldots \ldots \ldots \ldots$

2.5.2 The weakly $U$-regular case . . . . . . . . . . . . . 55

2.5.3 The weakly $B$-orthodox case . . . . . . . . . . 56

2.5.4 The Ehresmann case . . . . . . . . . . . . . . 56

2.6 Examples . . . . . . . . . . . . . . . . . . . . . . . . 57

3 Weakly $U$-superabundant semigroups with (C) 63

3.1 Weakly $U$-superabundant semigroups with $(\mathrm{C}) \ldots \ldots \ldots$

3.2 Completely $\widetilde{\mathcal{J}}_{U}$-simple semigroups $\ldots \ldots \ldots \ldots$. . . . . . 67

4 Representations for generalised orthogroups $\quad 70$

4.1 Fundamental inverse semigroups . . . . . . . . . . . . . 70

4.2 A fundamental orthogroup of $W_{B} \ldots \ldots \ldots \ldots \ldots \ldots$

4.3 A fundamental weakly $\bar{B}$-superabundant subsemigroup of $V_{B} \quad \ldots \quad 84$

4.4 A fundamental weakly $\bar{B}$-superabundant subsemigroup of $U_{B} \quad \ldots \quad 89$

4.5 A fundamental weakly $\bar{B}$-superabundant subsemigroup of $S_{B} \quad \ldots \quad 97$

5 Structure theorems for weakly $B$-orthodox semigroups 103

5.1 The least admissible Ehresmann congruence . . . . . . . . . . 103

5.2 A structure theorem for weakly $B$-orthodox semigroups $\ldots \ldots 106$

5.3 Weakly $B$-orthodox semigroups with (N) . . . . . . . . . 110

5.4 Weakly $B$-superabundant semigroups with $(\mathrm{C}) \ldots \ldots \ldots$

5.5 Examples . . . . . . . . . . . . . . . . . . . . 113 
6 Correspondence between algebraic structures and ordered structures 117

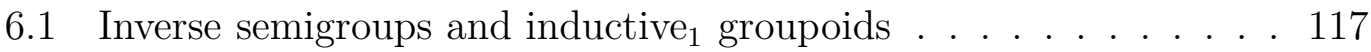

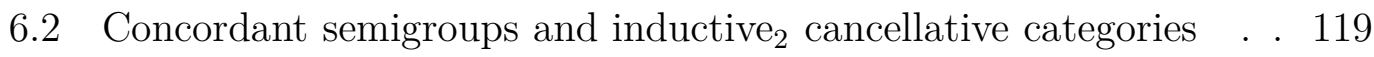

6.3 Ehresmann semigroups and Ehresmann categories . . . . . . . . 122

7 Beyond orthodox semigroups I: weakly $B$-orthodox semigroups $\begin{array}{ll}\text { and generalised categories } & 126\end{array}$

7.1 Inductive generalised categories . . . . . . . . . . . . . 126

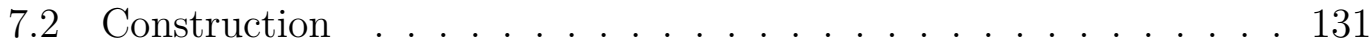

7.3 Correspondence . . . . . . . . . . . . . . . . 136

7.4 Special cases . . . . . . . . . . . . . . . . . . . . . 140

8 Trace of weakly $B$-orthodox semigroups $\quad 147$

8.1 Preliminaries . . . . . . . . . . . . . . . . . . . . . 147

8.2 Blocked Rees matrix semigroups . . . . . . . . . . . . . . . . 148

8.3 Primitive weakly $B$-orthodox semigroups $\ldots \ldots \ldots$. . . . . . . 152

8.4 Trace of weakly $B$-orthodox semigroups . . . . . . . . . . . 158

9 Beyond orthodox semigroups II: weakly $B$-orthodox semigroups $\begin{array}{ll}\text { and categories } & 161\end{array}$

9.1 Weakly orthodox categories . . . . . . . . . . . . . 161

9.2 Construction . . . . . . . . . . . . . . . . . . . 168

9.3 Correspondence . . . . . . . . . . . . . . . . . . . . . . . 175

$9.4 \quad$ Special cases . . . . . . . . . . . . . . . . . . . . . . . . 184

10 Weakly $U$-regular semigroups 195

10.1 Weakly regular categories _. . . . . . . . . . . . 195

10.2 Structure theorems . . . . . . . . . . . . . . . . . 203

10.3 Correspondence . . . . . . . . . . . . . . . . . . . 222

11 Special kinds of weakly $U$-regular semigroups 233

11.1 Weakly $U$-regular semigroups with (WIC) . . . . . . . . . 233

11.2 The abundant case . . . . . . . . . . . . . . . . . 235

11.3 The concordant case . . . . . . . . . . . . . . . . 238

11.4 The regular case . . . . . . . . . . . . . . . . . . 260 
Bibliography 


\section{List of Figures}

1 The structure of this thesis . . . . . . . . . . . . xii

1.1 The $\tau$-commutative condition . . . . . . . . . . . . . . 25

1.2 The natural transformation property . . . . . . . . . . . . 31

2.1 Classes of semigroups . . . . . . . . . . . . . . 50

5.1 The spined product . . . . . . . . . . . . . . 106

5.2 The structure of weakly $B$-orthodox semigroups . . . . . . . . . . 108

7.1 Maps .......................... 127

9.1 A natural transformation of $I_{\mathcal{W O}}$ and CS . . . . . . . . . . . 183

9.2 A natural transformation of $I_{\mathcal{W O C}}$ and SC . . . . . . . . . . . . 184

10.1 A natural transformation of $I_{\mathcal{W R S}}$ and CS . . . . . . . . . . 231

10.2 A natural transformation of $I_{\mathcal{W R C}}$ and SC . . . . . . . . . . 232 


\section{Preface}

The aim of this thesis is to investigate weakly $U$-abundant semigroups, using both techniques developed for regular and abundant semigroups, and new ones. The relevant definitions concerning the classes of semigroups in question are given in Chapter 2.

Fundamental semigroups, that is, regular semigroups having no non-trivial idempotent separating congruences, have played an important role in the structure theory of regular semigroups, especially, in the study of inverse semigroups. As an extension of Munn's approach to inverse semigroups, Hall constructed the fundamental semigroup $W_{B}$ depending only on $B$, which is a subsemigroup of $\mathcal{O P}(B / \mathcal{L}) \times \mathcal{O P}^{*}(B / \mathcal{R})$, where for any partially ordered set $X, \mathcal{O P}(X)$ is the monoid of its order preserving selfmaps, with dual $\mathcal{O P}^{*}$. Also, he showed that if $S$ is an orthodox semigroup with band of idempotents $B$, then there exists a morphism $\phi: S \rightarrow W_{B}$ whose kernel is $\mu$, the maximum idempotent separating congruence on $S$. Consequently, an orthodox semigroup $S$ with band of idempotents $B$ is fundamental if and only if it is isomorphic to a full subsemigroup of $W_{B}$. Besides, Hall-Yamada showed that a regular semigroup $S$ with band of idempotents $B$ is an orthodox semigroup if and only if it is isomorphic to the spined product of the Hall semigroup $W_{B}$ and $S / \gamma$, where $\gamma$ is the least inverse congruence on $S$.

In 1981, El-Qallali and Fountain [7] generalised this result to abundant semigroups with band of idempotents, and satisfying the idempotent connected condition (IC) [8]. They described such a semigroup $S$ having a band of idempotents $B$ as a spined product of $W_{B}$ and $S / \delta_{B}$, where $\delta_{B}$ is the analogue of the least inverse congruence $\left(\delta_{B}\right.$ is in fact the least type A, or ample, congruence on $S$ ). In [46] Ren, Shum and the author similarly extended this approach to describe weakly $B$-orthodox semigroups with a stronger version (PIC) of (IC). We note that an 
abundant semigroup has (PIC) if and only if it has (IC). Condition (PIC) is designed so that $W_{B}$ can be used in the spined product construction; but for weakly $B$-abundant semigroups it is stronger than (IC). The next step was made by ElQallali, Fountain and Gould in [6]. They built an analogous theory for weakly $B$-orthodox semigroups with (IC), or the yet weaker (WIC) [14], this time using semigroups $U_{B}$ and $V_{B}$ in place of $W_{B}$. Here $U_{B}$ and $V_{B}$ are the largest fundamental semigroups containing a band of idempotents $B$ in the given classes. To do this they make heavy use of the congruence $\delta_{B}$. Most recently, Gomes and Gould $[17,18]$ removed the idempotent connected condition (WIC) (or (IC)) from the results of [6] [7], making use of a completely fresh technology to construct a $B$-fundamental weakly $B$-orthodox subsemigroup $S_{B}$ of $\mathcal{O P}(B / \mathcal{L}) \times \mathcal{O P}^{*}(B / \mathcal{R})$. The missing step is the spined product result in the case no idempotent connected condition holds. The aim of Chapter 5 is to provide such a result.

From Chapter 6, we change our angle to investigate the connection between algebraic structures and ordered structures. Ehresmann-Schein-Nambooripad (ESN) built a correspondence between inverse semigroups and inductive groupoids. Here an inductive ${ }_{1}$ groupoid is a groupoid equipped with a partial order possessing restrictions and co-restrictions, and the set of idempotents forming a semilattice under the partial order. The subscript is used to distinguish this meaning of the word 'inductive' from others that will appear later in this thesis. Inverse semigroups are precisely regular semigroups in which the idempotents form a semilattice. Consequently, we can regard the set of idempotents of a regular semigroup as a generalisation of a semilattice. This idea is precisely described in the definition of a regular biordered set, introduced by Nambooripad [38]. In that article, Nambooripad set up a connection between regular semigroups and inductive ${ }_{2}$ groupoids. Such a groupoid is a (functorially) ordered groupoid equipped with the structure of a regular biordered set on its identities, which is compatible with the ordered groupoid structure. In 1988, Nambooripad's work for regular semigroups was extended by Armstrong [1] from regular to concordant semigroups, replacing ordered groupoids by ordered cancellative categories. A concordant semigroup is an abundant semigroup with a regular biordered set of idempotents and satisfying the extra condition of being idempotent connected (IC), which is a condition of a standard type that gives some control over the position of idempotents in products of elements of a semigroup. In 1991, Lawson [32] 
generalised the ESN theorem in a different direction to Ehresmann semigroups. In his work, he used two partial orders on an Ehresmann semigroup to overcome the lack of the idempotent connected condition, and established a correspondence between Ehresmann semigroups and Ehresmann categories. In Chapters 7 and 9 we concentrate on the connection between weakly $B$-orthodox semigroups and ordered structures, and in Chapter 10 we move away to the general case of weakly $U$-regular semigroups. These are weakly $U$-abundant semigroups with (C) and $U$ generating a regular subsemigroup whose set of idempotents is $U$.

The structure of the thesis is as follows.

Chapter 1 presents some basic definitions and results related to regular semigroups, biordered sets and categories.

Chapter 2 gives the basic definitions and fundamental notions concerning abundant semigroups and weakly $U$-abundant semigroups, where $U$ is a subset of idempotents of a semigroup.

Chapter 3 establishes the structure of completely $\widetilde{\mathcal{J}}_{U}$-simple semigroups which are weakly $U$-superabundant semigroups with a single $\widetilde{\mathcal{J}}_{U}$-class and satisfying the Congruence Condition (C). Here Condition (C) means the relations $\widetilde{\mathcal{R}}_{U}$ and $\widetilde{\mathcal{L}}_{U}$ are left and right congruences, respectively. We show that a completely $\widetilde{\mathcal{J}}_{U}$-simple semigroup is isomorphic to a rectangular band of monoids $M_{i \lambda}(i \in I$, $\lambda \in \lambda$ ) and satisfying Conditions called (R) and (L). Such conditions give some control over the position of idempotents in the $\widetilde{\mathcal{D}}_{U}$-class. Finally, we build on an existing result of Ren to show that a weakly $U$-superabundant semigroup with (C) is a semilattice of rectangular bands of monoids satisfying Conditions (R) and (L).

For the purpose of Chapter 5, we study in Chapter 4 fundamental weakly $B$ superabundant semigroups. We find the largest full completely regular subsemigroup of the Hall semigroup $W_{B}$, and correspondingly, weakly $B$-superabundant subsemigroups with (C) of $V_{B}$ (resp. $U_{B}, S_{B}$ ).

In Chapter 5, we obtain a general structure theorem for weakly $B$-orthodox semigroups as a spined product, which may be thought of as an analogue of the Hall-Yamada theorem. Our result is rather detailed, but simplifies drastically in the case $\gamma_{B}$, the analogue of the least inverse congruence, has the closed form $\delta_{B}$, and so in particular, if the band $B$ is normal or $S$ is weakly $B$-superabundant.

In Chapter 6 we briefly recall some of the historical achievements such as the 
Ehresmann-Schein-Nambooripad (ESN) Theorem, and its many extensions due to Armstrong [1,2], Lawson [32], Meakin [35,36] and Nambooripad [38-40]. These results set up a connection between algebraic structures and ordered structures.

In Chapter 7 we introduce a new approach. We define inductive generalised categories over bands and pseudo-functors. We show that the category of weakly $B$-orthodox semigroups is isomorphic to the category of inductive generalised categories. We then turn our attention to some special cases including orthodox semigroups, and in particular recover Lawson's work for Ehresmann semigroups. Our reasoning is briefly as follows. From a regular (concordant) semigroup one can produce a certain ordered category and then endow the category with a so-called pseudo-product. Unfortunately this need not produce the original semigroup: to do so requires factoring by a congruence. Our use of inductive generalised categories circumvents this latter inconvenience. A further point is that we could use partial orders on a semigroup as standard in this area, but to do so would be rather clumsy. It turns out that pre-orders provide an effective method.

In Chapter 8, we change our angle a little to discuss the trace of weakly $B$-orthodox semigroups. We show that the trace of a weakly $B$-orthodox semigroup is a primitive weakly $B$-orthodox semigroup and we investigate primitive weakly $B$-orthodox semigroups via blocked Rees matrix semigroups, which are introduced in [10].

The purpose of Chapter $\mathbf{9}$ is to revisit weakly $B$-orthodox semigroups and provide a correspondence with a class of categories (posscessing two orders) that is more akin to the original approach of Nambooripad and Armstrong. That is, we use triples such as in [1]. A significant point is that we continue to use two preorders instead of a partial order in our work and further new tricks to overcome the lack of an idempotent connected condition. It turns out that without the (IC) condition and without the idempotents forming a semilattice, pre-orders provide the most elegant approach. At the end of this chapter, we discuss some special cases including orthodox semigroups, and recover Lawson's work for Ehresmann semigroups.

Chapter 10 focuses on weakly $U$-regular semigroups. We investigate the correspondence between weakly $U$-regular semigroups and certain categories, by extending the techniques introduced in Chapter 9. 
In Chapter 11 we are concerned with some special kinds of weakly $U$ regular semigroups. We recover Armstrong's work for concordant semigroups and Nambooripad's work for regular semigroups.

We tried to keep some homogeneity in notation. Most of the time, we use Greek letters for functions, lower case letters for elements, capital letters for sets and bold face letters for categories. We write functions and functors on the right.

We use the term morphism for homomorphism. Semigroups are usually denoted by $S$ and monoids by $M$, but this notation is not frozen: we may also use $S$ for monoids and $M$ for semigroups if needed. In general, we use $B$ and $E$ (or $U)$ to denote a band and a set of idempotents, respectively.

The reader wishing to negotiate a pathway through this thesis can use the following diagram.

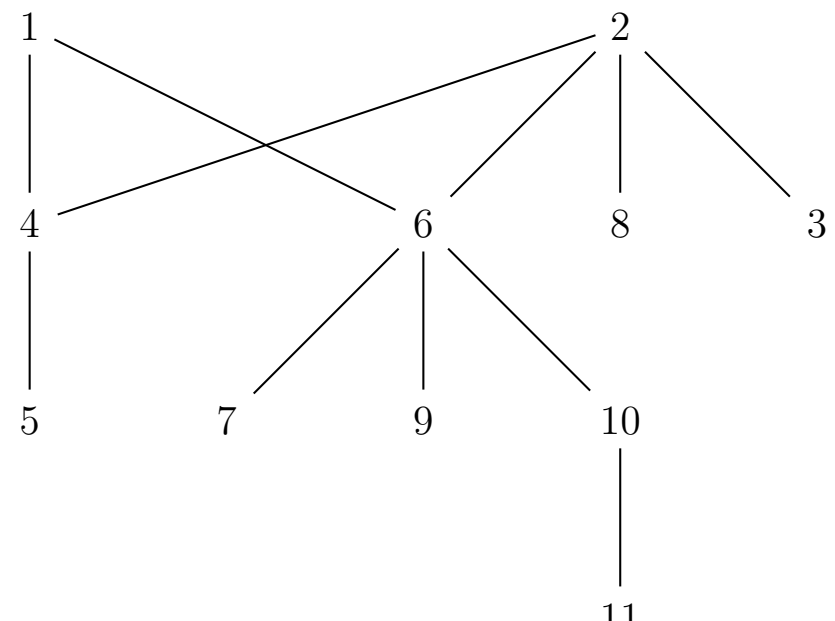

Figure 1: The structure of this thesis

The main result of Chapter 9 is in fact a special case of that of Chapter 10. However, Chapter 9 introduces many of the new techniques required, but in the more concrete content of a band, rather than a regular biordered set. The reader wishes to avoid the full technicalities of Chapter 10 may wish to focus on Chapter 9 . 


\section{Acknowledgements}

First of all, I would like to thank my supervisor Professor Victoria Gould. I am grateful for her advice, patience and encouragement. Her kind help regarding matters in my private life is also appreciated.

I would like to express gratitude to my department for the support that has been given especially by my TAP panel ( Professor Stephen Donkin, Professor Maxim Nazarov and Dr. Brent Everitt). In addition, I would like to thank Professor John Fountain for helping me to apply for external sources of funding and for his other help in other ways.

I have been supported by charitable sponsors in Hong Kong for three years and by the Annie Curry Williamson Scholarships for one year. As to the sponsors in Hong Kong, I would like to take this opportunity to thank Dr. Philip Po Him $\mathrm{Wu}$, Ms Katherine Hung, Mr Chim Pui Chung, the Wu Jieh Yee Charitable Foundation, the Yat Fei Hung Foundation, Mr Yi Hui Hung, Mr Billy Hung, Mr Peter Wong, Mr Robert Lai Kai Dong, Ms Tammy Tam, Mr Patrick Wong and Ms Ann Fok.

I would like to express my warmest thanks also to Professor Kar Ping Shum of the Institute of Mathematics at Yunnan University and my Master Degree supervisor Professor Xue Ming Ren for their encouragement and for having helped me with my application to the University of York.

It is a pleasure to express thanks to the organisers of NBSAN, the Groups and Semigroups: Interactions and Computations Conference, and the International Conference on Algebra 2010 for having given me the opportunity to give presentations.

Special thanks are due to Dr. Mark Kambites of the School of Mathematics at the University of Manchester for having given me very useful suggestions regarding my research, and to Dr. James D Mitchell and Dr. Yann H Peresse of 
School of Mathematics and Statistics at the University of St. Andrews for having supported me with their GAP skills. I should thank Mária B. Szendrei for her advice on a representation of an orthogroup to an orthogroup contained in the Hall semigroup.

I wish to thank my loving family and friends. I am grateful to my family and Tao for their support. I would also like to express my thanks to my friends, in particular to Lini Cao, Malcolm Connolly, Peng Li, Dr. Markus Neckenig, Wan Wan, Dandan Yang and Dr. Dirk Zeindler, all of whom have shown me so much encouragement and help not only in my study but also in my private life. 


\section{Author's Declaration}

Chapters 1 and 2 mainly present definitions and results by other authors. Chapter 1 is concerned with fundamental information about semigroup theory, biordered sets and category theory, mostly from [26], [38] and [34]. In Chapter 2, results about abundant semigroups and weakly $U$-abundant semigroups are introduced from [8], [10], [14], [32], [33] and [44]. Examples provided and results about weakly $B$-orthodox semigroups are from a joint paper [20] with Gould.

In Chapter 3, some results are stated and used from [44]. This chapter forms a part of [51].

In Chapter 4, the fundamental orthogroup in the Hall semigroup and its generalisation in $U_{B}, V_{B}$ and $S_{B}$ are constructed via two different methods. One method contributes to [49]. We are grateful to Professor Mária B. Szendrei for her advice with regard to the orthodox case and for giving us one technique.

Chapter 5 is a part of [51]. Examples provided are from [17], which are referenced accordingly.

Chapter 6 provides background information about the connection between algebraic structures and ordered structures, mostly from $[1,2],[32],[35,36]$ and [38-40].

The contents of Chapter 7 is from a joint paper [20] with Gould. The main result of this chapter is an extension of Lawson's work for Ehresmann semigroups [32].

In Chapter 8, definitions and results by Fountain [10] on blocked Rees matrix semigroups are presented. Results in Section 8.4 form a part of the joint paper [20] with Gould.

In Chapters 9 and 10, we use an adjustment of Armstrong's method for concordant semigroups [1] to build our results. Our modifications are to compensate for the lack of an idempotent connected condition and the fact on semigroups are 
only weakly $U$-abundant. Some of proofs are similar to those of Armstrong. The contents of Chapter 9 will form a paper [50].

In Chapter 11, we use the main result of Chapter 10 to recover Armstrong's work for concordant semigroups and Nambooripad's work for regular semigroups. 


\section{Chapter 1}

\section{Basic Theory I}

In this chapter, we mainly present certain basic definitions and results mostly taken from [26], [38] and [34]. For further details of semigroup theory, we refer the reader to [26], for biordered sets to [38] and for category theory to [34].

\section{$1.1 \quad$ Relations}

Let $X$ be a non-empty set. A subset $\rho$ of $X \times X$ is called a (binary) relation on $X$. It is worth specifically mentioning three special relations on $X$ : the empty subset $\phi$ of $X \times X$, the universal relation $X \times X$ and the identity relation

$$
1_{X}=\{(x, x): x \in X\}
$$

Let $\mathcal{B}(X)$ denote the set of all binary relations on $X$. We define a binary operation $\circ$ on $\mathcal{B}(X)$ by the rule that for any $\rho, \sigma \in \mathcal{B}(X)$,

$$
\rho \circ \sigma=\{(x, y) \in X \times X:(\exists z \in X)(x, z) \in \rho \text { and }(z, y) \in \sigma\} .
$$

Lemma 1.1. [26] The set $\mathcal{B}(X)$ forms a semigroup under $\circ$.

Let $\rho$ be a relation on $X$. We define the $\operatorname{domain} \operatorname{dom}(\rho)$ of $\rho$ by

$$
\operatorname{dom}(\rho)=\{x \in X:(\exists y \in X)(x, y) \in \rho\}
$$


and the co-domain $\operatorname{ran}(\rho)$ of $\rho$ by

$$
\operatorname{ran}(\rho)=\{y \in X:(\exists x \in X)(x, y) \in \rho\} .
$$

For any $x \in X$, we define

$$
x \rho=\{y \in X:(x, y) \in \rho\} .
$$

The inverse of a relation $\rho$ on $X$ is the relation $\rho^{-1}$ on $X$ defined by

$$
\rho^{-1}=\{(y, x) \in X \times X:(x, y) \in \rho\} .
$$

We pause to remark that for all $\rho, \rho_{1}, \ldots, \rho_{n}$ in $\mathcal{B}(X)$,

$$
\begin{gathered}
\left(\rho^{-1}\right)^{-1}=\rho \\
\left(\rho_{1} \circ \rho_{2} \circ \ldots \circ \rho_{n}\right)^{-1}=\rho_{n}^{-1} \circ \ldots \circ \rho_{2}^{-1} \circ \rho_{1}^{-1}
\end{gathered}
$$

and

$$
\operatorname{dom}\left(\rho^{-1}\right)=\operatorname{ran}(\rho), \operatorname{ran}\left(\rho^{-1}\right)=\operatorname{dom}(\rho) .
$$

If $\rho$ is a relation on $X$, we shall usually write ' $x \rho y$ ' for ' $(x, y) \in \rho$ '.

We say that a relation $\rho$ on $X$ is reflexive if $x \rho y$ for all $x$ in $X$, symmetric if $x \rho y$ implies $y \rho x$ for all $x, y$ in $X$, anti-symmetric if $x \rho y$ and $y \rho x$ together imply $x=y$, and transitive if $x \rho y$ and $y \rho z$ together imply that $x \rho z$ for all $x, y, z \in X$.

A relation $\rho$ on $X$ is said to be a pre-order if it is reflexive and transitive. A pre-order is also sometimes called a quasi-order. In this thesis, we prefer to call it a pre-order.

A relation $\rho$ on $X$ is called a partial order if it is reflexive, anti-symmetric and transitive.

A relation $\rho$ is an equivalence relation on $X$ if it is reflexive, symmetric and transitive. If $\rho$ is an equivalence relation, then the sets $x \rho$ are called $\rho$-classes or equivalence classes containing $x$, where $x \in X$. The set of all $\rho$-classes of $X$ is said to be the quotient set of $X$ by $\rho$ and is denoted by $X / \rho$. Clearly, the 
mapping $\rho^{\natural}: X \rightarrow X / \rho$ defined by

$$
x \rho^{\natural}=x \rho \quad(x \in X)
$$

is well-defined. We shall call it the natural mapping associated to $\rho$.

If $\left\{\rho_{i}: i \in I\right\}$ is a non-empty family of equivalences on a set $X$, then it is easy to see that the intersection $\bigcap\left\{\rho_{i}: i \in I\right\}$ is again an equivalence. If $\rho$ is a relation on $X$, then the family of equivalence relations containing $\rho$ is non-empty, since $X \times X$ is one such equivalence, and so the intersection of all the equivalences on $X$ containing $\rho$ is an equivalence, that is, the unique minimum equivalence on $X$ containing $\rho$. We shall call it the equivalence on $X$ generated by $\rho$ and denote it by $\rho^{e}$. It is routine to show that

$$
\rho^{e}=\left[\rho \cup \rho^{-1} \cup 1_{X}\right]^{t},
$$

where $R^{t}=\cup_{n=1}^{\infty} R^{n}$ is the transitive closure of an arbitrary relation $R$. If $\rho$ and $\sigma$ are equivalences on $X$, then the family of equivalence relations containing $\rho$ and $\sigma$ is non-empty, as $X \times X$ is one such equivalence. By definition, $(\rho \cup \sigma)^{e}$ is the least equivalence containing $\rho$ and $\sigma$. We will denote it by $\rho \vee \sigma$.

An extremely useful result is that:

Lemma 1.2. [26] If $\rho$ and $\sigma$ are equivalences on a set $X$ such that $\rho \circ \sigma=\sigma \circ \rho$, then $\rho \vee \sigma=\rho \circ \sigma$.

Note that if $\rho$ is a pre-order on $X$, then the relation $\equiv_{\rho}$ on $X$ given by

$$
x \equiv \rho y \text { if and only if } x \rho y \text { and } y \rho x \quad(x, y \in X) \text {, }
$$

is an equivalence relation. Since $\rho$ is a pre-order, that is, $\rho$ is reflexive and transitive, it immediately leads to $\equiv_{\rho}$ being an equivalence relation. For any $x \in X$, we will use $[x]$ to denote the $\equiv_{\rho}$-class of $X$ containing $x$.

In addition, the relation $\preceq_{\rho}$ on $X / \equiv_{\rho}$ defined by

$$
[x] \preceq_{\rho}[y] \text { if and only if } x \rho y
$$

is a well-defined partial order. Since if $x \equiv_{\rho} x^{\prime}, y \equiv_{\rho} y^{\prime}$ and $x \rho y$, then $x^{\prime} \rho x \rho y \rho y^{\prime}$, and so $x^{\prime} \rho y^{\prime}$, so that the choice of $x$ and $y$ does not mat- 
ter. Hence $\preceq_{\rho}$ is well-defined.

It is easy to see that $\preceq_{\rho}$ is reflexive and transitive as $\rho$ is reflexive and transitive. To show that $\preceq_{\rho}$ is anti-symmetric, we suppose that $[x] \preceq_{\rho}[y]$ and $[y] \preceq_{\rho}[x]$. Then $x \rho y$ and $y \rho x$, so that $x \equiv_{\rho} y$, that is, $[x]=[y]$.

\subsection{Ordered sets}

A partially ordered set $(X, \leq)$ is a non-empty set $X$ together with a partial order $\leq$. Let $X$ be a partially ordered set with respect to $\leq$ and let $Y$ be a non-empty subset of $X$. An element $a$ of $Y$ is called maximal if there is no element of $Y$ strictly greater than $a$, that is, if for any $y \in Y$ we have $a \leq y$, then $y=a$. An element $b$ of $Y$ is called maximum if $y \leq b$ for all $y \in Y$. Obviously, a maximum element is maximal, but the converse is not necessarily true. We say that an element $x$ in $X$ is a lower bound for $Y$ if $c \leq y$ for every $y \in Y$. If the set of lower bounds of $Y$ is non-empty and has a maximum element $d$, we refer to $d$ as the greatest lower bound, or meet of $Y$. The element $d$ is unique if it exists, and we write

$$
d=\bigwedge\{y: y \in Y\}
$$

In particular, if $Y=\{a, b\}$, we denote $d=a \wedge b$. If $X$ is a partially ordered set with respect to $\leq$ and such that $a \wedge b$ exists for every $a, b \in X$, we say that $(X, \leq)$ is a lower semilattice. In a lower semilattice $(X, \leq)$ we have that, for all $a, b \in X$,

$$
a \leq b \text { if and only if } a \wedge b=a .
$$

Analogously, we define the least upper bound or join

$$
\bigvee\{y: y \in Y\}
$$

of a non-empty subset $Y$ of $X$ and an upper semilattice. 


\subsection{Semigroups and Green's relations}

\subsubsection{Basic definitions}

Let $S$ be a non-empty set. A binary operation on $S$ is a mapping $\mu$ from $S \times S$ into $S$. The pair $(S, \mu)$ is said to be a semigroup if $\mu$ is associative, that is, for all $x, y, z \in S,((x, y) \mu, z) \mu=(x,(y, z) \mu) \mu$. To avoid the notation being rather cumbersome, we shall follow the usual practice in algebra to write $(x, y) \mu$ as $x y$ and usually call $x y$ the product of $x$ and $y$. In this case, the semigroup operation is called multiplication and the associative formula may be simply expressed as $(x y) z=x(y z)$.

A semigroup $(S, \mu)$ is a pair, but we shall usually say ' $S$ is a semigroup' and assume the binary operation is known.

An element $e$ of $S$ is called an idempotent if $e^{2}=e$. The set of idempotents of $S$ is denoted by $E(S)$.

An element $e$ of $S$ is said to be a left (resp. right) identity if, for all $x \in S$, $e x=x(x e=x)$. An element is an identity element or identity if it is a left and a right identity. It is easy to see that there exists at most one identity, which will be called the identity and denoted by 1 .

An element $e$ of $S$ is called a left (resp. right) zero if, for all $x \in S$, ex $=e$ $(x e=e)$. An element of $S$ is called a zero element or zero if it is a left and a right zero. There can be at most one zero, which is then called the zero and denoted by 0 .

Observe that a left (resp. right) identity is necessary idempotent, and so is a left (resp. right) zero.

A monoid is a semigroup with an identity. If $S$ is a semigroup, $S^{1}$ denotes the monoid equal to $S$ if $S$ is a monoid and to $S \cup\{1\}$ if $S$ is not a monoid. In the latter case, the operation of $S^{1}$ is completed by the rules

$$
1 x=x 1=x,
$$

for all $x \in S^{1}$. We say that $S^{1}$ is $S$ with an identity adjoined if necessary.

If $S$ is a semigroup with or without a zero element, we usually use $S^{0}$ to denote the semigroup with underlying set $S \cup\{0\}$ and multiplication extending 
that of $S$, with

$$
0 x=x 0=0 \quad(x \in S)
$$

and $00=0$. We say that $S^{0}$ is $S$ with a zero adjoined.

A monoid $M$ is a group if each of its elements has a group inverse, that is, for all $x \in M$, there exists $x^{\prime} \in M$ such that $x x^{\prime}=x^{\prime} x=1$. Here, $x^{\prime}$ is a group inverse of $x$. Note that if $x^{\prime \prime}$ is another group inverse of $x$, then $x^{\prime \prime}=x^{\prime \prime} 1=x^{\prime \prime} x x^{\prime}=1 x^{\prime}=x^{\prime}$, and so the group inverse of $x$ is unique, so that we shall say $x^{\prime}$ is the group inverse of $x$.

A subsemigroup $G$ of a semigroup $S$ is said to be a subgroup if $G$ is a group. A 0-group is a group $G$ with a zero adjoined.

A semigroup (resp. monoid, group) $S$ is said to be commutative if, for all $x, y \in S, x y=y x$.

A band is a semigroup $B$ in which every element is an idempotent, that is, $x^{2}=x$ for all $x \in B$.

In the following, we mention some special bands.

A band $B$ is a left zero band if, for all $x, y \in B, x y=x$. Symmetrically, a right zero band is defined.

A normal band is a band satisfying $x y z x=x z y x$ for all $x, y, z \in B$.

Let $E$ be a commutative semigroup of idempotents. We define a relation $\leq$ on $E$ by

$$
x \leq y \text { if and only if } x y=x \quad(x, y \in E) .
$$

It is easy to see that $\leq$ is a partial order on $E$. Indeed if $x \in E$, then $x^{2}=x$, and so $x \leq x$ so that $\leq$ is reflexive. Suppose that $x, y \in E$ with $x \leq y$ and $y \leq x$, then $x=x y=y x=y$, so $\leq$ is anti-symmetric. Furthermore, if $x \leq y$ and $y \leq z$ in $E$, then $x=x y$ and $y z=y$, so that $x z=(x y) z=x(y z)=x y=x$, whence $x \leq z$. Hence, $\leq$ is transitive and so $\leq$ is a partial order on $E$. We note that for any $x, y \in E, x y$ is the greatest lower bound of $x$ and $y$. Consequently, $(E, \leq)$ becomes a lower semilattice, that is, a partially ordered set in which every pair of elements has a greatest lower bound.

Conversely, if $(E, \leq)$ is a lower semilattice, then $E$, together with the binary operation $\wedge$, forms a commutative semigroup of idempotents. If $a, b, c \in E$, then

$$
(a \wedge b) \wedge c \leq a \wedge b \leq a,(a \wedge b) \wedge c \leq a \wedge b \leq b
$$


and

$$
(a \wedge b) \wedge c \leq c
$$

Thus $(a \wedge b) \wedge c$ is a lower bound of $\{a, b, c\}$. If $d$ is a lower bound of $\{a, b, c\}$, then $d \leq a, d \leq b$ and $d \leq c$. Hence, $d \leq a \wedge b$, and so $d \leq(a \wedge b) \wedge c$. Thus $(a \wedge b) \wedge c$ is the unique greatest lower bound of $\{a, b, c\}$. Similarly, we may show that $a \wedge(b \wedge c)$ is the unique greatest lower bound of $\{a, b, c\}$. Hence, $a \wedge(b \wedge c)=(a \wedge b) \wedge c$, so that $(E, \wedge)$ is a semigroup. Obviously, $a \wedge a=a$ for all $a \in E$ and $a \wedge b=b \wedge a$ for all $a, b \in E$. Thus $(E, \wedge)$ is a commutative semigroup of idempotents. Moreover, $a \wedge b=a$ if and only if $a \leq b$.

To sum up, we have:

Proposition 1.3. [26] Let $(E, \leq)$ be a lower semilattice. Then $(E, \wedge)$ is a commutative semigroup of idempotents and

$$
(\forall a, b \in E) a \leq b \text { if and only if } a \wedge b=a
$$

Let $(E, \cdot)$ be a commutative semigroup of idempotents. Then the relation $\leq$ on $E$ defined by

$$
a \leq b \text { if and only if } a b=a
$$

is a partial order on $E$ with respect to which $E$ is a lower semilattice. In $(E, \leq)$, the meet of $a$ and $b$ is their product $a b$.

As a consequence of Proposition 1.3, the notions of 'lower semilattice' and 'commutative semigroup of idempotents' are equivalent and interchangeable. We shall use the term semilattice to mean either concept, making free and frequent transfers between algebraic structure and ordered structure.

We close this section with the notion of a rectangular band. A band $B$ is said to be rectangular if, for all $x, y, z \in B, x y z=x z$. For example, given arbitrary non-empty sets $I$ and $J$, one can define a semigroup operation on $I \times J$ by putting

$$
(i, j) \cdot(k, \ell)=(i, \ell)
$$

The resulting semigroup $I \times J$ is a rectangular band as for any $(i, j) \in I \times J$,

$$
(i, j) \cdot(i, j)=(i, j)
$$


and for any $(i, j),(\lambda, \mu),(k, w) \in I \times J$, we have that

$$
(i, j) \cdot(\lambda, \mu) \cdot(k, w)=(i, w)
$$

Notice that any rectangular band is isomorphic to one so constructed.

We omit the proof of the next lemma as it will be shown in Section 1.3.6.

Lemma 1.4. [26] Every band is a semilattice of rectangular bands.

\subsubsection{Green's relations}

Let $S$ be a semigroup. A relation $\rho$ on $S$ is called left compatible if

$$
(\forall s, t, a \in S) \quad(s, t) \in \rho \Rightarrow(a s, a t) \in \rho ;
$$

the notion of right compatible is defined dually. It is called compatible if

$$
\left(\forall s, t, s^{\prime}, t^{\prime} \in S\right) \quad\left(s, s^{\prime}\right) \in \rho \text { and }\left(t, t^{\prime}\right) \in \rho \Rightarrow\left(s t, s^{\prime} t^{\prime}\right) \in \rho .
$$

A left (resp. right) compatible equivalence relation is called a left (resp. right) congruence. A compatible equivalence relation is called a congruence. It is easy to see that a relation $\rho$ on a semigroup $S$ is a congruence if and only if it is both a left and a right congruence.

It is necessary to mention the theorem below as it will be used in the later chapters.

Theorem 1.5. [26] If $\rho$ is a congruence on a semigroup $S$, then $S / \rho$ is a semigroup with respect to the operation defined by the rule that

$$
(x \rho)(y \rho)=(x y) \rho \quad(\forall x, y \in S)
$$

and the mapping $\rho^{\natural}: S \rightarrow S / \rho$ defined by

$$
x \rho^{\natural}=x \rho \quad(x \in S)
$$

is a morphism.

We pause here to make a short comment on Theorem 1.5. It is easy to see that the operation $(x \rho)(y \rho)=(x y) \rho$ is well-defined. Since for all $x, x^{\prime}, y, y^{\prime} \in S$, 
if $x \rho x^{\prime}$ and $y \rho y^{\prime}$, then $\left(x, x^{\prime}\right) \in \rho$ and $\left(y, y^{\prime}\right) \in \rho$, so that $\left(x y, x^{\prime} y^{\prime}\right) \in \rho$, that is, $x y \rho x^{\prime} y^{\prime}$.

Let $S$ be a semigroup and $\rho$ be a congruence on $S$. Then we shall call $\rho$ a $\mathcal{K}$-congruence if $S / \rho$ is a $\mathcal{K}$-semigroup. For example, if $S / \rho$ is a semilattice, then $\rho$ is called a semilattice congruence.

If $A$ and $B$ are subsets of a semigroup $S$, we write $A B$ for $\{a b: a \in A, b \in B\}$. It is easy to see that

$$
(\forall A, B, C \in S)(A B) C=A(B C)
$$

Hence notation such as $A B C, A_{1} A_{2} \cdots A_{n}$ are meaningful. To deal with singleton sets, we shall use the notational simplifications that are customary in algebra. For example, we will write $A b$ for $A\{b\}$.

A non-empty set $A$ of a semigroup $S$ is called a left ideal if $S A \subseteq A$, a right ideal if $A S \subseteq A$, and a (two-sided) ideal if it is both a left and a right ideal. Note that every (left, right) ideal is a subsemigroup; but not every subsemigroup is an ideal. Any semigroup is an ideal of itself.

Let $S$ be a semigroup without identity. For any $a \in S, S a$ will not in general contain $a$. However,

$$
\begin{aligned}
& S^{1} a=S a \cup\{a\}, \\
& a S^{1}=a S \cup\{a\}
\end{aligned}
$$

and

$$
S^{1} a S^{1}=S a S \cup S a \cup a S \cup\{a\} .
$$

Notice that $S^{1} a, a S^{1}$ and $S^{1} a S^{1}$ do not contain 1, so they are all subsets of $S$. Precisely, they are the smallest left, right and two-sided ideals of $S$ containing $a$, respectively. Commonly, $S^{1} a$ is called the principal left ideal generated by $a$. Dually, $a S^{1}$ is the principal right ideal generated by $a$ and $S^{1} a S^{1}$ is the principal ideal generated by a.

Built on these ideals mentioned above, we define relations $\leq_{\mathcal{L}}, \leq_{\mathcal{R}}$ and $\leq_{\mathcal{J}}$ on a semigroup $S$ as follows: for any $a, b \in S$,

$$
\begin{aligned}
& a \leq_{\mathcal{L}} b \text { if and only if } S^{1} a \subseteq S^{1} b, \\
& a \leq_{\mathcal{R}} b \text { if and only if } a S^{1} \subseteq b S^{1},
\end{aligned}
$$




$$
a \leq_{\mathcal{J}} b \text { if and only if } S^{1} a S^{1} \subseteq S^{1} b S^{1}
$$

It is easy to see that $\leq_{\mathcal{L}}, \leq_{\mathcal{R}}$ and $\leq_{\mathcal{J}}$ are pre-orders on $S$, respectively. Also, we note that $\leq_{\mathcal{R}}$ is left compatible. Since if $a, b, c \in S$ are such that $a \leq_{\mathcal{R}} b$, then $a S^{1} \subseteq b S^{1}$, and so $c a S^{1} \subseteq c b S^{1}$, that is, $c a \leq_{\mathcal{R}} c b$. Dually, $\leq_{\mathcal{L}}$ is right compatible.

The following lemma is immediate.

Lemma 1.6. Let $S$ be a semigroup with set of idempotents $E(S)$. For any e, $f \in$ $E(S)$,

(i) $e \leq_{\mathcal{R}} f$ if and only if $f e=e$;

(ii) $e \leq_{\mathcal{L}} f$ if and only if $e f=e$.

We denote the associated equivalences by $\mathcal{L}, \mathcal{R}$ and $\mathcal{J}$, respectively. So for any $a, b \in S$,

$$
\begin{aligned}
& a \mathcal{L} b \text { if and only if } S^{1} a=S^{1} b, \\
& a \mathcal{R} b \text { if and only if } a S^{1}=b S^{1}, \\
& a \mathcal{J} b \text { if and only if } S^{1} a S^{1}=S^{1} b S^{1} .
\end{aligned}
$$

In addition, we use $\mathcal{H}$ and $\mathcal{D}$ to denote the intersection and join of $\mathcal{L}$ and $\mathcal{R}$, respectively, that is,

$$
\mathcal{H}=\mathcal{L} \cap \mathcal{R} \text { and } \mathcal{D}=\mathcal{L} \vee \mathcal{R}
$$

As usual, these equivalence relations $\mathcal{L}, \mathcal{R}, \mathcal{H}, \mathcal{D}$ and $\mathcal{J}$ are called Green's relations [21].

The next proposition gives an alternative characterisation of Green's relations.

Proposition 1.7. [25] Let $S$ be a semigroup and $a, b \in S$. Then

(i) $a \mathcal{L} b$ if and only if there exist $x, y \in S^{1}$ such that $x a=b, y b=a$;

(ii) $a \mathcal{R} b$ if and only if there exist $u, v \in S^{1}$ such that $a u=b, b v=a$;

(iii) $a \mathcal{J} b$ if and only if there exist $x, y, u, v \in S^{1}$ such that $x a y=b, u b v=a$.

The following two lemmas give important properties of $\mathcal{L}$ and $\mathcal{R}$.

Lemma 1.8. [26] The relation $\mathcal{L}$ is a right congruence and $\mathcal{R}$ is a left congruence.

Lemma 1.9. [26] The relations $\mathcal{L}$ and $\mathcal{R}$ commute. 
In view of Lemma 1.2 and Lemma 1.9, we have

$$
\mathcal{D}=\mathcal{L} \circ \mathcal{R}=\mathcal{R} \circ \mathcal{L}
$$

We remark that in a group $G$, we have that

$$
\mathcal{H}=\mathcal{L}=\mathcal{R}=\mathcal{D}=\mathcal{J}=G \times G
$$

and in a commutative semigroup, we have that

$$
\mathcal{H}=\mathcal{L}=\mathcal{R}=\mathcal{D}=\mathcal{J}
$$

Before closing this section, we introduce partial orders amongst Green's equivalence classes. First, it is worth making a statement to simplify the notation of equivalence classes. We will denote the $\mathcal{L}$-class (resp. $\mathcal{R}$-class, $\mathcal{H}$-class, $\mathcal{D}$-class, $\mathcal{J}$-class) containing the element $a$ by $L_{a}$ (resp. $\left.R_{a}, H_{a}, D_{a}, J_{a}\right)$. Since $\mathcal{L}$, $\mathcal{R}$ and $\mathcal{J}$ are defined in terms of ideals, a partial order amongst the equivalence classes is induced by the inclusion order amongst these ideals.

Let $S$ be a semigroup and $a, b \in S$. Then

$$
\begin{aligned}
& L_{a} \leq L_{b} \text { if and only if } S^{1} a \subseteq S^{1} b, \\
& R_{a} \leq R_{b} \text { if and only if } a S^{1} \subseteq b S^{1}, \\
& J_{a} \leq J_{b} \text { if and only if } S^{1} a S^{1} \subseteq S^{1} b S^{1} .
\end{aligned}
$$

Hence, $S / \mathcal{L}, S / \mathcal{R}$ and $S / \mathcal{J}$ may be regarded as partially ordered sets.

If $T$ is a subsemigroup of $S$ and $\mathcal{K}$ is a relation on $S$, then $\mathcal{K}(T)$ is the relation on $T$ and $\mathcal{K}(S)$ is the relation on $S$.

\subsubsection{Regular semigroups}

The notion of regularity in a semigroup is derived from von Neumann's definition of a regular ring [42].

An element $a$ of a semigroup $S$ is said to be regular if there exists $x$ in $S$ such that $a x a=a$. A semigroup $S$ is called regular if all its elements are regular. Groups are of course regular semigroups, but the class of regular semigroups is vastly more extensive than the class of groups. As an analogue of the group inverse, we have the notion of a semigroup inverse of an element. If $a$ is an 
element of a semigroup $S$, we say that $a^{\prime}$ is an inverse of $a$ if

$$
a a^{\prime} a=a, \quad a^{\prime} a a^{\prime}=a^{\prime} .
$$

Notice that an element with an inverse is necessarily regular. Conversely, every regular element has an inverse. Since if $a x a=a$ then we put $a^{\prime}=x a x$ and it is routine to verify that $a^{\prime}$ is an inverse of $a$.

We remark that an element a may have more than one inverse, say, in rectangular bands every element is an inverse of every other element. We will use $V(a)$ to denote the set of all inverses of $a$.

Let $S$ be a regular semigroup and $a \in S$. Then there exists $x \in S$ such that $a=a x a$, from which it follows that $a \in S a(\operatorname{resp} . a S, S a S)$. In that case, Green's relations can be restated for regular semigroups as follows: for any $a, b \in S$,

$a \mathcal{L} b$ if and only if $S a=S b$,

$a \mathcal{R} b$ if and only if $a S=b S$,

$a \mathcal{J} b$ if and only if $S a S=S b S$.

The following theorem is very useful. Here, a $\mathcal{D}$-class is regular if all its elements are regular, or equivalently, it contains at least one regular element [26].

Theorem 1.10. [26] Let a be an element of a regular $\mathcal{D}$-class $D$ in a semigroup $S$.

(i) If $a^{\prime}$ is an inverse of a then $a^{\prime} \in D$ and the two $\mathcal{H}$-classes $R_{a} \cap L_{a^{\prime}}$, $L_{a} \cap R_{a^{\prime}}$ contain respectively the idempotents $a a^{\prime}$ and $a^{\prime} a$.

(ii) If $b \in D$ is such that $R_{a} \cap L_{b}, L_{a} \cap R_{b}$ contains idempotents e, $f$, respectively, then $H_{b}$ contains an inverse $a^{*}$ of a such that $a a^{*}=e, a^{*} a=f$.

(iii) No $\mathcal{H}$-class contains more than one inverse of a.

The next proposition is an immediate consequence of Theorem 1.10, which will be of considerable use in Chapter 7 .

Proposition 1.11. [26] Let $e, f$ be idempotents in a semigroup $S$. Then e $\mathcal{D} f$ if and only if there exists an element $a$ in $S$ and an inverse $a^{\prime}$ of a such that $a a^{\prime}=e$ and $a^{\prime} a=f$.

If $S$ is a semigroup with set of idempotents $E(S)$ we shall say that a congruence $\rho$ on $S$ is idempotent separating if it has the property that

$$
\rho \cap(E(S) \times E(S))=1_{E(S)}
$$


that is, no $\rho$-class contains more than one idempotent. In fact, it is of interest to recall the result, as Lallement [31] showed: on a regular semigroup a congruence is idempotent separating if and only if it is contained in $\mathcal{H}$.

A semigroup is fundamental if the largest idempotent separating congruence is trivial.

\subsubsection{Inverse semigroups}

The aim of this section is to cover the basic ideas and facts about inverse semigroups, which may be regarded as the class of semigroups closest to groups.

A semigroup $S$ is said to be inverse if every $a$ in $S$ has a unique inverse. We will denote the unique inverse of $a$ in $S$ by $a^{-1}$. Such a semigroup is certainly regular, but the converse need not be true.

There are some equivalent formulation for inverse semigroups.

Theorem 1.12. [26] Let $S$ be a semigroup with set of idempotents $E(S)$. Then the following statements are equivalent:

(i) $S$ is an inverse semigroup;

(ii) $S$ is regular and $E(S)$ is a semilattice;

(iii) every $\mathcal{L}$-class and every $\mathcal{R}$-class contains exactly one idempotent.

It is convenient to recall from [26] the following elementary properties of inverse semigroups.

Proposition 1.13. [26] Let $S$ be an inverse semigroup with semilattice of idempotents $E(S)$. Then

(i) $\left(a^{-1}\right)^{-1}=a$ for all $a \in S$;

(ii) $e^{-1}=e$ for all $e \in E(S)$;

(iii) $(a b)^{-1}=b^{-1} a^{-1}$ for all $a, b \in S$;

(iv) $a e a^{-1} \in E(S), a^{-1} e a \in E(S)$ for all $a \in S$ and $e \in E(S)$.

A significant feature of inverse semigroups is the natural partial order relation. Let $S$ be an inverse semigroup with semilattice of idempotents $E(S)$. Given $a, b \in S$, we define

$$
a \leq b \text { if and only if }(\exists e \in E(S)) a=e b .
$$

It is routine to verify that this relation $\leq$ is a partial order. 
The following list gives several alternative characterisations of $\leq$.

Proposition 1.14. [26] Let $S$ be an inverse semigroup with semilattice of idempotents $E(S)$ and $a, b \in S$. Then the following statements are equivalent:

(i) $a \leq b$;

(ii) $(\exists e \in E(S)) a=b e$;

(iii) $a a^{-1}=b a^{-1}$;

(iv) $a a^{-1}=a b^{-1}$;

(v) $a^{-1} a=b^{-1} a$;

(vi) $a^{-1} a=a^{-1} b$;

(vii) $a=a b^{-1} a$;

(viii) $a=a a^{-1} b$.

We note that the restriction of $\leq$ to the semilattice $E(S)$ of idempotents of an inverse semigroup $S$ is the natural semilattice order on $E(S)$ :

$$
e \leq f \text { if and only if } e f=e \quad(e, f \in E(S))
$$

Another crucial property of $\leq$ is that the order relation on any inverse semigroup $S$ is compatible with the operations of multiplication and inverse. Since if $a, b \in S$ with $a \leq b$, then there exists $e \in E(S)$, the semilattice of idempotents of $S$, such that $a=e b$, and so $a c=e b c$, so that $a c \leq b c$. The left compatibility follows dually from Proposition 1.14(ii).

Usually, we call $\leq$ the natural partial order on an inverse semigroup. In particular, the partial order $\leq$ is the identity relation on a group.

Another important notion for inverse semigroups is that of an idempotent separating congruence. Howie [27] gave an alternative description of this congruence:

Theorem 1.15. [27] If $S$ is an inverse semigroup with semilattice of idempotents $E(S)$, then the relation

$$
\mu=\left\{(a, b) \in S \times S:(\forall e \in E(S)) a^{-1} e a=b^{-1} e b\right\}
$$

is the greatest idempotent separating congruence on $S$.

As any inverse semigroup is regular, it follows from Lallement's result in Section 1.3.3 that $\mu$ is the largest congruence contained in $\mathcal{H}$.

\subsubsection{Orthodox semigroups}

An orthodox semigroup is a regular semigroup in which the set of idempotents forms a band. 
Due to Reilly and Scheiblich [43], we have:

Proposition 1.16. [43] If $S$ is a regular semigroup, then the following statements are equivalent:

(i) $S$ is orthodox;

(ii) for any $a, b$ in $S$, if $a^{\prime}$ is an inverse of $a$ and $b^{\prime}$ is an inverse of $b$, then $b^{\prime} a^{\prime}$ is an inverse of $a b$;

(iii) if e is idempotent then every inverse of e is idempotent;

(iv) for all $a, b \in S$, if $V(a) \cap V(b) \neq \emptyset$, then $V(a)=V(b)$.

Built on Proposition 1.16 (iv), Yamada [52] considered the equivalence relation

$$
\gamma=\{(x, y) \in S \times S: V(x)=V(y)\}
$$

on an orthodox semigroup $S$ in which the band $B$ of idempotents is normal, in the sense, $x y z t=x z y t$ for all $x, y, z, t \in B$. A further study in the general orthodox case by Hall [23] showed that:

Lemma 1.17. If $S$ is an orthodox semigroup with band of idempotents $B$, then the relation $\gamma$ defined above is a congruence on $S$ such that $\gamma \cap(B \times B)=\mathcal{J}(B)$. Moreover, it is the least inverse semigroup congruence on $S$.

\subsubsection{Completely regular semigroups}

A semigroup $S$ is said to be completely regular if there exists a unary operation $a \mapsto a^{-1}$ such that

$$
\left(a^{-1}\right)^{-1}=a, \quad a a^{-1} a=a, \quad a a^{-1}=a^{-1} a .
$$

The following result presents two alternative descriptions of completely regular semigroups.

Lemma 1.18. [26] Let $S$ be a semigroup. Then the following statements are equivalent:

(i) $S$ is completely regular;

(ii) every element of $S$ lies in a subgroup of $S$;

(iii) every $\mathcal{H}$-class in $S$ is a group. 
In view of Lemma 1.18 (iii), a completely regular semigroup is often referred to as a union of groups.

A crucial observation about completely regular semigroups is that:

Lemma 1.19. [26] Let $S$ be a completely regular semigroup. Then $\mathcal{D}=\mathcal{J}$ and $\mathcal{J}$ is a semilattice congruence on $S$.

Let $S$ be a completely regular semigroup. We denote the semilattice $S / \mathcal{J}$ by $Y$, and for each $\alpha \in Y$ we denote $\alpha\left(\mathcal{J}^{\natural}\right)^{-1}$ by $S_{\alpha}$. Each $S_{\alpha}$ is a $\mathcal{J}$-class of $S$ and a completely simple semigroup, in the sense that, it has no proper ideals and contains a primitive idempotent (by which we mean an idempotent which is minimal within the set of idempotents, here, for any $e, f \in E(S), e \leq f$ if and only if $e f=f e=e$ ).

To sum up, we have:

Theorem 1.20. [26] Every completely regular semigroup is a semilattice of completely simple semigroups.

Here, we need to mention a special subclass of the class of completely regular semigroups, that is, the class of Clifford semigroups. A semigroup is a Clifford semigroup if it is a semilattice of groups. These are precisely inverse semigroups $S$ with central idempotents, that is, in which $e a=a e$ for all $a \in S$ and $e \in E(S)$.

Notice that any band $B$ is a completely regular semigroup so that $\mathcal{J}$ is a semilattice congruence on $B$. As each $\mathcal{J}$-class of $B$ is a rectangular band, we obtain that every band is semilattice of rectangular bands, that is, $B=\bigcup_{\alpha \in Y} B_{\alpha}$, where $Y$ is the index set of the $\mathcal{J}$-classes of $B$ and each $B_{\alpha}$ is a $\mathcal{J}$-class.

For convenience, we will sometimes denote the $B_{\alpha}$ containing $e$ by $E(e)$. Then $E(e)$ is a rectangular band and hence $E(e) \subseteq V(e)$. By Theorem 1.10, $V(e) \subseteq E(e)$, and so $V(e)=E(e)$.

In what follows the reader should bear in mind that for a band $B$, two elements are $\mathcal{D}$-related if and only if they are mutually inverse.

To close this section, we present some results from [26], which will be useful subsequently.

Lemma 1.21. [26] Let $e, f$ be elements of a band $B$ such that $e \mathcal{D} f$. Then the maps

$$
\theta_{f}: x \mapsto f x f, \quad \theta_{e}: y \mapsto e y e
$$


are mutually inverse isomorphisms from $\langle e\rangle$ onto $\langle f\rangle$ and from $\langle f\rangle$ onto $\langle e\rangle$, respectively.

It is worth remarking that for a band $B$ and element $e$ of $B,\langle e\rangle=\{x \in$ $B: x e=e x=e\}$. If $e \mathcal{D} f$, then for any $x \in\langle e\rangle$ and any $y \in\langle f\rangle$, we have that $x \mathcal{D} f x f$ and $y \mathcal{D}$ eye. To make the domain of the map $\theta_{f}$ clear, we shall use the symbol $\left.\theta_{f}\right|_{\langle e\rangle}$ instead of simply $\theta_{f}$. Notice that the inverse of $\left.\theta_{f}\right|_{\langle e\rangle}$ is $\left.\theta_{e}\right|_{\langle f\rangle}$.

Lemma 1.22. [26] If $x, e, f$ are elements of a band $B$ such that $e \mathcal{D} f$, then $L_{e x f}=L_{x f}$ and $R_{e x f}=R_{e x}$.

\subsection{Biordered sets}

Let $E$ be a partial algebra, that is, a set $E$ together with a partial binary operation - on $E$. Usually, we omit the symbol '.', say, if $e, f \in E$ and $e \cdot f$ exists in $E$, then we write $e f$ for $e \cdot f$. We will express the term ' $e \cdot f$ exists in $E$ ' as ' $\exists e f$ '. Set

$$
D_{E}=\{(e, f) \in E \times E: \exists e f\},
$$

that is, $D_{E}$ is the domain of the partial binary operation. On $E$ we define

$$
\begin{gathered}
\omega^{r}=\{(e, f): f e=e\}, \quad \omega^{l}=\{(e, f): e f=e\}, \\
\mathcal{R}=\omega^{r} \cap\left(\omega^{r}\right)^{-1}, \quad \mathcal{L}=\omega^{l} \cap\left(\omega^{l}\right)^{-1} \text { and } \omega=\omega^{r} \cap \omega^{l} .
\end{gathered}
$$

In addition, for any $e \in E$, we define

$$
\omega^{r}(e)=\left\{f \in E: f \omega^{r} e\right\}
$$

and similarly for $\omega^{l}(e)$ and $\omega(e)$.

Definition 1.23. Let $E$ be a partial algebra as above. Then $E$ is a biordered set if it satisfies axioms (B1), (B21), (B22), (B31), (B32), (B4) and their duals, where $e, f, g, h$ denote arbitrary elements of $E$.

(B1) $\omega^{r}$ and $\omega^{l}$ are pre-orders on $E$ and $D_{E}=\left(\omega^{r} \cup \omega^{l}\right) \cup\left(\omega^{r} \cup \omega^{l}\right)^{-1}$;

(B21) $f \in \omega^{r}(e) \Rightarrow f \mathcal{R}$ fewe;

(B22) $g \omega^{l} f$ and $f, g \in \omega^{r}(e) \Rightarrow g e \omega^{l} f e$; 
(B31)

$$
\begin{aligned}
& g \omega^{r} f \omega^{r} e \Rightarrow g f=(g e) f \\
& g \omega^{l} f \text { and } f, g \in \omega^{r}(e) \Rightarrow(f g) e=(f e)(g e) .
\end{aligned}
$$

Let $M(e, f)$ denote the set $\omega^{l}(e) \cap \omega^{r}(f)$ for all $e, f \in E$. We define a relation $\prec$ on $M(e, f)$ by the rule that for any $g, h \in M(e, f)$,

$$
g \prec h \text { if and only if } e g \omega^{r} e h, g f \omega^{l} h f \text {. }
$$

It is easy to see that $\prec$ is a pre-order on $M(e, f)$. Since for any $g \in M(e, f)$, we have that $g \prec g$ and so it is reflexive. To show that $\prec$ is transitive, suppose that $g, h, k \in M(e, f)$ with $g \prec h, h \prec k$. Then

$$
\begin{gathered}
e g \omega^{r} e h, \quad g f \omega^{l} h f, \\
e h \omega^{r} e k \text { and } h f \omega^{l} k f .
\end{gathered}
$$

As $\omega^{r}$ and $\omega^{l}$ are pre-orders on $E$, we obtain that

$$
e g \omega^{r} e k \text { and } g f \omega^{l} k f
$$

that is, $g \prec k$. Hence, $\prec$ is transitive, so that $\prec$ is a pre-order.

We shall denote the set $M(e, f)$, together with the pre-order $\prec$, by $\mathcal{M}(e, f)$.

Then the set $S(e, f)=\{h \in \mathcal{M}(e, f): g \prec h, \forall g \in \mathcal{M}(e, f)\}$ is called the sandwich set of $e$ and $f$.

In particular, for any $e \in E, S(e, e)=\{e\}$. Since if $h \in S(e, e)$, then $h \omega^{l} e$ and $h \omega^{r} e$ so that $h \omega e$. As $e \in \mathcal{M}(e, e)$, we have that $e \prec h$, that is, $e \omega^{r} e h=h$ and $e \omega^{l} h e=h$, and so $e \omega h$. Consequently, $e=h$.

$$
f, g \in \omega^{r}(e) \Rightarrow S(f, g) e=S(f e, g e)
$$

The biordered set $E$ is regular if it also satisfies

(R) $S(e, f) \neq \emptyset, \forall e, f \in E$.

We pause to make some comments on the above axioms. By Axiom (B1), $D_{E}$ is symmetric. As the axioms for a biordered set are self-dual, the dual of any true proposition is also true. If $f \omega^{r} e$, then by (B1), $(f, e) \in D_{E}$, and so $\exists f e$. In (B31), if $g \omega^{r} f \omega^{r} e$, then by (B1), $\exists g e$ and by (B21), ge $\mathcal{R} g$, and so $g e \omega^{r} f$, 
so that by (B1), $\exists(g e) f$. In (B32), if $g \omega^{l} f$ and $f \in \omega^{r}(e)$, then by the dual of (B21), $\exists f g$ and $f g \omega f$, so that $f g \omega^{r} e$, which implies that $\exists(f g) e$ by (B1). As $g \omega^{l} f$ and $f, g \in \omega^{r}(e)$, by (B22), ge $\omega^{l} f e$, and so $\exists(f e)(g e)$ by (B1). In (B4), if $h \in S(f, g)$, then $h \omega^{r} g$. As $g \omega^{r} e$ and $\omega^{r}$ is a pre-order, we obtain that $h \omega^{r} e$, and so $\exists h e$, so that $S(f, g) e$ is well-defined.

A biordered subset $E^{\prime}$ of a biordered set $E$ is a biordered set which is a partial subalgebra in the usual sense. It is easy to see that for any $e \in E, \omega(e)$ is a biordered subset of $E$. In addition, if $E$ is regular, then for any $e \in E, \omega(e)$ is regular. Since if $g, k \in \omega(e)$ and $h \in S(g, k)$, then $h \omega^{l} g$ and $h \omega^{r} k$, and so $h \in \omega(e)$ so that $S(g, k) \subseteq \omega(e)$.

Lemma 1.24. Let $S$ be a semigroup with non-empty set of idempotents $E(S)$. Then $E(S)$ is a biordered set with respect to the pair of pre-orders $\leq_{\mathcal{R}}$ and $\leq_{\mathcal{L}}$ on $S$.

Proof. For (B1), let $D_{E(S)}=\left(\leq_{\mathcal{R}} \cup \leq_{\mathcal{L}}\right) \cup\left(\leq_{\mathcal{R}} \cup \leq_{\mathcal{L}}\right)^{-1}$. Now we show that $E(S)$ forms a partial algebra with respect to $D_{E(S)}$. Suppose that $e, f \in E(S)$. If $e \leq_{\mathcal{R}} f$, then by Lemma 1.6(i), we have $f e=e \in E(S)$ and $(e f)^{2}=$ efef $=$ $e(f e) f=e f \in E(S)$. Dually, if $e \leq_{\mathcal{L}} f$, then ef $=e \in E(S)$ and $f e \in E(S)$. Hence, $E(S)$ forms a partial algebra.

From the associativity of multiplication in $S$ and the idempotent property of $E(S)$, axioms (B21), (B22),(B31) and (B32) hold.

Finally, we show that (B4) holds. Suppose that $e, f, g \in E(S)$ are such that $f, g \leq_{\mathcal{R}} e$. We first verify that $S(f, g) e \subseteq S(f e, g e)$. Assume that $h \in S(f, g)$. Then $h \leq_{\mathcal{L}} f$ and $h \leq_{\mathcal{R}} g \leq_{\mathcal{R}} e$, and so $h e \in E(S)$ by Lemma 1.6. Put $h^{\prime}=h e$. As

$$
h^{\prime} f e=(h e) f e=h(e f) e=h f e=h e=h^{\prime}
$$

and

$$
g e h^{\prime}=g e(h e)=g(e h) e=g h e=h e=h^{\prime},
$$


we have that $h^{\prime} \in \mathcal{M}(f e, g e)$. Further, if $k^{\prime} \in \mathcal{M}(f e, g e)$ and $k=k^{\prime} f$, then

$$
\begin{array}{rlr}
k^{2} & =k^{\prime} f k^{\prime} f & \\
& =k^{\prime} f\left(e k^{\prime}\right) f & \\
& =k^{\prime}(f e) k^{\prime} f & \\
& =k^{\prime} f & \left(k^{\prime} \leq_{\mathcal{R}} g e \leq e\right) \\
& =k &
\end{array}
$$

and $g k=g k^{\prime} f=g(g e) k^{\prime} f=(g e) k^{\prime} f=k^{\prime} f=k$. Hence $k \in \mathcal{M}(f, g)$, so that $k \prec h$. Also,

$$
\begin{aligned}
h^{\prime} f & =h e f & & \\
& =h f & & \left(f \leq_{\mathcal{R}} e\right) \\
& =h & & \left(h \leq_{\mathcal{L}} f\right)
\end{aligned}
$$

and $k e=k^{\prime} f e=k^{\prime}$ since $k^{\prime} \leq_{\mathcal{L}} f e$. Therefore,

$$
\begin{aligned}
\left((f e) h^{\prime}\right)\left((f e) k^{\prime}\right) & =f e\left(h^{\prime} f\right) e k^{\prime} & & \\
& =f e h e k^{\prime} & & \left(h^{\prime} f=h\right) \\
& =f(e h) e k^{\prime} & & \\
& =f h e k^{\prime} & & \left(h \leq_{\mathcal{R}} g \leq_{\mathcal{R}} e\right) \\
& =f h e k e & & \left(k^{\prime}=k e\right) \\
& =f h e(g k) e & & (g k=k) \\
& =f h(e g) k e & & \\
& =f h g k e & & \left(g \leq_{\mathcal{R}} e\right) \\
& =f h k e & & (k=g k) \\
& =f(h f) k e & & \left(h \leq_{\mathcal{L}} f\right) \\
& =(f h)(f k) e & & (k \prec h) \\
& =f k e & & \left(k^{\prime} \leq_{\mathcal{R}} g e \leq e\right), \\
& =f k^{\prime}=(f e) k^{\prime} & & \text { f } \left.\leq^{\prime}\right)
\end{aligned}
$$

and so $(f e) k^{\prime} \leq_{\mathcal{R}}(f e) h^{\prime}$. Also, we have $k^{\prime}(g e) \leq_{\mathcal{L}} h^{\prime}(g e)$ as 


$$
\begin{aligned}
k^{\prime}(g e) h^{\prime}(g e) & =k^{\prime} g\left(e h^{\prime}\right) g e & & \\
& =k^{\prime} g h^{\prime} g e & & \left(h^{\prime}=h e \mathcal{R} h \leq_{\mathcal{R}} e\right) \\
& =k^{\prime} \text { ghege } & & \left(h^{\prime}=h e\right) \\
& =k^{\prime} \text { ghge } & & \left(g \leq_{\mathcal{R}} e\right) \\
& =\text { keghge } & & \left(k^{\prime}=k e\right) \\
& =k g h g e & & \left(g \leq_{\mathcal{R}} e\right) \\
& =k g e & & (k \prec h) \\
& =k e g e & & \left(g \leq_{\mathcal{R}} e\right) \\
& =k^{\prime}(g e) & & \left(k^{\prime}=k e\right) .
\end{aligned}
$$

So $k^{\prime} \prec h^{\prime}$ in $\mathcal{M}(f e, g e)$. Hence, $h^{\prime} \in S(f e, g e)$, and so $S(f, g) e \subseteq S(f e, g e)$.

To see the converse, suppose that $u^{\prime} \in S(f e, g e)$. Then $u^{\prime} \leq_{\mathcal{R}} g e \leq e$ and $u^{\prime} \leq_{\mathcal{L}} f e \leq e$, and so $u^{\prime} \leq e$. Put $u=u^{\prime} f$. Notice that

$$
\begin{aligned}
u^{2} & =u^{\prime} f u^{\prime} f & \\
& =u^{\prime} \text { feu } f & \left(u^{\prime} \leq e\right) \\
& =u^{\prime} f & \left(u^{\prime} \leq_{\mathcal{L}} f e, u^{\prime 2}=u^{\prime}\right) \\
& =u . &
\end{aligned}
$$

Also,

$$
\begin{aligned}
g u & =g u^{\prime} f & & \\
& =g e u^{\prime} f & & \left(u^{\prime} \leq e\right) \\
& =u^{\prime} f & & \left(u^{\prime} \leq_{\mathcal{R}} g e\right) \\
& =u . & &
\end{aligned}
$$

Together with $u \leq_{\mathcal{L}} f$, we obtain that $u \in \mathcal{M}(f, g)$. If $v^{\prime} \in \mathcal{M}(f, g)$ and $v=v^{\prime} e$, then

$$
\begin{aligned}
v^{2} & =v^{\prime} e v^{\prime} e \\
& =v^{\prime} e \quad\left(v^{\prime} \leq_{\mathcal{R}} g \leq_{\mathcal{R}} e\right) \\
& =v .
\end{aligned}
$$


In addition,

$$
\begin{aligned}
v f e & =v^{\prime} \text { efe } & & \\
& =v^{\prime} f e & & \left(f \leq_{\mathcal{R}} e\right) \\
& =v^{\prime} e & & \left(v^{\prime} \leq_{\mathcal{L}} f\right) \\
& =v & &
\end{aligned}
$$

and

$$
\begin{aligned}
\text { gev } & =\text { gev }^{\prime} e \\
& =v^{\prime} e \quad\left(v^{\prime} \leq_{\mathcal{R}} g \leq_{\mathcal{R}} e\right) \\
& =v .
\end{aligned}
$$

Thus, $v \in \mathcal{M}(f e, g e)$, and so $v \prec u^{\prime}$. Since $v f=v^{\prime} e f=v^{\prime}$, we have that

$$
\begin{aligned}
(f u)\left(f v^{\prime}\right) & =\left(f u^{\prime} f\right) f v^{\prime} & & \\
& =f u^{\prime} f v^{\prime} & & \\
& =f u^{\prime} f v f & & \\
& =\left(f e u^{\prime}\right)(f e v) f & & \left(u^{\prime}, v \leq_{\mathcal{R}} g e \leq e\right) \\
& =(f e v) f & & \left(v^{\prime} \prec u\right) \\
& =f v^{\prime} f & & \left(v \leq_{\mathcal{R}} g \leq e\right) \\
& =f v^{\prime}, & &
\end{aligned}
$$

so that $f v^{\prime} \leq_{\mathcal{R}} f u$. In addition, we have $v^{\prime} g \leq_{\mathcal{L}} u g$ as

$$
\begin{aligned}
\left(v^{\prime} g\right)(u g) & =v^{\prime}(g u) g & & \\
& =v^{\prime} u g & & (g u=u) \\
& =v f u g & & \left(v^{\prime}=v f\right) \\
& =v f e u g & & \left(u \leq_{\mathcal{R}} g \leq_{\mathcal{R}} e\right) \\
& =\text { vug } & & (v f e=v) \\
& =\text { vgeug } & & \left(u \leq_{\mathcal{R}} g \leq_{\mathcal{R}} e\right) \\
& =\text { vgeu'fg } & & \left(u=u^{\prime} f\right) \\
& =\text { vgeu feg } & & \left(g \leq_{\mathcal{R}} e\right) \\
& =\text { vgeu'g } & & \left(u^{\prime} \omega^{l} f e\right)
\end{aligned}
$$




$$
\begin{aligned}
& =\text { vgeu'geg } \quad\left(g \leq_{\mathcal{R}} e\right) \\
& =\text { vgeg } \quad\left(v \prec u^{\prime} \text { in } \mathcal{M}(f e, g e)\right) \\
& =v g \quad\left(g \leq_{\mathcal{R}} e\right) \\
& =v f e g \quad(v=v f e) \\
& =v^{\prime} g \quad\left(v^{\prime}=v f \text { and } g \leq_{\mathcal{R}} e\right) .
\end{aligned}
$$

Hence $v^{\prime} \prec u$. Thus, $u \in S(f, g)$. Observe that $u e=u^{\prime} f e=u^{\prime}$ as $u^{\prime} \leq_{\mathcal{L}} f e$, so that $S(f e, g e) \subseteq S(f, g) e$.

To the converse of Lemma 1.24, it is shown in [3] that every biordered set $E$ is the set of idempotents of some semigroup. In particular, the set of idempotents of any regular semigroup is a regular biordered set [38]; conversely, every regular biordered set is the set of idempotents of some regular semigroup. For further details the reader is referred to [38].

Definition 1.25. Let $E$ and $E^{\prime}$ be biordered sets, and $\theta: E \rightarrow E^{\prime}$ a mapping. Then $\theta$ is a morphism if it satisfies

(M) $(e, f) \in D_{E} \Rightarrow(e \theta, f \theta) \in D_{E^{\prime}}$ and $(e f) \theta=(e \theta)(f \theta)$.

$\theta$ is a regular morphism if it also satisfies

(RM1) $S(e, f) \theta \subseteq S^{\prime}(e \theta, f \theta)$

(RM2) $S(e, f) \neq \emptyset \Leftrightarrow S^{\prime}(e \theta, f \theta) \neq \emptyset, \forall e, f \in E$,

where $S^{\prime}(e \theta, f \theta)$ denotes the sandwich set of $e \theta$ and $f \theta$ in $E^{\prime}$.

It is easy to see that if $e \omega^{r} f$ then $e \theta \omega^{r} f \theta$ and dually for $\omega^{l}$.

We remark that if $E$ is a regular biordered set, then a morphism $\theta: E \rightarrow E^{\prime}$ is regular provided only that it satisfies (RM1), since in this case (RM2) follows automatically.

Here, we state that if $\theta_{1}: E_{1} \rightarrow E_{2}$ and $\theta_{2}: E_{2} \rightarrow E_{3}$ are two morphisms, then it is clear that $\theta_{1} \theta_{2}: E_{1} \rightarrow E_{3}$ is also a morphism and if $\theta_{1}$ and $\theta_{2}$ are regular, so is $\theta_{1} \theta_{2}$.

A (regular) morphism is said to be a (regular) isomorphism if it is bijective and so is the inverse. 
Lemma 1.26. (c.f. Corollary 2.15 [38]) A bijective morphism is an isomorphism if and only if it is regular.

In the following, we list some necessary properties of (regular) biordered sets which will be used in Chapter 10 .

Lemma 1.27. (c.f. Proposition 2.3 [38]) Let $E$ be a biordered set and $e, f \in E$ be such that $f \omega^{r}$ e, then for any $g \omega^{r} f$,

$$
(g f) e=g(f e)=(g e)(f e)
$$

Lemma 1.28. (c.f. Proposition 2.5 [38]) Let E be a biordered set and e, $e^{\prime}, f, f^{\prime} \in$ $E$ with $e \mathcal{L} e^{\prime}$ and $f \mathcal{R} f^{\prime}$. Then $\mathcal{M}(e, f)=\mathcal{M}\left(e^{\prime}, f^{\prime}\right)$. In particular, $S(e, f)=$ $S\left(e^{\prime}, f^{\prime}\right)$.

Lemma 1.29. (c.f. Proposition 2.2 [38]) Let $E$ be a biordered set and $(e, f) \in$ $D_{E}$, then ef $\in S(f, e)$.

Lemma 1.30. (c.f. Proposition 2.12 [38]) Let $E$ be a biordered set and let $e, f, g, h \in E$ be such that $g \in S(e, f)$ and $h \omega^{r} f$. Then $S(g, h) \subseteq S(e, h)$; further, $S(g, h) \neq \emptyset$ if and only if $S(e, h) \neq \emptyset$. Dually, if $g \in S(f, e)$ and $h \omega^{l} f$, then $S(h, g) \subseteq S(h, e)$, further, $S(h, g) \neq \emptyset$ if and only if $S(h, e) \neq \emptyset$.

Lemma 1.31. (c.f. Proposition 2.13 [38]) Let $E$ be a biordered set and let e, $g \in$ $E$ with $\alpha: \omega(f) \rightarrow \omega\left(f^{\prime}\right)$ being an isomorphism. If $h_{1} \in S(e, f), h_{2} \in S\left(f^{\prime}, g\right)$, $h_{1}^{\prime}=\left(h_{1} f\right) \alpha$ and $h_{2}^{\prime}=\left(f^{\prime} h_{2}\right) \alpha^{-1}$, then $\left(S\left(h_{1}, h_{2}^{\prime}\right) f\right) \alpha=f^{\prime} S\left(h_{1}^{\prime}, h_{2}\right)$.

Lemma 1.32. (c.f. Corollary 2.8 [38]) Let $E$ be a biordered set and $e, f \in E$ be such that either $e \mathcal{R} f$ or $e \mathcal{L} f$. Then the map $\tau(e, f): \omega(e) \rightarrow \omega(f)$, defined by the rule that for all $g \in \omega(e)$,

$$
g \tau(e, f)= \begin{cases}g f & \text { if } e \mathcal{R} f \\ f g & \text { if } e \mathcal{L} f\end{cases}
$$

is an isomorphism such that if either e $\mathcal{R} f \mathcal{R} g$ or e $\mathcal{L} f \mathcal{L} g$, then

$$
\tau(e, f) \tau(f, g)=\tau(e, g)
$$


and

$$
\tau(e, f)=(\tau(f, e))^{-1}
$$

Further, if $g, g^{\prime} \in \omega^{r}(e)$ and $g \mathcal{L} g^{\prime}$, then

$$
\tau\left(g, g^{\prime}\right) \tau\left(g^{\prime}, g e\right)=\tau(g, g e) \tau\left(g e, g^{\prime} e\right)
$$

At the end of this section, we should recall a notion which is related to biordered sets. In [38], an E-square is a $2 \times 2$ matrix $\left(\begin{array}{ll}e & f \\ g & h\end{array}\right)$, where $e, f, g, h \in$ $E$, a biordered set, with $e \mathcal{R} f, g \mathcal{R} h, e \mathcal{L} g$ and $f \mathcal{L} h$. An $E$-square is said to be singular if it has one of the following two forms:

$(i)\left(\begin{array}{cc}g & h \\ e g & e h\end{array}\right)$ where $g, h \in \omega^{l}(e)$ and $g \mathcal{R} h$-row-singular;

(ii) $\left(\begin{array}{ll}g & g e \\ h & h e\end{array}\right)$ where $g, h \in \omega^{r}(e)$ and $g \mathcal{L} h$ - column-singular.

An E-square $\left(\begin{array}{ll}e & f \\ g & h\end{array}\right)$ is $\tau$-commutative if the following diagram commutes:

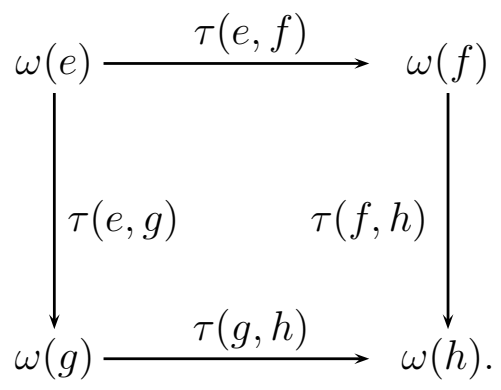

Figure 1.1: The $\tau$-commutative condition

We note that every singular $E$-square is $\tau$-commutative.

Lemma 1.33. (c.f. Proposition 2.9 [38]) Let $g, h \in \omega^{r}(e)$ and ge $\mathcal{L}$ he. Then there exists a unique E-square $G=\left(\begin{array}{ll}g & u \\ v & h\end{array}\right)$ satisfying the following conditions:

(i) $g e=u e$ and $v e=h e$;

(ii) $G$ is $\tau$-commutative, and so $h(k u)=(v k) h$ for all $k \in \omega(g)$.

Dually, we have: 
Lemma 1.34. Let $g, h \in \omega^{l}(e)$ and eg $\mathcal{R}$ eh. Then there exists a unique E-square $G=\left(\begin{array}{ll}g & u \\ v & h\end{array}\right)$ satisfying the following conditions:

(i) $e g=e v$ and $e u=e h$;

(ii) $G$ is $\tau$-commutative, and so $h(k u)=(v k) h$ for all $k \in \omega(g)$.

Lemma 1.35. Let $g, h \in S(e, f)$. Then there exists a unique E-square $G=$ $\left(\begin{array}{ll}g & u \\ v & h\end{array}\right)$, where $u, v \in S(e, f)$.

Proof. Suppose that $g, h \in S(e, f)$. Then $g, h \in \omega^{l}(e) \cap \omega^{r}(f)$, eg $\mathcal{R} e h$ and $g f \mathcal{L} h f$. Since $g, h \in \omega^{r}(f)$ and $g f \mathcal{L} h f$, it follows from Lemma 1.33 that there exists a unique $E$-square $G=\left(\begin{array}{ll}g & u \\ v & h\end{array}\right)$ such that $g f=u f, v f=h f$ and $G$ is $\tau$-commutative.

As $g, h \in \omega^{l}(e)$ and $e g \mathcal{R} e h$, therefore by Lemma 1.34, there exists a unique $E$-square $K=\left(\begin{array}{cc}g & i \\ j & h\end{array}\right)$ such that $e g=e j$, ei $=e h$ and $K$ is $\tau$-commutative. Now, we claim that $G=K$. Since $u \mathcal{L} h$, we have that eu $\mathcal{L} e h$. As eu $\mathcal{R} e g \mathcal{R}$ eh, we obtain that $e u=e h$, and so $e v \mathcal{R} e h=e u \mathcal{R} e g$. Since $g \mathcal{L} v$, we have that $e g=e v$. Hence, $G$ satisfies the conditions in Lemma 1.34, so that $G=K$.

Finally, we show that $u, v \in S(e, f)$. As $G=\left(\begin{array}{ll}g & u \\ v & h\end{array}\right)$ is an E-square and $g, h \in \omega^{l}(e) \cap \omega^{r}(f)$, we obtain that $u, v \in \omega^{l}(e) \cap \omega^{r}(f)$. Notice that $e u=e h$, uf $\mathcal{L} h f$, then $h \prec u$. As $h \in S(e, f)$, we have that for any $t \in \mathcal{M}(e, f), t \prec h$. Since $\prec$ is a pre-order, we succeed in obtaining that $t \prec u$. Thus, $u \in S(e, f)$. Similarly, we have that $v \in S(e, f)$.

We make a short comment on Lemma 1.35 that if $S(e, f) \neq \emptyset$ for all $e, f \in E$, then for any $g, h \in S(e, f)$, there exists $k \in S(e, f)$ such that $g \mathcal{R} k \mathcal{L} h$.

\subsection{Categories}

Category theory was first introduced by Eilenberg and Maclane in 1945 [5]. The key idea of category theory is to provide a fundamental and abstract way to describe mathematical entities and their relationships via 'objects' that are linked by 'arrows' (or 'morphisms'). Here, we recall some basic definitions and properties of a category [28] and [48]. 
Definition 1.36. A category $P$ consists of

(C1) a class $\mathrm{Ob}(P)$ of objects;

(C2) a class $\operatorname{Mor}(P)$ of morphisms (or arrows) between the objects. Each morphism $f$ has a unique domain $\mathbf{d}(f) \in \mathrm{Ob}(P)$ and codomain $\mathbf{r}(f) \in \mathrm{Ob}(P)$. Denote the Mor-class of all morphisms from $A \in \mathrm{Ob}(P)$ to $B \in \mathrm{Ob}(P)$ by $\operatorname{Mor}(A, B)$;

(C3) if $A, B, C, D \in \mathrm{Ob}(P)$, then there is a binary operation

$$
\operatorname{Mor}(A, B) \times \operatorname{Mor}(B, C) \rightarrow \operatorname{Mor}(A, C),(f, g) \mapsto f \circ g
$$

called composition of morphisms such that if $f \in \operatorname{Mor}(A, B), g \in \operatorname{Mor}(B, C)$ and $h \in \operatorname{Mor}(C, D)$, then $(f \circ g) \circ h=f \circ(g \circ h)$;

(C4) for each $A \in \mathrm{Ob}(P)$, there exists a morphism $1_{A} \in \operatorname{Mor}(A, A)$ such that if $B \in \mathrm{Ob}(P)$, and $f \in \operatorname{Mor}(A, B)$, then $1_{A} \circ f=f$ and $f \circ 1_{B}=f$.

A simple but very accessible example of a category is the category $\mathcal{S}$ et of sets, whose objects are sets and whose morphisms are functions from one set to another. Here, we should mention that the objects of a category need not be sets nor the morphisms functions. Particularly, a category is said to be 'concrete' if all objects are (structured) sets, morphisms from $A$ to $B$ are (structure preserving) mappings from $A$ to $B$, composition of morphisms is composition of mappings, and the identities are the identity mappings. In addition, a category $P$ is called small if both $\operatorname{Ob}(P)$ and $\operatorname{Mor}(P)$ are actually sets (small classes) and large otherwise. The category $\mathcal{S}$ et is a large category. In this thesis, the large categories will all be concrete, say, the category Semigp, consisting of all semigroups and semigroup morphisms, is a large category since the collection of all semigroups is not a set. Also, Semigp is a concrete category.

We now introduce the notion of a functor that is a special mapping between categories.

Definition 1.37. Let $P_{1}$ and $P_{2}$ be categories. A functor $F$ from $P_{1}$ to $P_{2}$ is a pair of maps, both denoted $F$, from $\mathrm{Ob}\left(P_{1}\right)$ to $\operatorname{Ob}\left(P_{2}\right)$ and from $\operatorname{Mor}\left(P_{1}\right)$ to $\operatorname{Mor}\left(P_{2}\right)$, such that the following conditions hold:

(F1) for any $f \in P_{1}, \mathbf{d}(f) F=\mathbf{d}(f F)$ and $\mathbf{r}(f) F=\mathbf{r}(f F)$;

(F2) if $\exists g \circ f$ in $P_{1}$, then $(g \circ f) F=g F \circ f F$;

(F3) for $A \in \mathrm{Ob}\left(P_{1}\right), 1_{A} F=1_{A F}$. 
We note that in (F2), if $\exists g \circ f$ in $P_{1}$, then $\mathbf{r}(g)=\mathbf{d}(f)$. By (F1), we have that $\mathbf{r}(g F)=\mathbf{r}(g) F=\mathbf{d}(f) F=\mathbf{d}(f F)$, and so $\exists g F \circ f F$ in $P_{2}$.

For any category $P$, we will use $I_{P}$ to denote the identity functor, which assigns each object and morphism to itself.

We view small categories as a generalisation of monoids. Clearly, a small category with one object is a monoid. In a small category $C$, we often identity $e \in \mathrm{Ob}(C)$ with $1_{e}$.

Let $G$ be a group with identity $1_{G}$ and $I$ be a non-empty set. We construct a small category $B(G, I)$ as follows:

$$
\mathrm{Ob}(B(G, I))=I
$$

and for any $e, f \in I$,

$$
\operatorname{Mor}(e, f)=\{(e, g, f): g \in G\}
$$

We define a partial binary operation on $B(G, I)$ by

$$
(e, g, f)(f, h, v)=(e, g h, v)
$$

Clearly, the operation is well-defined and associative when it is defined.

For any $e \in \operatorname{Ob}(B(G, I))$, there exists a unique identity $\left(e, 1_{G}, e\right)$ associated to $e$.

To sum up, $B(G, I)$ forms a category.

A semigroup $S$ with zero is categorical at zero or is $C$-semigroup if for all $a, b, c \in S$, if $a b \neq 0$ and $b c \neq 0$ then $a b c \neq 0$.

To construct a C-semigroup from $B(G, I)$, we adjoin a zero element 0 to $B(G, I)$. Then we define

$$
0 x=x 0=0
$$

and for any $x, y \in B(G, I)$, if $x \cdot y$ does not exist in $B(G, I)$, then $x \cdot y=0$, otherwise, $x \cdot y=x y$, where $x y$ is the product in $B(G, I)$. It is routine to verify that $B(G, I) \cup\{0\}$ becomes a C-semigroup, denoted by $B^{0}(G, I)$.

A cancellative category is a small category in which we have both right and left cancellation for morphisms. Note that any subcategory of a cancellative category is cancellative. 
A groupoid $G$ is a small category whose morphisms are all invertible, that is, for any $e, f \in \operatorname{Ob}(G)$ and $x \in \operatorname{Mor}(e, f)$, there exists $x^{-1} \in \operatorname{Mor}(f, e)$ such that $x \cdot x^{-1}=1_{e}$ and $x \cdot x^{-1}=1_{f}$. Any groupoid $G$ is cancellative. Since for any $x, y, z \in G$, if $\exists x \cdot y, \exists x \cdot z$ and $x \cdot y=x \cdot z$, then $\mathbf{r}(x)=\mathbf{d}(y)=\mathbf{d}(z)$ and $x^{-1} \cdot x \cdot y=x^{-1} \cdot x \cdot z$, that is, $1_{\mathbf{r}(x)} \cdot y=1_{\mathbf{r}(x)} \cdot z$, and so, $y=z$. Thus, $G$ is left cancellative. Dually, $G$ is right cancellative. One of the simplest examples of a groupoid is a group or $B(G, I)$.

For the reader's convenience, we will simplify the notation from $\operatorname{Mor}(P)$ to $P$. In addition, for the term ' $x \cdot y$ exists' we may use the expression ' $\exists x \cdot y$ ' or ' $x \cdot y$ is defined'.

It is a good position to mention the notion of ordered category. There exist two ways to define an ordered category. We list both in the following. In order to avoid ambiguity, we use subscripts.

Definition 1.38. A category $P$ with a partial order $\leq$ is ordered $_{1}$ if it satisfies the following conditions:

(OC1) if $x, y \in P$ with $x \leq y$, then $\mathbf{r}(x) \leq \mathbf{r}(y)$ and $\mathbf{d}(x) \leq \mathbf{d}(y)$;

(OC2) if $\mathbf{r}(x)=\mathbf{r}(y), \mathbf{d}(x)=\mathbf{d}(y)$ and $x \leq y$, then $x=y$;

(OC3) if $x^{\prime} \leq x, y^{\prime} \leq y$ and both $x^{\prime} \cdot y^{\prime}$ and $x \cdot y$ exist, then $x^{\prime} \cdot y^{\prime} \leq x \cdot y$.

An alternative description of ordered category is that:

Definition 1.39. A category $P$ with a partial order $\leq$ is ordered $_{2}$ if it satisfies Conditions (OC1), (OC3) and (OC4):

(OC4) ( $i$ ) for any $x \in P$ and $e \in \mathrm{P}$ with $e \leq \mathbf{d}(x)$, there exists a unique element ${ }_{e} \mid x$ such that ${ }_{e} \mid x \leq x$ and $\mathbf{d}\left({ }_{e} \mid x\right)=e$;

(ii) for any $x \in P$ and $f \in \mathrm{P}$ with $f \leq \mathbf{r}(x)$, there exists a unique element $\left.x\right|_{f}$ such that $\left.x\right|_{f} \leq x$ and $\mathbf{r}\left(\left.x\right|_{f}\right)=f$.

We pause to make a short comment on ordered ${ }_{1}$ and ordered $_{2}$ categories. It is easy to see that (OC4) implies (OC2). Since if $P$ is an $\operatorname{ordered}_{2}$ category and $x, y \in P$ with $\mathbf{r}(x)=\mathbf{r}(y), \mathbf{d}(x)=\mathbf{d}(y)$ and $x \leq y$, then by (OC4), $x=\mathbf{d}_{\mathbf{d}(x)} \mid y=$ $\mathbf{d}(y) \mid y=y$. So an ordered ${ }_{2}$ category is an ordered ${ }_{1}$ category. In (OC4), the unique element ${ }_{e} \mid x$ is called the restriction of $x$ to $e$ and dually, the unique element $\left.x\right|_{f}$ is called the co-restriction of $x$ to $f$.

A parallel definition of an ordered 2 groupoid is that: 


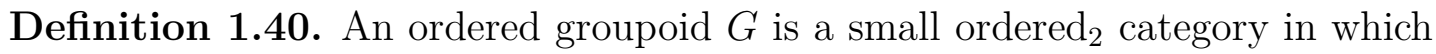
Condition $(\mathrm{G})$ holds:

(G) if $x, y \in G$ and $x \leq y$, then $x^{-1} \leq y^{-1}$.

If in addition,

(IG) $E$ is a semilattice.

Then $G=(G, \cdot, \leq)$ is called an inductive ${ }_{1}$ groupoid.

The subscript is used to distinguish this meaning of the word 'inductive' from both Ehresmann's use and a generalised definition which will occur in Chapter 6.

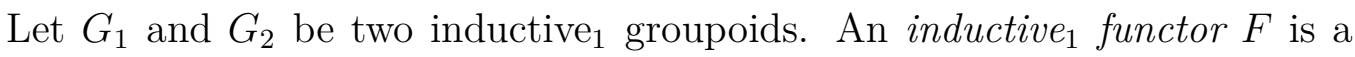
functor $F: G_{1} \rightarrow G_{2}$ preserving the order, restrictions and co-restrictions.

Next, we present a useful property of ordered $_{2}$ categories, which follows from the uniqueness of restrictions and co-restrictions.

Proposition 1.41. [1] Let $P$ be an ordered 2 category. Then for $x \in P$, $e \leq$ $\mathbf{d}(x)$ and $f \leq \mathbf{r}(x)$, we have that $f=\mathbf{r}\left({ }_{e} \mid x\right)$ if and only if $e=\mathbf{d}\left(\left.x\right|_{f}\right)$; moreover, ${ }_{e}|x=x|_{f}$.

Further:

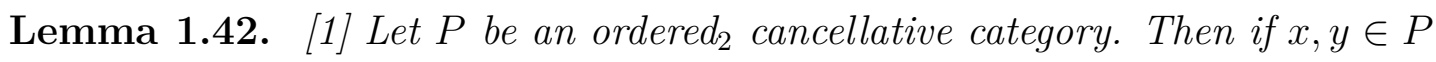
are such that $\mathbf{r}(x)=\mathbf{d}(y)$ and $e \leq \mathbf{d}(x)$, then $\left.{ }_{e}\left|(x \cdot y)=\left({ }_{e} \mid x\right) \cdot\left({ }_{\mathbf{r}(e} \mid x\right)\right| y\right)$.

Now, we introduce the notion of two categories being isomorphic.

Let $C$ and $D$ be two categories. We say that $C$ is isomorphic to $D$ if there exist functors: $F: C \rightarrow D$ and $G: D \rightarrow C$ such that $G F=I_{D}$ and $F G=I_{C}$, where $I_{D}$ and $I_{C}$ are the identity functors associated to $D$ and $C$, respectively.

Observe that two isomorphic categories are identical and differ only in the notation of their objects and morphisms. Sometimes this property is too strong, and so we need to introduce a weaker notion of equivalence.

Definition 1.43. Let $C$ and $D$ be categories and let $F$ and $G$ be functors from $C$ to $D$. Then a natural transformation $\eta$ from $F$ to $G$ consists of morphisms $\eta_{X}: X F \rightarrow X G$ for all $X \in C$, such that for every morphism $f: X \rightarrow Y$ in $C$ we have $f F \circ \eta_{Y}=\eta_{X} \circ f G$.

For ease of understand $f F \circ \eta_{Y}=\eta_{X} \circ f G$, we use the following commutative diagram: If, for all $X \in \mathrm{Ob}(C)$, the morphism $\eta_{X}$ is an isomorphism in $D$, then 


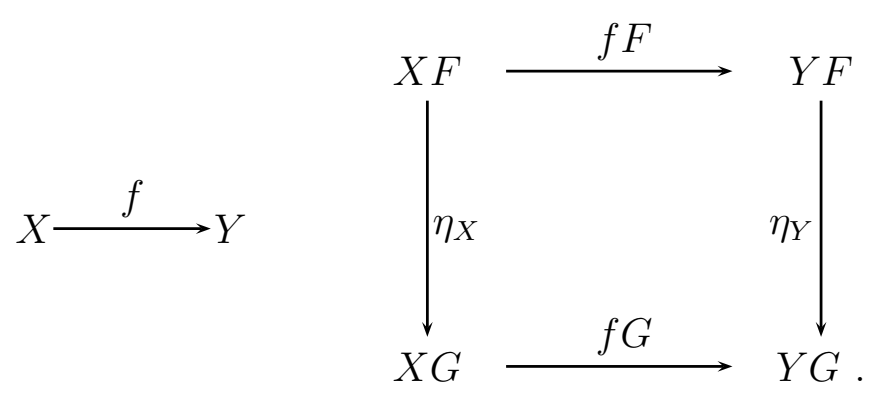

Figure 1.2: The natural transformation property

$\eta$ is said to be a natural isomorphism.

Let $C$ and $D$ be two categories. We say that $C$ and $D$ are equivalent if there exists a functor $F: C \rightarrow D$, a functor $G: D \rightarrow C$, and two natural isomorphisms $\varepsilon: G F \rightarrow I_{D}$ and $\eta: F G \rightarrow I_{C}$.

Clearly, for an equivalence of categories, it is not necessary to require $X G F=$ $X$, but only $X G F$ is isomorphic to $X$ in the category $D$, and the same for $Y F G$ and $Y$ in the category $C$.

To close this section, we cite from [53] an example of categories below, which are equivalent but not isomorphic.

Let $C$ be a category consisting of a single object $a$ and a single morphism $1_{a}$. Let $D$ be a category with $\operatorname{Ob}(D)=\{b, c\}$ and $\operatorname{Mor}(D)=\left\{1_{b}, 1_{c}, \alpha, \beta\right\}$, where $\alpha: b \rightarrow c$ and $\beta: c \rightarrow b$ are isomorphisms. Now, we define a map

$$
F: C \rightarrow D \text { by } a \mapsto b \text { and } 1_{a} \mapsto 1_{b}
$$

In addition, we define a map $G: D \rightarrow C$ by $X G=a$ and $f G=1_{a}$ for all $X \in \mathrm{Ob}(D)$ and $f \in \operatorname{Mor}(D)$. It is routine to verify that $F$ and $G$ are functors. Furthermore, $C$ and $D$ are equivalent but not isomorphic. 


\section{Chapter 2}

\section{Basic Theory II}

This chapter briefly recalls basic definitions and properties of abundant semigroups and weakly $U$-abundant semigroups that generalise the definitions and properties of regular semigroups we have introduced in Chapter 1.

\subsection{Abundant semigroups}

\subsubsection{Relations $\mathcal{L}^{*}, \mathcal{R}^{*}$}

Let $S$ be a semigroup. We define relations $\leq_{\mathcal{L}^{*}}$ and $\leq_{\mathcal{R}^{*}}$ on $S$ by the rule that for any $a, b \in S$,

$$
a \leq_{\mathcal{L}^{*}} b \text { if and only if } a \leq_{\mathcal{L}} b \text { in some over semigroup of } S
$$

and

$$
a \leq_{\mathcal{R}^{*}} b \text { if and only if } a \leq_{\mathcal{R}} b \text { in some over semigroup of } S \text {, }
$$

where $\leq_{\mathcal{L}}$ and $\leq_{\mathcal{R}}$ are defined in Section 1.3.2. As $\leq_{\mathcal{L}}$ and $\leq_{\mathcal{R}}$ are pre-orders, we have $\leq_{\mathcal{L}^{*}}$ and $\leq_{\mathcal{R}^{*}}$ are pre-orders, respectively. Since $\leq_{\mathcal{L}}$ is right compatible and $\leq_{\mathcal{R}}$ is left compatible, we have $\leq_{\mathcal{L}^{*}}$ is right compatible and $\leq_{\mathcal{R}^{*}}$ is left compatible.

Now, we denote the associated equivalences by $\mathcal{L}^{*}$ and $\mathcal{R}^{*}$, respectively. So, for any $a, b \in S, a \mathcal{L}^{*} b$ if and only if $a \mathcal{L} b$ in some oversemigroup of $S$. The relation $\mathcal{R}^{*}$ is defined dually. As usual, the intersection of the equivalence relations $\mathcal{L}^{*}$ and $\mathcal{R}^{*}$ is denoted by $\mathcal{H}^{*}$ and their join by $\mathcal{D}^{*}$.

For ease of description, the $\mathcal{L}^{*}$-class containing the element $a$ of a semigroup 
$S$ will be denoted by $L_{a}^{*}$. Then corresponding notation will be used for the classes of the other relations.

The following basic lemma is from [10].

Lemma 2.1. [10] Let $S$ be a semigroup and let $a, b \in S$. Then the following statements are equivalent:

(i) $(a, b) \in \mathcal{L}^{*}$;

(ii) for all $x, y \in S^{1}$, ax $=$ ay if and only if $b x=b y$.

Clearly, an idempotent $e$ of $S$ acts as a right identity within its $\mathcal{L}^{*}$-class. In that case, we have:

Lemma 2.2. If $e$ is an idempotent of a semigroup $S$, then the following statements are equivalent for $a \in S$ :

(i) $(e, a) \in \mathcal{L}^{*}$;

(ii) $a e=a$ and for all $x, y \in S^{1}$, ax $=$ ay implies $e x=e y$.

In view of its definition, $\mathcal{L}^{*}$ is a right congruence, and dually, $\mathcal{R}^{*}$ is a left congruence.

We pause to mention that $\mathcal{L} \subseteq \mathcal{L}^{*}$ on any semigroup $S$. For any regular elements $a, b \in S,(a, b) \in \mathcal{L}^{*}$ if and only if $(a, b) \in \mathcal{L}$. In particular, if $S$ is a regular semigroup, then $\mathcal{L}^{*}=\mathcal{L}$.

Another way to define relations $\mathcal{L}^{*}$ and $\mathcal{R}^{*}$ is using certain ideals. We now define a left (resp. right) ideal $I$ of a semigroup $S$ to be a left (resp. right) ${ }^{*}$-ideal of $S$ if $L_{a}^{*} \subseteq I$ (resp. $R_{a}^{*} \subseteq I$ ) for all $a \in I$. A subset $I$ of $S$ is a ${ }^{*}-i d e a l$ of $S$ if it is

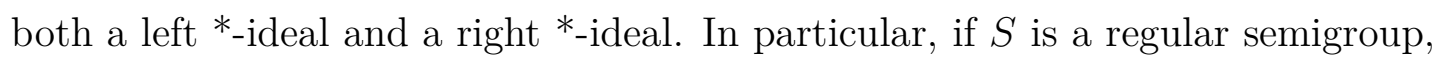
then every left (resp. right, two-sided) ideal of $S$ is a left (resp. right, two-sided) *-ideal. Observe that for any element $a$ of a semigroup $S, S$ is a *-ideal of itself. Here, there exists a smallest *-ideal containing $a$, a smallest left *-ideal containing $a$ and a smallest right ${ }^{*}$-ideal containing $a$. We will denote them by $J^{*}(a), L^{*}(a)$ and $R^{*}(a)$, respectively.

Lemma 2.3. [10] Let $S$ be a semigroup. For any $a, b \in S$,

(i) $a \mathcal{L}^{*} b$ if and only if $L^{*}(a)=L^{*}(b)$;

(ii) $a \mathcal{R}^{*} b$ if and only if $R^{*}(a)=R^{*}(b)$.

Finally, we define $\mathcal{J}^{*}$ by analogy with the characterisations of $\mathcal{L}^{*}$ and $\mathcal{R}^{*}$ given in Lemma 2.3, by saying that for any $a, b \in S, a \mathcal{J}^{*} b$ if $J^{*}(a)=J^{*}(b)$. 


\subsubsection{Abundant semigroups}

This section is concerned with a class of non-regular semigroups built using the relations $\mathcal{L}^{*}$ and $\mathcal{R}^{*}$.

We say that a semigroup $S$ is abundant if every $\mathcal{L}^{*}$-class and every $\mathcal{R}^{*}$-class contains at least one idempotent. If $S$ is a such semigroup and $a \in S$, then we denote idempotents in the $L_{a}^{*}$ and $R_{a}^{*}$ by $a^{*}$ and $a^{\dagger}$, respectively. Note that $a^{*}$ and $a^{\dagger}$ need not be unique.

As an analogue of orthodox semigroups in the class of abundant semigroups, we have quasi-adequate semigroups [7]. A quasi-adequate semigroup is an abundant semigroup whose set of idempotents forms a subsemigroup. In particular, if the set of idempotents of a quasi-adequate semigroup becomes a semilattice, then it is called an adequate semigroup [9]. Note that if $S$ is an adequate semigroup, then for any $a \in S, a^{*}$ and $a^{\dagger}$ are unique. Since if $a^{\circ}$ is another idempotent in the $\mathcal{L}^{*}$-class of $a$, then we have $a a^{\circ}=a$, and so $a^{*} a^{\circ}=a^{*}$ by Lemma 2.1 , that is, $a^{\circ} a^{*}=a^{*}$ as $E(S)$ is a semilattice. According to the comments succeeding Lemma 2.1, we have $a^{\circ}=a^{*}$. Dually, we show that $a^{\dagger}$ is unique. Thus, in an adequate semigroup $S$, we have unary operations $a \mapsto a^{*}$ and $a \mapsto a^{\dagger}$ for any $a \in S$. So adequate semigroups provide an abundant analogue of inverse semigroups, but see below.

An adequate semigroup $S$ with semilattice of idempotents $E(S)$ is said to be an ample semigroup or a type $A$ semigroup if it satisfies for all $a \in S$ and $e \in E(S)$ :

(i) $a e=(a e)^{\dagger} a ;$

(ii) $e a=a(e a)^{*}$.

In particular, an inverse semigroup is ample, where $a^{\dagger}=a a^{-1}$ and $a^{*}=a^{-1} a$. Ample semigroups are usually thought of as the appropriate abundant analogue of inverse semigroups.

It is easy to see that morphisms between semigroups preserve Green's relations. They need not, however, preserve $\mathcal{L}^{*}$ and $\mathcal{R}^{*}$. With this in mind we define the notion of good morphisms.

Let $S$ and $T$ be semigroups and let $\phi: S \rightarrow T$ be a morphism. Then $\phi$ is said to be good if for any $a, b \in S$,

$$
a \mathcal{L}^{*} b \text { implies } a \phi \mathcal{L}^{*} b \phi,
$$


$a \mathcal{R}^{*} b$ implies $a \phi \mathcal{R}^{*} b \phi$.

\subsection{Weakly $U$-abundant semigroups}

For ease of reference we gather together in this section some basic definitions and elementary observations concerning weakly $U$-abundant semigroups.

\subsubsection{Relations $\widetilde{\mathcal{L}}_{U}, \widetilde{\mathcal{R}}_{U}$}

Let $S$ be a semigroup. We denote as usual its set of idempotents by $E(S)$. Consider a non-empty subset $U \subseteq E(S)$; we will call it the set of distinguished idempotents. The relation $\leq_{\widetilde{\mathcal{L}}_{U}}$ on $S$ is defined by the rule that for all $a, b \in S$, $a \leq_{\widetilde{\mathcal{L}}_{U}} b$ if and only if

$$
\{e \in U: b e=b\} \subseteq\{e \in U: a e=a\}
$$

It is clear that $\leq_{\widetilde{\mathcal{L}}_{U}}$ is a pre-order. We denote the associated equivalence relation by $\widetilde{\mathcal{L}}_{U}$, so that for $a, b \in S, a \widetilde{\mathcal{L}}_{U} b$ if and only if

$$
\{e \in U: a e=a\}=\{e \in U: b e=b\}
$$

It is easy to see that $\mathcal{L} \subseteq \mathcal{L}^{*} \subseteq \widetilde{\mathcal{L}}_{U}$. In particular, we have:

Lemma 2.4. [46] Let $E(S)$ be the set of all idempotents of $S$. If $a, b$ are regular, then $a \widetilde{\mathcal{L}}_{E(S)} b$ if and only if $a \mathcal{L} b$.

It follows that if $S$ is regular and $U=E(S)$, then $\mathcal{L}=\mathcal{L}^{*}=\widetilde{\mathcal{L}}_{U}$. Although $\mathcal{L}$ and $\mathcal{L}^{*}$ are always right compatible, the same need not be true for $\widetilde{\mathcal{L}}_{U}$. The last fact is shown by a very simple example: the null semigroup of two elements with an adjoined identity, where the distinguished set $U=\{0,1\}$.

Notice that for $e, f \in U, e \leq_{\widetilde{\mathcal{L}}_{U}} f$ if and only if $e \leq_{\mathcal{L}} f$, so that $e \widetilde{\mathcal{L}}_{U} f$ if and only if $e \mathcal{L} f$. Another useful observation is that :

Lemma 2.5. [32] If $a \in S$ and $e \in U$, then $a \widetilde{\mathcal{L}}_{U} e$ if and only if $a e=a$ and for all $f \in U$, af $=a$ implies ef $=e$.

We observe that for any distinguished idempotent $a^{*}$ in the $\widetilde{\mathcal{L}}_{U}$-class of $a$, we have $a a^{*}=a$. 
The relations $\leq_{\widetilde{\mathcal{R}}_{U}}$ and $\widetilde{\mathcal{R}}_{U}$ are the left-right duals of $\leq_{\widetilde{\mathcal{L}}_{U}}$ and $\widetilde{\mathcal{L}}_{U}$. Also, if $a \in S$ and $a^{\dagger} \in U$ are such that $a \widetilde{\mathcal{R}}_{U} a^{\dagger}$, then we have $a^{\dagger} a=a$.

According to Lawson [33], there is another description of $\widetilde{\mathcal{L}}_{U}$ and $\widetilde{\mathcal{R}}_{U}$. The $\widetilde{\mathcal{L}}_{U^{-}}$-class and $\widetilde{\mathcal{R}}_{U}$-class containing $a$ will be denoted by $\widetilde{L}_{U, a}$ and $\widetilde{R}_{U, a}$, respectively, abbreviated as $\widetilde{L}_{a}$ and $\widetilde{R}_{a}$, where $U$ is clear. A left ideal $I$ of a semigroup $S$ is said to be a $U$-admissible left ideal if for every $a \in I, \widetilde{L}_{a} \subseteq I$. If $a$ is an element of $S$, then we define the principal $U$-admissible left ideal containing a to be the intersection of all $U$-admissible left ideals containing $a$, and we denote it by $\widetilde{L}(a)$. In particular, for any $e \in U, \widetilde{L}(e)=S e$. Dually, we define the principal $U$-admissible right ideal containing $a$ and we denote it by $\widetilde{R}(a)$. Following the above terminology, in [44], an ideal $I$ of $S$ is called $U$-admissible if $I$ is both a $U$-admissible right ideal and a $U$-admissible left ideal of $S$ and the principal $U$ admissible ideal containing $a$ is defined to be the intersection of all $U$-admissible ideals of $S$ containing $a$, denoted by $\widetilde{J}(a)$. Clearly, $\widetilde{L}(a) \subseteq \widetilde{J}(a)$ and $\widetilde{R}(a) \subseteq \widetilde{J}(a)$ for all $a$ in $S$. The following lemma concerning $\widetilde{\mathcal{L}}_{U}, \widetilde{\mathcal{R}}_{U}$ and $\widetilde{\mathcal{J}}_{U}$ is extracted from [44].

Lemma 2.6. [44] Let $S$ be a semigroup and $U$ be a non-empty subset of $E(S)$. Then for any $a, b \in S$,

$$
\begin{aligned}
& (a, b) \in \widetilde{\mathcal{L}}_{U} \text { if and only if } \widetilde{L}(a)=\widetilde{L}(b) ; \\
& (a, b) \in \widetilde{\mathcal{R}}_{U} \text { if and only if } \widetilde{R}(a)=\widetilde{R}(b) .
\end{aligned}
$$

Consequently, we define the relation $\widetilde{\mathcal{J}}_{U}$ by the rule that

$$
(a, b) \in \widetilde{\mathcal{J}}_{U} \quad \text { if and only if } \widetilde{J}(a)=\widetilde{J}(b) .
$$

To close this section, we define $\widetilde{\mathcal{H}}_{U}$ and $\widetilde{\mathcal{D}}_{U}$ as the intersection and the join of $\widetilde{\mathcal{L}}_{U}$ and $\widetilde{\mathcal{R}}_{U}$, respectively. Note that we do not always have that $\widetilde{\mathcal{D}}_{U}=\widetilde{\mathcal{L}}_{U} \circ \widetilde{\mathcal{R}}_{U}$.

\subsubsection{Weakly $U$-abundant semigroups}

In a manner analogous to the definition of an abundant semigroup, $S$ is said to be weakly $U$-abundant if every $\widetilde{\mathcal{L}}_{U^{-}}$lass and every $\widetilde{\mathcal{R}}_{U^{-}}$-class contains an idempotent of $U$. If $S$ is a such semigroup and $a \in S$, then we follow usual practice and 
denote idempotents in the $\widetilde{\mathcal{L}}_{U^{-}}$class and $\widetilde{\mathcal{R}}_{U^{-}}$class of $a$ by $a^{*}$ and $a^{\dagger}$, respectively. Note that there need not be a unique choice for $a^{*}$ and $a^{\dagger}$ unless $U$ is a semilattice.

We make some comments that if the distinguished set of idempotents of a weakly $U$-abundant semigroup $S$ is the whole set of idempotents $E(S)$, then usually, we call it a weakly abundant semigroup. Another point is that if we talk of a particular weakly $U$-abundant semigroup, then we are referring to a particular set of idempotents $U$; on the other hand, if we are talking of the class of all weakly $U$-abundant semigroups, the $U$ varies over all possible set of idempotents.

We will be interested in semigroups $S$ in which the relation $\widetilde{\mathcal{L}}_{U}$ is a right congruence and $\widetilde{\mathcal{R}}_{U}$ is a left congruence. In this case, we say that $S$ satisfies Congruence Condition $(C)$ (with respect to $U$ ). Indeed it seem very little theory can be developed if we do not assume the Congruence Condition. If $S$ is weakly $U$-abundant with $(\mathrm{C})$, then

$$
x y \widetilde{\mathcal{L}}_{U}(x y)^{*} \widetilde{\mathcal{L}}_{U}\left(x^{*} y\right)^{*} \widetilde{\mathcal{L}}_{U} x^{*} y
$$

for any $x^{*},(x y)^{*}$ and $\left(x^{*} y\right)^{*}$. Dually, we have

$$
x y \widetilde{\mathcal{R}}_{U}(x y)^{\dagger} \widetilde{\mathcal{R}}_{U}\left(x y^{\dagger}\right)^{\dagger} \widetilde{\mathcal{R}}_{U} x y^{\dagger}
$$

for any $y^{\dagger},(x y)^{\dagger}$ and $\left(x y^{\dagger}\right)^{\dagger}$.

The next lemma gives an equivalent description of weakly $U$-abundant semigroups.

Lemma 2.7. A semigroup $S$ is weakly $U$-abundant if and only if for any $a \in S$, there exist e, $f \in U$ such that $\widetilde{L}(a)=$ Se and $\widetilde{R}(a)=f S$.

Proof. For any distinguished idempotent $e$ in $U, \widetilde{L}(e)=S e$. In fact, for any $a, b \in S, a \widetilde{\mathcal{L}}_{U} b$ if and only if $\widetilde{L}(a)=\widetilde{L}(b)$. Thus, a semigroup is weakly $U$ abundant if and only if for any $a \in S$, there exists $e \in U$ such that $a \widetilde{\mathcal{L}}_{U} e$, that is, $\widetilde{L}(a)=\widetilde{L}(e)$, if and only if $\widetilde{L}(a)=S e$. Dually, $\widetilde{R}(a)=f S$.

For convenience, we give the next lemma.

Lemma 2.8. Let $S$ be a weakly $U$-abundant semigroup. For any $x, y \in S$ we have $(y x)^{*} \leq_{\mathcal{L}} x^{*}$ and $(x y)^{\dagger} \leq_{\mathcal{R}} x^{\dagger}$. Further, $(x y)^{\dagger} x^{\dagger} \mathcal{R}(x y)^{\dagger}$ and $y^{*}(x y)^{*} \mathcal{L}(x y)^{*}$ 
Proof. Let $x, y \in S$. Certainly, $y x x^{*}=y x$, and so $(y x)^{*} x^{*}=(y x)^{*}$ by definition of $(y x)^{*}$ so that $(y x)^{*} \leq_{\mathcal{L}} x^{*}$. Dually, we obtain that $(x y)^{\dagger} \leq_{\mathcal{R}} x^{\dagger}$.

In view of Lemma 1.24, the set $E(S)$ of idempotents of $S$ is a biordered set, and so by (B21) and its dual in Section $1.4,(x y)^{\dagger} x^{\dagger} \mathcal{R}(x y)^{\dagger}$ and $y^{*}(x y)^{*} \mathcal{L}(x y)^{*}$ in $S$.

Observe that morphisms between semigroups need not preserve $\mathcal{L}^{*}$ and $\mathcal{R}^{*}$ as mentioned in Section 2.1.2, nor $\widetilde{\mathcal{L}}_{U}$ and $\widetilde{\mathcal{R}}_{U}$. With this in mind we define the notion of admissible morphisms.

Let $S$ and $T$ be semigroups with distinguished subsets of idempotents $U$ and $V$, respectively, and let $\phi: S \rightarrow T$ be a morphism. Then $\phi$ is said to be $(U, V)$-admissible if for any $a, b \in S$,

$$
\begin{aligned}
& a \widetilde{\mathcal{L}}_{U} b \text { implies } a \phi \widetilde{\mathcal{L}}_{V} b \phi, \\
& a \widetilde{\mathcal{R}}_{U} b \text { implies } a \phi \widetilde{\mathcal{R}}_{V} b \phi,
\end{aligned}
$$

and $U \phi \subseteq V$. Briefly, we will refer to the notion of being $(U, V)$-admissible as admissible, where no ambiguity can occur.

Moreover, $\phi$ is said to be strongly admissible [17] if for any $a, b \in S$,

$$
a \widetilde{\mathcal{L}}_{U} b \text { if and only if } a \phi \widetilde{\mathcal{L}}_{U \phi} b \phi
$$

and

$$
a \widetilde{\mathcal{R}}_{U} b \text { if and only if } a \phi \widetilde{\mathcal{R}}_{U \phi} b \phi
$$

Naturally, a congruence $\rho$ on $S$ is said to be admissible if the natural morphism $\rho^{\natural}: S \rightarrow S / \rho$ is admissible, that is $(U, U \rho)$-admissible.

For admissible morphisms and congruences, the following lemmas are easy to see, making use of Lemma 2.5.

Lemma 2.9. [46] Let $S, T$ be semigroups with distinguished subsets of idempotents $U, V$ respectively. Suppose that $S$ is weakly $U$-abundant, and let $\phi: S \rightarrow T$ be a morphism. Then $\phi$ is admissible if and only if $U \phi \subseteq V$ and for any $a \in S$ there exist idempotents $f \in \widetilde{L}_{a} \cap U$ and $e \in \widetilde{R}_{a} \cap U$ such that a $\phi \widetilde{\mathcal{L}}_{V} f \phi$ and $a \phi \widetilde{\mathcal{R}}_{V} e \phi$. 
Lemma 2.10. [6] Let $S$ be a semigroup with subset of idempotents $U$ and let $\phi: S \rightarrow T$ be an admissible surjective morphism. If $S$ is weakly $U$-abundant, then $T$ is weakly $U \phi$-abundant.

Lemma 2.11. If $S$ is a weakly $U$-abundant semigroup with $(C)$ and $\rho$ is an admissible congruence on $S$, then $S / \rho$ has $(C)$ with respect to $U / \rho$.

Proof. To see that $S / \rho$ satisfies the Congruence Condition, we assume that a $\rho$ and $b \rho$ are elements of $S / \rho$ such that $a \rho \widetilde{\mathcal{L}}_{U / \rho} b \rho$. Since $S$ is a weakly $U$-abundant semigroup and $\rho$ is an admissible congruence on $S$, there exist $e \in \widetilde{L}_{a} \cap U$ and $f \in \widetilde{L}_{b} \cap U$ such that $a \rho \widetilde{\mathcal{L}}_{U / \rho} e \rho$ and $b \rho \widetilde{\mathcal{L}}_{U / \rho} f \rho$, respectively. It follows that $e \rho \widetilde{\mathcal{L}}_{U / \rho} f \rho$, that is, e $\rho \mathcal{L} f \rho$. Clearly, for any $c \rho \in S / \rho$, we have e $\rho c \rho \mathcal{L} f \rho c \rho$ because $\mathcal{L}$ is a right congruence. So $(e c) \rho \mathcal{L}(f c) \rho$. Observe that a $\widetilde{\mathcal{L}}_{U} e$ and $b \widetilde{\mathcal{L}}_{U} f$. It follows that $a c \widetilde{\mathcal{L}}_{U} e c$ and $b c \widetilde{\mathcal{L}}_{U} f c$ since $\widetilde{\mathcal{L}}_{U}$ is a right congruence on $S$. Also, $(a c) \rho \widetilde{\mathcal{L}}_{U / \rho}(e c) \rho$ and $(b c) \rho \widetilde{\mathcal{L}}_{U / \rho}(f c) \rho$ since $\rho$ is an admissible congruence. Thus a $\rho c \rho \widetilde{\mathcal{L}}_{U / \rho} b \rho c \rho$. Hence, $\widetilde{\mathcal{L}}_{U / \rho}$ is a right congruence on $S / \rho$. Dually, we can verify that $\widetilde{\mathcal{R}}_{U / \rho}$ is a left congruence on $S / \rho$. Consequently, $S / \rho$ satisfies the Congruence Condition (C).

A weakly $U$-abundant semigroup is $U$-fundamental if the largest admissible congruence contained in $\widetilde{\mathcal{H}}_{U}$ is trivial. For convenience we shall sometimes simplify the term ' $U$-fundamental weakly $U$-abundant semigroup' to 'fundamental weakly $U$-abundant semigroup'.

It is easy to see that if $S$ is a weakly $U$-abundant semigroup with (C) and $T$ is a $U$-full subsemigroup of $S$, in the sense that $U \subseteq T$, then $T$ satisfies (C). In addition, if $S$ is $U$-fundamental, then $T$ is $U$-fundamental.

\subsubsection{Weakly $U$-regular semigroups}

In this section we list some properties of weakly $U$-abundant semigroups with (C), where $U$ is a regular biordered set.

We first recall that if $U$ is a biordered set, then $\mathcal{M}(e, f)$ denote the preordered set $\left(\omega^{l}(e) \cap \omega^{r}(f), \prec\right)$, where

$$
g \prec h \Leftrightarrow e g \omega^{r} e h, g f \omega^{l} h f .
$$


The set $S(e, f)=\{h \in \mathcal{M}(e, f): g \prec h, \forall g \in \mathcal{M}(e, f)\}$ is called the sandwich set of $e$ and $f$. In particular, if $U$ is regular, then $S(e, f) \neq \emptyset$ for all $e, f \in U$.

Lemma 2.12. Let $S$ be a weakly $U$-abundant semigroup, where $U$ is a regular biordered set. For $e, f \in U$, we define

$$
S_{1}(e, f)=\{h \in \mathcal{M}(e, f): e h f=e f\},
$$

and

$$
S_{2}(e, f)=\{h \in \mathcal{M}(e, f): h(e f) h=h \text { and }(e f) h(e f)=e f\} .
$$

Then $S_{1}(e, f)=S_{2}(e, f) \subseteq S(e, f)$.

Proof. To show that $S_{1}(e, f)=S_{2}(e, f)$, we assume that $h \in \mathcal{M}(e, f)$. Then $h e=h=f h$, and so

$$
h(e f) h=(h e)(f h)=h \text { and }(e f) h(e f)=e(f h e) f=e h f .
$$

Obviously, ehf $=e f$ if and only if $(e f) h(e f)=e f$. Thus, $S_{1}(e, f)=S_{2}(e, f)$. We now turn to show that $S_{1}(e, f) \subseteq S(e, f)$. Suppose that $h \in S_{1}(e, f)$ and $g \in \mathcal{M}(e, f)$. Then

$$
(e h)(e g)=e(h e) g=e h g=e h(f g)=(e h f) g=e f g=e g
$$

and so $e g \omega^{r} e h$. Dually, $g f \omega^{l} h f$. Thus $g \prec h$ so that $h \in S(e, f)$.

Let $U$ be a set of idempotents of a semigroup $S$. We will use $\langle U\rangle$ to denote the semigroup generated by $U$. A weakly $U$-abundant semigroup with $(\mathrm{C})$ is said to be a weakly $U$-regular semigroup if $\langle U\rangle$ is a regular semigroup whose set of idempotents is $U$. We remind the reader that this terminology, based on existing convention, needs to be viewed with care: if we talk of a particular weakly $U$ regular semigroup, then we are referring to a particular set of idempotents $U$; on the other hand, if we are talking of the class of all weakly $U$-regular semigroups, the $U$ varies over all possible sets of idempotents. It is clear that the collection of weakly $U$-regular semigroups and admissible morphisms forms a category, which we denote by $\mathcal{W} \mathcal{R} \mathcal{S}$.

Lemma 2.13. Let $S$ be a weakly $U$-regular semigroup. Then $S_{1}(e, f)=S(e, f) \neq$ $\emptyset$ for all $e, f \in U$. 
Proof. Suppose that $e, f \in U$. Then $e f$ is a regular element in $\langle U\rangle$. If $a$ is an inverse of $e f$ in $\langle U\rangle$ and $h=$ fae, then $h \in\langle U\rangle$ and $h^{2}=($ fae $)($ fae $)=$ $f($ aefa $) e=f a e=h$. Together with $E(\langle U\rangle)=U$, we obtain that $h \in U$. Since, ehf $=$ efaef $=e f$, it follows that $h \in S_{1}(e, f)$, and so $S_{1}(e, f) \neq \emptyset$.

By Lemma 2.12, $S_{1}(e, f) \subseteq S(e, f)$. To show the converse, we assume that $g \in S(e, f)$. Let $h \in S_{1}(e, f)$. Then by Lemma 2.12, $h \in S(e, f)$, and so $e g \mathcal{R} e h$, gf $\mathcal{L} h f$. Thus, egf= egef $=(e g)(e h f)=(e g)(e h) f=e h f=e f$ so that $g \in S_{1}(e, f)$. Hence $S(e, f)=S_{1}(e, f)$.

\subsubsection{Weakly $B$-orthodox semigroups}

We recall that an orthodox semigroup is a regular semigroup $S$ such that $E(S)$ is a band. Consequently, a weakly $B$-abundant semigroup is said to be weakly $B$-orthodox if it has (C) and $B$ is a band. Whenever we talk of a particular weakly $B$-orthodox semigroup, then we are referring to a particular band $B$; on the other hand, if we are talking of the class of all weakly $B$-orthodox semigroups, the $B$ varies over all possible bands. It is clear that the collection of weakly $B$-orthodox semigroups and admissible morphisms forms a category, which we denote by $\mathcal{W O}$.

Lemma 2.14. Let $S$ be a weakly B-orthodox semigroup. For any $x \in S$ and $e, f, g, h \in B$,

(i) if $e \leq_{\mathcal{R}}\left(\right.$ resp. $\left.\leq_{\mathcal{L}}\right) g \mathcal{R} x^{\dagger}$, then ex $\widetilde{\mathcal{R}}_{B}$ e;

(ii) if $f \leq_{\mathcal{L}}\left(\right.$ resp. $\left.\leq_{\mathcal{R}}\right) h \mathcal{L} x^{*}$, then $x f \widetilde{\mathcal{L}}_{B} f$.

Proof. To prove $(i)$, suppose that $e \leq_{\mathcal{R}} g \mathcal{R} x^{\dagger}$, then ex $\widetilde{\mathcal{R}}_{B}$ ex $x^{\dagger} \widetilde{\mathcal{R}}_{B}$ eg $\mathcal{R}$ e, otherwise, $e \leq_{\mathcal{L}} g$, and so $e x \widetilde{\mathcal{R}}_{B} e g=e$. By a similar argument, we can show that $(i i)$ holds.

\subsubsection{Ehresmann semigroups}

Let $S$ be weakly $E$-abundant with (C). We say that $S$ is an Ehresmann semigroup (with distinguished semilattice $E$ ) if $E$ is a semilattice. It is straightforward to see that if $S$ is Ehresmann, then, for any $a \in S$, the elements $a^{*}$ and $a^{\dagger}$ are the unique elements of $E$ in the $\widetilde{\mathcal{L}}_{E^{-}}$class and the $\widetilde{\mathcal{R}}_{E^{-}}$class of $a$, respectively. We regard Ehresmann semigroups as algebras with signature $(2,1,1)$; as such, they 
form a variety $\mathcal{E}$. Indeed, $\mathcal{E}$ is the variety generated by $\mathcal{A}$, where $\mathcal{A}$ is the quasivariety of adequate semigroups [29]. The corresponding result is the one-side case may be found in [16] or [30].

An important property of Ehresmann semigroups is given below.

Lemma 2.15. [32] Let $S$ be an Ehresmann semigroup with semilattice of distinguished idempotents $E$. Then

(i) for all $x, y \in S,(x y)^{*} \leq y^{*}$ and $(x y)^{\dagger} \leq x^{\dagger}$;

(ii) for all $e \in E, e^{*}=e$ and $e^{\dagger}=e$;

(iii) for all $x, y \in S, x \widetilde{\mathcal{L}}_{E} y \Leftrightarrow x^{*}=y^{*}$ and $x \widetilde{\mathcal{R}}_{E} y \Leftrightarrow x^{\dagger}=y^{\dagger}$;

(iv) for all $x, y \in S,(x y)^{*}=\left(x^{*} y\right)^{*}$ and $(x y)^{\dagger}=\left(x y^{\dagger}\right)^{\dagger}$.

We introduce the notion of restriction semigroups as an analogue of ample semigroups. Consequently, a restriction semigroup has also been called a weakly E-ample semigroup, where $E$ is the distinguished semilattice of idempotents. There are four ways to define restriction semigroups: as varieties of algebras, representation by (partial) mappings, using generalised Green's relations $\widetilde{\mathcal{R}}_{E}$ and $\widetilde{\mathcal{L}}_{E}$, and inductive constellations. Here we define restriction semigroups by using $\widetilde{\mathcal{R}}_{E}$ and $\widetilde{\mathcal{L}}_{E}$.

An Ehresmann semigroup $S$ is left restriction with distinguished semilattice $E$ if it satisfies the left ample condition (AL).

(AL) $(\forall a \in S, e \in E) a e=(a e)^{\dagger} a$.

Similarly, an Ehresmann semigroup $S$ with distinguished semilattice of idempotents $E$ is right restriction if it satisfies the right ample condition (AR).

(AR) $(\forall a \in S, e \in E) e a=a(e a)^{*}$.

An Ehresmann semigroup is a restriction semigroup if it is both a left restriction and a right restriction semigroup.

\subsection{The idempotent connected condition}

We focus on the idempotent connected condition in this section. A fuller version of some of the ideas we present here is contained in [6], [8] and [48]. Essentially, all of the idempotent connected and ample (formely, type A) conditions extant give some control over the position of idempotents in products, usually facilitating results for abundant or weakly abundant semigroups reminiscent of those in the regular case. 


\subsection{1 (WIC), (IC) and (PIC)}

Let $S$ be a weakly $U$-abundant semigroup. For any $e \in U$, we put

$$
\langle e\rangle=\left\langle e u e: u \in\langle U\rangle \text { and }(e u e)^{2}=e u e\right\rangle
$$

or equivalently,

$$
\langle e\rangle=\left\{u_{1} \cdots u_{n}: u_{i} \in\langle U\rangle, u_{i}^{2}=u_{i} \text { and } u_{i} \leq e\right\} .
$$

Clearly, $\langle e\rangle$ is a subsemigroup with identity $e$.

We say that a weakly $U$-abundant semigroup $S$ satisfies the weak idempotent connected condition (WIC) (with respect to $U$ ) if for any $a \in S$ and some $a^{*}, a^{\dagger}$, if $x \in\left\langle a^{\dagger}\right\rangle$, then there exists $y \in\left\langle a^{*}\right\rangle$ with $x a=a y$; and dually, if $z \in\left\langle a^{*}\right\rangle$ then there exists $t \in\left\langle a^{\dagger}\right\rangle$ with $t a=a z$.

We pause here to make some comments on Condition (WIC). The phrase 'for some $a^{\dagger}, a^{*}$ ' may be replaced by 'for any $a^{\dagger}, a^{*}$. For suppose that $S$ has (WIC), $a \in S, a^{\dagger}$ is the chosen idempotent of $U$ in the $\widetilde{\mathcal{R}}_{U^{-}}$class of $a$, and $a^{+}$is another element of $U$ in the same $\widetilde{\mathcal{R}}_{U^{-}}$class. If $v \in\left\langle a^{+}\right\rangle$, then

$$
v=u_{1} \cdots u_{n} \quad\left(u_{i} \in\langle U\rangle, u_{i}=u_{i}^{2}, u_{i} \leq a^{+}\right) .
$$

So

$$
a^{\dagger} v a^{\dagger}=v a^{\dagger}=\left(u_{1} a^{\dagger}\right) \cdots\left(u_{n} a^{\dagger}\right) .
$$

Certainly, $u_{i} a^{\dagger} \in\langle U\rangle, u_{i} a^{\dagger} \leq a^{\dagger}$ and $\left(u_{i} a^{\dagger}\right)^{2}=u_{i} a^{\dagger} u_{i} a^{\dagger}=u_{i} a^{\dagger}$. Thus, $v a^{\dagger} \in\left\langle a^{\dagger}\right\rangle$, and so by (WIC), $v a=v a^{\dagger} a=a k$ for some $k \in\left\langle a^{*}\right\rangle$. Then $k=w_{1} \cdots w_{m}$ for some $w_{i} \in\langle U\rangle, w_{i}^{2}=w_{i}$ and $w_{i} \leq a^{*}$. As above, for any $a^{\circ} \in U$ lying in the $\widetilde{\mathcal{L}}_{U^{-}}$class of $a$, we have $a^{\circ} k=\left(a^{\circ} w_{1}\right) \cdots\left(a^{\circ} w_{m}\right) \in\left\langle a^{\circ}\right\rangle$, and so $v a=a k=a\left(a^{\circ} k\right)$. Thus in the condition of (WIC) we may choose the $y$ lie in any given $\left\langle a^{\circ}\right\rangle$, and dually, the $t$ to lie in any given $\left\langle a^{\dagger}\right\rangle$.

The following lemma provides an equivalent statement of Condition (WIC).

Lemma 2.16. Let $S$ be a weakly $U$-abundant semigroup. Then $S$ satisfies (WIC) if and only if for any $a \in S$ and some (any) $a^{*}, a^{\dagger}$, if $u \in\langle U\rangle, u^{2}=u$ and $u \leq a^{\dagger}$ then there exists $y \in\left\langle a^{*}\right\rangle$ with $u a=$ ay; and dually, if $v \in\langle U\rangle, v^{2}=v$ and $v \leq a^{*}$ then there exists $t \in\left\langle a^{\dagger}\right\rangle$ with $t a=a v$. 
Proof. The necessity is clear. We now show the sufficiency. If $x \in\left\langle a^{\dagger}\right\rangle$, then $x=e_{1} \cdots e_{n}$, where $e_{i} \in\langle U\rangle, e_{i}^{2}=e_{i}$ and $e_{i} \leq a^{\dagger}$. Then we have

$$
e_{i} a=a y_{i} \quad\left(y_{i} \in\left\langle a^{*}\right\rangle\right)
$$

and so

$$
x a=e_{1} \cdots e_{n} a=e_{1} \cdots e_{n-1}\left(e_{n} a\right)=e_{1} \cdots e_{n-1} a y_{n}=\cdots=a y_{1} \cdots y_{n}
$$

where $y_{1} \cdots y_{n} \in\left\langle a^{*}\right\rangle$. Dually, we show the second part holds.

We now present two stronger versions of Condition (WIC) which have been investigated in [6] and [8].

A weakly $U$-abundant semigroup $S$ satisfies Condition (IC) if for any $a \in S$ and for some $a^{\dagger}, a^{*}$, there exists an order isomorphism $\alpha:\left\langle a^{\dagger}\right\rangle \rightarrow\left\langle a^{*}\right\rangle$ such that for all $x \in\left\langle a^{\dagger}\right\rangle$,

$$
x a=a(x \alpha) .
$$

The order isomorphism given above is said to be a connecting order isomorphism.

Notice that we can replace 'some' in (IC) by 'any'. For suppose that $a \in S$, $a^{\dagger}, a^{+}$are idempotents of $U$ in the $\widetilde{\mathcal{R}}_{U^{-}}$class of $a$, then the map

$$
\rho_{a^{\dagger}}:\left\langle a^{+}\right\rangle \rightarrow\left\langle a^{\dagger}\right\rangle
$$

given by

$$
x \rho_{a^{\dagger}}=x a^{\dagger}
$$

for all $x \in\left\langle a^{+}\right\rangle$, is an isomorphism. Since if $x \in\left\langle a^{+}\right\rangle$, then

$$
x=u_{1} \cdots u_{n} \quad\left(u_{i} \in\langle U\rangle, u_{i}=u_{i}^{2}, u_{i} \leq a^{+}\right)
$$

and so

$$
x a^{\dagger}=\left(u_{1} a^{\dagger}\right) \cdots\left(u_{n} a^{\dagger}\right)
$$

where $u_{i} a^{\dagger} \in\langle U\rangle, u_{i} a^{\dagger} \leq a^{\dagger}$ and $\left(u_{i} a^{\dagger}\right)^{2}=u_{i} a^{\dagger}$, so that $x a^{\dagger} \in\left\langle a^{\dagger}\right\rangle$. Thus, $\rho_{a^{\dagger}}$ is well-defined. 
Let $y$ be another element in $\left\langle a^{+}\right\rangle$. Then we have

$$
(x y) \rho_{a^{\dagger}}=x y a^{\dagger}=x a^{\dagger} y a^{\dagger}=x \rho_{a^{\dagger}} y \rho_{a^{\dagger}},
$$

and so $\rho_{a^{\dagger}}$ is a morphism.

Certainly, $\rho_{a^{+}}:\left\langle a^{\dagger}\right\rangle \rightarrow\left\langle a^{+}\right\rangle$is a morphism. Further, for any $x \in\left\langle a^{+}\right\rangle$, we have

$$
x \rho_{a^{\dagger}} \rho_{a^{+}}=x a^{\dagger} a^{+}=x a^{+}=x
$$

and similarly, for any $z \in\left\langle a^{\dagger}\right\rangle$, we have

$$
z \rho_{a^{+}} \rho_{a^{\dagger}}=z
$$

Hence, $\rho_{a^{+}}$is an isomorphism.

Dually, if $a^{*}, a^{\circ}$ are idempotent of $U$ in the $\widetilde{\mathcal{L}}_{U}$-class of $a$, then the map

$$
\lambda_{a^{\circ}}:\left\langle a^{*}\right\rangle \rightarrow\left\langle a^{\circ}\right\rangle
$$

given by

$$
x \lambda_{a^{\circ}}=a^{\circ} x
$$

for any $x \in\left\langle a^{*}\right\rangle$, is an isomorphism.

Let $\alpha$ be an order isomorphism from $\left\langle a^{\dagger}\right\rangle \rightarrow\left\langle a^{*}\right\rangle$ such that $x a=a(x \alpha)$ for all $x \in\left\langle a^{\dagger}\right\rangle$. Then certainly, $\rho_{a^{\dagger}} \alpha \lambda_{a^{\circ}}$ is an order isomorphism from $\left\langle a^{+}\right\rangle$to $\left\langle a^{\circ}\right\rangle$ and also, for any $z \in\left\langle a^{+}\right\rangle$, we have

$$
z a=z a^{\dagger} a=a\left(\left(z a^{\dagger}\right) \alpha\right)=a a^{\circ}\left(\left(z a^{\dagger}\right) \alpha\right)=a\left(z \rho_{a^{\dagger}} \alpha \lambda_{a^{\circ}}\right) .
$$

Consequently, $\rho_{a^{\dagger}} \alpha \lambda_{a^{\circ}}:\left\langle a^{+}\right\rangle \rightarrow\left\langle a^{\circ}\right\rangle$ is an order isomorphism as required in Condition (IC).

Yet another version of Condition (WIC) is Condition (PIC). We say that a weakly $U$-abundant semigroup $S$ satisfies (PIC) if for all $a \in S$ and for some $a^{\dagger}, a^{*}$, there exists an isomorphism $\alpha:\left\langle a^{\dagger}\right\rangle \rightarrow\left\langle a^{*}\right\rangle$ such that for all $x \in\left\langle a^{\dagger}\right\rangle$,

$$
x a=a(x \alpha) .
$$

The isomorphism given above is said to be a connecting isomorphism. As 
with the definition of (WIC) (resp. (IC)), we can use the same method as that for Condition (IC) to replace 'some' by 'any', so we omit it.

The following Lemma is cited from [6].

Lemma 2.17. Let $T$ be a full subsemigroup of a weakly $U$-abundant semigroup S. If $S$ satisfies (WIC) (resp. (IC), (PIC)), then so does T.

\subsubsection{Special cases}

In this section we concentrate on some special kinds of weakly $U$-abundant semigroup with (WIC) (resp. (IC), (PIC)).

Let $S$ be a weakly $U$-regular semigroup. Since $E(\langle U\rangle)=U$, it follows that for any $e \in U$,

$$
\langle e\rangle=\langle e u e: u \in\langle U\rangle \text { and } \text { eue } \in U\rangle
$$

that is,

$$
\langle e\rangle=\langle v: v \in U \text { and } v \leq e\rangle
$$

or equivalently,

$$
\langle e\rangle=\left\{v_{1} \cdots v_{n}: v_{i} \in U \text { and } v_{i} \leq e\right\} .
$$

For a band $B$ and element $e$ of $B$, we have

$$
\langle e\rangle=\langle e u e: u \in B\rangle
$$

so that if $x=\left(e u_{1} e\right) \cdots\left(e u_{n} e\right) \in\langle e\rangle$, then $x \in B$ and $x \leq e$. Conversely, if $y \in B$ and $y \leq e$, then $y=e y e \in\langle e\rangle$. Thus $\langle e\rangle$ is the principal order ideal generated by $e$, that is

$$
\langle e\rangle=\{x \in B: x \leq e\}=\{x \in B: e x=x e=e\} .
$$

A weakly $B$-orthodox semigroup with (WIC) (resp. (IC), (PIC)) has been mentioned variously in [6], [11], [14], [15], [16], [17] and [19].

We now describe an important connection between Condition (WIC) and Conditions (AL) and (AR) on an Ehresmann semigroup below.

Lemma 2.18. Let $S$ be an Ehresmann semigroup with distinguished semilattice of idempotents $E$. Then $S$ has (WIC) if and only if it satisfies Conditions (AL) and $(A R)$, that is, $S$ is a restriction semigroup. 
Proof. Clearly, if $S$ satisfies Conditions (AL) and (AR), then it has (WIC).

Conversely, suppose that $S$ is an Ehresmann semigroup with distinguished semilattice of idempotents $E$ and satisfying (WIC). For any $a \in S$ and $e \in E$, we have $a e=a a^{*} e a^{*}=f a$ for some $f \in E$, from which it follows that $f a e=a e$, and so $f(a e)^{\dagger}=(a e)^{\dagger}$. Thus,

$$
(a e)^{\dagger} a=f(a e)^{\dagger} a=(a e)^{\dagger} f a=(a e)^{\dagger} a e=a e .
$$

Dually, $e a=a(e a)^{*}$. Hence, $S$ satisfies Conditions (AL) and (AR).

If $S$ is abundant, we replace the distinguished set of idempotents $U$ by the whole set of idempotents $E(S)$. For an element $e$ of $E(S)$,

$$
\begin{aligned}
\langle e\rangle & =\left\{\left(e u_{1} e\right) \cdots\left(e u_{n} e\right): u_{i} \in\langle E(S)\rangle, e u_{i} e \in E(S)\right\} \\
& =\left\{v_{1} \cdots v_{n}: v_{i} \in E(S), v_{i} \leq e\right\} \\
& =\langle f: f \in E(S), f \leq e\rangle .
\end{aligned}
$$

Thus, $\langle e\rangle$ is generated by all idempotents $f$ satisfying $f \leq e$.

In [8], El-Qallali and Fountain introduced the notion of Condition (IC) in the abundant case, as we describe below.

An abundant semigroup $S$ with set of idempotents $E(S)$ is idempotent connected (IC) if for any $a \in S$, and for some $a^{\dagger}, a^{*}$, there exists a bijection $\alpha:\left\langle a^{\dagger}\right\rangle \rightarrow\left\langle a^{*}\right\rangle$ such that $x a=a(x \alpha)$ for all $x \in\left\langle a^{\dagger}\right\rangle$.

It is easy to see that the bijection $\alpha$ in Condition (IC) must be an isomorphism. Since if $x, y \in\left\langle a^{\dagger}\right\rangle$, then $x y \in\left\langle a^{\dagger}\right\rangle$ and $(x y) a=a(x y) \alpha$. But also $x y a=$ $x a(y \alpha)=a(x \alpha)(y \alpha)$. So $a(x y) \alpha=a(x \alpha)(y \alpha)$. Since $(x y) \alpha,(x \alpha)(y \alpha) \in\left\langle a^{*}\right\rangle$ and $a \mathcal{L}^{*} a^{*}$, we have that $a^{*}(x y) \alpha=a^{*}(x \alpha)(y \alpha)$, and so $(x y) \alpha=(x \alpha)(y \alpha)$. Usually, we call such $\alpha$ a connecting isomorphism. Thus the notion of (IC) from [8] coincides with the notion of (IC) in Section 2.3.1. A further point is that the connecting isomorphism $\alpha:\left\langle a^{\dagger}\right\rangle \rightarrow\left\langle a^{*}\right\rangle$ is unique. Since if $\beta:\left\langle a^{\dagger}\right\rangle \rightarrow\left\langle a^{*}\right\rangle$ is another connecting isomorphism, then for any $x \in\left\langle a^{\dagger}\right\rangle$, we have $x a=a(x \alpha)=a(x \beta)$. As $a \mathcal{L}^{*} a^{*}$, we have that $a^{*}(x \alpha)=a^{*}(x \beta)$, and so $x \alpha=y \beta$ so that $\alpha=\beta$. Finally, we note that the notion of Condition (IC) defined by El-Qallali and Fountain coincides with Condition (PIC); in addition, Condition (WIC) and Condition (IC) coincide in the abundant case, as the following lemma demonstrates. 
Lemma 2.19. [8] Let $S$ be an abundant semigroup with set of idempotents $E(S)$. Then the following statements are equivalent:

(i) $S$ satisfies $(I C)$;

(ii) for each $a \in S$, the following two conditions hold:

(a) for each $e \leq a^{\dagger}$, there exists $f \leq a^{*}$ (resp. $f \in S$ ) such that $e a=a f$

(b) for each $g \leq a^{*}$, there exists $h \leq a^{\dagger}$ (resp. $h \in S$ ) such that $h a=a g$.

We make a short comment on Lemma 2.19. In part (a) of (ii), $f$ is unique. Since if $k \leq a^{*}$ and $e a=a k$, then we have $a k=a f$. As $a^{*} \mathcal{L}^{*} a$, we obtain that $a^{*} k=a^{*} f$, that is, $k=f$. Dually, in part (b) of $(i i), h$ is unique.

We say that an abundant semigroup is a concordant semigroup if it satisfies (IC) and the set of idempotents forms a regular biordered set. An abundant semigroup is a Type $W$ semigroup if it satisfies (IC) and the set of idempotents forms a band. In view of Lemma 2.18, it is easy to see that an adequate semigroup $S$ has (IC) if and only if it is an ample semigroup.

At the end of this section, we turn our attention to the regular case. If $S$ is a regular semigroup, then for any $a \in S$ and any inverse $a^{\prime}$ of $a$, there exists an isomorphism

$$
\alpha:\left\langle a a^{\prime}\right\rangle \rightarrow\left\langle a^{\prime} a\right\rangle
$$

defined by

$$
x \alpha=a^{\prime} x a
$$

for all $x \in\left\langle a a^{\prime}\right\rangle$. For suppose that if $x \in\left\langle a a^{\prime}\right\rangle$, then

$$
x=v_{1} \cdots v_{n} \quad\left(v_{i} \in E(S), v_{i} \leq a a^{\prime}\right),
$$

and so

$$
\begin{aligned}
a^{\prime} x a & =a^{\prime} v_{1} \cdots v_{n} a \\
& =a^{\prime} v_{1} a a^{\prime} v_{2} \cdots a a^{\prime} v_{n} a \\
& =w_{1} \cdots w_{n} \quad\left(w_{i}=a^{\prime} v_{i} a\right) .
\end{aligned}
$$


Now ,

$$
\begin{aligned}
w_{i}^{2} & =\left(a^{\prime} v_{i} a\right)\left(a^{\prime} v_{i} a\right) \\
& =a^{\prime}\left(v_{i} a a^{\prime} v_{i}\right) a \\
& =a^{\prime} v_{i}^{2} a \quad\left(v_{i} \leq a a^{\prime}\right) \\
& =a^{\prime} v_{i} a \quad\left(v_{i} \in E(S)\right) \\
& =w_{i}
\end{aligned}
$$

and clearly, $w_{i} \leq a^{\prime} a$, so that $x \alpha \in\left\langle a^{\prime} a\right\rangle$. Thus, $\alpha$ is well-defined. If also $y \in\left\langle a a^{\prime}\right\rangle$, then

$$
(x y) \alpha=a^{\prime} x y a=a^{\prime} x a a^{\prime} y a=(x \alpha)(y \alpha),
$$

and so $\alpha$ is a morphism. Similarly, $\beta:\left\langle a^{\prime} a\right\rangle \rightarrow\left\langle a a^{\prime}\right\rangle$ given by

$$
x \beta=a x a^{\prime} \quad\left(x \in\left\langle a^{\prime} a\right\rangle\right)
$$

is a morphism. Moreover,

$$
\alpha \beta=1_{\left\langle a a^{\prime}\right\rangle} \text { and } \beta \alpha=1_{\left\langle a^{\prime} a\right\rangle}
$$

so that $\alpha$ is an isomorphism. In addition, for any $x \in\left\langle a a^{\prime}\right\rangle$, we have $x a=a a^{\prime} x a=$ $a(x \alpha)$ and consequently, $S$ has (IC). 


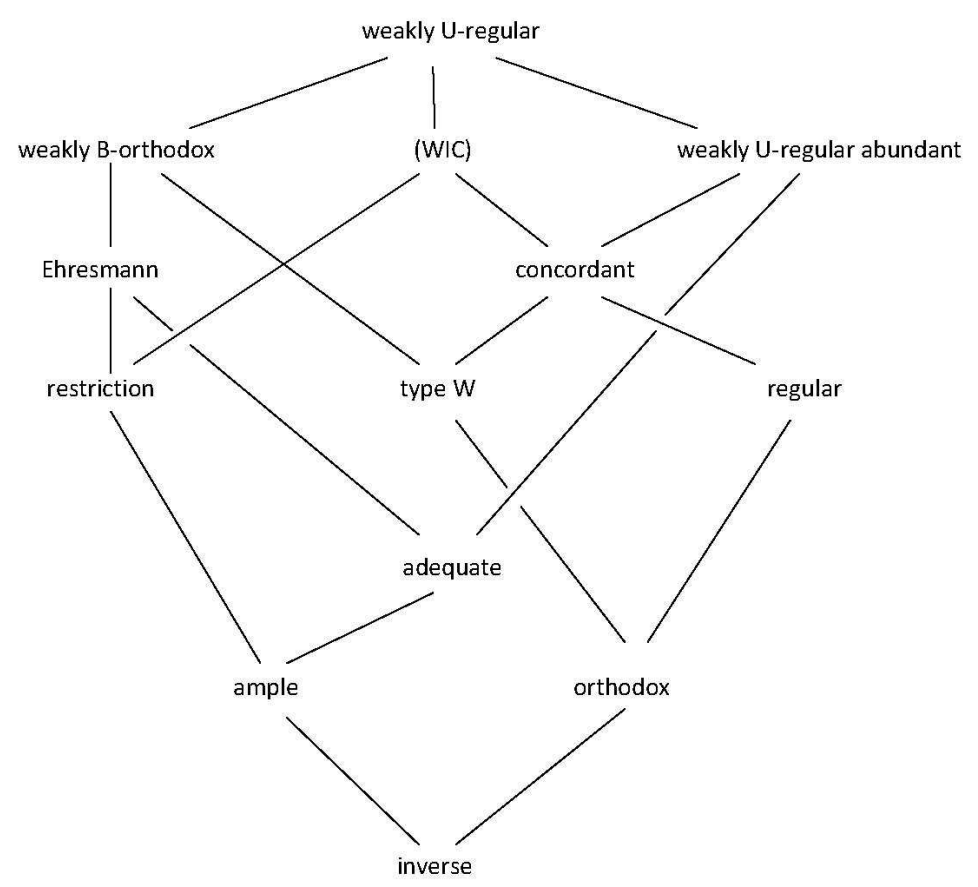

Figure 2.1: Classes of semigroups

In the above picture, (WIC) denotes weakly $U$-regular semigroups with (WIC).

\subsection{An analogue of the least inverse congruence}

We denote by $\gamma_{B}$ the analogue for a weakly $B$-orthodox semigroup $S$ of the least inverse congruence on an orthodox semigroup, that is, $\gamma_{B}$ is the smallest admissible congruence on $S$ such that $S / \gamma_{B}$ is Ehresmann with respect to $\bar{B}=$ $B / \gamma_{B}$. Since if $\left\{\rho_{i}: i \in I\right\}$ is a non-empty family of admissible congruences on $S$, then it is easy to see that the intersection $\bigcap\left\{\rho_{i}: i \in I\right\}$ is again an admissible congruence. We use $\gamma_{B}$ to denote the admissible congruence generated by

$$
\{(e, f): e \mathcal{D} f \text { in } B\}
$$

Then $S / \gamma_{B}$ is weakly $B / \gamma_{B}$-abundant with (C) by Lemma 2.11. If $\bar{e}, \bar{f} \in B / \gamma_{B}$, then

$$
\bar{e} \bar{f}=\overline{e f}=\overline{f e}=\bar{f} \bar{e} \quad(\text { as ef } \mathcal{D} f e)
$$


and so $S / \gamma_{B}$ is an Ehresmann semigroup with respect to $B / \gamma_{B}$.

In addition, suppose that $\rho$ is an admissible congruence on $S$ such that $S / \rho$ is an Ehresmann semigroup with respect to $B / \rho$. Let $e, f \in B$ be such that $e \mathcal{D} f$. Then

$$
\begin{aligned}
e \rho=(e f e) \rho & =e \rho f \rho e \rho=e \rho e \rho f \rho=e \rho f \rho \\
& =e \rho f \rho f \rho=f \rho e \rho f \rho=(f e f) \rho=f \rho,
\end{aligned}
$$

and so $e \rho f$ so that

$$
\{(e, f): e \mathcal{D} f\} \subseteq \rho
$$

Thus, $\gamma_{B} \subseteq \rho$. Hence, $\gamma_{B}$ is the smallest admissible congruence on $S$ such that $S / \gamma_{B}$ is an Ehresmann semigroup with respect to $B / \gamma_{B}$.

We wish to find a closed form for $\gamma_{B}$. These ideas have been investigated in [6], [7], [14] and [46].

Let $S$ be a weakly $B$-orthodox semigroup with (WIC) (resp. (IC), (PIC)). The relation $\delta_{B}$ is defined on $S$ as follows:

$$
a \delta_{B} b \text { if and only if } a=e b f, b=g a h \quad \text { for some } e, f, g, h \in B \text {. }
$$

Here we remind the reader that given a band $B, E(e)$ denotes the $\mathcal{D}$-class of $B$ containing $e$.

Lemma 2.20. [14] (cf. [45], Lemma 3.5) Let $S$ be a weakly B-abundant semigroup. The following conditions are equivalent:

(i) $a \delta_{B} b$;

(ii) $a=e b f$ and $b=$ gah for some $e \in E\left(b^{\dagger}\right), f \in E\left(b^{*}\right), g \in E\left(a^{\dagger}\right)$ and $h \in E\left(a^{*}\right)$;

(iii) $E\left(a^{\dagger}\right) a E\left(a^{*}\right)=E\left(b^{\dagger}\right) b E\left(b^{*}\right)$.

Moreover, if a $\delta_{B} b$, then

$$
E\left(a^{\dagger}\right)=E\left(b^{\dagger}\right) \text { and } E\left(a^{*}\right)=E\left(b^{*}\right)
$$

Further:

Lemma 2.21. [14] Let $S$ be a weakly B-abundant semigroup. For any e, $f \in B$, $e \delta_{B} f$ if and only if $e \mathcal{D} f$. 
Proof. Let $e, f \in B$. If $e \mathcal{D} f$, then $e=e f e$ and $f=f e f$, so that $e \delta_{B} f$.

Conversely, if $e \delta_{B} f$, then by Lemma 2.20, $E\left(e^{\dagger}\right)=E\left(f^{\dagger}\right)$ and so $e \mathcal{D} f$.

In view of these equivalent descriptions of $\delta_{B}$, we now show that if $S$ has (WIC), then it is a congruence on $S$, arguing as in [6], [14] and [22]. Here $\mathcal{D}$ refers to the band $B$.

Lemma 2.22. Let $S$ be a weakly B-orthodox semigroup with (WIC). Then the relation $\delta_{B}$ is the least $B / \mathcal{D}$-ample congruence on $S$.

Proof. In view of Lemma $2.20(\mathrm{iii})$, it is easy to see that $\delta_{B}$ is an equivalence. Suppose now that $a, b, c \in S$ with $a \delta_{B} b$, and $e, f, g, h \in B$ are such that $e, g \in$ $E\left(a^{\dagger}\right)=E\left(b^{\dagger}\right), f, h \in E\left(a^{*}\right)=E\left(b^{*}\right)$ satisfying that $a=e b f$ and $b=g a h$. Notice that for any $b^{\dagger}$ we have that $e b^{\dagger} \mathcal{D} b^{\dagger}$ in $B$, and as $\mathcal{D}$ is a semilattice congruence on $B, c^{*} e b^{\dagger} \mathcal{D} c^{*} b^{\dagger}$ for any $c^{*}$. Consequently,

$$
\begin{aligned}
c a & =c e b f \\
& =c c^{*} c b^{\dagger} b f \\
& =c\left(c^{*} e b^{\dagger}\right)\left(c^{*} b^{\dagger}\right)\left(c^{*} e b^{\dagger}\right) b f \\
& =c\left(c^{*} e b^{\dagger} c^{*}\right)\left(b^{\dagger} c^{*} e b^{\dagger}\right) b e \\
& =(x c)(b y) f \\
& =x(c b) y f
\end{aligned}
$$

for some $x, y \in B$, using (WIC). Similarly, $c b=u(c a) v$ for some $u, v \in B$. It follows that $c a \delta_{B} c b$ so that $\delta_{B}$ is a left congruence. Dually, $\delta_{B}$ is a right congruence.

Now, we show that $\delta_{B}$ is an admissible congruence. Suppose that $a \in S$ and $e, f, g \in B$ with $g \widetilde{\mathcal{R}}_{B} a \widetilde{\mathcal{L}}_{B} e$ in $S$. If $a \delta_{B} f \delta_{B}=a \delta_{B}$, then $a=g a f h$ for some $g \in E\left((a f)^{\dagger}\right)$ and $h \in E\left((a f)^{*}\right)$. As $f h \in B$, and so $a f h=a$, so that $e f h=e$ as a $\widetilde{\mathcal{L}}_{B}$ e. From a $\widetilde{\mathcal{L}}_{B}$ e, we obtain that af $\widetilde{\mathcal{L}}_{B}$ ef, and so $h \in E\left((e f)^{*}\right)=E(e f)$. Thus $e \delta_{B}$ ef, that is, $e \delta_{B} f \delta_{B}=e \delta_{B}$. Hence $a \delta_{B} \widetilde{\mathcal{L}}_{B / \mathcal{D}} e \delta_{B}$. Dually, $a \delta_{B} \widetilde{\mathcal{R}}_{B / \mathcal{D}} g \delta_{B}$.

To see that $\delta_{B}$ is a $B / \mathcal{D}$-ample congruence, we suppose that $a \in S, e \in B$ with $a \delta_{B} \in S / \delta_{B}$ and $e \delta_{B} \in B / \delta_{B}$ are such that $e \delta_{B} \leq a^{\dagger} \delta_{B}$. Then $e \delta_{B} a \delta_{B}=$ $a^{\dagger} \delta_{B} e \delta_{B} a \delta_{B}=\left(a^{\dagger} e a\right) \delta_{B}=\left(a^{\dagger} e a^{\dagger} a\right) \delta_{B}=(a f) \delta_{B}=a \delta_{B} f \delta_{B}$ as $a^{\dagger} e a^{\dagger} \leq a^{\dagger}$ and $S$ satisfies (WIC). As $B / \mathcal{D}$ is a semilattice, it follows from Lemma 2.18 that $S / \delta_{B}$ is a $B / \mathcal{D}$-ample semigroup. 
Finally, if $\tau$ is an arbitrary $B / \mathcal{D}$-ample congruence on $S$, then $\left.\tau\right|_{B}$ is a semilattice congruence on $B$. Since $\mathcal{D}$ is the least semilattice congruence on $B$, it follows that $\left.\mathcal{D} \subseteq \tau\right|_{B}$. For any $a, b \in S$ and $a \delta_{B} b$, we have $b=e a f$ for some $e \in E\left(a^{\dagger}\right)$ and $f \in E\left(a^{*}\right)$. As $e \mathcal{D} a^{\dagger}$ and $f \mathcal{D} a^{*}$, we obtain that $e \tau a^{\dagger}$ and $f \tau a^{*}$, and so $a^{\dagger} a a^{*} \tau$ eaf, that is, $a \tau b$. Thus, $\delta_{B} \subseteq \tau$, and hence $\delta_{B}$ is the least $B / \mathcal{D}$-ample congruence on $S$.

\section{$2.5 \quad$ Orders}

Our purpose in this section is to describe certain pre-orders and partial orders on a weakly $U$-abundant semigroup. The results present here are necessary for Chapters 7, 9 and 10 .

\subsubsection{The weakly $U$-abundant case}

The aim of this section is to present two pairs of relations on a weakly $U$-abundant semigroup.

Let $S$ be a weakly $U$-abundant semigroup. We define relations $\leq_{r}$ and $\leq_{l}$ by the rule that for any $x, y \in S$,

$$
x \leq_{r} y \text { if and only if } x=u y \text { for some } u \in\langle U\rangle
$$

and

$$
x \leq_{l} y \text { if and only if } x=y v \text { for some } v \in\langle U\rangle .
$$

The next lemma is immediate.

Lemma 2.23. On a weakly $U$-abundant semigroup $S$, the relations $\leq_{r}$ and $\leq_{l}$ given above are pre-orders.

In view of Lemma 2.8, we have:

Lemma 2.24. Let $S$ be a weakly $U$-abundant semigroup. Then for any $x, y \in S$,

(i) if $x \leq_{r} y$, then $x^{*} \leq_{\mathcal{L}} y^{*}$;

(ii) if $x \leq_{l} y$, then $x^{\dagger} \leq_{\mathcal{R}} y^{\dagger}$.

Further, a weakly $U$-abundant semigroup $S$ possesses a pair of relations $\leq_{r}^{\prime}$ and $\leq_{l}^{\prime}$ as follows: for any $x, y \in S$, 


$$
x \leq_{r}^{\prime} y \text { if and only if } x=e y \text { for some } e \in U \text { and } e \leq y^{\dagger}
$$

and

$$
x \leq_{l}^{\prime} y \text { if and only if } x=y f \text { for some } f \in U \text { and } f \leq y^{*} \text {. }
$$

We remark that if $x \leq_{r}^{\prime} y$ then $x^{\dagger} \leq_{\mathcal{R}} y^{\dagger}$. For suppose that $x \leq_{r}^{\prime} y$, then there exists $e \in U$ and $y^{\dagger}$ such that $e \leq y^{\dagger}$ and $x=e y$. Thus,

$$
x^{\dagger} \widetilde{\mathcal{R}}_{U} x=e y \widetilde{\mathcal{R}}_{U} e y^{\dagger}=e \leq y^{\dagger}
$$

and so $x^{\dagger} \leq_{\mathcal{R}} y^{\dagger}$. Dually, if $x \leq_{l}^{\prime} y$ then $x^{*} \leq_{\mathcal{L}} y^{*}$.

Lemma 2.25. On a weakly $U$-abundant semigroup $S$, the relations $\leq_{r}^{\prime}$ and $\leq_{l}^{\prime}$ given above are reflexive and anti-symmetric.

Proof. It is easy to see that $\leq_{r}^{\prime}$ is reflexive as for any $x \in S, x=x^{\dagger} x$. To show that $\leq_{r}^{\prime}$ is anti-symmetric, we suppose that $x \leq_{r}^{\prime} y \leq_{r}^{\prime} x$. Then $x=e y$ and $y=f x$, where $e, f \in U$ and $e \leq y^{\dagger}, f \leq x^{\dagger}$, and so by Lemma 2.8 or the comment above, we have

$$
x^{\dagger} \leq_{\mathcal{R}} e \leq y^{\dagger} \leq_{\mathcal{R}} f \leq x^{\dagger}
$$

so that $x^{\dagger} \mathcal{R} y^{\dagger} \mathcal{R}$ e $\mathcal{R} f$ and consequently, $x=e y=y$.

It is useful to make a short comment on the pair of relations $\leq_{r}^{\prime}$ and $\leq_{l}^{\prime}$. On a weakly $U$-abundant semigroup, $\leq_{r}^{\prime}$ is not transitive. Since if $x \leq_{r}^{\prime} y$ and $y \leq_{r}^{\prime} z$, then there exist $e, f \in U$ such that $e \leq y^{\dagger}, f \leq z^{\dagger}$, and $x=e y, y=f z$. Thus, $x=$ ef $z$. As $y^{\dagger} \widetilde{\mathcal{R}}_{U} y=f z \widetilde{\mathcal{R}}_{U} f z^{\dagger}=f$ and $e \leq y^{\dagger}$, we obtain that $e \leq_{\mathcal{R}} f$, and so by (B21) in Section 1.4, ef is an idempotent. But, we can not guarantee that ef $\in U$, and so $\leq_{r}^{\prime}$ is not transitive. Dually, $\leq_{l}^{\prime}$ is not transitive. Now, we use $\leq_{r}^{\prime t}$ and $\leq_{l}^{\prime t}$ to denote the transitive closures of $\leq_{r}^{\prime}$ and $\leq_{l}^{\prime}$, respectively.

Lemma 2.26. Let $S$ be a weakly $U$-abundant semigroup. Then relations $\leq_{r}^{t}$ and $\leq_{l}^{\prime t}$ are partial orders on $S$.

We call $\leq_{r}$ and $\leq_{l}$ (resp. $\leq_{r}^{\prime}$ and $\leq_{l}^{\prime}, \leq_{r}^{t}$ and $\leq_{l}^{\prime t}$ ) the natural pre-orders (resp. natural relaions, natural partial orders) of a weakly $U$-abundant semigroup $S$. 


\subsubsection{The weakly $U$-regular case}

Here we remind the reader that the natural pre-orders of a weakly $U$-abundant semigroup will be pre-orders on a weakly $U$-regular semigroup. Further the natural relations of a weakly $U$-abundant semigroup will become partial orders on a weakly $U$-regular semigroup, as we now demonstrate.

Lemma 2.27. On a weakly $U$-regular semigroup $S$, the relations $\leq_{r}^{\prime}$ and $\leq_{l}^{\prime}$ given in Section 2.5.1 are partial orders.

Proof. In view of Lemma 2.25, it is sufficient to show that $\leq_{r}^{\prime}$ is transitive and dually, $\leq_{l}^{\prime}$ is transitive. Suppose that $x \leq_{r}^{\prime} y$ and $y \leq_{r}^{\prime} z$. Then there exist $e, f \in U$ such that $e \leq y^{\dagger}, f \leq z^{\dagger}$, and $x=e y, y=f z$. So $x=e f z$. Referring to the comment succeeding Lemma 2.25, we have that $e \leq_{\mathcal{R}} f$ in $U$. As $U$ is a regular biordered set, we obtain that $e f \in U$ by (B1), and so $x \leq_{r}^{\prime} y$.

We call $\leq_{r}^{\prime}$ and $\leq_{l}^{\prime}$ the natural partial orders of a weakly $U$-regular semigroup.

The next lemma presents an equivalent statement for $\leq_{r}^{\prime}$ and $\leq_{l}^{\prime}$ on a weakly $U$-regular semigroup.

Lemma 2.28. Let $S$ be a weakly $U$-regular semigroup. For any $x, y \in S$, we have

(i) $x \leq_{r}^{\prime} y$ if and only if there exists $e \in U$ such that $x=$ ey and $x^{\dagger} \leq_{\mathcal{R}} y^{\dagger}$;

(ii) $x \leq_{l}^{\prime} y$ if and only if there exists $f \in U$ such that $x=y f$ and $x^{*} \leq_{\mathcal{L}} y^{*}$.

Proof. We first show that part $(i)$ holds and dually, part (ii) holds. Suppose that $x, y \in S$. if $x \leq_{r}^{\prime} \quad y$, then by the comment succeeding the definition of $\leq_{r}^{\prime}$ in Section 2.5.1, we have $x=e y$ for some $e \in U$ and $x^{\dagger} \leq_{\mathcal{R}} y^{\dagger}$.

Conversely, if $x=e y$ and $x^{\dagger} \leq_{\mathcal{R}} y^{\dagger}$, then by Lemma 2.8, we have $(e y)^{\dagger} \leq_{\mathcal{R}} e$, and so $x^{\dagger} \leq_{\mathcal{R}} e$. Note that

$$
\left(x^{\dagger} e y^{\dagger}\right)\left(x^{\dagger} e y^{\dagger}\right)=x^{\dagger} e\left(y^{\dagger} x^{\dagger}\right) e y^{\dagger}=x^{\dagger} e x^{\dagger} e y^{\dagger}=x^{\dagger} x^{\dagger} e y^{\dagger}=x^{\dagger} e y^{\dagger}
$$

Since $S$ is weakly $U$-regular, we have that $x^{\dagger} e y^{\dagger} \in U$. Clearly, $x^{\dagger} e y^{\dagger} \leq y^{\dagger}$. Also, we have

$$
x=x^{\dagger} x=x^{\dagger} e y=x^{\dagger} e y^{\dagger} y
$$

Thus, $x \leq_{r}^{\prime} y$. 


\subsubsection{The weakly $B$-orthodox case}

Observe that bands are regular biordered sets, and so a weakly $B$-orthodox semigroup is a special kind of weakly $U$-regular semigroup. Consequently, the natural pre-orders (resp. natural relations) defined in Section 2.5.1 are the natural preorders (resp. natural partial orders) of a weakly $B$-orthodox semigroup $S$. To be easily referred, we present these results in a lemma below.

Lemma 2.29. Let $S$ be a weakly B-orthodox semigroup. Then $\leq_{r}$ and $\leq_{l}$ are pre-orders; $\leq_{r}^{\prime}$ and $\leq_{l}^{\prime}$ are partial orders on $S$. If in addition $S$ satisfies Condition (WIC), then $\leq_{l}^{\prime}=\leq_{r}^{\prime}$.

Proof. Suppose now that $S$ has (WIC) and $x \leq_{r}^{\prime} y$. Then $x=e y$ for some $e \in B$ and $e \leq y^{\dagger}$. By the remark before Lemma 2.25 we have $x^{\dagger} \leq_{\mathcal{R}} y^{\dagger}$, and so $x=y^{\dagger} x=y^{\dagger} e y^{\dagger} y=y f$ for some $f \in B$, since $y^{\dagger} e y^{\dagger} \leq y^{\dagger}$. Clearly as $x=e y$ we have $x^{*} \leq_{\mathcal{L}} y^{*}$. Hence $x \leq_{l}^{\prime} y$. Dually, $\leq_{l}^{\prime} \subseteq \leq_{r}^{\prime}$, so that the two relations coincide.

\subsubsection{The Ehresmann case}

We remark that if $E$ is a semilattice, then a weakly $E$-orthodox semigroup is an Ehresmann semigroup (with distinguished semilattice $E$ ).

Lemma 2.30. Let $S$ be an Ehresmann semigroup with distinguished semilattice E. Then $\leq_{r}=\leq_{r}^{\prime}$ and $\leq_{l}=\leq_{l}^{\prime}$, so that $\leq_{r}$ and $\leq_{l}$ are partial orders.

Proof. In view of Lemma 2.29, $\leq_{r}^{\prime}$ and $\leq_{l}^{\prime}$ are partial orders. Further, we notice that if $x \leq_{r} y$, where $x=e y$ for some $e \in E$, then $x=e y^{\dagger} y$. As $e y^{\dagger} \leq y^{\dagger}$, we have that $x \leq_{r}^{\prime} y$; dually, $\leq_{l}=\leq_{l}^{\prime}$.

Let $S$ be an Ehresmann semigroup with distinguished semilattice of idempotents $E$. In [32], Lawson introduced a partial order on $S$ as follows, for all $x, y \in S$,

$$
x \leq_{e} y \text { if and only if } x=e y f \quad \text { for some } e, f \in E \text {. }
$$

Further, in an Ehresmann semigroup $S$,

$$
\leq_{l} \circ \leq_{r}=\leq_{e}=\leq_{r} \circ \leq_{l}
$$


The following lemma describes some properties of partial orders given above.

Lemma 2.31. [32] Let $S$ be an Ehresmann semigroup with semilattice of distinguished idempotents $E$.

(i) if $x \leq_{r} y$, then $x^{*} \leq y^{*}$ and $x^{\dagger} \leq y^{\dagger}$;

(ii) if $x \leq_{l} y$, then $x^{*} \leq y^{*}$ and $x^{\dagger} \leq y^{\dagger}$;

(iii) if $x \leq_{e} y$, then $x^{*} \leq y^{*}$ and $x^{\dagger} \leq y^{\dagger}$;

(iv) if $x \leq_{r} y, u \leq_{r} v, x^{*}=u^{\dagger}$ and $y^{*}=v^{\dagger}$, then $x u \leq_{r} y v$;

(v) if $x \leq_{l} y, u \leq_{l} v, x^{*}=u^{\dagger}$ and $y^{*}=v^{\dagger}$, then $x u \leq_{l} y v$.

Lemma 2.32. [32] Let $S$ be an Ehresmann semigroup with semilattice of distinguished idempotents $E$. Then

(i) if $e \leq x^{\dagger}$, then there exists a unique element $y \in S$ such that $y^{\dagger}=e$ and $y \leq r x$

(ii) if $e \leq x^{*}$, then there exists a unique element $y \in S$ such that $y^{*}=e$ and $y \leq_{l} x$.

We remark that in view of Lemma 2.18, Lemma 2.29 and Lemma 2.30, relations $\leq_{r}^{\prime}, \leq_{l}^{\prime}, \leq_{r}$ and $\leq_{l}$ coincide on a restriction semigroup, and so we use $\leq$ to denote the natural partial order on a restriction semigroup. In particular, on an inverse semigroup $S$, for all $a, b \in S$, we have

$$
a \leq b \text { if and only if } a=e b \quad(e \in E(S)) .
$$

\subsection{Examples}

This section is concerned with two examples We first show how a weakly $B$ orthodox semigroup may be naturally obtained from a monoid acting via morphisms on the left and right of a band with identity. This construction is reminiscent of that underlying the free ample monoid [13], and we believe will be of subsequent use.

Let $B$ be a band with 1 and let $T$ be a monoid acting on the left and right of $B$ by $\cdot$ and o via morphisms such that

$$
(t \cdot g) \circ t=(1 \circ t) g \text { and } t \cdot(g \circ t)=g(t \cdot 1),
$$


for all $g \in B$ and $t \in T$.

We note that as $T$ acts by morphisms, if $e, f \in B$ with $e \leq_{\mathcal{L}} f$, then for any $t \in T, t \cdot e=t \cdot e f=(t \cdot e)(t \cdot f) \leq_{\mathcal{L}} t \cdot f$, so that $\cdot$ preserves $\leq_{\mathcal{L}}$. Dually, o preserves $\leq_{\mathcal{R}}$.

Let $S=B *_{1} T=\left\{(e, t): e \leq_{\mathcal{L}} t \cdot 1\right\} \subseteq B \times T$ with semidirect product multiplication, i.e.

$$
(e, t)(f, s)=(e(t \cdot f), t s)
$$

Now if $e \leq_{\mathcal{L}} t \cdot 1$ and $f \leq_{\mathcal{L}} s \cdot 1$, then $t \cdot f \leq_{\mathcal{L}} t \cdot(s \cdot 1)=t s \cdot 1$, and so $e(t \cdot f) \leq_{\mathcal{L}} t s \cdot 1$. Thus $S$ is closed, and consequently, it is a semigroup.

We pause here to make a short comment on the above construction. We required the monoid $T$ acting on both sides of the band $B$, but when we constructed the semigroup $S$ we only used the action of $T$ on the left of $B$. The action of $T$ on the right of $B$ is helpful to show that each $\widetilde{\mathcal{L}}_{\bar{B}}$-class of $S$ contains an idempotent which appears below. Here $\bar{B}=\{(e, 1): e \in B\}$.

We now obtain a series of lemmas to verify that $S$ constructed above is a weakly $\bar{B}$-orthodox semigroup.

Lemma 2.33. The set $\bar{B}=\{(e, 1): e \in B\}$ is isomorphic to $B$.

Proof. Let $e, f \in B$. Then $e \leq_{\mathcal{L}} 1_{B}=1_{T} \cdot 1_{B}$ and $(e, 1)(f, 1)=(e(1 \cdot f), 1)=$ (ef, 1 ), whence it follows that $\bar{B}$ is a band isomorphic to $B$.

Lemma 2.34. For any $(e, t) \in S,(e, t) \widetilde{\mathcal{R}}_{\bar{B}}(e, 1)$.

Proof. Let $(e, t) \in S$. Then $(e, 1)(e, t)=(e(1 \cdot e), t)=(e, t)$ and if $(f, 1)(e, t)=$ $(e, t)$, then $(f e, t)=(e, t)$, so $f e=e$ and $(f, 1)(e, 1)=(e, 1)$. Thus, $(e, t) \widetilde{\mathcal{R}}_{\bar{B}}(e, 1)$.

Let $(e, t),(f, s) \in S$. By Lemmas 2.33 and 2.34,

$$
(e, t) \widetilde{\mathcal{R}}_{\bar{B}}(f, s) \Leftrightarrow e \mathcal{R} f
$$

Lemma 2.35. For any $(e, t) \in S,(e, t) \widetilde{\mathcal{L}}_{\bar{B}}(e \circ t, 1)$.

Proof. Let $(e, t) \in S$. Then

$$
\begin{aligned}
(e, t)(e \circ t, 1) & =(e(t \cdot(e \circ t)), t) \\
& =(e(e(t \cdot 1)), t) \\
& =(e, t) \quad\left(e \leq_{\mathcal{L}} t \cdot 1\right) .
\end{aligned}
$$


Further, if $(e, t)(f, 1)=(e, t)$, then $e(t \cdot f)=e$. Now

$$
\begin{aligned}
e \circ t & =(e(t \cdot f)) \circ t=(e \circ t)((t \cdot f) \circ t) \\
& =(e \circ t)(1 \circ t) f=((e 1) \circ t) f \\
& =(e \circ t) f,
\end{aligned}
$$

so $(e \circ t, 1)(f, 1)=(e \circ t, 1)$. Thus $(e, t) \widetilde{\mathcal{L}}_{\bar{B}}(e \circ t, 1)$.

Again by Lemma 2.33, $(e, t) \widetilde{\mathcal{L}}_{\bar{B}}(f, s)$ if and only if $e \circ t \mathcal{L} f \circ s$ in $B$.

Lemma 2.36. The semigroup $S$ is weakly $\bar{B}$-orthodox, where $\bar{B}=\{(e, 1): e \in$ $B\}$.

Proof. In view of Lemma 2.33, 2.34 and 2.35, it is sufficient to show that $S$ has (C). Suppose that $(e, t) \widetilde{\mathcal{R}}_{\bar{B}}(f, s)$ and $(g, u) \in S$. Then $(g, u)(e, t)=(g(u \cdot e), u t)$ and $(g, u)(f, s)=(g(u \cdot f), u s)$. As $e \mathcal{R} f$ we have $u \cdot e \mathcal{R} u \cdot f$ and then $g(u \cdot e) \mathcal{R} g(u \cdot f)$, so that $\widetilde{\mathcal{R}}_{\bar{B}}$ is a left congruence.

Now let $(e, t) \widetilde{\mathcal{L}}_{\bar{B}}(f, s)$ and $(g, u) \in S$. Then $(e, t)(g, u)=(e(t \cdot g), t u)$ and $(f, s)(g, u)=(f(s \cdot g), s u)$. We have

$$
\begin{aligned}
(e(t \cdot g)) \circ t & =(e \circ t)((t \cdot g) \circ t) \\
& =(e \circ t)(1 \circ t) g \\
& =(e \circ t) g \\
\mathcal{L} & (f \circ s) g=(f(s \cdot g) \circ s)
\end{aligned}
$$

so that $(e(t \cdot g)) \circ t u \mathcal{L}(f(s \cdot g)) \circ s u$. Thus $\widetilde{\mathcal{L}}_{\bar{B}}$ is a right congruence. Hence, $S$ is weakly $\bar{B}$-orthodox.

We now present another example which is that of a weakly $U$-regular semigroup, that is not necessarily weakly $B$-orthodox.

Let $M$ be a monoid and $I, \Lambda$ be non-empty sets. Let $P=\left(p_{\lambda i}\right)$ be a $\Lambda \times I$ matrix with entries being unit elements in $M$.

Let $S=I \times M \times \Lambda$ and define a composition on $S$ by

$$
(i, a, \lambda)(j, b, \mu)=\left(i, a p_{\lambda j} b, \mu\right)
$$

Then, we have: 
Lemma 2.37. The set $S$ forms a weakly $U$-abundant semigroup under the operation defined above, where $U=\left\{\left(i, p_{\lambda i}^{-1}, \lambda\right): i \in I, \lambda \in \Lambda\right\}$.

Proof. Clearly, $S$ forms a semigroup.

For any $\left(i, p_{\lambda i}^{-1}, \lambda\right) \in U$, we have

$$
\left(i, p_{\lambda i}^{-1}, \lambda\right)\left(i, p_{\lambda i}^{-1}, \lambda\right)=\left(i, p_{\lambda i}^{-1} p_{\lambda i} p_{\lambda i}^{-1}, \lambda\right)=\left(i, p_{\lambda i}^{-1}, \lambda\right)
$$

and so $U \subseteq E(S)$.

To show that $S$ is weakly $U$-abundant, we first show that for any $(i, a, \lambda) \in S$ and $\left(j, p_{\mu j}^{-1}, \mu\right) \in U$,

$$
(i, a, \lambda) \widetilde{\mathcal{R}}_{U}\left(j, p_{\mu j}^{-1}, \mu\right) \text { if and only if } i=j,
$$

and dually,

$$
(i, a, \lambda) \widetilde{\mathcal{L}}_{U}\left(j, p_{\mu j}^{-1}, \mu\right) \text { if and only if } \lambda=\mu \text {. }
$$

Suppose that $(i, a, \lambda) \widetilde{\mathcal{R}}_{U}\left(j, p_{\mu j}^{-1}, \mu\right)$, then we have

$$
\left(j, p_{\mu j}^{-1}, \mu\right)(i, a, \lambda)=(i, a, \lambda)
$$

that is,

$$
\left(j, p_{\mu j}^{-1} p_{\mu i} a, \lambda\right)=(i, a, \lambda)
$$

and so $i=j$.

Conversely, if $i=j$, then

$$
\left(i, p_{\mu i}^{-1}, \mu\right)(i, a, \lambda)=\left(i, p_{\mu i}^{-1} p_{\mu i} a, \lambda\right)=(i, a, \lambda) .
$$

Suppose that $\left(k, p_{\gamma k}^{-1}, \gamma\right) \in U$ is such that $\left(k, p_{\gamma k}^{-1}, \gamma\right)(i, a, \lambda)=(i, a, \lambda)$. Then we obtain that $\left(k, p_{\gamma k}^{-1} p_{\gamma i} a, \lambda\right)=(i, a, \lambda)$, and so we must have $k=i$ so that

$$
\begin{aligned}
\left(k, p_{\gamma k}^{-1}, \gamma\right)\left(i, p_{\mu i}^{-1}, \mu\right) & =\left(k, p_{\gamma k}^{-1} p_{\gamma i} p_{\mu i}^{-1}, \mu\right) \\
& =\left(i, p_{\gamma i}^{-1} p_{\gamma i} p_{\mu i}^{-1}, \mu\right) \quad(k=i) \\
& =\left(i, p_{\mu i}^{-1}, \mu\right)
\end{aligned}
$$

Thus, $(i, a, \lambda) \widetilde{\mathcal{R}}_{U}\left(i, p_{\mu i}^{-1}, \mu\right)$. Hence, $S$ is a weakly $U$-abundant semigroup. 
In view of the proof of Lemma 2.37, the next lemma is immediate.

Lemma 2.38. For any $(i, a, \lambda),(j, b, \mu) \in S$, we have

(i) $(i, a, \lambda) \widetilde{\mathcal{R}}_{U}(j, b, \mu)$ if and only if $i=j$;

(ii) $(i, a, \lambda) \widetilde{\mathcal{L}}_{U}(j, b, \mu)$ if and only if $\lambda=\mu$.

Lemma 2.39. The semigroup $S$ satisfies $(C)$.

Proof. Suppose that $(i, a, \lambda),(j, b, \mu),(k, c, \gamma) \in S$ are such that $(i, a, \lambda) \widetilde{\mathcal{R}}_{U}(j, b, \mu)$. Then $i=j$. Observe that

$$
\begin{array}{rlr}
(k, c, \gamma)(i, a, \lambda) & =\left(k, c p_{\gamma i} a, \lambda\right) & \\
& \widetilde{\mathcal{R}}_{U}\left(k, c p_{\gamma i} b, \mu\right) & \\
& =(k, c, \gamma)(i, b, \mu) & \\
& =(k, c, \gamma)(j, b, \mu) & (i=j)
\end{array}
$$

so that $\widetilde{\mathcal{R}}_{U}$ is a left congruence. Dually, we show that $\widetilde{\mathcal{L}}_{U}$ is a right congruence.

Lemma 2.40. If e, $f \in U$, then e $\mathcal{R}$ ef $\mathcal{L} f$ in $\langle U\rangle$.

Proof. Let $e=\left(i, p_{\lambda i}^{-1}, \lambda\right)$ and $f=\left(j, p_{\mu j}^{-1}, \mu\right) \in U$. Then

$$
\text { ef }=\left(i, p_{\lambda i}^{-1}, \lambda\right)\left(j, p_{\mu j}^{-1}, \mu\right)=\left(i, p_{\lambda i}^{-1} p_{\lambda j} p_{\mu j}^{-1}, \mu\right)
$$

Take $a=\left(j, p_{\lambda j}^{-1}, \lambda\right)\left(i, p_{\lambda i}^{-1}, \lambda\right) \in\langle U\rangle$. Then we have

$$
\begin{aligned}
e f a & =\left(i, p_{\lambda i}^{-1} p_{\lambda j} p_{\mu j}^{-1}, \mu\right)\left(j, p_{\lambda j}^{-1}, \lambda\right)\left(i, p_{\lambda i}^{-1}, \lambda\right) \\
& =\left(i, p_{\lambda i}^{-1} p_{\lambda j} p_{\mu j}^{-1} p_{\mu j} p_{\lambda j}^{-1} p_{\lambda i} p_{\lambda i}^{-1}, \lambda\right) \\
& =\left(i, p_{\lambda i}^{-1}, \lambda\right) \\
& =e
\end{aligned}
$$

and eef $=$ ef so that ef $\mathcal{R}$ e in $\langle U\rangle$. Dually, we have ef $\mathcal{L} f$ in $\langle U\rangle$.

Lemma 2.41. The semigroup $\langle U\rangle$ generated by $U$ is regular and $E(\langle U\rangle)=U$.

Proof. Let $(i, a, \lambda) \in\langle U\rangle$. Then

$$
(i, a, \lambda)=e_{1} \cdots e_{n} \quad\left(e_{1}, \cdots, e_{n} \in U\right)
$$


We now show that $(i, a, \lambda) \mathcal{R} e_{1}$ in $\langle U\rangle$ and dually, we have $a \mathcal{L} e_{n}$ in $\langle U\rangle$. By Lemma 2.40, if $n=2$, then certainly the statement holds. We assume that $e_{2} \cdots e_{n} \mathcal{R} e_{2}$ in $\langle U\rangle$. Then

$$
\begin{aligned}
(i, a, \lambda)=e_{1}\left(e_{2} \cdots e_{n}\right) & \mathcal{R} e_{1} e_{2} \\
& \mathcal{R} e_{1} \quad \text { (Lemma 2.40). }
\end{aligned}
$$

Hence, $\langle U\rangle$ is a regular semigroup.

With $(i, a, \lambda)$ as in the statement of this lemma, we have $(i, a, \lambda) \mathcal{R}\left(i, p_{\mu i}^{-1}, \mu\right)$ for any $\mu \in \Lambda$, and if $\left(k, p_{\lambda k}^{-1}, \lambda\right) \in U$, then we have $(i, a, \lambda) \mathcal{L}\left(k, p_{\lambda k}^{-1}, \lambda\right)$.

Now, we show that $E(\langle U\rangle)=U$. Clearly $U \subseteq E(\langle U\rangle)$. To show that $E(\langle U\rangle) \subseteq U$, we suppose that $(i, a, \lambda) \in E(\langle U\rangle)$. Then $(i, a, \lambda) \mathcal{H}\left(i, p_{\lambda i}^{-1}, \lambda\right)$. Since both $(i, a, \lambda)$ and $\left(i, p_{\lambda i}^{-1}, \lambda\right)$ are idempotent and each $\mathcal{H}$-class contains at most one idempotent, we must have that $(i, a, \lambda)=\left(i, p_{\lambda i}^{-1}, \lambda\right)$ so that $E(\langle U\rangle) \subseteq$ $U$.

To sum up, we have :

Theorem 2.42. The semigroup $S$ is a weakly $U$-regular semigroup, where $U=$ $\left\{\left(i, p_{\lambda i}^{-1}, \lambda\right): i \in I, \lambda \in \Lambda\right\}$. 


\section{Chapter 3}

\section{Weakly $U$-superabundant semigroups with $(\mathrm{C})$}

A weakly $U$-superabundant semigroup is a weakly $U$-abundant semigroup in which every $\widetilde{\mathcal{H}}_{U}$-class contains a distinguished idempotent of $U$. Such semigroups are analogous to completely regular semigroups. The purpose of this chapter is to build a complete construction modulo the semilattice decomposition for weakly $U$-superabundant semigroups with $(\mathrm{C})$.

We make the convention that $B$ will always denote a band. Green's relation $\mathcal{D}$ will always refer to $B$, unless stated otherwise. To avoid ambiguity, if $\mathcal{K}$ is a relation on a semigroup $S$, then we will use $\mathcal{K}(S)$ to denote the relation on $S$ in some places.

\subsection{Weakly $U$-superabundant semigroups with (C)}

In this section, we are concerned with some properties of weakly $U$-superabundant semigroups with $(\mathrm{C})$, which broadly determine their structure.

Lemma 3.1. [33] If $S$ is a weakly U-superabundant semigroup, then

$$
\widetilde{\mathcal{D}}_{U}=\widetilde{\mathcal{L}}_{U} \circ \widetilde{\mathcal{R}}_{U}=\widetilde{\mathcal{R}}_{U} \circ \widetilde{\mathcal{L}}_{U}
$$

Further: 
Lemma 3.2. Let $S$ be a weakly U-superabundant semigroup. If distinguished idempotents e, $f \in U$ are $\widetilde{\mathcal{D}}_{U}$-related, then there exists $h \in U$ such that e $\mathcal{L} h \mathcal{R} f$. Proof. Let $e, f \in U$ be such that $e \widetilde{\mathcal{D}}_{U} f$. By Lemma 3.1, there exists $x \in S$ such that $e \widetilde{\mathcal{L}}_{U} x \widetilde{\mathcal{R}}_{U} f$. As $S$ is weakly $U$-superabundant, there exists $h \in U$ such that $h \widetilde{\mathcal{H}}_{U} x$, and so $e \widetilde{\mathcal{L}}_{U} h \widetilde{\mathcal{R}}_{U} f$. Thus, e $\mathcal{L} h \mathcal{R} f$.

As an analogue of the fact that $\mathcal{J}=\mathcal{D}$ on a completely regular semigroup, we have:

Lemma 3.3. [44] If $S$ is a weakly $U$-superabundant semigroup with $(C)$, then $\widetilde{\mathcal{J}}_{U}=\widetilde{\mathcal{D}}_{U}$, and $\widetilde{\mathcal{J}}_{U}$ is a semilattice congruence on $S$.

From Lemma 3.3, if $S$ is a weakly $U$-superabundant semigroup with (C), then each $\widetilde{\mathcal{D}}_{U}$-class forms a semigroup. We can say more.

Lemma 3.4. If $a, b$ are $\widetilde{\mathcal{D}}_{U}$-equivalent elements in a weakly $U$-superabundant semigroup $S$ with $(C)$, then a $\widetilde{\mathcal{R}}_{U} a b \widetilde{\mathcal{L}}_{U}$ b.

Proof. Suppose that $a \widetilde{\mathcal{D}}_{U} b$ in $S$ and $e, f \in U$ with $e \widetilde{\mathcal{H}}_{U} a, f \widetilde{\mathcal{H}}_{U} b$. Then e $\widetilde{\mathcal{D}}_{U} f$. By Lemma 3.2, there exists $k \in U$ such that $e \mathcal{L} k \mathcal{R} f$, and so

$$
a b \widetilde{\mathcal{R}}_{U} \text { af } \widetilde{\mathcal{R}}_{U} a k=a \text { and } a b \widetilde{\mathcal{L}}_{U} \text { eb } \widetilde{\mathcal{L}}_{U} k b=b
$$

Hence, $a \widetilde{\mathcal{R}}_{U} a b \widetilde{\mathcal{L}}_{U} b$.

It is useful to mention the next lemma.

Lemma 3.5. [33] If a weakly $U$-abundant semigroup $S$ satisfies $(C)$ and $e \in U$, then $\widetilde{H}_{e}$ is a monoid with respect to $e$.

Lemma 3.6. Let $S$ be a weakly $U$-superabundant semigroup with $(C)$ and let $a \in S$ and $e, f, h \in U$ be such that $h \widetilde{\mathcal{H}}_{U}$ a and $e \widetilde{\mathcal{L}}_{U} a \widetilde{\mathcal{R}}_{U} f$. Then the right translations $\left.\rho_{f}\right|_{\widetilde{L}_{a}},\left.\rho_{h}\right|_{\widetilde{L}_{f}}$ are mutually inverse $\widetilde{\mathcal{R}}_{U}$-class preserving bijections from $\widetilde{L}_{a}$ onto $\widetilde{L}_{f}$ and $\widetilde{L}_{f}$ onto $\widetilde{L}_{a}$, respectively.

Proof. It is easy to see that $\left.\rho_{f}\right|_{\widetilde{L}_{a}}$ is a map from $\widetilde{L}_{a}$ to $\widetilde{L}_{f}$ since for any $x \in \widetilde{L}_{a}$, $x \rho_{f}=x f \widetilde{\mathcal{L}}_{U} h f=f$. Similarly, we can show that $\left.\rho_{h}\right|_{\widetilde{L}_{f}}$ is a map from $\widetilde{L}_{f}$ to $\widetilde{L}_{a}$. And we deduce that for any $x \in \widetilde{L}_{a}$ and $y \in \widetilde{L}_{f}$,

$$
x \rho_{f} \rho_{h}=x f h=x(f h)=x h=x \quad \text { and } \quad y \rho_{h} \rho_{f}=y h f=y(h f)=y f=y \text {. }
$$


Thus the right translations $\left.\rho_{f}\right|_{\widetilde{L}_{a}},\left.\rho_{h}\right|_{\widetilde{L}_{f}}$ are mutually inverse bijections from $\widetilde{L}_{a}$ onto $\widetilde{L}_{f}$ and $\widetilde{L}_{f}$ onto $\widetilde{L}_{a}$, respectively. Since $f \widetilde{\mathcal{R}}_{U} h$ and $\widetilde{\mathcal{R}}_{U}$ is a left congruence it follows that for any $x \in \widetilde{L}_{a}, x f \widetilde{\mathcal{R}}_{U} x h=x$, that is, the right translation $\left.\rho_{f}\right|_{\widetilde{L}_{a}}$ preserves the $\widetilde{\mathcal{R}}_{U}$-class. By a similar argument, we have the right translation $\left.\rho_{h}\right|_{\widetilde{L}_{f}}$ preserves the $\widetilde{\mathcal{R}}_{U}$-class.

We have a left-right dual featuring:

Lemma 3.7. Let $S$ be a weakly $U$-superabundant semigroup with $(C)$ and let $a \in S$ and $e, f, h \in U$ be such that $h \widetilde{\mathcal{H}}_{U}$ a and $e \widetilde{\mathcal{L}}_{U} a \widetilde{\mathcal{R}}_{U} f$. Then the left translations $\left.\lambda_{e}\right|_{\widetilde{R}_{a}},\left.\lambda_{h}\right|_{\widetilde{R}_{e}}$ are mutually inverse $\widetilde{\mathcal{L}}_{U}$-class preserving bijections from $\widetilde{R}_{a}$ onto $\widetilde{R}_{e}$ and $\widetilde{R}_{e}$ onto $\widetilde{R}_{a}$, respectively.

Lemma 3.8. If $a, b$ are $\widetilde{\mathcal{D}}_{U}$-equivalent elements in a weakly $U$-superabundant semigroup $S$ with $(C)$, then $\widetilde{H}_{a}$ is isomorphic to $\widetilde{H}_{b}$.

Proof. Suppose that $a \widetilde{\mathcal{D}}_{U} b$ in $S$ and $e, f \in U$ with $e \widetilde{\mathcal{H}}_{U} a, f \widetilde{\mathcal{H}}_{U} b$. By Lemma 3.1, there exists $c \in S$ such that $a \widetilde{\mathcal{R}}_{U} c \widetilde{\mathcal{L}}_{U} b$. Since $S$ is a weakly $U$-superabundant semigroup it follows that there is a distinguished idempotent $h \in U$ such that $h \widetilde{\mathcal{H}}_{U}$ c. Due to Lemma 3.6, $\left.\rho_{h}\right|_{\widetilde{H}_{a}}$ and $\left.\rho_{e}\right|_{\widetilde{H}_{c}}$ are mutually inverse bijections from $\widetilde{H}_{a}$ onto $\widetilde{H}_{c}$ and from $\widetilde{H}_{c}$ onto $\widetilde{H}_{a}$, respectively. By Lemma $3.7,\left.\lambda_{f}\right|_{\widetilde{H}_{c}}$ and $\left.\lambda_{h}\right|_{\widetilde{H}_{b}}$ are mutually inverse bijections from $\widetilde{H}_{c}$ onto $\widetilde{H}_{b}$ and from $\widetilde{H}_{b}$ onto $\widetilde{H}_{c}$, respectively. So we have that $\left.\left.\rho_{h}\right|_{\widetilde{H}_{a}} \lambda_{f}\right|_{\widetilde{H}_{c}}$ and $\left.\left.\lambda_{h}\right|_{\widetilde{H}_{b}} \rho_{e}\right|_{\widetilde{H}_{c}}$ are mutually inverse bijections from $\widetilde{H}_{a}$ onto $\widetilde{H}_{b}$ and $\widetilde{H}_{b}$ onto $\widetilde{H}_{a}$, respectively.

We still need to show that $\left.\left.\rho_{h}\right|_{\widetilde{H}_{a}} \lambda_{f}\right|_{\widetilde{H}_{c}}$ and $\left.\left.\lambda_{h}\right|_{\widetilde{H}_{b}} \rho_{e}\right|_{\widetilde{H}_{c}}$ are morphisms. To show that $\left.\left.\rho_{h}\right|_{\widetilde{H}_{a}} \lambda_{f}\right|_{\widetilde{H}_{c}}$ is a morphism, it is sufficient to prove that both $\left.\lambda_{f}\right|_{\widetilde{H}_{c}}$ and $\left.\rho_{h}\right|_{\widetilde{H}_{a}}$ are morphisms. If $x, y \in \widetilde{H}_{a}$, then $x \widetilde{\mathcal{R}}_{U} h \widetilde{\mathcal{R}}_{U} y$, and so $(x y) \rho_{h}=x y h=$ $x(h y) h=(x h)(y h)=\left(x \rho_{h}\right)\left(y \rho_{h}\right)$. Thus $\left.\rho_{h}\right|_{\widetilde{H}_{a}}$ is a morphism. Dually, we can show that $\left.\lambda_{f}\right|_{\widetilde{H}_{c}}$ is a morphism. Hence $\left.\left.\rho_{h}\right|_{\widetilde{H}_{a}} \lambda_{f}\right|_{\widetilde{H}_{c}}$ is a morphism as required. Similarly, we can show that the composition $\left.\left.\lambda_{h}\right|_{\widetilde{H}_{b}} \rho_{e}\right|_{\widetilde{H}_{c}}$ is a morphism. So $\widetilde{H}_{a}$ is isomorphic to $\widetilde{H}_{b}$ as required.

In view of Lemma 3.1 , we can use an egg-box picture to depict each $\widetilde{\mathcal{D}}_{U}$-class of a weakly $U$-superabundant semigroup $S$. Let $D$ denote a typical $\widetilde{\mathcal{D}}_{U}$-class of $S$. We denote the set of $\widetilde{\mathcal{R}}_{U}$-classes of $S$ in $D$ by $I$ and the set of $\widetilde{\mathcal{L}}_{U}$-classes of $S$ in $D$ by $\Lambda$. As a matter of notation we shall treat $I$ and $\Lambda$ as index sets and write the $\widetilde{\mathcal{R}}_{U^{-}}$classes as $\widetilde{R}_{i}(i \in I)$ and the $\widetilde{\mathcal{L}}_{U^{-}}$classes as $\widetilde{L}_{\lambda}(\lambda \in \Lambda)$. The $\widetilde{\mathcal{H}}_{U^{-}}$class $\widetilde{R}_{i} \cap \widetilde{L}_{\lambda}$ is denoted by $\widetilde{H}_{i \lambda}$. 
Lemma 3.9. If $S$ is a weakly $U$-superabundant semigroup with $(C)$, then each $\widetilde{\mathcal{D}}_{U}$-class of $S$ is a rectangular band of its $\widetilde{\mathcal{H}}_{U}$-classes, which are isomorphic monoids.

Proof. Let $D$ denote a typical $\widetilde{\mathcal{D}}_{U^{-}}$class of $S$. In view of Lemma 3.5 , each $\widetilde{\mathcal{H}}_{U^{-}}$ class is a monoid, and so $a \widetilde{\mathcal{H}}_{U} a^{2}$, for all $a \in S$. Let $i, j \in I$ and $\mu, \lambda \in \Lambda$. We assume that $a \in \widetilde{H}_{i \lambda}$ and $b \in \widetilde{H}_{j \mu}$. By Lemma 3.4, we have $a \widetilde{\mathcal{R}}_{U} a b \widetilde{\mathcal{L}}_{U} b$, that is, $a b \in \widetilde{H}_{i \mu}$, or equivalently, $\widetilde{H}_{i \lambda} \widetilde{H}_{j \mu} \subseteq \widetilde{H}_{i \mu}$. Thus each $\widetilde{\mathcal{D}}_{U}$-class is a rectangular band of monoids, which are isomorphic by Lemma 3.8.

Next, we present an equivalent statement for a weakly $B$-superabundant semigroup, where $B$ is a band.

Lemma 3.10. Let $S$ be a weakly B-abundant semigroup. For any $e, f \in B$,

$$
e \widetilde{\mathcal{D}}_{B} f \Leftrightarrow e \mathcal{D} f
$$

if and only if $S$ is a weakly B-superabundant semigroup.

Proof. In view of Lemma 3.2, it is sufficient to show the necessity. Suppose that $x \in S$. Certainly, we have that $x^{\dagger} \widetilde{\mathcal{R}}_{B} x \widetilde{\mathcal{L}}_{B} x^{*}$ for some $x^{\dagger}, x^{*} \in B$. It follows that $x^{\dagger} \widetilde{\mathcal{D}}_{B} x^{*}$. By the hypothesis, we get $x^{\dagger} \mathcal{D} x^{*}$. So $x^{\dagger} x^{*} \mathcal{L} x^{*} \widetilde{\mathcal{L}}_{B} x$ and $x^{\dagger} x^{*} \mathcal{R} x^{\dagger} \widetilde{\mathcal{R}}_{B} x$. Thus $x^{\dagger} x^{*} \widetilde{\mathcal{H}}_{B} x$. Hence $S$ is a weakly $B$-superabundant semigroup.

As an immediate consequence of Lemma 3.10, if $S$ is a weakly $B$-superabundant and $B$ is a band, then $\mathcal{D}(B)=\widetilde{\mathcal{D}}_{B}(B)$.

Lemma 3.11. A weakly B-orthodox semigroup $S$ is B-superabundant if and only if $S / \gamma$ is weakly $B / \mathcal{D}$-superabundant, where $\gamma$ is any admissible congruence on $S$ such that $\gamma \cap(B \times B)=\mathcal{D}$.

Proof. It is easy to see that the necessity holds. It remains to show the converse is true. Suppose that $S$ is a weakly $B$-orthodox semigroup and $S / \gamma$ is weakly $B / \mathcal{D}$-superabundant. Then for any $a \in S$, there exists an idempotent $e \in B$ such that $a \gamma \widetilde{\mathcal{H}}_{B / \mathcal{D}} e \gamma$. Of course, we have $a^{\dagger} \gamma \widetilde{\mathcal{R}}_{B / \mathcal{D}} a \gamma \widetilde{\mathcal{L}}_{B / \mathcal{D}} a^{*} \gamma$ for any $a^{\dagger} \in \widetilde{R}_{a} \cap B$, $a^{*} \in \widetilde{L}_{a} \cap B$. It follows that $a^{\dagger} \gamma \widetilde{\mathcal{R}}_{B / \mathcal{D}} e \gamma \widetilde{\mathcal{L}}_{B / \mathcal{D}} a^{*} \gamma$, that is, $a^{\dagger} \gamma \mathcal{R}_{B / \mathcal{D}} e \gamma \mathcal{L}_{B / \mathcal{D}} a^{*} \gamma$, and so $a^{\dagger} \gamma=e \gamma=a^{*} \gamma$ since $B / \gamma=B / \mathcal{D}$ is a semilattice. Therefore, $a^{\dagger} \mathcal{D}$ e $\mathcal{D} a^{*}$, 
which implies that $a^{\dagger} \mathcal{R} a^{\dagger} a^{*} \mathcal{L} a^{*}$, and so $a^{\dagger} a^{*} \widetilde{\mathcal{H}}_{B} a$. Hence, $S$ is weakly $B$ superabundant.

In Chapter 2, given a weakly $B$-orthodox semigroup $S$ with (WIC) (resp. (IC)), a relation $\delta_{B}$ defined by the rule that for any $a, b \in S, a \delta_{B} b$ if and only if $a=e b f, b=g a h$ for some $e, f, g, h \in B$, is an admissible congruence on $S$ with the property that $\delta_{B} \cap(B \times B)=\mathcal{D}$. It follows from Lemma 3.11 that a weakly $B$-orthodox semigroup $S$ with (WIC) (resp. (IC)) is weakly $B$-superabundant if and only if $S / \delta_{B}$ is weakly $B / \mathcal{D}$-superabundant.

\subsection{Completely $\widetilde{\mathcal{J}}_{U}$-simple semigroups}

A semigroup $S$ is called $\widetilde{\mathcal{J}}_{U}$-simple if $\widetilde{\mathcal{J}}_{U}$ is the universal relation on $S$. A weakly $U$-abundant semigroup $S$ is called completely $\widetilde{\mathcal{J}}_{U}$-simple if $S$ is a weakly $U$-superabundant semigroup with (C) and is $\widetilde{\mathcal{J}}_{U}$-simple.

In [44], Ren, Shum and Quo built the analogue for weakly $U$-superabundant semigroups with $(\mathrm{C})$ of the structure theorem for superabundant semigroups as follows.

Theorem 3.12. [44] A semigroup $S$ is a weakly $U$-superabundant semigroup with $(C)$ if and only if $S$ is a semilattice $Y$ of completely $\widetilde{\mathcal{J}}_{U}$-simple semigroups $S_{\alpha}(\alpha \in Y)$ such that for all $\alpha, \beta \in Y$, the following statements hold:

(i) for each $a \in S_{\alpha}, \widetilde{L}_{a}(S)=\widetilde{L}_{a}\left(S_{\alpha}\right)$ and $\widetilde{R}_{a}(S)=\widetilde{R}_{a}\left(S_{\alpha}\right)$;

(ii) for all $a, b \in S_{\alpha}$ and $x \in S_{\beta},(a, b) \in \widetilde{\mathcal{L}}_{U}\left(S_{\alpha}\right)$ implies $(a x, b x) \in \widetilde{\mathcal{L}}_{U}\left(S_{\alpha \beta}\right)$ and $(a, b) \in \widetilde{\mathcal{R}}_{U}\left(S_{\alpha}\right)$ implies $(x a, x b) \in \widetilde{\mathcal{R}}_{U}\left(S_{\alpha \beta}\right)$.

In the following, we construct a completely $\widetilde{\mathcal{J}}_{U}$-simple semigroup from a set of monoids. Together with Theorem 3.12, we succeed in obtaining a complete construction for weakly $U$-superabundant semigroups with (C).

Let $I, \Lambda$ be non-empty sets. For each $(i, \lambda) \in I \times \Lambda$, let $M_{i \lambda}$ be a monoid with identity $e_{i \lambda}$. We denote a rectangular band of monoids $M_{i \lambda}(i \in I, \lambda \in \Lambda$ ) by $T$ and denote by $U$ the set of $\left\{e_{i \lambda}: i \in I, \lambda \in \Lambda\right\}$. In order to consider the relations $\widetilde{\mathcal{R}}_{U}$ and $\widetilde{\mathcal{L}}_{U}$ on $T$, we make a convention that for $i, j \in I$ and $\lambda, \mu \in \Lambda$,

$(R)$ if $i=j$ then $e_{i \lambda} e_{j \mu}=e_{j \mu}$ and $e_{j \mu} e_{i \lambda}=e_{i \lambda}$;

$(L)$ if $\lambda=\mu$ then $e_{i \lambda} e_{j \mu}=e_{i \lambda}$ and $e_{j \mu} e_{i \lambda}=e_{j \mu}$.

The next lemma follows immediately. 
Lemma 3.13. If the rectangular band $T$ of monoids $M_{i \lambda}(i \in I, \lambda \in \Lambda)$ satisfies Conditions $(R)$ and $(L)$, then for any $a, b \in T$ with $a \in M_{i \lambda}$ and $b \in M_{j \mu}$,

$$
\begin{gathered}
a \widetilde{\mathcal{R}}_{U} b \Leftrightarrow i=j, \\
a \widetilde{\mathcal{L}}_{U} b \Leftrightarrow \lambda=\mu,
\end{gathered}
$$

and consequently,

$$
a \widetilde{\mathcal{H}}_{U} b \Leftrightarrow i=j \text { and } \lambda=\mu \text {. }
$$

Proof. We prove $\widetilde{\mathcal{R}}_{U}$ case. Dually, the $\widetilde{\mathcal{L}}_{U}$ case holds, and the $\widetilde{\mathcal{H}}_{U}$ case follows from the result for $\widetilde{\mathcal{R}}_{U}$ and $\widetilde{\mathcal{L}}_{U}$. Let $a \in M_{i \lambda}$ and $b \in M_{j \mu}$ be such that $a \widetilde{\mathcal{R}}_{U} b$. Suppose that $f \in U$ with $f a=a$. Then $f=e_{i \nu} \in M_{i \nu}$ for some $\nu \in \Lambda$. Since $a \widetilde{\mathcal{R}}_{U} b$, it follows that $f b=b$, which leads to $i=j$.

Conversely, suppose that $i=j$. Then by Condition $(R), e_{i \lambda} e_{i \mu}=e_{i \mu}$ and $e_{i \mu} e_{i \lambda}=e_{i \lambda}$, that is, $e_{i \lambda} \mathcal{R} e_{i \mu}$. Next, we shall claim that $a \widetilde{\mathcal{R}}_{U} e_{i \lambda}$ and $b \widetilde{\mathcal{R}}_{U} e_{i \mu}$. Certainly, we have $e_{i \lambda} a=a$. Suppose that $e_{k \varepsilon} \in U$ with $e_{k \varepsilon} a=a$. Then $k=i$ and by Condition $(R)$, we have $e_{k \varepsilon} e_{i \lambda}=e_{i \lambda}$. So a $\widetilde{\mathcal{R}}_{U} e_{i \lambda}$. Similarly, we could deduce that $b \widetilde{\mathcal{R}}_{U} e_{i \mu}$. Together with $e_{i \lambda} \mathcal{R} e_{i \mu}$, we have $a \widetilde{\mathcal{R}}_{U} b$.

Furthermore, we can get the next result.

Lemma 3.14. If the rectangular band $T$ of monoids $M_{i \lambda}(i \in I, \lambda \in \Lambda)$ satisfies Conditions $(R)$ and $(L)$, then $T$ is a completely $\widetilde{\mathcal{J}}_{U}$-simple semigroup.

Proof. In view of Lemma 3.13, it is easy to see that $T$ is a weakly $U$-superabundant semigroup. We now claim that $T$ satisfies the Congruence Condition. Suppose that $a, b, c \in T$ with $a \widetilde{\mathcal{R}}_{U} b$ and $c \in M_{k \nu}$. By Lemma 3.13, $a \in M_{i \lambda}$ and $b \in M_{i \mu}$ for some $i \in I, \lambda, \mu \in \Lambda$. Clearly, $c a \in M_{k \lambda}$ and $c b \in M_{k \mu}$. Again by Lemma 3.13, ca $\widetilde{\mathcal{R}}_{U} c b$. Thus, $\widetilde{\mathcal{R}}_{U}$ is a left congruence. Similarly, we have that $\widetilde{\mathcal{L}}_{U}$ is a right congruence.

We still need to show that $\widetilde{\mathcal{D}}_{U}$ is a universal relation on $T$. Let $a, b \in T$ with $a \in M_{i \lambda}$ and $b \in M_{j \mu}$. Since there exists an element $x \in M_{i \mu}$, it immediately follows from Lemma 3.13 that $a \widetilde{\mathcal{R}} x \widetilde{\mathcal{L}}_{U} b$, that is, $a \widetilde{\mathcal{D}}_{U} b$. Hence $T$ is a completely $\widetilde{\mathcal{J}}_{U}$-simple semigroup.

In a summary, we have the following structure theorem for completely $\widetilde{\mathcal{J}}_{U^{-}}$ simple semigroups. 
Theorem 3.15. A semigroup $S$ is completely $\widetilde{\mathcal{J}}_{U}$-simple if and only if it is a rectangular band of monoids $M_{i \lambda}(i \in I, \lambda \in \Lambda)$ and satisfies Conditions $(R)$ and $(L)$, where the monoids $M_{i \lambda}$ must be isomorphic.

Proof. In view of Lemma 3.14 , it is sufficient to show that a completely $\widetilde{\mathcal{J}}_{U}$-simple semigroup is a rectangular band of monoids and satisfies Conditions $(R)$ and $(L)$. Clearly, by Lemma 3.9 , a completely $\widetilde{\mathcal{J}}_{U}$-simple semigroup $S$ is a rectangular band of monoids $M_{i \lambda}(i \in I, \lambda \in \Lambda)$ which are isomorphic. Since the set of $\widetilde{\mathcal{R}}_{U}$-classes of $S$ is denoted by $I$ and the set of $\widetilde{\mathcal{L}}_{U}$-classes of $S$ is denoted by $\Lambda$ it follows that Conditions $(R)$ and $(L)$ hold.

Finally, we consider a special case. If the set $U$ of identities of each $M_{i \lambda}$ forms a band, then the next lemma is immediate.

Lemma 3.16. Let $T$ be the rectangular band of monoids $M_{i \lambda}$ with identities $e_{i \lambda}$ $(i \in I, \lambda \in \Lambda)$. If $U=\left\{e_{i \lambda}: i \in I, \lambda \in \Lambda\right\}$ forms a band, then $T$ satisfies Conditions $(R)$ and $(L)$.

So, we have:

Corollary 3.17. A semigroup $S$ is completely $\widetilde{\mathcal{J}}_{B}$-simple if and only if it is a rectangular band of monoids $M_{i \lambda}$ with identities $e_{i \lambda}(i \in I, \lambda \in \Lambda)$, where $B=\left\{e_{i \lambda}: i \in I, \lambda \in \Lambda\right\}$ forms a band and the monoids $M_{i \lambda}$ must be isomorphic. 


\section{Chapter 4}

\section{Representations for generalised orthogroups}

In this chapter we begin the study of fundamental semigroups and their analogues in the class of generalised regular semigroups. Precisely, we mainly describe orthogroups in the Hall semigroup $W_{B}$ and weakly $B$-superabundant subsemigroups with (C) of $V_{B}$ (resp. $\left.U_{B}, S_{B}\right)$, which is analogous to $W_{B}$.

\subsection{Fundamental inverse semigroups}

The results in this section are basic but important in the study of inverse semigroups. To make our discussion in the following sections easy to understand, we list them here. The details are referred to [26].

We recall that an inverse semigroup $S$ is fundamental if the maximum idempotent separating congruence $\mu$ is the identity congruence on $S$. Such inverse semigroups do exist, since $S / \mu$ is fundamental inverse for any inverse semigroup $S$ whatsoever. Specially, every semilattice and every symmetric inverse semigroup $\mathcal{I}(X)$ is fundamental.

Observe that every element $a$ in an inverse semigroup $S$ determines an isomorphism $\alpha_{a}$ from the principal ideal $E_{a a^{-1}}$ of $E$ onto the principal ideal $E_{a^{-1} a}$. The isomorphism $\alpha_{a}$ is defined by

$$
e \alpha_{a}=a^{-1} e a \quad\left(e \in E_{a a^{-1}}\right)
$$


Built on the above observation, Munn [37] constructed a fundamental inverse semigroup from any semilattice $E$, as follows.

Let $E$ be a semilattice and $\mathcal{U}$ be the equivalence relation on $E$ given by

$$
\mathcal{U}=\left\{(e, f) \in E \times E: E_{e} \simeq E_{f}\right\}
$$

If $(e, f) \in \mathcal{U}$ let $T_{e, f}$ be the set of all isomorphisms from $E_{e}$ onto $E_{f}$. Let

$$
T_{E}=\bigcup_{(e, f) \in \mathcal{U}} T_{e, f}
$$

Then $T_{E}$ is an inverse subsemigroup of $\mathcal{I}_{E}$ and is fundamental. We shall call it the Munn semigroup of the semilattice $E$.

The crucial fact concerning the Munn semigroup is that:

Theorem 4.1. [26] If $S$ is an inverse semigroup with semilattice of idempotents $E$, then there is a morphism $\phi: S \rightarrow T_{E}$ whose kernel is $\mu$, the maximum idempotent separating congruence on $S$. The morphism $\phi$ is defined by

$$
a \phi=\alpha_{a} \quad(a \in S)
$$

where $\alpha_{a}$ is given above.

We pause to mention that the Munn semigroup $T_{E}$ is determined by its semilattice of idempotents. In view of this, it is natural to be concerned with the influence of the properties of the idempotents of an inverse semigroup on the structure of the inverse semigroup as a whole. Keeping this in mind, we recall that an inverse semigroup $S$ is said to be a Clifford semigroup if the idempotents are central, that is, $e x=x e$ for every idempotent $e$ and every $x$ in $S$, or equivalently, a semilattice of groups.

Theorem 4.1 built a concrete morphism $\phi: S \rightarrow T_{E}$. If $S$ is a Clifford semigroup, then the morphism $\phi$ has kernel $\mu=\mathcal{H}$.

Theorem 4.2. If $S$ is a Clifford semigroup with semilattice of idempotents $E$, then $\mu=\mathcal{H}$ and $S / \mu$ is a semilattice, which must be embedded in $T_{E}$.

Proof. For every element $a$ in a Clifford semigroup $S$, there exists an inverse $a^{-1}$ of $a$ such that $a \mathcal{H} a^{-1}$, and so $a^{-1} a=a a^{-1}=g$, where $g$ is the idempotent in 
$H_{a}$. Then $\alpha_{a}$ is a morphism from $E_{g}$ to $E_{g}$. Since $S$ is a Clifford semigroup, the idempotents are central. Then for any $e \in E_{g}$,

$$
e \alpha_{a}=a^{-1} e a=a^{-1} a e=g e=e .
$$

So $\alpha_{a}$ is the identity map on $E_{g}$, from which it follows that if $a \mathcal{H} b$ in $S$, then $\alpha_{a}=\alpha_{b}$, that is, $a \phi=b \phi$. Thus, $\mathcal{H} \subseteq \operatorname{Ker} \phi$. Certainly, $\operatorname{Ker} \phi \subseteq \mathcal{H}$. Hence, $\operatorname{Ker} \phi=\mathcal{H}$. By Theorem 4.1, $\mu=\mathcal{H}$.

\subsection{A fundamental orthogroup of $W_{B}$}

In Section 4.1 we were able to find a morphism $\phi$ from an inverse semigroup with semilattice of idempotents $E$ to the Munn semigroup $T_{E}$. Moreover, if $S$ is a Clifford semigroup, then the image of $S$ in $T_{E}$ is $E$ (hence is particular also Clifford). If we are to find a generalisation of this to an orthodox semigroup (a regular semigroup whose set of idempotents forms a band), we need begin by recalling the appropriate analogue of the Hall semigroup [26].

Let $B$ be a band. We denote by $\langle e\rangle$ the principal order ideal generated by $e$ for all $e \in B$. We define

$$
\mathcal{U}=\{(e, f) \in B \times B:\langle e\rangle \simeq\langle f\rangle\}
$$

and write $W_{e, f}$ for the set of all isomorphisms from $\langle e\rangle$ onto $\langle f\rangle$. If $(e, f) \in \mathcal{U}$ and $\alpha \in W_{e, f}$, we may define $\alpha_{l} \in \mathcal{T}(B / \mathcal{L})$ and $\alpha_{r} \in \mathcal{T}^{*}(B / \mathcal{R})$ by the rule that

$$
L_{x} \alpha_{l}=L_{x \alpha}, \quad R_{x} \alpha_{r}=R_{x \alpha} \quad(x \in\langle e\rangle) .
$$

It is routine to verify that $\left(\alpha_{l}\right)^{-1}=\left(\alpha^{-1}\right)_{l}$ and $\left(\alpha_{r}\right)^{-1}=\left(\alpha^{-1}\right)_{r}$. In this case, we may use the notation $\alpha_{l}^{-1}, \alpha_{r}^{-1}$ without ambiguity.

Now, we put

$$
W_{B}=\left\{\left(\rho_{e} \alpha_{l}, \lambda_{f} \alpha_{r}^{-1}\right): \alpha \in W_{e, f},(e, f) \in \mathcal{U}\right\}
$$

where for any $x \in B$,

$$
L_{x} \rho_{e}=L_{e x e}, \quad R_{x} \lambda_{f}=R_{f x f} .
$$


In fact, $W_{B}$ is precisely the analogue of $T_{E}$ and it is a fundamental orthodox subsemigroup of $\mathcal{T}(B / \mathcal{L}) \times \mathcal{T}^{*}(B / \mathcal{R})$. We shall call it the Hall semigroup of the band $B$.

It is useful and convenient to present the following result.

Lemma 4.3. [26] If $e, f, g$ are elements of a band $B$ with $(e, f) \in \mathcal{U}$ and $g \in\langle e\rangle$, then $\langle g\rangle \alpha=\langle g \alpha\rangle$, where $\alpha \in W_{e, f}$.

In the case of an inverse semigroup, the key idea of conjugates of idempotents guarantees that there exists a representation which provides more useful information about the structure of the semigroup. We note that this idea is still available in the orthodox case, but it is necessary to take a new technique to deal with the inverse of every element, since it is not unique.

Observe that if $a$ is an element in an orthodox semigroup $S$ with band of idempotents $B$ and $a^{*}, a^{\prime}$ are inverses of $a$, then $a^{*} x a \mathcal{L} a^{\prime} x a$ and $a x a^{*} \mathcal{R} a x a^{\prime}$, where $x \in B$. Built on this observation, we have the following maps.

Let $S$ be an orthodox semigroup with band of idempotents $B$. For each $a$ in $S$, a mapping $\rho_{a}: B / \mathcal{L} \rightarrow B / \mathcal{L}$ is defined by

$$
L_{x} \rho_{a}=L_{a^{\prime} x a} \quad(x \in B)
$$

where $a^{\prime}$ is an arbitrary chosen inverse of $a$. By dual arguments we can define $\lambda_{a}: B / \mathcal{R} \rightarrow B / \mathcal{R}$ by

$$
R_{x} \lambda_{a}=R_{a x a^{\prime}} \quad(x \in B),
$$

where $a^{\prime}$ is an arbitrary chosen inverse of $a$.

By Proposition 1.16, $b^{\prime} a^{\prime}$ is an inverse of $a b$, and so $\rho_{a b}=\rho_{a} \rho_{b}$, for all $a, b$ in $S$. Dually, we have that $\lambda_{a b}=\lambda_{b} \lambda_{a}$. Moreover:

Theorem 4.4. [26] Let $S$ be an orthodox semigroup with band of idempotents $B$, and let $\psi$ be the mapping from $S$ into $\mathcal{T}(B / \mathcal{L}) \times \mathcal{T}^{*}(B / \mathcal{R})$ defined by

$$
a \psi=\left(\rho_{a}, \lambda_{a}\right)
$$

where $\rho_{a}, \lambda_{a}$ are given as above. Then $\psi$ is a morphism whose kernel is the maximum idempotent separating congruence $\mu$ on $S$.

The result we have achieved presents a representation in $\mathcal{T}(B / \mathcal{L}) \times \mathcal{T}^{*}(B / \mathcal{R})$ rather than $T_{E}$. We have seen that $W_{B}$ is an analogue of $T_{E}$ and an orthodox 
subsemigroup of $\mathcal{T}(B / \mathcal{L}) \times \mathcal{T}^{*}(B / \mathcal{R})$, having band of idempotents isomorphic to $B$. So to obtain an exact analogue of Theorem 4.1, we must rewrite the maps $\rho_{a}$ and $\lambda_{a}$ in the form used to define $W_{B}$.

Now let $S$ be an orthodox semigroup with band of idempotents $B$. If $a \in S$ and $a^{\prime}$ is an inverse of $a$, then denoting $a a^{\prime}$ by $e$ and $a^{\prime} a$ by $f$, we obtain that

$$
\left(\rho_{a}, \lambda_{a}\right)=\left(\rho_{e} \theta_{l}, \lambda_{f} \theta_{r}^{-1}\right)
$$

where $\theta$ is the mapping in $W_{e, f}$ given by

$$
x \theta=a^{\prime} x a \quad(x \in\langle e\rangle) .
$$

In this case, the range of the mapping $\psi: a \mapsto\left(\rho_{a}, \lambda_{a}\right)$ is thus contained in the Hall semigroup

$$
W_{B}=\left\{\left(\rho_{e} \alpha_{l}, \lambda_{f} \alpha_{r}^{-1}\right): \alpha \in W_{e, f},(e, f) \in \mathcal{U}\right\}
$$

Before moving on we pause to confine ourselves to a consideration of a special kind of orthodox semigroups analogous to Clifford semigroups. We have mentioned in Chapter 1 that a semigroup is completely regular if each of its elements is contained in some subgroup of $S$. An orthodox semigroup is an orthogroup if it is completely regular. Obviously, every Clifford semigroup is an orthogroup.

At the end of the previous section, we mentioned that there exists a representation from a Clifford semigroup $S$ to $T_{E}$ and the image of $S$ is the semilattice of idempotents of $T_{E}$. Certainly, every semilattice is a Clifford semigroup. So there exactly exists a representation from a Clifford semigroup to a Clifford subsemigroup of $T_{E}$. At a certain stage it becomes natural to ask whether there exists such a representation of an orthogroup to an orthogroup contained in the Hall semigroup $W_{B}$.

As a first step what we have to do is to find a subsemigroup of $W_{B}$ which is an orthogroup. In this case, it is necessary to make use of the statement [49] that an orthodox semigroup is completely regular if and only if its greatest inverse semigroup homomorphic image is completely regular, i.e. a Clifford semigroup.

If $B$ is a band, then it is a semilattice $Y$ of rectangular bands $B_{\alpha}(\alpha \in Y)$. It is not hard to verify that if $e, x \in B$ with $x \in\langle e\rangle$, then $e \in B_{\alpha}$ and $x \in B_{\xi}$ 
for some $\alpha, \xi \in Y$ with $\xi \leq \alpha$. In addition, if $e, f \in B$ with $e \in B_{\alpha}, f \in B_{\beta}$ $(\alpha, \beta \in Y)$ and $\iota:\langle e\rangle \rightarrow\langle f\rangle$ is an isomorphism, then there is an isomorphism $\iota^{\prime}: \alpha Y \rightarrow \beta Y$ corresponding to $\iota$, defined by the property that

$$
x \iota \in B_{\xi \iota^{\prime}} \quad\left(\xi \in \alpha Y, x \in\langle e\rangle \cap B_{\xi}\right) .
$$

As $Y$ is a semilattice, the Munn semigroup $T_{Y}$ certainly exists. Then for every element $\left(\rho_{e} \theta_{l}, \lambda_{f} \theta_{r}^{-1}\right)$ in $W_{B}, \theta$ is an isomorphism from $\langle e\rangle$ onto $\langle f\rangle$. Due to the above analysis, there is an isomorphism $\theta^{\prime}: \alpha Y \rightarrow \beta Y$ corresponding to $\theta$ in $T_{Y}$, where $e \in B_{\alpha}$ and $f \in B_{\beta}$. Hence, there is a map $\nu$ from $W_{B}$ to $T_{Y}$ defined by the rule that

$$
\left(\rho_{e} \theta_{l}, \lambda_{f} \theta_{r}^{-1}\right) \nu=\theta^{\prime} \quad\left(\left(\rho_{e} \theta_{l}, \lambda_{f} \theta_{r}^{-1}\right) \in W_{B}\right)
$$

Lemma 4.5. The map $\nu$ defined above is a morphism from $W_{B}$ to $T_{Y}$.

Proof. We first show that $\nu$ is well-defined. Let $\left(\rho_{e} \theta_{l}, \lambda_{f} \theta_{r}^{-1}\right),\left(\rho_{h} \sigma_{l}, \lambda_{k} \sigma_{r}^{-1}\right) \in W_{B}$ be such that $\left(\rho_{e} \theta_{l}, \lambda_{f} \theta_{r}^{-1}\right)=\left(\rho_{h} \sigma_{l}, \lambda_{k} \sigma_{r}^{-1}\right)$. Then for any $x \in B$, we have that $L_{x} \rho_{e} \theta_{l}=L_{x} \rho_{h} \sigma_{l}$, that is $($ exe $) \theta \mathcal{L}(h x h) \sigma$. Choose $x=e$. We obtain that $e \theta \mathcal{L}(h e h) \sigma$, and so $f \mathcal{L}(h e h) \sigma$ as $e \theta=f$. Since $\mathcal{L}$ is a right congruence and $(h e h) \sigma \leq k$, we succeed in obtaining that $f k \mathcal{L}(h e h) \sigma$. By Lemma 1.22, we have that $k f k \mathcal{L}(h e h) \sigma$. As $\sigma:\langle h\rangle \rightarrow\langle k\rangle$ is an isomorphism, we have that $(k f k) \sigma^{-1} \mathcal{L}$ heh. Also, for any $x \in B$, we have that $R_{x} \lambda_{f} \theta_{r}^{-1}=R_{x} \lambda_{k} \sigma_{r}^{-1}$, that is, $(f x f) \theta^{-1} \mathcal{R}(k x k) \sigma^{-1}$. Take $x=f$, we obtain that $f \theta^{-1} \mathcal{R}(k f k) \sigma^{-1}$. As $f \theta^{-1}=e$, we get that $e \mathcal{R}(k f k) \sigma^{-1}$. Together with $(k f k) \sigma^{-1} \mathcal{L} h e h$, we have that $e \mathcal{D}$ heh. Similarly, we obtain that $h \mathcal{D}$ ehe. Certainly, ehe $\mathcal{D}$ heh. Thus $e \mathcal{D} h$. Dually, we have that $f \mathcal{D} k$. Hence,

$$
\operatorname{dom}\left(\theta^{\prime}\right)=\operatorname{dom}\left(\sigma^{\prime}\right)=\alpha Y \text { and } \operatorname{im}\left(\theta^{\prime}\right)=\operatorname{im}\left(\sigma^{\prime}\right)=\beta Y,
$$

where $e, h \in B_{\alpha}$ and $f, k \in B_{\beta}$.

We still need show that for any $\xi \leq \alpha, \xi \theta^{\prime}=\xi \sigma^{\prime}$. To do this, we assume that $x \leq e$ and $x \in B_{\xi}$. Then there exists $y \in B$ such that $x=$ eye. Since $e \mathcal{D} h$, we have that $x=$ eye $\mathcal{D} h y h \leq h$, and so $h y h \in B_{\xi}$. As $x \theta=($ eye $) \theta \mathcal{L}(h y h) \sigma$ so that 
$x \theta \mathcal{D}(h y h) \sigma$. By the properties of $\theta^{\prime}$ and $\sigma^{\prime}$, we have that

$$
x \theta \in B_{\xi \theta^{\prime}} \text { and }(h y h) \sigma \in B_{\xi \sigma^{\prime}} .
$$

Thus $\xi \theta^{\prime}=\xi \sigma^{\prime}$. Consequently, $\nu$ is well-defined.

Next, we show that $\nu$ is a morphism. Let $\left(\rho_{e} \eta_{l}, \lambda_{f} \eta_{r}^{-1}\right),\left(\rho_{g} \sigma_{l}, \lambda_{h} \sigma_{r}^{-1}\right) \in W_{B}$ and let $e \in B_{\alpha}, f \in B_{\beta}, g \in B_{\gamma}$ and $h \in B_{\delta}$. According to [Chapter VI, Theorem 2.17, [26]], we have that

$$
\left(\rho_{e} \eta_{l}, \lambda_{f} \eta_{r}^{-1}\right)\left(\rho_{g} \sigma_{l}, \lambda_{h} \sigma_{r}^{-1}\right)=\left(\rho_{i} \tau_{l}, \lambda_{j} \tau_{r}^{-1}\right)
$$

where $i=(f g f) \eta^{-1}, j=(g f g) \sigma$ and $\tau=\left(\left.\eta\right|_{\langle i\rangle}\right)\left(\left.\theta_{g f g}\right|_{\langle f g f\rangle}\right)\left(\left.\sigma\right|_{\langle g f g\rangle}\right)$. In the following, we show that $\eta^{\prime} \circ \sigma^{\prime}=\tau^{\prime}$.

As $f g f \in B_{\beta \gamma}$, we have that $(f g f) \eta^{-1} \in B_{(\beta \gamma) \eta^{\prime-1}}$, and so $\operatorname{dom} \tau^{\prime}=(\beta \gamma) \eta^{\prime-1} Y$. Similarly, $\operatorname{im} \tau^{\prime}=(\beta \gamma) \sigma^{\prime} Y$. Observe that $\operatorname{im} \eta^{\prime}=\beta Y$ and $\operatorname{dom} \sigma^{\prime}=\gamma Y$. Thus

$$
\begin{aligned}
\operatorname{dom}\left(\eta^{\prime} \circ \sigma^{\prime}\right) & =\left(\operatorname{im} \eta^{\prime} \cap \operatorname{dom} \sigma^{\prime}\right) \eta^{\prime-1} \\
& =(\beta Y \cap \gamma Y) \eta^{\prime-1} \\
& =(\beta \gamma) Y \eta^{\prime-1} \\
& =(\beta \gamma) \eta^{-1} Y \quad \text { Lem } \\
& =\operatorname{dom} \tau^{\prime} .
\end{aligned}
$$

Similarly, $\operatorname{im}\left(\eta^{\prime} \circ \sigma^{\prime}\right)=\operatorname{im} \tau^{\prime}$.

Let $\xi \in \operatorname{dom}\left(\eta^{\prime} \circ \sigma^{\prime}\right)$ and $x \in B_{\xi} \cap\langle i\rangle$. Then

$$
x \tau=x\left(\left.\eta\right|_{\langle i\rangle}\right)\left(\left.\theta_{g f g}\right|_{\langle f g f\rangle}\right)\left(\left.\sigma\right|_{\langle g f g\rangle}\right) \in B_{\mu} \quad \text { for some } \mu \in Y,
$$

and so $\xi \tau^{\prime}=\mu$. Since we have remarked that $\left.\theta_{g f g}\right|_{\langle f g f\rangle}$ fixes $\mathcal{D}$-classes succeeding Lemma 1.21, it follows that $x\left(\left.\eta\right|_{\langle i\rangle}\right) \mathcal{D} x\left(\left.\eta\right|_{\langle i\rangle}\right)\left(\left.\theta_{g f g}\right|_{\langle f g f\rangle}\right)$ so that $\xi \eta^{\prime}=\omega$ and $\omega \sigma^{\prime}=\mu$, where $x\left(\left.\eta\right|_{\langle i\rangle}\right), x\left(\left.\eta\right|_{\langle i\rangle}\right)\left(\left.\theta_{g f g}\right|_{\langle f g f\rangle}\right) \in B_{\omega}$. Hence, $\xi\left(\eta^{\prime} \circ \sigma^{\prime}\right)=\mu$, and so $\eta^{\prime} \circ \sigma^{\prime}=\tau^{\prime}$.

We now pause to mention that if the image $\theta^{\prime}$ of the element $\left(\rho_{e} \theta_{l}, \lambda_{f} \theta_{r}^{-1}\right)$ of $W_{B}$ under $\nu$ is an idempotent, then $e, f$ are $\mathcal{D}$-related and $\theta^{\prime}$ is an identity map on $\alpha Y$, where $e \in B_{\alpha}$. Hence, the elements of $W_{B}$, whose images under $\nu$ are idempotent, induce a partial identity mapping on $Y$. Moreover, we have: 
Lemma 4.6. [49] The elements of $W_{B}$ whose images under $\nu$ are idempotent form an orthogroup in $W_{B}$. We denote this orthogroup by $O G_{1}$.

Proof. We first show that $O G_{1}$ is closed. Suppose that $\left(\rho_{e} \iota_{l}, \lambda_{f} \iota_{r}^{-1}\right)$ and $\left(\rho_{g} \tau_{l}, \lambda_{h} \tau_{r}^{-1}\right)$ are in $O G_{1}$. Then $\iota^{\prime}, \tau^{\prime} \in E\left(T_{Y}\right)$. Since $E\left(T_{Y}\right)$ is a semilattice, we have that $\iota^{\prime} \tau^{\prime} \in E\left(T_{Y}\right)$. As $O G_{1} \subseteq W_{B}$, we have that the product $\left(\rho_{e} \iota_{l}, \lambda_{f} \iota_{r}^{-1}\right)\left(\rho_{g} \tau_{l}, \lambda_{h} \tau_{r}^{-1}\right)$ is in $W_{B}$. Also,

$$
\left(\left(\rho_{e} \iota_{l}, \lambda_{f} \iota_{r}^{-1}\right)\left(\rho_{g} \tau_{l}, \lambda_{h} \tau_{r}^{-1}\right)\right) \nu=\left(\rho_{e} \iota_{l}, \lambda_{f} \iota_{r}^{-1}\right) \nu\left(\rho_{g} \tau_{l}, \lambda_{h} \tau_{r}^{-1}\right) \nu=\iota^{\prime} \circ \tau^{\prime} \in E\left(T_{Y}\right)
$$

Thus, $\left(\rho_{e} \iota_{l}, \lambda_{f} \iota_{r}^{-1}\right)\left(\rho_{g} \tau_{l}, \lambda_{h} \tau_{r}^{-1}\right) \in O G_{1}$, and so $O G_{1}$ is closed.

Clearly, $\bar{B}=\left\{\left(\rho_{e}, \lambda e\right): e \in B\right\}$ is contained in $O G_{1}$ and so $O G_{1}$ is an orthodox semigroup.

Finally, we claim that $O G_{1}$ is completely regular. Let $\left(\rho_{e} \iota_{l}, \lambda_{f} \iota_{r}^{-1}\right) \in O G_{1}$, $e \in B_{\alpha}$ and $f \in B_{\beta}(\alpha, \beta \in Y)$. Then $\iota^{\prime}: \alpha Y \rightarrow \beta Y$ is idempotent. In that case, we must have that $e \mathcal{D} f$, and so $e \mathcal{R}$ ef $\mathcal{L} f$ so that $\left(\rho_{e}, \lambda_{e}\right) \mathcal{R}\left(\rho_{e f}, \lambda_{e f}\right) \mathcal{L}\left(\rho_{f}, \lambda_{f}\right)$. As $\left(\rho_{e}, \lambda_{e}\right) \mathcal{R}\left(\rho_{e} \iota_{l}, \lambda_{f} \iota_{r}^{-1}\right) \mathcal{L}\left(\rho_{f}, \lambda_{f}\right)$, we obtain that $\left(\rho_{e} \iota_{l}, \lambda_{f} \iota_{r}^{-1}\right) \mathcal{H}\left(\rho_{e f}, \lambda_{e f}\right)$. Thus, $O G_{1}$ is an orthogroup.

Lemma 4.6 gives us an abstract description of an orthogroup in $W_{B}$. Next, we shall find a closed form for such an orthogroup in $W_{B}$ which coincides with $O G_{1}$.

For any $e \in B$, we write

$$
A_{e}=\left\{\alpha \in W_{e, e}: \text { for all } x \in\langle e\rangle, x \alpha \mathcal{D} x\right\}
$$

and put

$$
O G_{2}=\bigcup_{e \in B} W_{e}
$$

where $W_{e}=\left\{\left(\rho_{e} \alpha_{l}, \lambda_{e} \alpha_{r}^{-1}\right) \in W_{B}: \alpha \in A_{e}\right\}$.

Lemma 4.7. For any $e \in B$, the set $A_{e}$ forms a group.

Proof. Suppose that $\alpha \in A_{e}$. We claim that $\alpha^{-1} \in A_{e}$. For any $x \in\langle e\rangle$, certainly, $x \alpha^{-1} \in\langle e\rangle$. Since $\alpha \in A_{e}$, we have that $x=\left(x \alpha^{-1}\right) \alpha \mathcal{D} x \alpha^{-1}$. This implies that $\alpha^{-1} \in A_{e}$. Obviously, the identity map $1_{\langle e\rangle}$ is the identity of $A_{e}$ and $A_{e}$ is closed. Hence, $A_{e}$ forms a group. 
Further:

Lemma 4.8. For any $e \in B$, the set $W_{e}$ forms a subgroup of $W_{B}$ with identity $\left(\rho_{e}, \lambda_{e}\right)$.

Proof. Clearly, for any $e \in B,\left(\rho_{e}, \lambda_{e}\right) \in W_{e}$ and it is the identity of $W_{e}$. We now show that $W_{e}$ is closed. Suppose that $\left(\rho_{e} \alpha_{l}, \lambda_{e} \alpha_{r}^{-1}\right)$ and $\left(\rho_{e} \beta_{l}, \lambda_{e} \beta_{r}^{-1}\right)$ are in $W_{e}$. We first consider the product of $\rho_{e} \alpha_{l}$ and $\rho_{e} \beta_{l}$ because dually, we obtain the similar result for the product of $\lambda_{e} \alpha_{r}^{-1}$ and $\lambda_{e} \beta_{r}^{-1}$. For any $x \in B$, we have that

$$
L_{x} \rho_{e} \alpha_{l} \rho_{e} \beta_{l}=L_{(e[(e x e) \alpha] e) \beta}=L_{((e x e) \alpha) \beta}=L_{(e x e) \alpha \beta}=L_{x} \rho_{e}(\alpha \beta)_{l} .
$$

Similarly, we obtain that $R_{x} \lambda_{e} \beta_{r}^{-1} \lambda_{e} \alpha_{r}^{-1}=R_{x} \lambda_{e}(\alpha \beta)_{r}^{-1}$. As $\alpha, \beta \in A_{e}$, the composition $\alpha \beta$ certainly belongs to $A_{e}$ by Lemma 4.7 . Thus, $\left(\rho_{e}(\alpha \beta)_{l}, \lambda_{e}(\alpha \beta)_{r}^{-1}\right) \in$ $W_{e}$, and so $W_{e}$ is closed.

Next, we show that the group inverse of $\left(\rho_{e} \alpha_{l}, \lambda_{e} \alpha_{r}^{-1}\right)$ exists and lies in $W_{e}$. To do this, we assume that $\left(\rho_{e} \alpha_{l}, \lambda_{e} \alpha_{r}^{-1}\right) \in W_{e}$. Then $\alpha \in A_{e}$. By Lemma 4.7, we have that $\alpha^{-1} \in A_{e}$, and so $\left(\rho_{e} \alpha_{l}^{-1}, \lambda_{e} \alpha_{r}\right) \in W_{e}$. In addition, it is routine to check that $\left(\rho_{e} \alpha_{l}, \lambda_{e} \alpha_{r}^{-1}\right)$ and $\left(\rho_{e} \alpha_{l}^{-1}, \lambda_{e} \alpha_{r}\right)$ are mutually group inverse in $W_{e}$.

Returning now to the set $O G_{2}$ constructed above, we have:

Lemma 4.9. The set $O G_{2}$ forms an orthogroup with band of idempotents $\bar{B}=$ $\left\{\left(\rho_{e}, \lambda_{e}\right): e \in B\right\}$.

Proof. In view of Lemma 4.8, it is sufficient to show that for any $e, f \in B$, there exists $h \in B$ such that $W_{e} W_{f} \subseteq W_{h}$. Suppose that $\left(\rho_{e} \alpha_{l}, \lambda_{e} \alpha_{r}^{-1}\right) \in W_{e}$ and $\left(\rho_{f} \beta_{l}, \lambda_{f} \beta_{r}^{-1}\right) \in W_{f}$. Then $\alpha \in A_{e}, \beta \in A_{f}$, and so $(e f e) \alpha^{-1} \mathcal{D}$ efe and $(f e f) \beta \mathcal{D}$ fef so that $(e f e) \alpha^{-1} \mathcal{D}(f e f) \beta$ as efe $\mathcal{D} f e f$. Put

$$
\begin{aligned}
\gamma=\left(\left.\theta_{(e f e) \alpha^{-1}}\right|_{\left\langle(e f e) \alpha^{-1}(f e f) \beta\right\rangle}\right) & \left(\left.\alpha\right|_{\left\langle(e f e) \alpha^{-1}\right\rangle}\right) . \\
& \left(\left.\theta_{f e f}\right|_{\langle e f e\rangle}\right)\left(\left.\beta\right|_{\langle f e f\rangle}\right)\left(\left.\theta_{(e f e) \alpha^{-1}(f e f) \beta}\right|_{\langle(f e f) \beta\rangle}\right) .
\end{aligned}
$$

By Lemma 1.21, $\gamma \in W_{(e f e) \alpha^{-1}(f e f) \beta,(e f e) \alpha^{-1}(f e f) \beta}$. Again by the remark succeeding Lemma 1.21 and $\alpha, \beta$ fixing $\mathcal{D}$-classes, we have that $\gamma$ fixes $\mathcal{D}$-classes, and so

$$
\gamma \in A_{(e f e) \alpha^{-1}(f e f) \beta}
$$


Thus, $\left(\rho_{(e f e) \alpha^{-1}(f e f) \beta} \gamma_{l}, \lambda_{(e f e) \alpha^{-1}(f e f) \beta} \gamma_{r}^{-1}\right)$ belongs to $W_{(e f e) \alpha^{-1}(f e f) \beta}$.

We now show that

$$
\left(\rho_{e} \alpha_{l}, \lambda_{e} \alpha_{r}^{-1}\right)\left(\rho_{f} \beta_{l}, \lambda_{f} \beta_{r}^{-1}\right)=\left(\rho_{(e f e) \alpha^{-1}(f e f) \beta} \gamma_{l}, \lambda_{(e f e) \alpha^{-1}(f e f) \beta} \gamma_{r}^{-1}\right)
$$

For any $x \in B$,

$$
\begin{aligned}
& L_{x} \rho_{(e f e) \alpha^{-1}(f e f) \beta} \gamma_{l} \\
& =L_{(e f e) \alpha^{-1}(f e f) \beta x(e f e) \alpha^{-1}(f e f) \beta}\left(\left(\left.\theta_{(e f e) \alpha^{-1}}\right|_{\left\langle(e f e) \alpha^{-1}(f e f) \beta\right\rangle}\right) .\right. \\
& \left.\left(\left.\alpha\right|_{\left\langle(e f e) \alpha^{-1}\right\rangle}\right)\left(\left.\theta_{f e f}\right|_{\langle e f e\rangle}\right)\left(\left.\beta\right|_{\langle f e f\rangle}\right)\left(\left.\theta_{(e f e) \alpha^{-1}(f e f) \beta}\right|_{\langle(f e f) \beta\rangle}\right)\right)_{l} \\
& =L_{\left((e f e) \alpha^{-1} \cdot(e f e) \alpha^{-1}(f e f) \beta x(e f e) \alpha^{-1}(f e f) \beta \cdot(e f e) \alpha^{-1}\right)\left(\left.\alpha\right|_{\left\langle(e f e) \alpha^{-1}\right\rangle}\right)} . \\
& \left(\left.\theta_{f e f}\right|_{\langle e f e\rangle}\right)\left(\left.\beta\right|_{\langle f e f\rangle}\right)\left(\left.\theta_{(e f e) \alpha^{-1}(f e f) \beta}\right|_{\langle(f e f) \beta\rangle}\right) \\
& =L_{\left((e f e) \alpha^{-1}(f e f) \beta x(e f e) \alpha^{-1}\right)\left(\left.\alpha\right|_{\left\langle(e f e) \alpha^{-1}\right\rangle}\right)\left(\left.\theta_{f e f}\right|_{\langle e f e\rangle}\right)\left(\left.\beta\right|_{\langle f e f\rangle}\right)\left(\left.\theta_{(e f e) \alpha^{-1}(f e f) \beta}\right|_{\langle(f e f) \beta\rangle}\right)} \\
& \left((e f e) \alpha^{-1} \mathcal{D}(f e f) \beta\right) \\
& =L_{\left((e f e) \alpha^{-1}(f e f) \beta x(e f e) \alpha^{-1}\right)}\left(\left(\left.\alpha\right|_{\left\langle(e f e) \alpha^{-1}\right\rangle}\right)\left(\left.\theta_{f e f}\right|_{\langle e f e\rangle}\right)\left(\left.\beta\right|_{\langle f e f\rangle}\right) \cdot\right. \\
& \left.\left(\left.\theta_{(e f e) \alpha^{-1}(f e f) \beta}\right|_{\langle(f e f) \beta\rangle}\right)\right)_{l} \\
& =L_{\left(x(e f e) \alpha^{-1}\right)}\left(\left(\left.\alpha\right|_{\left\langle(e f e) \alpha^{-1}\right\rangle}\right)\left(\left.\theta_{f e f}\right|_{\langle e f e\rangle}\right)\left(\left.\beta\right|_{\langle f e f\rangle}\right)\left(\left.\theta_{(e f e) \alpha^{-1}(f e f) \beta}\right|_{\langle(f e f) \beta\rangle}\right)\right)_{l} \\
& =L_{\left((e f e) \alpha^{-1} x(e f e) \alpha^{-1}\right)\left(\left.\alpha\right|_{\left\langle(e f e) \alpha^{-1}\right\rangle}\right)\left(\left.\theta_{f e f}\right|_{\langle e f e\rangle}\right)\left(\left.\beta\right|_{\langle f e f\rangle}\right)\left(\left.\theta_{(e f e) \alpha^{-1}(f e f) \beta}\right|_{\langle(f e f) \beta\rangle}\right)} \\
& \text { (Lemma 1.22) } \\
& \left.=L_{\left((e f e) \alpha^{-1} \text { exe }(e f e) \alpha^{-1}\right)\left(\left.\alpha\right|_{\left\langle(e f e) \alpha^{-1}\right\rangle}\right)\left(\left.\theta_{f e f}\right|_{\langle e f e\rangle}\right)\left(\left.\beta\right|_{\langle f e f\rangle}\right)\left(\theta_{(e f e) \alpha^{-1}(f e f) \beta} \mid\langle(f e f) \beta\rangle\right.}\right) \\
& \left((e x e) \alpha^{-1} \in\langle e\rangle\right) \\
& =L_{\left((e f e) \alpha^{-1} \alpha(e x e) \alpha(e f e) \alpha^{-1} \alpha\right)\left(\left.\theta_{f e f}\right|_{\langle e f e\rangle}\right)\left(\left.\beta\right|_{\langle f e f\rangle}\right)\left(\left.\theta_{(e f e) \alpha^{-1}(f e f) \beta}\right|_{\langle(f e f) \beta\rangle}\right)} \\
& \left((e f e) \alpha^{-1}, \text { exe } \in\langle e\rangle\right) \\
& =L_{((e f e)(e x e) \alpha(e f e))\left(\left.\theta_{f e f}\right|_{\langle e f e\rangle}\right)\left(\left.\beta\right|_{\langle f e f\rangle}\right)\left(\left.\theta_{(e f e) \alpha-1(f e f) \beta}\right|_{\langle(f e f) \beta\rangle}\right)} \\
& \left.=L_{((f e f)(e f e)(e x e) \alpha(e f e)(f e f))(\beta \mid\langle f e f\rangle)\left(\theta_{(e f e) \alpha^{-1}(f e f) \beta} \mid\langle(f e f) \beta\rangle\right)}\right)
\end{aligned}
$$




$$
\begin{aligned}
& \left.=L_{((f e f e f)(e \cdot(e x e) \alpha \cdot e)(f e f e f))\left(\left.\beta\right|_{\langle f e f\rangle}\right)\left(\theta_{(e f e) \alpha^{-1}(f e f) \beta} \mid\langle(f e f) \beta\rangle\right.}\right)
\end{aligned}
$$

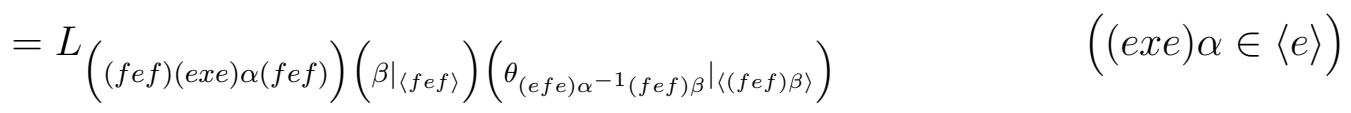

$$
\begin{aligned}
& \left.=L_{(((f e f)(e x e) \alpha(f e f)) \beta}\right)\left(\left.\theta_{(e f e) \alpha^{-1}(f e f) \beta}\right|_{\langle(f e f) \beta\rangle}\right) \\
& =L_{(((f e f) f((e x e) \alpha) f(f e f)) \beta)}\left(\theta_{(e f e) \alpha^{-1}(f e f) \beta}\langle\langle(f e f) \beta\rangle)\right. \\
& =L_{((f e f) \beta(f(e x e) \alpha f) \beta(f e f) \beta)}\left(\left.\theta_{(e f e) \alpha^{-1}(f e f) \beta}\right|_{\langle(f e f) \beta\rangle}\right) \quad(f e f, f(e x e) \alpha f \in\langle f\rangle)
\end{aligned}
$$

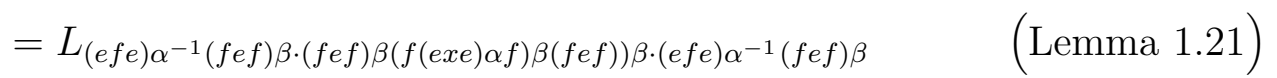

$$
\begin{aligned}
& =L_{(f(e x e) \alpha f) \beta(f e f)) \beta \cdot\left(\text { efe } \alpha^{-1}(f e f) \beta\right.} \quad\left((f e f) \beta \mathcal{D}(\text { efe }) \alpha^{-1}, \quad\right. \text { Lemma 1.22) } \\
& =L_{(f(e x e) \alpha f) \beta(f e f)) \beta} \quad\left((f e f) \beta \mathcal{D}(\text { efe }) \alpha^{-1}\right) \\
& =L_{(f(e x e) \alpha f \cdot f e f) \beta} \\
& =L_{(f(e x e) \alpha f e f) \beta} \\
& =L_{(f(e x e) \alpha \cdot e \cdot f e f) \beta} \quad((\text { exe }) \alpha \in\langle e\rangle) \\
& =L_{(f(e x e) \alpha e f) \beta} \\
& =L_{(f(e x e) \alpha f) \beta} \quad((\text { exe }) \alpha \in\langle e\rangle) \\
& =L_{x} \rho_{e} \alpha_{l} \rho_{f} \beta_{l} \text {. }
\end{aligned}
$$

Thus, $\rho_{(e f e) \alpha^{-1}(f e f) \beta} \gamma_{l}=\rho_{e} \alpha_{l} \cdot \rho_{f} \alpha_{l}$. Also, we deduce that

$$
\begin{aligned}
& R_{x} \lambda_{(e f e) \alpha^{-1}(f e f) \beta} \gamma_{r}^{-1} \\
& =R_{(e f e) \alpha^{-1}(f e f) \beta x(e f e) \alpha^{-1}(f e f) \beta}\left(\left(\left.\theta_{(e f e) \alpha^{-1}}\right|_{\left\langle(e f e) \alpha^{-1}(f e f) \beta\right\rangle}\right) .\right. \\
& \left.\left(\left.\alpha\right|_{\left\langle(e f e) \alpha^{-1}\right\rangle}\right)\left(\left.\theta_{f e f}\right|_{\langle e f e\rangle}\right)\left(\left.\beta\right|_{\langle f e f\rangle}\right)\left(\left.\theta_{(e f e) \alpha^{-1}(f e f) \beta}\right|_{\langle(f e f) \beta\rangle}\right)\right)_{r}^{-1}
\end{aligned}
$$

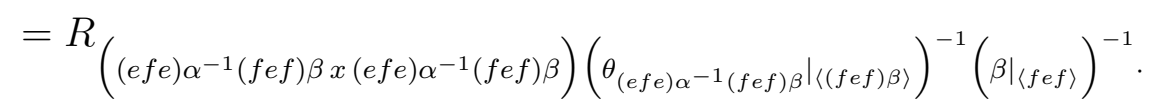

$$
\begin{aligned}
& \left(\left.\theta_{f e f}\right|_{\langle e f e\rangle}\right)^{-1}\left(\left.\alpha\right|_{\left\langle(e f e) \alpha^{-1}\right\rangle}\right)^{-1}\left(\left.\theta_{(e f e) \alpha^{-1}}\right|_{\left\langle(e f e) \alpha^{-1}(f e f) \beta\right\rangle}\right)^{-1} \\
& \left.=R_{\left((e f e) \alpha^{-1}(f e f) \beta x(e f e) \alpha^{-1}(f e f) \beta\right.}\right)\left(\left.\theta_{(f e f) \beta}\right|_{\left\langle(e f e) \alpha^{-1}(f e f) \beta\right\rangle}\right)\left(\left.\beta^{-1}\right|_{\langle(f e f) \beta\rangle}\right) . \\
& \left(\left.\theta_{e f e}\right|_{\langle f e f\rangle}\right)\left(\left.\alpha^{-1}\right|_{\langle e f e\rangle}\right)\left(\left.\theta_{(e f e) \alpha^{-1}(f e f) \beta}\right|_{\left\langle(e f e) \alpha^{-1}\right\rangle}\right)
\end{aligned}
$$




$$
\begin{aligned}
& \left.=R_{\left((f e f) \beta \cdot(e f e) \alpha^{-1}(f e f) \beta x(e f e) \alpha^{-1}(f e f) \beta \cdot(f e f) \beta\right.}\right)\left(\left.\beta^{-1}\right|_{\langle(f e f) \beta\rangle}\right)\left(\theta_{e f e}\langle\{f e f\rangle) .\right. \\
& \left(\left.\alpha^{-1}\right|_{\langle e f e\rangle}\right)\left(\left.\theta_{(e f e) \alpha^{-1}(f e f) \beta}\right|_{\left\langle(e f e) \alpha^{-1}\right\rangle}\right) \\
& =R_{\left((f e f) \beta x(e f e) \alpha^{-1}(f e f) \beta\right)\left(\left.\beta^{-1}\right|_{\langle(f e f) \beta\rangle}\right)\left(\left.\theta_{e f e}\right|_{\langle f e f\rangle}\right)\left(\left.\alpha^{-1}\right|_{\langle e f e\rangle}\right)\left(\left.\theta_{(e f e) \alpha^{-1}(f e f) \beta}\right|_{\left\langle(e f e) \alpha^{-1}\right\rangle}\right)} \\
& \left((\text { efe }) \alpha^{-1} \mathcal{D}(f e f) \beta\right) \\
& =R_{\left((f e f) \beta x(e f e) \alpha^{-1}(f e f) \beta\right)}\left(\left(\left.\beta^{-1}\right|_{\langle(f e f) \beta\rangle}\right)\left(\left.\theta_{\text {efe }}\right|_{\langle f e f\rangle}\right)\left(\alpha^{-1} \mid\langle e f e\rangle\right) .\right. \\
& \left.\left(\theta_{(e f e) \alpha^{-1}(f e f) \beta} \mid\left\langle(e f e) \alpha^{-1}\right\rangle\right)\right)_{r} \\
& =R_{((f e f) \beta x}\left(\left(\left.\beta^{-1}\right|_{\langle(f e f) \beta\rangle}\right)\left(\left.\theta_{e f e}\right|_{\langle f e f\rangle}\right)\left(\left.\alpha^{-1}\right|_{\langle e f e\rangle}\right)\left(\left.\theta_{(e f e) \alpha^{-1}(f e f) \beta}\right|_{\left\langle(e f e) \alpha^{-1}\right\rangle}\right)\right)_{r} \\
& \text { (Lemma 1.21) } \\
& =R_{((f e f) \beta x(f e f) \beta)\left(\left.\beta^{-1}\right|_{\langle(f e f) \beta\rangle}\right)\left(\left.\theta_{e f e}\right|_{\langle f e f\rangle}\right)\left(\left.\alpha^{-1}\right|_{\langle e f e\rangle}\right)\left(\left.\theta_{(e f e) \alpha^{-1}(f e f) \beta}\right|_{\left\langle(e f e) \alpha^{-1}\right\rangle}\right)} \\
& \text { (Lemma 1.22) } \\
& =R_{((f e f) \beta f x f(f e f) \beta)}\left(\left.\beta^{-1}\right|_{\langle(f e f) \beta\rangle}\right)\left(\left.\theta_{e f e}\right|_{\langle f e f\rangle}\right)\left(\left.\alpha^{-1}\right|_{\langle e f e\rangle}\right)\left(\left.\theta_{(e f e) \alpha^{-1}(f e f) \beta}\right|_{\left\langle(e f e) \alpha^{-1}\right\rangle}\right) \\
& ((f e f) \beta \in\langle f\rangle) \\
& \left.=R_{\left(((f e f) \beta f x f(f e f) \beta) \beta^{-1}\right.}\right)\left(\left.\theta_{e f e}\right|_{\langle f e f\rangle}\right)\left(\left.\alpha^{-1}\right|_{\langle e f e\rangle}\right)\left(\left.\theta_{(e f e) \alpha^{-1}(f e f) \beta}\right|_{\left\langle(e f e) \alpha^{-1}\right\rangle}\right) \\
& \left.=R_{\left((f e f) \beta \beta^{-1}(f x f) \beta^{-1}(f e f) \beta \beta^{-1}\right.}\right)\left(\left.\theta_{e f e}\right|_{\langle f e f\rangle}\right)\left(\left.\alpha^{-1}\right|_{\langle e f e\rangle}\right)\left(\left.\theta_{(e f e) \alpha^{-1}(f e f) \beta}\right|_{\left\langle(e f e) \alpha^{-1}\right\rangle}\right) \\
& ((f e f) \beta, f x f \in\langle f\rangle) \\
& =R_{\left((f e f)(f x f) \beta^{-1}(f e f)\right)\left(\left.\theta_{e f e}\right|_{\langle f e f\rangle}\right)\left(\left.\alpha^{-1}\right|_{\langle e f e\rangle}\right)\left(\left.\theta_{(e f e) \alpha^{-1}(f e f) \beta}\right|_{\left\langle(e f e) \alpha^{-1}\right\rangle}\right)} \\
& =R_{\left((e f e)(f e f)(f x f) \beta^{-1}(f e f)(e f e)\right)\left(\left.\alpha^{-1}\right|_{\langle e f e\rangle}\right)\left(\left.\theta_{(e f e) \alpha^{-1}(f e f) \beta}\right|_{\left\langle(e f e) \alpha^{-1}\right\rangle}\right)} \\
& \left.=R_{\left((e f e f e)\left(f \cdot(f x f) \beta^{-1} \cdot f\right)(e f e f e)\right.}\right)\left(\left.\alpha^{-1}\right|_{\langle e f e\rangle}\right)\left(\left.\theta_{(e f e) \alpha^{-1}(f e f) \beta}\right|_{\left\langle(e f e) \alpha^{-1}\right\rangle}\right) \\
& =R_{\left((e f e)(f x f) \beta^{-1}(e f e)\right)\left(\left.\alpha^{-1}\right|_{\langle e f e\rangle}\right)}\left(\left.\theta_{(e f e) \alpha^{-1}(f e f) \beta}\right|_{\left\langle(e f e) \alpha^{-1}\right\rangle}\right) \\
& =R_{\left(\left((e f e)(f x f) \beta^{-1}(e f e)\right) \alpha^{-1}\right)}\left(\left.\theta_{(e f e) \alpha^{-1}(f e f) \beta}\right|_{\left\langle(e f e) \alpha^{-1}\right\rangle}\right) \\
& =R_{\left(\left((e f e) e(f x f) \beta^{-1} e(e f e)\right) \alpha^{-1}\right)}\left(\left.\theta_{(e f e) \alpha^{-1}(f e f) \beta}\right|_{\left\langle(e f e) \alpha^{-1}\right\rangle}\right) \\
& =R_{\left((e f e) \alpha^{-1}\left(e(f x f) \beta^{-1} e\right) \alpha^{-1}(e f e) \alpha^{-1}\right)}\left(\left.\theta_{(e f e) \alpha^{-1}(f e f) \beta}\right|_{\left\langle(e f e) \alpha^{-1}\right\rangle}\right) \\
& \left(e f e, e(f x f) \beta^{-1} e \in\langle e\rangle\right)
\end{aligned}
$$




$$
\begin{array}{lr}
=R_{(e f e) \alpha^{-1}(f e f) \beta \cdot(e f e) \alpha^{-1}\left(e(f x f) \beta^{-1} e\right) \alpha^{-1}(e f e) \alpha^{-1} \cdot(e f e) \alpha^{-1}(f e f) \beta} & (\text { Lemma 1.21) } \\
=R_{(e f e) \alpha^{-1}\left(e(f x f) \beta^{-1} e\right) \alpha^{-1}(e f e) \alpha^{-1}(f e f) \beta} & \left((f e f) \beta \mathcal{D}(e f e) \alpha^{-1}\right) \\
=R_{(e f e) \alpha^{-1}\left(e(f x f) \beta^{-1} e\right) \alpha^{-1}} & \left((f x f) \beta \mathcal{D}(e f e) \alpha^{-1}\right. \text { and Lemma 1.22) } \\
=R_{\left(e f e \cdot e(f x f) \beta^{-1} e\right) \alpha^{-1}} & \\
=R_{\left(e f e \cdot e f(f x f) \beta^{-1} e\right) \alpha^{-1}} & \\
=R_{\left(e f(f x f) \beta^{-1} e\right) \alpha^{-1}} & \\
=R_{\left(e(f x f) \beta^{-1} e\right) \alpha^{-1}} & \\
=R_{x} \lambda_{f} \beta_{r}^{-1} \lambda_{e} \alpha_{r}^{-1} . &
\end{array}
$$

Thus, $\lambda_{(e f e) \alpha^{-1}(f e f) \beta} \gamma_{r}^{-1}=\lambda_{f} \beta_{r}^{-1} \cdot \lambda_{e} \alpha_{r}^{-1}$. Hence, $O G_{2}$ is a subsemigroup of $W_{B}$. By Lemma $4.8, \bar{B}=\left\{\left(\rho_{e}, \lambda_{e}\right): e \in B\right\}$ is contained in $O G_{2}$.

Observe that for any element $\left(\rho_{e} \theta_{l}, \lambda_{f} \theta_{r}^{-1}\right) \in O G_{1}$, we have that $e \mathcal{D} f$ according to the comments following Lemma 4.5. Whereas, if $\left(\rho_{e} \theta_{l}, \lambda_{f} \theta_{r}^{-1}\right) \in O G_{2}$, then $e=f$. In that case, we next show that $O G_{1}$ is equal to $O G_{2}$ by replacing $\left(\rho_{e} \theta_{l}, \lambda_{f} \theta_{r}^{-1}\right)$ with $\left(\rho_{g} \gamma_{l}, \lambda_{g} \gamma_{r}^{-1}\right)$ in $O G_{1}$ for some $g \in B$.

Lemma 4.10. The semigroup $O G_{1}$ coincides with $O G_{2}$.

Proof. We first show that $O G_{2} \subseteq O G_{1}$. Suppose that $\left(\rho_{e} \iota_{l}, \lambda_{e} \iota_{r}^{-1}\right) \in O G_{2}$. Since $B$ is a semilattice $Y$ of rectangular bands $B_{\alpha}(\alpha \in Y)$, we assume that $e \in B_{\alpha}$. According to the construction of $O G_{2}$, we have that $b \iota \mathcal{D} b$ for any $b \in\langle e\rangle$. Specifically, if $b \in\langle e\rangle \cap B_{\xi}$, where $\xi \in \alpha Y$, then $b \iota \mathcal{D} b$. As $b \iota \in B_{\xi \iota^{\prime}}$ and $b \in B_{\xi}$, we must have that $\xi \iota^{\prime}=\xi$, which implies that $\iota^{\prime}$ is the identity map on $\alpha Y$. So, $\left(\rho_{e} \iota_{l}, \lambda_{e} \iota_{r}^{-1}\right) \in O G_{1}$.

Conversely, suppose that $\iota \in W_{e, f}$ and here $\iota^{\prime}$ is an identity in $T_{Y}=T_{B / \mathcal{D}}$. Then, $e \mathcal{D} f$, and so $\left.\theta_{e}\right|_{\langle e f\rangle}$ and $\left.\theta_{e f}\right|_{\langle f\rangle}$ are well-defined isomorphisms from $\langle e f\rangle$ onto $\langle e\rangle$ and from $\langle f\rangle$ onto $\langle e f\rangle$, respectively. As $\iota \in W_{e, f}$, we have that $\gamma=$ $\left(\left.\theta_{e}\right|_{\langle e f\rangle}\right) \iota\left(\left.\theta_{e f}\right|_{\langle f\rangle}\right)$ is an automorphism of $\langle e f\rangle$. We now show that $\left(\rho_{e} \iota_{l}, \lambda_{f} \iota_{r}^{-1}\right)=$ $\left(\rho_{e f} \gamma_{l}, \lambda_{e f} \gamma_{r}^{-1}\right)$. For any $x \in B$,

$$
\begin{aligned}
L_{x} \rho_{e f} \gamma_{l} & =L_{x e f} \gamma_{l} \\
& =L_{(\text {efxef })\left(\left.\theta_{e}\right|_{\langle e f\rangle}\right) \iota\left(\left.\theta_{e f}\right|_{\langle f\rangle}\right)} \\
& =L_{(\text {eefxef.e }) \iota\left(\left.\theta_{e f}\right|_{\langle f\rangle}\right)} \\
& =L_{(\text {eefxef.e })}\left(\iota\left(\left.\theta_{e f}\right|_{\langle f\rangle}\right)\right)_{l}
\end{aligned}
$$




$$
\begin{aligned}
& =L_{\text {exe }}\left(\iota\left(\left.\theta_{\text {ef }}\right|_{\langle f\rangle}\right)\right)_{l} \\
& =L_{(e x e) \iota\left(\left.\theta_{e f}\right|_{\langle f\rangle}\right)} \\
& =L_{e f \cdot(e x e) \iota \cdot e f} \\
& =L_{(\text {exe }) \iota \cdot e f} \\
& =L_{((e x e) \iota) f \cdot e f} \quad((e x e) \iota \in\langle f\rangle) \\
& =L_{((\text {exe }) \iota) f} \quad(e \mathcal{D} f) \\
& =L_{(e x e) \iota} \\
& =L_{x} \rho_{e} \iota_{l} \text {. }
\end{aligned}
$$

Thus, $\rho_{e} \iota_{l}=\rho_{e f} \gamma_{l}$. Dually, $\lambda_{f} \iota_{r}^{-1}=\lambda_{e f} \gamma_{r}^{-1}$. Hence, $\left(\rho_{e} \iota_{l}, \lambda_{f} \iota_{r}^{-1}\right)=\left(\rho_{e f} \gamma_{r}, \lambda_{e f} \gamma_{r}^{-1}\right)$.

Next, we show that for any $x \in\langle e f\rangle, x \gamma \mathcal{D} x$. Suppose that $e, f \in B_{\alpha}$, where $\alpha \in Y$. According to the construction of $O G_{1}, \iota^{\prime}$ is the identity map on $\alpha Y$, that is, for any $\xi \in \alpha Y$ and $b \in\langle e\rangle \cap B_{\xi}$, we have that $b \iota \in B_{\xi \iota^{\prime}}=B_{\xi}$. Thus, $b \iota \mathcal{D} b$. By Lemma 1.21 and the remark following it, $\left.\theta_{e}\right|_{\langle e f\rangle}$ and $\left.\theta_{e f}\right|_{\langle f\rangle}$ are isomorphisms fixing $\mathcal{D}$-classes. Thus, $\gamma$ is an automorphism of $\langle e f\rangle$ such that for all $x \in\langle e f\rangle, x \gamma \mathcal{D} x$. So, $\left(\rho_{e f} \gamma_{l}, \lambda_{e f} \gamma_{r}^{-1}\right) \in O G_{2}$. Hence, $O G_{1} \subseteq O G_{2}$.

We return to our question of establishing a representation from an orthogroup to $O G_{2}\left(\right.$ resp. $\left.O G_{1}\right)$ as an analogue of Theorem 4.2, built on Theorem 4.4.

Theorem 4.11. If $S$ is an orthogroup with band of idempotents $B$, then there exists a representation $\psi: S \rightarrow O G_{2}$ whose kernel is $\mu$, the maximal idempotent separating congruence on $S$.

Proof. In view of Theorem 4.4, there exists a representation $\psi$ from an orthogroup $S$ to $W_{B}$. We now need to show that the image of $S$ under $\psi$ is contained in $O G_{2}$. Suppose that $a \in S$ and $a^{-1}$ is the inverse of $a$ in $H_{a}$. Then by Theorem 4.4 and the comments succeeding it, we have that

$$
a \psi=\left(\rho_{a}, \lambda_{a}\right)=\left(\rho_{e} \theta_{l}, \lambda_{f} \theta_{r}^{-1}\right),
$$

where $e=a a^{-1}, f=a^{-1} a$ and $\theta$ is an isomorphism in $W_{e, f}$ given by

$$
x \theta=a^{-1} x a \quad(x \in\langle e\rangle) .
$$


Since $a \mathcal{H} a^{-1}$ and every $\mathcal{H}$-class of an orthogroup is a group, we have that $a a^{-1}=$ $a^{-1} a$, and so $e=f \mathcal{H} a$. Then, for any $x \in\langle e\rangle, x=$ exe $\mathcal{D} a^{-1} x a$, that is, $x \mathcal{D} x \theta$, and so $\theta \in A_{e}$. Hence, $a \psi=\left(\rho_{e} \theta_{l}, \lambda_{e} \theta_{r}^{-1}\right) \in O G_{2}$ so that $S \theta \subseteq O G_{2}$, as required.

\subsection{A fundamental weakly $\bar{B}$-superabundant sub- semigroup of $V_{B}$}

The aim in this section is to move away from the regular case and consider a fundamental weakly $B$-superabundant semigroup $S$ with (C) and (IC). Here $B$ is a band. Recall that a weakly $B$-abundant semigroup is said to be weakly $B$-superabundant if every $\widetilde{\mathcal{H}}_{B}$-class contains a distinguished idempotent in $B$.

In [6], El-Qallali, Fountain and Gould constructed a fundamental weakly $B$ orthodox semigroup with (IC), namely, $V_{B}$, in a manner analogous to the Hall semigroup $W_{B}$. A brief description of the construction is necessary before we build a weakly $\bar{B}$-superabundant subsemigroup of $V_{B}$, where $\bar{B}$ is isomorphic to $B$.

For any $e, f \in B$ we define $V_{e, f}$ to be the set of all order isomorphisms $\alpha$ from $\langle e\rangle$ to $\langle f\rangle$ such that

$$
x \alpha y \alpha \mathcal{L}(x y) \alpha \text { and } u \alpha^{-1} v \alpha^{-1} \mathcal{R}(u v) \alpha^{-1},
$$

for all $x, y \in\langle e\rangle$ and $u, v \in\langle f\rangle$. For any $\alpha \in V_{e, f}$ we can define partial maps of $B / \mathcal{L}$ and $B / \mathcal{R}$ by

$$
L_{x} \alpha_{l}=L_{x \alpha} \text { and } R_{y} \alpha_{r}^{-1}=R_{y \alpha^{-1}}
$$

Due to [6], we have that if $e, f \in B$ and $\alpha \in V_{e, f}$, then for all $x, x^{\prime} \in\langle e\rangle$ and $y, y^{\prime} \in\langle f\rangle$,

$$
\begin{gathered}
x \leq_{\mathcal{L}} x^{\prime} \text { implies that } x \alpha \leq_{\mathcal{L}} x^{\prime} \alpha, \\
y \leq_{\mathcal{R}} y^{\prime} \text { implies that } y \alpha^{-1} \leq_{\mathcal{R}} y^{\prime} \alpha^{-1} .
\end{gathered}
$$

This fact is hard evidence showing that $\alpha_{l}$ and $\alpha_{r}^{-1}$ are well-defined and order preserving. 
Now, we put

$$
V_{B}=\left\{\left(\rho_{e} \alpha_{l}, \lambda_{f} \alpha_{r}^{-1}\right): e, f \in B, \alpha \in V_{e, f}\right\}
$$

Lemma 4.12. [17] The set $V_{B}$ is a fundamental weakly $\bar{B}$-orthodox semigroup with $(I C)$, where $\bar{B}=\left\{\left(\rho_{e}, \lambda_{e}\right): e \in B\right\}$.

We remark that for any $\left(\rho_{e} \alpha_{l}, \lambda_{f} \alpha_{r}^{-1}\right) \in V_{B}$, we have that

$$
\left(\rho_{f}, \lambda_{f}\right) \widetilde{\mathcal{L}}_{\bar{B}}\left(\rho_{e} \alpha_{l}, \lambda_{f} \alpha_{r}^{-1}\right) \widetilde{\mathcal{R}}_{\bar{B}}\left(\rho_{e}, \lambda_{e}\right)
$$

Considering the fact that $V_{B}$ is an analogue of the Hall semigroup $W_{B}$, we can extend the recipe in Section 4.2 from the Hall semigroup $W_{B}$ to $V_{B}$ to find a fundamental weakly $\bar{B}$-superabundant subsemigroup of $V_{B}$ as follows.

Let $B$ be a band. Then it is a semilattice $Y$ of rectangular bands $B_{\alpha}(\alpha \in Y)$. For every element $\left(\rho_{e} \theta_{l}, \lambda_{f} \theta_{r}^{-1}\right)$ in $V_{B}, \theta$ is an order isomorphism from $\langle e\rangle$ to $\langle f\rangle$. Referring to the statement before Lemma 4.5, if $e \in B_{\alpha}$ and $f \in B_{\beta}(\alpha, \beta \in Y)$, then there is an order isomorphism $\theta^{\prime}: \alpha Y \rightarrow \beta Y$ corresponding to $\theta$, defined by the property that

$$
x \theta \in B_{\xi \theta^{\prime}} \quad\left(\xi \in \alpha Y, x \in\langle e\rangle \cap B_{\xi}\right) .
$$

Since an order isomorphism of a semilattice is an isomorphism it follows that $\theta^{\prime} \in T_{Y}$.

Observe that for any $\left(\rho_{e} \theta_{l}, \lambda_{f} \theta_{r}^{-1}\right) \in V_{B}$, if $\theta^{\prime}$ is idempotent, then it induces a partial identity mapping on $Y$. We put

$$
K_{1}=\left\{\left(\rho_{e} \theta_{l}, \lambda_{f} \theta_{r}^{-1}\right) \in V_{B}: \theta^{\prime 2}=\theta^{\prime}\right\}
$$

The next lemma is an immediate consequence of Lemma 4.15, so we omit its proof.

Lemma 4.13. The set $K_{1}$ is a weakly $\bar{B}$-superabundant subsemigroup of $V_{B}$ with (C) and $(I C)$.

In Section 4.2 we gave a closed form for a subsemigroup of the Hall semigroup $W_{B}$ that is a particular orthogroup. In the following, We focus on a closed form 
for a fundamental weakly $\bar{B}$-superabundant subsemigroup of $V_{B}$ as an analogue of $O G_{2}$, beginning as follows.

For any $e \in B$, let

$$
O A_{e}=\left\{\alpha \in V_{e, e}: \text { for all } x \in\langle e\rangle, x \mathcal{D} x \alpha\right\}
$$

and

$$
K_{2}=\bigcup_{e \in B} V_{e}
$$

where $V_{e}=\left\{\left(\rho_{e} \alpha_{l}, \lambda_{e} \alpha_{r}^{-1}\right) \in V_{B}: e \in B, \alpha \in O A_{e}\right\}$.

The proof of the following lemma is similar to that of Lemma 4.9, and so we omit it. Here we remark that the steps using $\alpha$ etc. being a morphism can be replaced by the particular condition for $\alpha$ to lie in $V_{e, f}$.

Lemma 4.14. The set $K_{2}$ is a full subsemigroup of $V_{B}$. Consequently, $K_{2}$ is a fundamental weakly $\bar{B}$-superabundant semigroup with $(C)$ and $(I C)$.

The next lemma presents a relationship between $K_{1}$ and $K_{2}$.

Lemma 4.15. The semigroup $K_{2}$ coincides with $K_{1}$.

Proof. We first show that $K_{2} \subseteq K_{1}$. Suppose that $\left(\rho_{e} \iota_{l}, \lambda_{e} \iota_{r}^{-1}\right) \in K_{2}$. Since $B$ is a semilattice $Y$ of rectangular bands $B_{\alpha}(\alpha \in Y)$, we assume that $e \in$ $B_{\alpha}$. According to the construction of $K_{2}$, we have that $b \iota \mathcal{D} b$ for any $b \in\langle e\rangle$. Specifically, if $b \in\langle e\rangle \cap B_{\xi}$, where $\xi \in \alpha Y$, then $b \iota \mathcal{D} b$. As $b \iota \in B_{\xi \iota^{\prime}}$ and $b \in B_{\xi}$, we must have that $\xi \iota^{\prime}=\xi$, which implies that $\iota^{\prime}$ is the identity map on $\alpha Y$. So, $\left(\rho_{e} \iota_{l}, \lambda_{e} \iota_{r}^{-1}\right) \in K_{1}$.

Conversely, suppose that $\iota \in V_{e, f}$ and here $\iota^{\prime}$ is an identity in $T_{Y}=T_{B / \mathcal{D}}$. Then, $e \mathcal{D} f$, and so $\left.\theta_{e}\right|_{\langle e f\rangle}$ and $\left.\theta_{e f}\right|_{\langle f\rangle}$ are well-defined isomorphisms from $\langle e f\rangle$ onto $\langle e\rangle$ and from $\langle f\rangle$ onto $\langle e f\rangle$, respectively. As $\iota \in V_{e, f}$, we have that $\gamma=$ $\left(\left.\theta_{e}\right|_{\langle e f\rangle}\right) \iota\left(\left.\theta_{e f}\right|_{\langle f\rangle}\right)$ is an order automorphism of $\langle e f\rangle$. We now show that $\left(\rho_{e} \iota_{l}, \lambda_{f} \iota_{r}^{-1}\right)=$ $\left(\rho_{e f} \gamma_{l}, \lambda_{e f} \gamma_{r}^{-1}\right)$. For any $x \in B$,

$$
\begin{aligned}
L_{x} \rho_{e f} \gamma_{l} & =L_{x e f} \gamma_{l} \\
& =L_{(\text {efxef })\left(\left.\theta_{e}\right|_{\langle e f\rangle}\right) \iota\left(\left.\theta_{e f}\right|_{\langle f\rangle}\right)} \\
& =L_{(\text {e.efxef } \cdot e) \iota\left(\left.\theta_{e f}\right|_{\langle f\rangle}\right)} \\
& =L_{(\text {e.efxef } \cdot e)}\left(\iota\left(\left.\theta_{\text {ef }}\right|_{\langle f\rangle}\right)\right)_{l}
\end{aligned}
$$




$$
\begin{aligned}
& =L_{e x e}\left(\iota\left(\left.\theta_{e f}\right|_{\langle f\rangle}\right)\right)_{l} \\
& =L_{(e x e) \iota\left(\left.\theta_{e f}\right|_{\langle f\rangle}\right)} \\
& =L_{\text {ef }}(\text { exe }) \iota \cdot e f \\
& =L_{(e x e) \iota \cdot e f} \\
& =L_{((\text {exe }) \iota) f \cdot e f} \quad((\text { exe }) \iota \in\langle f\rangle) \\
& =L_{((\text {exe }) \iota) f} \quad(e \mathcal{D} f) \\
& =L_{(\text {exe }) \iota} \\
& =L_{x} \rho_{e} \iota_{l} \text {. }
\end{aligned}
$$

Thus, $\rho_{e} \iota_{l}=\rho_{e f} \gamma_{l}$. Dually, $\lambda_{f} \iota_{r}^{-1}=\lambda_{e f} \gamma_{r}^{-1}$. Hence, $\left(\rho_{e} \iota_{l}, \lambda_{f} \iota_{r}^{-1}\right)=\left(\rho_{e f} \gamma_{r}, \lambda_{e f} \gamma_{r}^{-1}\right)$.

Next, we show that for any $x \in\langle e f\rangle, x \gamma \mathcal{D} x$. Suppose that $e, f \in B_{\alpha}$, where $\alpha \in Y$. According to the construction of $K_{1}, \iota^{\prime}$ is the identity map on $\alpha Y$, that is, for any $\xi \in \alpha Y$ and $b \in\langle e\rangle \cap B_{\xi}$, we have that $b \iota \in B_{\xi \iota^{\prime}}=B_{\xi}$. Thus, $b \iota \mathcal{D} b$. By Lemma 1.21 and the remark succeeding it, $\left.\theta_{e}\right|_{\langle e f\rangle}$ and $\left.\theta_{e f}\right|_{\langle f\rangle}$ are isomorphisms preserving $\mathcal{D}$-classes. Thus, $\gamma$ is an order automorphism of $\langle e f\rangle$ such that for all $x \in\langle e f\rangle, x \gamma \mathcal{D} x$. So, $\left(\rho_{e f} \gamma_{r}, \lambda_{e f} \gamma_{r}^{-1}\right) \in K_{2}$. Hence, $K_{1} \subseteq K_{2}$.

We end this section with a representation of a weakly $B$-superabundant semigroup with (C) and (IC), which is analogous to Theorem 4.2 and Theorem 4.11. We first explain how a weakly $B$-orthodox semigroup with (IC) is represented in $V_{B}$.

Let $S$ be a weakly $B$-orthodox semigroup. For any $a \in S$, we define

$$
\alpha_{a}: B / \mathcal{L} \rightarrow B / \mathcal{L} \text { and } \beta_{a}: B / \mathcal{R} \rightarrow B / \mathcal{R}
$$

by

$$
L_{x} \alpha_{a}=L_{(x a)^{*}} \text { and } R_{x} \beta_{a}=R_{(a x)^{\dagger}} .
$$

Clearly, $\alpha_{a}$ and $\beta_{a}$ are well-defined. We note that for any $e \in B$,

$$
\left(\alpha_{e}, \beta_{e}\right)=\left(\rho_{e}, \lambda_{e}\right)
$$

where for any $x \in B$,

$$
L_{x} \rho_{e}=L_{x e} \text { and } R_{x} \lambda_{e}=R_{e x}
$$


Lemma 4.16. [17] Let $S$ be a weakly B-orthodox semigroup with (IC). The map

$$
\phi: S \rightarrow V_{B}
$$

given by

$$
a \phi=\left(\alpha_{a}, \beta_{a}\right)
$$

is a strongly B-admissible morphism with kernel $\mu_{B}$. Moreover, putting $\bar{B}=$ $\left\{\left(\rho_{e}, \lambda_{e}\right): e \in B\right\}$, we have that $\left.\theta\right|_{B}: B \rightarrow \bar{B}$ is an isomorphism.

We remark that for any $a \in S$, choose $a^{\dagger}, a^{*}$ and let $\alpha:\left\langle a^{\dagger}\right\rangle \rightarrow\left\langle a^{*}\right\rangle$ be an order isomorphism such that for all $x \in\left\langle a^{\dagger}\right\rangle$,

$$
x a=a(x \alpha),
$$

then $a \phi=\left(\alpha_{a}, \beta_{a}\right)=\left(\rho_{a^{\dagger}} \alpha_{l}, \lambda_{a^{*}} \alpha_{r}^{-1}\right)$.

The specialisation of Lemma 4.16 to the case of weakly $B$-superabundant semigroups $S$ with (C) and (IC) is of special interest here, since the image of $S$ under $\phi$ is contained in the fundamental weakly $\bar{B}$-superabundant semigroup $K_{2}$ with $(\mathrm{C})$ and $(\mathrm{IC})$.

Theorem 4.17. If $S$ is a weakly B-superabundant semigroup with (C) and (IC), then the image of $S$ under the map $\phi$ given in Lemma 4.16, is contained in $K_{2}$.

Proof. In view of Lemma 4.16, we show that the image of $S$ under $\phi$ is contained in $K_{2}$. By the remark succeeding Lemma 4.16, we have that for any $a \in S$,

$$
a \phi=\left(\rho_{e} \alpha_{l}, \lambda_{e} \alpha_{r}^{-1}\right),
$$

where $e \in B, e \widetilde{\mathcal{H}}_{B} a$ in $S$ and $\alpha:\langle e\rangle \rightarrow\langle e\rangle$ is an ordered isomorphism such that $x a=a(x \alpha)$ for all $x \in\langle e\rangle$. For any $x \in\langle e\rangle$, we have that

$$
x=x e \widetilde{\mathcal{R}}_{B} x a=a(x \alpha) \widetilde{\mathcal{L}}_{B} e(x \alpha)=x \alpha .
$$

Thus, $x \widetilde{\mathcal{D}}_{B} x \alpha$. By Lemma 3.10, we have that $x \mathcal{D} x \alpha$. Hence, $\alpha \in O A_{e}$. Consequently, $a \phi=\left(\rho_{e} \alpha_{l}, \lambda_{e} \alpha_{r}^{-1}\right) \in K_{2}$. 


\subsection{A fundamental weakly $\bar{B}$-superabundant sub- semigroup of $U_{B}$}

In Chapter 2 we introduced the weak idempotent connected condition (WIC), that coincides with (IC), defined by El-Qallali and Fountain, for abundant semigroups, but not for weakly $B$-abundant semigroups. Starting with a band $B$, El-Qallali, Fountain and Gould construct a weakly $B$-orthodox subsemigroup $U_{B}$ of $\mathcal{O P}(B / \mathcal{L}) \times \mathcal{O P}^{*}(B / \mathcal{R})$ satisfying (WIC). The semigroup $U_{B}$ plays the role of $W_{B}$ for the class of weakly $B$-abundant semigroups having a band of idempotents $B$. Our purpose here is to find a fundamental weakly $\bar{B}$-superabundant subsemigroup of $U_{B}$ with (C) and (WIC).

We refer the reader to [17] for more details, but for convenience we sketch the construction of $U_{B}$ as follows.

Let $B$ be a band. For any $e, f \in B$, we commonly denote a relation from $\langle e\rangle$ to $\langle f\rangle$, that is, a subset of $\langle e\rangle \times\langle f\rangle$, by $I^{e, f}$. We say that $I^{e, f}$ is connecting if $I^{e, f}$ is a subsemigroup of $\langle e\rangle \times\langle f\rangle$ and for every $\left(x, x^{\prime}\right),\left(y, y^{\prime}\right) \in I^{e, f}$ we have that

$$
x \leqslant_{\mathcal{L}} y \text { implies that } x^{\prime} \leqslant_{\mathcal{L}} y^{\prime}
$$

and

$$
x^{\prime} \leqslant_{\mathcal{R}} y^{\prime} \text { implies that } x \leqslant_{\mathcal{R}} y .
$$

Let $A, B$ be sets and $R \subseteq A \times B$ be a relation. Then $R$ is full if both projection maps are onto.

Lemma 4.18. [17] Let $I^{e, f}$ be full connecting. Then for any $(x, y),(z, t) \in I^{e, f}$,

$$
x \leqslant_{\mathcal{D}} z \text { if and only if } y \leqslant_{\mathcal{D}} t .
$$

Set

$$
U_{B}=\left\{\left(\rho_{e} I_{l}^{e, f}, \lambda_{f} I_{r}^{e, f}\right): e, f \in B, I^{e, f} \in\langle e\rangle \times\langle f\rangle \text { is full connecting }\right\}
$$

where $I_{l}^{e, f}$ is defined by

$$
L_{x} I_{l}^{e, f}=L_{y} \quad(x, y) \in I^{e, f}
$$


and $I_{r}^{e, f}$ is defined by

$$
R_{y} I_{r}^{e, f}=R_{x} \quad(x, y) \in I^{e, f} .
$$

Note that for any $e \in B$,

$$
\iota^{e, e}=\{(x, x): x \leq e\}
$$

is full connecting, and

$$
\left(\rho_{e} \ell_{l}^{e, e}, \lambda_{e} \iota_{l}^{e, e}\right)=\left(\rho_{e}, \lambda_{e}\right),
$$

so that $\bar{B}=\left\{\left(\rho_{e}, \lambda_{e}\right): e \in B\right\} \subseteq U_{B}$.

Lemma 4.19. [17] The set $U_{B}$ is a fundamental weakly $\bar{B}$-orthodox semigroup of $\mathcal{O P}(B / \mathcal{L}) \times \mathcal{O P}^{*}(B / \mathcal{R})$, with $(W I C)$.

We remark that for any $\left(\rho_{e} I_{l}^{e, f}, \lambda_{f} I_{r}^{e, f}\right) \in U_{B}$, we have that

$$
\left(\rho_{f}, \lambda_{f}\right) \widetilde{\mathcal{L}}_{\bar{B}}\left(\rho_{e} I_{l}^{e, f}, \lambda_{f} I_{r}^{e, f}\right) \widetilde{\mathcal{R}}_{\bar{B}}\left(\rho_{e}, \lambda_{e}\right) .
$$

Since Condition (WIC) gives us a very loose control over the position of idempotents, but does not impose artificially the existence of order isomorphisms, the idea used in previous sections to construct $O G_{2}$ and $K_{2}$ still works here, however, we need carefully deal with more complicated proofs. We first look at a concrete construction for a subsemigroup of $U_{B}$ as follows.

For any $e \in B$, we put

$$
Q_{1}=\bigcup_{e \in B} U_{e}
$$

where, $U_{e}=\left\{\left(\rho_{e} I_{l}^{e, e}, \lambda_{e} I_{r}^{e, e}\right) \in U_{B}\right.$ : for all $\left.(x, y) \in I^{e, e}, x \mathcal{D} y\right\}$.

Lemma 4.20. The set $Q_{1}$ forms a fundamental weakly $\bar{B}$-superabundant subsemigroup of $U_{B}$ with $(C)$ and $(W I C)$.

Proof. We first show that $Q_{1}$ is a semigroup. Let $\left(\rho_{e} I_{l}^{e, e}, \lambda_{e} I_{r}^{e, e}\right),\left(\rho_{f} J_{l}^{f, f}, \lambda_{f} J_{r}^{f, f}\right) \in$ $Q_{1}$. Since

$$
e f e \leq e \text { and } f e f \leq f,
$$

and $I^{e, e}, J^{f, f}$ are full connecting, there exist $(z, e f e) \in I^{e, e}$ and $(f e f, w) \in J^{f, f}$. 
By the construction of $Q_{1}, z \mathcal{D}$ efe $\mathcal{D}$ fef $\mathcal{D} w$. If follows from Lemma 1.21 that

$$
\left.\theta_{z}\right|_{\langle z w\rangle}:\langle z w\rangle \rightarrow\langle z\rangle,\left.\quad \theta_{f e f}\right|_{\langle e f e\rangle}:\langle e f e\rangle \rightarrow\langle f e f\rangle \text { and }\left.\theta_{z w}\right|_{\langle w\rangle}:\langle w\rangle \rightarrow\langle z w\rangle
$$

are $\mathcal{D}$-class preserving isomorphisms. We claim that $K^{z w, z w}$ is full connecting, where

$$
K^{z w, z w}=\left(\left.\theta_{z}\right|_{\langle z w\rangle}\right)\left(\left.I^{e, e}\right|_{\langle z\rangle}\right)\left(\left.\theta_{f e f}\right|_{\langle e f e\rangle}\right)\left(\left.J^{f, f}\right|_{\langle f e f\rangle}\right)\left(\left.\theta_{z w}\right|_{\langle w\rangle}\right) .
$$

To show that the projection maps to $\langle z w\rangle$ are onto, assume that $g \in B$ with $g \leq z w$. Since $\left.\theta_{z}\right|_{\langle z w\rangle}$ is an isomorphism from $\langle z w\rangle$ onto $\langle z\rangle$, we have that $\left.g \theta_{z}\right|_{\langle z w\rangle} \leq z$. As $z \leq e$ and $I^{e, e}$ is full connecting, there exists an element $\left(\left.g \theta_{z}\right|_{\langle z w\rangle}, t\right) \in I^{e, e}$. Now $\left.g \theta_{z}\right|_{\langle z w\rangle}=z g z$ and $I^{e, e}$ is a semigroup, so that

$$
\left(\left.g \theta_{z}\right|_{\langle z w\rangle}, \text { efetefe }\right)=(z, \text { efe })\left(\left.g \theta_{z}\right|_{\langle z w\rangle}, t\right)(z, \text { efe }) \in I^{e, e} .
$$

Clearly efetefe $\in\langle e f e\rangle$, so that

$$
\left.(\text { efetefe, } f(\text { efetefe }) f) \in \theta_{\text {fef }}\right|_{\langle e f e\rangle},
$$

that is,

$$
(\text { efetefe, }(f e f) \text { fetef }(f e f)) \in \theta_{\text {fef }} \mid\langle e f e\rangle \text {. }
$$

Now fete $f \in\langle f\rangle$; as $J^{f, f}$ is full connecting, there exists an element (fetef, $\left.k\right) \in$ $J^{f, f}$. Consequently,

$$
(f e f, w)(\text { fetef }, k)(f e f, w)=((f e f) \text { fetef }(f e f), w k w) \in J^{f, f} .
$$

Certainly, $w k w \in\langle w\rangle$, so that

$$
\left.(w k w, z w(w k w) z w) \in \theta_{z w}\right|_{\langle w\rangle} .
$$

It follows that

$$
(g, z w(w k w) z w) \in K^{z w, z w} .
$$

Dually, the projection of $K^{z w, z w}$ to the second coordinate is onto.

Since each of $\left.\theta_{z}\right|_{\langle z w\rangle}, I^{e, e},\left.\theta_{f e f}\right|_{\langle e f e\rangle}, J^{f, f}$ and $\left.\theta_{z w}\right|_{\langle w\rangle}$ is a subsemigroup of $B \times B$, it follows that the same is true of the composition, hence of $K^{z w, z w}$. As $\left.\theta_{z}\right|_{\langle z w\rangle}$ and $\left.\theta_{z w}\right|_{\langle w\rangle}$ are isomorphisms so that they preserve the partial order $\leq_{\mathcal{L}}$ 
and $\leq_{\mathcal{R}}$. Thus, $K^{z w, z w}$ is full connecting. Finally, since each of the relations concerned fixes the same $\mathcal{D}$-class, the same is clearly true of the composition, we have that $\left(\rho_{z w} K_{l}^{z w, z w}, \lambda_{z w} K_{r}^{z w, z w}\right) \in Q_{1}$.

Let $\left(\rho_{e} I_{l}^{e, e}, \lambda_{e} I_{r}^{e, e}\right),\left(\rho_{f} J_{l}^{f, f}, \lambda_{f} J_{r}^{f, f}\right) \in Q_{1}$. We claim that

$$
\left(\rho_{e} I_{l}^{e, e}, \lambda_{e} I_{r}^{e, e}\right)\left(\rho_{f} J_{l}^{f, f}, \lambda_{f} J_{r}^{f, f}\right)=\left(\rho_{z w} K_{l}^{z w, z w}, \lambda_{z w} K_{r}^{z w, z w}\right)
$$

To see this, let $x \in B$. we have that

$$
\begin{aligned}
L_{x} \rho_{e} I_{l}^{e, e} \rho_{f} J_{l}^{f, f} & =L_{e x e} I_{l}^{e, e} \rho_{f} J_{l}^{f, f} & & \\
& =L_{u} \rho_{f} J_{l}^{f, f} & & \text { where }(e x e, u) \in I^{e, e} \\
& =L_{f u f} J_{l}^{f, f} & & \\
& =L_{v} & & \text { where }(f u f, v) \in J^{f, f} .
\end{aligned}
$$

On the other hand,

$$
\begin{aligned}
& L_{x} \rho_{z w} K_{l}^{z w, z w} \\
& =L_{z w x z w}\left(\left(\left.\theta_{z}\right|_{\langle z w\rangle}\right)\left(\left.I^{e, e}\right|_{\langle z\rangle}\right)\left(\left.\theta_{f e f}\right|_{\langle e f e\rangle}\right)\left(J^{f, f} \mid\langle f e f\rangle\right)\left(\left.\theta_{z w}\right|_{\langle w\rangle}\right)\right)_{l} \\
& =L_{(z w x z w)}\left(\left(\left.\theta_{z}\right|_{\langle z w\rangle}\right)\left(\left.I^{e, e}\right|_{\langle z\rangle}\right)\left(\left.\theta_{f e f}\right|_{\langle e f e\rangle}\right)\left(\left.J^{f, f}\right|_{\langle f e f\rangle}\right)\left(\left.\theta_{z w}\right|_{\langle w\rangle}\right)\right) \\
& =L_{(z \cdot z w x z w \cdot z)}\left(\left(\left.I^{e, e}\right|_{\langle z\rangle}\right)\left(\left.\theta_{f e f}\right|_{\langle e f e\rangle}\right)\left(\left.J^{f, f}\right|_{\langle f e f\rangle}\right)\left(\left.\theta_{z w}\right|_{\langle w\rangle}\right)\right) \\
& =L_{(z w x z)}\left(\left(\left.I^{e, e}\right|_{\langle z\rangle}\right)\left(\left.\theta_{f e f \mid}\right|_{\langle e f e\rangle}\right)\left(\left.J^{f, f}\right|_{\langle f e f\rangle}\right)\left(\left.\theta_{z w}\right|_{\langle w\rangle}\right)\right) \quad(z \mathcal{D} \text { efe } \mathcal{D} \text { fef } \mathcal{D} w) \\
& =L_{(z x z)}\left(\left(\left.I^{e, e}\right|_{\langle z\rangle}\right)\left(\left.\theta_{f e f}\right|_{\langle e f e\rangle}\right)\left(\left.J^{f, f}\right|_{\langle f e f\rangle}\right)\left(\left.\theta_{z w}\right|_{\langle w\rangle}\right)\right) \\
& =L_{(z \text { exez })}\left(\left(I^{e, e} \mid\langle z\rangle\right)\left(\left.\theta_{f e f \mid}\right|_{\langle e f e\rangle}\right)\left(\left.J^{f, f}\right|_{\langle f e f\rangle}\right)\left(\left.\theta_{z w \mid}\right|_{\langle w\rangle}\right)\right) \quad(z \leq e) \\
& =L_{(\text {efeuefe })}\left(\left(\left.\theta_{f e f}\right|_{\langle e f e\rangle}\right)\left(\left.J^{f, f}\right|_{\langle f e f\rangle}\right)\left(\left.\theta_{z w}\right|_{\langle w\rangle}\right)\right) \quad\left((z, \text { efe }),(\text { exe }, u) \in I^{e, e}\right) \\
& =L_{(\text {fef } \cdot \text { efeuefe } \cdot f e f)}\left(\left(\left.J^{f, f}\right|_{\langle f e f\rangle}\right)\left(\left.\theta_{z w}\right|_{\langle w\rangle}\right)\right) \\
& =L_{(f e f \cdot f u f \cdot f e f)}\left(\left(\left.J^{f, f}\right|_{\langle f e f\rangle}\right)\left(\left.\theta_{z w}\right|_{\langle w\rangle}\right)\right) \\
& =L_{(w v w)}\left(\left.\theta_{z w}\right|_{\langle w\rangle}\right) \\
& =L_{z w w v w z w} \\
& (u \in\langle e\rangle) \\
& \left((f e f, w),(f u f, v) \in J^{f, f}\right)
\end{aligned}
$$




$$
\begin{array}{ll}
=L_{z w v w} & (z \mathcal{D} w) \\
=L_{w v w} & (\text { Lemma }
\end{array}
$$

Note that $(f u f, v),(f e f, w) \in J^{f, f}$, we obtain that $(f e f u f e f, w v w) \in J^{f, f}$ as $J^{f, f}$ is a subsemigroup. As $u \in\langle e\rangle$, and so fefufef $=$ fefeuefef $=$ feuef $=f u f$ so that $(f u f, w v w) \in J^{f, f}$. Since $J^{f, f}$ is full connecting and $(f u f, v) \in J^{f, f}$, it follows that $L_{v}=L_{w v w}$. Thus, $\rho_{z w} K_{l}^{z w, z w}=\rho_{e} I_{l}^{e, e} \rho_{f} J_{l}^{f, f}$. Dually, we obtain that $\lambda_{z w} K_{r}^{z w, z w}=\lambda_{f} I_{r}^{f, f} \lambda_{e} J_{r}^{e, e}$, so that

$$
\left(\rho_{e} I_{l}^{e, e}, \lambda_{e} I_{r}^{e, e}\right)\left(\rho_{f} J_{l}^{f, f}, \lambda_{f} J_{r}^{f, f}\right)=\left(\rho_{z w} K_{l}^{z w, z w}, \lambda_{z w} K_{r}^{z w, z w}\right)
$$

Hence, $Q_{1}$ forms a semigroup. In view of the remark following Lemma 4.19, we have that $\left(\rho_{e} I_{l}^{e, e}, \lambda_{e} I_{r}^{e, e}\right) \widetilde{\mathcal{H}}_{\bar{B}}\left(\rho_{e}, \lambda_{e}\right)$, and so $Q_{1}$ is weakly $\bar{B}$-superabundant.

As $\bar{B} \subseteq Q_{1}$, we have that $Q_{1}$ is a full subsemigroup of $U_{B}$, and so $Q_{1}$ is a fundamental weakly $\bar{B}$-superabundant subsemigroup of $U_{B}$ with $(\mathrm{C})$ and (WIC).

Another alternative characterisation of a fundamental weakly $\bar{B}$-superabundant subsemigroup $Q_{2}$ of $U_{B}$, satisfying (C) and (WIC), is available. The following construction is closely analogous to that of $K_{1}$ and $O G_{1}$.

Before describing the construction of $Q_{2}$, we mention an important fact.

Now, let $B$ be a band. Obviously, it is a semilattice $Y$ of rectangular bands $B_{\alpha}(\alpha \in Y)$. In addition, there is the Munn semigroup $T_{Y}$ corresponding to $Y$. If $e, f \in B$ with $e \in B_{\alpha}, f \in B_{\beta}(\alpha, \beta \in Y)$, and $I^{e, f}$ is a full connecting relation in $\langle e\rangle \times\langle f\rangle$, then it follows from Lemma 4.18 that there exists a mapping $\overline{I^{e, f}}$ from $\alpha Y$ onto $\beta Y$ with the property that

$$
(u, v) \in I^{e, f} \text { implies that }(u, v) \in B_{\xi} \times B_{\xi I^{e, f}},
$$

where $u \in\langle e\rangle \cap B_{\xi}$. In fact, $\overline{I^{e, f}}$ must be a bijection.

We define a map

$$
\chi: U_{B} \rightarrow T_{Y}
$$

by

$$
\left(\rho_{e} I_{l}^{e, f}, \lambda_{f} I_{r}^{e, f}\right) \chi=\overline{I^{e, f}}
$$

for any $\left(\rho_{e} I_{l}^{e, f}, \lambda_{f} I_{r}^{e, f}\right) \in U_{B}$ 
Lemma 4.21. The mapping $\chi$ is a morphism.

Proof. We first claim that $\chi$ is well-defined. To do this, let $\left(\rho_{e} I_{l}^{e, f}, \lambda_{f} I_{r}^{e, f}\right) \in U_{B}$. We first show that $\overline{I^{e, f}}$ is an isomorphism from $\alpha Y$ onto $\beta Y$, where $e \in B_{\alpha}$ and $f \in B_{\beta}$.

It is easy to see that $\overline{I^{e, f}}$ is well-defined since if $(x, y),(z, t) \in I^{e, f}$ with $x \mathcal{D} z$, then by Lemma 4.18, we have that $y \mathcal{D} t$. Again by Lemma 4.18, we have that $\overline{I^{e, f}}$ is injective. As $I^{e, f}$ is full, it is certainly true that $\overline{I^{e, f}}$ is surjective. In addition, $\overline{I^{e, f}}$ is a morphism because $I^{e, f}$ is a subsemigroup of $\langle e\rangle \times\langle f\rangle$.

If $\left(\rho_{g} J_{l}^{g, h}, \lambda_{h} J_{r}^{g, h}\right) \in U_{B}$ and $\left(\rho_{e} I_{l}^{e, f}, \lambda_{f} I_{r}^{e, f}\right)=\left(\rho_{g} J_{l}^{g, h}, \lambda_{h} J_{r}^{g, h}\right)$, then we show that $\overline{I^{e, f}}=\overline{J^{g, h}}$. We have remarked that

$$
\left(\rho_{e}, \lambda_{e}\right) \widetilde{\mathcal{R}}_{\bar{B}}\left(\rho_{e} I_{l}^{e, f}, \lambda_{f} I_{r}^{e, f}\right) \widetilde{\mathcal{L}}_{\bar{B}}\left(\rho_{f}, \lambda_{f}\right)
$$

Similarly, $\left(\rho_{g}, \lambda_{g}\right) \widetilde{\mathcal{R}}_{\bar{B}}\left(\rho_{g} J_{l}^{g, h}, \lambda_{h} J_{r}^{g, h}\right) \widetilde{\mathcal{L}}_{\bar{B}}\left(\rho_{h}, \lambda_{h}\right)$. Thus $\left(\rho_{e}, \lambda_{e}\right) \mathcal{R}\left(\rho_{g}, \lambda_{g}\right)$. Since $B$ is isomorphic to $\bar{B}$, we have that $e \mathcal{R} g$, and so $e \mathcal{D} g$. Similarly, $f \mathcal{D} h$. Hence,

$$
\operatorname{dom} \overline{I^{e, f}}=\operatorname{dom} \overline{J^{g, h}}=\alpha Y, \quad \operatorname{im} \overline{I^{e, f}}=\operatorname{im} \overline{J^{g, h}}=\beta Y,
$$

where $e, g \in B_{\alpha}$ and $f, h \in B_{\beta}$.

Let $\xi \in \operatorname{dom} \overline{I^{e, f}}$ and $x \in B_{\xi} \cap\langle e\rangle$. Then there exists $y \in B$ such that $x=e y e$. Since $e \mathcal{D} g$, we have that $x=$ eye $\mathcal{D} g y g \leq g$, and so gyg $\in B_{\xi}$. Observe that

$$
L_{y} \rho_{e} I_{l}^{e, f}=L_{\text {eye }} I_{l}^{e, f}=L_{z} \quad\left((\text { eye }, z) \in I^{e, f}\right)
$$

and

$$
L_{y} \rho_{g} J_{l}^{g, h}=L_{g y g} J_{l}^{g, h}=L_{u} \quad\left((g y g, u) \in J^{g, h}\right) .
$$

As $\rho_{e} I^{e, f}=\rho_{g} J^{g, h}$, we have that $z \mathcal{L} u$. Then $\xi \overline{I^{e, f}}=\xi \overline{J^{g, h}}=\eta$, where $z, u \in B_{\eta}$. Thus $\overline{I^{e, f}}=\overline{J^{e, f}}$, and so $\chi$ is well-defined.

In the following, we show that $\chi$ is a morphism. According to Lemma 4.3 of $[6]$, we have that

$$
\left(\rho_{e} I_{l}^{e, f}, \lambda_{f} I_{r}^{e, f}\right)\left(\rho_{g} J_{l}^{g, h}, \lambda_{h} J_{r}^{g, h}\right)=\left(\rho_{z} K_{l}^{z, w}, \lambda_{w} K_{r}^{z, w}\right)
$$


where $(z, f g f) \in I^{e, f},(g f g, w) \in J^{g, h}$ and

$$
K^{z, w}=\left(I^{e, f}\left(\left.\theta_{g f g}\right|_{\langle f g f\rangle}\right) J^{g, h}\right) \cap(\langle z\rangle \times\langle w\rangle) .
$$

Hence, it is sufficient to show that

$$
\overline{I^{e, f}} \circ \overline{J^{g, h}}=\overline{K^{z, w}} \text {. }
$$

We have remarked that $\operatorname{dom} \overline{I^{e, f}}=\alpha Y$, im $\overline{I^{e, f}}=\beta Y$. Similarly, $\operatorname{dom} \overline{J^{g, h}}=\gamma Y$, $\operatorname{im} \overline{J^{e, f}}=\delta Y$, where $g \in B_{\gamma}, h \in B_{\delta}$. Thus

$$
\begin{aligned}
& \xi \in \operatorname{dom} \overline{I^{e, f}} \circ \overline{J^{g, h}} \\
\Leftrightarrow & \xi \in\left(\operatorname{im} \overline{I^{e, f}} \cap \operatorname{dom} \overline{J^{g, h}}\right) \overline{I^{e, f}}-1 \\
\Leftrightarrow & \xi \in(\beta \gamma) \overline{I^{e, f}}-1 \\
\Leftrightarrow & \exists x \in B_{\xi} \cap\langle e\rangle,(x, y) \in I^{e, f}, y \in B_{\mu}, \mu \leq \beta \gamma \\
\Leftrightarrow & \xi \leq \tau \text { where } z \in B_{\xi} \text { since }(z, f g f) \in I^{e, f} \\
\Leftrightarrow & \xi \in \operatorname{dom} \overline{K^{z, w}} .
\end{aligned}
$$

Returning to the above, let $\xi \in \operatorname{dom}\left(\overline{I^{e, f}} \circ \overline{J^{g, h}}\right)$ and $x \in B_{\xi}$, so that $y \in B_{\xi \overline{I^{e, f}}}=\mu$. Now $(g f g) g f y f g(g f g) \in B_{\mu}($ as $\mu \leq \beta \gamma)$ and $((g f g) g f y f g(g f g), w h w) \in J^{g, h}$, so $\mu \overline{J^{g, h}}=\nu$, where $w h w \in B_{\gamma}$. Thus $\overline{\xi^{e, f}} \circ \overline{J^{g, h}}=\nu=\xi \overline{K^{z, w}}$, as $(x, w h w) \in K^{z, w}$. So

$$
\overline{I^{e, f}} \circ \overline{J^{g, h}}=\overline{K^{z, w}} \text {. }
$$

We omit the proof of the next lemma as it follows from Lemma 4.23.

Lemma 4.22. The set of the elements of $U_{B}$, whose images under $\chi$ are idempotent, forms a fundamental weakly $\bar{B}$-superabundant subsemigroup of $U_{B}$ with (C) and (WIC). We denote it by $Q_{2}$.

Lemma 4.23. The semigroup $Q_{2}$ coincides with $Q_{1}$.

Proof. It is easy to see that $Q_{1} \subseteq Q_{2}$. We show that $Q_{2} \subseteq Q_{1}$. Suppose that $\left(\rho_{e} I^{e, f}, \lambda_{f} I_{r}^{e, f}\right) \in Q_{2}$. Then $e \mathcal{D} f$ and $\overline{I^{e, f}}$ is the identity map from $\alpha Y$ to $\alpha Y$, where $e \in B_{\alpha}$. So for any $(u, v) \in I^{e, f}, u \mathcal{D} v$. Now let $J^{\text {ef,ef }}=$ 
$\left(\left.\theta_{e}\right|_{\langle e f\rangle}\right) I^{e, f}\left(\left.\theta_{e f}\right|_{\langle f\rangle}\right)$. We claim that $\left(\rho_{e f} J_{l}^{e f, e f}, \lambda_{e f} J_{r}^{e f, e f}\right)=\left(\rho_{e} I^{e, f}, \lambda_{f} I_{r}^{e, f}\right)$. For any $x \in B$, we have that

$$
\begin{aligned}
& L_{x} \rho_{e f} J_{l}^{e f, e f}=L_{(e f x e f) J^{e f, e f}} \\
& =L_{(e f x e f)\left(\left.\theta_{e}\right|_{\langle e f\rangle}\right) I^{e, f}\left(\left.\theta_{e f}\right|_{\langle f\rangle}\right)} \\
& =L_{(e \cdot e f x e f \cdot e) I^{e, f}\left(\left.\theta_{e f}\right|_{\langle f\rangle}\right)} \\
& =L_{(e \cdot e f x e f \cdot e)}\left(I^{e, f}\left(\theta_{e f} \mid\langle f\rangle\right)\right)_{l} \\
& =L_{\text {exe }}\left(I^{e, f}\left(\left.\theta_{\text {ef }}\right|_{\langle f\rangle}\right)\right)_{l} \quad(e \mathcal{D} f, \text { Lemma 1.22) } \\
& =L_{(e x e) I^{e, f}\left(\left.\theta_{e f}\right|_{\langle f\rangle}\right)} \\
& =L_{\left.y \theta_{e f}\right|_{\langle f\rangle}} \quad\left((e x e, y) \in I^{e, f}\right) \\
& =L_{\text {efyef }} \\
& =L_{y e f} \\
& =L_{y \cdot f \cdot e f} \quad(y \in\langle f\rangle) \\
& =L_{y f} \quad(f \mathcal{D} e) \\
& =L_{y} \\
& =L_{e x e} I_{l}^{e, f} \quad\left((e x e, y) \in I^{e, f}\right) \\
& =L_{x} \rho_{e} I_{l}^{e, f} \text {. }
\end{aligned}
$$

Thus, $\rho_{e f} J_{l}^{e f, e f}=\rho_{e} I_{l}^{e, f}$. Dually, we obtain that $\lambda_{e f} J_{r}^{e f, e f}=\lambda_{f} I_{r}^{e, f}$. Hence $\left(\rho_{e f} J_{l}^{e f, e f}, \lambda_{e f} J_{r}^{e f, e f}\right)=\left(\rho_{e} I_{l}^{e, f}, \lambda_{f} I_{r}^{e, f}\right)$. In addition, by the proof of Lemma 4.21, we have $\overline{J^{e f, e f}}=\overline{I^{e, f}}$. Consequently, $Q_{2} \subseteq Q_{1}$.

Lemma 4.24. [17] If $S$ is a weakly B-orthodox semigroup with (WIC), then the map $\theta: S \rightarrow U_{B}$ given by $a \theta=\left(\alpha_{a}, \beta_{a}\right)$, where $\alpha_{a}$ and $\beta_{a}$ are defined as Section 4.3, is a strongly B-admissible morphism with kernel $\mu_{B}$. Moreover, $\left.\theta\right|_{B}: B \rightarrow \bar{B}$ is an isomorphism.

Consequently, we have:

Theorem 4.25. If $S$ is a weakly B-superabundant semigroup with (WIC) and $(C)$, then the map $\phi: S \rightarrow U_{B}$ in Lemma 4.24 has image contained in $Q_{1}$.

Proof. Suppose that $a \in S$ and $e \in \widetilde{H}_{a} \cap B$. In view of [6], we know $a \phi=$ $\left(\alpha_{a}, \beta_{a}\right)=\left(\rho_{a^{\dagger}} I_{l}^{a^{\dagger}, a^{*}}, \lambda_{a^{*}} I_{r}^{a^{\dagger}}, a^{*}\right)$. Specially, we can use $e$ instead of $a^{\dagger}$ and $a^{*}$ so 
that we obtain $a \phi=\left(\rho_{e} I_{l}^{e, e}, \lambda_{e} I_{r}^{e, e}\right)$. In addition, for any $x \in\langle e\rangle$, we have that $x a=a y$, where $(x, y) \in I^{e, e}$. As

$$
x=x e \widetilde{\mathcal{R}}_{B} x a=a y \widetilde{\mathcal{L}}_{B} e y=y .
$$

We have that $x \widetilde{\mathcal{D}}_{B} y$. It follows from Lemma 3.10 that $x \mathcal{D} y$ in $B$. Thus $a \phi=$ $\left(\rho_{e} I_{l}^{e, e}, \lambda_{e} I_{r}^{e, e}\right)$ is contained in $Q_{1}$.

\subsection{A fundamental weakly $\bar{B}$-superabundant sub- semigroup of $S_{B}$}

The aim of this section is to remove the idempotent connected condition from the results of previous sections. We stress that to do so Gomes and Gould [17] used a completely fresh technique to construct from a band $B$ a weakly $B$-orthodox subsemigroup $S_{B}$ of $\mathcal{O P}\left(B^{1} / \mathcal{L}\right) \times \mathcal{O} \mathcal{P}^{*}\left(B^{1} / \mathcal{R}\right)$, with the property that any fundamental weakly $\bar{B}$-orthodox semigroup is a subsemigroup of $S_{B}$, where $\bar{B}$ is isomorphic to $B$. As a consequence, the fundamental semigroup $U_{B}$ constructed in last section, satisfying (C) and (WIC) is embedded into $S_{B}$.

To define $S_{B}$, we give some notation. For a set $X$, an equivalence $\kappa$ on $X$ and $\gamma: X / \kappa \rightarrow X / \kappa$, the relation $\bar{\gamma}$ is defined by

$$
\bar{\gamma}=\{(x, y) \in X \times X: y \in[x] \gamma\}
$$

where $[x]$ is the equivalence class containing $x$.

We put

$$
\begin{gathered}
S_{B}=\left\{(\alpha, \beta) \in \mathcal{O}^{1}(B): \text { for all } x \in B^{1}, x \bar{\alpha} \in L_{x} \alpha \text { and } x \bar{\beta} \in R_{x} \beta,\right. \\
\text { we have } \left.\beta \lambda_{x}=\lambda_{x \bar{\alpha}} \beta \lambda_{x} \text { and } \alpha \rho_{x}=\rho_{x \bar{\beta}} \alpha \rho_{x}\right\},
\end{gathered}
$$

where

$$
\mathcal{O}^{1}(B)=\left\{(\alpha, \beta) \in \mathcal{O P}\left(B^{1} / \mathcal{L}\right) \times \mathcal{O P}^{*}\left(B^{1} / \mathcal{R}\right): \operatorname{Im} \alpha \subseteq B / \mathcal{L}, \operatorname{Im} \beta \subseteq B / \mathcal{R}\right\}
$$

Lemma 4.26. [17] The set $S_{B}$ is weakly $\bar{B}$-orthodox and is $\bar{B}$-fundamental, where $\bar{B}=\left\{\left(\rho_{e}, \lambda_{e}\right): e \in B\right\}$. 
We remark that if $(\alpha, \beta) \in S_{B}, u \in L_{1} \alpha$ and $v \in R_{1} \beta$, then

$$
(\alpha, \beta) \widetilde{\mathcal{L}}_{\bar{B}}\left(\rho_{u}, \lambda_{u}\right) \text { and }(\alpha, \beta) \widetilde{\mathcal{R}}_{\bar{B}}\left(\rho_{v}, \lambda_{v}\right) .
$$

In the following we will construct a weakly $\bar{B}$-superabundant subsemigroup of $S_{B}$, that is fundamental and satisfies (C). Put

$$
\begin{aligned}
& N_{B}=\left\{(\alpha, \beta) \in S_{B} \text { : there exists } e \in L_{1} \alpha \cap R_{1} \beta \cap B\right. \text { such that for all } \\
& \left.\qquad x \in B^{1}, x \bar{\alpha} \in L_{x} \alpha \text { and } x \bar{\beta} \in R_{x} \beta \text {, we have } x \bar{\alpha} \mathcal{D} \text { exe } \mathcal{D} x \bar{\beta}\right\} .
\end{aligned}
$$

Lemma 4.27. The set $N_{B}$ is a subsemigroup of $S_{B}$ containing the band of idempotents $\bar{B}=\left\{\left(\rho_{e}, \lambda_{e}\right): e \in B\right\}$.

Proof. To show that $N_{B}$ is a subsemigroup of $S_{B}$, it is sufficient to show that $N_{B}$ is closed under the multiplication. We suppose that $(\alpha, \beta),(\gamma, \delta) \in N_{B}$. Then there exists $e \in L_{1} \alpha \cap R_{1} \beta \cap B$ such that for all $x \in B^{1}, x \bar{\alpha} \in L_{x} \alpha$ and $x \bar{\beta} \in R_{x} \beta$, we have that $x \bar{\alpha} \mathcal{D}$ exe $\mathcal{D} x \bar{\beta}$. Also, there exists $f \in L_{1} \gamma \cap R_{1} \delta \cap B$ such that for all $x \in B^{1}, x \bar{\gamma} \in L_{x} \gamma$ and $x \bar{\delta} \in R_{x} \delta$, we have that $x \bar{\gamma} \mathcal{D}$ fxf $\mathcal{D} x \bar{\delta}$. We consider the product of $(\alpha, \beta)$ and $(\gamma, \delta)$. Observe that

$$
L_{1} \alpha \gamma=L_{e} \gamma=L_{e \bar{\gamma}}, \quad \quad e \bar{\gamma} \mathcal{D} \text { fef } \mathcal{D} e \bar{\delta},
$$

and

$$
R_{1} \delta \beta=R_{f} \beta=R_{f \bar{\beta}}, \quad f \bar{\beta} \mathcal{D} \text { efe } \mathcal{D} f \bar{\alpha} .
$$

Since efe $\mathcal{D}$ fef, we have that

$$
L_{1} \alpha \gamma=L_{(f \bar{\beta})(e \bar{\gamma})}, \quad R_{1} \delta \beta=R_{(f \bar{\beta})(e \bar{\gamma})}, \quad \text { and }(f \bar{\beta})(e \bar{\gamma}) \mathcal{D} \text { ef }
$$

So, $(f \bar{\beta})(e \bar{\gamma}) \in L_{1} \alpha \gamma \cap R_{1} \delta \beta$. Now, we fix the choices of $f \bar{\beta}$ and $e \bar{\gamma}$.

For all $x \in B^{1}$, we have that $x \overline{\alpha \gamma} \in L_{x} \alpha \gamma$ and $x \overline{\delta \beta} \in R_{x} \delta \beta$. Hence,

$$
x \overline{\alpha \gamma} \mathcal{L}(x \bar{\alpha}) \bar{\gamma} \mathcal{D} f(x \bar{\alpha}) f \mathcal{D} \text { fexef }
$$

and

$$
x \bar{\delta} \mathcal{R}(x \bar{\delta}) \bar{\beta} \mathcal{D} e(x \bar{\delta}) \text { e } \mathcal{D} \text { ef xef. }
$$


As $(f \bar{\beta})(e \bar{\gamma}) \mathcal{D}$ ef, we obtain that

$$
x \overline{\alpha \gamma} \mathcal{D}(f \bar{\beta})(e \bar{\gamma}) x(f \bar{\beta})(e \bar{\gamma}) \mathcal{D} x \overline{\delta \beta}
$$

Thus, $(\alpha \gamma, \delta \beta) \in N_{B}$, and so $N_{B}$ forms a semigroup.

Finally, we should show that $N_{B}$ contains the band of idempotents $\bar{B}$. For any $e \in B,\left(\rho_{e}, \lambda_{e}\right) \in \bar{B}$. Clearly, $e \in L_{1} \rho_{e} \cap R_{1} \lambda_{e} \cap B$. For all $x \in B^{1}$, $x \overline{\rho_{e}} \in L_{x} \rho_{e}$ and $x \overline{\lambda_{e}} \in R_{x} \lambda_{e}$, we have that $x \overline{\rho_{e}} \mathcal{L}$ xe $\mathcal{D}$ ex $\mathcal{R} x \overline{\lambda_{e}}$. It follows that $x \overline{\rho_{e}} \mathcal{D}$ exe $\mathcal{D} x \overline{\lambda_{e}}$. Hence, $\left(\rho_{e}, \lambda_{e}\right) \in N_{B}$. Consequently, $\bar{B} \subseteq N_{B}$.

Further information about $S_{B}$ is obtained from the following result.

Theorem 4.28. The semigroup $N_{B}$ is a fundamental weakly $\bar{B}$-superabundant subsemigroup of $S_{B}$ with $(C)$.

Proof. For any $(\alpha, \beta) \in N_{B}$, there exists $e \in L_{1} \alpha \cap R_{1} \beta \cap B$. In view of the remark following Lemma 4.26 , we have that $(\alpha, \beta) \widetilde{\mathcal{H}}_{\bar{B}}\left(\rho_{e}, \lambda_{e}\right)$. Hence, $N_{B}$ is a weakly $\bar{B}$-superabundant subsemigroup of $S_{B}$.

Indeed $N_{B}$ is $\bar{B}$-fundamental with (C) as $\bar{B} \subseteq N_{B}$.

We now want to make full use of the approach of $O G_{1}$ to determine a subsemigroup of $S_{B}$ that is fundamental weakly $\bar{B}$-superabundant with (C). But in view of the fact we no longer have any idempotent connected condition, we can not find a useful mapping from $S_{B}$ to the Munn semigroup $T_{Y}$ to get this result, where $B$ is a semilattice $Y$ of rectangular bands $B_{\alpha}(\alpha \in Y)$. With this in mind, we decide to consider a mapping from $S_{B}$ to $C_{Y}$, where $C_{Y}$ is a fundamental Ehresmann semigroup with $(\mathrm{C})$. We refer the reader to [18] to acquire more details about $C_{Y}$. Here we provide some general facts about $C_{Y}$. We have

$$
\begin{gathered}
C_{Y}=\left\{(\alpha, \beta) \in \mathcal{O}_{1}\left(Y^{1}\right) \times \mathcal{O}_{1}^{*}\left(Y^{1}\right): \operatorname{Im} \alpha, \operatorname{Im} \beta \subseteq Y \text { and } \forall x \in Y^{1},\right. \\
\left.\rho_{x \alpha} \leqslant \beta \rho_{x} \alpha \text { and } \rho_{x \beta} \leqslant \alpha \rho_{x} \beta\right\}
\end{gathered}
$$

where for any $x \in Y, \rho: Y^{1} \rightarrow Y$ is the order preserving map given by $\xi \rho_{x}=\xi x=$ $x \xi$ and for $\gamma, \delta \in \mathcal{O P}\left(Y^{1}\right), \gamma \leqslant \delta$ means that $y \gamma \leqslant y \delta$ for all $y \in Y^{1}$. From [18], $C_{Y}$ is a fundamental Ehresmann semigroup with $(\mathrm{C})$ and is isomorphic to $\mathcal{S}_{Y}$, where

$$
\mathcal{S}_{Y}=\left\{(\alpha, \beta) \in \mathcal{O}_{1}\left(Y^{1}\right) \times \mathcal{O}_{1}^{*}\left(Y^{1}\right): \operatorname{Im} \alpha, \operatorname{Im} \beta \subseteq Y \text { and } \forall x \in Y^{1},\right.
$$




$$
\left.\beta \rho_{x}=\rho_{x \alpha} \beta \rho_{x} \text { and } \alpha \rho_{x}=\rho_{x \beta} \alpha \rho_{x}\right\}
$$

and the set of distinguished idempotents of $\mathcal{S}_{Y}$ is $\bar{Y}=\left\{\left(\rho_{\xi}, \lambda_{\xi}\right): \xi \in Y\right\}$.

We define a mapping $\phi: S_{B} \rightarrow \mathcal{S}_{Y}$ by the rule that for any $(\alpha, \beta) \in S_{B}$

$$
(\alpha, \beta) \phi=\left(\alpha^{\prime}, \beta^{\prime}\right)
$$

where $\left(\alpha^{\prime}, \beta^{\prime}\right)$ is a pair of mappings from $Y^{1}$ to $Y$ having the property that for any $x \in B^{1}$ with $x \in B_{\xi}^{1}, x \bar{\alpha} \in L_{x} \alpha$ and $x \bar{\beta} \in R_{x} \beta$, we have that $x \bar{\alpha} \in B_{\xi \alpha^{\prime}}^{1}$ and $x \bar{\beta} \in B_{\xi \beta^{\prime}}^{1}$.

Lemma 4.29. The mapping $\phi$ is a morphism.

Proof. Certainly, for any $(\alpha, \beta) \in S_{B}$, we have that $\operatorname{Im} \alpha^{\prime}, \operatorname{Im} \beta^{\prime} \subseteq Y$. To show that $\phi$ is well-defined, suppose that $\xi \in Y^{1}$ and $x \in B_{\xi}^{1}$. Then for any $x \bar{\alpha} \in L_{x} \alpha$ and $x \bar{\beta} \in R_{x} \beta$, we have that $\beta \lambda_{x}=\lambda_{x \bar{\alpha}} \beta \lambda_{x}$ and $\alpha \rho_{x}=\rho_{x \bar{\beta}} \alpha \rho_{x}$. For any $\kappa \in Y^{1}$ and $y \in B_{\kappa}^{1}$, we have that

$$
R_{y} \beta \lambda_{x}=R_{y} \lambda_{x \bar{\alpha}} \beta \lambda_{x} \quad \text { and } \quad L_{y} \alpha \rho_{x}=L_{y} \rho_{x \bar{\beta}} \alpha \rho_{x}
$$

which means $R_{y} \beta \lambda_{x}$ and $R_{y} \lambda_{x \bar{\alpha}} \beta \lambda_{x}$ are in the same $\mathcal{D}$-class and the same is true for $L_{y} \alpha \rho_{x}$ and $L_{y} \rho_{x \bar{\beta}} \alpha \rho_{x}$. Thus, $\kappa \beta^{\prime} \rho_{\xi}=\kappa \rho_{\xi \alpha^{\prime}} \beta^{\prime} \rho_{\xi}$ and $\kappa \alpha^{\prime} \rho_{\xi}=\kappa \rho_{\xi \beta^{\prime}} \alpha^{\prime} \rho_{\xi}$. Hence $\left(\alpha^{\prime}, \beta^{\prime}\right) \in S_{Y}$.

Clearly, $\phi$ is a morphism.

Lemma 4.30. The set of the elements of $S_{B}$ whose images under $\phi$ are distinguished idempotents of $\mathcal{S}_{Y}$, forms a fundamental weakly $\bar{B}$-superabundant subsemigroup of $S_{B}$ with $(C)$. We denote it by $N_{B}^{\prime}$.

Proof. Since $\phi$ is a morphism and the distinguished idempotents of $\mathcal{S}_{Y}$ form a semilattice, it follows that $N_{B}^{\prime}$ is closed. Also, it is easy to see that $\bar{B} \subseteq N_{B}^{\prime}$, which implies that $N_{B}^{\prime}$ has $(\mathrm{C})$ and is fundamental.

It remains to show that $N_{B}^{\prime}$ is weakly $\bar{B}$-superabundant. We assume that $(\alpha, \beta) \in N_{B}^{\prime}$. Then $\left(\alpha^{\prime}, \beta^{\prime}\right) \in \bar{E}\left(\mathcal{S}_{Y}\right)$. It follows that $\alpha^{\prime}=\beta^{\prime}$. Specially, $1 \alpha^{\prime}=1 \beta^{\prime}$, and so if $1 \bar{\alpha} \in B_{1 \alpha^{\prime}}$ and $1 \bar{\beta} \in B_{1 \beta^{\prime}}$, then, $1 \bar{\alpha} \mathcal{D} 1 \bar{\beta}$. Therefore, there exists an idempotent $e \in L_{1 \bar{\alpha}} \cap R_{1 \bar{\beta}}$, that is, $e \in L_{1} \alpha \cap R_{1} \beta$. By the remark following Lemma 4.26, we obtain that $(\alpha, \beta) \widetilde{\mathcal{H}}_{B}\left(\rho_{e}, \lambda_{e}\right)$. Consequently, $N_{B}^{\prime}$ is weakly $\bar{B}$-superabundant. 
To reveal the relationship between $N_{B}$ and $N_{B}^{\prime}$, we have:

Lemma 4.31. The semigroup $N_{B}$ coincides with $N_{B}^{\prime}$.

Proof. We begin by showing that $N_{B} \subseteq N_{B}^{\prime}$. Suppose that $(\alpha, \beta) \in N_{B}$. Then there exists $e \in L_{1} \alpha \cap R_{1} \beta \cap B$ such that for all $x \in B^{1}, x \bar{\alpha} \in L_{x} \alpha$ and $x \bar{\beta} \in R_{x} \beta$, we have that $x \bar{\alpha} \mathcal{D}$ exe $\mathcal{D} x \bar{\beta}$. It follows that $\alpha^{\prime}=\beta^{\prime}$ and for any $\varepsilon \in Y^{1}$, $\varepsilon \alpha^{\prime}=\left(1 \alpha^{\prime}\right) \varepsilon$, that is, $\alpha^{\prime}=\rho_{1 \alpha^{\prime}}$. Hence, $\alpha^{\prime}=\left(\alpha^{\prime}\right)^{2}$, and so $\left(\alpha^{\prime}, \beta^{\prime}\right)$ is an idempotent of $\mathcal{S}_{Y}$. Since there exists an idempotent $e \in L_{1} \alpha \cap R_{1} \beta$ with $e \in B_{\xi}$, it follows that $\varepsilon \alpha^{\prime}=\rho_{\xi} \varepsilon=\varepsilon \rho_{\xi}$. Therefore, $\left(\alpha^{\prime}, \beta^{\prime}\right)=\left(\rho_{\xi}, \lambda_{\xi}\right) \in \bar{E}\left(\mathcal{S}_{Y}\right)$. Consequently, $N_{B} \subseteq N_{B}^{\prime}$.

Conversely, suppose that $(\alpha, \beta) \in N_{B}^{\prime}$. Then there exists an idempotent $\left(\rho_{\tau}, \lambda_{\tau}\right)$ such that $\left(\rho_{\tau}, \lambda_{\tau}\right)=\left(\alpha^{\prime}, \beta^{\prime}\right)$ which implies that $\alpha^{\prime}=\beta^{\prime}$ and there exists $e \in L_{1} \alpha \cap R_{1} \beta$ with $e \in B_{\tau}$. For any $x \in B^{1}$ with $x \in B_{\xi}, x \bar{\alpha} \in L_{x} \alpha$ and $x \bar{\beta} \in$ $R_{x} \beta$, we have that $x \bar{\alpha}, x \bar{\beta} \in B_{\xi \rho_{\tau}}$. Thus, $x \bar{\alpha} \mathcal{D}$ ex $\mathcal{D} x \bar{\beta}$. Hence $N_{B}^{\prime} \subseteq N_{B}$.

Lemma 4.32. [17] Let $S$ be a weakly B-orthodox semigroup. Then $\theta: S \rightarrow S_{B}$ given by

$$
a \theta=\left(\alpha_{a}, \beta_{a}\right)
$$

where $\alpha_{a}$ and $\beta_{a}$ are defined in Section 4.3, is a strongly admissible morphism with kernel $\mu_{B}$. Moreover, $\left.\theta\right|_{B}: B \rightarrow \bar{B}$ is an isomorphism.

Corollary 4.33. Let $S$ be a weakly B-orthodox semigroup and let $K$ be a subsemigroup of $S_{B}$ containing $\bar{B}$. Then $K$ is a weakly $\bar{B}$-orthodox semigroup. If $K$ contains the image of $S$ under $\theta$ given as in Lemma 4.32, then $\theta: S \rightarrow K$ is a strongly admissible morphism with kernel $\mu_{B}$. Moreover, $\left.\theta\right|_{B}: B \rightarrow \bar{B}$ is an isomorphism.

Lemma 4.32 stated that there exists a strongly $B$-admissible morphism $\theta$ : $S \rightarrow S_{B}$. Consequently, we can improve on this fact to get a similar result for weakly $B$-superabundant semigroup with (C) as follows.

Theorem 4.34. If $S$ is a weakly B-superabundant semigroup with $(C)$, then the map $\theta: S \rightarrow S_{B}$ in Lemma 4.32 has the image contained in $N_{B}$.

Proof. Suppose that $a \in S$. Firstly, we need to find an idempotent which belongs to $L_{1} \alpha_{a}$ and $R_{1} \beta_{a}$. Of course, we have $L_{1} \alpha_{a}=L_{a^{*}}$ and $R_{1} \beta_{a}=R_{a^{\dagger}}$. Also, we 
have $a^{*} \mathcal{L} a^{\dagger} a^{*} \mathcal{R} a^{\dagger}$ since $S$ is weakly $B$-superabundant. It follows that

$$
a^{\dagger} a^{*} \in L_{1} \alpha_{a} \cap R_{1} \beta_{a}
$$

According to definitions of $\alpha_{a}$ and $\beta_{a}, L_{x} \alpha_{a}=L_{(x a)^{*}}$ and $R_{x} \beta_{a}=R_{(a x)^{\dagger}}$ for all $x \in B^{1}$. So $x \overline{\alpha_{a}} \mathcal{L}(x a)^{*}$ and $x \overline{\beta_{a}} \mathcal{R}(a x)^{\dagger}$. Then

$x \overline{\alpha_{a}} \mathcal{L}(x a)^{*} \widetilde{\mathcal{L}}_{B} x a \widetilde{\mathcal{R}}_{B} x a^{\dagger} \widetilde{\mathcal{R}}_{B} x a^{\dagger} a^{*} \mathcal{D} a^{\dagger} a^{*} x a^{\dagger} a^{*} \mathcal{D} a^{\dagger} a^{*} x \widetilde{\mathcal{L}}_{B} a x \widetilde{\mathcal{R}}_{B}(a x)^{\dagger} \mathcal{R} x \overline{\beta_{a}}$.

It follows that $x \overline{\alpha_{a}} \widetilde{\mathcal{D}}_{B} a^{\dagger} a^{*} x a^{\dagger} a^{*} \widetilde{\mathcal{D}}_{B} x \overline{\beta_{a}}$. By Lemma 3.10, we have

$$
x \overline{\alpha_{a}} \mathcal{D} a^{\dagger} a^{*} x a^{\dagger} a^{*} \mathcal{D} x \overline{\beta_{a}} \text { in } B .
$$

Hence $a \theta=\left(\alpha_{a}, \beta_{a}\right) \in N_{B}$. 


\section{Chapter 5}

\section{Structure theorems for weakly $B$-orthodox semigroups}

The goal of this chapter is to provide structure theorems for weakly $B$-orthodox semigroups, where $B$ is a band. We shall focus on providing a description of a weakly $B$-orthodox semigroup $S$ as a spined product of a weakly $B$-orthodox semigroup $S_{B}$ and $S / \gamma_{B}$, where $S_{B}$ is the fundamental weakly $\bar{B}$-orthodox semigroup constructed in Chapter 4 and $\gamma_{B}$ is the analogue of the least inverse congruence on an orthodox semigroup. This result is analogous to the Hall-Yamada theorem for orthodox semigroups.

Throughout this chapter Green's relation $\mathcal{D}$ always refer to $B$. Here $B$ denotes a band. To avoid ambiguity, if $\mathcal{K}$ is a relation on a semigroup $S$, then we will sometimes use $\mathcal{K}(S)$ to denote the relation on $S$.

\subsection{The least admissible Ehresmann congruence}

In Chapter 1 we mentioned that there exists the least inverse congruence $\gamma$ on any orthodox semigroup. As an analogue of the least inverse congruence, many articles have discussed the least $B / \mathcal{D}$-ample congruence $\delta_{B}$ on any weakly $B$ orthodox semigroup with certain idempotent connected condition, as mentioned in Chapter 2. Here, we concentrate on a correspondence congruence $\gamma_{B}$ on weakly $B$-orthodox semigroups. Such semigroups do not satisfy any idempotent connected condition.

The aim of this section is to find a closed form for $\gamma_{B}$, where $\gamma_{B}$ is the least 
admissible Ehresmann congruence on a weakly $B$-orthodox semigroup $S$. For any $a, b \in S$, we define

$$
a \delta_{B} b \text { if and only if } a=a^{\dagger} b a^{*} \text { and } b=b^{\dagger} a b^{*},
$$

for some $a^{\dagger}, a^{*}, b^{\dagger}, b^{*} \in B$ with $a^{\dagger} \widetilde{\mathcal{R}}_{B} a \widetilde{\mathcal{L}}_{B} a^{*}, b^{\dagger} \widetilde{\mathcal{R}}_{B} b \widetilde{\mathcal{L}}_{B} b^{*}$

Lemma 5.1. If $a \delta_{B} b$ in a weakly $B$-orthodox semigroup $S$, then $a^{\dagger} \mathcal{D} b^{\dagger}, a^{*} \mathcal{D} b^{*}$, for any $a^{\dagger}, b^{\dagger}, a^{*}$ and $b^{*}$. In particular, for any $e, f \in B, e \delta_{B} f$ if and only if $e \mathcal{D} f$.

Proof. Suppose that $a, b \in S$ are such that $a \delta_{B} b$. Then $a=a^{\dagger} b a^{*}, b=b^{\dagger} a b^{*}$, for some $a^{\dagger} \in \widetilde{R}_{a} \cap B, a^{*} \in \widetilde{L}_{a} \cap B, b^{\dagger} \in \widetilde{R}_{b} \cap B$ and $b^{*} \in \widetilde{L}_{b} \cap B$. From $a=a^{\dagger} b a^{*}$, we can deduce that

$$
a^{\dagger} b^{\dagger} a=a^{\dagger} b^{\dagger} a^{\dagger} b a^{*}=a^{\dagger} b^{\dagger} a^{\dagger} \cdot b^{\dagger} \cdot b a^{*}=a^{\dagger} b^{\dagger} b a^{*}=a^{\dagger} b a^{*}=a .
$$

Since $a \widetilde{\mathcal{R}}_{B} a^{\dagger}$, we have $a^{\dagger} b^{\dagger} a^{\dagger}=a^{\dagger}$. By a similar argument, we get $b^{\dagger} a^{\dagger} b^{\dagger}=b^{\dagger}$. Thus $a^{\dagger} \mathcal{D} b^{\dagger}$. Dually, we have $a^{*} \mathcal{D} b^{*}$. If $a^{\diamond}$ is another idempotent in $\widetilde{R}_{a} \cap B$ and $b^{\diamond}$ is another idempotent in $\widetilde{R}_{b} \cap B$, then $a^{\dagger} \mathcal{R} a^{\diamond}$ and $b^{\dagger} \mathcal{R} b^{\diamond}$. Together with $a^{\dagger} \mathcal{D} b^{\dagger}$, we obtain that $a^{\diamond} \mathcal{D} b^{\diamond}$. Dually, we have $a^{\circ} \mathcal{D} b^{\circ}$ for any $a^{\circ} \in \widetilde{L}_{a} \cap B$ and $b^{\circ} \in \widetilde{L}_{b} \cap B$.

Lemma 5.2. Let $S$ be a weakly B-orthodox semigroup and let $\theta: S \rightarrow T$ be an admissible morphism, where $T$ is an Ehresmann semigroup with respect to $B \theta$. Then $\delta_{B} \subseteq \operatorname{Ker} \theta$.

Proof. Suppose that $a, b \in S$ with $a \delta_{B} b$. Then $a=a^{\dagger} b a^{*}$ and $b=b^{\dagger} a b^{*}$, for some $a^{\dagger}, a^{*}, b^{\dagger}, b^{*} \in B$ with $a^{\dagger} \widetilde{\mathcal{R}}_{B} a \widetilde{\mathcal{L}}_{B} a^{*}, b^{\dagger} \widetilde{\mathcal{R}}_{B} b \widetilde{\mathcal{L}}_{B} b^{*}$. According to Lemma 5.1, we have $a^{*} \theta \mathcal{D}(B \theta) b^{*} \theta$ and $a^{\dagger} \theta \mathcal{D}(B \theta) b^{\dagger} \theta$ since $\theta$ is an admissible morphism. But, $B \theta$ is a semilattice, $a^{*} \theta=b^{*} \theta$ and $a^{\dagger} \theta=b^{\dagger} \theta$ so that $a \theta=\left(a^{\dagger} b a^{*}\right) \theta=a^{\dagger} \theta b \theta a^{*} \theta=$ $b^{\dagger} \theta b \theta b^{*} \theta=\left(b^{\dagger} b b^{*}\right) \theta=b \theta$. Thus, $(a, b) \in \operatorname{Ker} \theta$.

Corollary 5.3. If $S$ is a weakly $B$-orthodox semigroup, then $\delta_{B} \subseteq \gamma_{B}$.

Our aim is to show that, under certain conditions, $\delta_{B}=\gamma_{B}$ holds on weakly $B$-orthodox semigroups.

Lemma 5.4. Let $S$ be a weakly B-orthodox semigroup. Then the relation $\delta_{B}$ defined above is an equivalence relation. 
Proof. Clearly, $\delta_{B}$ is reflexive and symmetric. To show that $\delta_{B}$ is transitive we assume that $a, b, c \in S$ such that $a \delta_{B} b$ and $b \delta_{B} c$. Then $a=a^{\dagger} b a^{*}, b=b^{\dagger} a b^{*}$, $b=b^{\diamond} c b^{\circ}$ and $c=c^{\dagger} b c^{*}$, for some $a^{\dagger} \in \widetilde{R}_{a} \cap B, a^{*} \in \widetilde{L}_{a} \cap B, b^{\dagger}, b^{\diamond} \in \widetilde{R}_{b} \cap B$, $b^{*}, b^{\circ} \in \widetilde{L}_{b} \cap B, c^{\dagger} \in \widetilde{R}_{c} \cap B$ and $c^{*} \in \widetilde{L}_{c} \cap B$. By Lemma 5.1, we obtain that

$$
a^{\dagger} \mathcal{D} b^{\dagger} \mathcal{D} b^{\diamond} \mathcal{D} c^{\dagger} \text { and } a^{*} \mathcal{D} b^{*} \mathcal{D} b^{\circ} \mathcal{D} c^{*}
$$

Now

$$
\begin{aligned}
a=a^{\dagger} b a^{*} & =a^{\dagger} b^{\diamond} c b^{\circ} a^{*} \quad\left(b=b^{\diamond} c b^{\circ}\right) \\
& =a^{\dagger} b^{\diamond} \cdot c^{\dagger} \cdot c \cdot c^{*} \cdot b^{\circ} a^{*} \\
& =a^{\dagger} c^{\dagger} c c^{*} a^{*} \quad\left(a^{\dagger} \mathcal{D} b^{\diamond} \mathcal{D} c^{\dagger}, a^{*} \mathcal{D} b^{\circ} \mathcal{D} c^{*}\right) \\
& =a^{\dagger} c a^{*}
\end{aligned}
$$

Similarly, we get $c=c^{\dagger} a c^{*}$, and so $a \delta_{B} c$. Hence, $\delta_{B}$ is transitive.

Lemma 5.5. Let $S$ be a weakly B-orthodox semigroup. If the equivalence relation $\delta_{B}$ defined above is a congruence on $S$, then it is an admissible congruence on $S$.

Proof. Suppose that the relation $\delta_{B}$ is a congruence. We show that $\delta_{B}$ is an admissible congruence on $S$. Assume that $a \in S, e, f \in B$ are such that $e \widetilde{\mathcal{L}}_{B} a \widetilde{\mathcal{R}}_{B} f$ and $\left(a \delta_{B}\right)\left(k \delta_{B}\right)=a \delta_{B}$ for some $k \in B$. We want to show that $\left(e \delta_{B}\right)\left(k \delta_{B}\right)=e \delta_{B}$. From $(a k) \delta_{B}=a \delta_{B}$ and Lemma 5.1 , we get $(a k)^{*} \mathcal{D} a^{*}$. Since $\widetilde{\mathcal{L}}_{B}$ is a right congruence, $a^{*} k \widetilde{\mathcal{L}}_{B}(a k)^{*}$. Again, due to $a \widetilde{\mathcal{L}}_{B} e$, we obtain that $e \mathcal{D} a^{*}$. As $\mathcal{D}$ is a congruence on $B$, we have that $e k \mathcal{D} a^{*} k$. Thus $e \mathcal{D} a^{*} \mathcal{D} a^{*} k \mathcal{D} e k$. It immediately follows from Lemma 5.1 that $e \delta_{B} e k$, that is, $\left(e \delta_{B}\right)\left(k \delta_{B}\right)=e \delta_{B}$. Hence $a \delta \widetilde{\mathcal{L}}_{B \delta} e \delta$.

An argument that is completely dual gives that $a \delta_{B} \widetilde{\mathcal{R}}_{B \delta} f \delta_{B}$. Consequently, according to Lemma $2.9, \delta_{B}$ is an admissible congruence on $S$.

Corollary 5.6. If $\rho$ is an admissible congruence on a weakly B-orthodox semigroup $S$ satisfying that $B / \rho$ is a semilattice, then $S / \rho$ is an Ehresmann semigroup.

The following lemma is an immediate consequence of Lemma 5.5, Corollary 5.6 and Corollary 5.3.

Lemma 5.7. Let $S$ be a weakly B-orthodox semigroup. If the equivalence relation $\delta_{B}$ defined above is a congruence on $S$, then it is an admissible Ehresmann congruence on $S$. Moreover, $\delta_{B}=\gamma_{B}$. 
Lemma 5.8. If $S$ is a weakly B-orthodox semigroup, then $\widetilde{\mathcal{H}}_{B} \cap \delta_{B}=\iota$.

Proof. Suppose that $a, b \in S$ and $(a, b) \in \widetilde{\mathcal{H}}_{B} \cap \delta_{B}$. Then $a=a^{\dagger} b a^{*}$ for some $a^{\dagger} \in \widetilde{R}_{a} \cap B, a^{*} \in \widetilde{L}_{a} \cap B$. Since $a \widetilde{\mathcal{H}}_{B} b$, we have $a^{\dagger} \widetilde{\mathcal{R}}_{B} a \widetilde{\mathcal{R}}_{B} b$ and $a^{*} \widetilde{\mathcal{L}}_{B} a \widetilde{\mathcal{L}}_{B} b$. Thus $a=a^{\dagger} b a^{*}=b$ as required.

\subsection{A structure theorem for weakly $B$-orthodox semigroups}

The Hall-Yamada theorem presents a construction for orthodox semigroups, that is, any orthodox semigroup $S$ with band of idempotents $B$ is isomorphic to the spined product of $S / \gamma$ and the Hall semigroup $W_{B}$, where $\gamma$ is the least inverse congruence on $S$. So far, many articles have extended the Hall-Yamada theorem to weakly $B$-orthodox semigroups satisfying certain idempotent connected conditions [6], [46]. In Chapter 4 , given a band $B$, we built a fundamental weakly $\bar{B}$-orthodox semigroup $S_{B}$, which is an analogue of the Hall semigroup $W_{B}$, where $\bar{B}=\left\{\left(\rho_{e}, \lambda_{e}\right): e \in B\right\}$. In view of Lemma 4.32, Gomes and Gould found a representation from a weakly $B$-orthodox semigroup to $S_{B}$.

We stress that they did not attempt to produce an analogue of the HallYamada structure theorem for orthodox semigroups. With this in mind we give the following lemmas for weakly $B$-orthodox semigroups, from which we shall obtain a very general structure theorem for weakly $B$-orthodox semigroups. We then proceed to show this result can be applied in a number of cases of interest.

We begin by reminding the reader that if we have semigroups $S, T, H$ and morphisms $\phi$ and $\psi$ as follows,

$$
S \stackrel{T}{\phi} \quad \begin{aligned}
& \psi \\
& H
\end{aligned}
$$

Figure 5.1: The spined product

then the spined product $\mathcal{S}=\mathcal{S}(S, T, \phi, \psi)$ of $S$ and $T$ with respect to $H, \phi$ and $\psi$ is

$$
\mathcal{S}=\{(s, t) \in S \times T: s \phi=t \psi\}
$$


From now on, $S$ will be a weakly $B$-orthodox semigroup, $\gamma_{B}$ is the least admissible Ehresmann congruence on $S$ and $\gamma_{\bar{B}}$ is the least admissible Ehresmann congruence on $S_{B}$. Define a map $\psi: S / \gamma_{B} \rightarrow S_{B} / \gamma_{\bar{B}}$ by the rule that $s \gamma_{B} \psi=$ $s \theta \gamma_{\bar{B}}$ for any $s \in S$, where $\theta$ is defined as in Lemma 4.32 .

Lemma 5.9. The mapping $\psi: S / \gamma_{B} \rightarrow S_{B} / \gamma_{\bar{B}}$ defined above is an admissible morphism such that $\left.\psi\right|_{B / \gamma_{B}}: B / \gamma_{B} \rightarrow \bar{B} / \gamma_{\bar{B}}$ is an isomorphism, if and only if for any $a, b \in S, a \gamma_{B} b$ implies $a \theta \gamma_{\bar{B}} b \theta$ and $e \gamma_{B} f$ if and only if $e \theta \gamma_{\bar{B}}$ f $\theta$ for any $e, f \in B$, where $\theta$ is defined as in Lemma 4.32.

Proof. Necessity. Suppose that $a, b \in S$ and $a \gamma_{B} b$. It immediately follows that $a \gamma_{B} \psi=b \gamma_{B} \psi$. By the definition of $\psi$, we have $a \theta \gamma_{\bar{B}}=a \gamma_{B} \psi=b \gamma_{B} \psi=b \theta \gamma_{\bar{B}}$, that is, $a \theta \gamma_{\bar{B}} b \theta$. Consequently, e $\gamma_{B} f$ implies $e \theta \gamma_{\bar{B}} f \theta$ for any $e, f \in B$.

Conversely, if $e \theta \gamma_{\bar{B}} f \theta$ then by the definition of $\psi$, we have $e \gamma_{B} \psi=e \theta \gamma_{\bar{B}}=$ $f \theta \gamma_{\bar{B}}=f \gamma_{B} \psi$. Since $\left.\psi\right|_{B / \gamma_{B}}$ is an isomorphism it follows that $e \gamma_{B}=f \gamma_{B}$, that is, $e \gamma_{B} f$.

Sufficiency. According to the hypothesis, it is easy to see that the map $\psi: S / \gamma_{B} \rightarrow S_{B} / \gamma_{\bar{B}}$ defined by $s \gamma_{B} \psi=s \theta \gamma_{\bar{B}}$ for any $s \in S$ is well-defined and maps $B / \gamma_{B}$ to $\bar{B} / \gamma_{\bar{B}}$. Since $\theta, \gamma_{B}^{\natural}$ and $\gamma_{\bar{B}}^{\natural}$ are morphisms it follows that $\psi$ is a morphism.

Next, we claim that $\psi$ is an admissible morphism. Suppose that $a, b \in S$ and $a \gamma_{B} \widetilde{\mathcal{R}}_{B / \gamma_{B}} b \gamma_{B}$. Since $\gamma_{B}^{\natural}$ is admissible, we have

$$
a^{\dagger} \gamma_{B} \widetilde{\mathcal{R}}_{B / \gamma_{B}} a \gamma_{B} \widetilde{\mathcal{R}}_{B / \gamma_{B}} b \gamma_{B} \widetilde{\mathcal{R}}_{B / \gamma_{B}} b^{\dagger} \gamma_{B}
$$

for any $a^{\dagger} \in \widetilde{R}_{a} \cap B, b^{\dagger} \in \widetilde{R}_{b} \cap B$. Certainly, $a^{\dagger} \gamma_{B} \mathcal{R} b^{\dagger} \gamma_{B}$. As $B / \gamma_{B}$ is a semilattice, we get $a^{\dagger} \gamma_{B}=b^{\dagger} \gamma_{B}$. By the hypothesis, we obtain that $a^{\dagger} \theta \gamma_{\bar{B}} b^{\dagger} \theta$. Since $\theta$ and $\gamma_{B}^{\natural}$ are admissible morphisms, we have that

$$
a \gamma_{B} \psi=a \theta \gamma_{\bar{B}} \widetilde{\mathcal{R}}_{\bar{B} / \gamma_{\bar{B}}}\left(a \theta \gamma_{\bar{B}}\right)^{\dagger}=a^{\dagger} \theta \gamma_{\bar{B}}=b^{\dagger} \theta \gamma_{\bar{B}}=\left(b \theta \gamma_{\bar{B}}\right)^{\dagger} \widetilde{\mathcal{R}}_{\bar{B} / \gamma_{\bar{B}}} b \theta \gamma_{\bar{B}}=b \gamma_{B} \psi
$$

and so $\psi$ preserves $\widetilde{\mathcal{R}}_{B / \gamma_{B}}$. Dually, $\psi$ preserves $\widetilde{\mathcal{L}}_{B / \gamma_{B}}$. Thus $\psi$ is admissible.

It remains to show that $\left.\psi\right|_{B / \gamma_{B}}: B / \gamma_{B} \rightarrow \bar{B} / \gamma_{\bar{B}}$ is bijective. Certainly, $\left.\psi\right|_{B / \gamma_{B}}$ is onto. It is sufficient to check that $\left.\psi\right|_{B / \gamma_{B}}$ is injective. Suppose that $e \gamma_{B} \psi=f \gamma_{B} \psi$ for any $e \gamma_{B}, f \gamma_{B} \in B / \gamma_{B}$. Then by the definition of $\psi$ we have that $e \theta \gamma_{\bar{B}}=f \theta \gamma_{\bar{B}}$. Again by the hypothesis, we succeed in obtaining $e \gamma_{B} f$. 
Theorem 5.10. Let $S$ be a weakly B-orthodox semigroup and let $S_{B} * S / \gamma_{B}$ be the spined product of $S_{B}$ and $S / \gamma_{B}$ with respect to $S_{B} / \gamma_{\bar{B}}, \gamma_{\bar{B}}^{\natural}$ and $\psi$, where $\psi$ is defined above and satisfies the conditions in Lemma 5.9. Then the mapping $\phi$ : $a \mapsto\left(a \theta, a \gamma_{B}\right)$ is a monomorphism from $S$ to $S_{B} * S / \gamma_{B}$ if and only if $\mu_{B} \cap \gamma_{B}=\iota$, where $\mu_{B}=\operatorname{ker} \theta$, and $\theta$ is defined as in Lemma 4.32.

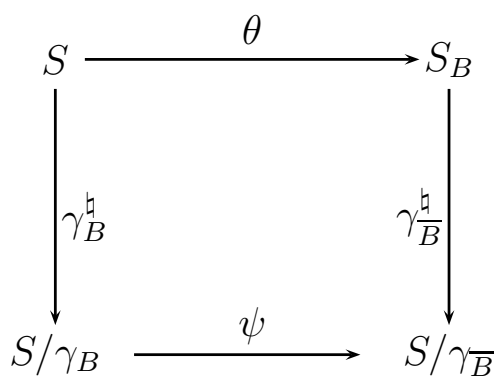

Figure 5.2: The structure of weakly $B$-orthodox semigroups

Proof. Clearly, $\phi$ is a morphism, since $\theta$ and $\gamma_{B}^{\natural}$ are admissible morphisms. Then $\phi$ is injective if and only if, for any $a, b \in S$ we have that $a \phi=b \phi$ if and only if $a=b$. That is, $\phi$ is injective if and only if $\operatorname{ker} \theta \cap \operatorname{ker} \gamma_{B}^{\natural}=\mu_{B} \cap \gamma_{B}=\iota$.

The next result is immediate.

Lemma 5.11. Let $S_{B} * S / \gamma_{B}$ be the spined product of $S_{B}$ and $S / \gamma_{B}$ with respect to $S_{B} / \gamma_{\bar{B}}, \gamma_{\bar{B}}^{\natural}$ and $\psi$, where $\psi$ is defined above and satisfies the conditions in Lemma 5.9. Then the mapping $\phi: a \mapsto\left(a \theta, a \gamma_{B}\right)$ is an epimorphism from $a$ weakly B-orthodox semigroup $S$ to $S_{B} * S / \gamma_{B}$ if and only if, if $x \in S_{B}$ and $\left(x, s \gamma_{B}\right) \in S_{B} * S / \gamma_{B}$ for some $s \in S$ then there exists $t \in S$ such that $x=t \theta$ and $t \gamma_{B}=s \gamma_{B}$, where $\theta$ is defined as in Lemma 4.32.

We now present the main result of this section. We will see later that in some special cases, it can be significantly simplified. We make a statement that $\mu_{B}=\operatorname{ker} \theta$, where $\mu_{B}$ is the largest congruence contained in $\widetilde{\mathcal{H}}_{B}$ and $\theta$ is defined as in Lemma 4.32 .

Theorem 5.12. Let $S$ be a weakly B-orthodox semigroup. The mapping $\phi: a \mapsto$ $\left(a \theta, a \gamma_{B}\right)$ is an isomorphism from $S$ to the spined product $S_{B} * S / \gamma_{B}$ of $S_{B}$ and 
$S / \gamma_{B}$ with respect to $S_{B} / \gamma_{\bar{B}}, \gamma_{\bar{B}}^{\natural}$ and $\psi$, where $\psi: S / \gamma_{B} \rightarrow S_{B} / \gamma_{\bar{B}}$ defined by $s \gamma_{B} \psi=s \theta \gamma_{\bar{B}}$ for any $s \in S$ is an admissible morphism and $\left.\psi\right|_{B / \gamma_{B}}: B / \gamma_{B} \rightarrow$ $\bar{B} / \gamma_{\bar{B}}$ is an isomorphism, if and only if:

(i) for any $a, b \in S, a \gamma_{B} b$ implies $a \theta \gamma_{\bar{B}} b \theta$ and $e \gamma_{B} f$ if and only if $e \theta \gamma_{\bar{B}} f \theta$ for any $e, f \in B$;

(ii) $\gamma_{B} \cap \mu_{B}=\iota$;

(iii) if $\left(x, s \gamma_{B}\right) \in S_{B} * S / \gamma_{B}$ for some $x \in S_{B}$ and $s \in S$, then there exists $t \in S$ such that $x=t \theta$ and $t \gamma_{B}=s \gamma_{B}$.

According to Corollary 4.33, if a weakly $B$-orthodox semigroup $S$ is embedded into a subsemigroup $K$ of $S_{B}$ containing $\bar{B}$ under $\theta$ given in Lemma 4.32, then the following result is immediate.

Corollary 5.13. Let $S$ be a weakly B-orthodox semigroup and let $K$ be a $\bar{B}$-full subsemigroup of $S_{B}$ such that $\operatorname{Im} \theta \subseteq K$. Let $\gamma_{\bar{B}}$ denote the least Ehresmann congruence on $K$. Then the mapping $\phi: a \mapsto\left(a \theta, a \gamma_{B}\right)$ is an isomorphism from $S$ to the spined product $K * S / \gamma_{B}$ of $K$ and $S / \gamma_{B}$ with respect to $K / \gamma_{\bar{B}}, \gamma_{\bar{B}}^{\natural}$ and $\psi$, where $\psi: S / \gamma_{B} \rightarrow K / \gamma_{\bar{B}}$ defined by $s \gamma_{B} \psi=s \theta \gamma_{\bar{B}}$ for any $s \in S$ is an admissible morphism and $\left.\psi\right|_{B / \gamma_{B}}: B / \gamma_{B} \rightarrow \bar{B} / \gamma_{\bar{B}}$ is an isomorphism, if and only if :

(i) for any $a, b \in S, a \gamma_{B} b$ implies $a \theta \gamma_{\bar{B}} b \theta$ and $e \gamma_{B} f$ if and only if e $\theta \gamma_{\bar{B}} f \theta$ for any $e, f \in B$;

(ii) $\gamma_{B} \cap \mu_{B}=\iota$;

(iii) if $\left(x, s \gamma_{B}\right) \in K * S / \gamma_{B}$ for some $x \in K$ and $s \in S$, then there exists $t \in S$ such that $x=t \theta$ and $t \gamma_{B}=s \gamma_{B}$.

Finally, we shall present an important and useful lemma for the following work. In Section 5.1, we defined an equivalence relation $\delta_{B}$ on a weakly $B$ orthodox semigroup $S$ and showed that if $\delta_{B}$ is a congruence then it is the least admissible Ehresmann congruence on $S$. In this case, i.e. when $\delta_{B}=\gamma_{B}$ and $\delta_{\bar{B}}=\gamma_{\bar{B}}$, Theorem 5.12 and Corollary 5.13 immediately simplify.

Lemma 5.14. Let $S$ be a weakly B-orthodox semigroup. If $\delta_{B}$ and $\delta_{\bar{B}}$ are congruences, then $\delta_{B}$ satisfies Conditions $(i)$, (ii) and (iii) in Theorem 5.12 (resp. Corollary 5.13).

Proof. To prove $(i)$, suppose that $a, b \in S$ are such that $a \delta_{B}=b \delta_{B}$. By the definition of $\delta_{B}$ we have that $a=a^{\dagger} b a^{*}$ and $b=b^{\dagger} a b^{*}$ for some $a^{\dagger}, a^{*}, b^{\dagger}, b^{*} \in B$ 
with $a^{\dagger} \widetilde{\mathcal{R}}_{B} a \widetilde{\mathcal{L}}_{B} a^{*}, b^{\dagger} \widetilde{\mathcal{R}}_{B} b \widetilde{\mathcal{L}}_{B} b^{*}$. It follows that $a \theta=\left(a^{\dagger} b a^{*}\right) \theta=\left(a^{\dagger} \theta\right)(b \theta)\left(a^{*} \theta\right)$ and $b \theta=\left(b^{\dagger} a b^{*}\right) \theta=\left(b^{\dagger} \theta\right)(a \theta)\left(b^{*} \theta\right)$. Since $\theta: S \rightarrow S_{B}$ is a strongly admissible morphism, we obtain that $a^{\dagger} \theta \widetilde{\mathcal{R}}_{\bar{B}} a \theta \quad \widetilde{\mathcal{L}}_{\bar{B}} a^{*} \theta$ and $b^{\dagger} \theta \widetilde{\mathcal{R}}_{\bar{B}} b \theta \widetilde{\mathcal{L}}_{\bar{B}} b^{*} \theta$. Thus $a \theta \delta_{\bar{B}} b \theta$.

According to Lemma 5.1 we know that $\left.\delta_{B}\right|_{B}=\mathcal{D}$, and since $\left.\theta\right|_{B}: B \rightarrow \bar{B}$ is an isomorphism in Lemma 4.32 it follows that $e \delta_{B} f$ if and only if $e \theta \delta_{\bar{B}} f \theta$ for any $e, f \in B$. Hence, Condition ( $i$ ) holds.

Next, we show that $\delta_{B}$ satisfies Condition (ii). Suppose that $a, b \in S$ are such that $a\left(\delta_{B} \cap \mu_{B}\right) b$. Since $\mu_{B} \subseteq \widetilde{\mathcal{H}}_{B}$, it follows that $a\left(\delta_{B} \cap \widetilde{\mathcal{H}}_{B}\right) b$. By Lemma 5.8 , we have that $a=b$, that is, $\delta_{B} \cap \mu_{B}=\iota$.

Finally, we claim that $\delta_{B}$ satisfies Condition (iii). Suppose that $\left(x, s \delta_{B}\right) \in$ $S_{B} * S / \delta_{B}$. Then $x \delta_{\bar{B}}=s \delta_{B} \psi=s \theta \delta_{\bar{B}}$. Thus $x=\alpha \cdot s \theta \cdot \beta, s \theta=\epsilon \cdot x \cdot \varepsilon$, where $\alpha, \beta, \epsilon, \varepsilon \in \bar{B}, \alpha \widetilde{\mathcal{R}}_{\bar{B}} x \widetilde{\mathcal{L}}_{\bar{B}} \beta, \epsilon \widetilde{\mathcal{R}}_{\bar{B}} s \theta \widetilde{\mathcal{L}}_{\bar{B}} \varepsilon$. Since $\theta$ is admissible, we can take $\epsilon=s^{\dagger} \theta, \varepsilon=s^{*} \theta$ for some $s^{\dagger} \in B \cap \widetilde{R}_{s}, s^{*} \in B \cap \widetilde{L}_{s}$. Let $\alpha=g \theta, \beta=h \theta$ for some $g, h \in B$. Then $x=(g s h) \theta$. By Lemma 5.1, we get $g \theta \mathcal{D}(\bar{B}) s^{\dagger} \theta$ and $h \theta \mathcal{D}(\bar{B}) s^{*} \theta$ in $\bar{B}$. Since $\left.\theta\right|_{B}: B \rightarrow \bar{B}$ is an isomorphism, it follows that $g \mathcal{D} s^{\dagger}$ and $h \mathcal{D} s^{*}$ in $B$. Thus $g \delta_{B} s^{\dagger}$ and $h \delta_{B} s^{*}$ so that $s=s^{\dagger} s s^{*} \delta_{B} g s h$. Hence, $(g s h) \theta=x$ and $(g s h) \delta_{B}=s \delta_{B}$. Condition (iii) holds.

\subsection{Weakly $B$-orthodox semigroups with (N)}

We recall that a band $B$ is called normal if $x y z t=x z y t$ for all $x, y, z, t \in B$. Let $S$ be a weakly $B$-orthodox semigroup with (N), that is, $B$ is a normal band. We show that the least admissible Ehresmann congruence $\gamma_{B}$ has the closed form $\delta_{B}$ given in Section 5.1. We can then apply Theorem 5.12 and Lemma 5.14 to give a structure theorem.

Lemma 5.15. Let $S$ be a weakly B-orthodox semigroup with $(N)$. Then the relation $\delta_{B}$ defined in Section 5.1 is a congruence on $S$.

Proof. In view of Lemma 5.4, it remains to show that the relation $\delta_{B}$ defined in Section 5.1 is compatible. It is sufficient to prove that $\delta_{B}$ is left compatible, because dually right compatibility will hold. Suppose that $a, b, c \in S$ and $a \delta_{B} b$. Then $a=a^{\dagger} b a^{*}$ and $b=b^{\dagger} a b^{*}$ for some $a^{\dagger} \in \widetilde{R}_{a} \cap B, a^{*} \in \widetilde{L}_{a} \cap B, b^{\dagger} \in \widetilde{R}_{b} \cap B$ and $b^{*} \in \widetilde{L}_{b} \cap B$. According to Lemma 5.1, we have that $a^{\dagger} \mathcal{D} b^{\dagger}$ and $a^{*} \mathcal{D} b^{*}$. Now, 
we can deduce that

$$
\begin{aligned}
& c a=(c a)^{\dagger} c a(c a)^{*}=(c a)^{\dagger} c\left(a^{\dagger} b a^{*}\right)(c a)^{*} \quad\left(a=a^{\dagger} b a^{*}\right) \\
& =(c a)^{\dagger} c c^{*}\left(a^{\dagger} b^{\dagger} a^{\dagger}\right) b a^{*}(c a)^{*} \quad\left(a^{\dagger} \mathcal{D} b^{\dagger}\right) \\
& =(c a)^{\dagger} c\left(c^{*} a^{\dagger} b^{\dagger} a^{\dagger}\right) b a^{*}(c a)^{*} \\
& =(c a)^{\dagger} c\left(c^{*} b^{\dagger} a^{\dagger}\right) b a^{*}(c a)^{*} \quad\left(\text { by }(N), c^{*} a^{\dagger} b^{\dagger} a^{\dagger}=c^{*} b^{\dagger} a^{\dagger}\right) \\
& =(c a)^{\dagger} c b^{\dagger} a^{\dagger} b a^{*}(c a)^{*} \\
& =(c a)^{\dagger} c b^{\dagger} a^{\dagger} b^{\dagger} b a^{*}(c a)^{*} \\
& =(c a)^{\dagger} c b^{\dagger} b a^{*}(c a)^{*} \quad\left(a^{\dagger} \mathcal{D} b^{\dagger}\right) \\
& =(c a)^{\dagger} c b a^{*}(c a)^{*} \text {. }
\end{aligned}
$$

By Lemma 2.8, $a^{*}(c a)^{*} \mathcal{L}(c a)^{*}$, so we get $a^{*}(c a)^{*} \in \widetilde{L}_{c a} \cap B$.

Similarly, we have that $c b=(c b)^{\dagger} c a b^{*}(c b)^{*}$, where $b^{*}(c b)^{*} \in \widetilde{L}_{c b} \cap B$. Thus $c a \delta_{B} c b$, that is, $\delta_{B}$ is left compatible. Consequently, $\delta_{B}$ is a congruence on $S$.

Due to Lemma 5.15 and Lemma 5.7, we immediately obtain the following result.

Lemma 5.16. Let $S$ be a weakly B-orthodox semigroup with (N). Then the relation $\delta_{B}$ defined in Section 5.1 is the least admissible Ehresmann congruence on $S$.

Since $S_{B}$ is constructed from a band $B$ and $\bar{B}$ is isomorphic to $B$, it follows that if $B$ is a normal band, so is $\bar{B}$. In this case, $S_{B}$ is a weakly $\bar{B}$-orthodox semigroup with $(\mathrm{N})$, so the relation $\delta_{B}$ given in Section 5.1 is also the least admissible Ehresmann congruence on $S_{B}$. We will denote it by $\delta_{\bar{B}}$.

Finally, according to Lemma 5.16, Lemma 5.14 and Theorem 5.12, we obtain a structure theorem for weakly $B$-orthodox semigroups with (N).

Theorem 5.17. A weakly B-orthodox semigroup $S$ with $(N)$ is isomorphic to the spined product $S_{B} * S / \delta_{B}$ of $S_{B}$ and $S / \delta_{B}$ with respect to $S_{B} / \delta_{\bar{B}}$, $\delta_{\bar{B}}^{\natural}$ and $\psi$, where $\psi: S / \delta_{B} \rightarrow S_{B} / \delta_{\bar{B}}$ defined by $s \delta_{B} \psi=s \theta \delta_{\bar{B}}$ for any $s \in S$ is an admissible morphism, and $\left.\psi\right|_{B / \delta_{B}}: B / \delta_{B} \rightarrow \bar{B} / \delta_{\bar{B}}$ is an isomorphism.

Theorem 5.17 describes $S$ as a spined product of $S_{B}$ ( an analogue of the Hall semigroup) and the greatest admissible Ehresmann image of $S$ (an analogue of the greatest inverse image of an orthodox semigroup). 


\subsection{Weakly $B$-superabundant semigroups with (C)}

We first make full use of Lemma 3.10 to check the equivalence relation $\delta_{B}$ defined in Section 5.1 is the least admissible Ehresmann congruence on a weakly $B$ superabundant semigroup $S$ with $(\mathrm{C})$.

Lemma 5.18. Let $S$ be a weakly B-superabundant semigroup with $(C)$. Then the relation $\delta_{B}$ on $S$ defined in Section 5.1 is the least admissible Ehresmann congruence on $S$.

Proof. In view of Lemma 5.4, it remains to show that the relation $\delta_{B}$ is compatible. We first claim that $\delta_{B}$ is right compatible. Dually, left compatibility holds. Suppose that $a, b, c \in S$ and $a \delta_{B} b$. Then $a=a^{\dagger} b a^{*}$ and $b=b^{\dagger} a b^{*}$ for some $a^{\dagger} \in \widetilde{R}_{a} \cap B, a^{*} \in \widetilde{L}_{a} \cap B, b^{\dagger} \in \widetilde{R}_{b} \cap B$ and $b^{*} \in \widetilde{L}_{b} \cap B$. By Lemma 5.1, we have that $a^{\dagger} \mathcal{D} b^{\dagger}$ and $a^{*} \mathcal{D} b^{*}$. Since $S$ is a weakly $B$-superabundant semigroup, it follows from Lemma 3.10 that $a^{\dagger} \mathcal{D} a^{*}$ and $b^{*} \mathcal{D} b^{\dagger}$, so that $a^{\dagger} \mathcal{D} a^{*} \mathcal{D} b^{*} \mathcal{D} b^{\dagger}$. Obviously, we have that

$$
a c=(a c)^{\dagger} a c(a c)^{*}=(a c)^{\dagger}\left(a^{\dagger} b a^{*}\right) c(a c)^{*} \quad\left(a=a^{\dagger} b a^{*}\right) .
$$

By Lemma 2.8, we obtain that $(a c)^{\dagger} a^{\dagger} \mathcal{R}(a c)^{\dagger}$, and so $(a c)^{\dagger} a^{\dagger} \widetilde{\mathcal{R}}_{B} a c$. Now we just need to remove $a^{*}$ from the right side of $a c=(a c)^{\dagger} a^{\dagger} b a^{*} c(a c)^{*}$. Observe that

$$
\begin{aligned}
a c=(a c)^{\dagger} a^{\dagger} b a^{*} c(a c)^{*} & =(a c)^{\dagger} a^{\dagger} b a^{*} c^{\dagger} c(a c)^{*} \\
& =(a c)^{\dagger} a^{\dagger} b\left((a c)^{\dagger} a^{\dagger} b\right)^{*}\left(a^{*} c^{\dagger}\right)\left(c(a c)^{*}\right)^{\dagger} c(a c)^{*} .
\end{aligned}
$$

Next, we show that $\left((a c)^{\dagger} a^{\dagger} b\right)^{*} \mathcal{D} a^{*} c^{\dagger} \mathcal{D}\left(c(a c)^{*}\right)^{\dagger}$. By the Congruence Condition, we get $(a c)^{\dagger} \widetilde{\mathcal{R}}_{B} a c \widetilde{\mathcal{L}}_{B} a^{*} c \widetilde{\mathcal{R}}_{B} a^{*} c^{\dagger}$, and $(a c)^{\dagger} \widetilde{\mathcal{R}}_{B} a c \widetilde{\mathcal{L}}_{B} a^{*} c \widetilde{\mathcal{L}}_{B}\left(a^{*} c\right)^{*}$, that is, $(a c)^{\dagger} \widetilde{\mathcal{D}}_{B} a^{*} c^{\dagger} \widetilde{\mathcal{D}}_{B}\left(a^{*} c\right)^{*}$. It follows from Lemma 3.10 that

$$
(a c)^{\dagger} \mathcal{D} a^{*} c^{\dagger} \mathcal{D}\left(a^{*} c\right)^{*}
$$

As $\mathcal{D}$ is a congruence on $B$, we have that $(a c)^{\dagger} a^{\dagger} b^{\dagger} \mathcal{D} a^{*} c^{\dagger} a^{\dagger} b^{\dagger}$, and so

$$
\left((a c)^{\dagger} a^{\dagger} b\right)^{*} \widetilde{\mathcal{L}}_{B}(a c)^{\dagger} a^{\dagger} b \widetilde{\mathcal{R}}_{B}(a c)^{\dagger} a^{\dagger} b^{\dagger} \mathcal{D} a^{*} c^{\dagger} a^{\dagger} b^{\dagger}
$$

Since $a^{*} \mathcal{D} a^{\dagger} \mathcal{D} b^{\dagger}$, it follows from Lemma 1.22 that $a^{*} c^{\dagger} a^{\dagger} b^{\dagger} \mathcal{R} a^{*} c^{\dagger}$. Thus, $\left((a c)^{\dagger} a^{\dagger} b\right)^{*} \widetilde{\mathcal{D}}_{B} a^{*} c^{\dagger}$, that is, $\left((a c)^{\dagger} a^{\dagger} b\right)^{*} \mathcal{D} a^{*} c^{\dagger}$ by Lemma 3.10. Since $\left(a^{*} c\right)^{*} \mathcal{D}\left(a^{*} c\right)^{\dagger}$, 
we have that

$$
\left(c(a c)^{*}\right)^{\dagger} \widetilde{\mathcal{R}}_{B} c(a c)^{*} \widetilde{\mathcal{L}}_{B} c^{*}(a c)^{*} \mathcal{D} c^{*}\left(a^{*} c\right)^{*} \mathcal{D} c^{*}\left(a^{*} c\right)^{\dagger} \mathcal{R} c^{*} a^{*} c^{\dagger}
$$

By Lemma 1.22, we obtain that $c^{*} a^{*} c^{*} \mathcal{L} a^{*} c^{*}$, so that $\left(c(a c)^{*}\right)^{\dagger} \widetilde{\mathcal{D}}_{B} a^{*} c^{\dagger}$, that is, $\left(c(a c)^{*}\right)^{\dagger} \mathcal{D} a^{*} c^{\dagger}$. Thus

$$
\begin{aligned}
a c & =(a c)^{\dagger} a^{\dagger} b\left((a c)^{\dagger} a^{\dagger} b\right)^{*}\left(a^{*} c^{\dagger}\right)\left(c(a c)^{*}\right)^{\dagger} c(a c)^{*} & & \\
& =(a c)^{\dagger} a^{\dagger} b\left((a c)^{\dagger} a^{\dagger} b\right)^{*}\left(c(a c)^{*}\right)^{\dagger} c(a c)^{*} & & \left(\left((a c)^{\dagger} a^{\dagger} b\right)^{*} \mathcal{D} a^{*} c^{\dagger} \mathcal{D}\left(c(a c)^{*}\right)^{\dagger}\right) \\
& =(a c)^{\dagger} a^{\dagger} b c(a c)^{*} & & \left((a c)^{\dagger} a^{\dagger} \widetilde{\mathcal{R}}_{B} a c\right) .
\end{aligned}
$$

Similarly, we have that $b c=(b c)^{\dagger} a c(b c)^{*}$ for some $(b c)^{\dagger} \widetilde{\mathcal{R}}_{B} b c \widetilde{\mathcal{L}}_{B}(b c)^{*}$. Hence $a c \delta_{B} b c$ and so $\delta_{B}$ is a congruence on $S$. Again by Lemma 5.7, we obtain that $\delta_{B}$ is the least admissible Ehresmann congruence on $S$ as required.

At the end of this section we want to build a structure theorem for weakly $B$ superabundant semigroups with $(\mathrm{C})$ as a spined product. But we can not use $S_{B}$ to get this result because we can not ensure that $S_{B}$ is a weakly $\bar{B}$-superabundant semigroup with (C) and therefore that $\gamma_{\bar{B}}=\delta_{\bar{B}}$ on $S_{B}$. With this in mind, we will make full use of the weakly $\bar{B}$-superabundant subsemigroup $N_{B}$ of $S_{B}$ constructed in Chapter 4.

According to Lemma 5.18, Lemma 5.14, Theorem 4.34 and Corollary 5.13, we build a structure theorem for weakly $B$-superabundant semigroups with (C) as follows.

Theorem 5.19. A weakly B-superabundant semigroup $S$ with $(C)$ is isomorphic to the spined product $N_{B} * S / \delta_{B}$ of $N_{B}$ and $S / \delta_{B}$ with respect to $N_{B} / \delta_{\bar{B}}$, $\delta_{\bar{B}}^{\natural}$ and $\psi$, where $\theta$ is defined in Lemma 4.32, $\psi: S / \delta_{B} \rightarrow N_{B} / \delta_{\bar{B}}$, defined by $s \delta \psi=s \theta \delta_{\bar{B}}$ for any $s \in S$, is an admissible morphism, and $\left.\psi\right|_{B / \delta_{B}}: B / \delta_{B} \rightarrow \bar{B} / \delta_{\bar{B}}$ is an isomorphism.

\subsection{Examples}

We now present a number of examples, allowing us to show that the weak idempotent connected condition (WIC) and the band of distinguished idempotents $B$ being a normal band are not equivalent in a weakly $B$-orthodox semigroup $S$. 
Let $S$ be a weakly $B$-orthodox semigroup. For any element $e$ of $B$ we denote by $\langle e\rangle$ the principal order ideal generated by $e$. We recall that $S$ satisfies the weakly idempotent connected condition (WIC) (with respect to $B$ ) if for any $a \in S$ and some $a^{\dagger}, a^{*}$, if $x \in\left\langle a^{\dagger}\right\rangle$ then there exists $y \in B$ with $x a=a y$; dually, if $z \in\left\langle a^{*}\right\rangle$ then there exists $t \in B$ with $t a=a z$.

\section{Example 5.20.}

We begin by citing an example [17] of a weakly $B$-orthodox semigroup with (WIC). Consider the three element band $B=\{1, a, b\}$ which is a two-element right zero band with an identity adjoined. We have $U_{B}=\{1, a, b, c\}$ and have table

\begin{tabular}{c|cccc}
$*$ & 1 & $c$ & $a$ & $b$ \\
\hline 1 & 1 & $c$ & $a$ & $b$ \\
$c$ & $c$ & 1 & $a$ & $b$ \\
$a$ & $a$ & $b$ & $a$ & $b$ \\
$b$ & $b$ & $a$ & $a$ & $b$.
\end{tabular}

Also, we can calculate that $1 * a * b * 1=b$ and $1 * b * a * 1=a$, so $B$ is not a normal band. From its very construction, $U_{B}$ is weakly $B$-orthodox with (WIC).

\section{Example 5.21.}

Now, we consider the normal band $B=\{e, f, 0\}$ which is a two-element right zero band with a zero adjoined having table

\begin{tabular}{c|ccc}
$*$ & $e$ & $f$ & 0 \\
\hline$e$ & $e$ & $f$ & 0 \\
$f$ & $e$ & $f$ & 0 \\
0 & 0 & 0 & 0.
\end{tabular}

We claim that $S_{B}$ does not have (WIC).

Lemma 5.22. [17] Any pair of the form $\left(c_{L}, d_{R}\right)$ lies in $S_{B}$, where $c_{L}$ and $d_{R}$ are the constant maps in $\mathcal{O P}\left(B^{1} / \mathcal{L}\right)$ and $\mathcal{O P}\left(B^{1} / \mathcal{R}\right)$ with images $L$ and $R$, respectively. 
Remark: In [Theorem 6.2, [17]] we obtain that for any $(\alpha, \beta) \in S_{B}$,

$$
(\alpha, \beta) \widetilde{\mathcal{R}}_{\bar{B}}\left(\rho_{v}, \lambda_{v}\right)
$$

where $v \in R_{1} \beta$.

Take $\left(c_{L_{f}}, d_{R_{f}}\right) \in S_{B}$. We have $R_{1} d_{R_{f}}=R_{f}$. So we choose $v=f$,

$$
\left\langle\left(\rho_{v}, \lambda_{v}\right)\right\rangle=\left(\rho_{v}, \lambda_{v}\right) \bar{B}\left(\rho_{v}, \lambda_{v}\right)=\left(\rho_{f}, \lambda_{f}\right) \bar{B}\left(\rho_{f}, \lambda_{f}\right)=\left\{\left(\rho_{f}, \lambda_{f}\right),\left(\rho_{0}, \lambda_{0}\right)\right\},
$$

since $\bar{B}=\left\{\left(\rho_{e}, \lambda_{e}\right),\left(\rho_{f}, \lambda_{f}\right),\left(\rho_{0}, \lambda_{0}\right)\right\}$ is isomorphic to $B$ under $b \mapsto\left(\rho_{b}, \lambda_{b}\right)$. We have

$$
\left(\rho_{0}, \lambda_{0}\right)\left(c_{L_{f}}, d_{R_{f}}\right)=\left(c_{L_{f}}, \lambda_{0}\right)
$$

and

$$
\left(c_{L_{f}}, d_{R_{f}}\right) \bar{B}=\left\{\left(c_{L_{e}}, d_{R_{f}}\right),\left(c_{L_{f}}, d_{R_{f}}\right),\left(\rho_{0}, d_{R_{f}}\right)\right\} .
$$

So $\left(\rho_{0}, \lambda_{0}\right)\left(c_{L_{f}}, d_{R_{f}}\right) \notin\left(c_{L_{f}}, d_{R_{f}}\right) \bar{B}$. Thus $S_{B}$ does not have (WIC).

The final example explains that not every weakly $B$-superabundant semigroup with (C) has (WIC).

\section{Example 5.23.}

Let $\langle a\rangle$ be a monogenic monoid generated by $a$ and $X=\left\{x_{i}: i \in \mathbb{N}\right\}$ be a right zero semigroup. Set $S=\langle a\rangle \cup X$. We define the operation $*$ as the following table:

\begin{tabular}{c|cccc}
$*$ & 1 & $a$ & $a^{n}$ & $x_{i}$ \\
\hline 1 & 1 & $a$ & $a^{n}$ & $x_{i}$ \\
$a$ & $a$ & $a^{2}$ & $a^{n+1}$ & $x_{i}$ \\
$a^{m}$ & $a^{m}$ & $a^{m+1}$ & $a^{m+n}$ & $x_{i}$ \\
$x_{j}$ & $x_{j}$ & $x_{j+1}$ & $x_{j+n}$ & $x_{i}$.
\end{tabular}

We can easily check that $S$ is a semigroup. It is easy to see that the $\widetilde{\mathcal{L}}_{B^{-}}$

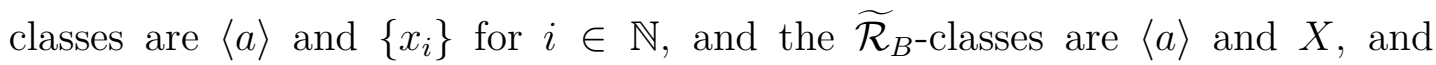
so the $\widetilde{\mathcal{H}}_{B}$-classes are $\langle a\rangle$ and $\left\{x_{i}\right\}$ for $i \in N$. It follows that $S$ is a weakly $B$ superabundant semigroup with distinguished band $\{1\} \cup X$. Moreover, $S$ satisfies 
the Congruence Condition. But we yet find that $a x_{1}=x_{1} \neq k a$ for any $k \in S$, so $S$ fails to have (WIC). 


\section{Chapter 6}

\section{Correspondence between algebraic structures and ordered structures}

Here we survey briefly some of interesting achievements such as the EhresmannSchein-Nambooripad (ESN) Theorem, and its many extensions due to Armstrong $[1,2]$, Lawson [32], Meakin [35,36] and Nambooripad [38-40]. These results set up a connection between algebraic structures and ordered structures.

\subsection{Inverse semigroups and inductive groupoids $_{1}$}

The correspondence between inverse semigroups and inductive ${ }_{1}$ groupoids has been widely investigated. Theorem A below is an amalgamation of Ehresmann [4], Nambooripad and Veeramony [41] and Schein [47].

As mentioned in Chapter 1 , an inductive ${ }_{1}$ groupoid is briefly described as a groupoid equipped with a partial order possessing restrictions and co-restrictions, and the set of idempotents forming a semilattice under the partial order. An

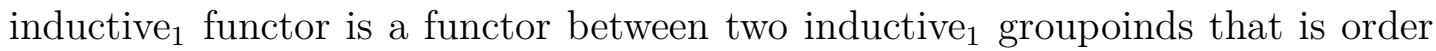
and meet preserving.

Let $G$ be an inductive 1 groupoid. We define a product $\otimes$ on $G$ by the rule that

$$
a \otimes b=\left(\left.a\right|_{\mathbf{r}(a) \wedge \mathbf{d}(b)}\right) \cdot(\mathbf{r}(a) \wedge \mathbf{d}(b) \mid b)
$$


Then, $G \mathbf{S}=(G, \otimes)$ is an inverse semigroup (having the same partial order as $G$ ). Commonly, the product $\otimes$ is called pseudo-product.

Conversely, let $S$ be an inverse semigroup with semilattice of idempotents $E$. We construct a category $S \mathbf{C}$ as follows:

$$
\mathrm{Ob}(S \mathbf{C})=E, \quad \operatorname{Mor}(S \mathbf{C})=S .
$$

For any $x \in S \mathbf{C}$, we put $\mathbf{d}(x)=x x^{\prime}$ and $\mathbf{r}(x)=x^{\prime} x$, where $x^{\prime}$ is the inverse of $x$ in $S$. If $x, y \in S \mathbf{C}$ and $\mathbf{r}(x)=\mathbf{d}(y)$, then we define $x \cdot y=x y$ in $S \mathbf{C}$, where $x y$ is the product of $x$ and $y$ in $S$. Certainly, the operation - is a partial binary operation and associative as a partial binary operation, so that $S \mathbf{C}$ becomes a category. In addition, it is easy to see that the inverse morphism of $x$ in $S \mathbf{C}$ is the inverse $x^{\prime}$ of $x$ in $S$ so that $S \mathbf{C}$ is a groupoid. We note that there exists a natural partial order in any inverse semigroup $S$, defined by the rule that for any $a, b \in S$,

$$
a \leq b \Leftrightarrow a=e b \quad \text { for some } e \in E \text {. }
$$

We make use of the natural partial order $\leq$ on $S$ to set up $S \mathbf{C}$ as an inductive 1 groupoid by defining restriction and co-restriction as follows:

$$
{ }_{e}|a=e a, a|_{f}=a f
$$

where $e, f \in E$ are such that $e \leq \mathbf{d}(a)=a a^{\prime}$ and $f \leq \mathbf{r}(a)=a^{\prime} a$. Then $S \mathbf{C}$ is

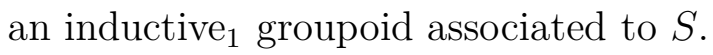

Further:

Theorem A (ESN Theorem) The category of inverse semigroups and morphisms

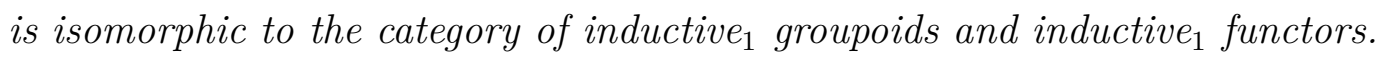

Inverse semigroups are regular semigroups in which the idempotents form a semilattice. Consequently, we can regard the set of idempotents of a regular semigroup as a generalisation of a semilattice. This idea is precisely described in the definition of a regular biordered set, introduced by Nambooripad [38]. In that article, Nambooripad defined an inductive 2 groupoid, as now we demonstrate.

An ordered $_{2}$ groupoid $(G, \leq)$ with a regular biordered set of objects $E$ is said to be inductive $_{2}$ if the following conditions and the duals $(\mathrm{IG} 1)^{\circ},(\mathrm{IG} 3)^{\circ},(\mathrm{IG} 4)^{\circ}$ and (IG5) of (IG1), (IG3), (IG4) and (IG5) hold: 
(IG1) if $e, f \in E$ are such that $e \mathcal{R} f$ or $e \mathcal{L} f$, then there exists a distinguished morphism $[e, f]$ from $e$ to $f$ such that $[e, e]=1_{e}$, the identity associated to $e$;

(IG2) for any $e, f \in E, e \omega f$ if and only if $1_{e} \leq 1_{f}$;

(IG3) if $e, f, g \in E$ are such that $e \mathcal{R} f \mathcal{R} g$, then $[e, f] \cdot[f, g]=[e, g]$;

(IG4) if $g, h, e \in E$ are such that $[g, h]$ exists and $e \omega g$, then $[e, h e h]$ exists and $[e, h e h] \leq[g, h]$

(IG5) let $x \in G$, and for $i=1,2$, let $e_{i}, f_{i} \in E$ be such that $e_{i} \leq \mathbf{d}(x)$ and $f_{i}=\mathbf{r}\left(e_{i} \mid x\right)$. If $e_{1} \omega^{r} e_{2}$, then $f_{1} \omega^{r} f_{2}$ and $\left[e_{1}, e_{1} e_{2}\right] \cdot\left(e_{1} e_{2} \mid x\right)=\left(e_{1} \mid x\right) \cdot\left[f_{1}, f_{1} f_{2}\right]$;

(IG6) if $\left(\begin{array}{ll}e & f \\ g & h\end{array}\right)$ is a singular E-square, then $[e, f] \cdot[f, h]=[e, g] \cdot[g, h]$.

This leads to a generalisation of Theorem A from a semilattice to a regular biordered set.

Theorem B (Nambooripad [38]) The category of regular semigroups and morphisms is equivalent to the category of inductive ${ }_{2}$ groupoids and inductive func- $_{2}$ tors.

The definition of an inductive $e_{2}$ functor is given in the next section in the more general content of inductive ${ }_{2}$ cancellative categories.

As we will give more details in the next section for the more general case of concordant semigroups, we omit the process of building Theorem B.

Note that for a technical reason, 'isomorphic' in Theorem A has been replaced by 'equivalent' in Theorem B. Of course, Theorem B may be specialised to orthodox semigroups.

\subsection{Concordant semigroups and inductive 2 can- cellative categories}

Theorem B was extended by Armstrong [1] from regular to concordant semigroups, replacing ordered $_{2}$ groupoids by more general kinds of ordered $_{2}$ categories.

Before giving Armstrong's result, we recall that a concordant semigroup is an abundant semigroup with a regular biordered set of idempotents and satisfying the extra condition of being idempotent connected (IC). That is, a concordant semigroup is a weakly $U$-regular semigroup for which $U=E(S), \mathcal{R}^{*}=\widetilde{\mathcal{R}}_{U}$, $\mathcal{L}^{*}=\widetilde{\mathcal{L}}_{U}$ and $(\mathrm{IC})$ holds. 
An ordered $_{2}$ cancellative category $(C, \leq)$ with a regular biordered set of objects $E$ is said to be an inductive $e_{2}$ cancellative category if the following conditions and the duals $(\mathrm{IC} 1)^{\circ},(\mathrm{IC} 3)^{\circ},(\mathrm{IC} 4)^{\circ}$ and $(\mathrm{IC} 5)^{\circ}$ of $(\mathrm{IC} 1),(\mathrm{IC} 3),(\mathrm{IC} 4)$ and (IC5) hold:

(IC1) if $e, f \in E$ are such that $e \mathcal{R} f$ or $e \mathcal{L} f$, then there exists a distinguished morphism $[e, f]$ from $e$ to $f$ such that $[e, e]=1_{e}$, the identity associated to $e$;

(IC2) for any $e, f \in E, e \omega f$ if and only if $1_{e} \leq 1_{f}$;

(IC3) if $e, f, g \in E$ are such that $e \mathcal{R} f \mathcal{R} g$, then $[e, f] \cdot[f, g]=[e, g]$;

(IC4) if $g, h, e \in E$ are such that $[g, h]$ exists and $e \omega g$, then $[e, h e h]$ exists and $[e, h e h] \leq[g, h]$

(IC5) let $x \in C$, and for $i=1,2$, let $e_{i}, f_{i} \in E$ be such that $e_{i} \leq \mathbf{d}(x)$ and $f_{i}=\mathbf{r}\left(e_{i} \mid x\right)$. If $e_{1} \omega^{r} e_{2}$, then $f_{1} \omega^{r} f_{2}$ and $\left[e_{1}, e_{1} e_{2}\right] \cdot\left(e_{1} e_{2} \mid x\right)=\left(e_{1} \mid x\right) \cdot\left[f_{1}, f_{1} f_{2}\right]$;

(IC6) if $\left(\begin{array}{ll}e & f \\ g & h\end{array}\right)$ is a singular E-square, then $[e, f] \cdot[f, h]=[e, g] \cdot[g, h]$.

We pause to make a short comment that an inductive 2 groupoid $(G, \leq)$ is an

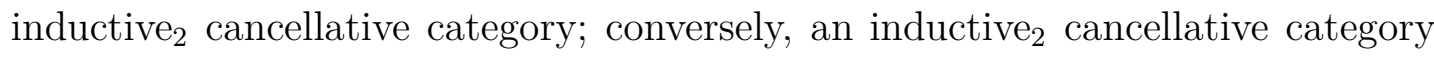
$(C, \leq)$ becomes an inductive en $_{2}$ groupoid if every morphism has an inverse. It is necessary to claim that Condition $(\mathrm{G})$ holds, that is, if $x \leq y$ in $C$, then $x^{-1} \leq y^{-1}$. Suppose that $x \leq y$. Then $\mathbf{d}(x) \omega \mathbf{d}(y)$ and $\mathbf{r}(x) \omega \mathbf{r}(y)$, that is, $\mathbf{r}(x) \omega \mathbf{d}\left(y^{-1}\right)$, and so by (OC4), there exists a unique element $\mathbf{r}(x) \mid y^{-1}$ such that $\mathbf{r}(x) \mid y^{-1} \leq y^{-1}$ and $\mathbf{d}\left(\mathbf{r}(x) \mid y^{-1}\right)=\mathbf{r}(x)$. So $x \cdot \mathbf{r}(x) \mid y^{-1}$ is defined and by (OC3), $x \cdot \mathbf{r}(x) \mid y^{-1} \leq y \cdot y^{-1}=1_{\mathbf{d}(y)}$. As $1_{\mathbf{d}(x)} \leq 1_{\mathbf{d}(y)}$ and $\mathbf{d}\left(1_{\mathbf{d}(x)}\right)=\mathbf{d}(x)=\mathbf{d}\left(x \cdot \mathbf{r}(x) \mid y^{-1}\right)$, it follows from (OC4) that $x \cdot \mathbf{r}(x) \mid y^{-1}=1_{\mathbf{d}(x)}$, which gives that $x^{-1}={ }_{\mathbf{r}(x)} \mid y^{-1}$. Thus. $x^{-1} \leq y^{-1}$.

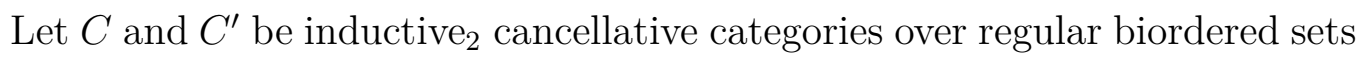
$E$ and $E^{\prime}$, respectively, and $\phi: C \rightarrow C^{\prime}$ be an order-preserving functor. Then $\phi$ is inductive $_{2}$ if

(IOF1) the map $\phi: E \rightarrow E^{\prime}$ is a regular morphism (in the sense of Definition 1.25);

(IOF2) if $e, f \in E$ and $[e, f]$ exists, then $[e, f] \phi=[e \phi, f \phi]$.

Now, we give an outline of the correspondence between concordant semi-

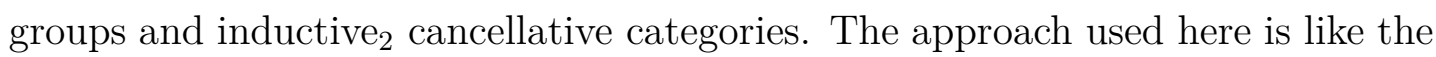
one in regular case.

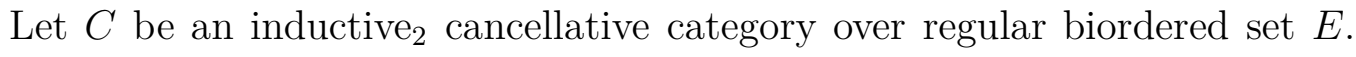


We define a relation $\rho$ on $C$ by the rule that for any $x, y \in C$,

$$
x \rho y \Leftrightarrow \mathbf{d}(x) \mathcal{R} \mathbf{d}(y), \mathbf{r}(x) \mathcal{L} \mathbf{r}(y) \text { and } x \cdot[\mathbf{r}(x), \mathbf{r}(y)]=[\mathbf{d}(x), \mathbf{d}(y)] \cdot y \text {. }
$$

It is routine to check that $\rho$ is an equivalence relation on $C$.

Suppose that $x \in C, h \omega^{r} \mathbf{d}(x)$ and $k \omega^{l} \mathbf{r}(x)$. We define

$$
h * x=[h, h \mathbf{d}(x)] \cdot{ }_{h \mathbf{d}(x)} \mid x \text { and } x \diamond k=\left.x\right|_{\mathbf{r}(x) k} \cdot[\mathbf{r}(x) k, k] .
$$

We then define a binary operation $\odot$ on $C / \rho$ by the rule that for all $x, y \in C$, $h \in S(\mathbf{r}(x), \mathbf{d}(y))$,

$$
\bar{x} \odot \bar{y}=\overline{(x \otimes y)_{h}},
$$

where $\bar{x}$ denotes the $\rho$-class of $x$ in $C$ and $(x \otimes y)_{h}=(x \diamond h) \cdot(h * y)$. It is proved in [1] that the set $C \mathbf{S}=(C / \rho, \odot)$ forms a concordant semigroup.

To see the converse, let $S$ be a concordant semigroup with regular biordered set of idempotents $E$. We build a category $S \mathbf{C}$ as follows:

$$
\mathrm{Ob}(S \mathbf{C})=E, \operatorname{Mor}(S \mathbf{C})=\left\{(e, a, f): e \in R_{a}^{*} \cap E, f \in L_{a}^{*} \cap E\right\}
$$

For any $(e, a, f) \in \operatorname{Mor}(S \mathbf{C})$, we set

$$
\mathbf{d}((e, a, f))=e(\text { abbreviated to } \mathbf{d}(e, a, f)=e)
$$

and

$$
\mathbf{r}((e, a, f))=f(\text { abbreviated to } \mathbf{r}(e, a, f)=f) .
$$

In addition, we define a partial binary operation on $S \mathbf{C}$ by

$$
(e, a, f) \cdot(g, b, h)= \begin{cases}(e, a b, h) & \text { if } f=g \\ \text { undefined } & \text { otherwise }\end{cases}
$$

where $a b$ is the product of $a$ and $b$ in $S$. Then $S \mathbf{C}$ becomes a category with regular biordered set of objects $E$.

Certainly, $S \mathbf{C}$ is cancellative. Since if $(e, a, f),(g, b, f),(f, c, v) \in S \mathbf{C}$ and $(e, a, f) \cdot(f, c, v)=(g, b, f) \cdot(f, c, v)$, then $(e, a c, v)=(g, b c, v)$ and so $e=g$ and $a c=b c$. As $f \mathcal{R}^{*} c$, we have that $a f=b f$, that is, $a=b$ as $a, b \mathcal{L}^{*} f$. Hence, 
$(e, a, f)=(g, b, f)$, so that $S \mathbf{C}$ is right cancellative. Dually, it is left cancellative, and consequently, $S \mathbf{C}$ is cancellative.

Since any concordant semigroup $S$ satisfies (IC), it follows from the statements in Section 2.3.2 that if $(e, a, f) \in S \mathbf{C}$, then there is a unique connecting isomorphism from $\langle e\rangle$ to $\langle f\rangle$. We remind the reader that if $k \in E$, then $\langle k\rangle$ means the subsemigroup of $E$ generated by the idempotents in $k\langle E\rangle k$. We can therefore define a relation on $S \mathbf{C}$ by the rule that for all $(e, a, f),(g, b, h) \in S \mathbf{C}$,

$$
(e, a, f) \leq(g, b, h) \Leftrightarrow e \omega g, a=e b \text { and } f=e \beta
$$

where $\beta:\langle e\rangle \rightarrow\langle f\rangle$ is a connecting isomorphism. It is routine to show that $\leq$ is a partial order on $S \mathbf{C}$.

If $(e, a, f) \in S \mathbf{C}, u, v \in E$ and $u \leq e$ and $v \leq f$, then we define the restriction and co-restriction as

$$
{ }_{u} \mid(e, a, f)=(u, u a, u \beta) \text { and }\left.(e, a, f)\right|_{v}=\left(v \beta^{-1}, a v, v\right),
$$

where $\beta:\langle e\rangle \rightarrow\langle f\rangle$ is a connecting isomorphism. Then $S \mathbf{C}$ becomes an ordered 2 cancellative category under $\leq$

Suppose that $e, f \in E$ are such that $e \mathcal{L} f$ or $e \mathcal{R} f$. Then we define $[e, f]=$ $(e, e f, f)$. Clearly, $[e, f]$ is well-defined and it belongs to $S \mathbf{C}$.

Now, we obtain that $(S \mathbf{C}, \leq)$ together with the restriction and co-restriction forms an inductive 2 cancellative category. Further details of the proof can be found in $[1]$.

To sum up, we have:

Theorem C (Armstrong [1]) The category of concordant semigroups and good morphisms is equivalent to the category of inductive ${ }_{2}$ cancellative categories and inductive functors. $^{2}$

\subsection{Ehresmann semigroups and Ehresmann cat- egories}

Theorem A was generalised in a different direction to Ehresmann semigroups by Lawson [32]. His use of two partial orders on an Ehresmann semigroup is an 
important observation for the idea discussed in Chapter 7.

We recall from [32] that an Ehresmann category $C=\left(C, \cdot \leq_{r}, \leq_{l}\right)$ is a category $(C, \cdot)$ with set of identities $E$, equipped with two relations $\leq_{l}$ and $\leq_{r}$ such that the following conditions, and the duals $(\mathrm{E} 1)^{\circ}$ and $(\mathrm{E} 5)^{\circ}$ of $(\mathrm{E} 1)$ and $(\mathrm{E} 5)$ hold:

(E1) $\left(C, \cdot, \leq_{r}\right)$ is an ordered ${ }_{1}$ category with restriction;

(E2) if $e, f \in E$, then $e \leq_{r} f \Leftrightarrow e \leq_{l} f$;

(E3) $E$ is a meet semilattice under $\leq_{r}\left(\right.$ or $\left.\leq_{l}\right)$;

(E4) $\leq_{r} \circ \leq_{l}=\leq_{l} \circ \leq_{r}$;

(E5) if $x \leq_{r} y$ and $f \in E$, then $\left.x\right|_{\mathbf{r}(x) f} \leq\left._{r} y\right|_{\mathbf{r}(y) f}$.

We note that [32] interchanges the symbols $\mathbf{r}$ and $\mathbf{d}$ and the notion of restriction and co-restriction, from the conventions of this thesis.

We now pause to make a short comment on Ehresmann categories $C=$ $\left(C, \cdot, \leq_{r}, \leq_{l}\right)$. Condition (E1) implies that if $x \leq_{r} y$ in $C$ then $\mathbf{d}(x) \leq \mathbf{d}(y)$ and $\mathbf{r}(x) \leq \mathbf{r}(y)$ by $(\mathrm{OC} 1)$. Since $\left(C, \cdot \leq_{r}, \leq_{l}\right)$ is an ordered $_{1}$ category with restriction and co-restriction, then there exists a unique element $\mathbf{d}(x) \mid y$ such that $\mathbf{d}(x) \mid y \leq_{r} y$ and $\mathbf{d}(\mathbf{d}(x) \mid y)=\mathbf{d}(x)$. Since $x \leq_{r} y$, and the uniqueness of restriction gives $x=\mathbf{d}(x) \mid y$. To the converse, if $x=e_{e} \mid y$, then by the definition of restriction, we certainly obtain that $x \leq_{r} y$. Hence, we have:

Lemma 6.1. Let $C=\left(C, \cdot, \leq_{r}, \leq_{l}\right)$ be an Ehresmann category over $E$. Then for any $x, y \in C$,

(i) $x \leq_{r} y$ if and only if $x={ }_{e} \mid y$ for some $e \in E$;

(ii) $x \leq_{l} y$ if and only if $x=\left.y\right|_{f}$ for some $f \in E$.

Let $\mathbf{C}=\left(C, \cdot, \leq_{r}, \leq_{l}\right)$ and $\mathbf{D}=\left(D, \cdot, \leq_{r}, \leq_{l}\right)$ be Ehresmann categories with semilattice $E_{C}$ and $E_{D}$ of identities, respectively. We say that a functor $F: \mathbf{C} \rightarrow$ $\mathbf{D}$ is strongly ordered if it satisfies the following conditions:

$(i)$ if $x \leq_{l} y\left(\operatorname{resp} . x \leq_{r} y\right)$, then $x F \leq_{l} y F\left(\operatorname{resp} . x F \leq_{r} y F\right)$;

(ii) if $e, f \in E_{C}$, then $(e f) F=e F f F$.

Given an Ehresmann semigroup $S$ with distinguished semilattice $E$, we have introduced two partial orders $\leq_{r}$ and $\leq_{l}$ on $S$ in Section 2.2.5. Lawson [32] showed that the category, consisting of the set of objects $E$ and the set of morphisms $S$, forms an Ehresmann category together with the partial binary operation · defined 
by the rule that for any $x, y \in S$,

$$
x \cdot y= \begin{cases}x y & \text { if } x^{*}=y^{\dagger} \\ \text { undefined } & \text { otherwise }\end{cases}
$$

where $x^{*}, y^{\dagger} \in E$ with $x \widetilde{\mathcal{L}}_{E} x^{*}$ and $y \widetilde{\mathcal{R}}_{E} y^{\dagger}$.

Conversely, let $C=\left(C, \cdot, \leq_{r}, \leq_{l}\right)$ be an Ehresmann category with set of identities $E$. For any $x, y \in C$, we define

$$
x \bar{\otimes} y=\left.x\right|_{\mathbf{r}(x) \wedge \mathbf{d}(y)} \cdot \mathbf{r}(x) \wedge \mathbf{d}(y) \mid y .
$$

Then $(C, \bar{\otimes})$ is an Ehresmann semigroup.

Theorem D (Lawson [32]) The category of Ehresmann semigroups and admissible morphisms is isomorphic to the category of Ehresmann categories and strongly ordered functors.

We recall that a restriction semigroup is an Ehresmann semigroup satisfying (WIC) by Lemma 2.18. On such semigroups the orders $\leq_{r}, \leq_{l}$ and $\leq_{e}$ coincide and we denote the unique order by $\leq$.

Notice that if $\leq_{r}=\leq_{l}$ on an Ehresmann category $\left(C, \cdot, \leq_{r}, \leq_{l}\right)$, then $(C, \leq)$ becomes an inductive ${ }_{1}$ category, that is, an ordered $_{2}$ category, in which the set of identities is a semilattice.

Corollary 6.2. [24] The category of restriction semigroups and admissible congruences is isomorphic to the category of inductive ${ }_{1}$ categories and strongly ordered functors.

We now turn to ample semigroups. We replace the distinguished semilattice of idempotents $E$ by the whole set of idempotents and use relations $\mathcal{R}^{*}$ and $\mathcal{L}^{*}$ instead of $\widetilde{\mathcal{R}}_{E}$ and $\widetilde{\mathcal{L}}_{E}$ in the definition of restriction semigroups. We thus obtain the class of ample semigroups whose set of idempotents forms a semilattice. An admissible morphism in this context is more usually referred to as a good morphism.

Corollary 6.3. [24] The category of ample semigroups and good morphisms is isomorphic to the category of inductive ${ }_{1}$ cancellative categories and strongly ordered functors. 
Corollary 6.3 is a little difference in Armstrong's paper [2] and [24].

If we use relations $\mathcal{R}$ and $\mathcal{L}$ to replace $\mathcal{R}^{*}$ and $\mathcal{L}^{*}$ in the definition of ample semigroups, we then obtain the class of inverse semigroups. A good morphism in this context is a morphism. Consequently, we obtain Theorem A.

Ehresmann semigroups have a semilattice of idempotents, need not be regular or even abundant, need not satisfy an (IC) condition, and indeed need not be restriction semigroups. Lawson overcomes the lack of an (IC) condition by using two partial order relations. Our aim of Chapter 7 is to extend Lawson's result to the class of weakly $B$-orthodox semigroups, which extend the class of Ehresmann semigroups by replacing semilattices by bands. In Chapter 7 we use a new technique of generalised categories. We could use triples such as in [1], and this is the approach we take in Chapter 9 and in the more general weakly $U$-regular case, in Chapter 10. 


\section{Chapter 7}

\section{Beyond orthodox semigroups I: weakly $B$-orthodox semigroups and generalised categories}

Our purpose of this chapter is to describe a class of weakly $B$-orthodox semigroups. In doing so we produce a new approach to characterising orthodox semigroups via inductive generalised groupoids. Here $B$ denotes a band of idempotents; we note that if $B$ is a semilattice then a weakly $B$-orthodox semigroup is exactly an Ehresmann semigroup. We build a correspondence between our work and a result of Lawson for Ehresmann semigroups [32].

For convenience we make the convention that $B$ will always denote a band. Green's relations and their associated pre-orders will always refer to $B$, unless stated otherwise. In particular, if $S$ is weakly $B$-orthodox and $e \in B$, then $R_{e}$ $\left(L_{e}\right)$ denote the $\mathcal{R}$-class $(\mathcal{L}$-class $)$ of $e$ in $B$.

\subsection{Inductive generalised categories}

Let $I, R, L$ and $D$ be disjoint sets and let $p$ denote a collection of four (welldefined) onto maps:

$$
\begin{aligned}
& I \rightarrow R, \quad I \rightarrow L, \quad R \rightarrow D \quad \text { and } \quad L \rightarrow D \\
& i \mapsto R_{i}, \quad i \mapsto L_{i}, \quad R_{i} \mapsto D_{i} \quad L_{i} \mapsto D_{i}
\end{aligned}
$$

such that 


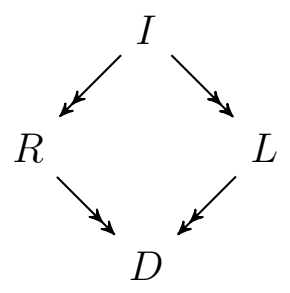

Figure 7.1: Maps

commutes. We denote this configuration by $(I, R, L, D, p)$ and refer to it as a context.

We pause to give our motivating example. Let $B$ be a band and $p$ denote the natural maps:

$$
B \rightarrow B / \mathcal{R}, \quad B \rightarrow B / \mathcal{L}, \quad B / \mathcal{R} \rightarrow B / \mathcal{D} \text { and } B / \mathcal{L} \rightarrow B / \mathcal{D} .
$$

Then $(B, B / \mathcal{R}, B / \mathcal{L}, B / \mathcal{D}, p)$ is a context. Of course, if $B$ is a semilattice, then all of Green's relations are trivial and the $p$-maps are essentially the identity maps.

Definition 7.1. A generalised category $P$ over a context $(I, R, L, D, p)$ consists of

(GC1) a class $\mathrm{Ob}(P)$ of objects $R \cup \dot{U}$;

$(\mathrm{GC} 2)$ a class $\operatorname{Mor}(P)$ of morphisms between the objects. Each morphism $x$ has a unique domain $\mathbf{d}(x) \in R$ and codomain $\mathbf{r}(x) \in L$. Denote the Mor-class of all morphisms from $R_{i} \in R$ to $L_{j} \in L$ by $\operatorname{Mor}\left(R_{i}, L_{j}\right)$;

(GC3) if $R_{i}, R_{k} \in R$ and $L_{j}, L_{h} \in L$ with $D_{j}=D_{k}$, then there is a binary operation

$$
\operatorname{Mor}\left(R_{i}, L_{j}\right) \times \operatorname{Mor}\left(R_{k}, L_{h}\right) \rightarrow \operatorname{Mor}\left(R_{i}, L_{h}\right),(x, y) \mapsto x \cdot y
$$

called composition of morphisms such that if $x \in \operatorname{Mor}\left(R_{i}, L_{j}\right), y \in \operatorname{Mor}\left(R_{k}, L_{h}\right)$, and $z \in \operatorname{Mor}\left(R_{m}, L_{n}\right)$, where $D_{j}=D_{k}$ and $D_{h}=D_{m}$, then $(x \cdot y) \cdot z=x \cdot(y \cdot z)$;

(GC4) for each $i \in I$, there exists a distinguished morphism, again denoted by $i$, such that $i \in \operatorname{Mor}\left(R_{i}, L_{i}\right)$ and if $\mathbf{d}(x)=R_{i}$ and $\mathbf{r}(y)=L_{i}$, then $i \cdot x=x$ and $y \cdot i=y$.

Let $P$ be a generalised category over a context $(I, R, L, D, p)$. Following the usual convention when building categories from semigroups, we may iden- 
tify $\operatorname{Mor}(P)$ with $P$. If $B$ is a band and $P$ is a generalised category over $(B, B / \mathcal{R}, B / \mathcal{L}, B / \mathcal{D}, p)$, where $p$ denote the natural maps, then we say simply $P$ is a generalised category over $B$.

Our notion of a generalised category is motivated by that of the "trace product' of a weakly $B$-orthodox semigroup. We explain this in Chapter 8 but comment briefly here on the special case of a band.

We have seen that if $B$ is a band, then $(B, B / \mathcal{R}, B / \mathcal{L}, B / \mathcal{D}, p)$ is a context. Define a generalised category $P$ over $B$ by putting $\operatorname{Mor}(P)=B$ and for $e \in B$, put $\mathbf{d}(e)=R_{e}$ and $\mathbf{r}(e)=L_{e}$. Let the partial binary operation be given by $e \cdot f=e f$, where $e \cdot f$ exists. Note the latter is true if and only if $D_{e}=D_{f}$. Thus the effect of our generalised category is to restrict the multiplication in $B$ to that within its $\mathcal{D}$-classes.

We now focus on generalised categories over a band $B$, in general more extensive than the example above, making use of the natural partial order in $B / \mathcal{R}$ and $B / \mathcal{L}$. Note that if $e \in B$ then by (GC4) we have that $e \in \operatorname{Mor}\left(R_{e}, L_{e}\right)$, so that $\mathbf{d}(e)=R_{e}$ and $\mathbf{r}(e)=L_{e}$.

We build on Definition 7.1 to define an inductive generalised category over

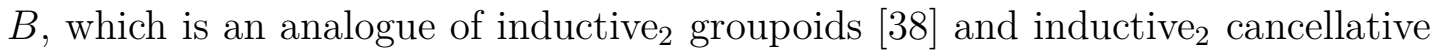
categories [1]. We will see that the elements of our inductive generalised category may be pre-ordered or partially ordered, in two ways, reflecting the approach of $[32]$.

Definition 7.2. Let $P$ be a generalised category over a band $B$. Then $P$ is an inductive generalised category if the following conditions and the duals (I1) ${ }^{\circ}$, $(\mathrm{I} 2)^{\circ}$, and $(\mathrm{I} 3)^{\circ}$ of (I1), (I2) and (I3) hold:

(I1) if $x \in P$ and $e, u \in B$ with $e \leq_{\mathcal{L}} u \in \mathbf{d}(x)$, then there exists an element ${ }_{e} \mid x$ in $P$, called the restriction of $x$ to $e$, such that $e \in \mathbf{d}\left({ }_{e} \mid x\right)$ and $\mathbf{r}\left({ }_{e} \mid x\right) \leq_{\mathcal{L}} \mathbf{r}(x)$; in particular, if $e \in \mathbf{d}(x)$, then ${ }_{e} \mid x=x$;

(I2) if $x \in P$ and $e, f, g, u \in B$ with $e \leq_{\mathcal{L}} g \mathcal{R} f \leq_{\mathcal{L}} u \in \mathbf{d}(x)$, then ${ }_{e f}\left|x=e_{e}\right|\left({ }_{f} \mid x\right)$;

(I3) if $x, y \in P$ and $e, u \in B$ with $x \cdot y$ defined in $P$ and $e \leq_{\mathcal{L}} u \in \mathbf{d}(x)$, then ${ }_{e} \mid(x \cdot y)=\left({ }_{e} \mid x\right) \cdot\left({ }_{f} \mid y\right)$, where $f \in \mathbf{r}\left({ }_{e} \mid x\right)$;

(I4) if $x, y \in P$ and $e_{1}, e_{2}, f_{1}, f_{2} \in B$ with $e_{1}, e_{2} \in \mathbf{r}(x)$ and $f_{1}, f_{2} \in \mathbf{d}(y)$, then $\left.x\right|_{e_{1} f_{1}} \cdot e_{1} f_{1}|y=x|_{e_{2} f_{2}} \cdot e_{2} f_{2} \mid y$;

(I5) if $x \in P$ and $e, f, u, v, g, h \in B$ with $g \in \mathbf{r}(x), h \in \mathbf{d}(x), u \in \mathbf{d}\left(\left.x\right|_{g f}\right)$ 
and $v \in \mathbf{r}\left({ }_{e h} \mid x\right)$, then ${ }_{e u}\left|\left(\left.x\right|_{g f}\right)=\left({ }_{e h} \mid x\right)\right|_{v f}$;

(I6) if $e, g, h, u, v \in B$ are such that $u \leq_{\mathcal{R}} g \mathcal{L} e$ and $v \leq_{\mathcal{L}} h \mathcal{R} e$, then $\left.e\right|_{u}=e u$ and ${ }_{v} \mid e=v e$.

We make some comments on the above definition. In (I3) let $\mathbf{r}(x)=L_{v}$ and $\mathbf{d}(y)=R_{w}$. Since there exists $x \cdot y$ we know that $v \mathcal{D} w$ so we have $\mathbf{r}(x)=L_{w v}$ and $\mathbf{d}(y)=R_{w v}$. Hence by (I1), $f \in \mathbf{r}\left({ }_{e} \mid x\right) \leq_{\mathcal{L}} L_{w v}$ and $w v \in \mathbf{d}(y)$, so that ${ }_{f} \mid y$ exists and $\mathbf{d}\left({ }_{f} \mid y\right)=R_{f}$. Hence $\left({ }_{e} \mid x\right) \cdot\left({ }_{f} \mid y\right)$ exists. To simplify the term ' $x \cdot y$ exists' may use the expression ' $\exists x \cdot y$ ' or ' $x \cdot y$ is defined'.

Suppose now that $P$ is a generalised category over a band $B$. We remarked above that if $e \in B$, then $\mathbf{d}(e)=R_{e}$ and $\mathbf{r}(e)=L_{e}$, so that if also $f \in B$ then $\exists e \cdot f$ if and only if $e \mathcal{D} f$. In this case, clearly $e \in \mathbf{d}(e), e \in \mathbf{d}(e \cdot f)$ and by (I1), $e \mid e=e$, so that $e \in \mathbf{r}\left({ }_{e} \mid e\right)$. Using (I1), (I3), (I6) and (GC4) we have

$$
e \cdot f={ }_{e} \mid(e \cdot f)=\left({ }_{e} \mid e\right) \cdot\left({ }_{e} \mid f\right)=e \cdot e f=e f .
$$

We pause to introduce a pair of pre-orders on an inductive generalised category $P$ over a band $B$ deduced from Definition 7.2. We make use of the restriction and co-restriction of $P$ to define relations $\leq_{r}$ and $\leq_{l}$ by the rule that for any $x, y \in P$,

$$
x \leq_{r} y \text { if and only if } x={ }_{e} \mid y \text { for some } e \in B,
$$

and

$$
x \leq_{l} y \text { if and only if } x=\left.y\right|_{f} \text { for some } f \in B .
$$

Lemma 7.3. The relations $\leq_{r}$ and $\leq_{l}$ are pre-orders on $P$.

Proof. To prove that $\leq_{r}$ is a pre-order on $P$, we first observe that $\leq_{r}$ is reflexive by (I1). It is necessary to show that $\leq_{r}$ is transitive. Assume that $x, y, z \in P$ with $x \leq_{r} y$ and $y \leq_{r} z$. Then there exist $e, f \in B$ such that $x={ }_{e} \mid y$ and $y={ }_{f} \mid z$. For $e \mid y$ and ${ }_{f} \mid z$ to exist we have $e \leq_{\mathcal{L}} g \in \mathbf{d}(y)=R_{f}$ and $f \leq_{\mathcal{L}} h \in \mathbf{d}(z)$. From (I2), $x={ }_{e}\left|\left({ }_{f} \mid z\right)=e_{e f}\right| z$. Hence $x \leq_{r} z$.

By the dual argument, we show that $\leq_{l}$ is a pre-order on $P$.

The reader might notice that previous authors have used partial orders rather than pre-orders. For our purpose, pre-orders are easier to use, but the partial orders are still there, as we now show.

We define $\leq_{r}^{\prime}$ and $\leq_{l}^{\prime}$ on $P$ by the rule that 


$$
x \leq_{r}^{\prime} y \text { if and only if } x={ }_{e} \mid y \text { for some } e \leq u \in \mathbf{d}(y),
$$

and

$$
x \leq_{l}^{\prime} y \text { if and only if } x=\left.y\right|_{f} \text { for some } f \leq v \in \mathbf{r}(y) .
$$

Lemma 7.4. The relations $\leq_{r}^{\prime}$ and $\leq_{l}^{\prime}$ are partial orders on $P$.

Proof. As in Lemma 7.3, $\leq_{r}^{\prime}$ is reflexive. If $x \leq_{r}^{\prime} y$ and $y \leq_{r}^{\prime} z$ then with $e, f$ as in Lemma 7.3, we have $e \leq g$ and $f \leq h$. Certainly, $x={ }_{e f} \mid z$ and $e f h=e f$, as $f \leq h$. Also, $e \leq g \mathcal{R} f \leq h$, so hef $=e f$. Hence ef $\leq h \in \mathbf{d}(z)$.

Finally, suppose that $x \leq_{r}^{\prime} y \leq_{r}^{\prime} x$. Then $x={ }_{e} \mid y$ and $y={ }_{f} \mid x$ for some $e \leq u \in \mathbf{d}(y)$ and $f \leq v \in \mathbf{d}(x)$. We have $e \leq u \mathcal{R} f$ and $f \leq v \mathcal{R} e$, so that $e \mathcal{R} f$ and $\mathbf{d}(x)=\mathbf{d}(y)$. Now $x={ }_{e} \mid y=y$, by (I1).

We say that $\leq_{r}$ and $\leq_{l}$ are the natural pre-orders associated with $P$ and $\leq_{r}^{\prime}$ and $\leq_{l}^{\prime}$ are the natural partial orders associated with $P$.

We end this section by showing that the class of inductive generalised categories over bands forms a category, together with certain maps referred to as pseudo-functors. They appear in the next definition.

Definition 7.5. Let $P_{1}$ and $P_{2}$ be inductive generalised categories over bands $B_{1}$ and $B_{2}$, respectively. A pseudo-functor $F$ from $P_{1}$ to $P_{2}$ is a pair of maps, both denoted $F$, from $B_{1}$ to $B_{2}$ and from $P_{1}$ to $P_{2}$, such that the following conditions and the dual $(\mathrm{F} 2)^{\circ}$ of (F2) hold:

(F1) the map $F$ is a morphism from $B_{1}$ to $B_{2}$;

(F2) if $e \in B_{1}$ and $e \leq_{\mathcal{L}} u \in \mathbf{d}(x)$ in $P_{1}$, then $\left.{ }_{e} \mid x\right) F={ }_{e F} \mid x F$;

(F3) if $\exists x \cdot y$ in $P_{1}$ then $\exists x F \cdot y F$ in $P_{2}$, and $(x \cdot y) F=x F \cdot y F$.

To see that (F2) makes sense, suppose that $u \in B_{1}, x \in P_{1}$ with $u \in \mathbf{d}(x)$. Then $R_{u}=\mathbf{d}(x)$ so that $\exists u \cdot x$ and $u \cdot x=x$. By (F3), $\exists u F \cdot x F$ and $u F \cdot x F=x F$. Hence $\mathbf{d}(x F)=\mathbf{d}(u F)=R_{u F}$, as $u F \in B_{2}$. Suppose also that $e \in B_{1}$, with $e \leq_{\mathcal{L}} u$ in $B_{1}$. Using (F1), we have $e F \leq_{\mathcal{L}} u F \in \mathbf{d}(x F)$ in $P_{2}$, and so $\exists_{e F} \mid x F$. Notice that we can define $F$ on $\mathrm{Ob}\left(P_{1}\right)$ by putting $R_{e} F=R_{e F}$ and $L_{e} F=L_{e F}$.

From the comments above, it is easy to check that Lemma 7.6 holds.

Lemma 7.6. Let $P_{1}, P_{2}$ and $P_{3}$ be inductive generalised categories over $B_{1}, B_{2}$ and $B_{3}$, respectively, and let $F_{1}: P_{1} \rightarrow P_{2}$ and $F_{2}: P_{2} \rightarrow P_{3}$ be pseudo-functors. Then $F_{1} F_{2}: P_{1} \rightarrow P_{3}$ is a pseudo-functor. 
The next observation follows immediately.

Lemma 7.7. The class of inductive generalised categories over bands, together with pseudo-functors, forms a category.

We refer to the category in the above lemma as $\mathcal{I G C}$.

\subsection{Construction}

Our primary interest in this section will be a construction of a weakly $B$-orthodox semigroup, built from an inductive generalised category over $B$.

Let $P$ be an inductive generalised category over a band $B$. We define a pseudo-product $\otimes$ on $P$ by

$$
x \otimes y=\left(\left.x\right|_{e f}\right) \cdot(e f \mid y),
$$

where $e \in \mathbf{r}(x), f \in \mathbf{d}(y)$. It follows from (I4) that the pseudo-product is independent of the choices of $e$ and $f$ and thus is well-defined. We will denote the set $P$, together with the pseudo-product $\otimes$, by $P \mathbf{S}$.

We pause to present our initial idea which follows Armstrong's steps, using the notion of sandwich set, simplifying a little here as our set of idempotents forms a band. We may define a pseudo-product $\otimes^{\prime}$ on $P$ by the rule that for any $x, y \in P$,

$$
x \otimes^{\prime} y=\left(\left.x\right|_{e f e}\right) \cdot(f e f \mid y),
$$

where $e \in \mathbf{r}(x)$ and $f \in \mathbf{d}(x)$. In that case, Condition (I5) is not enough to guarantee that $\otimes^{\prime}$ is associative in $P$. To achieve this it is necessary to add a stronger condition in place of (I5), which effectively says that $e \otimes^{\prime}\left(x \otimes^{\prime} y\right)=$ $\left(e \otimes^{\prime} x\right) \otimes^{\prime} y$ for any $x, y \in P$ and $e \in B$. This appears to us too contrived. Keeping this in mind we use the pseudo-product $\otimes$ defined as above.

We now present a series of lemmas related to $P$, which will help us to show our main result at the end of this section.

Lemma 7.8. If $x, y \in P$ with $\exists x \cdot y$, then $x \otimes y=x \cdot y$.

Proof. If $\exists x \cdot y$ then $\mathbf{r}(x)=L_{e}$ and $\mathbf{d}(y)=R_{f}$ say, where $e \mathcal{D} f$. Then $\mathbf{r}(x)=L_{f e}$ and $\mathbf{d}(y)=R_{f e}$, so $x \otimes y=\left(\left.x\right|_{f e f e}\right) \cdot\left({ }_{f e f e} \mid y\right)=\left(\left.x\right|_{f e}\right) \cdot\left({ }_{f e} \mid y\right)=x \cdot y$ by (I1). 
Lemma 7.9. If $e, f \in B$ then $e \otimes f=e f$.

Proof. We have

$$
\begin{aligned}
e \otimes f & =\left(\left.e\right|_{e f}\right) \cdot(\text { ef } \mid f) & & \\
& =e e f \cdot e f f & & (\text { by }(\mathrm{I} 6)) \\
& =e f \cdot e f=e f & & (\text { by }(\mathrm{GC} 4)) .
\end{aligned}
$$

Consequently, $B$ forms the same band under $\otimes$ and the original multiplication.

Lemma 7.10. If $x \in P$ and $e, f, u \in B$ with $u \mathcal{D} e \leq_{\mathcal{L}} f \in \mathbf{d}(x)$ then $u \cdot\left({ }_{e} \mid x\right)={ }_{u e} \mid x$.

Proof. Since $u \mathcal{D} e$, we deduce that

$$
\begin{aligned}
u \cdot{ }_{e} \mid x & =u \otimes_{e} \mid x & & \text { (Lemma 7.8) } \\
& =\left(\left.u\right|_{u e}\right) \cdot\left({ }_{u e} \mid\left({ }_{e} \mid x\right)\right) & & \\
& =u e \cdot\left({ }_{u e} \mid x\right) & & (\text { by }(\mathrm{I} 6),(\mathrm{I} 2)) \\
& ={ }_{u e} \mid x & & (\text { by }(\mathrm{GC} 4)) .
\end{aligned}
$$

Lemma 7.11. The set PS forms a semigroup under the operation $\otimes$.

Proof. It is sufficient to show that $P \mathbf{S}$ is associative. Suppose that $x, y, z \in P$ with $x^{*} \in \mathbf{r}(x), y^{\dagger} \in \mathbf{d}(y), y^{*} \in \mathbf{r}(y)$ and $z^{\dagger} \in \mathbf{d}(z)$. Then

$$
\begin{aligned}
& x \otimes(y \otimes z)=x \otimes\left(\left(\left.y\right|_{y^{*} z^{\dagger}}\right) \cdot\left(y^{*} z^{\dagger} \mid z\right)\right) \\
& =\left(\left.x\right|_{x^{*} u}\right) \cdot\left(_{x^{*} u} \mid\left(\left(\left.y\right|_{y^{*} z^{\dagger}}\right) \cdot\left(y_{y^{*} z^{\dagger}} \mid z\right)\right)\right) \quad\left(u \in \mathbf{d}\left(\left.y\right|_{y^{*} z^{\dagger}}\right)\right) \\
& =\left(\left.x\right|_{x^{*} u}\right) \cdot\left({ }_{x^{*} u} \mid\left(\left.y\right|_{y^{*} z^{\dagger}}\right)\right) \cdot\left({ }_{v} \mid\left(y_{y^{*} z^{\dagger}} \mid z\right)\right) \\
& \left(v \in \mathbf{r}\left(x_{x^{*} u} \mid\left(\left.y\right|_{y^{*} z^{\dagger}}\right)\right)\right. \text {, by (I3)). }
\end{aligned}
$$

Notice, by (I1), that $v \leq_{\mathcal{L}} y^{*} z^{\dagger} \in \mathbf{r}\left(\left.y\right|_{y^{*} z^{\dagger}}\right)$ and by (I5), that

$$
x^{*} u\left|\left(\left.y\right|_{y^{*} z^{\dagger}}\right)=\left({ }_{x^{*} y^{\dagger}} \mid y\right)\right|_{g z^{\dagger}},
$$


where $g \in \mathbf{r}\left({ }_{x^{*} y^{\dagger}} \mid y\right)$, and so $v \mathcal{L} g z^{\dagger}$ and $x^{*} u \in \mathbf{d}\left(\left.\left(_{x^{*} y^{\dagger}} \mid y\right)\right|_{g z^{\dagger}}\right)$. Thus

$$
\begin{aligned}
& x \otimes(y \otimes z)=\left(\left.x\right|_{x^{*} u}\right) \cdot\left(\left.\left(_{x^{*} y^{\dagger}} \mid y\right)\right|_{g z^{\dagger}}\right) \cdot\left({ }_{v} \mid\left(_{y^{*} z^{\dagger}} \mid z\right)\right) \\
& =\left(\left.x\right|_{x^{*} u}\right) \cdot\left(\left.\left({ }_{x^{*} y^{\dagger}} \mid y\right)\right|_{g z^{\dagger}}\right) \cdot\left({ }_{v y^{*} z^{\dagger}} \mid z\right) \quad\left(v \leq_{\mathcal{L}} y^{*} z^{\dagger}\right. \text {, by (I2)) } \\
& =\left(\left.x\right|_{x^{*} u}\right) \cdot\left(\left.\left(x_{x^{*} y^{\dagger}} \mid y\right)\right|_{g z^{\dagger}}\right) \cdot\left({ }_{v} \mid z\right) \quad\left(v \leq_{\mathcal{L}} y^{*} z^{\dagger}\right) \\
& =\left(\left.x\right|_{x^{*} u}\right) \cdot\left(\left.\left(_{x^{*} y^{\dagger}} \mid y\right)\right|_{g z^{\dagger}}\right) \cdot\left({ }_{v g z^{\dagger}} \mid z\right) \quad\left(v \mathcal{L} g z^{\dagger}\right) \\
& \left.=\left(\left.x\right|_{x^{*} u}\right) \cdot\left(\left.\left(_{x^{*} y^{\dagger}} \mid y\right)\right|_{g z^{\dagger}}\right) \cdot\left(v \cdot{ }_{g z^{\dagger}} \mid z\right)\right) \quad \text { (Lemma 7.10) } \\
& =\left(\left.x\right|_{x^{*} u}\right) \cdot\left(\left.\left({ }_{x^{*} y^{\dagger}} \mid y\right)\right|_{g z^{\dagger}} \cdot v\right) \cdot\left({ }_{g z^{\dagger}} \mid z\right) \\
& =\left(\left.x\right|_{x^{*} u}\right) \cdot\left(\left(\left.\left(_{x^{*} y^{\dagger}} \mid y\right)\right|_{g z^{\dagger}}\right) \cdot\left(g z^{\dagger} \mid z\right) \quad\left(v \mathcal{L} g z^{\dagger} \text {, by }(\mathrm{GC} 4)\right)\right. \text {. }
\end{aligned}
$$

Due to the dual of (I1), $u \in \mathbf{d}\left(\left.y\right|_{y^{*} z^{\dagger}}\right) \leq_{\mathcal{R}} \mathbf{d}(y)$, whence $x^{*} y^{\dagger} x^{*} u=x^{*} y^{\dagger} x^{*} y^{\dagger} u=$ $x^{*} y^{\dagger} u=x^{*} u$. So

$$
\begin{aligned}
x \otimes(y \otimes z) & =\left(\left.x\right|_{x^{*} y^{\dagger} x^{*} u}\right) \cdot\left(\left.\left(_{x^{*} y^{\dagger}} \mid y\right)\right|_{g z^{\dagger}}\right) \cdot\left({ }_{g z^{\dagger}} \mid z\right) \\
& =\left(\left.\left(\left.x\right|_{x^{*} y^{\dagger}}\right)\right|_{x^{*} u}\right) \cdot\left(\left(\left.\left(_{x^{*} y^{\dagger}} \mid y\right)\right|_{g z^{\dagger}}\right) \cdot\left({ }_{g z^{\dagger}} \mid z\right)\right. \\
& \left(\text { by }(\text { I2 })^{\circ}, \text { since } x^{*} u \leq \mathcal{R} x^{*} y^{\dagger}\right) \\
& \left(\left.\left(\left(\left.x\right|_{x^{*} y^{\dagger}}\right) \cdot\left({ }_{x^{*} y^{\dagger}} \mid y\right)\right)\right|_{g z^{\dagger}}\right) \cdot\left({ }_{g z^{\dagger}} \mid z\right) \\
& \left(x^{*} u \in \mathbf{d}\left(\left.\left(_{x^{*} y^{\dagger}} \mid y\right)\right|_{g z^{\dagger}}\right)\right) \\
& =\left(\left.(x \otimes y)\right|_{g z^{\dagger}}\right) \cdot\left({ }_{g z^{\dagger}} \mid z\right) \\
& =(x \otimes y) \otimes z .
\end{aligned}
$$

The following lemma shows that $P \mathbf{S}$ is a weakly $B$-abundant semigroup.

Lemma 7.12. Let $x \in P \mathbf{S}, e \in \mathbf{r}(x)$ and $g \in \mathbf{d}(x)$. Then $g \widetilde{\mathcal{R}}_{B} x \widetilde{\mathcal{L}}_{B} e$ in $P \mathbf{S}$.

Proof. By Lemma 7.8, we obtain that $x \otimes e=x \cdot e=x$. Suppose that $k \in B$ and $x \otimes k=x$. Then

$$
\begin{aligned}
x \otimes k & =\left(\left.x\right|_{e k}\right) \cdot\left({ }_{e k} \mid k\right) & & \\
& =\left(\left.x\right|_{e k}\right) \cdot e k & & (\text { by (I6)) } \\
& =\left.x\right|_{e k} & & (\text { by }(\mathrm{GC} 4)) .
\end{aligned}
$$


Thus $x=\left.x\right|_{e k}$, which implies that $e k \in \mathbf{r}(x)$, and so $e \mathcal{L} e k$. It follows that

$$
\begin{array}{rlrl}
e \otimes k & =e k & & (\text { Lemma 7.9) } \\
& =e e k & \\
& =e & & (e \mathcal{L} e k) .
\end{array}
$$

Consequently, $x \widetilde{\mathcal{L}}_{B} e$.

Similarly, we can show that $x \widetilde{\mathcal{R}}_{B} g$.

As an application of Lemma 7.12, we give a concrete description of relations $\leq_{\widetilde{\mathcal{R}}_{B}}$ and $\leq_{\widetilde{\mathcal{L}}_{B}}$ on $P \mathbf{S}$ as follows.

Lemma 7.13. For any $x, y \in P \mathrm{~S}$,

(i) $x \leq_{\widetilde{\mathcal{R}}_{B}} y$ if and only if $\mathbf{d}(x) \leq_{\mathcal{R}} \mathbf{d}(y)$;

(ii) $x \leq_{\widetilde{\mathcal{L}}_{B}} y$ if and only if $\mathbf{r}(x) \leq_{\mathcal{L}} \mathbf{r}(y)$.

Proof. We prove $(i)$. Let $x, y \in P$ and let $\mathbf{d}(x)=R_{e}$ and $\mathbf{d}(y)=R_{f}$. Then

$$
\begin{aligned}
x \leq_{\widetilde{\mathcal{R}}_{B}} y \text { in } P \mathbf{S} & \Leftrightarrow e \leq_{\widetilde{\mathcal{R}}_{B}} f \text { in } P \mathbf{S} \quad \text { (Lemma 7.12) } \\
& \Leftrightarrow e \leq_{\mathcal{R}} f \text { in } B \\
& \Leftrightarrow R_{e} \leq_{\mathcal{R}} R_{f} \\
& \Leftrightarrow \mathbf{d}(x) \leq_{\mathcal{R}} \mathbf{d}(y) .
\end{aligned}
$$

Now let us sum up results related to $P \mathbf{S}$ in the following theorem:

Theorem 7.14. If $P$ is an inductive generalised category over $B$, then $(P \mathbf{S}, \otimes)$ is a weakly B-orthodox semigroup. Further, the natural pre-orders and partial orders in $P$ and PS coincide.

Proof. We first show that $(P \mathbf{S}, \otimes)$ has $(\mathrm{C})$. Suppose that $x, y, z \in P \mathbf{S}$ and $x \widetilde{\mathcal{R}}_{B} y$. It follows from Lemma 7.13 that $\mathbf{d}(x)=\mathbf{d}(y)$. We deduce that $z \otimes x=\left(\left.z\right|_{v e}\right) \cdot\left({ }_{v e} \mid x\right)$ and $z \otimes y=\left(\left.z\right|_{v e}\right) \cdot\left({ }_{v e} \mid y\right)$, where $v \in \mathbf{r}(z)$ and $e \in \mathbf{d}(x)=\mathbf{d}(y)$. Hence, $\mathbf{d}(z \otimes x)=$ $\mathbf{d}\left(\left.z\right|_{v e}\right)=\mathbf{d}(z \otimes y)$. By Lemma 7.13, $z \otimes x \widetilde{\mathcal{R}}_{B} z \otimes y$. Dually, we can show that $\widetilde{\mathcal{L}}_{B}$ is a right congruence. 
Let $x, y \in P$ and suppose that $x \leq_{r} y$ in $P$. Then $x={ }_{e} \mid y$ for some $e \leq_{\mathcal{L}} u \in \mathbf{d}(y)$. Hence,

$$
e \otimes y=\left.e\right|_{e u} \cdot{ }_{e u}\left|y=e u \cdot{ }_{e u}\right| y={ }_{e u}\left|y={ }_{e}\right| y=x,
$$

so that $x \leq_{r} y$ in $P \mathbf{S}$.

If in addition we have $e \leq u$, so that $x \leq_{r}^{\prime} y$ in $P$, then from $x={ }_{e} \mid y$ we have $\mathbf{d}(x)=R_{e}$ and $x^{\dagger} \leq_{\mathcal{R}} y^{\dagger}$, by Lemma 7.13, so $x \leq_{r}^{\prime} y$ in $P \mathbf{S}$.

Conversely, if $x \leq_{r} y$ in $P \mathbf{S}$, then $x=f \otimes y$ for some $f \in B$. Hence,

$$
x=f \otimes y=\left.f\right|_{f y^{\dagger}} \cdot{ }_{f y^{\dagger}}\left|y=f y^{\dagger} \cdot{ }_{f y^{\dagger}}\right| y={ }_{f y^{\dagger}} \mid y,
$$

so that $x \leq_{r} y$ in $P$.

Further, if $x \leq_{r}^{\prime} y$ in $P \mathbf{S}$, then we have $x^{\dagger} \leq_{\mathcal{R}} y^{\dagger}$, so that $\mathbf{d}(x) \leq_{\mathcal{R}} \mathbf{d}(y)$, that is, $f y^{\dagger} \leq_{\mathcal{R}} y^{\dagger}$. Clearly then $f y^{\dagger} \leq y^{\dagger}$, so that $x \leq_{r}^{\prime} y$ in $P$.

The dual result holds for $\leq_{l}$ and $\leq_{l}^{\prime}$.

We can obtain an admissible morphism between weakly $B$-orthodox semigroups from a pseudo-functor between inductive generalised categories over bands. This is made more precise in the following lemma.

Lemma 7.15. Let $F: P_{1} \rightarrow P_{2}$ be a pseudo-functor between inductive generalised categories $P_{1}$ and $P_{2}$, where $P_{1}$ and $P_{2}$ are over bands $B_{1}$ and $B_{2}$, respectively. Then the map $F \mathbf{S}: P_{1} \mathbf{S} \rightarrow P_{2} \mathbf{S}$ defined by the rule that $x F \mathbf{S}=x F$, where $x \in P_{1} \mathbf{S}$, is an admissible morphism; moreover, if $F_{1}: P_{1} \rightarrow P_{2}$ and $F_{2}: P_{2} \rightarrow P_{3}$ are pseudo-functors, then $\left(F_{1} F_{2}\right) \mathbf{S}=F_{1} \mathbf{S} F_{2} \mathbf{S}$.

Proof. We claim first that $F \mathbf{S}$ is a semigroup morphism. Suppose that $x, y \in P_{1} \mathbf{S}$. Then by the definition of $F \mathbf{S}$,

$$
\begin{aligned}
(x \otimes y) F \mathbf{S} & =(x \otimes y) F & & \\
& =\left(\left(\left.x\right|_{f u}\right) \cdot\left({ }_{f u} \mid y\right)\right) F & & (f \in \mathbf{r}(x), u \in \mathbf{d}(y)) \\
& \left.=\left(\left.x\right|_{f u}\right) F \cdot{ }_{f u} \mid y\right) F & & (\text { by }(\mathrm{F} 3)) \\
& =\left(\left.x F\right|_{(f u) F}\right) \cdot\left({ }_{(f u) F} \mid y F\right) & & \left(\text { by }(\mathrm{F} 2),(\mathrm{F} 2)^{\circ}\right) \\
& =\left(\left.x F\right|_{f F u F}\right) \cdot\left({ }_{f F u F} \mid y F\right) & & (\text { by }(F 1)) .
\end{aligned}
$$


Since $f \in \mathbf{r}(x)$ and $u \in \mathbf{d}(y)$, it follows from the comments succeeding Definition 7.5 that $f F \in \mathbf{r}(x F)$ and $u F \in \mathbf{d}(y F)$. Thus,

$$
(x \otimes y) F \mathbf{S}=x F \otimes y F=x F \mathbf{S} \otimes y F \mathbf{S} .
$$

We now show that $F \mathbf{S}$ is admissible. Clearly, by (F1), $B_{1} F \mathbf{S} \subseteq B_{2}$. For any $e \in \mathbf{r}(x)$, we have $e \widetilde{\mathcal{L}}_{B_{1}} x$ and $e F \in \mathbf{r}(x F)$. Thus, eF $\widetilde{\mathcal{L}}_{B_{2}} x F$, that is, eF $\mathbf{S} \widetilde{\mathcal{L}}_{B_{2}} x F \mathbf{S}$. By a similar argument, we have that for any $k \in \mathbf{d}(x)$, $k F \mathbf{S} \widetilde{\mathcal{R}}_{B_{2}} x F \mathbf{S}$. By Lemma $2.9, F \mathbf{S}$ is an admissible morphism between weakly $B$-orthodox semigroups $P_{1} \mathbf{S}$ and $P_{2} \mathbf{S}$.

The final part of the lemma is clear.

Theorem 7.14 and Lemma 7.15 show that $\mathbf{S}: \mathcal{I G C} \rightarrow \mathcal{W O}$ is a functor.

\subsection{Correspondence}

In Section 7.2, we start with an inductive generalised category over $B$ and construct a weakly $B$-orthodox semigroup. Our present aim is to prove a converse to this result and thus provide a correspondence between the class of inductive generalised categories over bands and the class of weakly $B$-orthodox semigroups, i.e. between $\mathcal{I G C}$ and $\mathcal{W O}$.

Let $S$ be a weakly $B$-orthodox semigroup. We define $S \mathbf{C}$ to be the set $S$ equipped with the following partial binary operation:

$$
x \cdot y= \begin{cases}x y & \text { if } x^{*} \mathcal{D} y^{\dagger} \\ \text { undefined } & \text { otherwise }\end{cases}
$$

where $x y$ is the product of $x$ and $y$ in $S$. This is known as the trace product and denoted by $S \mathbf{C}=(S, \cdot)$.

It is an immediate result that if $e, f \in B$ and $x \in S$ are such that $e \widetilde{\mathcal{R}}_{B} x \widetilde{\mathcal{L}}_{B} f$ then $e \cdot x=x=x \cdot f$.

We now turn to give a number of basic properties of $S \mathbf{C}$, which will be found useful in the sequel.

Lemma 7.16. If $\exists x \cdot y$ in $S \mathbf{C}$, then $x \widetilde{\mathcal{R}}_{B} x y \widetilde{\mathcal{L}}_{B} y$ in $S$. 
Proof. Suppose that $x$ and $y$ are in $S$ such that $x \cdot y$ is defined in $S$ C. Then $x^{*} \mathcal{D} y^{\dagger}$. We assume that $x^{*} \mathcal{L} h \mathcal{R} y^{\dagger}$, where $h \in B$. Since $\widetilde{\mathcal{R}}_{B}$ is a left congruence and $\widetilde{\mathcal{L}}_{B}$ is a right congruence, it follows that $x y \widetilde{\mathcal{R}}_{B} x y^{\dagger} \widetilde{\mathcal{R}}_{B} x h=x$ and dually, $x y \widetilde{\mathcal{L}}_{B} x^{*} y \widetilde{\mathcal{L}}_{B} h y=y$. So $x \widetilde{\mathcal{R}}_{B} x y \widetilde{\mathcal{L}}_{B} y$, as required.

Lemma 7.17. If $S$ is a weakly $B$-orthodox semigroup, then $S \mathbf{C}$ is a generalised category over $B$ such that $\mathbf{d}(x)=R_{x^{\dagger}}$ and $\mathbf{r}(x)=L_{x^{*}}$.

Proof. We have $x \in \operatorname{Mor}\left(R_{e}, L_{f}\right)$ if and only if $x^{\dagger} \mathcal{R} e$ and $x^{*} \mathcal{L} f$ in $B$. If in addition $y \in \operatorname{Mor}\left(R_{g}, L_{h}\right)$, then $\exists x \cdot y$ in $S \mathbf{C}$ if and only if $x^{*} \mathcal{D} y^{\dagger}$, i.e. $D_{f}=D_{g}$. Moreover, if $\exists x \cdot y$, then $x \cdot y \in \operatorname{Mor}\left(R_{e}, L_{h}\right)$ by Lemma 7.16. Clearly Condition (GC3) holds.

For any $e \in B$, we take the distinguished morphism $e$ associated to $e$ to be itself, whose domain is $R_{e}$ and codomain is $L_{e}$. Certainly, if $e \in \mathbf{d}(x)$ (resp. $e \in$ $\mathbf{r}(x)$ ), then $e$ is a left (resp. right) identity of $x$. Hence, (GC4) holds.

We build on the above to show that $S \mathbf{C}$ may be equipped with restrictions and co-restrictions, under which it becomes an inductive generalised category.

For $x \in S$ and $e, f \in B$ with $e \leq_{\mathcal{L}} u \in \mathbf{d}(x)$ and $f \leq_{\mathcal{R}} v \in \mathbf{r}(x)$,

$$
{ }_{e} \mid x=e x \text { and }\left.x\right|_{f}=x f .
$$

Lemma 7.18. Let $S$ be a weakly B-orthodox semigroup. With the above definition of restriction and co-restriction, SC becomes an inductive generalised category over B. Further, the natural pre-orders and partial orders in $S$ and $S \mathbf{C}$ coincide.

Proof. In view of Lemma 7.17, it remains to show that $S \mathbf{C}$ with the restriction and co-restriction defined above satisfies Conditions (I1) to (I6) and the duals $(\mathrm{I} 1)^{\circ},(\mathrm{I} 2)^{\circ}$ and $(\mathrm{I} 3)^{\circ}$ of (I1), (I2) and (I3).

(I1) If $x \in S$ and $e, u \in B$ with $e \leq_{\mathcal{L}} u \in \mathbf{d}(x)$, then $e \mid x=e x$, and so by Lemmas 2.8 and 2.14, Condition (I1) is satisfied.

(I2) Since restriction and co-restriction are given by multiplication in $S$, it is clear that (I2) and its dual hold.

(I3) Suppose that $x, y \in S$ and $e, u \in B$ with $x \cdot y$ defined in $S \mathbf{C}$, let $e \leq_{\mathcal{L}}$ $u \in \mathbf{d}(x)$ and $f \in \mathbf{r}\left({ }_{e} \mid x\right)=L_{(e x)^{*}}$. Then ${ }_{e} \mid(x \cdot y)=e x y=\operatorname{exfy}=\left({ }_{e} \mid x\right) \cdot\left({ }_{f} \mid y\right)$. 
(I4) It is routine to check that Condition (I4) holds, both products being equal to $x y$.

(I5) As for (I4) this is again routine, with both sides of the equality we must verify being equal to $\operatorname{exf}$.

(I6) Clearly, it is satisfied by the definitions of the restriction and co-restriction, respectively.

Now, let $x, y \in S$. Then

$$
\begin{array}{rlrl}
x \leq_{r} y \text { in } S & \Leftrightarrow x=e y & & \text { some } e \in B \\
& \Leftrightarrow x=e y^{\dagger} y & & \text { some } e \in B, y^{\dagger} \in \mathbf{d}(y) \\
& \Leftrightarrow x={ }_{e y^{\dagger}} \mid y & & \text { some } e \in B, y^{\dagger} \in \mathbf{d}(y) \\
& \Leftrightarrow x={ }_{f} \mid y & & \text { some } f \in B \text { with } f \leq_{\mathcal{L}} u \in \mathbf{d}(y) \\
& \Leftrightarrow x \leq_{r} y \text { in } S \mathbf{C} . &
\end{array}
$$

In addition, with notation as above, if $x \leq_{r}^{\prime} y$ in $S$ we have that $x^{\dagger} \leq_{\mathcal{R}} y^{\dagger}$, so that $x=y^{\dagger} e y^{\dagger} y={ }_{y^{\dagger} e y^{\dagger}} \mid y$ and $y^{\dagger} e y^{\dagger} \leq y^{\dagger}$, and so $x \leq_{r}^{\prime} y$ in $S \mathbf{C}$. Conversely, if $x \leq_{r}^{\prime} y$ in $S \mathbf{C}$, then $x={ }_{g} \mid y$, where $g \leq y^{\dagger} \in \mathbf{d}(y)$. Then $x=g y$ in $S$, and $x^{\dagger} \mathcal{R} g \leq_{\mathcal{R}} y^{\dagger}$, so that $x \leq_{r}^{\prime} y$ in $S$.

Proposition 7.19. Let $S$ be a weakly $B$-orthodox semigroup and $P$ be an inductive generalised category over $B$. Then $S \mathbf{C S}=S$ and $P \mathbf{S C}=P$.

Proof. Let $S$ be a weakly $B$-orthodox semigroup. It follows from Lemma 7.18 that $S \mathbf{C}$ is an inductive generalised category over $B$ with multiplication a restriction of that in $S$ and $\mathbf{d}(x)=R_{x^{\dagger}}, \mathbf{r}(x)=L_{x^{*}}$, for any $x \in S$, and if $e \leq_{\mathcal{L}} u \in \mathbf{d}(x)$ and $f \leq_{r} v \in \mathbf{r}(x)$ then ${ }_{e} \mid x=e x$ and $\left.x\right|_{f}=x f$.

We now construct $S$ CS, which again has underlying set $S$, by defining the pseudo-product

$$
x \otimes y=\left(\left.x\right|_{v g}\right) \cdot\left({ }_{v g} \mid y\right),
$$

where $v \in \mathbf{r}(x)=L_{x^{*}}$ and $g \in \mathbf{d}(y)=R_{y^{\dagger}}$. Observe that

$$
x \otimes y=\left(\left.x\right|_{v g}\right) \cdot\left({ }_{v g} \mid y\right)=x v g v g y=x v g y=x y,
$$

so the operations in $S$ and $S$ CS are the same. Moreover, the distinguished bands of $S$ and $S$ CS are both $B$. Hence $S=S$ CS.

We now focus on the converse. Let $P$ be an inductive generalised category 
over $B$ with partial binary operation $\cdot$. We establish the weakly $B$-orthodox semigroup PS by defining the pseudo-product $\otimes$ of Theorem 7.14.

We temporarily use the notation $\odot$ for the partial binary operation in PSC. For any $x, y \in P$ we have

$$
\begin{aligned}
\exists x \odot y & \Leftrightarrow x^{*} \mathcal{D} y^{\dagger} \text { in } P \mathbf{S} \\
& \Leftrightarrow e \mathcal{D} f, \text { where } \mathbf{r}(x)=L_{e} \text { and } \mathbf{d}(y)=R_{f} \\
& \Leftrightarrow \exists x \cdot y \text { in } P .
\end{aligned}
$$

Further, if $\exists x \odot y$, then by Lemma 7.8 ,

$$
x \odot y=x \otimes y=x \cdot y .
$$

For $x \in P$ we have that $\mathbf{d}(x)=R_{x^{\dagger}}$ in $P$ SC, where $x \widetilde{\mathcal{R}}_{B} x^{\dagger}$ in $P \mathbf{S}$. But, the latter holds if and only if $x^{\dagger} \in \mathbf{d}(x)$ in $P$, i.e. $\mathbf{d}(x)=R_{x^{\dagger}}$ in $P$. Thus $\mathbf{d}$ in $P$ and $P$ SC coincide, and dually for $\mathbf{r}$.

Clearly, the distinguished morphisms in $P$ and PSC are the same.

Again as a temporary measure, we use $\|$ to denote restriction and corestriction in $P$ SC.

Let $x \in P$ and let $e, u \in B$ with $e \leq_{\mathcal{L}} u \in \mathbf{d}(x)$. Then in PSC,

$$
{ }_{e} \| x=e \otimes x=\left.e\right|_{e u} \cdot{ }_{e u}\left|x={ }_{e u}\right| x={ }_{e} \mid x
$$

and similarly for co-restrictions.

We now proceed to establish an isomorphism between $\mathcal{I} \mathcal{G C}$ and $\mathcal{W O}$.

The next lemma demonstrates that an admissible morphism between two weakly $B$-orthodox semigroups gives rise to a pseudo-functor.

Lemma 7.20. Let $S$ be a weakly $B_{1}$-orthodox semigroup and $T$ be a weakly $B_{2}$ orthodox semigroup. Suppose that $\theta$ is an admissible morphism. Then the map $\theta \mathbf{C}: S \mathbf{C} \rightarrow T \mathbf{C}$ given by the rule that $x \theta \mathbf{C}=x \theta$ for $x \in B_{1}$ and $x \in S$ is a pseudo-functor. Further, if $\theta_{1}: S \rightarrow T$ and $\theta_{2}: T \rightarrow Q$ are admissible morphisms, then $\left(\theta_{1} \theta_{2}\right) \mathbf{C}=\theta_{1} \mathbf{C} \theta_{2} \mathbf{C}$.

Proof. (F1) Since $\theta$ is an admissible morphism, it follows that $\theta$ is a morphism from $B_{1}$ to $B_{2}$. 
(F2) Suppose that $x \in S$ and $e, f \in B_{1}$ with $e \leq_{\mathcal{L}} f \in \mathbf{d}(x)$. Then ${ }_{e} \mid x$ is defined and $e_{e} \mid x=e x$. Since $\theta$ is admissible, it follows that $e \theta \leq_{\mathcal{L}} f \theta$ and $f \theta \widetilde{\mathcal{R}}_{B_{2}} x \theta$, that is, $f \theta \in \mathbf{d}(x \theta)$, which implies that ${ }_{e \theta} \mid x \theta$ is defined. Then $\left({ }_{e} \mid x\right) \theta \mathbf{C}=(e x) \theta \mathbf{C}=(e x) \theta=e \theta x \theta={ }_{e \theta}\left|x \theta={ }_{e \theta} \mathbf{C}\right| x \theta \mathbf{C}$.

(F3) If $\exists x \cdot y$ in $S \mathbf{C}$, then $x^{*} \mathcal{D} y^{\dagger}$. Hence there is an $h \in B$ with $x \widetilde{\mathcal{L}}_{B_{1}} h \widetilde{\mathcal{R}}_{B_{1}} y$. Since $\theta$ is admissible, $x \theta \widetilde{\mathcal{L}}_{B_{2}} h \theta \widetilde{\mathcal{R}}_{B_{2}} y \theta$ and $h \theta \in B_{2}$. Thus $\exists x \theta \cdot y \theta$ in $T \mathbf{C}$. Clearly, if $x \cdot y$ exists, $(x \cdot y) \theta=(x y) \theta=x \theta y \theta=x \theta \cdot y \theta$, since $\theta$ is a morphism.

It is routine to see that $\left(\theta_{1} \theta_{2}\right) \mathbf{C}=\theta_{1} \mathbf{C} \theta_{2} \mathbf{C}$.

The following result is easy to see, given Lemma 7.15 and 7.20.

Lemma 7.21. Let $\theta: S \rightarrow T$ be an admissible morphism of weakly $B$-orthodox semigroups, and $F: P_{1} \rightarrow P_{2}$ be a pseuo-functor of inductive generalised categories over bands. Then $\theta \mathbf{C S}=\theta$ and $F \mathbf{S C}=F$.

Lemmas 7.18 and 7.20 show that $\mathbf{C}: \mathcal{W O} \rightarrow \mathcal{I G C}$ is a functor and Proposition 7.19 and Lemma 7.21 give that $\mathbf{S}$ and $\mathbf{C}$ are mutually inverse. Hence we deduce our main result.

Theorem 7.22. The category $\mathcal{W O}$ of weakly $B$-orthodox semigroups and admissible morphisms is isomorphic to the category $\mathcal{I G C}$ of inductive generalised categories over bands and pseudo-functors.

\subsection{Special cases}

In this section, we concentrate on some special kinds of weakly $B$-orthodox semigroups. We now present a lemma which will be used in our first two cases.

Lemma 7.23. Let $P$ be an inductive generalised category over $B$. Suppose that for all $x \in E(P \mathbf{S})$ and $e \in \mathbf{d}(x), f \in \mathbf{r}(x)$ we have $e \mathcal{R}^{*} x \mathcal{L}^{*} f$ in $P \mathbf{S}$. Then $E(P \mathbf{S})=B$.

Proof. Suppose that $x \in P$ and $x \otimes x=x$. Assume that $f \in \mathbf{r}(x)$ and $e \in \mathbf{d}(x)$ with $e \mathcal{R}^{*} x \mathcal{L}^{*} f$, and so $f \otimes x=f$, that is, $\left.f\right|_{f e} \cdot{ }_{f e} \mid x=f$, or equivalently, ${ }_{f e} \mid x=f$, which implies that $f e \mathcal{R} f$. Dually, $f$ e $\mathcal{L}$ e. Thus, e $\mathcal{D} f$ so that e $\mathcal{R}$ ef $\mathcal{L} f$. Since $e \mathcal{R}^{*} x \mathcal{L}^{*} f$, we have $x \mathcal{H}^{*}$ ef. An $\mathcal{H}^{*}$ class contains at most an idempotent so that $x=e f$. 
An (inductive) generalised category $P$ is an (inductive) generalised groupoid if for all $x \in P$ with $\mathbf{d}(x)=R_{e}$ and $\mathbf{r}(x)=L_{f}$, there exists $y \in P$ with $\mathbf{d}(y)=R_{f}$ and $\mathbf{r}(y)=L_{e}$ such that $e=x \cdot y$ and $y \cdot x=f$.

Corollary 7.24. The category of orthodox semigroups and morphisms is isomorphic to the category of inductive generalised groupoids over bands and pseudofunctors.

Proof. Let $S$ be an orthodox semigroup with $B=E(S)$. Suppose that $x \in S \mathbf{C}$ with $\mathbf{d}(x)=R_{e}$ and $\mathbf{r}(x)=L_{f}$. Since $\mathcal{R}=\widetilde{\mathcal{R}}_{B}$ and $\mathcal{L}=\widetilde{\mathcal{L}}_{B}$, we have that $e \mathcal{R} x \mathcal{L} f$. It follows from the fact that $S$ is regular that there exists $y \in S$ with $e=x y$ and $y x=f$. We have that $e \mathcal{L} y \mathcal{R} f$ and so $\mathbf{d}(y)=R_{f}$ and $\mathbf{r}(y)=L_{e}$ and the products $x \cdot y, y \cdot x$ exist in $S \mathbf{C}$. Moreover, $x \cdot y=x y=e$ and $y \cdot x=y x=f$.

Conversely, let $P$ be an inductive generalised groupoid over $B$. Suppose that $x \in P$ and $\mathbf{d}(x)=R_{e}, \mathbf{r}(x)=L_{f}$. Then there exists $y \in P$ with $\mathbf{d}(y)=R_{f}$ and $\mathbf{r}(y)=L_{e}$ such that $f=y \cdot x$ and $e=x \cdot y$. And so $x \otimes y \otimes x=(x \cdot y) \otimes x=$ $e \otimes x=e \cdot x=x$. Thus, $P \mathbf{S}$ is regular. In addition, as $e=x \cdot y=x \otimes y$ and $x=e \otimes x$, we have that $e \mathcal{R} x$ in PS. Dually, $f \mathcal{L} x$ in PS. By Lemma 7.23, we have that $E(P \mathbf{S})=B$. Hence, $P \mathbf{S}$ is an orthodox semigroup.

Now, we focus on the class of abundant semigroups. We replace the distinguished set of idempotents $B$ by the whole set of idempotents and use relations $\mathcal{R}^{*}$ and $\mathcal{L}^{*}$ instead of $\widetilde{\mathcal{R}}_{B}$ and $\widetilde{\mathcal{L}}_{B}$ in the definition of weakly $B$-orthodox semigroups. We thus obtain the class of abundant semigroups whose set of idempotents forms a band. An admissible morphism in this context is more usually referred to as a good morphism. We define an inductive generalised category $P$ over a band $B$ to be abundant if it satisfies the following condition and its dual (I7) ${ }^{\circ}$ :

(I7) if $e, f, g \in B$ and $x, y, z \in P$ are such that $e, f \leq_{\mathcal{L}} g \in \mathbf{d}(x), e \in \mathbf{r}(y)$, $f \in \mathbf{r}(z)$ and $y \cdot{ }_{e}\left|x=z \cdot{ }_{f}\right| x$, then $y=z$.

Corollary 7.25. The category of abundant semigroups whose set of idempotents forms a band and good morphisms is isomorphic to the category of abundant inductive generalised categories over bands and pseudo-functors.

Proof. Let $P$ be an abundant inductive generalised category over a band $B$. Suppose that $x \in P, e \in \mathbf{d}(x)$ and $f \in \mathbf{r}(x)$. We know that $e \widetilde{\mathcal{R}}_{B} x$ in $P \mathbf{S}$, so that $e \otimes x=x$. Assume that $y, z \in P$ with $y \otimes x=z \otimes x$, giving that 
$\left(\left.y\right|_{y^{*} e}\right) \cdot\left({ }_{y^{*} e} \mid x\right)=\left(\left.z\right|_{z^{*} e}\right) \cdot\left({ }_{z^{*} e} \mid x\right)$, where $y^{*} \in \mathbf{r}(y)$ and $z^{*} \in \mathbf{r}(z)$. By (I7), we obtain that $\left.y\right|_{y^{*} e}=\left.z\right|_{z^{*} e}$. Thus, $y^{*} e \mathcal{L} z^{*} e$ in $B$. We have

$$
\begin{array}{rlrl}
y \otimes e & =\left.y\right|_{y^{*} e} \cdot y^{*} e & & \\
& =\left.z\right|_{z^{*} e} \cdot y^{*} e & & (\operatorname{by}(\mathrm{I} 6)) \\
& =\left.z\right|_{z^{*} e} & & \left(y^{*} e \mathcal{L} z^{*} e\right) \\
& =\left.z\right|_{z^{*} e} \cdot z^{*} e & \\
& =z \otimes e .
\end{array}
$$

This is enough to show that $e \mathcal{R}^{*} x$. Dually, we have that $f \mathcal{L}^{*} x$.

In view of Lemma 7.23 , we have that $E(P \mathbf{S})=B$.

Conversely, let $S$ be an abundant semigroup with $E(S)=B$. It follows that $\mathcal{R}^{*}=\widetilde{\mathcal{R}}_{B}$ and $\mathcal{L}^{*}=\widetilde{\mathcal{L}}_{B}$. In view of Lemma 7.18 , it is sufficient to claim that $S \mathbf{C}$ satisfies Conditions (I7), and dually, (I7) . Assume that $e, f, g \in B$ and $x, y, z \in P$ are such that $e, f \leq_{\mathcal{L}} g \in \mathbf{d}(x), e \in \mathbf{r}(y), f \in \mathbf{r}(z)$ and $y \cdot{ }_{e}\left|x=z \cdot{ }_{f}\right| x$. It follows that yex $=z f x$. Since $g \in \mathbf{d}(x)$, that is, $g \mathcal{R}^{*} x$ in $S$, we have that $y e g=z f g$, that is, ye $=z f$, as $e, f \leq_{\mathcal{L}} g$. Hence, $y=z$, as required. Dually, $(\mathrm{I} 7)^{\circ}$ holds.

We now discuss Ehresmann semigroups. Let $S$ be an Ehresmann semigroup with distinguished semilattice $E$. We mentioned in Lemma 2.30 that $\leq_{r}=\leq_{r}^{\prime}$ and $\leq_{l}=\leq_{l}^{\prime}$.

Let $P$ be an inductive generalised category over $E$. The context

$$
(E, E / \mathcal{R}, E / \mathcal{L}, E / \mathcal{D}, p)
$$

is essentially four copies of $E$ equipped with the identity map. We therefore identify $E$ with $E / \mathcal{R}, E / \mathcal{L}$ and $E / \mathcal{D}$ and note that $P$ becomes a category in the usual sense. Notice that as $P=P$ SC, we have that $\leq_{r}=\leq_{r}^{\prime}, \leq_{l}=\leq_{l}^{\prime}$ and $\leq_{r}$ and $\leq_{l}$ are partial orders on $P$.

Lemma 7.26. An inductive generalised category $P$ over a semilattice $E$ with $\leq_{r}$ forms an ordered 1 category with restriction.

Proof. From comments above $P$ is a category (with the appropriate identifications) and $\left(P, \leq_{r}\right)$ is a poset. 
(OC1) Suppose that $x, y \in P$ with $x \leq_{r} y$. Then there exists $e \in E$ such that $e \leq \mathbf{d}(y)$ and $x={ }_{e} \mid y$. Thus, $\mathbf{d}(x)=e \leq \mathbf{d}(y)$ and $\mathbf{r}(x) \leq \mathbf{r}(y)$ by (I1).

(OC2) Suppose that $x, y \in P$ with $\mathbf{r}(x)=\mathbf{r}(y), \mathbf{d}(x)=\mathbf{d}(y)$ and $x \leq_{r} y$. Then there exists $e \in E$ such that $e \leq \mathbf{d}(y)$ and $x={ }_{e} \mid y$. Certainly, $\mathbf{d}(x)=e$ and so $e=\mathbf{d}(y)$, whence from (I1), $x=y$.

(OC3) If $x^{\prime} \leq_{r} x$ and $y^{\prime} \leq_{r} y$, and both $x^{\prime} \cdot y^{\prime}$ and $x \cdot y$ exist, then there exist $e, f \in E$ such that $e \leq \mathbf{d}(x), f \leq \mathbf{d}(y), x^{\prime}={ }_{e} \mid x$ and $y^{\prime}={ }_{f} \mid y$. Thus, we have that $\mathbf{r}\left({ }_{e} \mid x\right)=\mathbf{r}\left(x^{\prime}\right)=\mathbf{d}\left(y^{\prime}\right)=\mathbf{d}\left({ }_{f} \mid y\right)=f$, and so $x^{\prime} \cdot y^{\prime}=\left({ }_{e} \mid x\right) \cdot\left({ }_{f} \mid y\right)={ }_{e} \mid(x \cdot y)$ by (I3). Hence, $x^{\prime} \cdot y^{\prime} \leq_{r} x \cdot y$.

Finally, we assume that $x \in P$ and $e \in E$ with $e \leq \mathbf{d}(x)$. Then $e \mid x$ is defined and $\mathbf{d}\left({ }_{e} \mid x\right)=e$. Also, ${ }_{e} \mid x \leq_{r} x$ by (I1). Further, ${ }_{e} \mid x$ is unique since if $z \leq_{r} x$ and $e=\mathbf{d}(z)$, then there exists $h \in E$ with $h \leq \mathbf{d}(x)$ and $z={ }_{h} \mid x$, which gives that $h=\mathbf{d}(z)$. Thus, $e=h$. Hence, $z={ }_{e} \mid x$.

As a dual result of Lemma 7.26, we have the following lemma.

Lemma 7.27. An inductive generalised category $P$ over a semilattice $E$ with $\leq_{l}$ forms an ordered 1 category with co-restriction.

Next we show that an inductive generalised category $P$ over a semilattice $E$ is an Ehresmann category as defined in [32] and explained in Chapter 6.

Lemma 7.28. An inductive generalised category $P$ over a semilattice $E$ with the pair of natural partial orders $\left(\leq_{r}, \leq_{l}\right)$ forms an Ehresmann category.

Conversely, an Ehresmann category $\left(C, \cdot, \leq_{r}, \leq_{l}\right)$ with semilattice of identities E, may be regarded as an inductive generalised category over $E$ with natural partial orders $\left(\leq_{r}, \leq_{l}\right)$.

Proof. Let $P$ be inductive generalised category over a semilattice $E$. In view of the above discussion, we have claimed that $P$ is a category with set of identities E. By Lemma 7.26 and Lemma 7.27, Conditions (E1) and (E1) ${ }^{\circ}$ are satisfied.

(E2) If $e, f \in E$ and $e \leq_{r} f$, then $e={ }_{e} \mid f=e f$ so that we must have $e \leq f$. Then $\left.f\right|_{e}$ is defined and $\left.f\right|_{e}=f e=e$ so that $e \leq_{l} f$. Together with the dual, we have that for $e, f \in E$,

$$
e \leq_{r} f \Leftrightarrow e \leq_{l} f \Leftrightarrow e \leq f
$$

so that in particular, (E2) holds.

(E3) Clearly, $E$ is a semilattice under $\leq_{r}=\leq_{l}=\leq$. 
(E4) To show that $\leq_{r} \circ \leq_{l} \subseteq \leq_{l} \circ \leq_{r}$, we assume that $x \leq_{r} \circ \leq_{l} y$. Then there exists $z \in P$ such that $x \leq_{r} z \leq_{l} y$. And so there exist $e, f \in E$ with $\mathbf{d}(x)=e \leq \mathbf{d}(z)=u$ and $\mathbf{r}(z)=f \leq \mathbf{r}(y)=v$, such that $x={ }_{e} \mid z$ and $z=\left.y\right|_{f}$. Thus, $x={ }_{e}\left|\left(\left.y\right|_{f}\right)={ }_{e u}\right|\left(\left.y\right|_{v f}\right)$. By (I4), we get that $x=\left.\left({ }_{e h} \mid y\right)\right|_{g f}$, where $h=\mathbf{d}(y)$ and $g=\mathbf{r}\left({ }_{e h} \mid y\right)$. Set $z^{\prime}={ }_{e h} \mid y$. Then $x \leq_{l} z^{\prime}$ and $z^{\prime} \leq_{r} y$. Consequently, $x \leq_{l} \circ \leq_{r} y$. With the dual, we obtain (E4).

(E5) Suppose that $x, y \in P$ and $f \in E$ with $x \leq_{r} y$. Then there exists $k \in E$ with $k \leq \mathbf{d}(y)$ and $x={ }_{k} \mid y$. So $\left.\left.\left.x\right|_{\mathbf{r}(x) f}={ }_{k} \mid y\right)\left.\right|_{\mathbf{r}(x) f}={ }_{k} \mathbf{d}(y) \mid y\right)\left.\right|_{\mathbf{r}(x) f}$. Let $h=\mathbf{d}\left(\left.y\right|_{\mathbf{r}(y) f}\right)$. By (I4), we obtain that $\left.{ }_{k k \mathbf{d}(y)} \mid y\right)\left.\right|_{\mathbf{r}(x) f}={ }_{k h} \mid\left(\left.y\right|_{\mathbf{r}(y) f}\right)$, so that $\left.x\right|_{\mathbf{r}(x) f} \leq\left._{r} y\right|_{\mathbf{r}(y) f}$.

Conversely, let $C=\left(C, \cdot, \leq_{r}, \leq_{l}\right)$ be an Ehresmann category with semilattice of identities $E$. Then $C=(C, \cdot)$ may also be regarded as generalised category over $E$.

We let $\leq$ denote the restriction of $\leq_{r}\left(\right.$ resp. $\left.\leq_{l}\right)$ to $E$. It is clear that the first part of (I1) holds, moreover, by uniqueness of restriction, ${ }_{e} \mid x=x$ if $e=\mathbf{d}(x)$.

For (I2), if $x \in C$ and $e, f, g, u \in E$, with $e \leq_{\mathcal{L}} g \mathcal{R} f \leq_{\mathcal{L}} u \in \mathbf{d}(x)$, then this simplifies to $e \leq f \leq \mathbf{d}(x)$. Now ${ }_{e f}\left|x={ }_{e}\right| x \leq_{r} x$ and $\mathbf{d}\left({ }_{e} \mid x\right)=e$; also, $e\left|\left({ }_{f} \mid x\right) \leq_{r}\right| x \leq_{r} x$ and $\mathbf{d}\left({ }_{e} \mid\left({ }_{f} \mid x\right)\right)=e$. By uniqueness of restriction, $e_{f}\left|x=e_{e}\right|\left({ }_{f} \mid x\right)$.

(I3) If $x, y \in C$ with $\exists x \cdot y$, then $\mathbf{r}(x)=\mathbf{d}(y)$. If $e \leq \mathbf{d}(x)$, then we have

$$
{ }_{e} \mid(x \cdot y) \leq_{r} x \cdot y \text { and } \mathbf{d}\left({ }_{e} \mid x \cdot y\right)=e
$$

and also

$$
\left({ }_{e} \mid x\right) \cdot\left({ }_{f} \mid y\right) \leq_{r} x \cdot y \text { and } \mathbf{d}\left(\left({ }_{e} \mid x\right) \cdot\left({ }_{f} \mid y\right)\right)=e,
$$

where $f=\mathbf{r}\left({ }_{e} \mid x\right)$. Hence, ${ }_{e} \mid(x \cdot y)=\left({ }_{e} \mid x\right) \cdot\left({ }_{f} \mid y\right)$.

(I4) This is clear.

(I5) Let $x \in C$ and $e, f, u, v, g, h \in E$ with $g=\mathbf{r}(x), h=\mathbf{d}(x), u=\mathbf{d}\left(\left.x\right|_{g f}\right)$ and $v=\mathbf{r}(e h \mid x)$. Then $(e \bar{\otimes} x) \bar{\otimes} f=e \bar{\otimes}(x \bar{\otimes} f)$, where $\bar{\otimes}$ is defined [32] and recalled in Chapter 6, by $x \bar{\otimes} y=\left(\left.x\right|_{k}\right) \cdot\left({ }_{k} \mid y\right)$, where $k=\mathbf{r}(x) \mathbf{d}(y)$. As shown in [32], $\bar{\otimes}$ is associative, hence, 


$$
\begin{aligned}
(e \bar{\otimes} x) \bar{\otimes} f & =\left.\left(\left(\left.e\right|_{e h}\right) \cdot\left({ }_{e h} \mid x\right)\right)\right|_{v f} \cdot\left({ }_{v f} \mid f\right) \\
& =\left.\left(e h \cdot\left({ }_{e h} \mid x\right)\right)\right|_{v f} \cdot v f \\
& =\left.(e h \mid x)\right|_{v f}
\end{aligned}
$$

and similarly, $e \bar{\otimes}(x \bar{\otimes} f)={ }_{e u} \mid\left(\left.x\right|_{g f}\right)$, so we obtain that $\left.\left(e_{e h} \mid x\right)\right|_{v f}={ }_{e u} \mid\left(\left.x\right|_{g f}\right)$.

(I6) Suppose that $e, g, h, u, v \in E$ are such that $u \leq_{\mathcal{R}} g \mathcal{L} e$ and $v \leq_{\mathcal{L}} h \mathcal{R} e$, which simplify to $u \leq e$ and $v \leq e$. Clearly, $\left.e\right|_{u}=u=e u$ and ${ }_{v} \mid e=v=v e$.

Let $\mathbf{C}=\left(C, \cdot, \leq_{r}, \leq_{l}\right)$ and $\mathbf{D}=\left(D, \cdot, \leq_{r}, \leq_{l}\right)$ be Ehresmann categories with semilattice $E_{C}$ and $E_{D}$ of identities, respectively. We recall from Chapter 6 that a strongly ordered functor [32] $F: \mathbf{C} \rightarrow \mathbf{D}$ is a functor which preserves $\leq_{r}, \leq_{l}$ and the binary operation of the semilattices. Hence $F$ is a morphism $E_{C} \rightarrow E_{D}$. As shown in [32], $F$ preserves restrictions and co-restrictions. Thus $F$ is a pseudofunctor in the sense of Definition 7.5.

On the other hand, if $G: \mathbf{C} \rightarrow \mathbf{D}$ is a pseudo-functor, then from the comments following Definition 7.5, $G$ is a functor, which by (F1) preserves $\wedge$. Suppose now that $x, y \in \mathbf{C}$ with $x \leq_{r} y$. Then $x={ }_{e} \mid y$ for some $e \in E$, and so by (F2), $x G=_{e G} \mid y G$ so that $x G \leq_{r} y G$. Dually, $G$ preserves $\leq_{l}$, so that $G$ is a strongly ordered functor. Theorem 7.22, Lemma 7.18 and the comments above now give us Lawson's result from [32], Theorem D.

Corollary 7.29. [6, Theorem 4.24] The category of Ehresmann semigroups and admissible morphisms is isomorphic to the category of Ehresmann categories and strongly ordered functors.

We now turn to weakly $B$-superabundant semigroups with (C), which are weakly $B$-orthodox semigroups such that each $\widetilde{\mathcal{H}}_{B}$-class contains a distinguished idempotent in $B$. We say that a generalised category $P$ over a band $B$ is a super-generalised category if it is an inductive generalised category and satisfies the following condition:

(I8) if $x \in P, e \in \mathbf{d}(x)$ and $f \in \mathbf{r}(x)$, then $e \mathcal{D} f$.

Corollary 7.30. The category of weakly B-superabundant semigroups with $(C)$ and admissible morphisms is isomorphic to the category of super-generalised categories over $B$ and pseudo-functors. 
Proof. Let $S$ be a weakly $B$-superabundant semigroup with (C). It follows from Lemma 7.18 that it is only necessary to show that $S \mathbf{C}$ satisfies Condition (I8). Suppose that $x \in S, e \in \mathbf{d}(x)$ and $f \in \mathbf{r}(x)$. Then $e \widetilde{\mathcal{R}}_{B} x \widetilde{\mathcal{L}}_{B} f$ in $S$. As $S$ is a weakly $B$-superabundant semigroup, it follows that there exists $h \in B$ such that $h \widetilde{\mathcal{H}}_{B} x$. Thus, $e \mathcal{R} h \mathcal{L} f$, which implies that $e \mathcal{D} f$.

Conversely, let $P$ be a super-generalised category over $B$. It is sufficient to show that $P \mathbf{S}$ is weakly $B$-superabundant. Suppose that $x \in P, e \in \mathbf{d}(x)$ and $f \in \mathbf{r}(x)$. Then by (I8), e $\mathcal{D} f$, that is, e $\mathcal{R}$ ef $\mathcal{L} f$. As $e \widetilde{\mathcal{R}}_{B} x \widetilde{\mathcal{L}}_{B} f$ in $P \mathbf{S}$, we get that $x \widetilde{\mathcal{H}}_{B}$ ef. Hence $P \mathbf{S}$ is a weakly $B$-superabundant semigroup with (C).

Finally in this chapter, we discuss the class of weakly $B$-orthodox semigroups which have Condition (WIC) mentioned in Chapter 2. We define an inductive generalised category $P$ over a band $B$ to be connected if it satisfies the following condition and its dual (I9) :

(I9) if $x \in P$ and $e \leq u \in \mathbf{d}(x)$ then there exists $f \leq v \in \mathbf{r}(x)$ such that ${ }_{e}|x=x|_{f}$.

Corollary 7.31. The category of weakly B-orthodox semigroups with (WIC) and admissible morphisms is isomorphic to the category of connected inductive generalised categories over bands and pseudo-functors.

Proof. Let $S$ be a weakly $B$-orthodox semigroup with (WIC). In view of Lemma 7.18, it remains to show that $S \mathbf{C}$ satisfies Conditions (I9) and (I9) ${ }^{\circ}$. We will show that (I9) holds, dually, (I9) ${ }^{\circ}$ holds. Suppose that $x \in S$ and $e \leq u \in \mathbf{d}(x)$. Then ${ }_{e} \mid x=e x$. Since $S$ has (WIC), it follows that there exists $f \in B$ such that $e x=x f$. Then $e x=x v f v$, where $v \in \mathbf{r}(x)$. Thus, $e x=x v f v=\left.x\right|_{v f v}$.

Conversely, let $P$ be a connected inductive generalised category over a band $B$. Suppose that $x \in P$ and $e \leq u \in \mathbf{d}(x)$. Then it follows from (I9) that there exists $f \leq v \in \mathbf{r}(x)$ such that ${ }_{e}|x=x|_{f}$. Thus $e \otimes x={ }_{e}|x=x|_{f}=x \otimes f$. Together with the dual argument, we have shown that $P \mathbf{S}$ has (WIC). 


\section{Chapter 8}

\section{Trace of weakly $B$-orthodox semigroups}

A weakly $B$-orthodox semigroup $S$ with zero is primitive if for any $e, f \in B$, $e \leq f$ implies that $e=0$ or $e=f$. Here $e \leq f$ if and only if $e f=f e=e$. The purpose of this chapter is to show that the trace of a weakly $B$-orthodox semigroup is a primitive weakly $B$-orthodox semigroup and investigate primitive weakly $B$-orthodox semigroups via blocked Rees matrix semigroups, which are introduced in [10].

\subsection{Preliminaries}

In this section our aim is to list some properties of primitive weakly $B$-orthodox semigroups. Throughout this section we use $S$ to denote a primitive weakly $B$ orthodox semigroup, unless stated otherwise. Green's relations will always refer to $B$. For any subset $T$ of a semigroup $S$, we will use $T^{*}$ to denote the set of non-zero elements of $T$.

Lemma 8.1. Let $S$ be a weakly $U$-abundant semigroup with zero and $x, y$ be nonzero elements in $S$ with $x \widetilde{\mathcal{R}}_{U}$ e and $y \widetilde{\mathcal{L}}_{U} f$, where $e, f \in U$. Then $y x=0$ if and only if $f e=0$.

Proof. From $x \widetilde{\mathcal{R}}_{U} e$, we have $y x \widetilde{\mathcal{R}}_{U} y e$, and so if $y x=0$, then $y e=0$. Again, by y $\widetilde{\mathcal{L}}_{U} f$, we have ye $\widetilde{\mathcal{L}}_{U} f e$, and so $f e=0$.

Conversely, if $f e=0$ then $y x=y$ fex $=0$. 
Lemma 8.2. If $e, f$ are distinguished idempotents in $S$ and $e S \subseteq f S$ (resp. Se $\subseteq$ $S f)$, then $e S=f S($ resp. $S e=S f)$ or $e=0$.

Proof. Suppose that $e, f \in B$ and $e S \subseteq f S$. Then $e \in f S$, and so $f e=e$, which implies that $e \mathcal{R}$ ef $\leq f$. Since $S$ is primitive, we obtain that ef $=0$ or ef $=f$. In the former case, we have that $e=e e=e f e=0$, and in the latter case, we obtain that $e \mathcal{R} f$, that is, $e S=f S$.

Lemma 8.3. For any $e \in B^{*}$ and $a \in S^{*}$, a $\widetilde{\mathcal{L}}_{B} e$ (resp. a $\left.\widetilde{\mathcal{R}}_{B} e\right)$ if and only if $a \in S e$ (resp. $a \in e S$ ) and Se (resp. eS) is contained in every distinguished idempotent-generated left (resp. right) ideal containing a.

Proof. Suppose that $a \widetilde{\mathcal{L}}_{B}$ e. By Lemma 2.6, $\widetilde{L}(a)=\widetilde{L}(e)$. As $\widetilde{L}(e)=S e, a \in S e$. If $a \in S f$ for some $f \in B$, then $a f=a$, and so $e f=e$ giving $S e \subseteq S f$.

Conversely, suppose that $a \in S e$ and that for any $f \in B, a \in S f$ implies that $S e \subseteq S f$. Then $\widetilde{L}(a) \subseteq S e$ since $S e$ is a left $B$-admissible ideal containing a. As $S$ is weakly $B$-orthodox, it follows from Lemma 2.7 that $\widetilde{L}(a)=S f$ for some $f \in B$. Hence, by Lemma $8.2, S e=S f$, and so a $\widetilde{\mathcal{L}}_{B} e$.

The next lemma is an immediate consequence of Lemma 8.2 and Lemma 8.3.

Lemma 8.4. Let $a \in S$ and $e \in B^{*}$. Then a $\widetilde{\mathcal{L}}_{B}$ e (resp. a $\widetilde{\mathcal{R}}_{B}$ e) if and only if $a \neq 0$ and $a \in S e(r e s p . a \in e S)$.

Lemma 8.5. If $x, y$ are non-zero elements in $S$ such that $x y \neq 0$, then $x y \in$ $\widetilde{R}_{x} \cap \widetilde{L}_{y}$.

Proof. Suppose that $e$ and $f$ are distinguished idempotents such that $e \widetilde{\mathcal{R}}_{B} x$ and $f \widetilde{\mathcal{L}}_{B} y$. Then by Lemma 2.7, $x \in e S$ and $y \in S f$. Thus, $x y \in e S \cap S f$. Again by Lemma 8.4, $x y \in \widetilde{R}_{e} \cap \widetilde{L}_{f}$, that is, $x y \in \widetilde{R}_{x} \cap \widetilde{L}_{y}$.

\subsection{Blocked Rees matrix semigroups}

As a tool for the following sections we recall some concepts and results from [10] about blocked Rees matrix semigroups and their structure. Blocked Rees matrix semigroups [10] are a generalisation of Rees matrix semigroups over a monoid. We refer the reader to [10] for more details. 
Let $I, \Lambda$ and $\Gamma$ be non-empty sets. Suppose that $\Gamma$ indexes partitions of $\Lambda$ and $I$ as follows:

$$
P(\Lambda)=\left\{\Lambda_{\alpha}: \alpha \in \Gamma\right\}, \quad P(I)=\left\{I_{\beta}: \beta \in \Gamma\right\}
$$

For convenience, $i, j, k, h$ will denote members of $I ; \lambda, \mu, \nu, \rho$ will denote members of $\Lambda$, and $\alpha, \beta, \gamma, \delta$ will denote members of $\Gamma$.

We recall from [12] that a non-empty set $M$ is a partial semigroup if there is a partial binary operation on $M$ such that for all $a, b, c \in M,(a b) c$ is defined if and only if $a(b c)$ is defined, and if $(a b) c$ is defined, then $(a b) c=a(b c)$.

Let $M=\bigcup\left\{M_{\alpha \beta}: \alpha, \beta \in \Gamma\right\}$ be a partial semigroup such that for $x, y \in M$, $x y$ is defined if and only if $x \in M_{\alpha \beta}, y \in M_{\beta \gamma}$ for some $\alpha, \beta, \gamma \in \Gamma$ and then in this case, $x y \in M_{\alpha \gamma}$. Suppose also that for all $\alpha \in \Gamma, M_{\alpha \alpha}=T_{\alpha}$ is a monoid with identity $g_{\alpha}$ and for all $\alpha, \beta \in \Gamma, M_{\alpha \beta}=\emptyset$ or is a $\left(T_{\alpha}, T_{\beta}\right)$-bisystem, that is, $T_{\alpha}$ acts on $M_{\alpha \beta}$ on the left, $T_{\beta}$ acts on $M_{\alpha \beta}$ on the right and $(t m) t^{\prime}=t\left(m t^{\prime}\right)$ for all $t \in T_{\alpha}, m \in M_{\alpha \beta}, t^{\prime} \in T_{\beta}$.

We remark that if for any $\alpha, \beta \in \Gamma, M_{\alpha \beta}$ is regarded as the set of morphisms from $\alpha$ to $\beta$, then $M$ forms a category with set of objects $\Gamma$ and set of morphisms $M$.

Let 0 (zero) be a symbol not in any $M_{\alpha \beta}$ and let $P=\left(p_{\lambda i}\right)$ be a $\Lambda \times I$ matrix over $M \cup\{0\}$, where for $\lambda \in \Lambda_{\alpha}, i \in I_{\beta}, p_{\lambda i} \in M_{\alpha \beta} \cup\{0\}$.

Let

$$
\begin{aligned}
\mathcal{M}^{0} & =\mathcal{M}^{0}(M ; I, \Lambda, \Gamma ; P) \\
& =\left\{(i, a, \lambda): a \in M_{\alpha \beta},(i, \lambda) \in I_{\alpha} \times \Lambda_{\beta},(\alpha, \beta) \in \Gamma \times \Gamma\right\} \cup\{0\} .
\end{aligned}
$$

In order to be able to define a multiplication on $\mathcal{M}^{0}(M ; I, \Lambda, \Gamma ; P)$, we say that $0 x=x 0=0$ for every element $x$ of $\mathcal{M}^{0}$. Now we define a product on non-zero elements of $\mathcal{M}^{0}(M ; I, \Lambda, \Gamma ; P)$ by the rule that for any $(i, a, \lambda),(j, b, \mu) \in \mathcal{M}^{0}$,

$$
(i, a, \lambda)(j, b, \mu)= \begin{cases}\left(i, a p_{\lambda j} b, \mu\right) & \text { if } p_{\lambda j} \neq 0 \\ 0 & \text { if } p_{\lambda j}=0\end{cases}
$$

It is routine to show that this product is associative and is categorical at zero, and so we obtain a semigroup called a blocked Rees matrix semigroup. A blocked Rees 
matrix semigroup $\mathcal{M}^{0}=\mathcal{M}^{0}(M ; I, \Lambda, \Gamma ; P)$ is called a weakly orthodox blocked Rees matrix semigroup or WO-B Rees matrix semigroup if the following condition holds:

(B) all non-zero entries of $P$ are in the diagonal blocks, and if $(i, \lambda) \in I_{\alpha} \times \Lambda_{\alpha}$ then $p_{\lambda i}$ is the identity $g_{\alpha}$ of $T_{\alpha}$.

Proposition 8.6. Let $\mathcal{M}^{0}=\mathcal{M}^{0}(M ; I, \Lambda, \Gamma ; P)$ be a WO-B Rees matrix semigroup and $B=\left\{\left(i, g_{\alpha}, \lambda\right): \alpha \in \Gamma,(i, \lambda) \in I_{\alpha} \times \Lambda_{\alpha}\right\} \cup\{0\}$. Then

(i) for any $\left(i, g_{\alpha}, \lambda\right) \in B,\left(i, g_{\alpha}, \lambda\right)$ is an idempotent;

(ii) the set $B$ forms a band;

(iii) for any $\left(i, g_{\alpha}, \lambda\right) \in B \backslash\{0\},\left(i, g_{\alpha}, \lambda\right)$ is primitive in $B$;

(iv) for any non-zero elements $(i, a, \lambda),(j, b, \mu) \in \mathcal{M}^{0},(i, a, \lambda) \widetilde{\mathcal{R}}_{B}(j, b, \mu)$ if and only if $i=j$;

(v) for any non-zero elements $(i, a, \lambda),(j, b, \mu) \in \mathcal{M}^{0},(i, a, \lambda) \widetilde{\mathcal{L}}_{B}(j, b, \mu)$ if and only if $\lambda=\mu$.

Proof. ( $i)$ If $\left(i, g_{\alpha}, \lambda\right) \in B$, then we have

$$
\begin{aligned}
\left(i, g_{\alpha}, \lambda\right)\left(i, g_{\alpha}, \lambda\right) & =\left(i, g_{\alpha} p_{\lambda i} g_{\alpha}, \lambda\right) \\
& =\left(i, g_{\alpha} g_{\alpha} g_{\alpha}, \lambda\right) \quad\left(\text { since }(i, \lambda) \in I_{\alpha} \times \Lambda_{\alpha}, p_{\lambda i}=g_{\alpha}\right) \\
& =\left(i, g_{\alpha}, \lambda\right) .
\end{aligned}
$$

(ii) Suppose that $\left(i, g_{\alpha}, \lambda\right),\left(j, g_{\beta}, \mu\right) \in B \backslash\{0\}$. Then

$$
\left(i, g_{\alpha}, \lambda\right)\left(j, g_{\beta}, \mu\right)=\left(i, g_{\alpha} p_{\lambda j} g_{\beta}, \mu\right)
$$

If $\alpha=\beta$, then $p_{\lambda j}=g_{\alpha}=g_{\beta}$, and so $\left(i, g_{\alpha}, \lambda\right)\left(i, g_{\beta}, \mu\right)=\left(i, g_{\alpha}, \mu\right) \in B$. If $\alpha \neq \beta$, then $p_{\lambda j}=0$, and so $\left(i, g_{\alpha}, \lambda\right)\left(j, g_{\beta}, \mu\right)=0 \in B$. Hence, $B$ is closed. Again by $(i), B$ forms a band.

(iii) Suppose that $\left(i, g_{\alpha}, \lambda\right),\left(j, g_{\beta}, \mu\right) \in B \backslash\{0\}$ are such that $\left(i, g_{\alpha}, \lambda\right) \leq$ $\left(j, g_{\beta}, \mu\right)$. Then

$$
\left(i, g_{\alpha}, \lambda\right)\left(j, g_{\beta}, \mu\right)=\left(i, g_{\alpha}, \lambda\right)=\left(j, g_{\beta}, \mu\right)\left(i, g_{\alpha}, \lambda\right)
$$

which implies that $i=j$ and $\lambda=\mu$, and so $\alpha=\beta$ so that $g_{\alpha}=g_{\beta}$. Hence $\left(i, g_{\alpha}, \lambda\right)=\left(j, g_{\beta}, \mu\right)$. 
(iv) Suppose that $(i, a, \lambda) \in \mathcal{M}^{0} \backslash\{0\}$. Then there exist $\alpha, \beta \in \Gamma$ such that $(i, \lambda) \in I_{\alpha} \times \Lambda_{\beta}$ and $a \in M_{\alpha \beta}$. We now claim that for any $\nu \in \Lambda_{\alpha}$ and $\left(i, g_{\alpha}, \nu\right) \in B,\left(i, g_{\alpha}, \nu\right) \widetilde{\mathcal{R}}_{B}(i, a, \lambda)$. Clearly,

$$
\begin{aligned}
\left(i, g_{\alpha}, \nu\right)(i, a, \lambda)=\left(i, g_{\alpha} p_{\nu i} a, \lambda\right) & =\left(i, g_{\alpha} g_{\alpha} a, \lambda\right) \quad\left((i, \nu) \in I_{\alpha} \times \Lambda_{\alpha}, p_{\nu i}=g_{\alpha}\right) \\
& =\left(i, g_{\alpha} a, \lambda\right)=(i, a, \lambda) .
\end{aligned}
$$

Let $\gamma \in \Gamma,(k, \rho) \in I_{\gamma} \times \Lambda_{\gamma}$ and $\left(k, g_{\gamma}, \rho\right) \in B$ be such that

$$
\left(k, g_{\gamma}, \rho\right)(i, a, \lambda)=(i, a, \lambda)
$$

Then $k=i$, and so $\alpha=\gamma$. Thus,

$$
\begin{aligned}
\left(k, g_{\gamma}, \rho\right)\left(i, g_{\alpha}, \nu\right) & =\left(k, g_{\gamma} p_{\rho i} g_{\alpha}, \nu\right) \\
& =\left(i, g_{\alpha} g_{\alpha} g_{\alpha}, \nu\right) \quad(\alpha=\gamma, k=i) \\
& =\left(i, g_{\alpha}, \nu\right) .
\end{aligned}
$$

Hence $\left(i, g_{\alpha}, \nu\right) \widetilde{\mathcal{R}}_{B}(i, a, \lambda)$.

Let $\eta, \delta \in \Gamma$ and $(j, \mu) \in I_{\eta} \times \Lambda_{\delta}$. If $(j, b, \mu) \in \mathcal{M}^{0} \backslash\{0\}$, then

$$
(j, b, \mu) \widetilde{\mathcal{R}}_{B}\left(j, g_{\eta}, \sigma\right)
$$

where $\sigma \in \Lambda_{\eta}$ and $\left(j, g_{\eta}, \sigma\right) \in B$. So

$$
\begin{aligned}
(i, a, \lambda) \widetilde{\mathcal{R}}_{B}(j, b, \mu) & \Leftrightarrow\left(i, g_{\alpha}, \nu\right) \widetilde{\mathcal{R}}_{B}\left(j, g_{\eta}, \sigma\right) \\
& \Leftrightarrow\left(i, g_{\alpha}, \nu\right) \mathcal{R}\left(j, g_{\eta}, \sigma\right) \\
& \Leftrightarrow i=j .
\end{aligned}
$$

(v) It is the dual proof of $(i v)$.

The following lemma is an immediate consequence of Proposition 8.6.

Lemma 8.7. Let $\mathcal{M}^{0}=\mathcal{M}^{0}(M ; I, \Lambda, \Gamma ; P)$ be a WO-B Rees matrix semigroup and $B=\left\{\left(g_{\alpha}\right)_{i \lambda}: \alpha \in \Gamma,(i, \lambda) \in I_{\alpha} \times \Lambda_{\alpha}\right\} \cup\{0\}$. Then $\mathcal{M}^{0}$ is a primitive weakly $B$-orthodox semigroup.

Proof. In view of $(i v)$ and $(v)$ of Proposition 8.6, it is easy to see that $\mathcal{M}^{0}$ satisfies the Congruence Condition $(\mathrm{C})$. 


\subsection{Primitive weakly $B$-orthodox semigroups}

Throughout this section $S$ denotes a given fixed primitive weakly $B$-orthodox semigroup with zero. Our aim, achieved in Theorem 8.13, is to show that $S$ is isomorphic to a WO-B Rees matrix semigroup.

Lemma 8.8. Let $e \in B^{*}$ and let

$$
\begin{aligned}
& X=\bigcup\left\{\widetilde{L}_{f}: f \in B^{*} \text { and } f \mathcal{D} e\right\} \\
& Y=\bigcup\left\{\widetilde{R}_{g}: g \in B^{*} \text { and } g \mathcal{D} e\right\}
\end{aligned}
$$

and $Z=X \cap Y$. Then

(i) for any $a, b \in Z,(a, b) \in \widetilde{\mathcal{L}}_{B} \circ \widetilde{\mathcal{R}}_{B}$ and $(a, b) \in \widetilde{\mathcal{R}}_{B} \circ \widetilde{\mathcal{L}}_{B}$;

(ii) if $a \in \widetilde{L}_{f} \cap \widetilde{R}_{g} \subseteq Z$, then $g f \in \widetilde{H}_{a} \cap B^{*}$ is such that $\rho_{f}: \widetilde{H}_{g} \rightarrow \widetilde{H}_{a}$, given by $x \rho_{f}=x f$ for any $x \in \widetilde{H}_{g}$, and $\lambda_{g}: \widetilde{H}_{f} \rightarrow \widetilde{H}_{a}$, given by $y \lambda_{g}=$ gy for any $y \in \widetilde{H}_{f}$, are bijective;

(iii) for any $a, b \in Z,\left|\widetilde{H}_{a}\right|=\left|\widetilde{H}_{b}\right|$;

(iv) if $g, h \in B^{*}$ and $\widetilde{H}_{g}$ and $\widetilde{H}_{h}$ contained in $Z$, then $\widetilde{H}_{g}$ and $\widetilde{H}_{h}$ are isomorphic monoids.

Proof. (i) Suppose that $a, b \in Z$. Then there exist $g, f \in B^{*}$ such that $a \widetilde{\mathcal{L}}_{B} f$, $b \widetilde{\mathcal{R}}_{B} g$ and $g \mathcal{D} f \mathcal{D}$ e. Since $B$ is a band, we have $f \mathcal{L} g f \mathcal{R} g$, and so a $\widetilde{\mathcal{L}}_{B}$ gf $\widetilde{\mathcal{R}}_{B} b$, that is, $(a, b) \in \widetilde{\mathcal{L}}_{B} \circ \widetilde{\mathcal{R}}_{B}$. Similarly, we have $(a, b) \in \widetilde{\mathcal{R}}_{B} \circ \widetilde{\mathcal{L}}_{B}$.

(ii) Let $a \in \widetilde{L}_{f} \cap \widetilde{R}_{g} \subseteq Z$. Then $f \mathcal{D}$ e $\mathcal{D} g$, and so $g f \in B^{*}$ and $f \mathcal{L} g f \mathcal{R} g$ so that $g f \widetilde{\mathcal{H}}_{B} a$. We now show that $\rho_{f}: \widetilde{H}_{g} \rightarrow \widetilde{H}_{a}$ is a well-defined bijection. For any $x \in \widetilde{H}_{g}$, we have

$$
x \rho_{f}=x f \widetilde{\mathcal{L}}_{B} \text { gf } \widetilde{\mathcal{H}}_{B} a
$$

and

$$
x \rho_{f}=x f=x g f \mathcal{R} x g=x \widetilde{\mathcal{R}}_{B} g \widetilde{\mathcal{R}}_{B} a,
$$

and so $x \rho_{f} \in \widetilde{H}_{a}$ so that $\rho_{f}$ is well-defined.

It is easy to see that $\rho_{f}$ is injective. Since if $x_{1}, x_{2} \in \widetilde{H}_{g}$ and $x_{1} \rho_{f}=x_{2} \rho_{f}$, 
then $x_{1} f=x_{2} f$. We have

$$
\begin{aligned}
x_{1}=x_{1} g & =x_{1} g f g \quad(f \mathcal{D} g) \\
& =x_{1} f g=x_{2} f g=x_{2} g f g=x_{2} g=x_{2} .
\end{aligned}
$$

To show $\rho_{f}$ is surjective, we suppose that $z \in \widetilde{H}_{a}=\widetilde{L}_{f} \cap \widetilde{R}_{g}$. Then we have

$$
z g=z f g \mathcal{R} z f=z \widetilde{\mathcal{R}}_{B} g \quad(f g \mathcal{R} f)
$$

and

$$
z g \widetilde{\mathcal{L}}_{B} f g \mathcal{L} g
$$

and so $z g \in \widetilde{H}_{g}$. Also, $z g \rho_{f}=z g f=z$ as $g f \mathcal{R} g \widetilde{\mathcal{R}}_{B} z$. Thus, $\rho_{f}$ is a bijection.

By a similar argument, we show that $\lambda_{g}: \widetilde{H}_{f} \rightarrow \widetilde{H}_{a}$ is a well-defined bijection.

(iii) Suppose that $a, b \in Z$. By $(i)$, there exists $c \in Z$ such that $a \widetilde{\mathcal{L}}_{B} c \widetilde{\mathcal{R}}_{B} b$. Also, there exists $f, g \in B^{*}$ such that $f \mathcal{D}$ e $\mathcal{D} g$ and $c \in \widetilde{L}_{f} \cap \widetilde{R}_{g}$. By $(i i)$, we have

$$
\left|\widetilde{H}_{a}\right|=\left|\widetilde{H}_{f}\right|=\left|\widetilde{H}_{c}\right|=\left|\widetilde{H}_{g}\right|=\left|\widetilde{H}_{b}\right| .
$$

(iv) Suppose that $g, h \in B^{*}$ are such that $\widetilde{H}_{g}$ and $\widetilde{H}_{h}$ are contained in $Z$. Then by $(i)$, there exists $a \in Z$ such that $g \widetilde{\mathcal{L}}_{B} a \widetilde{\mathcal{R}}_{B} h$. By $(i i), \rho_{g}: \widetilde{H}_{h} \rightarrow \widetilde{H}_{a}$ and $\lambda_{h}: \widetilde{H}_{g} \rightarrow \widetilde{H}_{a}$ are bijective. Using the same method as $(i i)$, we can show that $\lambda_{g}: \widetilde{H}_{a} \rightarrow \widetilde{H}_{g}$ is the inverse of $\lambda_{h}$. Then $\rho_{g} \lambda_{g}: \widetilde{H}_{h} \rightarrow \widetilde{H}_{g}$ is a bijection. By Lemma 3.5, $\widetilde{H}_{h}$ and $\widetilde{H}_{g}$ are monoids. If $x_{1}, x_{2} \in \widetilde{H}_{h}$, then

$$
\begin{aligned}
\left(x_{1} x_{2}\right) \rho_{g} \lambda_{g}=g x_{1} x_{2} g & =g x_{1} h x_{2} g \quad\left(h \widetilde{\mathcal{R}}_{B} x_{2}\right) \\
& =g x_{1} h g g h x_{2} g \quad(h \mathcal{D} g) \\
& =g x_{1} g g x_{2} g=\left(x_{1} \rho_{g} \lambda_{g}\right)\left(x_{2} \rho_{g} \lambda_{g}\right) .
\end{aligned}
$$

Hence $\rho_{g} \lambda_{g}$ is an isomorphism, and so $\widetilde{H}_{h}$ is isomorphic to $\widetilde{H}_{g}$.

We pause to make a short comment on Lemma 8.8. If $e, f \in B^{*}$ with $e \mathcal{D} f$, then by $(i v), \rho_{f} \lambda_{f}$ is an isomorphism from $\widetilde{H}_{e}$ onto $\widetilde{H}_{f}$. It is easy to see that $Z$ is a union of $\widetilde{\mathcal{H}}_{B}$-classes and due to part $(i), Z$ can be depicted by an egg-box picture. 
Lemma 8.9. If $e, f \in B^{*}$ and $\widetilde{H}=\widetilde{R}_{e} \cap \widetilde{L}_{f} \neq \emptyset$, then $\widetilde{H}$ is an $\left(\widetilde{H}_{e}, \widetilde{H}_{f}\right)$-bisystem with respect to the multiplication in $S$.

Proof. Suppose that $x \in \widetilde{H}=\widetilde{R}_{e} \cap \widetilde{L}_{f}$ and $t \in \widetilde{H}_{e}$. Then

$$
t x \widetilde{\mathcal{R}}_{B} t e=t \widetilde{\mathcal{R}}_{B} e
$$

and

$$
t x \widetilde{\mathcal{L}}_{B} e x=x \widetilde{\mathcal{L}}_{B} f
$$

and so $t x \in \widetilde{H}$. Dually, we show that for any $u \in \widetilde{H}_{f}, x u \in \widetilde{H}$. Since the multiplication in $S$ is associative, we have $t(x u)=(t x) u$. Thus, $\widetilde{H}$ is an $\left(\widetilde{H}_{e}, \widetilde{H}_{f}\right)$ bisystem.

Lemma 8.10. Suppose that $e, f \in B^{*}$ are such that e $\mathcal{D} f$. If $a \in \widetilde{L}_{e}$ (resp. $\widetilde{R}_{e}$ ), then $\widetilde{L}_{f} \cap \widetilde{R}_{a} \neq \emptyset$ (resp. $\widetilde{R}_{f} \cap \widetilde{L}_{a} \neq \emptyset$ ) and there exists a bijection $\theta: \widetilde{H}_{a} \rightarrow \widetilde{L}_{f} \cap \widetilde{R}_{a}$ (resp. $\left.\theta: \widetilde{H}_{a} \rightarrow \widetilde{R}_{f} \cap \widetilde{L}_{a}\right)$ such that $(x t) \theta=(x \theta)\left(t \rho_{f} \lambda_{f}\right)\left(\right.$ resp. $\left.(t x) \theta=\left(t \rho_{f} \lambda_{f}\right) x \theta\right)$, where $x \in \widetilde{H}_{a}, t \in \widetilde{H}_{e}$. In addition, if $a \in \widetilde{R}_{g}$ (resp. $\widetilde{L}_{g}$ ) for some $g \in B^{*}$, then $(r x) \theta=r(x \theta)($ resp. $(x r) \theta=(x \theta) r)$ for all $x \in \widetilde{H}_{a}, r \in \widetilde{H}_{g}$.

Proof. If $e \mathcal{D} f$ in $B^{*}$ and $a \in \widetilde{L}_{e}$, then $f \mathcal{L}$ ef $\mathcal{R} e$, and so af $=a$ ef $\widetilde{\mathcal{R}}_{B} a e=a$ and $a f=$ aef $\widetilde{\mathcal{L}}_{B}$ eef $=$ ef $\mathcal{L} f$ so that $a f \in \widetilde{L}_{f} \cap \widetilde{R}_{a}$. Thus $\widetilde{L}_{f} \cap \widetilde{R}_{a} \neq \emptyset$.

We now claim that $\rho_{f}: \widetilde{H}_{a} \rightarrow \widetilde{L}_{f} \cap \widetilde{R}_{a}$, given by $x \rho_{f}=x f$ for all $x \in \widetilde{H}_{a}$, is a bijection. Since if $x \in \widetilde{H}_{a}$, then $x \in \widetilde{L}_{e}$, and so by the above statement, we have $x \rho_{f} \in \widetilde{L}_{f} \cap \widetilde{R}_{a}$.

To see that $\rho_{f}$ is injective, we suppose that $x_{1}, x_{2} \in \widetilde{H}_{a}$ and $x_{1} \rho_{f}=x_{2} \rho_{f}$. Then $x_{1} f=x_{2} f$, and so

$$
\begin{aligned}
x_{1}=x_{1} e & =x_{1} \text { efe } \quad(e \mathcal{D} f) \\
& =x_{1} f e \quad\left(x_{1} \in \widetilde{L}_{e}\right) \\
& =x_{2} f e=x_{2} \text { efe }=x_{2} e=x_{2}
\end{aligned}
$$

so that $\rho_{f}$ is injective.

For any $y \in \widetilde{L}_{f} \cap \widetilde{R}_{a}$, we have $y e=y f e \widetilde{\mathcal{R}}_{B} y f=y \widetilde{\mathcal{R}}_{B} a$ and $y e \widetilde{\mathcal{L}}_{B}$ fe $\mathcal{L}$ e $\widetilde{\mathcal{L}}_{B} a$, and so ye $\in \widetilde{H}_{a}$. Also, $(y e) \rho_{f}=y e f=y f e f=y f=y$. Thus, $\rho_{f}$ is surjective. 
Let $x \in \widetilde{H}_{a}$ and $t \in \widetilde{H}_{e}$. By Lemma 8.9, we have $x t \in \widetilde{H}_{a}$. We also have that

$$
\begin{array}{rlrl}
(x t) \rho_{f}=x t f & =x e t f & & \left(x \in \widetilde{L}_{e}\right) \\
& =x e f e t f \quad & (e \mathcal{D} f) \\
& =x f t f \quad\left(x \in \widetilde{L}_{e}, t \in \widetilde{H}_{e}\right) \\
& =\left(x \rho_{f}\right)\left(t \rho_{f} \lambda_{f}\right) .
\end{array}
$$

Finally, if $g \in B^{*}$ and $a \in \widetilde{R}_{g}$, then $\widetilde{H}_{a}$ and $\widetilde{L}_{f} \cap \widetilde{R}_{a}$ are both left $\widetilde{H}_{g}$-systems and for $x \in \widetilde{H}_{a}, r \in \widetilde{H}_{g}$ we have $(r x) \rho_{f}=r x f=r\left(x \rho_{f}\right)$.

Let $I$ (resp. $\Lambda$ ) index the non-zero $\widetilde{\mathcal{R}}_{B}$ (resp. $\widetilde{\mathcal{L}}_{B}$ )-classes of $S$. For $i \in I$ and $\lambda \in \Lambda$, we will denote the intersection of $\widetilde{R}_{i}$ and $\widetilde{L}_{\lambda}$ by $\widetilde{H}_{i \lambda}$. Then

$$
S \backslash\{0\}=\bigcup\left\{\widetilde{H}_{i \lambda}:(i, \lambda) \in I \times \Lambda\right\} .
$$

Let $\Gamma$ index the $\mathcal{D}$-classes of $B$. For each $\alpha \in \Gamma$, we define

$$
I_{\alpha}=\left\{i \in I: D_{\alpha} \cap \widetilde{R}_{i} \neq \emptyset\right\}
$$

and

$$
\Lambda_{\alpha}=\left\{\lambda \in \Lambda: D_{\alpha} \cap \widetilde{L}_{\lambda} \neq \emptyset\right\} .
$$

We remark that for any $i \in I$, there exists a unique $\alpha \in \Gamma$ such that $i \in I_{\alpha}$. Since each $\widetilde{\mathcal{R}}_{B}$-class of $S$ contains at least one idempotent lying in $B$ and $\Gamma$ indexes the $\mathcal{D}$-classes of $B$. Dually, for any $\lambda \in \Lambda$, there exists a unique $\alpha \in \Gamma$ such that $\lambda \in \Lambda_{\alpha}$. Thus, if $\alpha, \beta \in \Gamma$ with $\alpha \neq \beta$, then $I_{\alpha} \cap I_{\beta}=\emptyset$ and $\Lambda_{\alpha} \cap \Lambda_{\beta}=\emptyset$. Hence, $\Gamma$ indexes partitions of $I$ and $\Lambda$ as follows:

$$
P(I)=\left\{I_{\alpha}: \alpha \in \Gamma\right\} \text { and } P(\Lambda)=\left\{\Lambda_{\alpha}: \alpha \in \Gamma\right\} .
$$

Obviously, if an $\widetilde{\mathcal{H}}_{B}$-class $\widetilde{H}$ contains a distinguished idempotent $e$, then there exists $\alpha \in \Gamma$ and $(i, \lambda) \in I_{\alpha} \times \Lambda_{\alpha}$ such that $\widetilde{H}=\widetilde{R}_{i} \cap \widetilde{L}_{\lambda}$. Conversely, each $\widetilde{\mathcal{R}}_{B}$-class and each $\widetilde{\mathcal{L}}_{B}$-class contains a distinguished idempotent, so for each $\alpha \in \Gamma$ and $e \in D_{\alpha}$, we can choose a pair $(i(\alpha), \lambda(\alpha)) \in I_{\alpha} \times \Lambda_{\alpha}$ such that $\widetilde{H}_{i(\alpha) \lambda(\alpha)}=\widetilde{R}_{i(\alpha)} \cap \widetilde{L}_{\lambda(\alpha)}$ is an $\widetilde{\mathcal{H}}_{B}$-class containing $e$. By Lemma 3.5, $\widetilde{H}_{i(\alpha) \lambda(\alpha)}$ is a monoid. We denote it by $M_{\alpha \alpha}$ or $T_{\alpha}$. 
By Lemma $8.8(i i)$, if $e \mathcal{D} f$ in $B^{*}$, then $\widetilde{H}_{e}$ is isomorphic to $\widetilde{H}_{f}$, and so for any $\alpha \in \Gamma$, the monoid structure of $T_{\alpha}$ is independent of the choices for $\widetilde{\mathcal{H}}_{B^{-}}$ classes containing a distinguished idempotent, indexed by a pair $(i, \lambda) \in I_{\alpha} \times \Lambda_{\alpha}$. For convenience, we will use $g_{\alpha}$ to denote the distinguished idempotent contained in $T_{\alpha}$. Certainly, $g_{\alpha}$ is the identity of $T_{\alpha}$.

Notice that for any $\alpha \in \Gamma$ and for each pair $(i, \lambda) \in I_{\alpha} \times \Lambda_{\alpha}, \widetilde{H}_{i \lambda} \neq \emptyset$ by Lemma $8.8(i)$. According to Lemma 8.8 (ii), there exist distinguished idempotents $r_{i}^{\alpha}$ and $q_{\lambda}^{\alpha}$ in $\widetilde{H}_{i \lambda(\alpha)}$ and $\widetilde{H}_{i(\alpha) \lambda}$, respectively, such that $x \mapsto r_{i}^{\alpha} x$ is a bijection from $\widetilde{H}_{i(\alpha) \lambda(\alpha)}$ onto $\widetilde{H}_{i \lambda(\alpha)}$ and $y \mapsto y q_{\lambda}^{\alpha}$ is a bijection from $\widetilde{H}_{i \lambda(\alpha)}$ onto $\widetilde{H}_{i \lambda}$. Thus once we have choose $\left\{r_{i}^{\alpha} \in B^{*}: i \in I_{\alpha}, \alpha \in \Gamma\right\}$ and $\left\{q_{\lambda}^{\alpha} \in B^{*}: \lambda \in \Lambda_{\alpha}, \alpha \in \Gamma\right\}$ we have a unique expression $r_{i}^{\alpha} x q_{\lambda}^{\alpha}\left(x \in T_{\alpha}=\widetilde{H}_{i(\alpha) \lambda(\alpha)}\right)$ for each element $a$ of $\widetilde{H}_{i \lambda}$, where $(i, \lambda) \in I_{\alpha} \times \Lambda_{\alpha}$.

For $\alpha, \beta \in \Gamma$ with $\alpha \neq \beta$, we put $M_{\alpha \beta}=\widetilde{H}_{i(\alpha) \lambda(\beta)}$. Notice that for any $(i, \lambda) \in I_{\alpha} \times \Lambda_{\beta}, \widetilde{H}_{i \lambda} \neq \emptyset$ if and only if $\widetilde{H}_{i(\alpha) \lambda(\beta)} \neq \emptyset$ by Lemma 8.10. Assume that $M_{\alpha \beta} \neq \emptyset$. Then by Lemma $8.9, M_{\alpha \beta}$ is a $\left(T_{\alpha}, T_{\beta}\right)$-bisystem. Also, if $(i, \lambda) \in I_{\alpha} \times \Lambda_{\beta}$ and $r_{i}^{\alpha}, q_{\lambda}^{\beta}$ are distinguished idempotents in $\widetilde{H}_{i \lambda(\alpha)}$ and $\widetilde{H}_{i(\beta) \lambda}$, respectively, then by Lemma 8.10 , we have that $x \mapsto x q_{\lambda}^{\beta}$ is a bijection from $\widetilde{H}_{i(\alpha) \lambda(\beta)}$ onto $\widetilde{H}_{i(\alpha) \lambda}$ and that $y \mapsto r_{i}^{\alpha} y$ is a bijection from $\widetilde{H}_{i(\alpha) \lambda}$ onto $\widetilde{H}_{i \lambda}$. Hence every element $a$ of $\widetilde{H}_{i \lambda}$ with $(i, \lambda) \in I_{\alpha} \times \Lambda_{\beta}$ may be written uniquely as $r_{i}^{\alpha} m q_{\lambda}^{\beta}$, where $m \in M_{\alpha \beta}$. Further, $\widetilde{H}_{i \lambda}$ is a $\left(T_{\alpha}, T_{\beta}\right)$-system under the actions

$$
t_{\alpha} \cdot\left(r_{i}^{\alpha} m q_{\lambda}^{\beta}\right)=r_{i}^{\alpha} t_{\alpha} m q_{\lambda}^{\beta} \text { and }\left(r_{i}^{\alpha} m q_{\lambda}^{\beta}\right) \cdot t_{\beta}=r_{i}^{\alpha} m t_{\beta} q_{\lambda}^{\beta},
$$

and so $\widetilde{H}_{i \lambda}$ is $\left(T_{\alpha}, T_{\beta}\right)$-isomorphic to $M_{\alpha \beta}$, that is, there exists a $\left(T_{\alpha}, T_{\beta}\right)$-isomorphism from $\widetilde{H}_{i \lambda}$ onto $M_{\alpha \beta}$. In addition, it follows from Lemma 8.8 and Lemma 8.10 that the bisystem structure of $M_{\alpha \beta}$ is independent of the possible choices for $T_{\alpha}$ and $T_{\beta}$.

We now put

$$
M=\left\{M_{\alpha \beta}:(\alpha, \beta) \in \Gamma \times \Gamma\right\} .
$$

Lemma 8.11. The set $M$ is a partial semigroup.

Proof. Let $a, b, c \in M \backslash\{0\}$. Then there exist $\alpha, \beta, \gamma, \delta, \eta, \varepsilon \in \Gamma$ such that $a \in$ $M_{\alpha \beta}, b \in M_{\gamma \delta}$ and $c \in M_{\eta \varepsilon}$. Note that $a^{*} \in D_{\beta}, b^{\dagger} \in D_{\gamma}, b^{*} \in D_{\delta}$ and $c^{\dagger} \in D_{\eta}$. Then $a b$ is defined if and only if $a b \neq 0$ if and only if $a^{*} b^{\dagger} \neq 0$ by Lemma 8.1. As $B$ is primitive, $a^{*} b^{\dagger} \neq 0$ if and only if $a^{*} \mathcal{D} b^{\dagger}$, if and only if $\beta=\gamma$. If $(a b) c$ is 
defined, then $a b \neq 0$ and $(a b) c \neq 0$, and so $\beta=\gamma$. By Lemma 8.5, $a b \widetilde{\mathcal{L}}_{B} b$, and so $(a b)^{*} \mathcal{L} b^{*}$ so that $(a b)^{*} \in D_{\delta}$. From $(a b) c \neq 0$, we obtain that $(a b)^{*} \mathcal{D} c^{\dagger}$, and so $\delta=\eta$. Thus, $b c$ is defined and by Lemma $8.5, b c \widetilde{\mathcal{R}}_{B} b$ so that $(b c)^{\dagger} \mathcal{R} b^{\dagger}$, and so $(b c)^{\dagger} \in D_{\gamma}$. As $\gamma=\beta$, we have that $a(b c)$ is defined. Since the multiplication of $S$ is associative, we have $(a b) c=a(b c)$. Dually, if $a(b c)$ is defined, then we have $(a b) c$ is defined and $(a b) c=a(b c)$.

We now define $P$ to be the $\Lambda \times I$ matrix $\left(p_{\lambda i}\right)$, where for $(\lambda, i) \in I_{\alpha} \times \Lambda_{\beta}$, $p_{\lambda i}=q_{\lambda}^{\alpha} r_{i}^{\beta}$. Now $q_{\lambda}^{\alpha} \in \widetilde{H}_{i(\alpha) \lambda}$ and $r_{i}^{\beta} \in \widetilde{H}_{i \lambda(\beta)}$ so that $q_{\lambda}^{\alpha} \in \widetilde{R}_{i(\alpha)}$ and $r_{\lambda}^{\beta} \in \widetilde{L}_{\lambda(\beta)}$, and hence either $q_{\lambda}^{\alpha} r_{i}^{\beta}=0$ or by Lemma 8.5, $q_{\lambda}^{\alpha} r_{i}^{\beta} \in \widetilde{R}_{i(\alpha)} \cap \widetilde{L}_{\lambda(\beta)}=\widetilde{H}_{i(\alpha) \lambda(\beta)}=$ $M_{\alpha \beta}$. Consequently, any non-zero entry in $(\alpha, \beta)$-block of $P$ is a member of $M_{\alpha \beta}$.

We now have the necessary ingredients to form a blocked Rees matrix semigroup $\mathcal{M}^{0}=\mathcal{M}^{0}(M ; I, \Lambda, \Gamma ; P)$.

Lemma 8.12. The blocked Rees matrix semigroup $\mathcal{M}^{0}=\mathcal{M}^{0}(M ; I, \Lambda, \Gamma ; P)$ constructed above satisfies $(B)$ and consequently, $\mathcal{M}^{0}(M ; I, \Lambda, \Gamma ; P)$ forms a WO-B blocked Rees matrix semigroup.

Proof. It is sufficient to show Condition (B) holds. Let $\alpha, \beta \in \Gamma$ and $(\lambda, i) \in$ $\Lambda_{\alpha} \times I_{\alpha}$. Since $q_{\lambda}^{\alpha}$ and $r_{i}^{\beta}$ are distinguished idempotents in $B^{*}$ and $B$ forms a band, it follows that $p_{\lambda i}=q_{\lambda}^{\alpha} r_{i}^{\beta} \neq 0$ if and only if $\alpha=\beta$, and so all non-zero entries in $P$ are in the diagonal blocks and each non-zero entry from $T_{\alpha}$ is the identity $g_{\alpha}$ of $T_{\alpha}$. Furthermore for any $\alpha \in \Gamma$ and $(i, \lambda) \in I_{\alpha} \times \Lambda_{\alpha}, p_{\lambda i}=q_{\lambda}^{\alpha} r_{i}^{\beta}=g_{\alpha} g_{\alpha}=g_{\alpha} \neq 0$, and so Condition (B) holds.

Finally, we have:

Theorem 8.13. If $S$ is a primitive weakly B-orthodox semigroup, then $S$ is isomorphic to a WO-B Rees matrix semigroup.

Proof. Observe that $S \backslash\{0\}=\bigcup\left\{\widetilde{H}_{i \lambda}:(i, \lambda) \in I \times \Lambda\right\}$ and thus the map $\phi$ : $\mathcal{M}^{0} \rightarrow S$ defined by $0 \phi=0$ and

$$
(i, a, \lambda) \phi=r_{i}^{\alpha} a q_{\lambda}^{\beta}
$$

for $(i, \lambda) \in I_{\alpha} \times \Lambda_{\beta}$ and $a \in M_{\alpha \beta}$ is a bijection.

It is routine to show that $\phi$ is a morphism. 
We now consider the abundant case. An abundant semigroup is called primitive if the non-zero idempotents are primitive under the natural partial order on the idempotents. As a special case of Theorem 8.13, the following result cited from [10] can be derived. It is a little different in Fountain's paper.

Theorem 8.14. [10] A semigroup $S$ is a primitive abundant semigroup with zero, whose set of idempotents forms a band if and only if it is isomorphic to a blocked Rees matrix semigroup $\mathcal{M}^{0}(M ; I, \Lambda, \Gamma ; P)$ satisfying $(C),(R)$ and (IB):

(C) if $a, a_{1}, a_{2} \in M_{\alpha \beta}, b, b_{1}, b_{2} \in M_{\beta \gamma}$, then $a b_{1}=a b_{2}$ implies $b_{1}=b_{2}$; $a_{1} b=a_{2} b$ implies $a_{1}=a_{2}$;

(R) if $M_{\alpha \beta}, M_{\beta \alpha}$ are both non-empty where $\alpha \neq \beta$, then $a b a \neq a$ for all $a \in M_{\alpha \beta}, b \in M_{\beta \alpha}$;

(IB) all non-zero entries of $P$ are in the diagonal blocks, and if $(i, \lambda) \in I_{\alpha} \times \Lambda_{\alpha}$ then $p_{i \lambda}$ is the identity $g_{\alpha}$ of $T_{\alpha}$.

We remark that in [10], a blocked Rees matrix semigroup $\mathcal{M}^{0}(M ; I, \Lambda, \Gamma ; P)$ is a $P A$ blocked Rees matrix semigroup if $(\mathrm{C}),(\mathrm{R})$ and $(\mathrm{U})$ hold:

(U) for each $\alpha \in \Gamma$ and each $\lambda \in \Lambda_{\alpha}$ (resp. $i \in I_{\alpha}$ ) there is a member $i$ of $I_{\alpha}\left(\right.$ resp. $\lambda$ of $\Lambda$ ) such that $p_{\lambda i}$ is a unit in $T_{\alpha}$.

Note that if Condition (IB) holds, then Condition (U) holds.

\subsection{Trace of weakly $B$-orthodox semigroups}

First, we define the trace of a weakly $B$-orthodox semigroup to be $S \mathbf{C}=(S, \cdot)$, as in Chapter 7. Remark that $S \mathbf{C}$ contains $B \mathbf{C}=(B, \cdot)$ as a substructure, where $B \mathrm{C}$ is the band $B$ with multiplication restricted to $\mathcal{D}$-classes.

Now let $P$ be any generalised category over $B$. Define $\odot$ on $P^{0}=P \cup \dot{\cup}\{0\}$ by the rule that

$$
x \odot y= \begin{cases}x \cdot y & \text { if } \exists x \cdot y \text { in } P \\ 0 & \text { otherwise. }\end{cases}
$$

Lemma 8.15. The set $\left(P^{0}, \odot\right)$ is a semigroup containing a band $\left(B^{0}, \odot\right)$ as a subsemigroup, where $\left(B^{0}, \odot\right)$ is the 0-direct union of the $\mathcal{D}$-classes of $B$. Further, $P^{0}$ is primitive weakly $B^{0}$-orthodox, in the sense that distinguished idempotents are all primitive in $B^{0}$. 
Proof. Let $x, y, z \in P^{0}$. If any of $x, y, z$ is 0 , then clearly $x \odot(y \odot z)=(x \odot y) \odot z=$ 0 . Suppose that $x, y, z \in P$. Then

$$
\begin{aligned}
x \odot(y \odot z) & = \begin{cases}x \odot(y \cdot z) & \text { if } \exists y \cdot z \\
0 & \text { otherwise }\end{cases} \\
& = \begin{cases}x \cdot(y \cdot z) & \text { if } \exists y \cdot z \text { and } \exists x \cdot(y \cdot z) \\
0 & \text { otherwise }\end{cases} \\
& = \begin{cases}x \cdot(y \cdot z) & \text { if } \exists y \cdot z \text { and } \exists x \cdot y \\
0 & \text { otherwise }\end{cases} \\
& =(x \odot y) \odot z
\end{aligned}
$$

for reasons of symmetry. Clearly $B^{0}$ is a subsemigroup of $P^{0}$.

Let $x \in P^{0}$. If $x=0$, then $x \widetilde{R}_{B^{0}} 0$. If $x \in P$, then choosing $e \in \mathbf{d}(x)$ we have $\exists e \cdot x$ and $e \cdot x=x$, so that $e \odot x=x$. If $f \in B^{0}$ and $f \odot x=x$, then clearly $f \in B$ and $\exists f \cdot x$ with $f \cdot x=x$. Hence $R_{f}=\mathbf{d}(x)=R_{e}$ so that $e \mathcal{R} f$ and $f \odot e=e$. Hence $x \widetilde{\mathcal{R}}_{B^{0}} e$ and it follows that $P^{0}$ is weakly $B^{0}$-abundant.

Notice that $x \widetilde{\mathcal{R}}_{B^{0}} f$ where $f \in B$ if and only if $\mathbf{d}(x)=R_{f}$. If follows that $x \widetilde{\mathcal{R}}_{B^{0}} y$ if and only if $\mathbf{d}(x)=\mathbf{d}(y)$. Thus for any $z \in P, z \odot x=0$ if and only if $z \odot y=0$, and if $z \odot x \neq 0$, then $\mathbf{d}(z \odot x)=\mathbf{d}(z)=\mathbf{d}(z \odot y)$. It is clear that (C) holds and $P^{0}$ is weakly $B^{0}$-orthodox. It is immediate that $P^{0}$ is primitive.

Let $S$ be weakly $B$-orthodox. From Lemma $7.17, S \mathbf{C}=(S, \cdot)$ is an inductive generalised category over $B$. Then $S \mathbf{C}^{0}$ is a primitive weakly $B^{0}$-orthodox semigroup; $S \mathbf{C}^{0}$ is also sometimes called the trace of $S$. From Lemma 7.18, $S \mathbf{C}$, and with a little adjustment, $S \mathbf{C}^{0}$, can be endowed with an inductive structure from which we can recover $S$.

The natural partial orders in any primitive weakly $B$-orthodox semigroup with 0 are trivial, in the following sense:

Lemma 8.16. Let $S$ be a primitive weakly B-orthodox semigroup with 0, where $0 \in B$. Then $B$ is a 0-disjoint union of $\mathcal{D}$-classes. If $x, y \in S$, then $x \leq_{r}^{\prime} y$ if and only if $x=0$ or $x=y$.

Proof. We know that $B$ is a semilattice $Y$ of $\mathcal{D}$-classes $D_{\alpha}, \alpha \in Y$. We must have that $Y$ contains a zero $\tau$ and $D_{\tau}=\{0\}$. If $\tau<\alpha<\beta$, let $e \in D_{\alpha}$ and 
$f \in D_{\beta}$. Then $f e f \in D_{\alpha}$ and $0<f e f<f$, a contradiction. It follows that $B$ is a 0 -disjoint union of its $\mathcal{D}$-classes.

If $x \neq 0$ and $x \leq_{r}^{\prime} y$, then $x=e y$ for some $e \in B$ and $x^{\dagger} \leq_{\mathcal{R}} y^{\dagger}$. Thus $x^{\dagger} y^{\dagger} \leq y^{\dagger}$ so that $x^{\dagger} y^{\dagger}=y^{\dagger}$. Also, $x^{\dagger} \leq_{\mathcal{R}} e$ so that similarly, $x^{\dagger} e=x^{\dagger}$. Now $x=e y=x^{\dagger} e y=x^{\dagger} y=x^{\dagger} y^{\dagger} y=y^{\dagger} y=y$.

In view of Theorem 8.13, we have:

Theorem 8.17. If $S$ is a weakly B-orthodox semigroup, then $S \mathbf{C}^{0}$ is isomorphic to a WO-B Rees matrix semigroup $\mathcal{M}^{0}(M ; I, \Lambda, \Gamma ; P)$ satisfying $(B)$. 


\section{Chapter 9}

\section{Beyond orthodox semigroups II: weakly $B$-orthodox semigroups and categories}

The aim of this chapter is to construct weakly $B$-orthodox semigroups via an adjustment of Armstrong's method for concordant semigroups as mentioned in Chapter 6. Our modifications are to allow for the fact that $B$ is a band, and to compensate for the lack of an idempotent connected condition.

For convenience we make the convention that $B$ will always denote a band. Green's relations and their associated pre-orders will always refer to $B$, unless stated otherwise.

\subsection{Weakly orthodox categories}

The purpose in this section is to introduce the notions of a band category and a weakly orthodox category over a band $B$, and to present a pair of pre-orders which are deduced from the definition of band categories.

Let $B$ be a band. A subset $K$ of $B$ is a representative of $B$ if maps $\phi: K \rightarrow$ $B / \mathcal{L}$ given by $e \mapsto L_{e}$ and $\psi: K \rightarrow B / \mathcal{R}$ given by $e \mapsto R_{e}$ are bijective. So for any $e \in B$, there exists a unique $k \in K$ such that $e \mathcal{L} k$ in $B$ and there exists a unique $h \in K$ such that $e \mathcal{R} h$ in $B$. For convenience, we will denote $k$ and $h$ by $e^{\star}$ and $e^{+}$, respectively. 
Definition 9.1. Let $P$ be a category in which $\mathrm{Ob}(P)$ is the underlying set of a band $B$, and let $K$ be a representative of $B$. Suppose that for $e, f \in B$ with $e \mathcal{D} f$, there exists a distinguished morphism $[e, f]$ from $e$ to $f$ such that $[e, e]=1_{e}$, the identity associated to the object $e$. Then $P$ is a band category if the following conditions and their duals $(\mathrm{OB} 1)^{\circ},(\mathrm{OB} 2)^{\circ},(\mathrm{OB} 3)^{\circ}$ and $(\mathrm{OB} 4)^{\circ}$ of (OB1), (OB2), (OB3) and (OB4) hold:

(OB1) if $x \in P$ and $e \in B$ with $e \leq_{\mathcal{L}} \mathbf{d}(x)$, then there exists an element ${ }_{e} \mid x$ in $P$, called the restriction of $x$ to $e$, such that $\mathbf{d}(e \mid x)=e$ and $\mathbf{r}\left({ }_{e} \mid x\right) \leq_{\mathcal{L}} \mathbf{r}(x)$; also, if $e=\mathbf{d}(x)$, then $\mathbf{r}\left({ }_{e} \mid x\right) \mathcal{L} \mathbf{r}(x)$ and ${ }_{e} \mid x \cdot\left[\mathbf{r}\left({ }_{e} \mid x\right), \mathbf{r}(x)\right]=x$;

(OB2) if $x \in P$ and $e, f \in B$ with $e \leq_{\mathcal{L}} f \leq_{\mathcal{L}} \mathbf{d}(x)$, then $e_{e}\left|\left({ }_{f} \mid x\right)={ }_{e}\right| x$; moreover, if $e \mathcal{L} f \leq_{\mathcal{L}} \mathbf{d}(x)$, then $[e, f] \cdot{ }_{f}\left|x={ }_{e}\right| x$;

(OB3) if $x, y \in P$ and $e \in B$ with $\exists x \cdot y$ in $P$ and $e \leq_{\mathcal{L}} \mathbf{d}(x)$, then ${ }_{e} \mid(x \cdot y)=$ ${ }_{e}\left|x \cdot{ }_{f}\right| y$, where $f=\mathbf{r}\left({ }_{e} \mid x\right)$;

(OB4) if $e, f, h \in B$ with $e \mathcal{D} f$ and $h \leq_{\mathcal{L}} e$, then ${ }_{h} \mid[e, f]=\left[h,(h f)^{\star}\right]$;

$(\mathrm{OB} 5)$ if $e, f, g \in B$ are such that $e \mathcal{D} f \mathcal{D} g$, then $[e, f] \cdot[f, g]=[e, g]$.

We make some comments on the above definition. In (OB2), since $[e, f] \cdot{ }_{f} \mid x=$ ${ }_{e} \mid x$ we obtain that $\mathbf{r}\left({ }_{f} \mid x\right)=\mathbf{r}\left({ }_{e} \mid x\right)$. In (OB3) since $\exists x \cdot y$ we know that $\mathbf{r}(x)=\mathbf{d}(y)$. By (OB1), $f=\mathbf{r}\left({ }_{e} \mid x\right) \leq_{\mathcal{L}} \mathbf{r}(x)=\mathbf{d}(y)$, so that ${ }_{f} \mid y$ exists and $\mathbf{d}\left({ }_{f} \mid y\right)=f$. Hence ${ }_{e}\left|x \cdot{ }_{f}\right| y$ is defined. In (OB4) since $e \mathcal{D} f$ we obtain that $e \mathcal{R}$ ef. Hence he $\mathcal{R}$ he $f$, that is, $h \mathcal{R} h f$ as $h \leq_{\mathcal{L}} e$, so that $h \mathcal{R} h f \mathcal{L}(h f)^{\star}$, and consequently, $h \mathcal{D}(h f)^{\star}$. Hence $\left[h,(h f)^{\star}\right]$ exists.

We note that a band category $P$ depends on the choice of the band $B$ which is the set of objects of $P$. In order to emphasize that the set of objects is a particular band $B$, we can express the term 'band category' as 'band category over $B$ '.

Let $P$ be a band category over $B$. Using the technique in [1], we define a relation $\rho$ on $P$ by the rule that for all $x, y \in P$,

$$
x \rho y \Leftrightarrow \mathbf{d}(x) \mathcal{R} \mathbf{d}(y), \mathbf{r}(x) \mathcal{L} \mathbf{r}(y) \text { and } x \cdot[\mathbf{r}(x), \mathbf{r}(y)]=[\mathbf{d}(x), \mathbf{d}(y)] \cdot y,
$$

that is equal to

$$
x \rho y \Leftrightarrow(\exists u, v \in B) v \mathcal{R} \mathbf{d}(y), \mathbf{r}(x) \mathcal{L} u \text { and } x \cdot[\mathbf{r}(x), u]=[v, \mathbf{d}(y)] \cdot y .
$$


We note that if $x \rho y$ in $P$, then we have that

$$
\begin{aligned}
& x \cdot[\mathbf{r}(x), \mathbf{r}(y)]=[\mathbf{d}(x), \mathbf{d}(y)] \cdot y \\
\Leftrightarrow & x \cdot[\mathbf{r}(x), \mathbf{r}(y)] \cdot[\mathbf{r}(y), \mathbf{r}(x)]=[\mathbf{d}(x), \mathbf{d}(y)] \cdot y \cdot[\mathbf{r}(y), \mathbf{r}(x)] \\
\Leftrightarrow & x \cdot[\mathbf{r}(x), \mathbf{r}(x)]=[\mathbf{d}(x), \mathbf{d}(y)] \cdot y \cdot[\mathbf{r}(y), \mathbf{r}(x)] \quad(\text { by }(\text { OB } 5)) \\
\Leftrightarrow & x=[\mathbf{d}(x), \mathbf{d}(y)] \cdot y \cdot[\mathbf{r}(y), \mathbf{r}(x)] \quad\left([\mathbf{r}(x), \mathbf{r}(x)]=1_{\mathbf{r}(x)}\right) .
\end{aligned}
$$

In particular, if $x, y \in P$ are such that $\mathbf{d}(x)=\mathbf{d}(y)$, then $x \rho y$ if and only if $\mathbf{r}(x) \mathcal{L} \mathbf{r}(y)$ and

$$
x=y \cdot[\mathbf{r}(y), \mathbf{r}(x)] \text { or indeed } x \cdot[\mathbf{r}(x), \mathbf{r}(y)]=y .
$$

Dually, if $\mathbf{r}(x)=\mathbf{r}(y)$, then $x \rho y$ if and only if $\mathbf{d}(x) \mathcal{R} \mathbf{d}(y)$ and

$$
x=[\mathbf{d}(x), \mathbf{d}(y)] \cdot y \text { or indeed } y=[\mathbf{d}(y), \mathbf{d}(x)] \cdot x .
$$

Built on the above statement, it is easy to see that for any $e \in B,\left[e^{+}, e\right] \rho 1_{e}$. Since

$$
\mathbf{d}\left(\left[e^{+}, e\right]\right)=e^{+} \mathcal{R} e=\mathbf{d}\left(1_{e}\right), \quad \mathbf{r}\left(\left[e^{+}, e\right]\right)=e=\mathbf{r}\left(1_{e}\right)
$$

and

$$
\left[e, e^{+}\right] \cdot\left[e^{+}, e\right]=[e, e]=1_{e},
$$

we have that $\left[e^{+}, e\right] \rho 1_{e}$. Dually, $\left[e, e^{\star}\right] \rho 1_{e}$.

Lemma 9.2. The relation $\rho$ defined above is an equivalence on $P$ such that if $x, y \in \operatorname{Mor}(e, f)$ and $x \rho y$, then $x=y$. In particular, no two identities of $P$ are $\rho$-equivalent.

Proof. Clearly, $\rho$ is reflexive.

In order to show that $\rho$ is symmetric, we assume that $x, y \in P$ with $x \rho y$. Then $\mathbf{d}(x) \mathcal{R} \mathbf{d}(y), \mathbf{r}(x) \mathcal{L} \mathbf{r}(y)$ and $x \cdot[\mathbf{r}(x), \mathbf{r}(y)]=[\mathbf{d}(x), \mathbf{d}(y)] \cdot y$. Since $\mathcal{R}$ and 
$\mathcal{L}$ are symmetric, $[\mathbf{r}(y), \mathbf{r}(x)]$ and $[\mathbf{d}(y), \mathbf{d}(x)]$ exist. Thus,

$$
\begin{array}{rlr} 
& x \cdot[\mathbf{r}(x), \mathbf{r}(y)]=[\mathbf{d}(x), \mathbf{d}(y)] \cdot y & \\
\Rightarrow & x=[\mathbf{d}(x), \mathbf{d}(y)] \cdot y \cdot[\mathbf{r}(y), \mathbf{r}(x)] & (\text { by the statements above Lemma 9.2) } \\
\Rightarrow & {[\mathbf{d}(y), \mathbf{d}(x)] \cdot x=[\mathbf{d}(y), \mathbf{d}(x)] \cdot[\mathbf{d}(x), \mathbf{d}(y)] \cdot y \cdot[\mathbf{r}(y), \mathbf{r}(x)]} \\
\Rightarrow & {[\mathbf{d}(y), \mathbf{d}(x)] \cdot x=[\mathbf{d}(y), \mathbf{d}(y)] \cdot y \cdot[\mathbf{r}(y), \mathbf{r}(x)] \quad(\text { by }(\text { OB } 5))} \\
\Rightarrow & {[\mathbf{d}(y), \mathbf{d}(x)] \cdot x=y \cdot[\mathbf{r}(y), \mathbf{r}(x)]} & \left([\mathbf{d}(y), \mathbf{d}(y)]=1_{\mathbf{d}(y)}\right) .
\end{array}
$$

So, $y \rho x$.

Finally, if $x \rho y$ and $y \rho z$, then $\mathbf{d}(x) \mathcal{R} \mathbf{d}(z)$ and $\mathbf{r}(x) \mathcal{L} \mathbf{r}(z)$ as $\mathcal{R}$ and $\mathcal{L}$ are transitive. Hence, $[\mathbf{d}(x), \mathbf{d}(z)]$ and $[\mathbf{r}(x), \mathbf{r}(z)]$ exist. Then we have that

$$
\begin{aligned}
x \cdot[\mathbf{r}(x), \mathbf{r}(z)] & =x \cdot[\mathbf{r}(x), \mathbf{r}(y)] \cdot[\mathbf{r}(y), \mathbf{r}(z)] & & \text { by }(\text { OB } 5), \mathbf{r}(x) \mathcal{L} \mathbf{r}(y) \mathcal{L} \mathbf{r}(z)) \\
& =[\mathbf{d}(x), \mathbf{d}(y)] \cdot y \cdot[\mathbf{r}(y), \mathbf{r}(z)] & & (x \rho y) \\
& =[\mathbf{d}(x), \mathbf{d}(y)] \cdot[\mathbf{d}(y), \mathbf{d}(z)] \cdot z & & (y \rho z) \\
& =[\mathbf{d}(x), \mathbf{d}(z)] \cdot z & & (\text { by }(\text { OB } 5)) .
\end{aligned}
$$

Thus, $x \rho z$.

As $[e, e]=1_{e}$ for all $e \in B$, certainly, if $\mathbf{d}(x)=\mathbf{d}(y), \mathbf{r}(x)=\mathbf{r}(y)$ and $x \rho y$, then $x=y$.

We now present a pair of pre-orders on a band category over $B$ built on the relation $\rho$ given above.

Let $P$ be a band category over $B$. We make use of the restriction and corestriction of $P$ to define relations $\leq_{r}$ and $\leq_{\ell}$ by the rule that for all $x, y \in P$,

$$
x \leq_{r} y \text { if and only if } x \rho_{e} \mid y \quad \text { for some } e \in B,
$$

and

$$
x \leq_{\ell} y \text { if and only if }\left.x \rho y\right|_{f} \text { for some } f \in B \text {. }
$$

Lemma 9.3. The relations $\leq_{r}$ and $\leq_{\ell}$ are pre-orders on $P$.

Proof. We first show that $\leq_{r}$ is a pre-order on $P$. Notice that for any $x \in P$, if $e=\mathbf{d}(x)$, then $[\mathbf{d}(x), \mathbf{d}(e \mid x)]=[e, e]=1_{e}$, and so $\leq_{r}$ is reflexive by (OB1). It is sufficient to show that $\leq_{r}$ is transitive. Suppose that $x, y, z \in P$ with 
$x \leq_{r} y$ and $y \leq_{r} z$. Then there exist $e, f \in B$ such that $x \rho_{e} \mid y$ and $y \rho_{f} \mid z$. Thus, $\mathbf{d}(x) \mathcal{R} e \leq_{\mathcal{L}} \mathbf{d}(y) \mathcal{R} f \leq_{\mathcal{L}} \mathbf{d}(z)$, and so $\mathbf{d}(x) \mathcal{R} e=e \mathbf{d}(y) \mathcal{R}$ ef. Set $g=e f$. Since $g=e f \leq_{\mathcal{L}} f \leq_{\mathcal{L}} \mathbf{d}(z),{ }_{g} \mid z$ is well-defined. Now our aim is to show that $x \rho_{g} \mid z$. From $y \rho_{f} \mid z$, we have $\mathbf{d}(y) \mathcal{R} f, \mathbf{r}(y) \mathcal{L} \mathbf{r}\left({ }_{f} \mid z\right)$ and $y \cdot\left[\mathbf{r}(y), \mathbf{r}\left({ }_{f} \mid z\right)\right]=[\mathbf{d}(y), f] \cdot\left({ }_{f} \mid z\right)$. Hence, $y=[\mathbf{d}(y), f] \cdot{ }_{f} \mid z \cdot\left[\mathbf{r}\left({ }_{f} \mid z\right), \mathbf{r}(y)\right]$. For ${ }_{e} \mid y$ and ${ }_{f} \mid z$ to exist, we have that $e \leq_{\mathcal{L}} \mathbf{d}(y)$ and $f \leq_{\mathcal{L}} \mathbf{d}(z)$, so that ${ }_{e} \mid[\mathbf{d}(y), f]$ exists and ${ }_{e} \mid[\mathbf{d}(y), f]=\left[e,(e f)^{\star}\right]$ by (OB4). As $(e f)^{\star} \mathcal{L}$ ef $\leq_{\mathcal{L}} f \leq_{\mathcal{L}} \mathbf{d}(z)$, we obtain that ${ }_{(e f)^{\star}} \mid\left({ }_{f} \mid z\right)$ is defined and $(e f)^{\star}\left|\left({ }_{f} \mid z\right)={ }_{(e f)^{\star}}\right| z$ by $(\mathrm{OB} 2)$. Then we have that

$$
\begin{aligned}
e_{e} \mid y= & e_{e} \mid\left([\mathbf{d}(y), f] \cdot{ }_{f} \mid z \cdot\left[\mathbf{r}\left({ }_{f} \mid z\right), \mathbf{r}(y)\right]\right) \\
= & { }_{e}\left|[\mathbf{d}(y), f] \cdot{ }_{h}\right|\left({ }_{f} \mid z\right) \cdot{ }_{k} \mid\left[\mathbf{r}\left({ }_{f} \mid z\right), \mathbf{r}(y)\right] \\
& \quad\left(\operatorname{by}(\mathrm{OB} 3), h=\mathbf{r}(e \mid[\mathbf{d}(y), f]), k=\mathbf{r}\left({ }_{h} \mid\left({ }_{f} \mid z\right)\right)\right) \\
= & {\left[e,(e f)^{\star}\right] \cdot{ }_{(e f)^{\star}} \mid\left({ }_{f} \mid z\right) \cdot\left[k,(k \mathbf{r}(y))^{\star}\right] \quad(\text { by }(\mathrm{OB} 4)) } \\
= & {\left[e,(e f)^{\star}\right] \cdot{ }_{(e f)^{\star}} \mid z \cdot\left[k, k^{\star}\right] \quad }
\end{aligned}
$$$$
\text { (by (OB2), } \left.k=\mathbf{r}\left({ }_{h} \mid\left({ }_{f} \mid z\right)\right) \leq_{\mathcal{L}} \mathbf{r}\left({ }_{f} \mid z\right) \mathcal{L} \mathbf{r}(y)\right) \text {. }
$$

Hence, $\mathbf{r}(e \mid y)=k^{\star}$. From $x \rho_{e} \mid y$, we have that $\mathbf{r}(x) \mathcal{L} k^{\star}, \mathbf{d}(x) \mathcal{R} e$ and

$$
\begin{aligned}
x \cdot\left[\mathbf{r}(x), k^{\star}\right] & =[\mathbf{d}(x), e] \cdot e_{e} \mid y \\
& =[\mathbf{d}(x), e] \cdot\left[e,(e f)^{\star}\right] \cdot(e f)^{\star} \mid z \cdot\left[k, k^{\star}\right] \\
& =\left[\mathbf{d}(x),(e f)^{\star}\right] \cdot(e f)^{\star} \mid z \cdot\left[k, k^{\star}\right]
\end{aligned}
$$

Thus,

$$
\begin{aligned}
x \cdot[\mathbf{r}(x), k] & =x \cdot\left[\mathbf{r}(x), k^{\star}\right] \cdot\left[k^{\star}, k\right] & & (\text { by }(\text { OB } 5)) \\
& =\left[\mathbf{d}(x),(e f)^{\star}\right] \cdot(e f)^{\star} \mid z \cdot\left[k, k^{\star}\right] \cdot\left[k^{\star}, k\right] & & \\
& =[\mathbf{d}(x), g] \cdot\left[g,(e f)^{\star}\right] \cdot(e f)^{\star} \mid z \cdot[k, k] & & \left(\mathbf{d}(x) \mathcal{R} g \mathcal{L}(e f)^{\star}, \text { by }(\text { OB } 5)\right) \\
& =[\mathbf{d}(x), g] \cdot\left[g,(e f)^{\star}\right] \cdot(e f)^{\star} \mid z & & \left(k=\mathbf{r}\left((e f)^{\star} \mid z\right)\right) \\
& =[\mathbf{d}(x), g] \cdot{ }_{g} \mid z & & (\text { by }(\text { OB2 })) .
\end{aligned}
$$

It follows that $k=\mathbf{r}\left({ }_{g} \mid z\right)$. As $\mathbf{r}(x) \mathcal{L} \mathbf{r}\left({ }_{e} \mid y\right)=k^{\star} \mathcal{L} k=\mathbf{r}(g \mid z)$ and $\mathbf{d}(x) \mathcal{R} g=$ $\mathbf{d}(g \mid z)$, we have that $x \rho_{g} \mid z$. Hence, $x \leq_{r} z$.

By the dual argument, we show that $\leq_{\ell}$ is a pre-order on $P$. 
We remark that if $B$ is a semilattice, then the relation $\rho$ on $P$ is precisely the identity relation so that relations $\leq_{r}$ and $\leq_{\ell}$ may be expressed as follows: for all $x, y \in P$,

$$
x \leq_{r} y \text { if and only if } x={ }_{e} \mid y \quad \text { for some } e \in B,
$$

and

$$
x \leq_{\ell} y \text { if and only if } x=\left.y\right|_{f} \quad \text { for some } f \in B .
$$

In addition, the relations $\leq_{r}$ and $\leq_{\ell}$ become partial orders. Since if $x \leq_{r} y \leq_{r} x$, then $x={ }_{e} \mid y$ and $y={ }_{f} \mid x$ for some $e \leq \mathbf{d}(y)$ and $f \leq \mathbf{d}(x)$. We have that $\mathbf{d}(x)=e \leq \mathbf{d}(y)=f \leq \mathbf{d}(x)$ so $\mathbf{d}(x)=\mathbf{d}(y)$. By (OB1), $\mathbf{r}(\mathbf{d}(y) \mid y) \mathcal{L} \mathbf{r}(y)$, and so $\mathbf{r}(\mathbf{d}(y) \mid y)=\mathbf{r}(y)$ as $B$ is a semilattice. Thus, $x={ }_{e}\left|y={ }_{\mathbf{d}(y)}\right| y$. By (OB1), $\mathbf{d}(y) \mid y \cdot[\mathbf{r}(\mathbf{d}(y) \mid y), \mathbf{r}(y)]=y$, so that

$$
x={ }_{e}\left|y={ }_{\mathbf{d}(y)}\right| y={ }_{\mathbf{d}(y)} \mid y \cdot\left[\mathbf{r}\left({ }_{\mathbf{d}(y)} \mid y\right), \mathbf{r}(y)\right]=y \quad\left(\mathbf{r}\left({ }_{\mathbf{d}(y)} \mid y\right)=\mathbf{r}(y)\right) .
$$

We pause here to make some further comments on Definition 9.1. In (OB1) let $e=\mathbf{d}(x)$. Then $\mathbf{r}\left({ }_{e} \mid x\right) \mathcal{L} \mathbf{r}(x)$ and $x={ }_{e} \mid x \cdot\left[\mathbf{r}\left({ }_{e} \mid x\right), \mathbf{r}(x)\right]$. Hence due to the definition of $\rho$, we obtain that $x \rho_{e} \mid x$. This fact makes it impossible to define a partial order $\leq_{r}{ }^{\prime}$ on $P$ by the rule that for all $x, y \in P, x \leq_{r}{ }^{\prime} y$ if and only if $x={ }_{e} \mid y$ for some $e \leq \mathbf{d}(y)$ because it is not reflexive; even if $x={ }_{e} \mid y$ is replaced by $x \rho_{e} \mid y$, we still cannot guarantee that $\leq_{r}{ }^{\prime}$ is a partial order since $\leq_{r}{ }^{\prime}$ becomes reflexive but not anti-symmetric.

As an analogue of inductive generalised categories over $B$ in Chapter 7 , we will make use of the relation $\rho$ given above to define weakly orthodox categories over $B$, which are built on Definition 9.1.

Definition 9.4. A band category $P$ over $B$ is weakly orthodox if for any $x \in P$ and $e, f \in B,_{e u}\left|\left(\left.x\right|_{g f}\right) \rho\left({ }_{e h} \mid x\right)\right|_{v f}$, where $g=\mathbf{r}(x), h=\mathbf{d}(x), u=\mathbf{d}\left(\left.x\right|_{g f}\right)$ and $v=\mathbf{r}\left({ }_{e h} \mid x\right)$.

It is worth considering how the class of weakly orthodox categories over bands forms a category, together with certain functors referred to as orthodox functors. They are described in the next definition.

Definition 9.5. Let $P_{1}$ and $P_{2}$ be weakly orthodox categories over $B_{1}$ and $B_{2}$, respectively. An orthodox functor $F$ from $P_{1}$ to $P_{2}$ is a functor consisting of a 
pair of maps, both denoted $F$, from $B_{1}$ to $B_{2}$ and from $P_{1}$ to $P_{2}$, such that the following conditions and the dual (S3) of (S3) hold:

(S1) the map $F$ is a morphism from $B_{1}$ to $B_{2}$;

$(\mathrm{S} 2)$ if $e, f \in B_{1}$ with $e \mathcal{D} f$, then $[e, f]_{P_{1}} F=[e F, f F]_{P_{2}}$;

(S3) if $x \in P_{1}$ and $e \in B_{1}$ with $e \leq_{\mathcal{L}} \mathbf{d}(x)$, then $\left({ }_{e} \mid x\right) F \rho_{e F} \mid x F$.

We pause here to make a short comment on Definition 9.5. In (S2), if $e, f \in$ $B_{1}$ with $e \mathcal{D} f$, then by $(\mathrm{S} 1), e F \mathcal{D} f F$, so that both $[e, f]_{P_{1}}$ and $[e F, f F]_{P_{2}}$ are defined. In (S3), if $e \leq_{\mathcal{L}} \mathbf{d}(x)$, then $e F \leq_{\mathcal{L}} \mathbf{d}(x F)$ as $F$ is a functor, so that both $e_{e} \mid x$ and ${ }_{e F} \mid x F$ are well-defined. In addition, the fact that $\left({ }_{e} \mid x\right) F \rho_{e F} \mid x F$ gives in particular that $\mathbf{r}\left(\left(_{e} \mid x\right) F\right) \mathcal{L} \mathbf{r}\left({ }_{e F} \mid x F\right)$. For $\mathbf{d}$, we have the corresponding result as $F$ is a functor.

The next lemma is useful for Lemma 9.7.

Lemma 9.6. Let $P_{1}$ and $P_{2}$ be weakly orthodox categories over $B_{1}$ and $B_{2}$, respectively and let $F: P_{1} \rightarrow P_{2}$ be an orthodox functor. If $x \rho y$ in $P_{1}$, then $x F \rho y F$ in $P_{2}$.

Proof. Suppose that $x, y \in P_{1}$ and $x \rho y$. Then

$$
\begin{gathered}
\quad \mathbf{d}(x) \mathcal{R} \mathbf{d}(y), \mathbf{r}(x) \mathcal{L} \mathbf{r}(y) \text { and } x \cdot[\mathbf{r}(x), \mathbf{r}(y)]=[\mathbf{d}(x), \mathbf{d}(y)] \cdot y \\
\Rightarrow \mathbf{d}(x) F \mathcal{R} \mathbf{d}(y) F, \mathbf{r}(x) F \mathcal{L} \mathbf{r}(y) F \text { and } \\
x F \cdot[\mathbf{r}(x), \mathbf{r}(y)] F=[\mathbf{d}(x), \mathbf{d}(y)] F \cdot y F \\
\Rightarrow \mathbf{d}(x) F \mathcal{R} \mathbf{d}(y) F, \mathbf{r}(x) F \mathcal{L} \mathbf{r}(y) F \text { and } \\
x F \cdot[\mathbf{r}(x) F, \mathbf{r}(y) F]=[\mathbf{d}(x) F, \mathbf{d}(y) F] \cdot y F \quad(\text { by }(S 2)) \\
\Rightarrow \mathbf{d}(x F) \mathcal{R} \mathbf{d}(y F), \mathbf{r}(x F) \mathcal{L} \mathbf{r}(y F) \text { and } \\
x F \cdot[\mathbf{r}(x F), \mathbf{r}(y F)]=[\mathbf{d}(x F), \mathbf{d}(y F)] \cdot y F
\end{gathered}
$$$$
\text { (by } F \text { being a functor). }
$$

Hence, $x F \rho y F$.

Lemma 9.7. Let $P_{1}$ and $P_{2}$ be weakly orthodox categories over $B_{1}$ and $B_{2}$, respectively, and let $F_{1}: P_{1} \rightarrow P_{2}$ and $F_{2}: P_{2} \rightarrow P_{3}$ be orthodox functors. Then $F_{1} F_{2}: P_{1} \rightarrow P_{3}$ is an orthodox functor.

Proof. (S1) Certainly, $F_{1} F_{2}$ is a functor from $P_{1}$ to $P_{3}$ and a morphism from $B_{1}$ to $B_{3}$. 
(S2) Suppose that $e, f \in B_{1}$ are such that $e \mathcal{D} f$. Then $[e, f]_{P_{1}}$ is defined and using (S2) for $F_{1}$ and $F_{2}$,

$$
[e, f]_{P 1} F_{1} F_{2}=\left([e, f]_{P 1} F_{1}\right) F_{2}=\left[e F_{1}, f F_{1}\right]_{P_{2}} F_{2}=\left[e F_{1} F_{2}, f F_{1} F_{2}\right]_{P 3} .
$$

(S3) Suppose that $x \in P_{1}$ and $e \in B_{1}$ with $e \leq_{\mathcal{L}} \mathbf{d}(x)$. According to the comment succeeding Definition 9.5, we have that ${ }_{e}\left|x,{ }_{e F_{1}}\right| x F_{1}$ and ${ }_{e F_{1} F_{2}} \mid x F_{1} F_{2}$ are well-defined. By (S3), $(e \mid x) F_{1} \rho_{e F_{1}} \mid x F_{1}$ and $\left(e F_{1} \mid x F_{1}\right) F_{2} \rho_{e F_{1} F_{2}} \mid x F_{1} F_{2}$. From $\left({ }_{e} \mid x\right) F_{1} \rho_{e F_{1}} \mid x F_{1}$, we obtain that $\left.{ }_{e} \mid x\right) F_{1} F_{2} \rho\left({ }_{e F_{1}} \mid x F_{1}\right) F_{2}$ by Lemma 9.6. Hence, $(e \mid x) F_{1} F_{2} \rho_{e F_{1} F_{2}} \mid x F_{1} F_{2}$.

An immediate observation from Lemma 9.7 is that the class of weakly orthodox categories over bands and orthodox functors forms a category. We refer to it as $\mathcal{W O C}$.

\subsection{Construction}

Our aim in this section is to build a weakly $B$-orthodox semigroup from a weakly orthodox category over $B$. This result is analogous to Armstrong's work [1]

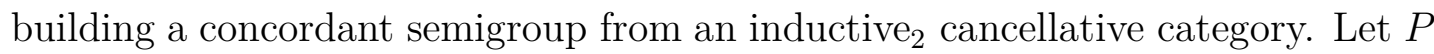
be a weakly orthodox category over $B$. For any $x, y \in P$, we define

$$
x \otimes y=\left.x\right|_{\mathbf{r}(x) \mathbf{d}(y)} \cdot \mathbf{r}(x) \mathbf{d}(y) \mid y .
$$

Before we give a list of lemmas which are necessary to prove our main theorem, we make a comment that since our set of idempotents forms a band it is an advantage to use the product $\otimes$ given above to avoid the notion of sandwich set, which is needed in [1].

Lemma 9.8. If $P$ is a weakly orthodox category over $B$ and $x \rho x^{\prime}, y \rho y^{\prime}$ in $P$, then $x \otimes y \rho x^{\prime} \otimes y^{\prime}$.

Proof. Suppose that $x \rho x^{\prime}$ and $y \rho y^{\prime}$ in $P$. Then

$$
\mathbf{d}(x) \mathcal{R} \mathbf{d}\left(x^{\prime}\right), \mathbf{r}(x) \mathcal{L} \mathbf{r}\left(x^{\prime}\right) \text { and } x \cdot\left[\mathbf{r}(x), \mathbf{r}\left(x^{\prime}\right)\right]=\left[\mathbf{d}(x), \mathbf{d}\left(x^{\prime}\right)\right] \cdot x^{\prime}
$$


whence $x=\left[\mathbf{d}(x), \mathbf{d}\left(x^{\prime}\right)\right] \cdot x^{\prime} \cdot\left[\mathbf{r}\left(x^{\prime}\right), \mathbf{r}(x)\right]$. The same happens to $y$ and $y^{\prime}$ as follows

$$
\mathbf{d}(y) \mathcal{R} \mathbf{d}\left(y^{\prime}\right), \mathbf{r}(y) \mathcal{L} \mathbf{r}\left(y^{\prime}\right) \text { and } y \cdot\left[\mathbf{r}(y), \mathbf{r}\left(y^{\prime}\right)\right]=\left[\mathbf{d}(y), \mathbf{d}\left(y^{\prime}\right)\right] \cdot y^{\prime},
$$

whence $y=\left[\mathbf{d}(y), \mathbf{d}\left(y^{\prime}\right)\right] \cdot y^{\prime} \cdot\left[\mathbf{r}\left(y^{\prime}\right), \mathbf{r}(y)\right]$. So

$$
\mathbf{r}(x) \mathbf{d}(y) \mathcal{R} \mathbf{r}(x) \mathbf{d}\left(y^{\prime}\right) \mathcal{L} \mathbf{r}\left(x^{\prime}\right) \mathbf{d}\left(y^{\prime}\right) \mathcal{R} \mathbf{r}\left(x^{\prime}\right) \mathbf{d}(y)
$$

As $\mathbf{r}(x) \mathbf{d}(y) \leq_{\mathcal{R}} \mathbf{r}(x),\left.x\right|_{\mathbf{r}(x) \mathbf{d}(y)}$ is defined and we have that

$$
\begin{aligned}
& x \mid \mathbf{r}(x) \mathbf{d}(y) \\
& =\left.\left(\left[\mathbf{d}(x), \mathbf{d}\left(x^{\prime}\right)\right] \cdot x^{\prime} \cdot\left[\mathbf{r}\left(x^{\prime}\right), \mathbf{r}(x)\right]\right)\right|_{\mathbf{r}(x) \mathbf{d}(y)} \\
& =\left.\left[\mathbf{d}(x), \mathbf{d}\left(x^{\prime}\right)\right]\right|_{k} \cdot\left(\left.x^{\prime}\right|_{\left(\mathbf{r}\left(x^{\prime}\right) \mathbf{r}(x) \mathbf{d}(y)\right)^{+}}\right) \cdot\left[\left(\mathbf{r}\left(x^{\prime}\right) \mathbf{r}(x) \mathbf{d}(y)\right)^{+}, \mathbf{r}(x) \mathbf{d}(y)\right] \\
& \text { (by }(\mathrm{OB} 4)^{\circ},(\mathrm{OB} 3)^{\circ}, k=\mathbf{d}\left(\left.x^{\prime}\right|_{\left.\left(\mathbf{r}\left(x^{\prime}\right) \mathbf{r}(x) \mathbf{d}(y)\right)^{+}\right)}\right) \\
& =\left[(\mathbf{d}(x) k)^{+}, k\right] \cdot\left(\left.x^{\prime}\right|_{\left(\mathbf{r}\left(x^{\prime}\right) \mathbf{d}(y)\right)^{+}}\right) \cdot\left[\left(\mathbf{r}\left(x^{\prime}\right) \mathbf{d}(y)\right)^{+}, \mathbf{r}(x) \mathbf{d}(y)\right] \\
& \text { (by } \left.(\mathrm{OB} 4)^{\circ}, \mathbf{r}(x) \mathcal{L} \mathbf{r}\left(x^{\prime}\right)\right) \\
& =\left[k^{+}, k\right] \cdot\left(\left.x^{\prime}\right|_{\left(\mathbf{r}\left(x^{\prime}\right) \mathbf{d}(y)\right)^{+}}\right) \cdot\left[\left(\mathbf{r}\left(x^{\prime}\right) \mathbf{d}(y)\right)^{+}, \mathbf{r}(x) \mathbf{d}(y)\right] \\
& \text { (by } \left.(\mathrm{OB} 1)^{\circ}, k \leq_{\mathcal{R}} \mathbf{d}\left(x^{\prime}\right) \mathcal{R} \mathbf{d}(x)\right) \text {. }
\end{aligned}
$$

Similarly, we have that

$$
\mathbf{r}(x) \mathbf{d}(y) \mid y=\left[\mathbf{r}(x) \mathbf{d}(y),\left(\mathbf{r}(x) \mathbf{d}\left(y^{\prime}\right)\right)^{\star}\right] \cdot\left(\left(\mathbf{r}(x) \mathbf{d}\left(y^{\prime}\right)\right)^{\star} \mid y^{\prime}\right) \cdot\left[g, g^{\star}\right]
$$


where $g=\mathbf{r}\left(\left(\mathbf{r}(x) \mathbf{d}\left(y^{\prime}\right)\right)^{\star} \mid y^{\prime}\right)$. So

$$
\begin{aligned}
& x \otimes y=\left.x\right|_{\mathbf{r}(x) \mathbf{d}(y)} \cdot \mathbf{r}(x) \mathbf{d}(y) \mid y \\
& =\left[k^{+}, k\right] \cdot\left(\left.x^{\prime}\right|_{\left(\mathbf{r}\left(x^{\prime}\right) \mathbf{d}(y)\right)^{+}}\right) \cdot\left[\left(\mathbf{r}\left(x^{\prime}\right) \mathbf{d}(y)\right)^{+}, \mathbf{r}(x) \mathbf{d}(y)\right] \cdot \\
& {\left[\mathbf{r}(x) \mathbf{d}(y),\left(\mathbf{r}(x) \mathbf{d}\left(y^{\prime}\right)\right)^{\star}\right] \cdot\left(\left(\mathbf{r}(x) \mathbf{d}\left(y^{\prime}\right)\right)^{\star} \mid y^{\prime}\right) \cdot\left[g, g^{\star}\right]} \\
& =\left[k^{+}, k\right] \cdot\left(\left.x^{\prime}\right|_{\left(\mathbf{r}\left(x^{\prime}\right) \mathbf{d}(y)\right)^{+}}\right) \cdot\left[\left(\mathbf{r}\left(x^{\prime}\right) \mathbf{d}(y)\right)^{+},\left(\mathbf{r}(x) \mathbf{d}\left(y^{\prime}\right)\right)^{\star}\right] \cdot \\
& \left(\left(\mathbf{r}(x) \mathbf{d}\left(y^{\prime}\right)\right)^{\star} \mid y^{\prime}\right) \cdot\left[g, g^{\star}\right] \\
& =\left[k^{+}, k\right] \cdot\left(\left.x^{\prime}\right|_{\left(\mathbf{r}\left(x^{\prime}\right) \mathbf{d}(y)\right)^{+}}\right) \cdot\left[\left(\mathbf{r}\left(x^{\prime}\right) \mathbf{d}(y)\right)^{+}, \mathbf{r}\left(x^{\prime}\right) \mathbf{d}\left(y^{\prime}\right)\right] \cdot \\
& {\left[\mathbf{r}\left(x^{\prime}\right) \mathbf{d}\left(y^{\prime}\right),\left(\mathbf{r}(x) \mathbf{d}\left(y^{\prime}\right)\right)^{\star}\right] \cdot\left(\left(\mathbf{r}(x) \mathbf{d}\left(y^{\prime}\right)\right)^{\star} \mid y^{\prime}\right) \cdot\left[g, g^{\star}\right] \quad(\text { by }(\mathrm{OB} 5))} \\
& =\left[k^{+}, k\right] \cdot\left(\left.x^{\prime}\right|_{\mathbf{r}\left(x^{\prime}\right) \mathbf{d}\left(y^{\prime}\right)}\right) \cdot\left({ }_{\mathbf{r}\left(x^{\prime}\right) \mathbf{d}\left(y^{\prime}\right)} \mid y^{\prime}\right) \cdot\left[g, g^{\star}\right] \quad\left(\text { by }(\mathrm{OB} 2),(\mathrm{OB} 2)^{\circ}\right) \\
& =\left[k^{+}, k\right] \cdot\left(x^{\prime} \otimes y^{\prime}\right) \cdot\left[g, g^{\star}\right] \text {. }
\end{aligned}
$$

Obviously, $\mathbf{d}(x \otimes y)=k^{+} \mathcal{R} k=\mathbf{d}\left(x^{\prime} \otimes y^{\prime}\right)$ and $\mathbf{r}(x \otimes y)=g^{\star} \mathcal{L} g=\mathbf{r}\left(x^{\prime} \otimes y^{\prime}\right)$. It follows from the observation succeeding the definition of $\rho$ that $x \otimes y \rho x^{\prime} \otimes y^{\prime}$.

Lemma 9.9. If $x, y \in P$ with $\exists x \cdot y$ in $P$, then $x \otimes y \rho x \cdot y$.

Proof. If $\exists x \cdot y$ in $P$, then $\mathbf{r}(x)=\mathbf{d}(y)$. So

$$
x \otimes y=\left.x\right|_{\mathbf{r}(x) \mathbf{d}(y)} \cdot \mathbf{r}(x) \mathbf{d}(y)|y=x|_{\mathbf{r}(x)} \cdot \mathbf{d}(y) \mid y
$$

By $(\mathrm{OB} 1)$ and $(\mathrm{OB} 1)^{\circ}$, we have that $[\mathbf{d}(x), k] \cdot\left(\left.x\right|_{\mathbf{r}(x)}\right)=x$, where $\mathbf{d}(x) \mathcal{R} k=$ $\mathbf{d}\left(\left.x\right|_{\mathbf{r}(x)}\right)=\mathbf{d}(x \otimes y)$ and $\left.{ }_{\mathbf{d}(y)} \mid y\right) \cdot[g, \mathbf{r}(y)]=y$, where $\mathbf{r}(y) \mathcal{L} g=\mathbf{r}\left(_{\mathbf{d}(y)} \mid y\right)=$ $\mathbf{r}(x \otimes y)$. Thus,

$$
\left.x\right|_{\mathbf{r}(x)}=[k, \mathbf{d}(x)] \cdot x \text { and } \mathbf{d}(y) \mid y=y \cdot[\mathbf{r}(y), g] .
$$

So, $x \otimes y=[k, \mathbf{d}(x)] \cdot(x \cdot y) \cdot[\mathbf{r}(y), g]$, that is, $(x \otimes y) \cdot[g, \mathbf{r}(y)]=[k, \mathbf{d}(x)] \cdot(x \cdot y)$. Hence, $x \otimes y \rho x \cdot y$.

The next lemma is an immediate consequence of Lemma 9.8 and Lemma 9.9.

Lemma 9.10. Let $x, x^{\prime}, y, y^{\prime} \in P$ be such that $x \rho x^{\prime}$ and $y \rho y^{\prime}$. If $x \cdot y$ and $x^{\prime} \cdot y^{\prime}$ exist in $P$, then $x \cdot y \rho x^{\prime} \cdot y^{\prime}$.

Let $P$ be a weakly orthodox category over $B$ and let $\rho$ be the equivalence 
given in Section 9.1. We define

$$
P \mathbf{S}=P / \rho,
$$

and

$$
\bar{x} \circ \bar{y}=\overline{x \otimes y}
$$

where $x, y \in P$ and $\bar{x}$ denotes the $\rho$-class of $P$ containing $x$.

We remark that by Lemma 9.8, the product o defined above is well-defined.

Our next task is to show that $P \mathbf{S}$ is a weakly $\bar{B}$-orthodox semigroup, where $\bar{B}=\left\{\overline{1_{e}}: e \in B\right\}$.

Lemma 9.11. If e, $f \in B$, then $\overline{1_{e}} \circ \overline{1_{f}}=\overline{1_{e f}}$. Further, the map $\varphi: B \rightarrow \bar{B}$, given by $e \varphi=\overline{1_{e}}$ for any $e \in B$, is an isomorphism, where $\bar{B}=\left\{\overline{1_{e}}: e \in B\right\}$.

Proof. If $e, f \in B$, then $\overline{1_{e}} \circ \overline{1_{f}}=\overline{1_{e} \otimes 1_{f}}$. Notice that

$$
\begin{array}{rlrl}
1_{e} \otimes 1_{f} & =\left(1_{e} \mid e f\right) \cdot\left({ }_{e f} \mid 1_{f}\right) & \\
& =\left(\left.[e, e]\right|_{e f}\right) \cdot\left({ }_{e f} \mid[f, f]\right) & & \\
& =\left[(e f)^{+}, e f\right] \cdot\left[e f,(e f)^{\star}\right] & & \left(\text { by }(\mathrm{OB} 4),(\mathrm{OB} 4)^{\circ}\right) \\
& =\left[(e f)^{+},(e f)^{\star}\right] & & (\text { by }(\mathrm{OB} 5)) .
\end{array}
$$

Since $(e f)^{+} \mathcal{R}$ ef $\mathcal{L}(e f)^{\star}$ and $\left[(e f)^{+}, e f\right] \cdot[e f, e f]=\left[(e f)^{+},(e f)^{\star}\right] \cdot\left[(e f)^{\star}, e f\right]$, we obtain that $\left[(e f)^{+},(e f)^{\star}\right] \rho[e f, e f]$, that is, $1_{e} \otimes 1_{f} \rho 1_{e f}$. Hence, $\overline{1_{e}} \circ \overline{1_{f}}=\overline{1_{e f}}$, and so $\varphi$ is a morphism.

It follows from Lemma 9.2 that if $e, f \in B$ and $\overline{1_{e}}=\overline{1_{f}}$, then $e=f$ so that $\varphi$ is injective. Clearly, $\varphi$ is surjective. Consequently, $\varphi$ is an isomorphism.

Lemma 9.12. If $P$ is a weakly orthodox category over $B$, then $P \mathbf{S}$ is a semigroup.

Proof. Suppose that $x, y, z \in P$. Then

$$
\begin{aligned}
\bar{x} \circ(\bar{y} \circ \bar{z}) & =\bar{x} \circ \overline{y \otimes z} \\
& =\bar{x} \circ \overline{y|\mathbf{r}(y) \mathbf{d}(z) \cdot \mathbf{r}(y) \mathbf{d}(z)| z}
\end{aligned}
$$




$$
\begin{aligned}
& =\overline{x \otimes\left(\left.y\right|_{\mathbf{r}(y) \mathbf{d}(z)} \cdot \mathbf{r}(y) \mathbf{d}(z) \mid z\right)}
\end{aligned}
$$

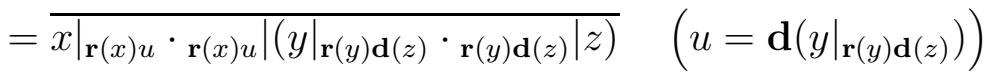

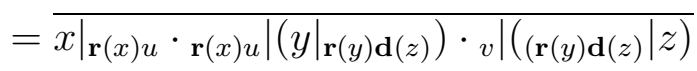

$$
\begin{aligned}
& \left(v=\mathbf{r}\left(\mathbf{r}(x) u \mid\left(\left.y\right|_{\mathbf{r}(y) \mathbf{d}(z)}\right)\right)\right. \text {, by (OB3)). }
\end{aligned}
$$

Since $P$ is weakly orthodox, it follows that $\mathbf{r}(x) u\left|\left(\left.y\right|_{\mathbf{r}(y) \mathbf{d}(z)}\right) \rho(\mathbf{r}(x) \mathbf{d}(y) \mid y)\right|_{g \mathbf{d}(z)}$, where $g=\mathbf{r}(\mathbf{r}(x) \mathbf{d}(y) \mid y)$. Hence, if we put $k=\mathbf{d}\left(\left.(\mathbf{r}(x) \mathbf{d}(y) \mid y)\right|_{g \mathbf{d}(z)}\right)$, then $\mathbf{r}(x) u \mathcal{R} k$, $v \mathcal{L} g \mathbf{d}(z)$ and

$$
\mathbf{r}(x) u\left|\left(\left.y\right|_{\mathbf{r}(y) \mathbf{d}(z)}\right) \cdot[v, g \mathbf{d}(z)]=[\mathbf{r}(x) u, k] \cdot(\mathbf{r}(x) \mathbf{d}(y) \mid y)\right|_{g \mathbf{d}(z)}
$$

whence

$$
\mathbf{r}(x) u\left|\left(\left.y\right|_{\mathbf{r}(y) \mathbf{d}(z)}\right)=[\mathbf{r}(x) u, k] \cdot(\mathbf{r}(x) \mathbf{d}(y) \mid y)\right|_{g \mathbf{d}(z)} \cdot[g \mathbf{d}(z), v] .
$$

So, we go back to the beginning of this proof,

$$
\begin{aligned}
& \bar{x} \circ(\bar{y} \circ \bar{z}) \\
& =\overline{\left.\left.x\right|_{\mathbf{r}(x) u} \cdot\left(\left.[\mathbf{r}(x) u, k] \cdot\left({ }_{\mathbf{r}(x) \mathbf{d}(y)} \mid y\right)\right|_{g \mathbf{d}(z)} \cdot[g \mathbf{d}(z), v]\right) \cdot{ }_{v}\left|{ }_{\mathbf{r}(y) \mathbf{d}(z)}\right| z\right)} \\
& =\overline{\left.x\right|_{\mathbf{r}(x) u} \cdot\left(\left.[\mathbf{r}(x) u, k] \cdot(\mathbf{r}(x) \mathbf{d}(y) \mid y)\right|_{g \mathbf{d}(z)} \cdot[g \mathbf{d}(z), v]\right) \cdot{ }_{v} \mid z} \\
& \left(v=\mathbf{r}\left(\mathbf{r}(x) u \mid\left(\left.y\right|_{\mathbf{r}(y) \mathbf{d}(z)}\right)\right) \leq_{\mathcal{L}} \mathbf{r}(y) \mathbf{d}(z) \leq_{\mathcal{L}} \mathbf{d}(z), \text { by }(\mathrm{OB} 2)\right) \\
& =\overline{\left.\left(\left.x\right|_{\mathbf{r}(x) u} \cdot[\mathbf{r}(x) u, k]\right) \cdot(\mathbf{r}(x) \mathbf{d}(y) \mid y)\right|_{g \mathbf{d}(z)} \cdot[g \mathbf{d}(z), v] \cdot{ }_{v} \mid z} \\
& =\overline{\left.\left.x\right|_{k} \cdot(\mathbf{r}(x) \mathbf{d}(y) \mid y)\right|_{g \mathbf{d}(z)} \cdot\left([g \mathbf{d}(z), v] \cdot{ }_{v} \mid z\right)} \quad\left(k \mathcal{R} \mathbf{r}(x) u \leq_{\mathcal{R}} \mathbf{r}(x) \text {, by }(\mathrm{OB} 2)^{\circ}\right) \\
& =\overline{\left.\left.x\right|_{k} \cdot(\mathbf{r}(x) \mathbf{d}(y) \mid y)\right|_{g \mathbf{d}(z) \cdot g \mathbf{d}(z) \mid z}} \quad\left(g \mathbf{d}(z) \mathcal{L} v \leq_{\mathcal{L}} \mathbf{d}(z) \text {, by }(\mathrm{OB} 2)\right)
\end{aligned}
$$

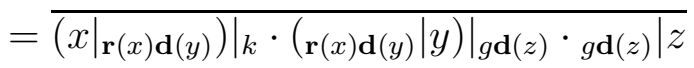

$$
\begin{aligned}
& \left(\text { by }(\mathrm{OB} 1)^{\circ}, k=\mathbf{d}\left(\left.(\mathbf{r}(x) \mathbf{d}(y) \mid y)\right|_{g \mathbf{d}(z)}\right) \leq_{\mathcal{R}} \mathbf{r}(x) \mathbf{d}(y) \leq_{\mathcal{R}} \mathbf{r}(x) \text {, by }(\mathrm{OB} 2)^{\circ}\right)
\end{aligned}
$$

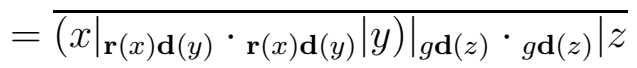

$$
\begin{aligned}
& \left(g \mathbf{d}(z) \leq_{\mathcal{R}} g=\mathbf{r}(\mathbf{r}(x) \mathbf{d}(y) \mid y), k=\mathbf{d}\left(\left.\left(_{\mathbf{r}(x) \mathbf{d}(y)} \mid y\right)\right|_{g \mathbf{d}(z)}\right), \text { by }(\mathrm{OB} 3)^{\circ}\right) \\
& =\overline{\left.(x \otimes y)\right|_{g \mathbf{d}(z)} \cdot g \mathbf{d}(z) \mid z} \\
& =\overline{(x \otimes y) \otimes z} \\
& =\overline{(x \otimes y)} \circ \bar{z} \\
& =(\bar{x} \circ \bar{y}) \circ \bar{z} \text {. }
\end{aligned}
$$

Lemma 9.13. If $P$ is a weakly orthodox category over $B$ and $x \in P$, then 
$\overline{1_{\mathrm{d}(x)}} \widetilde{\mathcal{R}}_{\bar{B}} \bar{x} \widetilde{\mathcal{L}}_{\bar{B}} \overline{1_{\mathbf{r}(x)}}$.

Proof. Let $x \in P$. By Lemma 9.9, we have that

$$
\overline{1_{\mathbf{d}(x)}} \circ \bar{x}=\overline{1_{\mathbf{d}(x)} \otimes x}=\overline{1_{\mathbf{d}(x)} \cdot x}=\bar{x} .
$$

Suppose that $k \in B$ is such that $\overline{1_{k}} \circ \bar{x}=\bar{x}$. Then

$$
\begin{aligned}
\overline{1_{k}} \circ \bar{x} & =\overline{1_{k} \otimes x}=\overline{\left.1_{k}\right|_{k \mathbf{d}(x)} \cdot k \mathbf{d}(x)} \mid x \\
& =\overline{[k, k]||_{k \mathbf{d}(x)} \cdot k \mathbf{d}(x) \mid x} \\
& =\overline{\left[(k \mathbf{d}(x))^{+}, k \mathbf{d}(x)\right] \cdot{ }_{k \mathbf{d}(x)} \mid x}
\end{aligned}
$$

So $\mathbf{d}(x) \mathcal{R}(k \mathbf{d}(x))^{+} \mathcal{R} k \mathbf{d}(x)$, which implies that $k \mathbf{d}(x)=\mathbf{d}(x)$. Thus

$$
\begin{aligned}
\overline{1_{k}} \circ \overline{1_{\mathbf{d}(x)}} & =\overline{1_{k \mathbf{d}(x)}} & & (\text { Lemma 9.11) } \\
& =\overline{1_{\mathbf{d}(x)}} & & (k \mathbf{d}(x)=\mathbf{d}(x)) .
\end{aligned}
$$

Hence, $\bar{x} \widetilde{\mathcal{R}}_{\bar{B}} \overline{1_{\mathbf{d}(x)}}$.

By the dual argument, we show that $\bar{x} \widetilde{\mathcal{L}}_{\bar{B}} \overline{1_{\mathbf{r}(x)}}$.

Now, using Lemma 9.11 and Lemma 9.13, we obtain a criterion for $\widetilde{\mathcal{R}}_{\bar{B}}$ and $\widetilde{\mathcal{L}}_{\bar{B}}$ on $P \mathbf{S}$.

Lemma 9.14. Let $P$ be a weakly orthodox category over $B$ and $x, y \in P$. Then (i) $\bar{x} \widetilde{\mathcal{R}}_{\bar{B}} \bar{y}$ in PS if and only if $\mathbf{d}(x) \mathcal{R} \mathbf{d}(y)$ in $B$;

(ii) $\bar{x} \widetilde{\mathcal{L}}_{\bar{B}} \bar{y}$ in PS if and only if $\mathbf{r}(x) \mathcal{L} \mathbf{r}(y)$ in $B$.

Lemma 9.15. If $x \in P$ and $u, v \in B$ are such that $u \mathcal{R} v$, then $\mathbf{d}\left(\left.x\right|_{\mathbf{r}(x) u}\right)=$ $\mathbf{d}\left(\left.x\right|_{\mathbf{r}(x) v}\right)$.

Proof. Suppose that $x \in P$ and $u, v \in B$ are such that $u \mathcal{R} v$. Then $\mathbf{r}(x) u \mathcal{R} \mathbf{r}(x) v$. So,

$$
\begin{aligned}
\left.x\right|_{\mathbf{r}(x) u} & =\left.x\right|_{\mathbf{r}(x) u} \cdot[\mathbf{r}(x) u, \mathbf{r}(x) u] & \left([\mathbf{r}(x) u, \mathbf{r}(x) u]=1_{\mathbf{r}(x) u}\right) \\
& =\left.x\right|_{\mathbf{r}(x) u} \cdot[\mathbf{r}(x) u, \mathbf{r}(x) v] \cdot[\mathbf{r}(x) v, \mathbf{r}(x) u] & (\text { by }(\mathrm{OB} 5)) \\
& =\left.x\right|_{\mathbf{r}(x) v} \cdot[\mathbf{r}(x) v, \mathbf{r}(x) u] & \left(\mathbf{r}(x) u \mathcal{R} \mathbf{r}(x) v, \text { by }(\mathrm{OB} 2)^{\circ}\right) .
\end{aligned}
$$

Hence, $\mathbf{d}\left(\left.x\right|_{\mathbf{r}(x) u}\right)=\mathbf{d}\left(\left.x\right|_{\mathbf{r}(x) v}\right)$. 
It is a convenient position from which to build our main theorem.

Theorem 9.16. If $P$ is a weakly orthodox category over $B$, then $(P \mathbf{S}, \circ)$ is a weakly $\bar{B}$-orthodox semigroup, where $\bar{B}=\left\{\overline{1_{e}}: e \in B\right\}$.

Proof. In view of Lemma 9.12 and Lemma 9.13, it is only necessary to show that $P \mathbf{S}$ has $(\mathrm{C})$. Suppose that $\bar{x} \widetilde{\mathcal{R}}_{\bar{B}} \bar{y}$ and $\bar{z} \in P \mathbf{S}$. We have that $\bar{z} \circ \bar{x}=$ $\overline{z \otimes x}=\overline{\left.z\right|_{\mathbf{r}(z) \mathbf{d}(x)} \cdot \mathbf{r}(z) \mathbf{d}(x)} \mid x$. Similarly, $\bar{z} \circ \bar{y}=\overline{z \otimes y}=\overline{\left.z\right|_{\mathbf{r}(z) \mathbf{d}(y) \cdot \mathbf{r}(z) \mathbf{d}(y)} \mid y}$. As $\bar{x} \widetilde{\mathcal{R}}_{\bar{B}} \bar{y}$, we obtain that $\mathbf{d}(x) \mathcal{R} \mathbf{d}(y)$ from Lemma 9.14, and so by Lemma 9.15, $\mathbf{d}\left(\left.z\right|_{\mathbf{r}(z) \mathbf{d}(x)}\right)=\mathbf{d}\left(\left.z\right|_{\mathbf{r}(z) \mathbf{d}(y)}\right)$. It follows from Lemma 9.14 that $\bar{z} \circ \bar{y} \widetilde{\mathcal{R}}_{\bar{B}} \bar{z} \circ \bar{x}$, and consequently, $\widetilde{\mathcal{R}}_{\bar{B}}$ is a left congruence. Dually, $\widetilde{\mathcal{L}}_{\bar{B}}$ is a right congruence.

We end this section by producing an admissible morphism between weakly $B$-orthodox semigroups from an orthodox functor. This appears in the next lemma.

Lemma 9.17. Let $P_{1}$ and $P_{2}$ be weakly orthodox categories over $B_{1}$ and $B_{2}$, respectively, and let $F: P_{1} \rightarrow P_{2}$ be an orthodox functor. Then the map FS: $P_{1} \mathbf{S} \rightarrow P_{2} \mathbf{S}$ defined by the rule that $\bar{x} F \mathbf{S}=\overline{x F}$, where $\bar{x} \in P_{1} \mathbf{S}$ and $x \in P_{1}$, is an admissible morphism. Further, if $F_{1}: P_{1} \rightarrow P_{2}$ and $F_{2}: P_{2} \rightarrow P_{3}$ are orthodox functors, then $\left(F_{1} F_{2}\right) \mathbf{S}=F_{1} \mathbf{S} F_{2} \mathbf{S}$.

Proof. In view of Lemma 9.6, if $\bar{x}=\bar{y}$ in $P_{1} \mathbf{S}$, that is, $x \rho y$ in $P_{1}$, then $x F \rho y F$ in $P_{2}$. Hence, $F \mathrm{~S}$ is well-defined.

We now show that $F \mathbf{S}$ is a semigroup morphism. Suppose that $x, y \in P_{1}$. Then

$$
\begin{aligned}
& (\bar{x} \circ \bar{y}) F \mathbf{S}=\left(\overline{\left.x\right|_{\mathbf{r}(x) \mathbf{d}(y)} \cdot \mathbf{r}(x) \mathbf{d}(y) \mid y}\right) F \mathbf{S} \\
& =\overline{\left(\left.x\right|_{\mathbf{r}(x) \mathbf{d}(y)} \cdot \mathbf{r}(x) \mathbf{d}(y) \mid y\right) F} \\
& =\overline{\left(\left.x\right|_{\mathbf{r}(x) \mathbf{d}(y)) F \cdot(\mathbf{r}(x) \mathbf{d}(y)} \mid y\right) F} \\
& =\overline{\left.x F\right|_{(\mathbf{r}(x) \mathbf{d}(y)) F^{\cdot}(\mathbf{r}(x) \mathbf{d}(y)) F} \mid y F} \quad \text { (by }(S 3) \text { and Lemma 9.10) } \\
& \left.=\overline{\left.x F\right|_{\mathbf{r}(x) F \mathbf{d}(y) F} \cdot \mathbf{r}(x) F \mathbf{d}(y) F \mid y F} \quad \text { (by }(S 1)\right) \\
& =\overline{\left.x F\right|_{\mathbf{r}(x F) \mathbf{d}(y F)} \cdot \mathbf{r}(x F) \mathbf{d}(y F)} \mid y F \\
& =\overline{x F \otimes y F} \\
& =\overline{x F} \circ \overline{y F} \\
& =\bar{x} F \mathbf{S} \circ \bar{y} F \mathbf{S} \text {. }
\end{aligned}
$$


Next, we show that $F \mathbf{S}$ is admissible. For any $x \in P_{1}$, we have that $\overline{1_{\mathbf{d}(x)}} \widetilde{\mathcal{R}}_{\bar{B}_{1}} \bar{x} \widetilde{\mathcal{L}}_{\bar{B}_{1}} \overline{1_{\mathbf{r}(x)}}$. Then

$$
\begin{aligned}
\overline{1_{\mathbf{d}(x)}} F \mathbf{S} & =\overline{1_{\mathbf{d}(x)} F}=\overline{[\mathbf{d}(x), \mathbf{d}(x)] F} \\
& =\overline{[\mathbf{d}(x) F, \mathbf{d}(x) F]} \\
& =\overline{[\mathbf{d}(x F), \mathbf{d}(x F)]} \\
& =\overline{1_{\mathbf{d}(x F)}} \widetilde{\mathcal{R}}_{\bar{B}_{2}} \overline{x F}=\bar{x} F \mathbf{S} .
\end{aligned}
$$

Dually, we have that $\overline{1_{\mathbf{r}(x)}} F \mathbf{S} \widetilde{\mathcal{L}}_{\bar{B}_{2}} \bar{x} F \mathbf{S}$.

Finally, $\overline{1_{e}} F \mathbf{S}=\overline{1_{e} F}=\overline{1_{e F}}$ as $F$ is a functor, so that $\overline{B_{1}} F \mathbf{S} \subseteq \overline{B_{2}}$. Since $F: B_{1} \rightarrow B_{2}$ is a morphism, by Lemma 9.11 , we have that $F \mathbf{S}$ is a morphism from $\overline{B_{1}}$ to $\overline{B_{2}}$.

To sum up, we have that $F \mathbf{S}$ is an admissible morphism from $P_{1} \mathbf{S}$ to $P_{2} \mathbf{S}$.

Consequently, $\mathbf{S}: \mathcal{W O C} \rightarrow \mathcal{W O}$ is a functor by Theorem 9.16 and Lemma 9.17.

\subsection{Correspondence}

In this section, our purpose is, starting with a weakly $B$-orthodox semigroup, to build a converse to Theorem 9.16. These results present a correspondence between weakly orthodox categories over bands and weakly $B$-orthodox semigroups.

Let $S$ be a weakly $B$-orthodox semigroup and let $K$ be a representative of $B$. For any $e \in B$, we will use $e^{\star}$ and $e^{+}$to denote the elements of $K$ which are $\mathcal{L}$-related to $e$ in $B$ and $\mathcal{R}$-related to $e$ in $B$, respectively. Set

$$
S \mathbf{C}=\left\{(e, x, f): e \widetilde{\mathcal{R}}_{B} x \widetilde{\mathcal{L}}_{B} f, \quad e, f \in B\right\} \subseteq B \times S \times B
$$

We put

$$
\mathbf{d}((e, x, f))=e(\text { abbreviated to } \mathbf{d}(e, x, f)=e)
$$

and

$$
\mathbf{r}((e, x, f))=f(\text { abbreviated to } \mathbf{r}(e, x, f)=f)
$$

for all $(e, x, f) \in S \mathbf{C}$, and define a partial binary operation $\cdot$ on $S \mathbf{C}$ by the rule that

$$
(e, x, f) \cdot(f, y, v)=(e, x y, v)
$$


where $(e, x, f),(f, y, v) \in S \mathbf{C}$ and $x y$ is the product of $x$ and $y$ in $S$. If $e, f \in B$ with $e \mathcal{D} f$, then we define $[e, f]=(e, e f, f)$. Obviously, $[e, f] \in S \mathbf{C}$. For any $(e, x, f) \in S \mathbf{C}$ and $u, v \in B$ with $u \leq_{\mathcal{L}} e$ and $v \leq_{\mathcal{R}} f$, we define

$$
{ }_{u} \mid(e, x, f)=\left(u, u x,(u x)^{\star}\right) \text { and }\left.(e, x, f)\right|_{v}=\left((x v)^{+}, x v, v\right) .
$$

Lemma 9.18. The set $S \mathbf{C}$ is a weakly orthodox category over $B$ with restriction and co-restriction defined above.

Proof. It is easy to see that $S \mathbf{C}$ forms a category with set of objects $B$ and morphisms the triples as given. For any $e \in B,[e, e]=(e, e, e)$ is the identity map associated to $e$.

(OB1) Suppose that $(e, x, f) \in S \mathbf{C}$ and $u \leq_{\mathcal{L}} e$ in $B$. Then ${ }_{u} \mid(e, x, f)=$ $\left(u, u x,(u x)^{\star}\right)$ and so by Lemma $2.8,(u x)^{\star} \leq_{\mathcal{L}} f$. In particular, if $u=e$, then ${ }_{e} \mid(e, x, f)=\left(e, x, x^{\star}\right)$ and $\mathbf{r}(e \mid(e, x, f))=x^{\star} \widetilde{\mathcal{L}}_{B} x \widetilde{\mathcal{R}}_{B} f$, so that

$$
e_{e}(e, x, f) \cdot\left[x^{\star}, f\right]=\left(e, x, x^{\star}\right) \cdot\left(x^{\star}, x^{\star}, f\right)=(e, x, f),
$$

as required.

(OB2) If $(e, x, f) \in S \mathbf{C}$ and $g, h \in B$ with $g \leq_{\mathcal{L}} h \leq_{\mathcal{L}} e$, then

$$
{ }_{g}\left|\left({ }_{h} \mid(e, x, f)\right)={ }_{g}\right|\left(h, h x,(h x)^{\star}\right)=\left(g, g h x,(g h x)^{\star}\right)=\left(g, g x,(g x)^{\star}\right)={ }_{g} \mid(e, x, f) .
$$

In addition, if $g \mathcal{L} h \leq_{\mathcal{L}} e$, then $[g, h]$ is defined and $[g, h]=(g, g, h)$. Thus

$$
\begin{aligned}
{[g, h] \cdot{ }_{h} \mid(e, x, f) } & =(g, g, h) \cdot\left(h, h x,(h x)^{\star}\right) \\
& =\left(g, g h x,(h x)^{\star}\right) \\
& =\left(g, g x,(h x)^{\star}\right) \\
& =\left(g, g x,(g x)^{\star}\right) \\
& ={ }_{g} \mid(e, x, f) .
\end{aligned}
$$

(OB3) If $(e, x, f),(f, y, v) \in S \mathbf{C}$ and $u \in B$ with $u \leq_{\mathcal{L}} e$, then

$$
\begin{gathered}
{ }_{u}\left|((e, x, f) \cdot(f, y, v))={ }_{u}\right|(e, x y, v)=\left(u, u x y,(u x y)^{\star}\right), \\
{ }_{u} \mid(e, x, f)=\left(u, u x,(u x)^{\star}\right)
\end{gathered}
$$


and by Lemma $2.8,(u x)^{\star} \leq_{\mathcal{L}} f$, we have that

$$
(u x)^{\star} \mid(f, y, v)=\left((u x)^{\star},(u x)^{\star} y,\left((u x)^{\star} y\right)^{\star}\right) .
$$

So

$$
\begin{aligned}
{ }_{u}\left|(e, x, f) \cdot{ }_{(u x)^{\star}}\right|(f, y, v) & =\left(u, u x,(u x)^{\star}\right) \cdot\left((u x)^{\star},(u x)^{\star} y,\left((u x)^{\star} y\right)^{\star}\right) \\
& =\left(u, u x y,\left((u x)^{\star} y\right)^{\star}\right) .
\end{aligned}
$$

Since $u x \widetilde{\mathcal{L}}_{B}(u x)^{\star}$, we have that $u x y \widetilde{\mathcal{L}}_{B}(u x)^{\star} y$. Thus $(u x y)^{\star}=\left((u x)^{\star} y\right)^{\star}$, and SO ${ }_{u}\left|((e, x, f) \cdot(f, y, v))={ }_{u}\right|(e, x, f) \cdot{ }_{(u x)^{\star}} \mid(f, y, v)$.

(OB4) If $e, f, h \in B$ with $e \mathcal{D} f$ and $h \leq_{\mathcal{L}} e$, then ${ }_{h} \mid[e, f]$ exists and

$$
{ }_{h}\left|[e, f]={ }_{h}\right|(e, e f, f)=\left(h, h e f,(h e f)^{\star}\right)=\left(h, h f,(h f)^{\star}\right) .
$$

As $h \leq_{\mathcal{L}} e \mathcal{D} f$, we have that $h=h e \mathcal{D} h f \mathcal{L}(h f)^{\star}$, and so $\left[h,(h f)^{\star}\right]$ exists. In addition, $\left[h,(h f)^{\star}\right]=\left(h, h(h f)^{\star},(h f)^{\star}\right)$. We note that

$$
\begin{aligned}
h f & =h f(h f)^{\star} & & \left(h f \mathcal{L}(h f)^{\star}\right) \\
& =h h f(h f)^{\star} & & \left(h^{2}=h\right) \\
& =h e h f(h f)^{\star} & & \left(h \leq_{\mathcal{L}} e\right) \\
& =h e(h f)^{\star} & & \left(h e \mathcal{D} h f \mathcal{D}(h f)^{\star}\right) \\
& =h(h f)^{\star} & & \left(h \leq_{\mathcal{L}} e\right),
\end{aligned}
$$

whence, $\left(h, h f,(h f)^{\star}\right)=\left(h, h(h f)^{\star},(h f)^{\star}\right)$, that is, ${ }_{h} \mid[e, f]=\left[h,(h f)^{\star}\right]$.

(OB5) If $e, f, g \in B$ are such that $e \mathcal{D} f \mathcal{D} g$, then $[e, f],[f, g]$ and $[e, g]$ are defined. Further, we have that

$$
[e, f] \cdot[f, g]=(e, e f, f) \cdot(f, f g, g)=(e, e f f g, g)=(e, e g, g)=[e, g]
$$

Hence, (OB5) holds.

Finally, we show that $S \mathbf{C}$ is weakly orthodox. Assume that $(e, x, f) \in S \mathbf{C}$ and $g, h \in B$. Then

$$
\left.(e, x, f)\right|_{f h}=\left((x f h)^{+}, x f h, f h\right)=\left((x h)^{+}, x h, f h\right),
$$




$$
\begin{aligned}
g(x h)^{+} \mid\left(\left.(e, x, f)\right|_{f h}\right) & ={ }_{g(x h)^{+}} \mid\left((x h)^{+}, x h, f h\right) \\
& =\left(g(x h)^{+}, g(x h)^{+} x h,\left(g(x h)^{+} x h\right)^{\star}\right) \\
& =\left(g(x h)^{+}, g x h,(g x h)^{\star}\right) \quad\left((x h)^{+} \widetilde{\mathcal{R}}_{B} x h\right), \\
g e \mid(e, x, f) & =\left(\text { ge, gex },(g e x)^{\star}\right)=\left(g e, g x,(g x)^{\star}\right)
\end{aligned}
$$

and

$$
\begin{aligned}
\left.{ }_{g e} \mid(e, x, f)\right)\left.\right|_{(g x)^{\star} h} & =\left.\left(g e, g x,(g x)^{\star}\right)\right|_{(g x)^{\star} h} \\
& =\left(\left(g x(g x)^{\star} h\right)^{+}, g x(g x)^{\star} h,(g x)^{\star} h\right) \\
& =\left((g x h)^{+}, g x h,(g x)^{\star} h\right) \quad\left((g x)^{\star} \widetilde{\mathcal{L}}_{B} g x\right) .
\end{aligned}
$$

Notice that

$$
\mathbf{d}\left(g(x h)^{+} \mid\left(\left.(e, x, f)\right|_{f h}\right)\right)=g(x h)^{+} \widetilde{\mathcal{R}}_{B} g x h \widetilde{\mathcal{R}}_{B}(g x h)^{+}=\mathbf{d}\left(\left.(g e \mid(e, x, f))\right|_{(g x)^{\star} h}\right)
$$

and

$$
\mathbf{r}\left(g(x h)^{+} \mid\left(\left.(e, x, f)\right|_{f h}\right)\right)=(g x h)^{\star} \widetilde{\mathcal{L}}_{B} g x h \widetilde{\mathcal{L}}_{B}(g x)^{\star} h=\mathbf{r}\left(\left.\left(_{g e} \mid(e, x, f)\right)\right|_{\left.(g x)^{\star} h\right) .}\right.
$$

Further,

$$
\begin{aligned}
& {\left.\left[g(x h)^{+},(g x h)^{+}\right] \cdot\left({ }_{g e} \mid(e, x, f)\right)\right|_{(g x)^{\star} h} } \\
= & \left(g(x h)^{+},(g x h)^{+},(g x h)^{+}\right) \cdot\left((g x h)^{+}, g x h,(g x)^{\star} h\right) \\
= & \left(g(x h)^{+}, g x h,(g x)^{\star} h\right) \\
= & \left(g(x h)^{+}, g x h,(g x h)^{\star}\right) \cdot\left((g x h)^{\star},(g x h)^{\star},(g x)^{\star} h\right) \\
= & g(x h)^{+} \mid\left(\left.(e, x, f)\right|_{f h}\right) \cdot\left[(g x h)^{\star},(g x)^{\star} h\right] .
\end{aligned}
$$

Thus, $g(x h)^{+}\left|\left(\left.(e, x, f)\right|_{f h}\right) \rho\left({ }_{g e} \mid(e, x, f)\right)\right|_{(g x)^{\star} h}$.

In Section 9.2, orthodox functors between weakly orthodox categories over bands give rise to admissible morphisms. In the following, we produce a converse to this result and so provide a functor $\mathrm{C}: \mathcal{W O} \rightarrow \mathcal{W O C}$.

Lemma 9.19. Let $S$ be a weakly $B_{1}$-orthodox semigroup and $T$ be a weakly $B_{2}$ orthodox semigroup. Suppose that $\theta: S \rightarrow T$ is an admissible morphism. Then the map $\theta \mathbf{C}: S \mathbf{C} \rightarrow T \mathbf{C}$ given by the rule that $e \theta \mathbf{C}=e \theta$ and $(e, x, f) \theta \mathbf{C}=$ $(e \theta, x \theta, f \theta)$ is an orthodox functor. Further, if $\theta_{1}: S \rightarrow T$ and $\theta_{2}: T \rightarrow Q$ are admissible morphisms, then $\left(\theta_{1} \theta_{2}\right) \mathbf{C}=\theta_{1} \mathbf{C} \theta_{2} \mathbf{C}$. 
Proof. Clearly, $\theta \mathbf{C}$ is a morphism from $B_{1}$ to $B_{2}$ and it is a functor as it preserves products, identities and the domain and co-domain of any morphism in $S \mathbf{C}$.

(S2) Suppose that $e, f \in B_{1}$ are such that $e \mathcal{D} f$. Then $[e, f]=(e, e f, f)$, and so

$$
\begin{aligned}
{[e, f] \theta \mathbf{C} } & =(e, e f, f) \theta \mathbf{C}=(e \theta,(e f) \theta, f \theta) \\
& =(e \theta, e \theta f \theta, f \theta) \\
& =[e \theta, f \theta] \\
& =[e \theta \mathbf{C}, f \theta \mathbf{C}] .
\end{aligned}
$$

(S3) If $(e, x, f) \in S \mathbf{C}$ and $u \in B_{1}$ with $u \leq_{\mathcal{L}} e$, then ${ }_{u} \mid(e, x, f)=\left(u, u x,(u x)^{\star}\right)$, and so $\left.{ }_{u} \mid(e, x, f)\right) \theta \mathbf{C}=\left(u \theta,(u x) \theta,(u x)^{\star} \theta\right)$. In addition,

$$
\begin{aligned}
{ }_{u \theta \mathbf{C}} \mid(e, x, f) \theta \mathbf{C} & ={ }_{u \theta} \mid(e \theta, x \theta, f \theta) \\
& =\left(u \theta, u \theta x \theta,(u \theta x \theta)^{\star}\right) \\
& =\left(u \theta,(u x) \theta,((u x) \theta)^{\star}\right) .
\end{aligned}
$$

Since $\theta$ is admissible, $((u x) \theta)^{\star} \mathcal{L}(u x)^{\star} \theta$ and

$$
\begin{aligned}
\left(u \theta,(u x) \theta,(u x)^{\star} \theta\right) & =\left(u \theta,(u x) \theta,((u x) \theta)^{\star}\right) \cdot\left(((u x) \theta)^{\star},(u x)^{\star} \theta,(u x)^{\star} \theta\right) \\
& =\left(u \theta,(u x) \theta,((u x) \theta)^{\star}\right) \cdot\left[((u x) \theta)^{\star},(u x)^{\star} \theta\right] .
\end{aligned}
$$

Hence, $\left({ }_{u} \mid(e, x, f)\right) \theta \mathbf{C} \rho_{u \theta \mathbf{C}} \mid(e, x, f) \theta \mathbf{C}$.

It is routine to see that $\left(\theta_{1} \theta_{2}\right) \mathbf{C}=\theta_{1} \mathbf{C} \theta_{2} \mathbf{C}$.

We close this section by establishing a correspondence between the category of weakly $B$-orthodox semigroups and the category of weakly orthodox categories over bands.

Lemma 9.20. If $S$ is a weakly B-orthodox semigroup, then there exists an isomorphism $\eta_{S}$ from $S$ to $S$ CS.

Proof. Let $x \in S$ and $e, f \in B$ with $e \widetilde{\mathcal{R}}_{B} x \widetilde{\mathcal{L}}_{B} f$. Then $(e, x, f) \in S \mathbf{C}$. We define a mapping $\eta_{S}: S \rightarrow S$ CS by $x \mapsto \overline{(e, x, f)}$. If $u \widetilde{\mathcal{R}}_{B} x \widetilde{\mathcal{L}}_{B} v$, then $u \mathcal{R} e$, $v \mathcal{L} f$ and $[u, e] \cdot(e, x, f)=(u, e, e) \cdot(e, x, f)=(u, x, f)=(u, x, v) \cdot[v, f]$. Thus, $(e, x, f) \rho(u, x, v)$. Hence, $\eta_{S}$ is well-defined.

Clearly, $\eta_{S}$ is surjective. To show that $\eta_{S}$ is injective, we suppose that $x, y \in$ $S$ with $x \eta_{S}=y \eta_{S}$. Then $\overline{(e, x, f)}=\overline{(g, y, h)}$, where $e \widetilde{\mathcal{R}}_{B} x \widetilde{\mathcal{L}}_{B} f$ and $g \widetilde{\mathcal{R}}_{B}$ y $\widetilde{\mathcal{L}}_{B} h$. 
So $e \mathcal{R} g, f \mathcal{L} h$ and $[e, g] \cdot(g, y, h)=(e, x, f) \cdot[f, h]$, that is, $(e, y, h)=(e, x, h)$. Obviously, $x=y$.

We now show that $\eta_{S}$ is a morphism. Assume that $x, y \in S$ are such that $e \widetilde{\mathcal{R}}_{B} x \widetilde{\mathcal{L}}_{B} f$ and $g \widetilde{\mathcal{R}}_{B} y \widetilde{\mathcal{L}}_{B} h$. Then $x g \widetilde{\mathcal{R}}_{B} x y$, and so $(x g)^{+}=(x y)^{+}$. Dually, $(f y)^{\star}=(x y)^{\star}$. Thus,

$$
\begin{aligned}
x \eta_{S} y \eta_{S} & =\overline{(e, x, f)} \circ \overline{(g, y, h)}=\overline{(e, x, f) \otimes(g, y, h)} \\
& =\overline{\left.(e, x, f)\right|_{f g} \cdot f g \mid(g, y, h)} \\
& =\overline{\left((x g)^{+}, x g, f g\right) \cdot\left(f g, f y,(f y)^{\star}\right)} \\
& =\overline{\left((x g)^{+}, x g f y,(f y)^{\star}\right)} \quad(x g f y=x f g f g y=x f g y=x y) \\
& =\overline{\left((x g)^{+}, x y,(f y)^{\star}\right)} \\
& =\overline{\left((x y)^{+}, x y,(x y)^{\star}\right)} \quad\left((x g)^{+}=(x y)^{+},(f y)^{\star}=(x y)^{\star}\right) \\
& =(x y) \eta_{S} .
\end{aligned}
$$

Finally, we note that $\eta_{S}$ preserves the distinguished band as

$$
e \eta_{S}=\overline{(e, e, e)}=\overline{1_{e}}
$$

for all $e \in B$.

Conversely, we have:

Lemma 9.21. Let $P$ be a weakly orthodox category over $B$. Then there exists an isomorphism $\tau_{P}$ from $P$ to PSC, where an isomorphism between two weakly orthodox categories means a bijective orthodox functor.

Proof. We define a map $\tau_{P}: P \rightarrow P$ SC by the rule that $e \tau_{P}=\overline{1_{e}}$ and $x \tau_{P}=$ $\left(\overline{1_{\mathbf{d}(x)}}, \bar{x}, \overline{1_{\mathbf{r}(x)}}\right)$ for all $e \in B=\mathrm{Ob}(P)$ and $x \in P=\operatorname{Mor}(P)$. Clearly, $\tau_{P}$ maps $P$ into PSC.

Notice that the distinguished band of $P \mathrm{~S}$ is $\bar{B}$, which is the set of objects of PSC. By Lemma 9.11, $\tau_{P}: B \rightarrow \bar{B}: e \mapsto \overline{1_{e}}$ is an isomorphism.

Now, we show that $\tau_{P}$ preserves $\mathbf{d}$ and $\mathbf{r}$. Suppose that $x \in P$. Then by the definition of $\tau_{P}$,

$$
\mathbf{d}(x) \tau_{P}=\overline{1_{\mathbf{d}(x)}}, \mathbf{r}(x) \tau_{P}=\overline{1_{\mathbf{r}(x)}}
$$

and

$$
x \tau_{P}=\left(\overline{1_{\mathbf{d}(x)}}, \bar{x}, \overline{1_{\mathbf{r}(x)}}\right),
$$


so that $\mathbf{d}\left(x \tau_{P}\right)=\overline{1_{\mathbf{d}(x)}}=\mathbf{d}(x) \tau_{P}$ and dually for $\mathbf{r}$. Thus, $\tau_{P}$ preserves $\mathbf{d}$ and $\mathbf{r}$.

If $x, y \in P$ with $x \cdot y$ defined in $P$, then $\mathbf{r}(x)=\mathbf{d}(y)$ and so $x \tau_{P} y \tau_{P}$ is defined in PSC and

$$
\begin{aligned}
x \tau_{P} y \tau_{P} & =\left(\overline{1_{\mathbf{d}(x)}}, \bar{x}, \overline{1_{\mathbf{r}(x)}}\right) \cdot\left(\overline{1_{\mathbf{d}(y)}}, \bar{y}, \overline{1_{\mathbf{r}(y)}}\right) \\
& =\left(\overline{1_{\mathbf{d}(x)}}, \bar{x} \circ \bar{y}, \overline{1_{\mathbf{r}(y)}}\right) \\
& =\left(\overline{1_{\mathbf{d}(x)}}, \overline{x \otimes y}, \overline{1_{\mathbf{r}(y)}}\right. \\
& =\left(\overline{1_{\mathbf{d}(x)}}, \overline{x \cdot y}, \overline{1_{\mathbf{r}(y)}}\right) \quad(\mathbf{r}(x)=\mathbf{d}(y), \text { Lemma } 9.9) \\
& =\left(\overline{1_{\mathbf{d}(x \cdot y)}}, \overline{x \cdot y}, \overline{1_{\mathbf{r}(x \cdot y)}}\right) \\
& =(x \cdot y) \tau_{P}
\end{aligned}
$$

which implies that $\tau_{P}$ preserves products. Also, $\tau_{P}$ preserves identities since $1_{e} \tau_{P}=\left(\overline{1_{e}}, \overline{1_{e}}, \overline{1_{e}}\right)=1_{\overline{1_{e}}}=1_{e \tau_{P}}$. Thus, $\tau_{P}$ is a functor.

Let $e, f \in B$ be such that $e \mathcal{D} f$. Then $[e, f] \tau_{P}=\left(\overline{1_{e}}, \overline{[e, f]}, \overline{1_{f}}\right)$. As $e \mathcal{R}$ ef $\mathcal{L} f$ and $[e, e f] \cdot 1_{e f}=[e, e f]=[e, f] \cdot[f, e f]$, we have that $\overline{[e, f]}=\overline{1_{e f}}$. Thus,

$$
\begin{aligned}
{[e, f] \tau_{P} } & =\left(\overline{1_{e}}, \overline{1_{e f}}, \overline{1_{f}}\right) \\
& =\left(\overline{1_{e}}, \overline{1_{e}} \circ \overline{1_{f}}, \overline{1_{f}}\right) \quad(\text { Lemma } 9.11) \\
& =\left[\overline{1_{e}}, \overline{1_{f}}\right] \\
& =\left[e \tau_{P}, f \tau_{P}\right] .
\end{aligned}
$$

Hence, $\tau_{P}$ satisfies Condition (S2).

To show that (S3) holds, we assume that $x \in P$ and $e \in B$ with $e \leq_{\mathcal{L}} \mathbf{d}(x)$. Then $e \tau_{P} \leq_{\mathcal{L}} \mathbf{d}(x) \tau_{P}=\mathbf{d}\left(x \tau_{P}\right)$ as $\tau_{P}$ is an isomorphism from $B$ to $\bar{B}$ shown above. Hence, ${ }_{e} \mid x$ and ${ }_{e \tau_{P}} \mid x \tau_{P}$ are defined. Observe that $\left({ }_{e} \mid x\right) \tau_{P}=\left(\overline{1_{e}}, \bar{e} \mid x, \overline{\left.1_{\mathbf{r}(e} \mid x\right)}\right)$ and

$$
\begin{aligned}
{ }_{e \tau_{P}} \mid x \tau_{P} & =\overline{1_{e}} \mid\left(\overline{1_{\mathbf{d}(x)}}, \bar{x}, \overline{1_{\mathbf{r}(x)}}\right) \\
& =\left(\overline{1_{e}}, \overline{1_{e}} \circ \bar{x},\left(\overline{1_{e}} \circ \bar{x}\right)^{\star}\right) \\
& =\left(\overline{1_{e}}, \overline{1_{e} \otimes x},\left(\overline{1_{e} \otimes x}\right)^{\star}\right) \\
& =\left(\overline{1_{e}}, \overline{\left.[e, e]\right|_{e \mathbf{d}(x)} \cdot e \mathbf{d}(x) \mid x}, \overline{\left(\overline{\left.[e, e]\right|_{e \mathbf{d}(x)} \cdot e \mathbf{d}(x) \mid x}\right)^{\star}}\right) \\
& =\left(\overline{1_{e}}, \overline{\left.[e, e]\right|_{e} \cdot{ }_{e} \mid x},\left(\overline{[e, e]\left|{ }_{e} \cdot{ }_{e}\right| x}\right)^{\star}\right) \quad\left(e \leq_{\mathcal{L}} \mathbf{d}(x)\right) \\
& =\left(\overline{1_{e}}, \overline{\left[e^{+}, e\right] \cdot{ }_{e} \mid x},\left(\overline{\left[e^{+}, e\right] \cdot{ }_{e} \mid x}\right)^{\star}\right) \quad\left(\operatorname{by}(\mathrm{OB} 4)^{\circ}\right) \\
& =\left(\overline{1_{e}}, \overline{[e, e] \cdot{ }_{e} \mid x},\left(\overline{[e, e] \cdot{ }_{e} \mid x}\right)^{\star}\right) \quad\left(\left[e^{+}, e\right] \rho 1_{e}=[e, e],\right. \text { Lemma 9.10) }
\end{aligned}
$$




$$
=\left(\overline{1_{e}}, \bar{e} \overline{\mid x},(\bar{e} \bar{x})^{\star}\right) \quad\left([e, e]=1_{e}\right) .
$$

Clearly,

$$
\begin{gathered}
\mathbf{d}\left(\left({ }_{e} \mid x\right) \tau_{P}\right)=\overline{1_{e}}=\mathbf{d}\left({ }_{e \tau_{P}} \mid x \tau_{P}\right), \\
\mathbf{r}\left(\left({ }_{e} \mid x\right) \tau_{P}\right)=\overline{1_{\mathbf{r}(e \mid x)}} \widetilde{\mathcal{L}}_{\bar{B}} \overline{{ }_{e} \mid x} \widetilde{\mathcal{L}}_{\bar{B}}\left(\overline{{ }_{e} \mid x}\right)^{\star}=\mathbf{r}\left({ }_{e \tau_{P}} \mid x \tau_{P}\right)
\end{gathered}
$$

and

$$
\begin{aligned}
& \left({ }_{e} \mid x\right) \tau_{P} \cdot\left[\mathbf{r}\left((e \mid x) \tau_{P}\right), \mathbf{r}\left(e \tau_{P} \mid x \tau_{P}\right)\right] \\
& =\left(\overline{1_{e}}, \bar{e} \mid x, \overline{1_{\mathbf{r}(e \mid x)}}\right) \cdot\left[\overline{1_{\mathbf{r}(e \mid x)}},(\bar{e} \mid x)^{\star}\right] \\
& =\left(\overline{1_{e}}, \overline{{ }_{e} \mid x}, \overline{1_{\mathbf{r}(e \mid x)}}\right) \cdot\left(\overline{1_{\mathbf{r}(e \mid x)}}, \overline{1_{\mathbf{r}(e \mid x)}} \circ\left(\overline{{ }_{e} \mid x}\right)^{\star},(\bar{e} \mid x)^{\star}\right) \\
& =\left(\overline{1_{e}}, \bar{e} \bar{e}, \overline{1_{\mathbf{r}(e \mid x)}}\right) \cdot\left(\overline{1_{\mathbf{r}(e \mid x)}}, \overline{1_{\mathbf{r}(e \mid x)}},(\overline{e \mid x})^{\star}\right) \quad\left(\overline{1_{\mathbf{r}(e \mid x)}} \mathcal{L}\left(\overline{e_{e} \mid x}\right)^{\star}\right) \\
& =\left(\overline{1_{e}}, \overline{{ }_{e} \mid x} \circ \overline{1_{\mathbf{r}(e \mid x)}},\left(\overline{e_{e} \mid x}\right)^{\star}\right) \\
& =\left(\overline{1_{e}}, \bar{e} \mid x,\left(\overline{{ }_{e} \mid x}\right)^{\star}\right) \quad\left(\overline{1_{\mathbf{r}(e \mid x)}} \widetilde{\mathcal{L}}_{\bar{B}} \overline{e_{e} \mid x}\right) \\
& ={ }_{e \tau_{P}} \mid x \tau_{P},
\end{aligned}
$$

so that $\left.{ }_{e} \mid x\right) \tau_{P} \rho_{e \tau_{P}} \mid x \tau_{P}$ and (S3) holds.

Next, suppose that $x, y \in P$ with $x \tau_{P}=y \tau_{P}$. Then $\left(\overline{1_{\mathbf{d}(x)}}, \bar{x}, \overline{1_{\mathbf{r}(x)}}\right)=$ $\left(\overline{1_{\mathbf{d}(y)}}, \bar{y}, \overline{1_{\mathbf{r}(y)}}\right)$, which implies that $\bar{x}=\bar{y}$, and also $\mathbf{d}(x)=\mathbf{d}(y), \mathbf{r}(x)=\mathbf{r}(y)$ by Lemma 9.11. Further, by Lemma 9.2, $x=y$.

We now show that $\tau_{P}$ is surjective. Let $\left(\overline{1_{e}}, \bar{x}, \overline{1_{f}}\right)$ be in PSC. Then $\overline{1_{e}} \widetilde{\mathcal{R}}_{\bar{B}} \bar{x} \widetilde{\mathcal{R}}_{\bar{B}} \overline{1_{\mathbf{d}(x)}}$ and $\overline{1_{f}} \widetilde{\mathcal{L}}_{\bar{B}} \bar{x} \widetilde{\mathcal{L}}_{\bar{B}} \overline{1_{\mathbf{r}(x)}}$, that is, $\overline{1_{e}} \mathcal{R} \overline{1_{\mathbf{d}(x)}}$ and $\overline{1_{f}} \mathcal{L} \overline{1_{\mathbf{r}(x)}}$ so that by Lemma 9.11, e $\mathcal{R} \mathbf{d}(x)$ and $f \mathcal{L} \mathbf{r}(x)$. Put $x^{\prime}=[e, \mathbf{d}(x)] \cdot x \cdot[\mathbf{r}(x), f]$. Certainly, $x^{\prime} \rho x$, that is, $\overline{x^{\prime}}=\bar{x}$. Thus, $x^{\prime} \tau_{P}=\left(\overline{1_{e}}, \overline{x^{\prime}}, \overline{1_{f}}\right)=\left(\overline{1_{e}}, \bar{x}, \overline{1_{f}}\right)$, and consequently, $\tau_{P}$ is surjective.

Lemma 9.22. For any $S \in O b(\mathcal{W O})$, we define $S \eta=\eta_{S}$, where $\eta_{S}$ is defined in Lemma 9.20. Then $\eta$ is a natural equivalence of the functors $I_{\mathcal{W O}}$ and $\mathbf{C S}$.

Proof. Let $\theta: S_{1} \rightarrow S_{2}$ in $\mathcal{W O}$, where $S_{1}$ and $S_{2}$ are over $B_{1}$ and $B_{2}$, respectively. Then for any $x \in S_{1}$, we have by the definition of $\eta_{S}$ in Lemma 9.20 that

$$
\begin{aligned}
\left(x \eta_{S_{1}}\right) \theta \mathbf{C S} & =\overline{(e, x, f)} \theta \mathbf{C S} \quad\left(e \widetilde{\mathcal{R}}_{B_{1}} x \widetilde{\mathcal{L}}_{B_{1}} f\right) \\
& =\overline{(e, x, f) \theta \mathbf{C}} \\
& =\overline{(e \theta, x \theta, f \theta)} \\
& =(x \theta) \eta_{S_{2}} \quad\left(e \theta \widetilde{\mathcal{R}}_{B_{2}} x \theta \widetilde{\mathcal{L}}_{B_{2}} f \theta\right) .
\end{aligned}
$$

Thus the diagram below commutes, and so $\eta=\left(\eta_{S}\right)$ is a natural morphism of 
$I_{\mathcal{W O}}$ and CS.

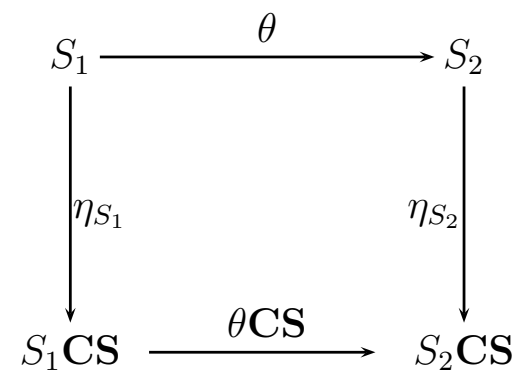

Figure 9.1: A natural transformation of $I_{\mathcal{W O}}$ and CS

Similarly, we have:

Lemma 9.23. For any $P \in O b(\mathcal{W O C})$, we define $P \tau=\tau_{P}$, where $\tau_{P}$ is defined in Lemma 9.21. Then $\tau$ is a natural equivalence of the functors $I_{\mathcal{W O C}}$ and $\mathbf{S C}$.

Proof. Let $F: P_{1} \rightarrow P_{2}$ in $\mathcal{W O C}$, where $P_{1}$ and $P_{2}$ are over $B_{1}$ and $B_{2}$, respectively. Then for any $x \in P_{1}$, we have by the definition of $\tau_{P}$ in Lemma 9.21 that

$$
\begin{aligned}
\left(x \tau_{P_{1}}\right) F \mathbf{S C} & =\left(\overline{1_{\mathbf{d}(x)}}, \bar{x}, \overline{1_{\mathbf{r}(x)}}\right) F \mathbf{S C} \\
& =\left(\overline{1_{\mathbf{d}(x)}} F \mathbf{S}, \bar{x} F \mathbf{S}, \overline{1_{\mathbf{r}(x)}} F \mathbf{S}\right) \\
& =\left(\overline{1_{\mathbf{d}(x)} F}, \overline{x F}, \overline{1_{\mathbf{r}(x)} F}\right) \\
& =\left(\overline{1_{\mathbf{d}(x F)}}, \overline{x F}, \overline{1_{\mathbf{d}(x F)}}\right) \\
& =(x F) \tau_{P_{2}}
\end{aligned}
$$

and

$$
\begin{aligned}
\left(e \tau_{P_{1}}\right) F \mathbf{S C} & =\overline{1_{e}} F \mathbf{S C} \\
& =\overline{1_{e}} F \mathbf{S} \\
& =\overline{1_{e} F} \\
& =\overline{1_{e F}} \\
& =(e F) \tau_{P_{2}} .
\end{aligned}
$$

Thus the diagram below commutes, and so $\tau=\left(\tau_{P}\right)$ is a natural morphism of $I_{\mathcal{W O C}}$ and SC. 


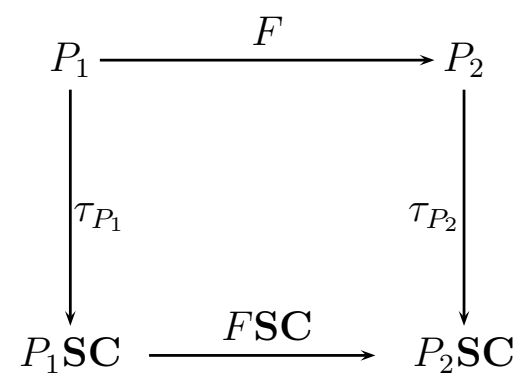

Figure 9.2: A natural transformation of $I_{\mathcal{W O C}}$ and $\mathbf{S C}$

To sum up, we have:

Theorem 9.24. The category $\mathcal{W O}$ of weakly $B$-orthodox semigroups and admissible morphisms is equivalent to the category $\mathcal{W O C}$ of weakly orthodox categories over bands and orthodox functors.

\subsection{Special cases}

Our purpose in this section is to investigate a certain kinds of weakly $B$-orthodox semigroups.

Lemma 9.25. Let $P$ be a weakly orthodox category over B. Suppose that for all $\bar{x} \in E(P \mathbf{S})$ we have that $\overline{1_{\mathbf{d}(x)}} \mathcal{R}^{*} \bar{x} \mathcal{L}^{*} \overline{1_{\mathbf{r}(x)}}$ in PS. Then $E(P \mathbf{S})=\bar{B}$.

Proof. Suppose that $x \in P$ and $\bar{x} \circ \bar{x}=\bar{x}$. As $\bar{x} \mathcal{L}^{*} \overline{1_{\mathbf{r}(x)}}$, we have that $\overline{1_{\mathbf{r}(x)}} \circ \bar{x}=$ $\overline{1_{\mathbf{r}(x)}}$. Thus

$$
\begin{aligned}
& \overline{1_{\mathbf{r}(x)} \otimes x}=\overline{1_{\mathbf{r}(x)}} \\
& \Rightarrow \overline{1_{\mathbf{r}(x)}|\mathbf{r}(x) \mathbf{d}(x) \cdot \mathbf{r}(x) \mathbf{d}(x)| x}=\overline{1_{\mathbf{r}(x)}} \\
& \Rightarrow \overline{\left[(\mathbf{r}(x) \mathbf{d}(x))^{+}, \mathbf{r}(x) \mathbf{d}(x)\right] \cdot \mathbf{r}(x) \mathbf{d}(x) \mid x}=\overline{[\mathbf{r}(x), \mathbf{r}(x)]} \\
& \Rightarrow(\mathbf{r}(x) \mathbf{d}(x))^{+} \mathcal{R} \mathbf{r}(x) \\
& \Rightarrow \mathbf{r}(x) \mathbf{d}(x) \mathcal{R} \mathbf{r}(x) \text {. }
\end{aligned}
$$

Dually, $\mathbf{r}(x) \mathbf{d}(x) \mathcal{L} \mathbf{d}(x)$. Hence, $\mathbf{r}(x) \mathcal{D} \mathbf{d}(x)$, and so $\mathbf{d}(x) \mathcal{R} \mathbf{d}(x) \mathbf{r}(x) \mathcal{L} \mathbf{r}(x)$. By Lemma 9.11, $\overline{1_{\mathbf{d}(x)}} \mathcal{R} \overline{1_{\mathbf{d}(x) \mathbf{r}(x)}} \mathcal{L} \overline{1_{\mathbf{r}(x)}}$. Again by $\overline{1_{\mathbf{d}(x)}} \mathcal{R}^{*} \bar{x} \mathcal{L}^{*} \overline{1_{\mathbf{r}(x)}}$, we have 
that $\bar{x} \mathcal{H}^{*} \overline{1_{\mathbf{d}(x) \mathbf{r}(x)}}$. Since $\mathcal{H}^{*}$-class contains at most one idempotent, we have that $\bar{x}=\overline{1_{\mathbf{d}(x) \mathbf{r}(x)}}$.

A weakly orthodox category $P$ over $B$ is an orthodox groupoid over $B$ if for all $x \in P$, there exists $y \in P$ with $\mathbf{d}(y)=\mathbf{r}(x)$ and $\mathbf{r}(y)=\mathbf{d}(x)$ such that $1_{\mathbf{d}(x)}=x \cdot y$ and $y \cdot x=1_{\mathbf{r}(x)}$.

Corollary 9.26. The category of orthodox semigroups and morphisms is equivalent to the category of orthodox groupoids over bands and orthodox functors.

Proof. Let $S$ be an orthodox semigroup with $B=E(S)$. Suppose that $(e, x, f) \in$ $S$ C. Since $\mathcal{R}=\widetilde{\mathcal{R}}_{B}$ and $\mathcal{L}=\widetilde{\mathcal{L}}_{B}$, we have that $e \mathcal{R} x \mathcal{L} f$. It follows from the fact that $S$ is regular that there exists $y \in S$ with $e \mathcal{L} y \mathcal{R} f, e=x y$ and $y x=f$. Then $(f, y, e) \in S \mathbf{C}$ and the products $(e, x, f) \cdot(f, y, e),(f, y, e) \cdot(e, x, f)$ exist in $S$ C. Moreover $(e, x, f) \cdot(f, y, e)=(e, x y, e)=(e, e, e)=[e, e]=1_{e}$ and similarly, $(f, y, e) \cdot(e, x, f)=1_{f}$.

Conversely, let $P$ be an orthodox groupoid over $B$. Suppose that $x \in P$. Then there exists $y \in P$ with $\mathbf{d}(y)=\mathbf{r}(x)$ and $\mathbf{r}(y)=\mathbf{d}(x)$ such that $1_{\mathbf{r}(x)}=y \cdot x$ and $1_{\mathbf{d}(x)}=x \cdot y$. So $\overline{1_{\mathbf{d}(x)}}=\overline{x \cdot y}=\overline{x \otimes y}=\bar{x} \circ \bar{y}$. Hence, $\bar{x} \circ \bar{y} \circ x=(\bar{x} \circ \bar{y}) \circ \bar{x}=$ $\overline{1_{\mathbf{d}(x)}} \circ \bar{x}=\bar{x}$ so that $P \mathbf{S}$ is regular. In addition, as $\overline{1_{\mathbf{d}(x)}}=\bar{x} \circ \bar{y}$ and $\bar{x}=\overline{1_{\mathbf{d}(x)}} \circ \bar{x}$, we have that $\overline{1_{\mathbf{d}(x)}} \mathcal{R} \bar{x}$ in PS. Dually, $\overline{1_{\mathbf{r}(x)}} \mathcal{L} \bar{x}$ in PS. By Lemma 9.25, we have that $E(P \mathbf{S})=\bar{B}$. Hence, $P \mathbf{S}$ is an orthodox semigroup.

We now concentrate on the class of abundant semigroups. We replace the distinguished set of idempotents $B$ by the whole set of idempotents and use relations $\mathcal{R}^{*}$ and $\mathcal{L}^{*}$ instead of $\widetilde{\mathcal{R}}_{B}$ and $\widetilde{\mathcal{L}}_{B}$. In addition, an admissible morphism in this context is more usually referred to as a good morphism. We define a weakly orthodox category $P$ over $B$ to be ${ }^{*}$-orthodox if it satisfies Condition (OB6) and its dual $(\mathrm{OB} 6)^{\circ}$ :

(OB6) if $y \otimes x \rho z \otimes x$, then $\left.\left.y\right|_{\mathbf{r}(y) \mathbf{d}(x)} \rho z\right|_{\mathbf{r}(z) \mathbf{d}(x)}$.

Corollary 9.27. The category of abundant semigroups whose set of idempotents forms a band and good morphisms is equivalent to the category of *-orthodox categories over bands and orthodox functors.

Proof. Let $P$ be a ${ }^{*}$-orthodox category over a band $B$. Suppose that $\bar{x} \in P$ S and $x \in P$. We know that $\overline{1_{\mathbf{d}(x)}} \widetilde{\mathcal{R}}_{\bar{B}} \bar{x}$, so that $\overline{1_{\mathbf{d}(x)}} \otimes \bar{x}=\bar{x}$. Assume that $y, z \in P$ with 
$\bar{y} \circ \bar{x}=\bar{z} \circ \bar{x}$. Then $y \otimes x \rho z \otimes x$. By (OB6), we obtain that $\left.\left.y\right|_{\mathbf{r}(y) \mathbf{d}(x)} \rho z\right|_{\mathbf{r}(z) \mathbf{d}(x)}$, and so $\mathbf{r}(y) \mathbf{d}(x) \mathcal{L} \mathbf{r}(z) \mathbf{d}(x), \mathbf{d}\left(\left.y\right|_{\mathbf{r}(y) \mathbf{d}(x)}\right) \mathcal{R} \mathbf{d}\left(\left.z\right|_{\mathbf{r}(z) \mathbf{d}(x)}\right)$, and we have

$$
\begin{aligned}
& \left(\left.y\right|_{\mathbf{r}(y) \mathbf{d}(x)}\right) \cdot[\mathbf{r}(y) \mathbf{d}(x), \mathbf{r}(z) \mathbf{d}(x)]=\left[\mathbf{d}\left(\left.y\right|_{\mathbf{r}(y) \mathbf{d}(x)}\right), \mathbf{d}\left(\left.z\right|_{\mathbf{r}(z) \mathbf{d}(x)}\right)\right] \cdot\left(\left.z\right|_{\mathbf{r}(z) \mathbf{d}(x)}\right) \\
& \Rightarrow\left(\left.y\right|_{\mathbf{r}(y) \mathbf{d}(x)}\right) \cdot[\mathbf{r}(y) \mathbf{d}(x), \mathbf{r}(z) \mathbf{d}(x)] \cdot\left({ }_{\mathbf{r}(z) \mathbf{d}(x)} \mid 1_{\mathbf{d}(x)}\right) \\
& =\left[\mathbf{d}\left(\left.y\right|_{\mathbf{r}(y) \mathbf{d}(x)}\right), \mathbf{d}\left(\left.z\right|_{\mathbf{r}(z) \mathbf{d}(x)}\right)\right] \cdot\left(\left.z\right|_{\mathbf{r}(z) \mathbf{d}(x)}\right) \cdot\left(\mathbf{r}(z) \mathbf{d}(x) \mid 1_{\mathbf{d}(x)}\right) \\
& \Rightarrow\left(\left.y\right|_{\mathbf{r}(y) \mathbf{d}(x)}\right) \cdot\left({ }_{\mathbf{r}(y) \mathbf{d}(x)} \mid 1_{\mathbf{d}(x)}\right)=\left[\mathbf{d}\left(\left.y\right|_{\mathbf{r}(y) \mathbf{d}(x)}\right), \mathbf{d}\left(\left.z\right|_{\mathbf{r}(z) \mathbf{d}(x)}\right)\right] \cdot\left(z \otimes 1_{\mathbf{d}(x)}\right) \\
& (\mathbf{r}(y) \mathbf{d}(x) \mathcal{L} \mathbf{r}(z) \mathbf{d}(x), \text { by }(\mathrm{OB} 2)) \\
& \Rightarrow y \otimes 1_{\mathbf{d}(x)}=\left[\mathbf{d}\left(\left.y\right|_{\mathbf{r}(y) \mathbf{d}(x)}\right), \mathbf{d}\left(\left.z\right|_{\mathbf{r}(z) \mathbf{d}(x)}\right)\right] \cdot\left(z \otimes 1_{\mathbf{d}(x)}\right) .
\end{aligned}
$$

Hence, $y \otimes 1_{\mathbf{d}(x)} \rho z \otimes 1_{\mathbf{d}(x)}$, that is, $\bar{y} \circ \overline{1_{\mathbf{d}(x)}}=\bar{z} \circ \overline{1_{\mathbf{d}(x)}}$.

Now, let $x \in P$. Then $\overline{1_{\mathbf{d}(x)}} \mathcal{R}^{*} \bar{x} \mathcal{L}^{*} \overline{1_{\mathbf{r}(x)}}$, and so by Lemma 9.25 , we have that $E(P \mathbf{S})=B$. Hence, $P \mathbf{S}$ is an abundant semigroup whose set of idempotents forms a band.

Conversely, in view of Lemma 9.18, it is necessary to show that $S \mathbf{C}$ satisfies Condition (OB6) and its dual. Assume that $(e, x, f),(u, y, v),(g, z, h) \in S \mathbf{C}$ such that $(u, y, v) \otimes(e, x, f) \rho(g, z, h) \otimes(e, x, f)$. Then

$$
\left.\left.(u, y, v)\right|_{v e} \cdot{ }_{v e}(e, x, f) \rho(g, z, h)\right|_{h e} \cdot{ }_{h e} \mid(e, x, f),
$$

that is,

$$
\left((y e)^{+}, y e, v e\right) \cdot\left(v e, v x,(v x)^{\star}\right) \rho\left((z e)^{+}, z e, h e\right) \cdot\left(h e, h x,(h x)^{\star}\right)
$$

or equivalently,

$$
\left((y e)^{+}, y x,(v x)^{\star}\right) \rho\left((z e)^{+}, z x,(h x)^{\star}\right),
$$

as yev $=$ yvevex $=y v e x=y x$ and similarly, $z e h x=z x$.

Thus, $(y e)^{+} \mathcal{R}(z e)^{+}$and $(v x)^{\star} \mathcal{L}(h x)^{\star}$, that is, $(y e)^{+}=(z e)^{+}$and $(v x)^{\star}=$ $(h x)^{\star}$ since they are unique. By Lemma 9.2, we have that $\left((y e)^{+}, y x,(v x)^{\star}\right)=$ $\left((z e)^{+}, z x,(h x)^{\star}\right)$, and so $y x=z x$. As $e \mathcal{R}^{*} x$ in $S$, we have that $y e=z e$, and so 
$v e \mathcal{L}^{*}$ ye $=$ ze $\mathcal{L}^{*}$ he. Thus,

$$
\begin{array}{rlr}
\left.(u, y, v)\right|_{v e} & =\left((y e)^{+}, y e, v e\right) & \\
& =\left((y e)^{+}, z e, v e\right) & \\
= & {\left[(y e)^{+},(z e)^{+}\right] \cdot\left((z e)^{+}, z e, v e\right)} & \\
= & {\left[(y e)^{+},(z e)^{+}\right] \cdot\left((z e)^{+}, z e, h e\right) \cdot[h e, v e]} & (v e \mathcal{L} h e) \\
= & {\left.\left[(y e)^{+},(z e)^{+}\right] \cdot(g, z, h)\right|_{h e} \cdot[h e, v e] .}
\end{array}
$$

So $\left.\left.(u, y, v)\right|_{v e} \rho(g, z, h)\right|_{h e}$. Hence, (OB6) holds.

Next, we discuss Ehresmann semigroups.

Let $P$ be a weakly orthodox category over a semilattice $E$. According to the remark succeeding Lemma 9.3, the relation $\rho$ is the identity on $P$ and $\leq_{r}$ and $\leq_{\ell}$ are partial orders on $P$. Then $P \mathbf{S}=P$, and so we will identify $\bar{x}$ with $x$ for all $x \in P$. In that case, for any $x, y \in P$,

$$
x \circ y=x \otimes y=\left.x\right|_{\mathbf{r}(x) \mathbf{d}(y)} \cdot \mathbf{r}(x) \mathbf{d}(y) \mid y .
$$

Lemma 9.28. A weakly orthodox category $P$ over a semilattice $E$ with $\leq_{r}$ forms an ordered 1 category with restriction.

Proof. Certainly, in view of the comments in Section 9.1, a weakly orthodox category $P$ over a semilattice E forms a poset under $\leq_{r}$.

(OC1) Suppose that $x, y \in P$ with $x \leq_{r} y$. Then there exists $e \in E$ such that $e \leq \mathbf{d}(y)$ and $x={ }_{e} \mid y$. Thus, $\mathbf{d}(x)=e \leq \mathbf{d}(y)$ and $\mathbf{r}(x) \leq \mathbf{r}(y)$ by (OB1).

(OC2) Suppose that $x, y \in P$ with $\mathbf{r}(x)=\mathbf{r}(y), \mathbf{d}(x)=\mathbf{d}(y)$ and $x \leq_{r} y$. Then there exists $e \in E$ such that $e \leq \mathbf{d}(y)$ and $x={ }_{e} \mid y$. Certainly, $\mathbf{d}(x)=e$, and so $e=\mathbf{d}(y)$, whence from (OB1),

$$
\begin{aligned}
y & ={ }_{e} \mid y \cdot\left[\mathbf{r}\left({ }_{e} \mid y\right), \mathbf{r}(y)\right] \\
& ={ }_{e} \mid y \cdot\left[\mathbf{r}\left({ }_{e} \mid y\right), \mathbf{r}\left({ }_{e} \mid y\right)\right] \quad\left(\mathbf{r}(y)=\mathbf{r}(x)=\mathbf{r}\left({ }_{e} \mid y\right)\right) \\
& ={ }_{e} \mid y=x .
\end{aligned}
$$

(OC3) If $x^{\prime} \leq_{r} x, y^{\prime} \leq_{r} y$, and both $x^{\prime} \cdot y^{\prime}$ and $x \cdot y$ exist, then there exist $e, f \in E$ such that $e \leq \mathbf{d}(x), f \leq \mathbf{d}(y), x^{\prime}={ }_{e} \mid x$ and $y^{\prime}={ }_{f} \mid y$. Thus, we have that 
$\mathbf{r}\left({ }_{e} \mid x\right)=\mathbf{r}\left(x^{\prime}\right)=\mathbf{d}\left(y^{\prime}\right)=\mathbf{d}\left({ }_{f} \mid y\right)=f$, and so $x^{\prime} \cdot y^{\prime}={ }_{e}\left|x \cdot{ }_{f}\right| y={ }_{e} \mid(x \cdot y)$ by (OB3). Hence, $x^{\prime} \cdot y^{\prime} \leq_{r} x \cdot y$.

Finally, we assume that $x \in P$ and $e \in E$ with $e \leq \mathbf{d}(x)$. Then ${ }_{e} \mid x$ is defined and $\mathbf{d}\left({ }_{e} \mid x\right)=e$. Also, $e_{e} \mid x \leq_{r} x$. Further, $e_{e} \mid x$ is unique since if $z \leq_{r} x$ and $e=\mathbf{d}(z)$, then there exists $h \in E$ with $h \leq \mathbf{d}(x)$ and $z={ }_{h} \mid x$, which gives that $h=\mathbf{d}(z)$. Thus, $e=h$, and so $z={ }_{e} \mid x$.

As a dual result to Lemma 9.28, we have the following lemma.

Lemma 9.29. A weakly orthodox category $P$ over a semilattice $E$ with $\leq_{\ell}$ forms an ordered 1 category with co-restriction.

Next we show that a weakly orthodox category $P$ over a semilattice $E$ is an Ehresmann category as mentioned in Chapter 6.

Lemma 9.30. A weakly orthodox category $P$ over a semilattice $E$ with the pair of natural partial orders $\left(\leq_{r}, \leq_{\ell}\right)$ defined in Section 9.1 forms an Ehresmann category $\left(P, \cdot, \leq_{r}, \leq_{\ell}\right)$.

Conversely, an Ehresmann category $\left(C, \cdot, \leq_{r}, \leq_{l}\right)$ with semilattice of identities $E$, may be regarded as a weakly orthodox category over $E$ with natural partial $\operatorname{orders}\left(\leq_{r}, \leq_{l}\right)$.

Proof. Let $P$ be a weakly orthodox category over a semilattice. In view of Lemma 9.28 and Lemma 9.29, Conditions (E1) and $(\mathrm{E} 1)^{\circ}$ are satisfied. Now, we identify $e$ with $1_{e}$ for all $e \in E$.

(E2) If $e, f \in E$ and $e \leq_{r} f$, then $e={ }_{e} \mid f$ so that we must have $e \leq f$. Then $\left.f\right|_{e}$ is defined and $\left.f\right|_{e}=\left.[f, f]\right|_{e}=\left[(f e)^{+}, e\right]=[e, e]=e$ so that $e \leq_{\ell} f$. Together with the dual, (E2) holds.

(E3) Clearly, $E$ is a semilattice under $\leq_{r}=\leq_{\ell}=\leq$.

(E4) To show that $\leq_{r} \circ \leq_{\ell} \subseteq \leq_{\ell} \circ \leq_{r}$, we assume that $x \leq_{r} \circ \leq_{\ell} y$. Then there exists $z \in P$ such that $x \leq_{r} z \leq_{\ell} y$. So there exist $e, f \in E$ with $\mathbf{d}(x)=$ $e \leq \mathbf{d}(z)=u$ and $\mathbf{r}(z)=f \leq \mathbf{r}(y)=v$, such that $x={ }_{e} \mid z$ and $z=\left.y\right|_{f}$. Thus, $x={ }_{e}\left|\left(\left.y\right|_{f}\right)={ }_{e u}\right|\left(\left.y\right|_{v f}\right)$. Since $P$ is weakly orthodox, we obtain that $\left.x \rho\left({ }_{e h} \mid y\right)\right|_{g f}$, where $h=\mathbf{d}(y)$ and $g=\mathbf{r}(e h \mid y)$. As $\rho$ is the identity by the remark following Lemma 9.3, we have that $x=\left.\left({ }_{e h} \mid y\right)\right|_{g f}$. Set $z^{\prime}={ }_{e h} \mid y$. Then $x \leq_{\ell} z^{\prime}$ and $z^{\prime} \leq_{r} y$. Consequently, $x \leq_{\ell} \circ \leq_{r} y$. 
(E5) Suppose that $x, y \in P$ and $f \in E$ with $x \leq_{r} y$. Then there exists $k \in E$ with $k \leq \mathbf{d}(y)$ and $x={ }_{k} \mid y$. So $\left.x\right|_{\mathbf{r}(x) \wedge f}=\left.\left({ }_{k} \mid y\right)\right|_{\mathbf{r}(x) \wedge f}$. As $P$ is weakly orthodox and $\rho$ is the identity, we obtain that $\left.\left({ }_{k} \mid y\right)\right|_{\mathbf{r}(x) \wedge f}={ }_{n k} \mid\left(\left.y\right|_{\mathbf{r}(y) \wedge f}\right)$, where $n=\mathbf{d}\left(\left.y\right|_{\mathbf{r}(y) \wedge f}\right)$, that is, $\left.x\right|_{\mathbf{r}(x) \wedge f}={ }_{n k} \mid\left(\left.y\right|_{\mathbf{r}(y) \wedge f)}\right.$. Thus, $\left.x\right|_{\mathbf{r}(x) \wedge f} \leq\left._{r} y\right|_{\mathbf{r}(y) \wedge f}$.

Conversely, let $C=\left(C, \cdot \leq_{r}, \leq_{l}\right)$ be an Ehresmann category with semilattice of identities $E$. If $e \mathcal{D} f$ in $E$, then $e=f$ and we put $[e, e]=e$ ( $E$ is identified with the set of identities at $E$ ).

From (E2) and (E3), $\leq_{r}$ and $\leq_{l}$ coincide on $E$, making $E$ a semilattice: we let $\leq$ denote the restriction of $\leq_{r}$ (resp. $\left.\leq_{l}\right)$ to $E$. It is clear that the first part of (OB1) holds, moreover, by uniqueness of restriction, $e_{e} \mid x=x$ if $e=\mathbf{d}(x)$, so that the second part of (OB1) holds.

For (OB2), if $x \in C$ and $e, f \in E$, with $e \leq_{\mathcal{L}} f \leq_{\mathcal{L}} \mathbf{d}(x)$, then $e \leq f \leq \mathbf{d}(x)$. Now ${ }_{e f}\left|x={ }_{e}\right| x \leq_{r} x$ and $\mathbf{d}\left({ }_{e} \mid x\right)=e$; also, $e_{e}\left|\left({ }_{f} \mid x\right) \leq_{r f}\right| x \leq_{r} x$ and $\mathbf{d}\left({ }_{e} \mid\left({ }_{f} \mid x\right)\right)=e$. By uniqueness of restriction, $e_{e f}\left|x={ }_{e}\right|\left({ }_{f} \mid x\right)$, that is, $e_{e}\left|x={ }_{e}\right|\left(_{f} \mid x\right)$. In particular, if $e \mathcal{L} f$, then $e=f$ and obviously, $[e, f] \cdot{ }_{f}\left|x=[f, f] \cdot{ }_{f}\right| x={ }_{f}\left|x={ }_{e}\right| x$.

(OB3) If $x, y \in C$ with $\exists x \cdot y$, then $\mathbf{r}(x)=\mathbf{d}(y)$. If $e \leq \mathbf{d}(x), \mathbf{r}\left({ }_{e} \mid x\right) \leq$ $\mathbf{r}(x)=\mathbf{d}(y)$ and we have that

$$
{ }_{e} \mid(x \cdot y) \leq_{r} x \cdot y \text { and } \mathbf{d}\left({ }_{e} \mid x \cdot y\right)=e
$$

and also

$$
{ }_{e}\left|x \cdot{ }_{f}\right| y \leq_{r} x \cdot y \text { and } \mathbf{d}\left({ }_{e}\left|x \cdot{ }_{f}\right| y\right)=e,
$$

where $f=\mathbf{r}\left({ }_{e} \mid x\right)$. Hence, ${ }_{e}\left|(x \cdot y)={ }_{e}\right| x \cdot{ }_{f} \mid y$, by uniqueness of restriction.

It is easy to see that (OB4) and (OB5) hold.

Finally, we show that $C$ is weakly orthodox. Let $x \in C$ and $e, f, u, v, g, h \in E$ with $g=\mathbf{r}(x), h=\mathbf{d}(x), u=\mathbf{d}\left(\left.x\right|_{g f}\right)$ and $v=\mathbf{r}(e h \mid x)$. Then $(e \bar{\otimes} x) \bar{\otimes} f=$ $e \bar{\otimes}(x \bar{\otimes} f)$, where $\bar{\otimes}$ is defined [32], by $x \bar{\otimes} y=\left.x\right|_{k} \cdot{ }_{k} \mid y$, where $k=\mathbf{r}(x) \wedge \mathbf{d}(x)$. As shown in $[32], \bar{\otimes}$ is associative. We have

$$
\begin{aligned}
(e \bar{\otimes} x) \bar{\otimes} f & =\left.\left(\left.e\right|_{e h} \cdot{ }_{e h} \mid x\right)\right|_{v f} \cdot{ }_{v f} \mid f \\
& =\left.\left(e h \cdot\left({ }_{e h} \mid x\right)\right)\right|_{v f} \cdot v f \\
& =\left.\left({ }_{e h} \mid x\right)\right|_{v f}
\end{aligned}
$$

and similarly, $e \bar{\otimes}(x \bar{\otimes} f)={ }_{e u} \mid\left(\left.x\right|_{g f}\right)$, so by associativity we obtain that $\left.(e h \mid x)\right|_{v f}=$ 
${ }_{e u} \mid\left(\left.x\right|_{g f}\right)$.

In view of Lemma 6.1, the partial order $\leq_{r}$ and $\leq_{\ell}$ defined in a weakly orthodox category over a semilattice coincide with $\leq_{r}$ and $\leq_{l}$ defined in an Ehresmann category, respectively.

Let $\mathbf{C}=\left(C, \cdot \leq_{r}, \leq_{l}\right)$ and $\mathbf{D}=\left(D, \cdot \leq_{r}, \leq_{l}\right)$ be Ehresmann categories with semilattice $E_{C}$ and $E_{D}$ of identities, respectively. A strongly ordered functor [32] $F: \mathbf{C} \rightarrow \mathbf{D}$ is a functor which preserves $\leq_{r}, \leq_{l}$ and $\wedge$. Since $F$ preserves $\wedge$, $F$ is a morphism $E_{C} \rightarrow E_{D}$. As shown in [32], $F$ preserves restrictions and co-restrictions. Thus $F$ is an orthodox functor in the sense of Definition 9.5.

On the other hand, if $G: \mathbf{C} \rightarrow \mathbf{D}$ is an orthodox functor, then by (S1), it preserves $\wedge$. Suppose now that $x, y \in \mathbf{C}$ with $x \leq_{r} y$. Then $x={ }_{e} \mid y$ for some $e \in E$, so that by (S3), $x G={ }_{e} \mid y G$ so that $x G \leq_{r} y G$. Dually, $G$ preserves $\leq_{l}$, so that $G$ is a strongly ordered functor. Theorem 9.24 and the comments above now give us

Corollary 9.31. The category of Ehresmann categories and strongly ordered functors is isomorphic to the category of weakly orthodox categories over semilattices and orthodox functors.

Let $S$ be an Ehresmann semigroup with distinguished semilattice of idempotents $E$. Indeed for any $x \in S$, there exists a unique $e \in E$ such that $e \widetilde{\mathcal{R}}_{E} x$ and a unique $f \in E$ such that $x \widetilde{\mathcal{L}}_{E} f$. Thus the map $\mathbf{C}: S \rightarrow S \mathbf{C}$ given by $x \mapsto(e, x, f)$ is bijective. In that case, we identify $S \mathbf{C}$ with $(S, \cdot)$ and so the partial binary operation - on $S \mathbf{C}$ is slightly modified to

$$
x \cdot y=x y
$$

where $x, y \in S$ satisfying $x^{*}=y^{\dagger}$ and $x y$ is the product of $x$ and $y$ in $S$. Then

Lemma 9.32. If $S$ is an Ehresmann semigroup with distinguished semilattice of idempotents $E$ and $P$ is a weakly orthodox category over $E$. Then $S \mathbf{C S}=S$ and $P \mathrm{SC}=P$.

Proof. Let $S$ be an Ehresmann semigroup over $E$. It follows from Lemma 9.18 that $S$ is a weakly orthodox category over $E$ with a restriction of that in $S$ and $\mathbf{d}(x)=x^{\dagger}, \mathbf{r}(x)=x^{*}$, for any $x \in S$, and if $e \leq x^{\dagger}$ and $f \leq x^{*}$ then ${ }_{e} \mid x=e x$ and $\left.x\right|_{f}=x f$. 
We now construct $S \mathbf{C S}$, which again has underlying set $S$ as the relation $\rho$ on a weakly orthodox category over a semilattice $E$ is trivial, by defining a product

$$
x \circ y=x \otimes y=\left.x\right|_{x^{*} y^{\dagger}} \cdot x^{*} y^{\dagger} \mid y .
$$

Observe that

$$
\begin{aligned}
x \circ y & =\left.x\right|_{x^{*} y^{\dagger} \cdot x^{*} y^{\dagger}} \mid y \\
& =x x^{*} y^{\dagger} \cdot x^{*} y^{\dagger} y \\
& =x x^{*} y^{\dagger} y \\
& =x y,
\end{aligned}
$$

so the operation in $S$ and $S \mathbf{C S}$ are the same. Moreover, the distinguished semilattices of $S$ and $S$ CS are both $E$. Hence $S=S$ CS.

Conversely, let $P$ be a weakly orthodox category over a semilattice $E$ with partial binary operation $\cdot$. We build the Ehresmann semigroup PS by modifying the product $\circ$ in Theorem 9.16 as

$$
x \circ y=\left.x\right|_{x^{*} y^{\dagger} \cdot x^{*} y^{\dagger}} \mid y \text {. }
$$

We temporarily use the notation $\odot$ for the partial binary operation in PSC. For any $x, y \in P$, we have

$$
\begin{aligned}
\exists x \odot y & \Leftrightarrow x^{*}=y^{\dagger} \text { in } P \mathbf{S} \\
& \Leftrightarrow \exists x \cdot y \text { in } P .
\end{aligned}
$$

Further, if $\exists x \odot y$, then by Lemma 9.9,

$$
x \odot y=x \otimes y=x \cdot y .
$$

For any $x \in P$ we have that $\mathbf{d}(x)=x^{\dagger}$ in PSC, where $x \widetilde{\mathcal{R}}_{B} x^{\dagger}$ in $P \mathbf{S}$. But the latter holds if and only if $x^{\dagger}=\mathbf{d}(x)$ in $P$. Thus $\mathbf{d}$ in $P$ and $P$ SC coincide, and dually for $\mathbf{r}$.

Clearly, the distinguished morphisms in $P$ and PSC are the same.

Again as a temporary measure, we use $\|$ to denote restriction and corestriction in $P$ SC. 
Let $x \in P$ and $e \in B$ with $e \leq \mathbf{d}(x)$. Then in PSC,

$$
{ }_{e} \| x=e \circ x=\left.e\right|_{e \mathbf{d}(x)} \cdot{ }_{e \mathbf{d}(x)}\left|x={ }_{e}\right| x
$$

and similarly for co-restriction.

The following result is easy to see, given Lemma 9.17 and Lemma 9.19.

Lemma 9.33. Let $\theta: S \rightarrow T$ be an admissible morphism of Ehresmann semigroups, and $F: P_{1} \rightarrow P_{2}$ be an orthodox functor of weakly orthodox categories over semilattices. Then $\theta \mathbf{C S}=\theta$ and $F \mathbf{S C}=F$.

As a immediate consequence of Lemma 9.32 and Lemma 9.33, we have that $\mathbf{S C}=I_{\mathcal{W O C}}$ and $\mathbf{C S}=I_{\mathcal{W O}}$, so that $\mathbf{S}$ and $\mathbf{C}$ are mutually inverse. Hence we have:

Corollary 9.34. The category of Ehresmann semigroups and admissible morphisms is isomorphic to the category of weakly orthodox categories over semilattices and orthodox functors.

In view of Corollary 9.31 and Corollary 9.34, we succeed in obtaining Lawson's result [32].

Corollary 9.35. [Theorem 4.24, [32]] The category of Ehresmann semigroups and admissible morphisms is isomorphic to the category of Ehresmann categories and strongly ordered functors.

We now look at weakly $B$-superabundant semigroups with (C), which are weakly $B$-orthodox semigroups such that each $\widetilde{\mathcal{H}}_{B}$-class contains a distinguished idempotent in $B$. We say that a weakly orthodox category over $B$ is superorthodox if it satisfies the following condition:

(OB7) if $x \in P$, then $\mathbf{d}(x) \mathcal{D} \mathbf{r}(x)$.

Corollary 9.36. The category of weakly B-superabundant semigroups with $(C)$ and admissible morphisms is equivalent to the category of weakly super-orthodox categories over $B$ and orthodox functors. 
Proof. Let $S$ be a weakly $B$-superorthodox semigroup. It follows from Lemma 9.18 that it is sufficient to show that $S \mathbf{C}$ satisfies Condition (OB7). Suppose that $\alpha=(e, x, f) \in S \mathbf{C}$. Then $e \widetilde{\mathcal{R}}_{B} x \widetilde{\mathcal{L}}_{B} f$. As $S$ is a weakly $B$-superabundant semigroup, there exists $h \in B$ such that $h \widetilde{\mathcal{H}}_{B} x$. Thus, $e \mathcal{R} h \mathcal{L} f$, which implies that $e \mathcal{D} f$, that is, $\mathbf{d}(\alpha) \mathcal{D} \mathbf{r}(\alpha)$.

Conversely, let $P$ be a weakly super-orthodox category over $B$. It is necessary to show that each $\widetilde{\mathcal{H}}_{\bar{B}}$-class of $P \mathbf{S}$ has a distinguished idempotent belonging to $\bar{B}$. Suppose that $x \in P$. By $(\mathrm{OB} 7), \mathbf{d}(x) \mathcal{D} \mathbf{r}(x)$. Then, $\mathbf{d}(x) \mathcal{R} \mathbf{d}(x) \mathbf{r}(x) \mathcal{L} \mathbf{r}(x)$. By Lemma 9.11, we have that $\overline{1_{\mathbf{d}(x)}} \mathcal{R} \overline{1_{\mathbf{d}(x) \mathbf{r}(x)}} \mathcal{L} \overline{1_{\mathbf{r}(x)}}$. As $\overline{1_{\mathbf{d}(x)}} \widetilde{\mathcal{R}}_{\bar{B}} \bar{x} \widetilde{\mathcal{L}}_{\bar{B}} \overline{1_{\mathbf{r}(x)}}$, we obtain that $\bar{x} \widetilde{\mathcal{H}}_{\bar{B}} \overline{1_{\mathbf{d}(x) \mathbf{r}(x)}}$. Hence, $P \mathbf{S}$ is a weakly $\bar{B}$-superabundant semigroup with $(\mathrm{C})$.

We now turn to the class of weakly $B$-orthodox semigroups, which have (WIC) mentioned in Chapter 2. We define a weakly orthodox category over $B$ to be weakly connected if it satisfies the following condition and its dual (OB8) :

(OB8) if $x \in P$ and $e \leq \mathbf{d}(x)$, then there exists $f \leq \mathbf{r}(x)$ such that $e_{e}|x \rho x|_{f}$.

Corollary 9.37. The category of weakly B-orthodox semigroups with (WIC) and admissible morphisms is equivalent to the category of weakly connected categories over $B$ and orthodox functors.

Proof. Starting with a weakly $B$-orthodox semigroup $S$ with (WIC), we show that $S \mathbf{C}$ satisfies Condition (OB8) and its dual. We show that (OB8) holds. Suppose that $(e, x, f) \in S \mathbf{C}$ and $u \leq e$. Then ${ }_{u} \mid(e, x, f)=\left(u, u x,(u x)^{\star}\right)$. Since $S$ has (WIC) it follows that there exists $v \in B$ such that $u x=x v$ and we can choose $v \leq f$. Then $(u x)^{\star} \widetilde{\mathcal{L}}_{B} x v \widetilde{\mathcal{L}}_{B} f v=v$ and $u=u e \widetilde{\mathcal{R}}_{B} u x \widetilde{\mathcal{R}}_{B}(x v)^{+}$. In addition,

$$
\begin{aligned}
{ }_{u} \mid(e, x, f) \cdot\left[(u x)^{\star}, v\right] & =\left(u, u x,(u x)^{\star}\right) \cdot\left((u x)^{\star},(u x)^{\star}, v\right) \\
& =(u, u x, v) \\
& =\left[u,(x v)^{+}\right] \cdot\left((x v)^{+}, x v, v\right) \\
& =\left.\left[u,(x v)^{+}\right] \cdot(e, x, f)\right|_{v} .
\end{aligned}
$$

Thus, ${ }_{u}|(e, x, f) \rho(e, x, f)|_{v}$.

Conversely, let $P$ be a weakly connected category over $B$. Suppose that $\bar{x} \in P \mathbf{S}$ and $\overline{1_{e}} \leq \overline{1_{\mathbf{d}(x)}}$. Then $\bar{x} \widetilde{\mathcal{R}}_{\bar{B}} \overline{1_{\mathbf{d}(x)}}$. By (OB8), there exists $f \leq \mathbf{r}(x)$ such 
that ${ }_{e}|x \rho x|_{f}$. Thus,

$$
\overline{1_{e}} \circ \bar{x}=\overline{1_{e} \otimes x}=\overline{1_{e} \cdot{ }_{e} \mid x}=\bar{e} \mid x=\overline{\left.x\right|_{f}}=\overline{\left.x\right|_{f} \cdot 1_{f}}=\bar{x} \circ \overline{1_{f}} .
$$

Together with the dual argument, we obtain that PS has (WIC). 


\section{Chapter 10}

\section{Weakly $U$-regular semigroups}

A weakly $U$-regular semigroup is a weakly $U$-abundant semigroup with (C) and $U$ generating a regular subsemigroup whose set of idempotents is $U$. The purpose of this chapter is to investigate a correspondence between weakly $U$-regular semigroups and certain categories, by using the techniques introduced in Chapter 9.

\subsection{Weakly regular categories}

The goal of this section is to develop the idea of weakly orthodox categories over a band constructed in Chapter 9 to introduce a category with set of objects a regular biordered set $U$.

Let $U$ be a regular biordered set. A subset $K$ of $U$ is a representative of $U$ if maps $\phi: K \rightarrow U / \mathcal{L}$ given by $e \mapsto L_{e}$ and $\psi: K \rightarrow U / \mathcal{R}$ given by $e \mapsto R_{e}$ are bijective. So for any $e \in U$, there exists a unique $k \in K$ such that $e \mathcal{L} k$ in $U$ and there exists a unique $h \in K$ such that $e \mathcal{R} h$ in $U$. For convenience, we will denote $k$ and $h$ by $e^{\star}$ and $e^{+}$, respectively.

Definition 10.1. Let $P$ be a category in which $\mathrm{Ob}(P)$ is the underlying set of a regular biordered set $U$, and let $K$ be a representative of $U$. Suppose that for $e, f \in U$ satisfying $e \mathcal{R} f$ or $e \mathcal{L} f$, there exists a distinguished morphism $[e, f]$ from $e$ to $f$, such that $[e, e]=1_{e}$, the identity associated to $e$. Then $P$ is an $R B S$ category if the following conditions and the duals $(\mathrm{P} 2)^{\circ},(\mathrm{P} 3)^{\circ},(\mathrm{P} 4)^{\circ}$, and $(\mathrm{P} 5)^{\circ}$ of (P2), (P3), (P4) and (P5) hold:

$(\mathrm{P} 1)$ if $e \mathcal{R} f \mathcal{R} g$ or $e \mathcal{L} f \mathcal{L} g$, then $[e, f] \cdot[f, g]=[e, g]$; 
(P2) if $x \in P, h \in U$ and $h \omega^{l} \mathbf{d}(x)$, then there exists an element ${ }_{h} \mid x$ in $P$, called the restriction of $x$ to $h$, such that $\mathbf{d}\left({ }_{h} \mid x\right)=h$ and $\mathbf{r}\left({ }_{h} \mid x\right) \omega^{l} \mathbf{r}(x)$; also, if $h=\mathbf{d}(x)$, then $\mathbf{r}\left({ }_{h} \mid x\right) \mathcal{L} \mathbf{r}(x)$ and ${ }_{h} \mid x \cdot\left[\mathbf{r}\left({ }_{h} \mid x\right), \mathbf{r}(x)\right]=x$;

(P3) if $g \omega e$ and $e \mathcal{R} f$ or $e \mathcal{L} f$, then ${ }_{g} \mid[e, f]=[g, g f] \cdot\left[g f,(g f)^{\star}\right]$; and if $g \omega^{l} e$ and $e \mathcal{L} f$, then ${ }_{g} \mid[e, f]=\left[g, g^{\star}\right]$

(P4) if $x \in P$ and $e, f \in U$ with $e \omega^{l} f \omega^{l} \mathbf{d}(x)$, then ${ }_{e}\left|\left({ }_{f} \mid x\right)={ }_{e}\right| x$; also, if $e \mathcal{L} f \omega^{l} \mathbf{d}(x)$, then $[e, f] \cdot{ }_{f}\left|x={ }_{e}\right| x ;$

(P5) if $x, y \in P$ and $h \in U$ with $h \omega^{l} \mathbf{d}(x)$ and $\exists x \cdot y$ in $P$, then ${ }_{h} \mid(x \cdot y)=$ ${ }_{h}\left|x \cdot{ }_{g}\right| y$, where $g=\mathbf{r}\left({ }_{h} \mid x\right)$;

(P6) if $\left(\begin{array}{ll}e & f \\ g & h\end{array}\right)$ is a singular $U$-square, then $[e, f] \cdot[f, h]=[e, g] \cdot[g, h]$.

Let us pause to make some simple but necessary comments on Definition 10.1. In (P3) since $g \omega e$, we know that if $e \mathcal{R} f$ (resp. $e \mathcal{L} f$ ), then $g \omega^{r} f$ (resp. $g \omega^{l} f$ ), and so by (B21) $g \mathcal{R} g f$ (resp. $g=g f$ ). Thus $[g, g f]$ exists. In (P5) since $\exists x \cdot y$ we know that $\mathbf{r}(x)=\mathbf{d}(y)$. By (P2), $g=\mathbf{r}\left({ }_{h} \mid x\right) \omega^{l} \mathbf{r}(x)=\mathbf{d}(y)$, so that ${ }_{g} \mid y$ is defined and $\mathbf{d}\left({ }_{g} \mid y\right)=g$. Hence, ${ }_{h}\left|x \cdot{ }_{g}\right| y$ is defined. Condition (P6) implies that

$$
[g, e] \cdot[e, f] \cdot[f, h] \cdot[h, f]=[g, e] \cdot[e, g] \cdot[g, h] \cdot[h, f],
$$

that is,

$$
[g, e] \cdot[e, f] \cdot[f, f]=[g, g] \cdot[g, h] \cdot[h, f]
$$

by (P1). Thus,

$$
[g, e] \cdot[e, f]=[g, h] \cdot[h, f] .
$$

Comparing with Definition 9.1, we note that Conditions (P1) and (P6) correspond to Condition (OB5); Conditions (P2), (P4) and (P5) are similar to Conditions (OB1), (OB2) and (OB3), respectively; Condition (P3) is a generalisation of Condition (OB4).

Notice that an RBS category $P$ depends on the choice of the regular biordered set $U$ which is the set of objects of $P$. In order to avoid the ambiguity, we will express the term 'RBS category' as 'RBS category over $U$ '.

Let $P$ be an RBS category over a regular biordered set $U$. We define a relation $\rho$ on $P$ by the rule that for any $x, y \in P$,

$x \rho y$ if and only if 


$$
\mathbf{d}(x) \mathcal{R} \mathbf{d}(y), \mathbf{r}(x) \mathcal{L} \mathbf{r}(y) \text { and } x \cdot[\mathbf{r}(x), \mathbf{r}(y)]=[\mathbf{d}(x), \mathbf{d}(y)] \cdot y
$$

It is worth making the point that

$$
\begin{aligned}
& x \cdot[\mathbf{r}(x, \mathbf{r}(y)]=[\mathbf{d}(x), \mathbf{d}(y)] \cdot y \\
\Leftrightarrow & {[\mathbf{d}(y), \mathbf{d}(x)] \cdot x \cdot[\mathbf{r}(x), \mathbf{r}(y)]=y } \\
\Leftrightarrow & {[\mathbf{d}(y), \mathbf{d}(x)] \cdot x=y \cdot[\mathbf{r}(y), \mathbf{r}(x)] } \\
\Leftrightarrow & x=[\mathbf{d}(x), \mathbf{d}(y)] \cdot y \cdot[\mathbf{r}(y), \mathbf{r}(x)],
\end{aligned}
$$

so that if $\mathbf{d}(x)=\mathbf{d}(y)$, then $x \rho y$ if and only if $\mathbf{r}(x) \mathcal{L} \mathbf{r}(y)$ and

$$
x=y \cdot[\mathbf{r}(y), \mathbf{r}(x)] \text { or indeed } x \cdot[\mathbf{r}(x), \mathbf{r}(y)]=y .
$$

Dually, if $\mathbf{r}(x)=\mathbf{r}(y)$, then $x \rho y$ if and only if $\mathbf{d}(x) \mathcal{R} \mathbf{d}(y)$ and

$$
x=[\mathbf{d}(x), \mathbf{d}(y)] \cdot y \text { or indeed } y=[\mathbf{d}(y), \mathbf{d}(x)] \cdot x .
$$

Further, we have:

Lemma 10.2. Let $e, f, g \in U$ be such that e $\mathcal{R} f \mathcal{L} g$. Then $[e, f] \rho 1_{f} \rho[f, g]$. Proof. As e $\mathcal{R} f$, we have that

$$
\begin{gathered}
\mathbf{d}([e, f])=e \mathcal{R} f=\mathbf{d}\left(1_{f}\right), \\
\mathbf{r}([e, f])=f=\mathbf{r}\left(1_{f}\right)
\end{gathered}
$$

and

$$
[e, f] \cdot 1_{f}=[e, f]
$$

so that $[e, f] \rho 1_{f}$. Dually, $[f, g] \rho 1_{f}$.

The proof of the next lemma is the same as that of Lemma 9.2 so we omit it.

Lemma 10.3. The relation $\rho$ defined above is an equivalence on $P$ such that if $x, y \in \operatorname{Mor}(e, f)$ and $x \rho y$, then $x=y$. In particular, no two identities of $P$ are $\rho$-equivalent. 
We now present a pair of pre-orders on an RBS category over $U$ built on the relation $\rho$ given above.

Let $P$ be an RBS category over $U$. We make use of the restriction and corestriction of $P$ to define relations $\leq_{r}{ }^{\prime}$ and $\leq_{\ell}{ }^{\prime}$ by the rule that for all $x, y \in P$,

$$
x \leq_{r}^{\prime} y \text { if and only if } x \rho_{e} \mid y \quad \text { for some } e \in U \text { and } e \omega \mathbf{d}(y)
$$

and

$$
x \leq_{\ell}^{\prime} y \text { if and only if }\left.x \rho y\right|_{f} \text { for some } f \in U \text { and } f \omega \mathbf{r}(y) \text {. }
$$

Lemma 10.4. The relations $\leq_{r}{ }^{\prime}$ and $\leq_{\ell}{ }^{\prime}$ are pre-orders on $P$.

Proof. We first show that $\leq_{r}^{\prime}$ is a pre-order on $P$. Notice that for any $x \in P$, if $e=\mathbf{d}(x)$, then $\left[\mathbf{d}(x), \mathbf{d}\left({ }_{e} \mid x\right)\right]=[e, e]=1_{e}$, and so $\leq_{r}{ }^{\prime}$ is reflexive by (P2). It is sufficient to show that $\leq_{r}{ }^{\prime}$ is transitive. Suppose that $x, y, z \in P$ with $x \leq_{r}{ }^{\prime} y$ and $y \leq_{r}{ }^{\prime} z$. Then there exist $e, f \in U$ such that $e \omega \mathbf{d}(y), f \omega \mathbf{d}(z)$, and $x \rho_{e} \mid y$ and $y \rho_{f} \mid z$. Thus, $\mathbf{d}(y) \mathcal{R} f, \mathbf{r}(y) \mathcal{L} \mathbf{r}\left({ }_{f} \mid z\right)$ and $y \cdot\left[\mathbf{r}(y), \mathbf{r}\left({ }_{f} \mid z\right)\right]=[\mathbf{d}(y), f] \cdot\left({ }_{f} \mid z\right)$. Hence, $y=[\mathbf{d}(y), f] \cdot{ }_{f} \mid z \cdot\left[\mathbf{r}\left({ }_{f} \mid z\right), \mathbf{r}(y)\right]$. As $e \omega \mathbf{d}(y) \mathcal{R} f$, we have that $e \mathcal{R}$ ef $\omega f$. In addition, $e \mid[\mathbf{d}(y), f]$ exists and $\left.e_{e} \mid \mathbf{d}(y), f\right]=[e, e f] \cdot\left[e f,(e f)^{\star}\right]$ by (P3). Since $(e f)^{\star} \mathcal{L}$ ef $\omega f \omega \mathbf{d}(z)$, we obtain that ${ }_{(e f)^{\star}} \mid\left(_{f} \mid z\right)$ is defined and ${ }_{(e f)^{\star}}\left|\left({ }_{f} \mid z\right)={ }_{(e f)^{\star}}\right| z$ by $(\mathrm{P} 4)$. Then we have that

$$
\begin{aligned}
e_{e} \mid y= & { }_{e} \mid\left([\mathbf{d}(y), f] \cdot{ }_{f} \mid z \cdot\left[\mathbf{r}\left({ }_{f} \mid z\right), \mathbf{r}(y)\right]\right) \\
= & \left.{ }_{e}\left|[\mathbf{d}(y), f] \cdot{ }_{h}\right|\left({ }_{f} \mid z\right) \cdot{ }_{k}|| \mathbf{r}\left({ }_{f} \mid z\right), \mathbf{r}(y)\right] \\
& \quad\left(\operatorname{by}(\mathrm{P} 5), h=\mathbf{r}\left({ }_{e} \mid[\mathbf{d}(y), f]\right), k=\mathbf{r}\left({ }_{h} \mid\left({ }_{f} \mid z\right)\right)\right) \\
= & {[e, e f] \cdot\left[e f,(e f)^{\star}\right] \cdot{ }_{(e f)^{\star}} \mid\left({ }_{f} \mid z\right) \cdot\left[k, k^{\star}\right] } \\
& \quad\left(\operatorname{ass}=\mathbf{r}\left({ }_{h} \mid\left({ }_{f} \mid z\right)\right) \omega^{l} \mathbf{r}\left({ }_{f} \mid z\right) \mathcal{L} \mathbf{r}(y), \text { by }(\mathrm{P} 3)\right) \\
= & {[e, e f] \cdot\left[e f,(e f)^{\star}\right] \cdot{ }_{(e f)^{\star}} \mid z \cdot\left[k, k^{\star}\right] \quad\left(\text { by }(\mathrm{P} 4),(e f)^{\star} \mathcal{L} \text { ef } \omega f \omega \mathbf{d}(z)\right) . }
\end{aligned}
$$

Hence, $\mathbf{r}(e \mid y)=k^{\star}$. From $x \rho_{e} \mid y$, we have that $\mathbf{r}(x) \mathcal{L} k^{\star}, \mathbf{d}(x) \mathcal{R} e$ and

$$
\begin{aligned}
x \cdot\left[\mathbf{r}(x), k^{\star}\right] & =[\mathbf{d}(x), e] \cdot e_{e} \mid y \\
& =[\mathbf{d}(x), e] \cdot[e, e f] \cdot\left[e f,(e f)^{\star}\right] \cdot(e f)^{\star} \mid z \cdot\left[k, k^{\star}\right] \\
& =[\mathbf{d}(x), e f] \cdot\left[e f,(e f)^{\star}\right] \cdot(e f)^{\star} \mid z \cdot\left[k, k^{\star}\right] \quad(\mathbf{d}(x) \mathcal{R} \text { eef, by }(\mathrm{P} 1)) \\
& =[\mathbf{d}(x), e f] \cdot{ }_{\text {ef }} \mid z \cdot\left[k, k^{\star}\right] \quad\left(\text { by }(\mathrm{P} 4), \text { ef } \mathcal{L}(e f)^{\star} \omega^{l} \mathbf{d}(z)\right) .
\end{aligned}
$$


Thus,

$$
\begin{aligned}
x \cdot[\mathbf{r}(x), k] & =x \cdot\left[\mathbf{r}(x), k^{\star}\right] \cdot\left[k^{\star}, k\right] & & (\text { by }(\mathrm{P} 1)) \\
& =[\mathbf{d}(x), e f] \cdot e_{e f} \mid z \cdot\left[k, k^{\star}\right] \cdot\left[k^{\star}, k\right] & & \\
& =[\mathbf{d}(x), e f] \cdot e_{f} \mid z \cdot[k, k] & & \left(k \mathcal{L} k^{\star}\right) \\
& =[\mathbf{d}(x), e f] \cdot e_{e f} \mid z & & \left(k=\mathbf{r}\left({ }_{e f} \mid z\right)\right) .
\end{aligned}
$$

It follows that $k=\mathbf{r}\left({ }_{e f} \mid z\right)$. As $\mathbf{r}(x) \mathcal{L} \mathbf{r}\left({ }_{e} \mid y\right)=k^{\star} \mathcal{L} k=\mathbf{r}\left({ }_{e f} \mid z\right)$ and $\mathbf{d}(x) \mathcal{R}$ ef $=$ $\mathbf{d}(e f \mid z)$, we have that $x \rho_{\text {ef }} \mid z$. Together with ef $\omega f \omega \mathbf{d}(z)$, we obtain that $x \leq_{r}{ }^{\prime} z$.

By the dual argument, we show that $\leq_{\ell}{ }^{\prime}$ is a pre-order on $P$.

In addition, there exists another way to define a pair of pre-orders on an RBS category $P$ over $U$.

Let $P$ be an RBS category over $U$. For any $x, y \in P$, we define

$$
x \leq_{r} y \text { if and only if } x \rho_{e} \mid y \text { for some } e \in U
$$

and

$$
x \leq_{l} y \text { if and only if }\left.x \rho y\right|_{f} \text { for some } f \in U .
$$

By (P2) and its dual, relations $\leq_{r}$ and $\leq_{l}$ are reflexive, but in general they are not transitive and symmetric. We recall that the transitive closure of a relation $\theta$ is denoted by $\theta^{t}$. We have:

Lemma 10.5. Let $P$ be an $R B S$ category over $U$. Then relations $\leq_{r}^{t}$ and $\leq_{l}^{t}$ are pre-orders on $P$.

As the comments succeeding Lemma 9.3, it is impossible to define a pair of partial orders on $P$.

Let $P$ be an RBS category over a regular biordered set $U$. By (B1), $\omega^{r}$ and $\omega^{l}$ are pre-orders on $U$, and $D_{U}=\left(\omega^{r} \cup \omega^{l}\right) \cup\left(\omega^{r} \cup \omega^{l}\right)^{-1}$. Suppose that $x \in P$ and $h \omega^{r} \mathbf{d}(x)$. We define

$$
h * x=[h, h \mathbf{d}(x)] \cdot{ }_{h \mathbf{d}(x)} \mid x .
$$

Clearly, $\mathbf{d}(h * x)=h$. In particular, if $h \omega \mathbf{d}(x)$, then $h * x={ }_{h} \mid x$. 
Observe that if $h \omega^{r} \mathbf{d}(x)$, then by (B1), $h \mathbf{d}(x)$ exists in $U$. Again by (B21), $h \mathcal{R} h \mathbf{d}(x) \omega \mathbf{d}(x)$, so that $[h, h \mathbf{d}(x)]$ exists and by $(\mathrm{P} 2), h \mathbf{d}(x) \mid x$ is well-defined.

Dually, if $k \omega^{l} \mathbf{r}(x)$, then we define

$$
x \diamond k=\left.x\right|_{\mathbf{r}(x) k} \cdot[\mathbf{r}(x) k, k]
$$

Note that $\mathbf{r}(x \diamond k)=k$. In particular, if $k \omega \mathbf{r}(x)$, then $x \diamond k=\left.x\right|_{k}$.

We stop here to make some comments on the above operations $*$ and $\diamond$. The initial idea of defining operations $*$ and $\diamond$ is to define a binary operation on an RBS category or a weakly regular category introduced below, via the sandwich set. Let $P$ be an RBS category. Suppose that $x, y \in P$ and $h \in S(\mathbf{r}(x), \mathbf{d}(y))$. Then $h \omega^{l} \mathbf{r}(x)$ and $h \omega^{r} \mathbf{d}(y)$. Our purpose is to use restrictions and co-restrictions to define a product. Note that $h \mathcal{L} \mathbf{r}(x) h \omega \mathbf{r}(x)$ and $h \mathcal{R} h \mathbf{d}(y) \omega \mathbf{d}(y)$ so that we can define a binary operation on $P$ by the rule that

$$
x y=\left.x\right|_{\mathbf{r}(x) h} \cdot[\mathbf{r}(x) h, h] \cdot[h, h \mathbf{d}(y)] \cdot h \mathbf{d}(y) \mid y .
$$

Certainly, it is well defined. For convenience, we defined $*$ and $\diamond$ above.

To maintain the analogy with weakly orthodox categories, we have weakly regular categories described as follows:

Definition 10.6. An RBS category $P$ over a regular biordered set $U$ is weakly regular if it satisfies the following condition:

(P7) for $x \in P, e, f \in U, h_{1} \in S(e, \mathbf{d}(x))$ and $h_{2} \in S(\mathbf{r}(x), f)$, we put $h_{1}^{\prime}=$ $\mathbf{r}\left(h_{1} \mathbf{d}(x) \mid x\right)$ and $h_{2}^{\prime}=\mathbf{d}\left(\left.x\right|_{\mathbf{r}(x) h_{2}}\right)$. Then there exist $h \in S\left(h_{1}, h_{2}^{\prime}\right)$ and $h^{\prime} \in S\left(h_{1}^{\prime}, h_{2}\right)$ such that

$$
\left(\left(h_{1} * x\right) \diamond h^{\prime}\right) \cdot\left[h^{\prime}, h^{\prime} h_{2}\right] \rho\left[h_{1} h, h\right] \cdot\left(h *\left(x \diamond h_{2}\right)\right) .
$$

It is a good place to make some necessary comments on Definition 10.6. For any $e, f \in U, S(e, f)$ denotes the sandwich set of $e$ and $f$. In $(\mathrm{P} 7), h_{1} \in S(e, \mathbf{d}(x))$ implies that $h_{1} \omega^{r} \mathbf{d}(x)$, and so by (B21) in Section $1.4, h_{1} \mathcal{R} h_{1} \mathbf{d}(x) \omega \mathbf{d}(x)$ so that $h_{1} \mathbf{d}(x) \mid x$ is well-defined and $h_{1} * x$ exists. Put $h_{1}^{\prime}=\mathbf{r}\left(h_{1} \mathbf{d}(x) \mid x\right)$. Similarly, $\left.x\right|_{\mathbf{r}(x) h_{2}}$ and $x \diamond h_{2}$ are well-defined. We put $h_{2}^{\prime}=\mathbf{d}\left(\left.x\right|_{\mathbf{r}(x) h_{2}}\right)$. Since $U$ is regular, we obtain that $S\left(h_{1}, h_{2}^{\prime}\right) \neq \emptyset$ and $S\left(h_{1}^{\prime}, h_{2}\right) \neq \emptyset$. If $h^{\prime} \in S\left(h_{1}^{\prime}, h_{2}\right)$, then $h^{\prime} \omega^{l} h_{1}^{\prime}=$ $\mathbf{r}\left(h_{1} \mathbf{d}(x) \mid x\right)=\mathbf{r}\left(h_{1} * x\right)$ and $h^{\prime} \omega^{r} h_{2}$, so that $\left(h_{1} * x\right) \diamond h^{\prime}$ is defined and by (B21), $h^{\prime} h_{2} \mathcal{R} h^{\prime}$. Hence $\left[h^{\prime}, h^{\prime} h_{2}\right]$ exists. By a similar argument, $h *\left(x \diamond h_{2}\right)$ is defined and $h_{1} h \mathcal{L} h$ so that $\left[h_{1} h, h\right]$ exists. 
We are now ready to say that the class of weakly regular categories over regular biordered sets forms a category, together with certain functors, namely RBS functors, which appear in the next definition.

Definition 10.7. Let $P_{1}$ and $P_{2}$ be weakly regular categories over regular biordered sets $U_{1}$ and $U_{2}$, respectively, and $F: P_{1} \rightarrow P_{2}$ be a functor. Then $F$ is said to be $R B S$ if

(PF1) $F: U_{1} \rightarrow U_{2}$ is a regular morphism;

$(\mathrm{PF} 2)$ if $e \mathcal{R} f$ or $e \mathcal{L} f$ in $U$, then $[e, f]_{P_{1}} F=[e F, f F]_{P_{2}}$;

(PF3) if $x \in P_{1}$ and $h, k \in U_{1}$ with $h \omega^{l} \mathbf{d}(x)$ and $k \omega^{r} \mathbf{r}(x)$, then $\left({ }_{h} \mid x\right) F \rho_{h F} \mid x F$ and $\left.\left(\left.x\right|_{k}\right) F \rho x F\right|_{k F}$.

We pause here to make a short comment on Definition 10.7. In (PF3), if $h \omega^{l} \mathbf{d}(x)$, then $h F \omega^{l} \mathbf{d}(x F)$ as $F$ is a biordered set morphism and is a functor, so that both ${ }_{h} \mid x$ and ${ }_{h F} \mid x F$ are well-defined. In addition, the fact that $\left({ }_{h} \mid x\right) F \rho_{h F} \mid x F$ gives in particular that $\mathbf{r}\left({ }_{h} \mid x\right) F \mathcal{L} \mathbf{r}\left({ }_{h F} \mid x F\right)$. For $\mathbf{d}$, as $F$ is a functor, we have that $\mathbf{d}\left(\left({ }_{h} \mid x\right) F\right)=\mathbf{d}\left({ }_{h} \mid x\right) F=h F=\mathbf{d}\left({ }_{h F} \mid x F\right)$.

The next lemma is useful for Lemma 10.9.

Lemma 10.8. Let $P_{1}$ and $P_{2}$ be weakly regular categories over $U_{1}$ and $U_{2}$, respectively and let $F: P_{1} \rightarrow P_{2}$ be an $R B S$ functor. If $x \rho y$ in $P_{1}$, then $x F \rho y F$ in $P_{2}$.

Proof. Suppose that $x, y \in P_{1}$ and $x \rho y$. Then

$$
\mathbf{d}(x) \mathcal{R} \mathbf{d}(y), \mathbf{r}(x) \mathcal{L} \mathbf{r}(y) \text { and } x \cdot[\mathbf{r}(x), \mathbf{r}(y)]=[\mathbf{d}(x), \mathbf{d}(y)] \cdot y .
$$

Thus,

$$
\mathbf{d}(x) F \mathcal{R} \mathbf{d}(y) F, \mathbf{r}(x) F \mathcal{L} \mathbf{r}(y) F \text { and } x F \cdot[\mathbf{r}(x), \mathbf{r}(y)] F=[\mathbf{d}(x), \mathbf{d}(y)] F \cdot y F
$$

This gives

$$
\mathbf{d}(x) F \mathcal{R} \mathbf{d}(y) F, \mathbf{r}(x) F \mathcal{L} \mathbf{r}(y) F \text { and } x F \cdot[\mathbf{r}(x) F, \mathbf{r}(y) F]=[\mathbf{d}(x) F, \mathbf{d}(y) F] \cdot y F,
$$

by (PF2), and so

$$
\mathbf{d}(x F) \mathcal{R} \mathbf{d}(y F), \mathbf{r}(x F) \mathcal{L} \mathbf{r}(y F) \text { and } x F \cdot[\mathbf{r}(x F), \mathbf{r}(y F)]=[\mathbf{d}(x F), \mathbf{d}(y F)] \cdot y F \text {. }
$$


Hence, $x F \rho y F$.

Lemma 10.9. Let $P_{1}$ and $P_{2}$ be weakly regular categories over $U_{1}$ and $U_{2}$, respectively, and let $F_{1}: P_{1} \rightarrow P_{2}$ and $F_{2}: P_{2} \rightarrow P_{3}$ be RBS functors. Then $F_{1} F_{2}: P_{1} \rightarrow P_{3}$ is an $R B S$ functor.

Proof. (PF1) Certainly, $F_{1} F_{2}$ is a functor from $P_{1}$ to $P_{3}$ and a regular morphism from $U_{1}$ to $U_{3}$.

(PF2) Suppose that $e, f \in U_{1}$ are such that $e \mathcal{R} f$. Then $[e, f]_{P_{1}}$ is defined, and $e F_{1} \mathcal{R} f F_{1}$ and $e F_{1} F_{2} \mathcal{R} f F_{1} F_{2}$ by the comment succeeding the definition of regular morphism in Chapter 1 . Using $(\mathrm{PF} 2)$ for $F_{1}$ and $F_{2}$, we have that

$$
[e, f]_{P 1} F_{1} F_{2}=\left([e, f]_{P 1} F_{1}\right) F_{2}=\left[e F_{1}, f F_{1}\right]_{P_{2}} F_{2}=\left[e F_{1} F_{2}, f F_{1} F_{2}\right]_{P 3} .
$$

Dually, if $e \mathcal{L} f$, then $[e, f]_{P 1} F_{1} F_{2}=\left[e F_{1} F_{2}, f F_{1} F_{2}\right]_{P 3}$.

(PF3) Assume that $x \in P_{1}$ and $e \in U_{1}$ with $e \omega^{l} \mathbf{d}(x)$. According to the comment succeeding Definition 10.7, we have that ${ }_{e}\left|x, e F_{1}\right| x F_{1}$ and $e_{e F_{1} F_{2}} \mid x F_{1} F_{2}$ are well-defined. By (PF3), $(e \mid x) F_{1} \rho_{e F_{1}} \mid x F_{1}$ and $\left(e F_{1} \mid x F_{1}\right) F_{2} \rho_{e F_{1} F_{2}} \mid x F_{1} F_{2}$. From $\left.{ }_{e} \mid x\right) F_{1} \rho_{e F_{1}} \mid x F_{1}$, we obtain that $(e \mid x) F_{1} F_{2} \rho\left({ }_{e} F_{1} \mid x F_{1}\right) F_{2}$ by Lemma 10.8 . Hence, $(e \mid x) F_{1} F_{2} \rho e_{e F_{1} F_{2}} \mid x F_{1} F_{2}$.

An immediate observation from Lemma 10.9 is that the class of weakly regular categories over regular biordered sets and RBS functors forms a category. We refer to it as $\mathcal{W} \mathcal{R C}$.

We close this section with an important property of RBS fuctors.

Lemma 10.10. If $P_{1}$ and $P_{2}$ are weakly regular categories over regular biordered sets $U_{1}$ and $U_{2}$, respectively, and $F: P_{1} \rightarrow P_{2}$ is an $R B S$ functor. Then for any $h, k \in U_{1}$ and $x \in P_{1}$, we have

(i) if $h \omega^{r} \mathbf{d}(x)$, then $(h * x) F \rho h F * x F$;

(ii) if $k \omega^{l} \mathbf{r}(x)$, then $(x \diamond k) F \rho x F \diamond k F$.

Proof. To prove $(i)$, suppose that $x \in P_{1}, h \in U_{1}$ and $h \omega^{r} \mathbf{d}(x)$. Then

$$
h * x=[h, h \mathbf{d}(x)] \cdot(h \mathbf{d}(x) \mid x) .
$$

Since $F$ is RBS, it follows that $F: U_{1} \rightarrow U_{2}$ is a regular morphism, and so 
$h F \omega^{r} \mathbf{d}(x) F$ so that $h F * \mathbf{d}(x) F$ exists. In addition, $(h \mathbf{d}(x)) F=h F \mathbf{d}(x) F$ and

$$
[h, h \mathbf{d}(x)] F=[h F,(h \mathbf{d}(x)) F]=[h F, h F \mathbf{d}(x) F] .
$$

According to the comments following Definition 10.7, we have that

$$
\mathbf{d}\left(\left(_{h \mathbf{d}(x)} \mid x\right) F\right)=(h \mathbf{d}(x)) F=h F \mathbf{d}(x) F=\mathbf{d}\left({ }_{(h F \mathbf{d}(x) F} \mid x F\right) .
$$

By (PF3),

$$
(h \mathbf{d}(x) \mid x) F \rho_{(h \mathbf{d}(x)) F}\left|x F={ }_{h F \mathbf{d}(x) F}\right| x F .
$$

Thus, $\mathbf{r}((h \mathbf{d}(x) \mid x) F) \mathcal{L} \mathbf{r}\left({ }_{h F \mathbf{d}(x) F} \mid x F\right)$ and

$$
\left({ }_{h \mathbf{d}(x)} \mid x\right) F={ }_{h F \mathbf{d}(x) F} \mid x F \cdot\left[\mathbf{r}\left({ }_{h F \mathbf{d}(x) F} \mid x F\right), \mathbf{r}\left(\left(_{h \mathbf{d}(x)} \mid x\right) F\right)\right],
$$

so that

$$
\begin{aligned}
& (h * x) F \\
& =\left([h, h \mathbf{d}(x)] \cdot{ }_{h \mathbf{d}(x)} \mid x\right) F \\
& =[h, h \mathbf{d}(x)] F \cdot(h \mathbf{d}(x) \mid x) F \\
& \left.=[h F, h F \mathbf{d}(x) F] \cdot{ }_{h F \mathbf{d}(x) F} \mid x F\right) \cdot\left[\mathbf{r}\left({ }_{h F \mathbf{d}(x) F} \mid x F\right), \mathbf{r}\left(\left(_{h \mathbf{d}(x)} \mid x\right) F\right)\right] \\
& \left.\left.=[h F, h F \mathbf{d}(x F)] \cdot{ }_{h F \mathbf{d}(x F)} \mid x F\right) \cdot\left[\mathbf{r}{ }_{h F \mathbf{d}(x F)} \mid x F\right), \mathbf{r}\left(\left(_{h \mathbf{d}(x)} \mid x\right) F\right)\right] \\
& \left.=(h F * x F) \cdot\left[\mathbf{r}\left(_{h F \mathbf{d}(x F)} \mid x F\right), \mathbf{r}\left({ }_{h \mathbf{d}(x)} \mid x\right) F\right)\right] \\
& =(h F * x F) \cdot[\mathbf{r}(h F * x F), \mathbf{r}((h * x) F)] \text {. }
\end{aligned}
$$

Consequently, $(h * x) F \rho h F * x F$. Similarly, part (ii) holds.

\subsection{Structure theorems}

In preparation for the main theorem at the end of this section, we have to list some necessary lemmas concerning a weakly regular category over a regular biordered set. Throughout this section, we will use $P$ to denote a weakly regular category over a regular biordered set $U$.

Lemma 10.11. Let $x \in P$ and $g, h \in U$ be such that $g \omega^{r} h \omega^{r} \mathbf{d}(x)$ and $g \mathbf{d}(x) \omega h \mathbf{d}(x)$. Then $g *(h * x)=g * x$.

Proof. By the hypothesis, $g *(h * x)$ and $g * x$ are well-defined. By (B21), 
$g h \omega h \mathcal{R} h \mathbf{d}(x)$. So,

$$
\begin{array}{rlrl} 
& g *(h * x) & \\
= & {[g, g h] \cdot{ }_{g h} \mid(h * x)} & & \\
= & {[g, g h] \cdot{ }_{g h} \mid\left([h, h \mathbf{d}(x)] \cdot{ }_{h \mathbf{d}(x)} \mid x\right)} & & \left(\text { by }(\mathrm{P} 5), k=\mathbf{r}\left({ }_{g h} \mid[h, h \mathbf{d}(x)]\right)\right) \\
= & {[g, g h] \cdot{ }_{g h}\left|[h, h \mathbf{d}(x)] \cdot{ }_{k}\right|\left({ }_{h \mathbf{d}(x)} \mid x\right)} & & \left(\text { by }(\mathrm{P} 2), k \omega^{l} h \mathbf{d}(x), \text { now use }(\mathrm{P} 4)\right) \\
= & {[g, g h] \cdot{ }_{g h}\left|[h, h \mathbf{d}(x)] \cdot{ }_{k}\right| x} & & \left(\text { by }(\mathrm{P} 3), u=(g h)(h \mathbf{d}(x)), k=u^{*}\right) \\
= & {[g, g h] \cdot[g h, u] \cdot\left[u, u^{\star}\right] \cdot{ }_{u^{\star}} \mid x} & & (g \mathcal{R} g h \mathcal{R} u, \text { by }(\mathrm{P} 1)) \\
= & {[g, u] \cdot\left[u, u^{\star}\right] \cdot{ }_{u^{\star}} \mid x} & & (\text { by }(\mathrm{P} 4)) . \\
= & {[g, u] \cdot{ }_{u} \mid x} & &
\end{array}
$$

As

$$
\begin{aligned}
& u=(g h)(h \mathbf{d}(x)) \\
& =g(h(h \mathbf{d}(x))) \\
& =g(h \mathbf{d}(x)) \\
& \left(g \omega^{r} h \mathcal{R} h \mathbf{d}(x),\right. \text { Lemma 1.27) } \\
& (h \mathbf{d}(x) \mathcal{R} h) \\
& =(g \mathbf{d}(x))(h \mathbf{d}(x)) \\
& \text { (by Lemma 1.27) } \\
& =g \mathbf{d}(x) \\
& (g \mathbf{d}(x) \omega h \mathbf{d}(x)),
\end{aligned}
$$

we obtain that $g *(h * x)=[g, g \mathbf{d}(x)] \cdot g \mathbf{d}(x) \mid x=g * x$.

Dually, we have:

Lemma 10.12. If $x \in P$ and $g, h \in U$ with $g \omega^{l} h \omega^{l} \mathbf{r}(x)$ and $\mathbf{r}(x) g \omega \mathbf{r}(x) h$, then $(x \diamond h) \diamond g=x \diamond g$.

Now, let $x, y \in P$ and $h \in S(\mathbf{r}(x), \mathbf{d}(y))$. We define

$$
(x \otimes y)_{h}=(x \diamond h) \cdot(h * y) .
$$

Since $h \in S(\mathbf{r}(x), \mathbf{d}(y))$, it follows that $h \omega^{l} \mathbf{r}(x)$ and $h \omega^{r} \mathbf{d}(y)$ so that $x \diamond h$ and $h * y$ exist. As $\mathbf{r}(x \diamond h)=h$ and $\mathbf{d}(h * y)=h,(x \otimes y)_{h}=(x \diamond h) \cdot(h * y)$ is well-defined.

Lemma 10.13. Let $x, y, z \in P, h_{1} \in S(\mathbf{r}(x), \mathbf{d}(y))$ and $h_{2} \in S(\mathbf{r}(y), \mathbf{d}(z))$. Set $h_{1}^{\prime}=\mathbf{r}\left(h_{1} * y\right)$ and $h_{2}^{\prime}=\mathbf{d}\left(y \diamond h_{2}\right)$. Then there exist $h \in S\left(\mathbf{r}(x), h_{2}^{\prime}\right)$ and 
$h^{\prime} \in S\left(h_{1}^{\prime}, \mathbf{d}(z)\right)$ such that $\left((x \otimes y)_{h_{1}} \otimes z\right)_{h^{\prime}}=\left(x \otimes(y \otimes z)_{h_{2}}\right)_{h}$.

Proof. Since $h_{1}^{\prime}=\mathbf{r}\left(h_{1} * y\right)=\mathbf{r}\left(h_{1} \mathbf{d}(y) \mid y\right) \omega^{l} \mathbf{r}(y)$ and $h_{2} \in S(\mathbf{r}(y), \mathbf{d}(z))$, it follows from Lemma 1.30 that $S\left(h_{1}^{\prime}, h_{2}\right) \subseteq S\left(h_{1}^{\prime}, \mathbf{d}(z)\right)$. Similarly, as $h_{2}^{\prime}=\mathbf{d}\left(y \diamond h_{2}\right)=$ $\mathbf{d}\left(\left.y\right|_{\mathbf{r}(y) h_{2}}\right) \omega^{r} \mathbf{d}(y)$ and $h_{1} \in S(\mathbf{r}(x), \mathbf{d}(y))$, by the same result, we have that $S\left(h_{1}, h_{2}^{\prime}\right) \subseteq S\left(\mathbf{r}(x), h_{2}^{\prime}\right)$.

Since $h_{1} \in S(\mathbf{r}(x), \mathbf{d}(y)), h_{2} \in S(\mathbf{r}(y), \mathbf{d}(z)), h_{1}^{\prime}=\mathbf{r}\left(h_{1} * y\right)$ and $h_{2}^{\prime}=$ $\mathbf{d}\left(y \diamond h_{2}\right)$, by $(\mathrm{P} 7)$, there exist $h^{\prime} \in S\left(h_{1}^{\prime}, h_{2}\right)$ and $h \in S\left(h_{1}, h_{2}^{\prime}\right)$ such that

$$
\left(\left(h_{1} * y\right) \diamond h^{\prime}\right) \cdot\left[h^{\prime}, h^{\prime} h_{2}\right] \rho\left[h_{1} h, h\right] \cdot\left(h *\left(y \diamond h_{2}\right)\right) .
$$

Put $g=\mathbf{d}\left(\left(h_{1} * y\right) \diamond h^{\prime}\right)$ and $k=\mathbf{r}\left(h *\left(y \diamond h_{2}\right)\right)$. We obtain that $g \mathcal{R} h_{1} h, h^{\prime} h_{2} \mathcal{L} k$, and

$$
\left[h_{1} h, g\right] \cdot\left(\left(h_{1} * y\right) \diamond h^{\prime}\right) \cdot\left[h^{\prime}, h^{\prime} h_{2}\right]=\left[h_{1} h, h\right] \cdot\left(h *\left(y \diamond h_{2}\right)\right) \cdot\left[k, h^{\prime} h_{2}\right] .
$$

Now, we deduce that

$$
\begin{aligned}
& \left((x \otimes y)_{h_{1}} \otimes z\right)_{h^{\prime}} \\
= & \left((x \otimes y)_{h_{1}} \diamond h^{\prime}\right) \cdot\left(h^{\prime} * z\right) \quad\left(h_{1}^{\prime}=\mathbf{r}\left(h_{1} * y\right), h^{\prime} \in S\left(h_{1}^{\prime}, \mathbf{d}(z)\right)\right) \\
= & \left(\left(\left(x \diamond h_{1}\right) \cdot\left(h_{1} * y\right)\right) \diamond h^{\prime}\right) \cdot\left(h^{\prime} * z\right) \\
= & \left.\left(\left(x \diamond h_{1}\right) \cdot\left(h_{1} * y\right)\right)\right|_{h_{1}^{\prime} h^{\prime}} \cdot\left[h_{1}^{\prime} h^{\prime}, h^{\prime}\right] \cdot\left(h^{\prime} * z\right) \\
= & \left.\left.\left(x \diamond h_{1}\right)\right|_{g} \cdot\left(h_{1} * y\right)\right|_{h_{1}^{\prime} h^{\prime}} \cdot\left[h_{1}^{\prime} h^{\prime}, h^{\prime}\right] \cdot\left(h^{\prime} * z\right) \\
& \quad\left(\mathrm{by}(\mathrm{P} 5)^{\circ}, g=\mathbf{d}\left(\left(h_{1} * y\right) \diamond h^{\prime}\right)=\mathbf{d}\left(\left.\left(h_{1} * y\right)\right|_{h_{1}^{\prime} h^{\prime}}\right)\right) \\
= & \left.\left(x \diamond h_{1}\right)\right|_{g} \cdot\left(\left(h_{1} * y\right) \diamond h^{\prime}\right) \cdot\left(h^{\prime} * z\right) \\
= & \left.\left(x \diamond h_{1}\right)\right|_{g} \cdot\left(\left(h_{1} * y\right) \diamond h^{\prime}\right) \cdot\left[h^{\prime}, h^{\prime}\right] \cdot\left(h^{\prime} * z\right) \\
= & \left.\left(x \diamond h_{1}\right)\right|_{g} \cdot\left(\left(h_{1} * y\right) \diamond h^{\prime}\right) \cdot\left[h^{\prime}, h^{\prime} h_{2}\right] \cdot\left[h^{\prime} h_{2}, h^{\prime}\right] \cdot\left(h^{\prime} * z\right) \\
= & \left(x \diamond h_{1}\right) \mid g \cdot[g, g] \cdot\left(\left(h_{1} * y\right) \diamond h^{\prime} h_{2}, \quad \text { by }(\mathrm{P} 1)\right) \\
= & \left(x \diamond h_{1}\right) \mid g \cdot\left[g, h_{1} h\right] \cdot\left[h_{1} h, g\right] \cdot\left(\left(h_{1} * y\right) \diamond h^{\prime}\right) \cdot\left[h^{\prime}, h^{\prime} h_{2}\right] \cdot\left[h^{\prime} h_{2}, h^{\prime}\right] \cdot\left(h^{\prime} * z\right) \\
& \quad\left(g \mathcal{R} h_{1} h, \quad \text { by }(\mathrm{P} 1)\right) \\
= & \left(x \diamond h_{1}\right) \mid g \cdot\left[g, h_{1} h\right] \cdot\left[h_{1} h, h\right] \cdot\left(h *\left(y \diamond h_{2}\right)\right) \cdot\left[k, h^{\prime} h_{2}\right] \cdot\left[h^{\prime} h_{2}, h^{\prime}\right] \cdot\left(h^{\prime} * z\right) \\
& \left(\text { as }\left[h_{1} h, g\right] \cdot\left(\left(h_{1} * y\right) \diamond h^{\prime}\right) \cdot\left[h^{\prime}, h^{\prime} h_{2}\right]=\left[h_{1} h, h\right] \cdot\left(h *\left(y \diamond h_{2}\right)\right) \cdot\left[k, h^{\prime} h_{2}\right]\right)
\end{aligned}
$$




$$
\begin{aligned}
& =\left.\left(x \diamond h_{1}\right)\right|_{h_{1} h} \cdot\left[h_{1} h, h\right] \cdot\left(h *\left(y \diamond h_{2}\right)\right) \cdot\left[k, h^{\prime} h_{2}\right] \cdot\left[h^{\prime} h_{2}, h^{\prime}\right] \cdot\left(h^{\prime} * z\right) \\
& \quad\left(g \mathcal{R} h_{1} h, \text { by }(\mathrm{P} 4)^{\circ}\right) \\
& =\left(\left(x \diamond h_{1}\right) \diamond h\right) \cdot\left(h *\left(y \diamond h_{2}\right)\right) \cdot\left[k, h^{\prime} h_{2}\right] \cdot\left[h^{\prime} h_{2}, h^{\prime}\right] \cdot\left(h^{\prime} * z\right) \quad\left(h \omega^{l} h_{1}\right) .
\end{aligned}
$$

From $h \in S\left(h_{1}, h_{2}^{\prime}\right)$, we have that $h \omega^{l} h_{1}$ and $h \omega^{r} h_{2}^{\prime}$. As $h_{1} \in S(\mathbf{r}(x), \mathbf{d}(y))$ and $h_{2}^{\prime}=\mathbf{d}\left(y \diamond h_{2}\right)$, we have that $h_{1} \omega^{l} \mathbf{r}(x)$ and $h_{2}^{\prime} \omega^{r} \mathbf{d}(y)$. Thus $h \omega^{l} h_{1} \omega^{l} \mathbf{r}(x)$ and $h \omega^{r} h_{2} \omega^{r} \mathbf{d}(y)$, so that $h \in \mathcal{M}(\mathbf{r}(x), \mathbf{d}(y))$. Since $h_{1} \in S(\mathbf{r}(x), \mathbf{d}(y))$, it follows that $\mathbf{r}(x) h \omega^{r} \mathbf{r}(x) h_{1}$. Again by $\mathbf{r}(x) h \mathcal{L} h \omega^{l} h_{1} \mathcal{L} \mathbf{r}(x) h_{1}$, we have that $\mathbf{r}(x) h \omega \mathbf{r}(x) h_{1}$. By Lemma 10.12, $\left(x \diamond h_{1}\right) \diamond h=x \diamond h$. Hence,

$$
\left((x \otimes y)_{h_{1}} \otimes z\right)_{h^{\prime}}=(x \diamond h) \cdot\left(h *\left(y \diamond h_{2}\right)\right) \cdot\left[k, h^{\prime} h_{2}\right] \cdot\left[h^{\prime} h_{2}, h^{\prime}\right] \cdot\left(h^{\prime} * z\right)
$$

Also,

$$
\begin{aligned}
& \left(x \otimes(y \otimes z)_{h_{2}}\right)_{h} \\
& =(x \diamond h) \cdot\left(h *(y \otimes z)_{h_{2}}\right) \\
& =(x \diamond h) \cdot\left(h *\left(\left(y \diamond h_{2}\right) \cdot\left(h_{2} * z\right)\right)\right) \\
& =(x \diamond h) \cdot\left[h, h h_{2}^{\prime}\right] \cdot h h_{2}^{\prime} \mid\left(\left(y \diamond h_{2}\right) \cdot\left(h_{2} * z\right)\right) \\
& \left.=(x \diamond h) \cdot\left[h, h h_{2}^{\prime}\right] \cdot{ }_{h h_{2}^{\prime}}\left|\left(y \diamond h_{2}\right) \cdot{ }_{k}\right|\left(h_{2} * z\right)\right) \\
& \left(h_{2} \in S(\mathbf{r}(y), \mathbf{d}(z))\right) \\
& \text { (by } \left.(\mathrm{P} 5), k=\mathbf{r}\left({ }_{h h_{2}^{\prime}} \mid\left(y \diamond h_{2}\right)\right)=\mathbf{r}\left(h *\left(y \diamond h_{2}\right)\right)\right) \\
& =(x \diamond h) \cdot\left(h *\left(y \diamond h_{2}\right)\right) \cdot{ }_{k} \mid\left(h_{2} * z\right) \quad\left(h \omega^{r} h_{2}^{\prime}=\mathbf{d}\left(y \diamond h_{2}\right), k=\mathbf{r}\left(h *\left(y \diamond h_{2}\right)\right)\right) \\
& =(x \diamond h) \cdot\left(h *\left(y \diamond h_{2}\right)\right) \cdot[k, k] \cdot{ }_{k} \mid\left(h_{2} * z\right) \\
& =(x \diamond h) \cdot\left(h *\left(y \diamond h_{2}\right)\right) \cdot\left[k, h^{\prime} h_{2}\right] \cdot\left[h^{\prime} h_{2}, k\right] \cdot{ }_{k} \mid\left(h_{2} * z\right) \quad\left(k \mathcal{L} h^{\prime} h_{2} \text {, by }(\mathrm{P} 1)\right) \\
& =(x \diamond h) \cdot\left(h *\left(y \diamond h_{2}\right)\right) \cdot\left[k, h^{\prime} h_{2}\right] \cdot h^{\prime} h_{2} \mid\left(h_{2} * z\right) \quad\left(k \mathcal{L} h^{\prime} h_{2}, \text { by }(\mathrm{P} 4)\right) \\
& =(x \diamond h) \cdot\left(h *\left(y \diamond h_{2}\right)\right) \cdot\left[k, h^{\prime} h_{2}\right] \cdot\left[h^{\prime} h_{2}, h^{\prime} h_{2}\right] \cdot{ }_{h^{\prime} h_{2}} \mid\left(h_{2} * z\right) \\
& =(x \diamond h) \cdot\left(h *\left(y \diamond h_{2}\right)\right) \cdot\left[k, h^{\prime} h_{2}\right] \cdot\left[h^{\prime} h_{2}, h^{\prime}\right] \cdot\left[h^{\prime}, h^{\prime} h_{2}\right] \cdot{ }_{h^{\prime} h_{2}} \mid\left(h_{2} * z\right) \\
& \left(h^{\prime} \mathcal{R} h^{\prime} h_{2} \text {, by }(\mathrm{P} 1)\right) \\
& =(x \diamond h) \cdot\left(h *\left(y \diamond h_{2}\right)\right) \cdot\left[k, h^{\prime} h_{2}\right] \cdot\left[h^{\prime} h_{2}, h^{\prime}\right] \cdot\left(h^{\prime} *\left(h_{2} * z\right)\right) \\
& \left(h^{\prime} \omega^{r} h_{2}=\mathbf{d}\left(h_{2} * z\right)\right) \text {. }
\end{aligned}
$$

Since $h^{\prime} \omega^{r} h_{2} \omega^{r} \mathbf{d}(z)$, it follows that $h^{\prime} \mathbf{d}(z) \mathcal{R} h^{\prime} \omega^{r} h_{2} \mathcal{R} h_{2} \mathbf{d}(z)$. As

$$
h^{\prime} \in \mathcal{M}(\mathbf{r}(y), \mathbf{d}(z)) \text { and } h_{2} \in S(\mathbf{r}(y), \mathbf{d}(z))
$$


we have that $h^{\prime} \mathbf{d}(z) \omega^{l} h_{2} \mathbf{d}(z)$. So $h^{\prime} \mathbf{d}(z) \omega h_{2} \mathbf{d}(z)$. By Lemma 10.11, $h^{\prime} *\left(h_{2} * z\right)=$ $h^{\prime} * z$. Thus,

$$
\left(x \otimes(y \otimes z)_{h_{2}}\right)_{h}=(x \diamond h) \cdot\left(h *\left(y \diamond h_{2}\right)\right) \cdot\left[k, h^{\prime} h_{2}\right] \cdot\left[h^{\prime} h_{2}, h^{\prime}\right] \cdot\left(h^{\prime} * z\right) .
$$

Hence, $\left((x \otimes y)_{h_{1}} \otimes z\right)_{h^{\prime}}=\left(x \otimes(y \otimes z)_{h_{2}}\right)_{h}$.

Lemma 10.14. If $x, y \in P$ and $x \rho y$, then $h * x \rho h * y$ and $x \diamond k \rho y \diamond k$, where $h \omega^{r} \mathbf{d}(x)$ and $k \omega^{l} \mathbf{r}(x)$.

Proof. We first show that $x \rho y$ and $h \omega^{r} \mathbf{d}(x)$ imply $h * x \rho h * y$. Dually, the second part holds. Clearly, if $x \rho y$, then $\mathbf{d}(x) \mathcal{R} \mathbf{d}(y)$ and $\mathbf{r}(x) \mathcal{L} \mathbf{r}(y)$. Write $h_{1}=\mathbf{r}(h * x)=\mathbf{r}(h \mathbf{d}(x) \mid x)$. We deduce that

$$
\begin{aligned}
& h \mathbf{d}(x) *(x \cdot[\mathbf{r}(x), \mathbf{r}(y)])={ }_{h \mathbf{d}(x)} \mid(x \cdot[\mathbf{r}(x), \mathbf{r}(y)]) \quad(h \mathbf{d}(x) \omega \mathbf{d}(x)) \\
& ={ }_{h \mathbf{d}(x)}\left|x \cdot{ }_{h_{1}}\right|[\mathbf{r}(x), \mathbf{r}(y)] \quad\left(h_{1}=\mathbf{r}\left({ }_{h \mathbf{d}(x)} \mid x\right), \text { by }(\mathrm{P} 5)\right) \\
& ={ }_{h \mathbf{d}(x)} \mid x \cdot\left[h_{1}, h_{1}^{\star}\right] \quad\left(h_{1} \omega^{l} \mathbf{r}(x) \mathcal{L} \mathbf{r}(y), \text { by }(\mathrm{P} 3)\right)
\end{aligned}
$$

and

$$
\begin{aligned}
& h \mathbf{d}(x) *([\mathbf{d}(x), \mathbf{d}(y)] \cdot y) \\
= & { }_{h \mathbf{d}(x)} \mid([\mathbf{d}(x), \mathbf{d}(y)] \cdot y) \\
= & { }_{h \mathbf{d}(x)}\left|[\mathbf{d}(x), \mathbf{d}(y)] \cdot{ }_{g}\right| y \quad(h \mathbf{d}(x) \omega \mathbf{d}(x)) \\
= & {[h \mathbf{d}(x),(h \mathbf{d}(x)) \mathbf{d}(y)] \cdot\left[(h \mathbf{d}(x)) \mathbf{d}(y),((h \mathbf{d}(x)) \mathbf{d}(y))^{\star}\right] \cdot{ }_{g} \mid y } \\
& (h \mathbf{d}(x) \omega \mathbf{d}(x) \mathcal{R} \mathbf{d}(y), \quad \text { by }(\mathrm{P} 3)) \\
= & {\left.\left.[h \mathbf{d}(x), h \mathbf{d}(y)] \cdot\left[h \mathbf{d}(y),(h \mathbf{d}(y))^{\star}\right] \cdot{ }_{g} \mid y \quad \mathbf{r}\left({ }_{h \mathbf{d}}|| \mathbf{d}(x), \mathbf{d}(y)\right]\right)\right) } \\
& \left(h \omega^{r} \mathbf{d}(x) \mathcal{R} \mathbf{d}(y), \text { by }(\mathrm{B} 31), \text { so } g=(h \mathbf{d}(y))^{\star}\right) \\
= & {[h \mathbf{d}(x), h \mathbf{d}(y)] \cdot h \mathbf{d}(y) \mid y \quad(\operatorname{by}(\mathrm{P} 4)) . }
\end{aligned}
$$

Since $x \rho y$, we have that $x \cdot[\mathbf{r}(x), \mathbf{r}(y)]=[\mathbf{d}(x), \mathbf{d}(y)] \cdot y$. Thus,

$$
h \mathbf{d}(x) *(x \cdot[\mathbf{r}(x), \mathbf{r}(y)])=h \mathbf{d}(x) *([\mathbf{d}(x), \mathbf{d}(y)] \cdot y) .
$$


So ${ }_{h \mathbf{d}(x)}\left|x \cdot\left[h_{1}, h_{1}^{\star}\right]=[h \mathbf{d}(x), h \mathbf{d}(y)] \cdot{ }_{h \mathbf{d}(y)}\right| y$. Hence,

$$
\begin{aligned}
& (h * x) \cdot\left[h_{1}, h_{1}^{\star}\right] \\
= & {[h, h \mathbf{d}(x)] \cdot h \mathbf{d}(x) \mid x \cdot\left[h_{1}, h_{1}^{\star}\right] } \\
= & {[h, h \mathbf{d}(x)] \cdot[h \mathbf{d}(x), h \mathbf{d}(y)] \cdot h \mathbf{d}(y) \mid y } \\
= & {[h, h \mathbf{d}(y)] \cdot h \mathbf{d}(y) \mid y \quad(h \mathcal{R} h \mathbf{d}(x) \mathcal{R} h \mathbf{d}(y), \text { by }(\mathrm{P} 1)) } \\
= & h * y
\end{aligned}
$$

which implies that $h_{1}^{\star}=\mathbf{r}(h * y)$, so that

$$
\begin{aligned}
(h * x) \cdot\left[h_{1}, h_{1}^{\star}\right] & =(h * x) \cdot[\mathbf{r}(h * x), \mathbf{r}(h * y)] \\
& =h * y \\
& =[h, h] \cdot(h * y) \\
& =[\mathbf{d}(h * x), \mathbf{d}(h * y)] \cdot(h * y) .
\end{aligned}
$$

Consequently, $h * x \rho h * y$.

Lemma 10.15. If $x \rho x^{\prime}, y \rho y^{\prime}$ and $h \in S(\mathbf{r}(x), \mathbf{d}(y))$, then $(x \otimes y)_{h} \rho\left(x^{\prime} \otimes y^{\prime}\right)_{h}$.

Proof. Since $x \rho x^{\prime}$ and $y \rho y^{\prime}$, we have that $\mathbf{r}(x) \mathcal{L} \mathbf{r}\left(x^{\prime}\right)$ and $\mathbf{d}(y) \mathcal{R} \mathbf{d}\left(y^{\prime}\right)$, which gives from Lemma 1.28 that $S(\mathbf{r}(x), \mathbf{d}(y))=S\left(\mathbf{r}\left(x^{\prime}\right), \mathbf{d}\left(y^{\prime}\right)\right)$. Thus, $\left(x^{\prime} \otimes y^{\prime}\right)_{h}$ is well-defined.

In view of Lemma 10.14, we have that $x \diamond h \rho x^{\prime} \diamond h$. So

$$
\begin{gathered}
\mathbf{d}\left(\left(x^{\prime} \otimes y^{\prime}\right)_{h}\right)=\mathbf{d}\left(x^{\prime} \diamond h\right) \mathcal{R} \mathbf{d}(x \diamond h)=\mathbf{d}\left((x \otimes y)_{h}\right), \\
\mathbf{r}\left(x^{\prime} \diamond h\right) \mathcal{L} \mathbf{r}(x \diamond h)
\end{gathered}
$$

and

$$
\left(x^{\prime} \diamond h\right) \cdot\left[\mathbf{r}\left(x^{\prime} \diamond h\right), \mathbf{r}(x \diamond h)\right]=\left[\mathbf{d}\left(x^{\prime} \diamond h\right), \mathbf{d}(x \diamond h)\right] \cdot(x \diamond h),
$$

that is,

$$
\left(x^{\prime} \diamond h\right) \cdot[h, h]=\left[\mathbf{d}\left(x^{\prime} \diamond h\right), \mathbf{d}(x \diamond h)\right] \cdot(x \diamond h),
$$

so, $x^{\prime} \diamond h=\left[\mathbf{d}\left(x^{\prime} \diamond h\right), \mathbf{d}(x \diamond h)\right] \cdot(x \diamond h)$.

Dually,

$$
\mathbf{r}\left(\left(x^{\prime} \otimes y^{\prime}\right)_{h}\right)=\mathbf{r}\left(h * y^{\prime}\right) \mathcal{L} \mathbf{r}(h * y)=\mathbf{d}\left((x \otimes y)_{h}\right)
$$


and $h * y^{\prime}=(h * y) \cdot\left[\mathbf{r}(h * y), \mathbf{r}\left(h * y^{\prime}\right)\right]$. Now, we have that

$$
\begin{aligned}
& \left(x^{\prime} \otimes y^{\prime}\right)_{h}=\left(x^{\prime} \diamond h\right) \cdot\left(h * y^{\prime}\right) \\
= & {\left[\mathbf{d}\left(x^{\prime} \diamond h\right), \mathbf{d}(x \diamond h)\right] \cdot(x \diamond h) \cdot(h * y) \cdot\left[\mathbf{r}(h * y), \mathbf{r}\left(h * y^{\prime}\right)\right] } \\
= & {\left[\mathbf{d}\left(x^{\prime} \diamond h\right), \mathbf{d}(x \diamond h)\right] \cdot(x \otimes y)_{h} \cdot\left[\mathbf{r}(h * y), \mathbf{r}\left(h * y^{\prime}\right)\right] . }
\end{aligned}
$$

Thus,

$$
\begin{aligned}
& {\left[\mathbf{d}(x \diamond h), \mathbf{d}\left(x^{\prime} \diamond h\right)\right] \cdot\left(x^{\prime} \otimes y^{\prime}\right)_{h} } \\
= & {\left[\mathbf{d}(x \diamond h), \mathbf{d}\left(x^{\prime} \diamond h\right)\right] \cdot\left[\mathbf{d}\left(x^{\prime} \diamond h\right), \mathbf{d}(x \diamond h)\right] \cdot(x \otimes y)_{h} \cdot\left[\mathbf{r}(h * y), \mathbf{r}\left(h * y^{\prime}\right)\right] } \\
= & {[\mathbf{d}(x \diamond h), \mathbf{d}(x \diamond h)] \cdot(x \otimes y)_{h} \cdot\left[\mathbf{r}(h * y), \mathbf{r}\left(h * y^{\prime}\right)\right] \quad(\text { by }} \\
= & (x \otimes y)_{h} \cdot\left[\mathbf{r}(h * y), \mathbf{r}\left(h * y^{\prime}\right)\right],
\end{aligned}
$$

together with $\mathbf{d}\left(x^{\prime} \diamond h\right) \mathcal{R} \mathbf{d}(x \diamond h)$ and $\mathbf{r}\left(h * y^{\prime}\right) \mathcal{L} \mathbf{r}(h * y)$, we have that $(x \otimes y)_{h} \rho\left(x^{\prime} \otimes y^{\prime}\right)_{h}$.

Lemma 10.16. If $x, y \in P$ and $h, h^{\prime} \in S(\mathbf{r}(x), \mathbf{d}(y))$, then $(x \otimes y)_{h}=(x \otimes y)_{h^{\prime}}$. Proof. Since $h, h^{\prime} \in S(\mathbf{r}(x), \mathbf{d}(y))$, we can set

$$
\begin{array}{ll}
h_{1}=\mathbf{d}(x \diamond h)=\mathbf{d}\left((x \otimes y)_{h}\right), & h_{2}=\mathbf{r}(h * y)=\mathbf{r}\left((x \otimes y)_{h}\right), \\
h_{1}^{\prime}=\mathbf{d}\left(x \diamond h^{\prime}\right)=\mathbf{d}\left((x \otimes y)_{h^{\prime}}\right), & h_{2}^{\prime}=\mathbf{r}\left(h^{\prime} * y\right)=\mathbf{r}\left((x \otimes y)_{h^{\prime}}\right) .
\end{array}
$$

Suppose that $h \mathcal{R} h^{\prime}$. Then $h \mathbf{d}(y) \mathcal{R} h \mathcal{R} h^{\prime} \mathcal{R} h^{\prime} \mathbf{d}(y)$. But $h, h^{\prime} \in S(\mathbf{r}(x), \mathbf{d}(y))$, so that $h \prec h^{\prime}$ and $h^{\prime} \prec h$, which imply that $h \mathbf{d}(y) \omega^{l} h^{\prime} \mathbf{d}(y)$ and $h^{\prime} \mathbf{d}(y) \omega^{l} h \mathbf{d}(y)$. Thus $h \mathbf{d}(y) \mathcal{L} h^{\prime} \mathbf{d}(y)$. Hence, $h \mathbf{d}(y)=h^{\prime} \mathbf{d}(y)$, and so $h \mathbf{d}(y)\left|y={ }_{h^{\prime}} \mathbf{d}(y)\right| y$, which implies that $h_{2}=h_{2}^{\prime}$. In addition,

$$
\begin{array}{rlrl}
h^{\prime} * y & =\left[h^{\prime}, h^{\prime} \mathbf{d}(y)\right] \cdot{ }_{h^{\prime} \mathbf{d}(y)} \mid y & \\
& =\left[h^{\prime}, h\right] \cdot\left[h, h^{\prime} \mathbf{d}(y)\right] \cdot{ }_{h^{\prime} \mathbf{d}(y)} \mid y & & \left(h \mathcal{R} h^{\prime} \mathcal{R} h^{\prime} \mathbf{d}(y), \text { by }(\mathrm{P} 1)\right) \\
& =\left[h^{\prime}, h\right] \cdot[h, h \mathbf{d}(y)] \cdot{ }_{h \mathbf{d}(y)} \mid y & & \left(h^{\prime} \mathbf{d}(y)=h \mathbf{d}(y)\right) \\
& =\left[h^{\prime}, h\right] \cdot(h * y) . & &
\end{array}
$$

Since $h, h^{\prime} \in S(\mathbf{r}(x), \mathbf{d}(y))$, we have that $h, h^{\prime} \omega^{l} \mathbf{r}(x)$, and so $\mathbf{r}(x) h \mathcal{L} h$ and 
$\mathbf{r}(x) h^{\prime} \mathcal{L} h^{\prime}$. As $h \mathcal{R} h^{\prime}$, by $(\mathrm{B} 32)^{\circ}$, we have that

$$
(\mathbf{r}(x) h)\left(\mathbf{r}(x) h^{\prime}\right)=\mathbf{r}(x)\left(h h^{\prime}\right)=\mathbf{r}(x) h^{\prime} \text { and }\left(\mathbf{r}(x) h^{\prime}\right)(\mathbf{r}(x) h)=\mathbf{r}(x) h,
$$

that is, $\mathbf{r}(x) h \mathcal{R} \mathbf{r}(x) h^{\prime}$. Now, we obtain a row-singular $U$-square $\left(\begin{array}{cc}h & h^{\prime} \\ \mathbf{r}(x) h & \mathbf{r}(x) h^{\prime}\end{array}\right)$. By the comments succeeding Definition 10.1, we have

$$
[\mathbf{r}(x) h, h] \cdot\left[h, h^{\prime}\right]=\left[\mathbf{r}(x) h, \mathbf{r}(x) h^{\prime}\right] \cdot\left[\mathbf{r}(x) h^{\prime}, h^{\prime}\right],
$$

and so

$$
\begin{aligned}
(x \diamond h) \cdot\left[h, h^{\prime}\right] & =\left.x\right|_{\mathbf{r}(x) h} \cdot[\mathbf{r}(x) h, h] \cdot\left[h, h^{\prime}\right] \\
& =\left.x\right|_{\mathbf{r}(x) h} \cdot\left[\mathbf{r}(x) h, \mathbf{r}(x) h^{\prime}\right] \cdot\left[\mathbf{r}(x) h^{\prime}, h^{\prime}\right] \\
& =\left.x\right|_{\mathbf{r}(x) h^{\prime}} \cdot\left[\mathbf{r}(x) h^{\prime}, h^{\prime}\right] \quad\left(\mathbf{r}(x) h \mathcal{R} \mathbf{r}(x) h^{\prime}, \text { by }(P 4)^{\circ}\right) \\
& =x \diamond h^{\prime} .
\end{aligned}
$$

Thus, $\mathbf{d}(x \diamond h)=\mathbf{d}\left(x \diamond h^{\prime}\right)$, that is, $h_{1}^{\prime}=h_{1}$. So

$$
\begin{aligned}
(x \otimes y)_{h^{\prime}}=\left(x \diamond h^{\prime}\right) \cdot\left(h^{\prime} * y\right) & \\
=(x \diamond h) \cdot\left[h, h^{\prime}\right] \cdot\left[h^{\prime}, h\right] \cdot(h * y) & \\
=(x \diamond h) \cdot[h, h] \cdot(h * y) & \left(h \mathcal{R} h^{\prime}, \text { by }(\mathrm{P} 1)\right) \\
& =(x \diamond h) \cdot(h * y) \\
& =(x \otimes y)_{h} .
\end{aligned}
$$

Dually, if $h \mathcal{L} h^{\prime}$ we can show that $(x \otimes y)_{h}=(x \otimes y)_{h^{\prime}}$. By the comment succeeding Lemma 1.35, if $h, h^{\prime} \in S(\mathbf{r}(x), \mathbf{d}(y))$, there exists $k \in S(\mathbf{r}(x), \mathbf{d}(y))$ such that $h \mathcal{R} k \mathcal{L} h^{\prime}$. Thus the lemma holds in all cases.

Let $P \mathbf{S}=P / \rho$. For $x, y \in P, h \in S(\mathbf{r}(x), \mathbf{d}(y))$, we define

$$
\bar{x} \odot \bar{y}=\overline{(x \otimes y)_{h}},
$$

where $\bar{x}$ denotes the $\rho$-class of $x$ in $P$.

Lemma 10.17. The set $P \mathbf{S}=P / \rho$ forms a semigroup under $\odot$ defined above. 
Proof. Let $x, x^{\prime}, y, y^{\prime} \in P$ with $x \rho x^{\prime}$ and $y \rho y^{\prime}$. If $h, h^{\prime} \in S(\mathbf{r}(x), \mathbf{d}(y))=$ $S\left(\mathbf{r}\left(x^{\prime}\right), \mathbf{d}\left(y^{\prime}\right)\right)$, then by Lemma 10.15 and Lemma 10.16 , we have that

$$
(x \otimes y)_{h} \rho\left(x^{\prime} \otimes y^{\prime}\right)_{h}=\left(x^{\prime} \otimes y^{\prime}\right)_{h^{\prime}} .
$$

Thus, $\overline{(x \otimes y)_{h}}=\overline{\left(x^{\prime} \otimes y^{\prime}\right)_{h^{\prime}}}$, and so the product is well-defined.

In order to show that the operation $\odot$ is associative, we assume that $x, y, z \in$ $P, h_{1} \in S(\mathbf{r}(x), \mathbf{d}(y))$ and $h_{2} \in S(\mathbf{r}(y), \mathbf{d}(z))$. By Lemma 10.13, there exist $h \in S\left(\mathbf{r}(x), \mathbf{d}\left((y \otimes z)_{h_{2}}\right)\right)$ and $h^{\prime} \in S\left(\mathbf{r}\left((x \otimes y)_{h_{1}}\right), \mathbf{d}(z)\right)$ such that $\left((x \otimes y)_{h_{1}} \otimes z\right)_{h^{\prime}}=$ $\left(x \otimes(y \otimes z)_{h_{2}}\right)_{h}$. Thus

$$
\begin{aligned}
(\bar{x} \odot \bar{y}) \odot \bar{z} & =\overline{(x \otimes y)_{h_{1}}} \odot \bar{z}=\overline{\left((x \otimes y)_{h_{1}} \otimes z\right)_{h^{\prime}}} \\
& =\overline{\left(x \otimes(y \otimes z)_{h_{2}}\right)_{h}}=\bar{x} \odot \overline{(y \otimes z)_{h_{2}}}=\bar{x} \odot(\bar{y} \odot \bar{z}) .
\end{aligned}
$$

Hence, $P \mathbf{S}$ is a semigroup.

Lemma 10.18. If $e, h \in U$ are such that $h \omega^{r} e$, then

$$
h * 1_{e} \rho 1_{h e} \rho\left(1_{h} \otimes 1_{e}\right)_{h},
$$

so that $\overline{1_{h}} \odot \overline{1_{e}}=\overline{1_{h e}}$.

Proof. Suppose that $e, h \in U$ with $h \omega^{r} e$. Then $h \mathcal{R} h e$ and

$$
\begin{aligned}
{[h e, h] \cdot\left(h * 1_{e}\right) } & =[h e, h] \cdot(h *[e, e]) \\
& =[h e, h] \cdot[h, h e] \cdot\left[h e,(h e)^{\star}\right] \\
& =[h e, h e] \cdot\left[h e,(h e)^{\star}\right] \\
& =1_{h e} \cdot\left[h e,(h e)^{\star}\right] .
\end{aligned}
$$

Certainly, $\mathbf{r}\left(h * 1_{e}\right)=(h e)^{\star} \mathcal{L} h e$. Thus, $h * 1_{e} \rho 1_{h e}$.

According to Lemma 1.29, we have that $e h=h \in S(h, e)$, and so $\left(1_{h} \otimes 1_{e}\right)_{h}$ is well-defined. Then

$$
\begin{array}{rlr}
\left(1_{h} \otimes 1_{e}\right)_{h} & =([h, h] \otimes[e, e])_{h} \\
& =([h, h] \diamond h) \cdot(h *[e, e]) \\
& =\left[h^{+}, h\right] \cdot[h, h e] \cdot\left[h e,(h e)^{\star}\right] \\
& =\left[h^{+}, h e\right] \cdot\left[h e,(h e)^{\star}\right] \quad\left(h^{+} \mathcal{R} h \mathcal{R} h e, \text { by }(\mathrm{P} 1)\right) .
\end{array}
$$


Further,

$$
\begin{aligned}
{\left[h^{+}, h e\right] \cdot 1_{h e} } & =\left[h^{+}, h e\right] \cdot[h e, h e] \\
& =\left[h^{+}, h e\right] \cdot\left[h e,(h e)^{\star}\right] \cdot\left[(h e)^{\star}, h e\right] \\
& =\left(1_{h} \otimes 1_{e}\right)_{h} \cdot\left[(h e)^{\star}, h e\right] .
\end{aligned}
$$

Hence, $1_{h e} \rho\left(1_{h} \otimes 1_{e}\right)_{h}$.

Dually, we have:

Lemma 10.19. If $e, h \in U$ are such that $h \omega^{l} e$, then

$$
1_{e} \diamond h \rho 1_{e h} \rho\left(1_{e} \otimes 1_{h}\right)_{h}
$$

so that $\overline{1_{e}} \odot \overline{1_{h}}=\overline{1_{e h}}$.

Lemma 10.20. If $P$ is a weakly regular category over $U$ and $x, y \in P$ with $x \cdot y$ defined, then $\bar{x} \odot \bar{y}=\overline{x \cdot y}$. Moreover, if $e \in U$, then $\overline{1_{e}} \in E(P \mathbf{S})$.

Proof. Suppose that $x, y \in P$ are such that $x \cdot y$ is defined. Then $\mathbf{r}(x)=\mathbf{d}(y)$, from which it follows that $S(\mathbf{r}(x), \mathbf{d}(y))=\{\mathbf{r}(x)\}$. Thus,

$$
\bar{x} \odot \bar{y}=\overline{(x \otimes y)_{\mathbf{r}(x)}}=\overline{(x \diamond \mathbf{r}(x)) \cdot(\mathbf{r}(x) * y)}=\overline{\left.x\right|_{\mathbf{r}(x) \cdot \mathbf{r}(x)} \mid y}
$$

Let $h=\mathbf{d}(y)=\mathbf{r}(x)$. From $(\mathrm{P} 2)$ and $(\mathrm{P} 2)^{\circ}$, we have $\mathbf{d}\left(\left.x\right|_{h}\right) \mathcal{R} \mathbf{d}(x), \mathbf{r}\left({ }_{h} \mid y\right) \mathcal{L} \mathbf{r}(y)$ and

$$
{ }_{h} \mid y \cdot\left[\mathbf{r}\left({ }_{h} \mid y\right), \mathbf{r}(y)\right]=y,
$$

so

$$
x \cdot{ }_{h} \mid y \cdot\left[\mathbf{r}\left({ }_{h} \mid y\right), \mathbf{r}(y)\right]=x \cdot y .
$$

But

$$
x=\left.\left[\mathbf{d}(x), \mathbf{d}\left(\left.x\right|_{h}\right)\right] \cdot x\right|_{h},
$$

So

$$
\left.\left[\mathbf{d}(x), \mathbf{d}\left(\left.x\right|_{h}\right)\right] \cdot x\right|_{h} \cdot{ }_{h} \mid y \cdot\left[\mathbf{r}\left({ }_{h} \mid y\right), \mathbf{r}(y)\right]=x \cdot y
$$

hence,

$$
\left.\left[\mathbf{d}\left(\left.x\right|_{h}\right), \mathbf{d}(x)\right] \cdot\left[\mathbf{d}(x), \mathbf{d}\left(\left.x\right|_{h}\right)\right] \cdot x\right|_{h} \cdot{ }_{h} \mid y \cdot\left[\mathbf{r}\left({ }_{h} \mid y\right), \mathbf{r}(y)\right]=\left[\mathbf{d}\left(\left.x\right|_{h}\right), \mathbf{d}(x)\right] \cdot(x \cdot y),
$$


that is,

$$
\left.\left[\mathbf{d}\left(\left.x\right|_{h}\right), \mathbf{d}\left(\left.x\right|_{h}\right)\right] \cdot x\right|_{h} \cdot{ }_{h} \mid y \cdot\left[\mathbf{r}\left({ }_{h} \mid y\right), \mathbf{r}(y)\right]=\left[\mathbf{d}\left(\left.x\right|_{h}\right), \mathbf{d}(x)\right] \cdot(x \cdot y),
$$

or equivalently,

$$
\left.x\right|_{h} \cdot{ }_{h} \mid y \cdot\left[\mathbf{r}\left({ }_{h} \mid y\right), \mathbf{r}(y)\right]=\left[\mathbf{d}\left(\left.x\right|_{h}\right), \mathbf{d}(x)\right] \cdot(x \cdot y),
$$

as $h=\mathbf{d}(y)=\mathbf{r}(x)$, we obtain that

$$
\left(\left.x\right|_{\mathbf{r}(x)} \cdot \mathbf{r}(x) \mid y\right) \cdot[\mathbf{r}(\mathbf{r}(x) \mid y), \mathbf{r}(y)]=\left[\mathbf{d}\left(\left.x\right|_{\mathbf{r}(x)}\right), \mathbf{d}(x)\right] \cdot(x \cdot y) .
$$

Hence, $\left.x\right|_{\mathbf{r}(x)} \cdot \mathbf{r}(x) \mid y \rho x \cdot y$, and so $\bar{x} \odot \bar{y}=\overline{x \cdot y}$.

Since $e \in U$ and $1_{e} \cdot 1_{e}=1_{e}$, it follows that $\overline{1_{e}} \in E(P \mathbf{S})$.

Lemma 10.21. Let $x \in P$ and $e \in U$ be such that $e \omega^{l} \mathbf{d}(x)$. Then $\bar{e} \bar{x}=\overline{1_{e}} \odot \bar{x}$.

Proof. As $e \omega^{l} \mathbf{d}(x)$, we have that $e \mathcal{L} \mathbf{d}(x) e \omega \mathbf{d}(x)$, and so

$$
\begin{aligned}
& \overline{1_{e}} \odot \bar{x}=\overline{[e, e]} \odot \bar{x} \\
& =\overline{[e, \mathbf{d}(x) e]} \odot \bar{x} \quad(e \mathcal{L} \mathbf{d}(x) e, \text { Lemma 10.2) } \\
& =\overline{[e, \mathbf{d}(x) e]} \odot \overline{1_{\mathbf{d}(x) e}} \odot \bar{x} \\
& =\overline{[e, \mathbf{d}(x) e]} \odot \overline{\left(1_{\mathbf{d}(x) e} \otimes x\right)_{\mathbf{d}(x) e}} \\
& (\mathbf{d}(x) e \omega \mathbf{d}(x) \text {, by Lemma 1.29, } \mathbf{d}(x) e \in S(\mathbf{d}(x) e, \mathbf{d}(x))) \\
& =\overline{[e, \mathbf{d}(x) e]} \odot \overline{\left(1_{\mathbf{d}(x) e} \diamond \mathbf{d}(x) e\right) \cdot(\mathbf{d}(x) e * x)} \\
& =\overline{[e, \mathbf{d}(x) e]} \odot \overline{1_{\mathbf{d}(x) e} \diamond \mathbf{d}(x) e} \odot \overline{\mathbf{d}(x) e * x} \\
& \text { (Lemma 10.20) } \\
& =\overline{[e, \mathbf{d}(x)] e} \odot \overline{1_{\mathbf{d}(x) e}} \odot \overline{\mathbf{d}(x) e * x} \\
& =\overline{[e, \mathbf{d}(x) e]} \odot \overline{\mathbf{d}(x) e * x} \\
& =\overline{[e, \mathbf{d}(x) e]} \odot \overline{[\mathbf{d}(x) e,(\mathbf{d}(x) e) \mathbf{d}(x)] \cdot(\mathbf{d}(x) e) \mathbf{d}(x) \mid x} \\
& =\overline{[e, \mathbf{d}(x) e]} \odot \overline{[\mathbf{d}(x) e, \mathbf{d}(x) e] \cdot \mathbf{d}(x) e \mid x} \quad(\mathbf{d}(x) e \omega \mathbf{d}(x)) \\
& =\overline{[e, \mathbf{d}(x) e]} \odot \overline{\mathbf{d}(x) e \mid x} \\
& =\overline{[e, \mathbf{d}(x) e] \cdot \mathbf{d}(x) e \mid x} \\
& =\overline{{ }^{\mid} \mid x} \\
& (e \mathcal{L} \mathbf{d}(x) e \text {, by }(\mathrm{P} 4)) \text {. }
\end{aligned}
$$


Lemma 10.22. Let $x, y \in P$ and $e \in U$. If $\bar{x}=\overline{1_{e}} \odot \bar{y}$, then $x \leq_{r} h * y$ and $h * y \leq_{r} y$ in $P$, where $h \in S(e, \mathbf{d}(y))$.

Proof. We note that

$$
\begin{aligned}
\bar{x} & =\overline{1_{e} \odot \bar{y}} \\
& =\overline{\left(1_{e} \otimes y\right)_{h}} \quad(h \in S(e, \mathbf{d}(y))) \\
& =\overline{\left(1_{e} \diamond h\right) \cdot(h * y)} \\
& =\overline{1_{e} \diamond h \odot \overline{h * y}} \quad(\text { Lemma 10.20) } \\
& =\overline{1_{e h} \odot \overline{h * y}} \quad(\text { Lemma 10.19) } \\
& =\overline{{ }_{e h} \mid(h * y)} \quad(\text { eh } \mathcal{L} h=\mathbf{d}(h * y), \text { Lemma 10.21), }
\end{aligned}
$$

and so $x \rho_{e h} \mid(h * y)$, that is, $x \leq_{r} h * y$. Notice that

$$
\begin{aligned}
& \overline{h * y}=\overline{[h, h \mathbf{d}(y)] \cdot h \mathbf{d}(y) \mid y} \\
& =\overline{[h, h \mathbf{d}(y)]} \odot \overline{h \mathbf{d}(y) \mid y} \quad(\text { Lemma 10.20) } \\
& =\overline{[h \mathbf{d}(y), h \mathbf{d}(y)]} \odot \overline{h \mathbf{d}(y) \mid y} \quad(h \mathcal{R} h \mathbf{d}(y), \text { Lemma 10.2) } \\
& =\overline{h \mathbf{d}(y) \mid y} \text {, }
\end{aligned}
$$

and so $h * y \rho_{h \mathbf{d}(y)} \mid y$ so that $h * y \leq_{r} y$.

Lemma 10.23. If $P$ is a weakly regular category over $U$ and $x \in P$, then $\overline{1_{\mathbf{d}(x)}} \widetilde{\mathcal{R}}_{\bar{U}} \bar{x} \widetilde{\mathcal{L}}_{\bar{U}} \overline{1_{\mathbf{r}(x)}}$, where $\bar{U}=\left\{\overline{1_{e}}: e \in U\right\}$.

Proof. In view of Lemma 10.20, $\bar{U}$ is a subset of idempotents of PS. Now, we claim that $\bar{x} \widetilde{\mathcal{R}}_{\bar{U}} \overline{1_{\mathbf{d}(x)}}$, and dually, we have that $\bar{x} \widetilde{\mathcal{L}}_{\bar{U}} \overline{1_{\mathbf{r}(x)}}$. Clearly, by Lemma 10.20, $\overline{1_{\mathbf{d}(x)}} \odot \bar{x}=\bar{x}$. Assume that $g \in U$ and $\overline{1_{g}} \odot \bar{x}=\bar{x}$. Then $\left(1_{g} \otimes x\right)_{h} \rho x$, where $h \in S(g, \mathbf{d}(x))$, which gives that $\left(1_{g} \diamond h\right) \cdot(h * x) \rho x$, that is,

$$
\left[(g h)^{+}, g h\right] \cdot[g h, h] \cdot(h * x) \rho x,
$$

which implies that $g h \mathcal{R}(g h)^{+} \mathcal{R} \mathbf{d}(x)$. So

$$
\begin{aligned}
& \overline{1_{g}} \odot \overline{1_{\mathbf{d}(x)}}=\overline{\left(1_{g} \otimes 1_{\mathbf{d}(x)}\right)_{h}} \\
& =\overline{\left(1_{g} \diamond h\right) \cdot\left(h * 1_{\mathbf{d}(x)}\right)} \\
& =\overline{1_{g} \diamond h} \odot \overline{h * 1_{\mathbf{d}(x)}} \\
& (h \in S(g, \mathbf{d}(x))) \\
& \text { (Lemma 10.20) }
\end{aligned}
$$




$$
\begin{aligned}
& =\overline{\left(1_{g} \otimes 1_{h}\right)_{h}} \odot \overline{\left(1_{h} \otimes 1_{\mathbf{d}(x))_{h}}\right.} \quad(\text { Lemma } 10.18, \text { Lemma 10.19) } \\
& =\left(\overline{1_{g}} \odot \overline{1_{h}}\right) \odot\left(\overline{1_{h}} \odot \overline{1_{\mathbf{d}(x)}}\right) \\
& =\overline{1_{g}} \odot \overline{1_{h}} \odot \overline{1_{\mathbf{d}(x)}} \\
& =\left(\overline{1_{g}} \odot \overline{1_{h}}\right) \odot \overline{1_{\mathbf{d}(x)}} \\
& =\overline{\left(1_{g} \otimes 1_{h}\right)_{h}} \odot \overline{1_{\mathbf{d}(x)}} \\
& =\overline{1_{g h}} \odot \overline{1_{\mathbf{d}(x)}} \quad\left(h \omega^{l} g,\right. \text { Lemma 10.19) } \\
& =\overline{\left(1_{g h} \otimes 1_{\mathbf{d}(x)}\right)_{g h}} \\
& (g h \mathcal{R} \mathbf{d}(x) \text {, so by Lemma } 1.29, g h \in S(g h, \mathbf{d}(x))) \\
& =\overline{1_{(g h)} \mathbf{d}(x)} \\
& =\overline{1_{\mathbf{d}(x)}} \quad(g h \mathcal{R} \mathbf{d}(x)) .
\end{aligned}
$$

Let $\bar{U}=\left\{\overline{1_{e}}: e \in U\right\}$. For any $\overline{1_{e}}, \overline{1_{f}} \in \bar{U}$, we have

$$
\overline{1_{e}} \omega^{r} \overline{1_{f}} \text { if and only if } \overline{1_{f}} \odot \overline{1_{e}}=\overline{1_{e}},
$$

and dually,

$$
\overline{1_{e}} \omega^{l} \overline{1_{f}} \text { if and only if } \overline{1_{e}} \odot \overline{1_{f}}=\overline{1_{e}} .
$$

Lemma 10.24. The map $\chi: U \rightarrow \bar{U}: e \mapsto \overline{1_{e}}$ is a regular isomorphism.

Proof. Suppose that $(e, f) \in D_{U}=\left(\omega^{r} \cup \omega^{l}\right) \cup\left(\omega^{r} \cup \omega^{l}\right)^{-1}$. If $e \omega^{r} f$, then by Lemma 10.18, $\overline{1_{e}} \odot \overline{1_{f}}=\overline{1_{e f}}$. Again, by Lemma 1.29, ef $\in S(f, e)$. Thus,

$$
\begin{aligned}
& \overline{1_{f} \odot \overline{1_{e}}} \\
= & \overline{\left(1_{f} \otimes 1_{e}\right)_{e f}} \\
= & \overline{\left(1_{f} \diamond e f\right) \cdot\left(e f * 1_{e}\right)} \\
= & \overline{\left.[f, f]\right|_{f e f} \cdot[f e f, e f] \cdot[e f, e f e] \cdot e_{e f e} \mid[e, e]} \\
= & \overline{\left.[f, f]\right|_{e f} \cdot[e f, e f] \cdot[e f, e] \cdot e \mid[e, e]} \quad(e \mathcal{R} \text { ef } \omega f) \\
= & \overline{\left[(e f)^{+}, e f\right] \cdot[e f, e f] \cdot[e f, e] \cdot\left[e, e^{\star}\right]} \\
= & \overline{\left[(e f)^{+}, e\right] \cdot\left[e, e^{\star}\right]} \quad\left((e f)^{+} \mathcal{R} \text { ef } \mathcal{R} \text { e, by }(\mathrm{P} 1)\right) .
\end{aligned}
$$


Note that $(e f)^{+} \mathcal{R}$ ef $\mathcal{R} e, e^{\star} \mathcal{L} e$ and using the above we have

$$
\begin{aligned}
{\left[(e f)^{+}, e\right] \cdot 1_{e} } & =\left[(e f)^{+}, e\right] \cdot[e, e] \\
& =\left[(e f)^{+}, e\right] \cdot\left[e, e^{\star}\right] \cdot\left[e^{\star}, e\right] \\
& =\left(1_{f} \otimes 1_{e}\right)_{e f} \cdot\left[e^{\star}, e\right] .
\end{aligned}
$$

We have that $\overline{1_{f}} \odot \overline{1_{e}}=\overline{1_{e}}=\overline{1_{f e}}$.

Dually, if $e \omega^{l} f$, then $\overline{1_{e}} \odot \overline{1_{f}}=\overline{1_{e f}}$ and $\overline{1_{f}} \odot \overline{1_{e}}=\overline{1_{f e}}$. Thus $\chi$ is a morphism.

To show that $\chi$ is a regular morphism, we assume that $h \in S(e, f)$. Then

$$
\begin{aligned}
& \overline{1_{e}} \odot \overline{1_{f}}=\overline{\left(1_{e} \otimes 1_{f}\right)_{h}} \\
& =\overline{\left(1_{e} \diamond h\right) \cdot\left(h * 1_{f}\right)} \\
& =\overline{1_{e} \diamond h} \odot \overline{h * 1_{f}} \\
& =\overline{1_{e h}} \odot \overline{1_{h f}} \\
& =\left(\overline{1_{e}} \odot \overline{1_{h}}\right) \odot\left(\overline{1_{h}} \odot \overline{1_{f}}\right) \\
& =\overline{1_{e}} \odot \overline{1_{h}} \odot \overline{1_{f}} \text {. }
\end{aligned}
$$

Thus, by Lemma 10.23 and Lemma 2.12, we have $\overline{1_{h}} \in S_{1}\left(\overline{1_{e}}, \overline{1_{f}}\right) \subseteq S\left(\overline{1_{e}}, \overline{1_{f}}\right)$, and so $\chi$ is a regular morphism.

Since no two identities of $P$ are $\rho$-equivalent by Lemma 10.3, $\chi$ is injective. Clearly, $\chi$ is surjective. Thus, $\chi$ is a bijective regular morphism, and so by Lemma 1.26, we succeed in obtaining that $\chi$ is a regular isomorphism.

The next lemma is useful for Lemma 10.27.

Lemma 10.25. Let $e, f \in U$ and $h \in S(e, f)$. Then $\overline{1_{e h}} \mathcal{R} \overline{1_{e}} \odot \overline{1_{f}} \mathcal{L} \overline{1_{h f}}$ in $\langle\bar{U}\rangle$, where $\langle\bar{U}\rangle$ is the semigroup generated by $\bar{U}$.

Proof. We first show that $\overline{1_{e h}} \mathcal{R} \overline{1_{e}} \odot \overline{1_{f}}$. Notice that

$$
\begin{aligned}
& \overline{1_{e}} \odot \overline{1_{f}}=\overline{\left(1_{e} \otimes 1_{f}\right)_{h}} \quad(h \in S(e, f)) \\
& =\overline{\left(1_{e} \diamond h\right) \cdot\left(h * 1_{f}\right)} \\
& =\overline{1_{e} \diamond h} \odot \overline{h * 1_{f}} \quad \text { (Lemma 10.20) } \\
& =\overline{1_{e h}} \odot \overline{1_{h f}} \quad \text { (Lemma } 10.18 \text { and Lemma 10.19). }
\end{aligned}
$$


In addition, we have

$$
\overline{1_{e h}} \odot\left(\overline{1_{e}} \odot \overline{1_{f}}\right)=\overline{1_{e h}} \odot \overline{1_{e h}} \odot \overline{1_{h f}}=\overline{1_{e h}} \odot \overline{1_{h f}}=\overline{1_{e}} \odot \overline{1_{f}}
$$

and

$$
\begin{aligned}
& \left(\overline{1_{e}} \odot \overline{1_{f}}\right) \odot{\overline{1_{h}}}^{2}=\overline{1_{e h}} \odot \overline{1_{h f}} \odot \overline{1_{h}} \odot \overline{1_{h}} \\
& =\overline{[e h, h] \cdot[h, h f]} \odot \overline{[h f, h]} \odot \overline{[h, e h]} \\
& (\text { eh } \mathcal{L} h \mathcal{R} h f, \text { Lemma 10.2) } \\
& =\overline{[e h, h] \cdot[h, h f] \cdot[h f, h] \cdot[h, e h]} \quad \text { (by Lemma 10.20) } \\
& =\overline{[e h, h] \cdot[h, h] \cdot[h, e h]} \quad(\text { by }(\mathrm{P} 1)) \\
& =\overline{[e h, e h]} \\
& \text { (by }(\mathrm{P} 1) \text { ) } \\
& =\overline{1_{e h}} \text {. }
\end{aligned}
$$

Thus, $\overline{1_{e h}} \mathcal{R} \overline{1_{e}} \odot \overline{1_{f}}$ in $\langle\bar{U}\rangle$. Dually we have that $\overline{1_{e}} \odot \overline{1_{f}} \mathcal{L} \overline{1_{h} f}$ in $\langle\bar{U}\rangle$.

We pause here to make a short comment on Lemma 10.25. Suppose that $\bar{x}=\overline{1_{e}} \odot \overline{1_{f}}$. As

$$
\begin{array}{rlrl}
\overline{1_{e}} \odot \overline{1_{f}} & =\overline{1_{e h} \odot \overline{1_{h f}}} \\
& =\overline{[e h, h]} \odot \overline{[h, h f]} & & (\text { eh } \mathcal{L} h \mathcal{R} h f, \text { Lemma 10.2) } \\
& =\overline{[e h, h] \cdot[h, h f]} \quad & & (\text { Lemma 10.20) }
\end{array}
$$

we have that $\mathbf{d}(x) \mathcal{R}$ eh. By Lemma 10.24, we have that $\overline{1_{\mathbf{d}(x)}} \mathcal{R} \overline{1_{e h}}$. Since $\overline{1_{e h}} \mathcal{R} \overline{1_{e}} \odot \overline{1_{f}}$ in $\langle\bar{U}\rangle$, we obtain that $\overline{1_{\mathbf{d}(x)}} \mathcal{R} \overline{1_{e}} \odot \overline{1_{f}}$ in $\langle\bar{U}\rangle$, that is, $\overline{1_{\mathbf{d}(x)}} \mathcal{R} \bar{x}$ in $\langle\bar{U}\rangle$. By a dual argument, we have that $\overline{1_{\mathbf{r}(x)}} \mathcal{L} \bar{x}$ in $\langle\bar{U}\rangle$.

Lemma 10.26. Let $P$ be a weakly regular category over $U$. Suppose that for $x \in E(P \mathbf{S})$, we have $\overline{1_{\mathbf{d}(x)}} \mathcal{R}^{*} \bar{x} \mathcal{L}^{*} \overline{1_{\mathbf{r}(x)}}$ in PS. Then $x \in \bar{U}$.

Proof. Suppose that $x \in P$ and $\bar{x} \odot \bar{x}=\bar{x}$. As $\bar{x} \mathcal{L}^{*} \overline{1_{\mathbf{r}(x)}}$, we have that $\overline{1_{\mathbf{r}(x)}} \odot \bar{x}=$ $\overline{1_{\mathbf{r}(x)}}$. Let $h \in S(\mathbf{r}(x), \mathbf{d}(x))$. Then, we have

$$
\begin{aligned}
& \overline{1_{\mathbf{r}(x)}} \odot \bar{x}=\overline{1_{\mathbf{r}(x)}} \\
\Rightarrow & \overline{\left(1_{\mathbf{r}(x)} \otimes x\right)_{h}}=\overline{1_{\mathbf{r}(x)}} \\
\Rightarrow & \overline{\left(1_{\mathbf{r}(x)} \diamond h\right) \cdot(h * x)}=\overline{1_{\mathbf{r}(x)}}
\end{aligned}
$$




$$
\begin{aligned}
& \Rightarrow \overline{1_{\mathbf{r}(x)} \mid \mathbf{r}(x) h \cdot[\mathbf{r}(x) h, h] \cdot(h * x)}=\overline{1_{\mathbf{r}(x)}} \\
& \Rightarrow \overline{\left[(\mathbf{r}(x) h)^{+}, \mathbf{r}(x) h\right] \cdot[\mathbf{r}(x) h, h] \cdot(h * x)}=\overline{1_{\mathbf{r}(x)}} \\
& \Rightarrow \mathbf{r}(x) h \mathcal{R}(\mathbf{r}(x) h)^{+} \mathcal{R} \mathbf{r}(x) .
\end{aligned}
$$

As $h \omega^{l} \mathbf{r}(x)$, we have that $\mathbf{r}(x) h \omega \mathbf{r}(x)$, and so we must have that $\mathbf{r}(x) h=\mathbf{r}(x)$. Also, we have $h \mathbf{r}(x)=h$, and so $h \mathcal{L} \mathbf{r}(x)$. By Lemma 10.24, we obtain that $\overline{1_{h}} \mathcal{L} \overline{1_{\mathbf{r}(x)}}$. Since $\bar{x} \mathcal{L}^{*} \overline{1_{\mathbf{r}(x)}}$, we succeed in obtaining that $\bar{x} \mathcal{L}^{*} \overline{1_{h}}$. Dually, we have that $\bar{x} \mathcal{R}^{*} \overline{1_{h}}$ so that $\bar{x} \mathcal{H}^{*} \overline{1_{h}}$. As $\bar{x}$ is an idempotent and each $\mathcal{H}^{*}$-class contains at most one idempotent, we must have $\bar{x}=\overline{1_{h}}$.

Lemma 10.27. The set $\bar{U}=\left\{\overline{1_{e}}: e \in U\right\}$ generates a regular subsemigroup $\langle\bar{U}\rangle$ of PS and satisfies $E(\langle\bar{U}\rangle)=\bar{U}$.

Proof. To show that $E(\langle\bar{U}\rangle)=\bar{U}$, we first verify that for $\bar{x} \in\langle\bar{U}\rangle, \overline{1_{\mathbf{d}(x)}} \mathcal{R} \bar{x} \mathcal{L} \overline{1_{\mathbf{r}(x)}}$ by induction. Suppose that $\bar{x}=\overline{1_{e}} \odot \overline{1_{f}}$, where $e, f \in U$. Then by the comment succeeding Lemma 10.25, we have that $\overline{1_{\mathbf{d}(x)}} \mathcal{R} \bar{x}$ in $\langle\bar{U}\rangle$.

Now, we assume that if $\bar{x}=\overline{1_{e_{1}}} \odot \overline{1_{e_{2}}} \odot \cdots \odot \overline{1_{e_{n}}}$, then $\overline{1_{\mathbf{d}(x)}} \mathcal{R} \bar{x}$ in $\langle\bar{U}\rangle$. Let

$$
\bar{y}=\overline{1_{e_{1}}} \odot \overline{1_{e_{2}}} \odot \cdots \odot \overline{1_{e_{n}}} \odot \overline{1_{e_{n+1}}} .
$$

Put

$$
\bar{z}=\overline{1_{e_{2}}} \odot \overline{1_{e_{3}}} \odot \cdots \odot \overline{1_{e_{n+1}}} .
$$

Then by the hypothesis, $\overline{1_{\mathbf{d}(z)}} \mathcal{R} \bar{z}$ in $\langle\bar{U}\rangle$, and so there exists $\bar{t} \in\langle\bar{U}\rangle$ such that $\bar{z} \odot \bar{t}=\overline{1_{\mathbf{d}(z)}}$, so that

$$
\bar{y} \odot \bar{t}=\overline{1_{e_{1}}} \odot \bar{z} \odot \bar{t}=\overline{1_{e_{1}}} \odot \overline{1_{\mathbf{d}(z)}} .
$$

If $k \in S\left(e_{1}, \mathbf{d}(z)\right)$, by Lemma 10.25 , we have that $\overline{1_{e_{1}}} \odot \overline{1_{\mathbf{d}(z)}} \mathcal{R} \overline{1_{e_{1} k}}$ in $\langle\bar{U}\rangle$, that is, $\bar{y} \odot \bar{t} \mathcal{R} \overline{1_{e_{1} k}}$ in $\langle\bar{U}\rangle$. Thus, there exists $\bar{u} \in\langle\bar{U}\rangle$ such that

$$
\bar{y} \odot \bar{t} \odot \bar{u}=\overline{1_{e_{1} k}},
$$

where $\bar{t} \odot \bar{u} \in\langle\bar{U}\rangle$. 
Observe that

$$
\begin{aligned}
\bar{y} & =\overline{1_{e_{1}} \odot \bar{z}} & & \\
& =\overline{\left(1_{e_{1}} \otimes z\right)_{k}} & & \left(k \in S\left(e_{1}, \mathbf{d}(z)\right)\right) \\
& =\overline{\left(1_{e_{1}} \diamond k\right) \cdot(k * z)} & & \\
& =\overline{1_{e_{1}} \diamond k \odot \overline{k * z}} & & (\text { Lemma 10.20) } \\
& =\overline{1_{e_{1} k} \odot \overline{k * z}} \quad & & (\text { Lemma 10.19), }
\end{aligned}
$$

and so $\overline{1_{e_{1}}} \odot \bar{y}=\bar{y}$, so that $\overline{1_{e_{1} k}} \mathcal{R} \bar{y}$ in $\langle\bar{U}\rangle$. As $e_{1} k \mathcal{L} k$, by Lemma 10.2 , we have $\overline{1_{e_{1} k}}=\overline{\left[e_{1} k, k\right]}$, and so

$$
\bar{y}=\overline{1_{e_{1} k}} \odot \overline{k * z}=\overline{\left[e_{1} k, k\right]} \odot \overline{k * z}=\overline{\left[e_{1} k, k\right] \cdot(k * z)}
$$

so that $\mathbf{d}(y) \mathcal{R} e_{1} k$, and so $\overline{1_{e_{1}}} \mathcal{R} \overline{1_{\mathbf{d}(y)}}$. Hence $\overline{1_{\mathbf{d}(y)}} \mathcal{R} \bar{y}$ in $\langle\bar{U}\rangle$. Dually, we have that $\bar{y} \mathcal{L} \overline{1_{\mathbf{r}(y)}}$ in $\langle\bar{U}\rangle$.

For any $\bar{x} \in E(\langle\bar{U}\rangle)$, we have that $\overline{1_{\mathbf{d}(x)}} \mathcal{R} \bar{x} \mathcal{L} \overline{1_{\mathbf{r}(x)}}$, and so by Lemma 10.26, we have $\bar{x} \in \bar{U}$. Together with $\bar{U} \subseteq E(\langle\bar{U}\rangle)$, we obtain that $E(\langle\bar{U}\rangle)=\bar{U}$.

Lemma 10.28. If $P$ is a weakly regular category over $U$, then for any $\bar{x}, \bar{y} \in P \mathbf{S}$,

(i) $\bar{x} \widetilde{\mathcal{L}}_{\bar{U}} \bar{y}$ if and only if $\mathbf{r}(x) \mathcal{L} \mathbf{r}(y)$;

(ii) $\bar{x} \widetilde{\mathcal{R}}_{\bar{U}} \bar{y}$ if and only if $\mathbf{d}(x) \mathcal{R} \mathbf{d}(y)$.

Proof. To prove $(i)$, suppose that $\bar{x}, \bar{y} \in P \mathbf{S}$ and $x, y \in P$. Then

$$
\begin{array}{rlrl}
\bar{x} \widetilde{\mathcal{L}}_{\bar{U}} \bar{y} & \Leftrightarrow \overline{1_{\mathbf{r}(x)}} \widetilde{\mathcal{L}}_{\bar{U}} \overline{1_{\mathbf{r}(y)}} & & \text { (by Lemma 10.23) } \\
& \Leftrightarrow \overline{1_{\mathbf{r}(x)}} \mathcal{L} \overline{1_{\mathbf{r}(y)}} & & \\
& \Leftrightarrow \mathbf{r}(x) \mathcal{L} \mathbf{r}(y) & \quad \text { (by Lemma 10.24). }
\end{array}
$$

Similarly, we show that part (ii) holds.

Lemma 10.29. If $P$ is a weakly regular category over $U$, then $P \mathbf{S}$ satisfies the Congruence Condition (C).

Proof. Let $\bar{x}, \bar{y}, \bar{z} \in P \mathbf{S}$ be such that $\bar{x} \widetilde{\mathcal{L}}_{\bar{U}} \bar{y}$. Then by Lemma $10.28, \mathbf{r}(x) \mathcal{L} \mathbf{r}(y)$. According to Lemma 1.28, we have that $S(\mathbf{r}(x), \mathbf{d}(z))=S(\mathbf{r}(y), \mathbf{d}(z))$. Suppose that $h \in S(\mathbf{r}(x), \mathbf{d}(z))$. Then

$$
\bar{x} \odot \bar{z}=\overline{(x \otimes z)_{h}}=\overline{(x \diamond h) \cdot(h * z)} \text { and } \bar{y} \odot \bar{z}=\overline{(y \otimes z)_{h}}=\overline{(y \diamond h) \cdot(h * z)} .
$$


As $\mathbf{r}((x \diamond h) \cdot(h * z))=\mathbf{r}(h * z)=\mathbf{r}((y \diamond h) \cdot(h * z))$, it follows from Lemma 10.28 that $\bar{x} \odot \bar{z} \widetilde{\mathcal{L}}_{\bar{E}} \bar{y} \odot \bar{z}$, and so $\widetilde{\mathcal{L}}_{\bar{U}}$ is a right congruence on $P \mathbf{S}$. Dually, $\widetilde{\mathcal{R}}_{\bar{U}}$ is a left congruence on $\mathrm{PS}$.

To sum up, we obtain the following result.

Theorem 10.30. Let $P$ be a weakly regular category over a regular biordered set $U$. Then PS is a weakly $\bar{U}$-regular semigroup, where $\bar{U}=\left\{\overline{1_{e}}: e \in U\right\}$. Moreover, pre-orders $\leq_{r}^{\prime}$ and $\leq_{\ell}^{\prime}$ on $P$ correspond to partial orders $\leq_{r}^{\prime}$ and $\leq_{l}^{\prime}$ on $P \mathbf{S}$, and pre-orders $\leq_{r}^{t}$ and $\leq_{l}^{t}$ on $P$ correspond to pre-orders $\leq_{r}$ and $\leq_{l}$ on PS.

Proof. It is sufficient to consider these orders on $P$ and PS. Let $x, y \in P$. We have that

$$
\begin{aligned}
x \leq_{r}^{\prime} y \text { in } P & \Leftrightarrow x \rho_{e} \mid y \quad \text { for some } e \omega \mathbf{d}(y) \\
& \Leftrightarrow \bar{x}=\bar{e} \mid y \text { in } P \mathbf{S} \quad \text { for some } e \omega \mathbf{d}(y) \\
& \Leftrightarrow \bar{x}=\overline{1_{e}} \odot \bar{y} \text { in } P \mathbf{S} \quad \text { for some } e \omega \mathbf{d}(y) \\
& \Leftrightarrow \bar{x} \leq_{r}^{\prime} \bar{y} \text { in } P \mathbf{S} .
\end{aligned}
$$

Dually, we have that $x \leq_{\ell}^{\prime} y$ in $P$ if and only if $\bar{x} \leq_{l}^{\prime} \bar{y}$ in $P \mathbf{S}$.

In addition,

$$
\begin{aligned}
& x \leq_{r}^{t} y \text { in } P \\
\Rightarrow & x=y_{0} \leq_{r} y_{1} \leq_{r} y_{2} \leq_{r} \cdots \leq_{r} y_{n}=y \text { in } P \quad \text { for some } n \geq 1 \\
\Rightarrow & x \rho_{e_{1}}\left|y_{1}, y_{1} \rho_{e_{2}}\right| y_{2}, \cdots, y_{n-1} \rho_{e_{n}}\left|y_{n}=e_{n}\right| y \quad \text { for some } e_{1}, e_{2}, \cdots, e_{n} \in U \\
\Rightarrow & \bar{x}=\overline{e_{1} \mid y_{1}}, y_{1}=\overline{e_{2} \mid y_{2}}, \cdots, y_{n-1}=\overline{e_{n} \mid y} \text { in } P \mathbf{S} \\
\Rightarrow & \bar{x}=\overline{1_{e_{1}}} \odot \overline{y_{1}}, \overline{y_{1}}=\overline{1_{e_{2}}} \odot \overline{y_{2}}, \cdots, \overline{y_{n-1}}=\overline{1_{e_{n}}} \odot \bar{y} \quad(\text { Lemma } 10.21) \\
\Rightarrow & \bar{x}=\overline{1_{e_{1}}} \odot \overline{1_{e_{2}}} \odot \cdots \odot \overline{1_{e_{n}}} \odot \bar{y} \text { in } P \mathbf{S} \\
\Rightarrow & \bar{x} \leq_{r} \bar{y} \text { in } P \mathbf{S} .
\end{aligned}
$$

Conversely, if $\bar{x} \leq_{r} \bar{y}$ in $P \mathbf{S}$, then there exist $\overline{1_{e_{1}}}, \overline{1_{e_{2}}}, \cdots, \overline{1_{e_{n}}} \in \bar{U}$ such that

$$
\bar{x}=\overline{1_{e_{1}}} \odot \overline{1_{e_{2}}} \odot \cdots \odot \overline{1_{e_{n}}} \odot \bar{y} .
$$

Let

$$
\overline{y_{n-1}}=\overline{1_{e_{n}}} \odot \bar{y}, \overline{y_{n-2}}=\overline{1_{e_{n-1}}} \odot \overline{y_{n-1}}, \cdots, \bar{x}=\overline{1_{e_{1}}} \odot \overline{y_{1}},
$$


and let $h_{n} \in S\left(e_{n}, \mathbf{d}(y)\right), h_{n-1} \in S\left(e_{n-1}, \mathbf{d}\left(y_{n-1}\right)\right), \cdots, h_{1} \in S\left(e_{1}, \mathbf{d}\left(y_{1}\right)\right)$. Then by Lemma 10.22, we have that

$$
\begin{gathered}
x \leq_{r} h_{1} * y_{1}, \quad h_{1} * y_{1} \leq_{r} y_{1}, \cdots \\
y_{n-2} \leq_{r} h_{n-1} * y_{n-1}, \quad h_{n-1} * y_{n-1} \leq_{r} y_{n-1}, \\
y_{n-1} \leq_{r} h_{n} * y, \quad h_{n} * y \leq_{r} y
\end{gathered}
$$

that is, $x \leq_{r}{ }^{t} y$ in $P$.

Similarly, $x \leq_{l}^{t} y$ in $P$ if and only if $\bar{x} \leq_{l} \bar{y}$ in PS.

We close this section by constructing an admissible morphism between weakly $U$-regular semigroups from an RBS functor.

Lemma 10.31. If $P_{1}$ and $P_{2}$ are weakly regular categories over regular biordered sets $U_{1}$ and $U_{2}$, respectively, and $F: P_{1} \rightarrow P_{2}$ is an $R B S$ functor, then the map $F \mathbf{S}: P_{1} \mathbf{S} \rightarrow P_{2} \mathbf{S}$ defined by the rule that $\bar{x} F \mathbf{S}=\overline{x F}$ is an admissible morphism; moreover, if $F_{1}: P_{1} \rightarrow P_{2}$ and $F_{2}: P_{2} \rightarrow P_{3}$ are $R B S$ functors, then $\left(F_{1} F_{2}\right) \mathbf{S}=F_{1} \mathbf{S} F_{2} \mathbf{S}$.

Proof. It follows from Lemma 10.8 that if $x, y \in P_{1}$ and $\bar{x}=\bar{y}$, that is, $x \rho y$ in $P_{1}$, then $x F \rho y F$, so that $F \mathbf{S}$ is well-defined.

Now, we claim that $F \mathbf{S}$ is a semigroup morphism. Suppose that $\bar{x}, \bar{y} \in P_{1} \mathbf{S}$ and $h \in S(\mathbf{r}(x), \mathbf{d}(y))$. Then $h F \in S(\mathbf{r}(x F), \mathbf{d}(y F))$, and

$$
\begin{aligned}
& (\bar{x} \odot \bar{y}) F \mathbf{S}=\overline{(x \otimes y)_{h}} F \mathbf{S} \quad(h \in S(\mathbf{r}(x), \mathbf{d}(y))) \\
& =\overline{(x \diamond h) \cdot(h * y)} F \mathbf{S} \\
& =\overline{((x \diamond h) \cdot(h * y)) F} \\
& =\overline{(x \diamond h) F \cdot(h * y) F} \\
& =\overline{(x \diamond h) F} \odot \overline{(h * y) F} \\
& =\overline{x F \diamond h F} \odot \overline{h F * y F} \\
& =\overline{(x F \diamond h F) \cdot(h F * y F)} \\
& =\overline{(x F \otimes y F)_{h F}} \\
& (h F \in S(\mathbf{r}(x F), \mathbf{d}(y F))) \\
& =\overline{x F} \odot \overline{y F} \\
& =\bar{x} F \mathbf{S} \cdot \bar{y} F \mathbf{S} \text {. }
\end{aligned}
$$


Next, we present the proof that $F \mathbf{S}$ is admissible. By Lemma 10.23, for any $x \in P_{1}$, we have that $\overline{1_{\mathbf{d}(x)}} \widetilde{\mathcal{R}}_{\overline{U_{1}}} \bar{x} \widetilde{\mathcal{L}}_{\overline{U_{1}}} \overline{1_{\mathbf{r}(x)}}$. Then

$$
\begin{aligned}
\overline{1_{\mathbf{d}(x)}} F \mathbf{S} & =\overline{1_{\mathbf{d}(x)} F} \\
& =\overline{1_{\mathbf{d}(x F)}} \widetilde{\mathcal{R}_{\overline{U_{2}}}} \overline{x F}=\bar{x} F \mathbf{S} .
\end{aligned}
$$

Dually, we have that $\overline{1_{\mathbf{r}(x)}} F \mathbf{S} \widetilde{\mathcal{L}}_{\overline{U_{2}}} \bar{x} F \mathbf{S}$.

Finally, $\overline{1_{e}} F \mathbf{S}=\overline{1_{e} F}=\overline{1_{e F}}$ as $F$ is a functor, so that $\overline{U_{1}} F \mathbf{S} \subseteq \overline{U_{2}}$.

To sum up, we have that $F \mathbf{S}$ is an admissible morphism from $P_{1} \mathbf{S}$ to $P_{2} \mathbf{S}$.

It is routine to show that $\left(F_{1} F_{2}\right) \mathbf{S}=F_{1} \mathbf{S} F_{2} \mathbf{S}$.

It is an immediate consequence of Theorem 10.30 and Lemma 10.31 that $\mathbf{S}: \mathcal{W R C} \rightarrow \mathcal{W R \mathcal { S }}$ is a functor.

\subsection{Correspondence}

The aim of this section is to present a converse to Theorem 10.30.

Let $S$ be a weakly $U$-regular semigroup and let $K$ be a representative of $U$. For any $e \in U$, we will use $e^{\star}$ and $e^{+}$to denote the elements of $K$ which are $\mathcal{L}$-related to $e$ in $U$ and $\mathcal{R}$-related to $e$ in $U$, respectively. Set

$$
S \mathbf{C}=\left\{(e, x, f): e \widetilde{\mathcal{R}}_{U} x \widetilde{\mathcal{L}}_{U} f, \quad e, f \in U\right\} \subseteq U \times S \times U
$$

We put

$$
\mathbf{d}((e, x, f))=e(\text { abbreviated to } \mathbf{d}(e, x, f)=e)
$$

and

$$
\mathbf{r}((e, x, f))=f(\text { abbreviated to } \mathbf{r}(e, x, f)=f)
$$

for all $(e, x, f) \in S \mathbf{C}$, and define a partial binary operation $\cdot$ on $S \mathbf{C}$ by the rule that

$$
(e, x, f) \cdot(f, y, v)=(e, x y, v)
$$

where $(e, x, f),(f, y, v) \in S \mathbf{C}$ and $x y$ is the product of $x$ and $y$ in $S$. Since

$e \widetilde{\mathcal{R}}_{U} x=x f \widetilde{\mathcal{R}}_{U} x y$ and $x y \widetilde{\mathcal{L}}_{U} f y=y \widetilde{\mathcal{L}}_{U} v$, we have that $e \widetilde{\mathcal{R}}_{U} x y \widetilde{\mathcal{L}}_{U} v$, and so $(e, x y, v) \in S \mathbf{C}$. If $e, f \in U$ with $e \mathcal{R} f$ or $e \mathcal{L} f$, then we define $[e, f]=(e, e f, f)$. Obviously, $[e, f] \in S \mathbf{C}$. For any $(e, x, f) \in S \mathbf{C}$ and $u, v \in B$ with $u \leq_{\mathcal{L}} e$ and 
$v \leq_{\mathcal{R}} f$, we define

$$
{ }_{u} \mid(e, x, f)=\left(u, u x,(u x)^{\star}\right) \text { and }\left.(e, x, f)\right|_{v}=\left((x v)^{+}, x v, v\right) .
$$

Notice that $u=u e \widetilde{\mathcal{R}}_{U} u x \widetilde{\mathcal{L}}_{U}(u x)^{\star}$ and $(x v)^{+} \widetilde{\mathcal{R}}_{U} x v \widetilde{\mathcal{L}}_{U} f v=v$. So $\left(u, u x,(u x)^{\star}\right)$ and $\left((x v)^{+}, x v, v\right)$ are in $S \mathbf{C}$, that is, ${ }_{u} \mid(e, x, f)$ and $\left.(e, x, f)\right|_{v}$ are well-defined.

Lemma 10.32. The set $S \mathbf{C}$, together with the restriction, co-restriction and the distinguished morphisms given as above, forms a weakly regular category over $U$.

Proof. Clearly, $S \mathbf{C}$ forms a category with set of objects $U$ and morphisms the triples are given as above. For any $e \in U,[e, e]=(e, e, e)$ is the identity associated to $e$. It is necessary to show that $S \mathbf{C}$ satisfies $(\mathrm{P} 1)$ to $(\mathrm{P} 7)$ and their duals.

(P1) If $e, f, g \in U$ with $e \mathcal{R} f \mathcal{R} g$, then

$$
[e, f] \cdot[f, g]=(e, f, f) \cdot(f, g, g)=(e, g, g)=[e, g]
$$

Similarly, if e $\mathcal{L} f \mathcal{L} g$, then $[e, f] \cdot[f, g]=[e, g]$.

(P2) Suppose that $(e, x, f) \in S \mathbf{C}, h \in U$ and $h \leq_{\mathcal{L}} e$. Then ${ }_{h} \mid(e, x, f)=$ $\left(h, h x,(h x)^{\star}\right)$ and $\mathbf{d}\left({ }_{h} \mid(e, x, f)\right)=h$. By Lemma $2.14,(h x)^{\star} \leq_{\mathcal{L}} f$. In particular, if $h=e$, then $e_{e}(e, x, f)=\left(e, x, x^{\star}\right)$. Certainly, $x^{\star} \mathcal{L} f$ and

$$
\left(e, x, x^{\star}\right) \cdot\left(x^{\star}, x^{\star}, f\right)=\left(e, x x^{\star}, f\right)=(e, x, f),
$$

that is, $e_{e} \mid(e, x, f) \cdot\left[x^{\star}, f\right]=(e, x, f)$.

(P3) If $g \leq e$ and $e \mathcal{R} f$, then $g \leq_{\mathcal{R}} f$, and so $g f \in U$ and

$$
\begin{aligned}
{ }_{g} \mid[e, f] & ={ }_{g} \mid(e, f, f)=\left(g, g f,(g f)^{\star}\right) \\
& =(g, g f, g f) \cdot\left(g f, g f,(g f)^{\star}\right) \\
& =[g, g f] \cdot\left[g f,(g f)^{\star}\right] .
\end{aligned}
$$

If $g \leq e$ and $e \mathcal{L} f$, then $g \leq_{\mathcal{L}} f$, and so $g f=g$. Thus

$$
{ }_{g}\left|[e, f]={ }_{g}\right|(e, e, f)=\left(g, g, g^{\star}\right)=\left[g, g^{\star}\right] \text { and }[g, g f] \cdot\left[g f,(g f)^{\star}\right]=\left[g, g^{\star}\right]
$$

so that $g \mid[e, f]=[g, g f] \cdot\left[g f,(g f)^{\star}\right]$. 
If $g \leq_{\mathcal{L}} e$ and $e \mathcal{L} f$, then ${ }_{g}\left|[e, f]={ }_{g}\right|(e, e, f)=\left(g, g, g^{\star}\right)=\left[g, g^{\star}\right]$.

(P4) if $(g, x, h) \in S \mathbf{C}$ and $e, f \in U$ with $e \leq_{\mathcal{L}} f \leq_{\mathcal{L}} g$, then

$$
\begin{aligned}
e_{e} \mid\left(f_{f} \mid(g, x, h)\right) & ={ }_{e} \mid\left(f, f x,(f x)^{\star}\right)=\left(e, e f x,(e f x)^{\star}\right) \\
& =\left(e, e x,(e x)^{\star}\right)={ }_{e} \mid(g, x, h) .
\end{aligned}
$$

In particular, if $e \mathcal{L} f \omega^{l} g$, then

$$
[e, f] \cdot\left({ }_{f} \mid(g, x, h)\right)=(e, e, f) \cdot\left(f, f x,(f x)^{\star}\right)=\left(e, e f x,(f x)^{\star}\right)=\left(e, e x,(f x)^{\star}\right)
$$

and

$$
{ }_{e} \mid(g, x, h)=\left(e, e x,(e x)^{\star}\right) .
$$

As $(f x)^{\star} \widetilde{\mathcal{L}}_{U} f x \widetilde{\mathcal{L}}_{U}$ ex $\widetilde{\mathcal{L}}_{U}(e x)^{\star}$, we have that $(e x)^{\star}=(f x)^{\star}$.

(P5) If $(e, x, f),(f, y, k) \in S \mathbf{C}$ and $h \leq_{\mathcal{L}} e$, then

$$
\begin{gathered}
{ }_{h}\left|((e, x, f) \cdot(f, y, k))={ }_{h}\right|(e, x y, k)=\left(h, h x y,(h x y)^{\star}\right), \\
{ }_{h} \mid(e, x, f)=\left(h, h x,(h x)^{\star}\right)
\end{gathered}
$$

and

$$
\begin{aligned}
{ }_{h}\left|(e, x, f) \cdot{ }_{(h x)^{\star} \mid}\right|(f, y, k) & =\left(h, h x,(h x)^{\star}\right) \cdot\left((h x)^{\star},(h x)^{\star} y,\left((h x)^{\star} y\right)^{\star}\right) \\
& =\left(h, h x(h x)^{\star} y,\left((h x)^{\star} y\right)^{\star}\right) \\
& =\left(h, h x y,\left((h x)^{\star} y\right)^{\star}\right) .
\end{aligned}
$$

But, $(h x y)^{\star} \widetilde{\mathcal{L}}_{U} h x y \widetilde{\mathcal{L}}_{U}\left((h x)^{\star} y\right)^{\star}$, and so $(h x y)^{\star}=\left((h x)^{\star} y\right)^{\star}$. Hence, ${ }_{h}\left|((e, x, f) \cdot(f, y, k))={ }_{h}\right|(e, x, f) \cdot{ }_{(h x)^{\star}} \mid(f, y, k)$.

(P6) We know that singular $U$-squares are of the form:

(a) $\left(\begin{array}{cc}g & h \\ e g & e h\end{array}\right)$ where, $g, h \in \omega^{l}(e),(g, h) \in \mathcal{R}$, or

(b) $\left(\begin{array}{ll}g & g e \\ h & h e\end{array}\right)$ where, $g, h \in \omega^{r}(e),(g, h) \in \mathcal{L}$.

Firstly, consider $(a)$. By $g \omega^{l} e$ and $(g, h) \in \mathcal{R}$, we have that

$$
[g, h] \cdot[h, e h]=(g, h, h) \cdot(h, h, e h)=(g, h, e h)
$$


and

$$
[g, e g] \cdot[e g, e h]=(g, g, e g) \cdot(e g, e h, e h)=(g, g e h, e h)=(g, g h, e h)=(g, h, e h)
$$

Thus $[g, h] \cdot[h, e h]=[g, e g] \cdot[e g, e h]$. Similarly, we prove $(b)$.

(P7) If $(u, x, v) \in S \mathbf{C}, h_{1} \in S(e, u), h_{2} \in S(v, f)$. Then

$$
h_{1} u \mid(u, x, v)=\left(h_{1} u, h_{1} x,\left(h_{1} x\right)^{\star}\right) \text { and }\left.(u, x, v)\right|_{v h_{2}}=\left(\left(x h_{2}\right)^{+}, x h_{2}, v h_{2}\right) .
$$

Put $h_{1}^{\prime}=\left(h_{1} x\right)^{\star}$ and $h_{2}^{\prime}=\left(x h_{2}\right)^{+}$. Take $h^{\prime} \in S\left(h_{1}^{\prime}, h_{2}\right)$ and $h \in S\left(h_{1}, h_{2}^{\prime}\right)$. Then

$$
\begin{aligned}
& \left(\left(h_{1} *(u, x, v)\right) \diamond h^{\prime}\right) \cdot\left[h^{\prime}, h^{\prime} h_{2}\right] \\
= & \left(\left(\left[h_{1}, h_{1} u\right] \cdot h_{1} u \mid(u, x, v)\right) \diamond h^{\prime}\right) \cdot\left[h^{\prime}, h^{\prime} h_{2}\right] \\
= & \left(\left(\left[h_{1}, h_{1} u\right] \cdot\left(h_{1} u, h_{1} x, h_{1}^{\prime}\right)\right) \diamond h^{\prime}\right) \cdot\left[h^{\prime}, h^{\prime} h_{2}\right] \\
= & \left(\left(\left(h_{1}, h_{1} u, h_{1} u\right) \cdot\left(h_{1} u, h_{1} x, h_{1}^{\prime}\right)\right) \diamond h^{\prime}\right) \cdot\left[h^{\prime}, h^{\prime} h_{2}\right] \quad\left(h_{1} \mathcal{R} h_{1} u\right) \\
= & \left(\left(h_{1}, h_{1} x, h_{1}^{\prime}\right) \diamond h^{\prime}\right) \cdot\left[h^{\prime}, h^{\prime} h_{2}\right] \\
= & \left.\left(h_{1}, h_{1} x, h_{1}^{\prime}\right)\right|_{h_{1}^{\prime} h^{\prime}} \cdot\left[h_{1}^{\prime} h^{\prime}, h^{\prime}\right] \cdot\left[h^{\prime}, h^{\prime} h_{2}\right] \quad\left(h_{1}^{\prime} h^{\prime} \mathcal{L} h^{\prime} \mathcal{R} h^{\prime} h_{2}\right) \\
= & \left(\left(h_{1} x h^{\prime}\right)^{+}, h_{1} x h^{\prime}, h_{1}^{\prime} h^{\prime}\right) \cdot\left(h_{1}^{\prime} h^{\prime}, h_{1}^{\prime} h^{\prime}, h^{\prime}\right) \cdot\left(h^{\prime}, h^{\prime} h_{2}, h^{\prime} h_{2}\right) \\
= & \left(\left(h_{1} x h^{\prime}\right)^{+}, h_{1} x h^{\prime}, h_{1}^{\prime} h^{\prime}\right) \cdot\left(h_{1}^{\prime} h^{\prime}, h_{1}^{\prime} h^{\prime} h_{2}, h^{\prime} h_{2}\right) \\
= & \left(\left(h_{1} x h^{\prime}\right)^{+}, h_{1} x h^{\prime} h_{1}^{\prime} h^{\prime} h_{2}, h^{\prime} h_{2}\right) \quad\left(h^{\prime} \mathcal{L} h_{1}^{\prime} h^{\prime}\right) \\
= & \left(\left(h_{1} x h^{\prime}\right)^{+}, h_{1} x h^{\prime} h_{2}, h^{\prime} h_{2}\right) \\
= & \left(\left(h_{1} x h^{\prime}\right)^{+}, h_{1} x h_{2}, h^{\prime} h_{2}\right) \\
& \left(h_{1} x h^{\prime} h_{2}=h_{1} x\left(h_{1} x\right)^{\star} h^{\prime} h_{2}=h_{1} x h_{2} \text { as } h^{\prime} \mathcal{L} h_{1}^{\prime} h^{\prime}=\left(h_{1} x\right)^{\star} h^{\prime}\right) .
\end{aligned}
$$

Similarly, we have that

$$
\left[h_{1} h, h\right] \cdot\left(h *\left((u, x, v) \diamond h_{2}\right)\right)=\left(h_{1} h, h_{1} x h_{2},\left(h_{1} x h_{2}\right)^{\star}\right) .
$$

Obviously, $\left(h_{1} x h^{\prime}\right)^{+} \widetilde{\mathcal{R}}_{U} h_{1} x h_{2} \widetilde{\mathcal{R}}_{U} h_{1} h$ and $h^{\prime} h_{2} \widetilde{\mathcal{L}}_{U} h_{1} x h_{2} \widetilde{\mathcal{L}}_{U}\left(h_{1} x h_{2}\right)^{\star}$. Thus, $\left(h_{1} x h^{\prime}\right)^{+} \mathcal{R} h_{1} h$ and $h^{\prime} h_{2} \mathcal{L}\left(h x h_{2}\right)^{\star}$, that is,

$$
\mathbf{d}\left(\left(\left(h_{1} *(u, x, v)\right) \diamond h^{\prime}\right) \cdot\left[h^{\prime}, h^{\prime} h_{2}\right]\right) \mathcal{R} h_{1} h
$$


and

$$
h^{\prime} h_{2} \mathcal{L} \mathbf{r}\left(\left[h_{1}, h\right] \cdot\left(h *\left((u, x, v) \diamond h_{2}\right)\right)\right)
$$

In addition, we have that

$$
\begin{aligned}
& \left(\left(h_{1} *(u, x, v)\right) \diamond h^{\prime}\right) \cdot\left[h^{\prime}, h^{\prime} h_{2}\right] \cdot\left[h^{\prime} h_{2},\left(h_{1} x h_{2}\right)^{\star}\right] \\
= & \left(\left(h_{1} x h^{\prime}\right)^{+}, h_{1} x h_{2}, h^{\prime} h_{2}\right) \cdot\left[h^{\prime} h_{2},\left(h_{1} x h_{2}\right)^{\star}\right] \\
= & \left(\left(h_{1} x h^{\prime}\right)^{+}, h_{1} x h_{2}, h^{\prime} h_{2}\right) \cdot\left(h^{\prime} h_{2}, h^{\prime} h_{2},\left(h_{1} x h_{2}\right)^{\star}\right) \\
= & \left(\left(h_{1} x h^{\prime}\right)^{+}, h_{1} x h_{2},\left(h_{1} x h_{2}\right)^{\star}\right)
\end{aligned}
$$

and

$$
\begin{aligned}
& {\left[\left(h_{1} x h^{\prime}\right)^{+}, h_{1} h\right] \cdot\left[h_{1} h, h\right] \cdot\left(h *\left((u, x, v) \diamond h_{2}\right)\right) } \\
= & \left(\left(h_{1} x h^{\prime}\right)^{+}, h_{1} h, h_{1} h\right) \cdot\left(h_{1} h, h_{1} x h_{2},\left(h_{1} x h_{2}\right)^{\star}\right) \\
= & \left(\left(h_{1} x h^{\prime}\right)^{+}, h_{1} x h_{2},\left(h_{1} x h_{2}\right)^{\star}\right) .
\end{aligned}
$$

Consequently, $\left(\left(h_{1} *(u, x, v)\right) \diamond h^{\prime}\right) \cdot\left[h^{\prime}, h^{\prime} h_{2}\right] \rho\left[h_{1}, h\right] \cdot\left(h *\left((u, x, v) \diamond h_{2}\right)\right)$.

In Lemma 10.31, we constructed an admissible morphism from an RBS functor. Next, we produce a converse to this result.

Lemma 10.33. Let $S$ be a weakly $U_{1}$-regular semigroup, and let $T$ be a weakly $U_{2}$-regular semigroup. If $\phi$ is an admissible morphism from $S$ to $T$, then the map $\phi \mathbf{C}$ defined by $e \phi \mathbf{C}=e \phi$ and $(e, x, f) \phi \mathbf{C}=(e \phi, x \phi, f \phi)$ is an $R B S$ functor from $S \mathbf{C}$ to TC. Further, if $\phi_{1}: S \rightarrow T$ and $\phi_{2}: T \rightarrow Q$ are admissible morphisms, then $\left(\phi_{1} \phi_{2}\right) \mathbf{C}=\phi_{1} \mathbf{C} \phi_{2} \mathbf{C}$.

Proof. As $\phi$ is an admissible morphism, it is clear that $\phi$ is a regular morphism from $U_{1}$ to $U_{2}$. Since $\phi$ preserves products and identities, it is a functor.

To show that (PF2) holds, suppose that $e, f \in U$. If $e \mathcal{R} f$, then $e \phi \mathcal{R} f \phi$ as $\phi$ is an admissible morphism. Thus, $[e, f] \phi \mathbf{C}=(e \phi, f \phi, f \phi)=[e \phi, f \phi]=$ $[e \phi \mathbf{C}, f \phi \mathbf{C}]$. Dually, if $e \mathcal{L} f$, then $[e, f] \phi \mathbf{C}=[e \phi, f \phi]=[e \phi \mathbf{C}, f \phi \mathbf{C}]$.

Finally, we show that (PF3) holds. Suppose that $(e, x, f) \in S \mathbf{C}$ and $h \in U_{1}$ with $h \leq_{\mathcal{L}} e$. Then ${ }_{h} \mid(e, x, f)=\left(h, h x,(h x)^{\star}\right)$, and so

$$
\begin{aligned}
\left({ }_{h} \mid(e, x, f)\right) \phi \mathbf{C} & =\left(h, h x,(h x)^{\star}\right) \phi \mathbf{C} \\
& =\left(h \phi,(h x) \phi,(h x)^{\star} \phi\right) .
\end{aligned}
$$


Also, we have that $h \phi \omega^{l} e \phi$ and

$$
\begin{aligned}
{ }_{h \phi} \mid(e \phi, x \phi, f \phi) & =\left(h \phi, h \phi x \phi,(h \phi x \phi)^{\star}\right) \\
& =\left(h \phi,(h x) \phi,((h x) \phi)^{\star}\right) .
\end{aligned}
$$

Thus, $((h x) \phi)^{\star} \widetilde{\mathcal{L}}_{U_{2}}(h x) \phi \widetilde{\mathcal{L}}_{U_{2}}(h x)^{\star} \phi$, and so $((h x) \phi)^{\star} \mathcal{L}(h x)^{\star} \phi$,

$$
\begin{aligned}
& \left({ }_{h} \mid(e, x, f)\right) \phi \mathbf{C} \cdot\left[(h x)^{\star} \phi,((h x) \phi)^{\star}\right] \\
= & \left(h \phi,(h x) \phi,(h x)^{\star} \phi\right) \cdot\left((h x)^{\star} \phi,(h x)^{\star} \phi,((h x) \phi)^{\star}\right) \\
= & \left(h \phi,(h x) \phi,((h x) \phi)^{\star}\right) \\
= & { }_{h \phi} \mathbf{C} \mid(e, x, f) \phi \mathbf{C} .
\end{aligned}
$$

Hence, $\left({ }_{h} \mid(e, x, f)\right) \phi \mathbf{C} \rho_{h \phi \mathbf{C}} \mid(e, x, f) \phi \mathbf{C}$.

Dually, if $k \in U_{1}$ and $k \leq_{\mathcal{R}} f$, then $\left.\left(\left.(e, x, f)\right|_{k}\right) \phi \mathbf{C} \rho(e, x, f) \phi \mathbf{C}\right|_{k \phi \mathbf{C}}$. Consequently, $\phi \mathbf{C}$ is an RBS functor from $S \mathbf{C}$ to $T \mathbf{C}$.

It is routine to show that $\left(\phi_{1} \phi_{2}\right) \mathbf{C}=\phi_{1} \mathbf{C} \phi_{2} \mathbf{C}$.

Now, we have that $\mathbf{C}: \mathcal{W R S} \rightarrow \mathcal{W R C}$ is a functor.

At the end of this section, we build a correspondence between the category $\mathcal{W R S}$ of weakly $U$-regular semigroups and the category $\mathcal{W} \mathcal{R C}$ of weakly regular categories over regular biordered sets.

Lemma 10.34. Let $S$ be a weakly $U$-regular semigroup. Then the mapping $\eta_{S}$ : $S \rightarrow S$ CS given by $x \eta_{S}=\overline{(e, x, f)}$, where $e \widetilde{\mathcal{R}}_{U} x \widetilde{\mathcal{L}}_{U} f$, is an isomorphism.

Proof. Let $x \in S, e, g \in \widetilde{R}_{x} \cap U$ and $f, h \in \widetilde{L}_{x} \cap U$. Then $e \mathcal{R} g, f \mathcal{L} h$ and

$$
(e, x, f) \cdot[f, h]=(e, x, f) \cdot(f, f, h)=(e, x, h)
$$

and

$$
[e, g] \cdot(g, x, h)=(e, g, g) \cdot(g, x, h)=(e, x, h) .
$$

Thus $(e, x, f) \rho(g, x, h)$, and so $\eta_{S}$ is well-defined.

To show that $\eta_{S}$ is injective, we assume that $x \eta_{S}=y \eta_{S}$. Then $\overline{(e, x, f)}=$ $\overline{(u, y, v)}$, where $e \widetilde{\mathcal{R}}_{U} x \widetilde{\mathcal{L}}_{U} f$ and $u \widetilde{\mathcal{R}}_{U} y \widetilde{\mathcal{L}}_{U} v$. Thus $e \mathcal{R} u$ and $f \mathcal{L} v$. Further,

$$
[e, u] \cdot(u, y, v)=(e, x, f) \cdot[f, v]
$$


that is, $(e, y, v)=(e, x, v)$, which implies that $x=y$. Hence $\eta_{S}$ is injective, as claimed.

Clearly $\eta_{S}$ is onto. It remains to show that $\eta_{S}$ is a morphism. Suppose that $x, y \in S, e \widetilde{\mathcal{R}}_{U} x \widetilde{\mathcal{L}}_{U} f, u \widetilde{\mathcal{R}}_{U} y \widetilde{\mathcal{L}}_{U} v$ and $h \in S(f, u)$. Then

$$
\begin{aligned}
x \eta_{S} \odot y \eta_{S} & =\overline{(e, x, f) \odot \overline{(u, y, v)}} \\
& =\overline{((e, x, f) \otimes(u, y, v))_{h}} \\
& =\overline{((e, x, f) \diamond h) \cdot(h *(u, y, v))} \\
& =\overline{\left.(e, x, f)\right|_{f h} \cdot[f h, h] \cdot[h, h u] \cdot h u \mid(u, y, v)} \\
& =\overline{\left((x f h)^{+}, x f h, f h\right) \cdot(f h, f h, h) \cdot(h, h u, h u) \cdot\left(h u, h u y,(h u y)^{\star}\right)} \\
& =\overline{\left((x h)^{+}, x h, h\right) \cdot\left(h, h y,(h y)^{\star}\right)} \quad\left(f \widetilde{\mathcal{L}}_{U} x \text { and } u \widetilde{\mathcal{R}}_{U} y\right) \\
& =\overline{\left((x h)^{+}, x h y,(h y)^{\star}\right)} \\
& =\overline{\left((x h)^{+}, x y,(h y)^{\star}\right)} \\
& \quad\left(h \in S(f, u)=S_{1}(f, u), \text { so } x h y=x f h u y=x y\right) \\
& =(x y) \eta_{S} .
\end{aligned}
$$

In addition, $\eta_{S}$ preserves the distinguished set as $e \eta_{S}=\overline{(e, e, e)}=\overline{1_{e}}$ for all $e \in U$. Thus $\eta_{S}$ is an isomorphism.

Finally, as $\eta_{S}$ is an isomorphism, it preserves the pre-orders and partialorders.

To the converse, we have:

Lemma 10.35. Let $P$ be a weakly regular category over $U$. Then the map $\tau_{P}$ : $P \rightarrow P$ SC defined by the rule that $e \tau_{P}=\overline{1_{e}}$ and $x \tau_{P}=\left(\overline{1_{\mathbf{d}(x)}}, \bar{x}, \overline{1_{\mathbf{r}(x)}}\right)$ for all $e \in U=O b(P)$ and $x \in P=\operatorname{Mor}(P)$, is an isomorphism from $P$ to PSC.

Proof. Note the distinguished subset of $P \mathbf{S}$ is $\bar{U}$, which is the set of objects of PSC. By Lemma 10.24, $\tau_{P}: U \rightarrow \bar{U}: e \mapsto \overline{1_{e}}$ is a regular isomorphism.

Now, we show that $\tau_{P}$ preserves $\mathbf{d}$ and $\mathbf{r}$. Suppose that $x \in P$. Then by the definition of $\tau_{P}$,

$$
\mathbf{d}(x) \tau_{P}=\overline{1_{\mathbf{d}(x)}}, \mathbf{r}(x) \tau_{P}=\overline{1_{\mathbf{r}(x)}}
$$

and

$$
x \tau_{P}=\left(\overline{1_{\mathbf{d}(x)}}, \bar{x}, \overline{1_{\mathbf{r}(x)}}\right) .
$$


Thus, $\tau_{P}$ preserves $\mathbf{d}$ and $\mathbf{r}$.

If $x, y \in P$ with $x \cdot y$ defined in $P$, then $\mathbf{r}(x)=\mathbf{d}(y)$ and so $\bar{x} \odot \bar{y}=\bar{x} \cdot y$.

Here, we temporarily use $\circ$ to denote the partial binary operation in PSC. Thus,

$$
\begin{aligned}
x \tau_{P} \circ y \tau_{P} & =\left(\overline{1_{\mathbf{d}(x)}}, \bar{x}, \overline{1_{\mathbf{r}(x)}}\right) \circ\left(\overline{1_{\mathbf{d}(y)}}, \bar{y}, \overline{1_{\mathbf{r}(y)}}\right) \\
& =\left(\overline{1_{\mathbf{d}(x)}}, \bar{x} \odot \bar{y}, \overline{1_{\mathbf{r}(y)}}\right) \\
& =\left(\overline{1_{\mathbf{d}(x)}}, \overline{x \cdot y}, \overline{1_{\mathbf{r}(y)}}\right) \quad(\text { Lemma } \\
& =\left(\overline{1_{\mathbf{d}(x \cdot y)}}, \overline{x \cdot y}, \overline{1_{\mathbf{r}(x \cdot y)}}\right) \\
& =(x \cdot y) \tau_{P}
\end{aligned}
$$

which implies that $\tau_{P}$ preserves products. Also, $\tau_{P}$ preserves identities since $1_{e} \tau_{P}=\left(\overline{1_{e}}, \overline{1_{e}}, \overline{1_{e}}\right)=1_{\overline{1_{e}}}$. Thus, $\tau_{P}$ is a functor.

Let $e, f \in U$ with $e \mathcal{R} f$ in $U$. Then $[e, f] \tau_{P}=\left(\overline{1_{e}}, \overline{[e, f]}, \overline{1_{f}}\right)$. Since $e \mathcal{R} f$, it is easy to see that $[e, f] \rho[f, f]$. Thus,

$$
[e, f] \tau_{P}=\left(\overline{1_{e}}, \overline{1_{f}}, \overline{1_{f}}\right)=\left(\overline{1_{e}}, \overline{1_{e}} \odot \overline{1_{f}}, \overline{1_{f}}\right)=\left[\overline{1_{e}}, \overline{1_{f}}\right]=\left[e \tau_{P}, f \tau_{P}\right] .
$$

Dually, if $e \mathcal{L} f$ in $U$, then $[e, f] \tau_{P}=\left[e \tau_{P}, f \tau_{P}\right]$. Hence, $\tau_{P}$ satisfies Condition (PF2).

To show that (PF3) holds, We assume that $x \in P$ and $e \in U$ with $e \omega^{l} \mathbf{d}(x)$. Then $e \tau_{P} \omega^{l} \mathbf{d}(x) \tau_{P}$ as $\tau_{P}$ is a regular isomorphism from $U$ to $\bar{U}$ shown above. Hence, $e_{e} \mid x$ and ${ }_{e \tau_{P}} \mid x \tau_{P}$ are well-defined. Observe that

$$
\left({ }_{e} \mid x\right) \tau_{P}=\left(\overline{1_{e}}, \bar{e} \mid x, \overline{1_{\mathbf{r}(e \mid x)}}\right)
$$

and

$$
\begin{aligned}
& e \tau_{P} \mid x \tau_{P} \\
= & \overline{1_{e}} \mid\left(\overline{1_{\mathbf{d}(x)}}, \bar{x}, \overline{1_{\mathbf{r}(x)}}\right) \\
= & \left(\overline{1_{e}}, \overline{1_{e}} \odot \bar{x},\left(\overline{1_{e}} \odot \bar{x}\right)^{\star}\right) \\
= & \left(\overline{1_{e}}, \bar{e} \mid \bar{x},(\bar{e} \mid x)^{\star}\right) \quad\left(e \omega^{l} \mathbf{d}(x), \text { Lemma } 10.21\right) .
\end{aligned}
$$

Clearly,

$$
\begin{gathered}
\mathbf{d}\left(\left(e_{e} \mid x\right) \tau_{P}\right)=\mathbf{d}\left({ }_{e} \mid x\right) \tau_{P}=\overline{1_{e}}=\mathbf{d}\left({ }_{e \tau_{P}} \mid x \tau_{P}\right) \\
\mathbf{r}\left(\left({ }_{e} \mid x\right) \tau_{P}\right)=\mathbf{r}\left({ }_{e} \mid x\right) \tau_{P}=\overline{1_{\mathbf{r}(e \mid x)}} \widetilde{\mathcal{L}}_{\bar{B}} \overline{e_{e} \mid x} \widetilde{\mathcal{L}}_{\bar{B}}(\bar{e} \mid x)^{\star}=\mathbf{r}\left({ }_{e \tau_{P}} \mid x \tau_{P}\right)
\end{gathered}
$$


and

$$
\begin{aligned}
& \left({ }_{e} \mid x\right) \tau_{P} \cdot\left[\mathbf{r}\left(\left({ }_{e} \mid x\right) \tau_{P}\right), \mathbf{r}\left({ }_{e \tau_{P}} \mid x \tau_{P}\right)\right] \\
& =\left(\overline{1_{e}}, \bar{e} \mid x, \overline{1_{\mathbf{r}(e \mid x)}}\right) \cdot\left[\overline{1_{\mathbf{r}(e \mid x)}},(\overline{e \mid x})^{\star}\right] \\
& =\left(\overline{1_{e}}, \bar{e} \mid x, \overline{1_{\mathbf{r}(e \mid x)}}\right) \cdot\left(\overline{1_{\mathbf{r}(e \mid x)}}, \overline{1_{\mathbf{r}(e \mid x)}} \odot\left(\overline{e^{\mid x}}\right)^{\star},(\overline{(e \mid x})^{\star}\right) \\
& =\left(\overline{1_{e}}, \bar{e} \mid x, \overline{1_{\mathbf{r}(e \mid x)}}\right) \cdot\left(\overline{1_{\mathbf{r}(e \mid x)}}, \overline{1_{\mathbf{r}(e \mid x)}},(\overline{e \mid x})^{\star}\right) \\
& =\left(\overline{1_{e}}, \overline{{ }_{e} \mid x} \odot \overline{1_{\mathbf{r}(e \mid x)}},\left(\overline{e^{\mid x}}\right)^{\star}\right) \\
& =\left(\overline{1_{e}}, \bar{e} \mid x,(\bar{e} \mid x)^{\star}\right) \\
& ={ }_{e \tau_{P}} \mid x \tau_{P},
\end{aligned}
$$

so that $\left({ }_{e} \mid x\right) \tau_{P} \rho_{e \tau_{P}} \mid x \tau_{P}$ and (PF3) holds.

Next, suppose that $x, y \in P$ with $x \tau_{P}=y \tau_{P}$. Then $\left(\overline{1_{\mathbf{d}(x)}}, \bar{x}, \overline{1_{\mathbf{r}(x)}}\right)=$ $\left(\overline{1_{\mathbf{d}(y)}}, \bar{y}, \overline{1_{\mathbf{r}(y)}}\right)$, which implies that $\bar{x}=\bar{y}$, and also $\mathbf{d}(x)=\mathbf{d}(y), \mathbf{r}(x)=\mathbf{r}(y)$ by Lemma 10.24. Further, by Lemma 10.3, $x=y$.

We now show that $\tau_{P}$ is surjective. Let $\left(\overline{1_{e}}, \bar{x}, \overline{1_{f}}\right)$ be in PSC. Then $\overline{1_{e}} \widetilde{\mathcal{R}}_{\bar{U}} \bar{x} \widetilde{\mathcal{R}}_{\bar{U}} \overline{1_{\mathbf{d}(x)}}$ and $\overline{1_{f}} \widetilde{\mathcal{L}}_{\bar{U}} \bar{x} \widetilde{\mathcal{L}}_{\bar{U}} \overline{1_{\mathbf{r}(x)}}$, that is, $\overline{1_{e}} \mathcal{R} \overline{1_{\mathbf{d}(x)}}$ and $\overline{1_{f}} \mathcal{L} \overline{1_{\mathbf{r}(x)}}$ so that by Lemma 10.24, e $\mathcal{R} \mathbf{d}(x)$ and $f \mathcal{L} \mathbf{r}(x)$. Put $x^{\prime}=[e, \mathbf{d}(x)] \cdot x \cdot[\mathbf{r}(x), f]$. Certainly, $x^{\prime} \rho x$, that is, $\overline{x^{\prime}}=\bar{x}$. Thus, $\tau_{P}\left(x^{\prime}\right)=\left(\overline{1_{e}}, \overline{x^{\prime}}, \overline{1_{f}}\right)=\left(\overline{1_{e}}, \bar{x}, \overline{1_{f}}\right)$, and consequently, $\tau_{P}$ is surjective.

As we have shown $\tau_{P}$ is an RBS functor, we succeed in obtaining that $\tau_{P}$ preserves the two pairs of pre-orders on $P$ by Lemma 10.8 .

Lemma 10.36. For any $S \in O b(\mathcal{W R S})$, define $S \eta=\eta_{S}$, where $\eta_{S}$ is defined in Lemma 10.34. Then $\eta$ is a natural equivalence of the functors $I_{\mathcal{W R S}}$ and $\mathbf{C S}$.

Proof. Let $\theta: S_{1} \rightarrow S_{2}$ in $\mathcal{W} \mathcal{R} \mathcal{S}$, where $S_{1}$ and $S_{2}$ are over $U_{1}$ and $U_{2}$, respectively. Then for any $x \in S_{1}$, we have by the definition of $\eta_{S}$ in Lemma 10.34 that

$$
\begin{aligned}
\left(x \eta_{S_{1}}\right) \theta \mathbf{C S} & =\overline{(e, x, f)} \theta \mathbf{C S} \quad\left(e \widetilde{\mathcal{R}}_{U_{1}} x \widetilde{\mathcal{L}}_{U_{1}} f\right) \\
& =\overline{(e, x, f) \theta \mathbf{C}} \\
& =\overline{(e \theta, x \theta, f \theta)} \\
& =(x \theta) \eta_{S_{2}} \quad\left(e \theta \widetilde{\mathcal{R}}_{U_{2}} x \theta \widetilde{\mathcal{L}}_{U_{2}} f \theta\right)
\end{aligned}
$$

Thus the diagram below commutes, and so $\eta=\left(\eta_{S}\right)$ is a natural isomorphism between $I_{\mathcal{W R S}}$ and $\mathrm{CS}$. 


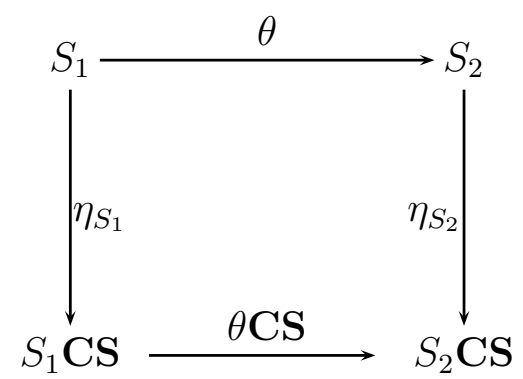

Figure 10.1: A natural transformation of $I_{\mathcal{W R S}}$ and CS

Similarly, we have:

Lemma 10.37. For any $P \in O b(\mathcal{W R C})$, define $P \tau=\tau_{P}$, where $\tau_{P}$ is defined in Lemma 10.35. Then $\tau$ is a natural equivalence of the functors $I_{\mathcal{W R C}}$ and $\mathbf{S C}$.

Proof. Let $F: P_{1} \rightarrow P_{2}$ in $\mathcal{W R C}$, where $P_{1}$ and $P_{2}$ are over $U_{1}$ and $U_{2}$, respectively. Then for any $x \in P_{1}$, we have by the definition of $\tau_{P}$ in Lemma 10.35 that

$$
\begin{aligned}
\left(x \tau_{P_{1}}\right) F \mathbf{S C} & =\left(\overline{1_{\mathbf{d}(x)}}, \bar{x}, \overline{1_{\mathbf{r}(x)}}\right) F \mathbf{S C} \\
& =\left(\left(\overline{1_{\mathbf{d}(x)}}\right) F \mathbf{S},(\bar{x}) F \mathbf{S},\left(\overline{1_{\mathbf{r}(x)}}\right) F \mathbf{S}\right) \\
& =\left(\overline{1_{\mathbf{d}(x)} F}, \overline{x F}, \overline{1_{\mathbf{r}(x)} F}\right) \\
& =\left(\overline{1_{\mathbf{d}(x F)}}, \overline{x F}, \overline{1_{\mathbf{r}(x F)}}\right) \\
& =(x F) \tau_{P_{2}}
\end{aligned}
$$

and

$$
\begin{aligned}
\left(e \tau_{P_{1}}\right) F \mathbf{S C} & =\overline{1_{e}} F \mathbf{S C} \\
& =\overline{1_{e}} F \mathbf{S} \\
& =\overline{1_{e} F} \\
& =\overline{1_{e F}} \\
& =(e F) \tau_{P_{2}} .
\end{aligned}
$$

Thus the diagram below commutes, and so $\tau=\left(\tau_{P}\right)$ is a natural morphism of $I_{\mathcal{W R C}}$ and SC. 


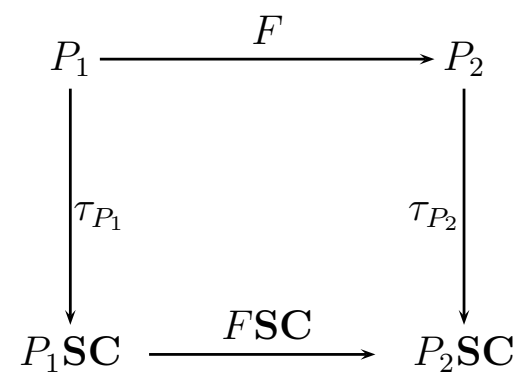

Figure 10.2: A natural transformation of $I_{\mathcal{W R C}}$ and SC

To sum up, we have:

Theorem 10.38. The category $\mathcal{W} \mathcal{R S}$ of weakly $U$-regular semigroups and admissible morphisms is equivalent to the category $\mathcal{W R C}$ of weakly regular categories over regular biordered sets and $R B S$ functors. 


\section{Chapter 11}

\section{Special kinds of weakly $U$-regular semigroups}

In this section we focus on some special kinds of weakly $U$-regular semigroups. We recover Armstrong's result for concordant semigroups and Nambooripad's result for regular semigroups.

\subsection{Weakly $U$-regular semigroups with (WIC)}

As mentioned In Chapter 2, a weakly $U$-regular semigroup satisfies (WIC) (with respect to $U$ ) if for any $a \in S$ and some (any) $a^{*}, a^{\dagger}$, if $x \in\left\langle a^{\dagger}\right\rangle$, then there exists $y \in\left\langle a^{*}\right\rangle$ with $x a=a y$; and dually, if $z \in\left\langle a^{*}\right\rangle$ then there exists $t \in\left\langle a^{\dagger}\right\rangle$ with $t a=a z$.

We say that a weakly regular category $P$ over $U$ has (WIC) if the following condition and its dual $(\mathrm{W})^{\circ}$ hold:

(W) if $x \in P$ and $u \in U$ with $u \omega \mathbf{d}(x)$, then there exists $v_{1}, \cdots, v_{n} \in U$ such that $v_{i} \omega \mathbf{r}(x)$ for $i=1, \cdots, n$ and $\overline{{ }_{u} \mid x}=\bar{x} \odot \overline{1_{v_{1}}} \odot \cdots \odot \overline{1_{v_{n}}}$.

Corollary 11.1. The category of weakly U-regular semigroups satisfying (WIC) and admissible morphisms, is equivalent to the category of weakly regular categories with (WIC) and RBS functors.

Proof. Let $S$ be a weakly $U$-regular semigroup with (WIC). In view of Lemma 10.32, it is sufficient to show that $S \mathbf{C}$ satisfies Condition (W) and its dual. 
Suppose that $(e, x, f) \in S \mathbf{C}$ and $u \in U$ with $u \leq e$. Then ${ }_{u} \mid(e, x, f)=$ $\left(u, u x,(u x)^{\star}\right)$. Since $S$ satisfies (WIC), it follows that there exist $v_{1}, \cdots, v_{n} \in U$ such that $v_{i} \leq f$ for $i=1, \cdots, n$ and $u x=x v_{1} \cdots v_{n}$.

By Lemma 10.34, $\eta_{S}: S \rightarrow S$ CS, given by $x \mapsto \overline{\left(x^{\dagger}, x, x^{*}\right)}$, is an isomorphism. Thus, for any $x, y \in S$, we have $x \eta_{S} \odot y \eta_{S}=(x y) \eta_{S}$, that is,

$$
\overline{\left(x^{\dagger}, x, x^{*}\right)} \odot \overline{\left(y^{\dagger}, y, y^{*}\right)}=\overline{(x y)^{\dagger}, x y,(x y)^{*}}
$$

so

$$
\overline{(e, x, f)} \odot \overline{1_{v_{1}}} \odot \cdots \odot \overline{1_{v_{n}}}=\overline{\left(\left(x v_{1} \cdots v_{n}\right)^{+}, x v_{1} \cdots v_{n},\left(x v_{1} \cdots v_{n}\right)^{\star}\right)} .
$$

As $u x=x v_{1} \cdots v_{n}$, we have that $u \widetilde{\mathcal{R}}_{U} u x=x v_{1} \cdots v_{n} \widetilde{\mathcal{R}}_{U}\left(x v_{1} \cdots v_{n}\right)^{+}$and $(u x)^{\star}=\left(x v_{1} \cdots v_{n}\right)^{\star}$. In addition, we have

$$
\begin{aligned}
& {\left[u,\left(x v_{1} \cdots v_{n}\right)^{+}\right] \cdot\left(\left(x v_{1} \cdots v_{n}\right)^{+}, x v_{1} \cdots v_{n},\left(x v_{1} \cdots v_{n}\right)^{\star}\right) } \\
= & \left(u,\left(x v_{1} \cdots v_{n}\right)^{+},\left(x v_{1} \cdots v_{n}\right)^{+}\right) \cdot\left(\left(x v_{1} \cdots v_{n}\right)^{+}, x v_{1} \cdots v_{n},\left(x v_{1} \cdots v_{n}\right)^{\star}\right) \\
= & \left.\left(u, x v_{1} \cdots v_{n},\left(x v_{1} \cdots v_{n}\right)^{\star}\right)\right) \\
= & \left(u, u x,(u x)^{\star}\right) \quad\left(u x=x v_{1} \cdots v_{n}\right)
\end{aligned}
$$

so that $\overline{u \mid(e, x, f)}=\overline{(e, x, f)} \odot \overline{1_{v_{1}}} \odot \cdots \odot \overline{1_{v_{n}}}$, and so Condtion (W) holds.

Conversely, suppose that $P$ is a weakly regular category over $U$ with (WIC) and $x \in P$. In view of Theorem 10.38, it is sufficient to show that for all $u \in$ $\left\langle\overline{1_{\mathbf{d}(x)}}\right\rangle$, there exists $v \in\left\langle\overline{1_{\mathbf{r}(x)}}\right\rangle$ satisfying $u \odot \bar{x}=\bar{x} \odot v$. Suppose that $\overline{1_{e}} \in\left\langle\overline{1_{\mathbf{d}(x)}}\right\rangle$. Then $\overline{1_{e}}=\overline{1_{e_{1}}} \odot \cdots \odot \overline{1_{e_{n}}}$, where $e_{i} \omega \mathbf{d}(x)$ for $i=1, \cdots, n$. By $(\mathrm{W})$, for any $e_{i}$, there exist $f_{i 1}, \cdots, f_{i m_{i}} \in \omega(\mathbf{r}(x))$ such that

$$
\overline{e_{i} \mid x}=\bar{x} \odot \overline{1_{f_{i 1}}} \odot \cdots \odot \overline{1_{f_{i m_{i}}}},
$$

that is,

$$
\overline{1_{e_{i}}} \odot \bar{x}=\bar{x} \odot \overline{1_{f_{i 1}}} \odot \cdots \odot \overline{1_{f_{i m_{i}}}}
$$

by Lemma 10.21, and so

$$
\begin{aligned}
& \overline{1_{e}} \odot \bar{x}=\overline{1_{e_{1}}} \odot \cdots \odot \overline{1_{e_{n}}} \odot \bar{x} \\
& =\overline{1_{e_{1}}} \odot \cdots \odot \overline{1_{e_{n-1}}} \odot \bar{x} \odot \overline{1_{f_{n 1}}} \odot \cdots \odot \overline{1_{f_{n m_{n}}}} \\
& =\bar{x} \odot \overline{1_{f_{11}}} \odot \cdots \odot \overline{1_{f_{1 m_{1}}}} \odot \cdots \odot \overline{1_{f_{n 1}}} \odot \cdots \odot \overline{1_{f_{n m_{n}}}} \text {, }
\end{aligned}
$$




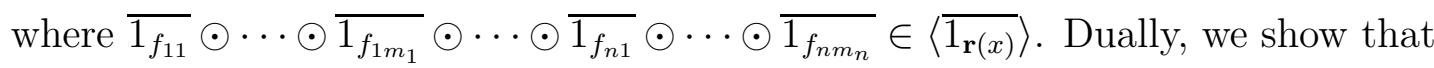
for any $g \in\left\langle\overline{1_{\mathbf{r}(x)}}\right\rangle$, there exists $k \in\left\langle\overline{1_{\mathbf{d}(x)}}\right\rangle$ satisfying $k \odot \bar{x}=\bar{x} \odot g$.

\subsection{The abundant case}

In this section we concentrate on the class of abundant semigroups. We replace the distinguished set of idempotents $U$ by the whole set of idempotents and use relations $\mathcal{R}^{*}$ and $\mathcal{L}^{*}$ instead of $\widetilde{\mathcal{R}}_{U}$ and $\widetilde{\mathcal{L}}_{U}$ in the definition of weakly $U$ regular semigroups. We thus obtain the class of abundant semigroups whose set of idempotents generates a regular semigroup. As mentioned in Chapter 7, an admissible morphism in this context is more usually referred to as a good morphism.

A weakly regular category $P$ over $U$ is an abundant category if it satisfies the following condition and its dual (P8) ${ }^{\circ}$ :

(P8) if $x, y, z \in P, h \in S(\mathbf{r}(x), \mathbf{d}(y))$ and $h^{\prime} \in S(\mathbf{r}(x), \mathbf{d}(z))$ are such that

$$
(x \otimes y)_{h} \rho(x \otimes z)_{h^{\prime}},
$$

then $\mathbf{r}(x) h \mathcal{R} \mathbf{r}(x) h^{\prime}$.

Corollary 11.2. The category of abundant semigroups whose set of idempotents generates a regular subsemigroup and good morphisms, is equivalent to the category of abundant cancellative categories and RBS functors.

Proof. Suppose that $S$ is an abundant semigroup whose set of idempotents generates a regular subsemigroup of $S$. In view of Lemma 10.32, it is sufficient to show that $S \mathbf{C}$ satisfies (P8). Dually, $(\mathrm{P} 8)^{\circ}$ holds. Let $(e, x, f),(u, y, v)$ and $(g, z, k) \in S \mathbf{C}$, and let $h \in S(f, u)$ and $h^{\prime} \in S(f, g)$ be such that

$$
((e, x, f) \otimes(u, y, v))_{h} \rho((e, x, f) \otimes(g, z, k))_{h^{\prime}}
$$

Notice that

$$
\begin{aligned}
& ((e, x, f) \otimes(u, y, v))_{h} \\
= & ((e, x, f) \diamond h) \cdot(h *(u, y, v))
\end{aligned}
$$




$$
\begin{aligned}
& =\left.(e, x, f)\right|_{f h} \cdot[f h, h] \cdot[h, h u] \cdot{ }_{h u} \mid(u, y, v) \\
& =\left((x f h)^{+}, x f h, f h\right) \cdot(f h, f h, h) \cdot(h, h u, h u) \cdot\left(h u, h u y,(h u y)^{\star}\right) \\
& =\left((x f h)^{+}, x f h, h\right) \cdot\left(h, h u y,(h u y)^{\star}\right) \\
& =\left((x h)^{+}, x h, h\right) \cdot\left(h, h y,(h y)^{\star}\right) \quad\left(f \mathcal{L}^{*} x, u \mathcal{R}^{*} y\right) \\
& =\left((x h)^{+}, x h y,(h y)^{\star}\right) \quad(x h y=x f h u y=x f u y=x y), \\
& =\left((x h)^{+}, x y,(h y)^{\star}\right) \quad
\end{aligned}
$$

and so, $(x h)^{+} \mathcal{R}^{\star} x y \mathcal{L}^{*}(h y)^{\star}$. Similarly, we have

$$
((e, x, f) \otimes(g, z, k))_{h^{\prime}}=\left(\left(x h^{\prime}\right)^{+}, x z,\left(h^{\prime} z\right)^{\star}\right)
$$

and $\left(x h^{\prime}\right)^{+} \mathcal{R}^{\star} x z \mathcal{L}^{*}\left(h^{\prime} z\right)^{\star}$. Since

$$
((e, x, f) \otimes(u, y, v))_{h} \rho((e, x, f) \otimes(g, z, k))_{h^{\prime}}
$$

we have that $\left(x h^{\prime}\right)^{+} \mathcal{R}(x h)^{+},(h y)^{\star} \mathcal{L}\left(h^{\prime} z\right)^{\star}$ and

$$
\left[(x h)^{+},\left(x h^{\prime}\right)^{+}\right] \cdot\left(\left(x h^{\prime}\right)^{+}, x z,\left(h^{\prime} z\right)^{\star}\right)=\left((x h)^{+}, x y,(h y)^{\star}\right) \cdot\left[(h y)^{\star},\left(h^{\prime} z\right)^{\star}\right]
$$

From $\left(x h^{\prime}\right)^{+} \mathcal{R}(x h)^{+}$, we obtain that $\left(x h^{\prime}\right)^{+}=(x h)^{+}$by the uniqueness. Similarly, we have $(h y)^{\star}=\left(h^{\prime} z\right)^{\star}$. Thus,

$$
\left(\left(x h^{\prime}\right)^{+}, x z,\left(h^{\prime} z\right)^{\star}\right)=\left((x h)^{+}, x y,(h y)^{\star}\right)
$$

and so $x y=x z$. Since $f \mathcal{L}^{*} x$, we have that $f y=f z$. As $u \mathcal{R}^{*} y$ and $h \omega^{r} u$, we obtain that $h \mathcal{R} h u$, and so $f h \mathcal{R}$ fhu $\mathcal{R}^{*}$ fhy $=$ fhuy $=$ fuy $=$ fy. Similarly, $f h^{\prime} \mathcal{R}^{*} f z$, and so $f h \mathcal{R}^{*} f y=f z \mathcal{R}^{*} f h^{\prime}$ so that $f h \mathcal{R} f h^{\prime}$. Hence, (P8) holds.

We now show that $S \mathbf{C}$ is cancellative. Suppose that $(e, x, f),(u, y, f)$ and $(f, z, v) \in S \mathbf{C}$ are such that $(e, x, f)(f, z, v)=(u, y, f)(f, z, v)$. Then $(e, x z, v)=$ $(u, y z, v)$, and so $x z=y z$. As $f \mathcal{R}^{*} z$, we have $x f=y f$ and so $x=y$. Thus, $S \mathbf{C}$ is right cancellative, dually, we show that $S \mathbf{C}$ is left cancellative.

Conversely, suppose that $P$ is an abundant cancellative category over $U$. Due to Theorem 10.30, it is necessary to show that $P \mathbf{S}$ is abundant. For any $x \in P$, we want to show that $\overline{1_{\mathbf{d}(x)}} \mathcal{R}^{*} \bar{x} \mathcal{L}^{*} \overline{1_{\mathbf{r}(x)}}$. First, we show that $\bar{x} \mathcal{L}^{*} \overline{1_{\mathbf{r}(x)}}$. Clearly, 
$\bar{x} \odot \overline{1_{\mathbf{r}(x)}}=\bar{x}$. Suppose that $a, b \in P$ and $\bar{x} \odot \bar{a}=\bar{x} \odot \bar{b}$. Notice that

$$
\begin{gathered}
\bar{x} \odot \bar{a}=\bar{x} \odot \bar{b} \\
\Rightarrow \overline{(x \otimes a)_{h}}=\overline{(x \otimes b)_{h^{\prime}}} \quad\left(h \in S(\mathbf{r}(x), \mathbf{d}(a)), h^{\prime} \in S(\mathbf{r}(x), \mathbf{d}(b))\right) \\
\Rightarrow \overline{(x \diamond h) \cdot(h * a)}=\overline{\left(x \diamond h^{\prime}\right) \cdot\left(h^{\prime} * b\right)} \\
\Rightarrow \mathbf{d}(x \diamond h) \mathcal{R} \mathbf{d}\left(x \diamond h^{\prime}\right), \mathbf{r}(h * a) \mathcal{L} \mathbf{r}\left(h^{\prime} * b\right) \text { and } \\
(x \diamond h) \cdot(h * a) \cdot\left[\mathbf{r}(h * a), \mathbf{r}\left(h^{\prime} * b\right)\right]=\left[\mathbf{d}(x \diamond h), \mathbf{d}\left(x \diamond h^{\prime}\right)\right] \cdot\left(x \diamond h^{\prime}\right) \cdot\left(h^{\prime} * b\right) .
\end{gathered}
$$

Also, we have

$$
\begin{array}{rlrl}
x \diamond h & =\left.x\right|_{\mathbf{r}(x) h} \cdot[\mathbf{r}(x) h, h] & \\
& =\left.x\right|_{\mathbf{r}(x) h} \cdot\left[\mathbf{r}(x) h,(\mathbf{r}(x) h)^{+}\right] \cdot\left[(\mathbf{r}(x) h)^{+}, \mathbf{r}(x) h\right] \cdot[\mathbf{r}(x) h, h] & & (\text { by }(P 1)) \\
& =\left.x\right|_{(\mathbf{r}(x) h)^{+}} \cdot\left[(\mathbf{r}(x) h)^{+}, \mathbf{r}(x) h\right] \cdot[\mathbf{r}(x) h, h] & & \left(\text { by }(\mathrm{P} 4)^{\circ}\right) \\
& =\left.x\right|_{(\mathbf{r}(x) h)^{+}} \cdot([\mathbf{r}(x), \mathbf{r}(x)] \diamond h) & \\
& =\left.x\right|_{(\mathbf{r}(x) h)^{+}} \cdot\left(1_{\mathbf{r}(x)} \diamond h\right) . &
\end{array}
$$

Similarly, $x \diamond h^{\prime}=\left.x\right|_{\left(\mathbf{r}(x) h^{\prime}\right)^{+}} \cdot\left(1_{\mathbf{r}(x)} \diamond h^{\prime}\right)$. Thus, we can write (10.1) into the following form:

$$
\begin{aligned}
& \left.x\right|_{(\mathbf{r}(x) h)^{+}} \cdot\left(1_{\mathbf{r}(x)} \diamond h\right) \cdot(h * a) \cdot\left[\mathbf{r}(h * a), \mathbf{r}\left(h^{\prime} * b\right)\right] \\
= & {\left.\left[\mathbf{d}(x \diamond h), \mathbf{d}\left(x \diamond h^{\prime}\right)\right] \cdot x\right|_{\left(\mathbf{r}(x) h^{\prime}\right)^{+}} \cdot\left(1_{\mathbf{r}(x)} \diamond h^{\prime}\right) \cdot\left(h^{\prime} * b\right) . }
\end{aligned}
$$

Since $(x \otimes a)_{h} \rho(x \otimes b)_{h^{\prime}}$, it follows from $(\mathrm{P} 8)$ that $\mathbf{r}(x) h \mathcal{R} \mathbf{r}(x) h^{\prime}$. Thus $(\mathbf{r}(x) h)^{+}=\left(\mathbf{r}(x) h^{\prime}\right)^{+}$, which implies that $\left.x\right|_{(\mathbf{r}(x) h)^{+}}=\left.x\right|_{\left(\mathbf{r}(x) h^{\prime}\right)^{+}}$, and so

$$
\mathbf{d}(x \diamond h)=\mathbf{d}\left(\left.x\right|_{(\mathbf{r}(x) h)^{+}}\right)=\mathbf{d}\left(\left.x\right|_{\left(\mathbf{r}(x) h^{\prime}\right)^{+}}\right)=\mathbf{d}\left(x \diamond h^{\prime}\right) .
$$

Thus we can write (10.2) into the following form:

$\left.x\right|_{(\mathbf{r}(x) h)^{+}} \cdot\left(1_{\mathbf{r}(x)} \diamond h\right) \cdot(h * a) \cdot\left[\mathbf{r}(h * a), \mathbf{r}\left(h^{\prime} * b\right)\right]=\left.x\right|_{\left(\mathbf{r}(x) h^{\prime}\right)^{+}} \cdot\left(1_{\mathbf{r}(x)} \diamond h^{\prime}\right) \cdot\left(h^{\prime} * b\right)$.

As $P$ is cancellative and $\left.x\right|_{(\mathbf{r}(x) h)^{+}}=\left.x\right|_{\left(\mathbf{r}(x) h^{\prime}\right)^{+}}$, we have that

$$
\left(1_{\mathbf{r}(x)} \diamond h\right) \cdot(h * a) \cdot\left[\mathbf{r}(h * a), \mathbf{r}\left(h^{\prime} * b\right)\right]=\left(1_{\mathbf{r}(x)} \diamond h^{\prime}\right) \cdot\left(h^{\prime} * b\right)
$$


Together with $\mathbf{d}\left(1_{\mathbf{r}(x)} \diamond h\right)=(\mathbf{r}(x) h)^{+}=\left(\mathbf{r}(x) h^{\prime}\right)^{+}=\mathbf{d}\left(1_{\mathbf{r}(x)} \diamond h^{\prime}\right)$ and $\mathbf{r}(h * a) \mathcal{L} \mathbf{r}\left(h^{\prime} * b\right)$, we obtain that

$$
\left(1_{\mathbf{r}(x)} \diamond h\right) \cdot(h * a) \rho\left(1_{\mathbf{r}(x)} \diamond h^{\prime}\right) \cdot\left(h^{\prime} * b\right)
$$

that is, $\left(1_{\mathbf{r}(x)} \otimes a\right)_{h} \rho\left(1_{\mathbf{r}(x)} \otimes b\right)_{h^{\prime}}$, and so $\overline{1_{\mathbf{r}(x)}} \odot \bar{a}=\overline{1_{\mathbf{r}(x)}} \odot \bar{b}$.

Suppose that $c \in P$ is such that $\bar{x} \odot \bar{c}=\bar{x}$, then $\bar{x} \odot \bar{c}=\bar{x} \odot \overline{1_{\mathbf{r}(x)}}$. Using the same method as above, we have that $\overline{1_{\mathbf{r}(x)}} \odot \bar{c}=\overline{1_{\mathbf{r}(x)}}$. Hence, $\bar{x} \mathcal{L}^{*} \overline{1_{\mathbf{r}(x)}}$. Dually, we can show that $\bar{x} \mathcal{R}^{*} \overline{1_{\mathbf{d}(x)}}$.

For any $\bar{x} \in E(P \mathbf{S})$, we have $\overline{1_{\mathbf{d}(x)}} \mathcal{R}^{*} \bar{x} \mathcal{L}^{*} \overline{1_{\mathbf{r}(x)}}$. By Lemma 10.26 , we obtain that $E(P \mathbf{S})=\bar{U}$.

\subsection{The concordant case}

The aim of this section is to investigate concordant semigroups. We recall that such semigroups satisfy (IC), defined by El-Qallali and Fountain, which coincides with (WIC) in abundant case. Notice that Condition (P7) is a complicated condition, that we would like to omit. To this end, we first define an $I C$ - $R B S$ category. The difference between a weakly regular category and an IC-RBS category is that Condition (P7) is replaced by Conditions (PC1), (PC2) and the duals $(\mathrm{PC} 1)^{\circ}$, $(\mathrm{PC} 2)^{\circ}$ of $(\mathrm{PC} 1)$ and $(\mathrm{PC} 2)$, respectively.

An RBS cancellative category $P$ over $U$ is said to be $I C-R B S$ if the following conditions and the duals $(\mathrm{PC} 1)^{\circ},(\mathrm{PC} 2)^{\circ}$ of $(\mathrm{PC} 1)$ and $(\mathrm{PC} 2)$ hold:

(PC1) if $x \in P$ and $h \in U$ with $h \omega \mathbf{d}(x)$, then there exists a unique $k \in U$ such that $k \omega \mathbf{r}(x)$ and ${ }_{h}|x \rho x|_{k}$; in particular, if $h=\mathbf{d}(x)$, then ${ }_{h} \mid x \rho x$;

(PC2) let $x \in P$ and for $i=1,2, e_{i}, f_{i} \in U$ be such that $e_{i} \omega \mathbf{d}(x), f_{i} \omega \mathbf{r}(x)$ and $e_{e_{i}}|x \rho x|_{f_{i}}$. If $e_{1} \omega^{r} e_{2}$, then $f_{1} \omega^{r} f_{2}$, and $e_{e_{1} e_{2}}|x \rho x|_{f_{1} f_{2}}$. If $e_{1} \omega^{l} e_{2}$, then $f_{1} \omega^{l} f_{2}$ and ${ }_{e_{2} e_{1}}|x \rho x|_{f_{2} f_{1}}$.

We pause to make a necessary comment on Condition (PC2). From $e_{1} \boldsymbol{\omega} \mathbf{d}(x)$, $f_{i} \omega \mathbf{r}(x)$ and $e_{i}|x \rho x|_{f_{i}}$, we obtain that $e_{i}$ and $f_{i}$ are unique by (PC1) and its dual $(\mathrm{PC} 1)^{\circ}$. If $f_{1}, f_{2} \omega \mathbf{r}(x)$ and $f_{1} \omega^{r} f_{2}$, then $f_{1} f_{2} \omega f_{2}$, and so $f_{1} f_{2} \omega \mathbf{r}(x)$ so that $\left.x\right|_{f_{1} f_{2}}$ is well-defined. Dually, if $f_{1} \omega^{l} f_{2}$, then $\left.x\right|_{f_{2} f_{1}}$ is well-defined.

There exist two approaches to build a correspondence between concordant semigroups and IC-RBS categories. We could show without mention of semigroups that if $P$ is an $R B S$ cancellative category, then $P$ has $(\mathrm{W}),(\mathrm{W})^{\circ},(\mathrm{P} 7)$ 
and (P8) if and only if $P$ has (PC1), (PC2) and the duals $(\mathrm{PC} 1)^{\circ},(\mathrm{PC} 2)^{\circ}$ of $(\mathrm{PC} 1)$ and (PC2), respectively. However, we are going to prove the correspondence between concordant semigroups and IC-RBS categories using Theorem 10.38.

Lemma 11.3. Let $P$ be an $I C$-RBS category over $U$. If $x \in P$ and for $i=1,2$, $e_{i}, f_{i} \in U$ are such that $e_{i} \omega \mathbf{d}(x), f_{i} \omega \mathbf{r}(x), e_{i}|x \rho x|_{f_{i}}$ and $e_{1} \omega^{r} e_{2}$, then $\left[e_{1}, e_{1} e_{2}\right] \cdot\left(e_{1} e_{2} \mid x\right) \cdot\left[\mathbf{r}\left(e_{e_{1} e_{2}} \mid x\right), f_{1} f_{2}\right]=\left(e_{1} \mid x\right) \cdot\left[\mathbf{r}\left(e_{1} \mid x\right), f_{1}\right] \cdot\left[f_{1}, f_{1} f_{2}\right]$.

Proof. By (PC2), we have that $f_{1} \omega^{r} f_{2}$ and $e_{1} e_{2}|x \rho x|_{f_{1} f_{2}}$. Then $e_{1} e_{2} \mathcal{R} \mathbf{d}\left(\left.x\right|_{f_{1} f_{2}}\right)$. Certainly, $\left[e_{1}, e_{1} e_{2}\right]$ and $\left[f_{1}, f_{1} f_{2}\right]$ exist. As $e_{i}|x \rho x|_{f_{i}}$, we obtain that $e_{i} \mathcal{R} \mathbf{d}\left(\left.x\right|_{f_{i}}\right)$, $f_{i} \mathcal{L} \mathbf{r}\left(e_{i} \mid x\right)$ and

$$
e_{i}\left|x \cdot\left[\mathbf{r}\left(e_{i} \mid x\right), f_{i}\right]=\left[e_{i}, \mathbf{d}\left(\left.x\right|_{f_{i}}\right)\right] \cdot x\right|_{f_{i}} .
$$

Thus,

$$
\begin{array}{rlrl} 
& e_{1} \mid x \cdot\left[\mathbf{r}\left(e_{1} \mid x\right), f_{1}\right] \cdot\left[f_{1}, f_{1} f_{2}\right] & \\
= & {\left.\left[e_{1}, \mathbf{d}\left(\left.x\right|_{f_{1}}\right)\right] \cdot x\right|_{f_{1}} \cdot\left[f_{1}, f_{1} f_{2}\right]} & \\
= & {\left.\left[e_{1}, \mathbf{d}\left(\left.x\right|_{f_{1}}\right)\right] \cdot x\right|_{f_{1} f_{2}}} & & \left(f_{1} \mathcal{R} f_{1} f_{2}, \text { by }(\mathrm{P} 4)^{\circ}\right) \\
= & {\left.\left[e_{1}, \mathbf{d}\left(\left.x\right|_{f_{1} f_{2}}\right)\right] \cdot x\right|_{f_{1} f_{2}}} & & \left(\mathbf{d}\left(\left.x\right|_{f_{1}}\right)=\mathbf{d}\left(\left.x\right|_{f_{1} f_{2}}\right)\right) \\
= & {\left.\left[e_{1}, e_{1} e_{2}\right] \cdot\left[e_{1} e_{2}, \mathbf{d}\left(\left.x\right|_{f_{1} f_{2}}\right)\right] \cdot x\right|_{f_{1} f_{2}}} & & \left(e_{1} \mathcal{R} e_{1} e_{2} \mathcal{R} \mathbf{d}\left(\left.x\right|_{f_{1} f_{2}}\right)\right) \\
= & {\left[e_{1}, e_{1} e_{2}\right] \cdot e_{e_{1} e_{2}} \mid x \cdot\left[\mathbf{r}\left(e_{1 e_{2}} \mid x\right), f_{1} f_{2}\right]} & & \left(e_{1} e_{2}|x \rho x|_{f_{1} f_{2}}\right) .
\end{array}
$$

The following lemma is necessary for Lemma 11.5. Here we recall from Section 1.4 that if $E$ is a regular biordered set, then for any $e \in E, \omega(e)$ is a regular biordered set.

Lemma 11.4. Let $P$ be an $I C-R B S$ category over $U$. For any $x \in P$, the map $\sigma_{x}: \omega(\mathbf{d}(x)) \rightarrow \omega(\mathbf{r}(x))$, defined by $\sigma_{x}=k$, is an isomorphism, where $k \omega \mathbf{r}(x)$ and ${ }_{e}|x \rho x|_{k}$.

Proof. Clearly, $\sigma_{x}$ does map into $\omega(\mathbf{r}(x))$ by (PC1). Now, we define

$$
\tau_{x}: \omega(\mathbf{r}(x)) \rightarrow \omega(\mathbf{d}(x))
$$

by $f \tau_{x}=g$, where $g \omega \mathbf{d}(x)$ and $\left.x\right|_{f} \rho_{g} \mid x$. By $(\mathrm{PC} 1)^{\circ}, \tau_{x}$ is well-defined. If 
$e \in \omega(\mathbf{d}(x))$, then it follows from (PC1) and its dual that $e \sigma_{x} \tau_{x}=k \tau_{x}=e$, where $k \omega \mathbf{r}(x)$ and ${ }_{e}|x \rho x|_{k}$. Thus, $\sigma_{x}=\tau_{x}^{-1}$, and so $\sigma_{x}$ is a bijection.

To show that $\sigma_{x}$ is a morphism, suppose that $e_{1}, e_{2} \in \omega(\mathbf{d}(x))$ and $e_{1} \omega^{r} e_{2}$. Write $f_{1}=e_{1} \sigma_{x}$ and $f_{2}=e_{2} \sigma_{x}$. Then, by (PC2), $f_{1} \omega^{r} f_{2}$ and $\left.x\right|_{f_{1} f_{2}} \rho_{e_{1} e_{2}} \mid x$. Thus, $\left(e_{1} e_{2}\right) \sigma_{x}=f_{1} f_{2}=e_{1} \sigma_{x} e_{2} \sigma_{x}$. If $e_{1} \omega^{l} e_{2}$, then by (PC2), $f_{1} \omega^{l} f_{2}$, and so $\left(e_{1} e_{2}\right) \sigma_{x}=e_{1} \sigma_{x}=f_{1}=f_{1} f_{2}=e_{1} \sigma_{x} e_{2} \sigma_{x}$.

To show that $\sigma_{x}$ is regular, we suppose that $e, f \in \omega(\mathbf{d}(x)), h \in S(e, f)$ and $k \in S\left(e \sigma_{x}, f \sigma_{x}\right)$. Then $h \sigma_{x} \in \mathcal{M}\left(e \sigma_{x}, f \sigma_{x}\right)$ and $k \tau_{x} \in \mathcal{M}(e, f)$, and so $k \tau_{x} \prec h$ in $\mathcal{M}(e, f)$, that is,

$$
e\left(k \tau_{x}\right) \omega^{r} \text { eh and }\left(k \tau_{x}\right) f \omega^{l} h f .
$$

As $\sigma_{x}$ is a morphism, we obtain that

$$
\left(e\left(k \tau_{x}\right)\right) \sigma_{x} \omega^{r}(e h) \sigma_{x} \text { and }\left(\left(k \tau_{x}\right) f\right) \sigma_{x} \omega^{l}(h f) \sigma_{x}
$$

that is,

$$
e \sigma_{x}\left(k \tau_{x}\right) \sigma_{x} \omega^{r} e \sigma_{x} h \sigma_{x} \text { and }\left(k \tau_{x}\right) \sigma_{x} f \sigma_{x} \omega^{l} h \sigma_{x} f \sigma_{x}
$$

or equivalently,

$$
e \sigma_{x} k \omega^{r} e \sigma_{x} h \sigma_{x} \text { and } k\left(f \sigma_{x}\right) \omega^{l} h \sigma_{x} f \sigma_{x},
$$

and so $h \sigma_{x} \in S\left(e \sigma_{x}, f \sigma_{x}\right)$. By Lemma 1.26, $\sigma_{x}$ is an isomorphism.

Before we discuss the relationship amongst IC-RBS categories, concordant semigroups and inductive ${ }_{2}$ cancellative categories (defined in [1] and mentioned in Chapter 6) we show that:

Lemma 11.5. If $P$ is an IC-RBS category over $U$, then it is a weakly regular category.

Proof. It is sufficient to show that Condition (P7) holds. Suppose that $x \in P$, $e, f \in U, h_{1} \in S(e, \mathbf{d}(x))$ and $h_{2} \in S(\mathbf{r}(x), f)$. We put

$$
h_{1}^{\prime}=\mathbf{r}\left(h_{1} \mathbf{d}(x) \mid x\right) \text { and } h_{2}^{\prime}=\mathbf{d}\left(\left.x\right|_{\mathbf{r}(x) h_{2}}\right)
$$

As $U$ is regular, there exists $h \in S\left(h_{1}, h_{2}^{\prime}\right)$. Since $h_{1} \omega^{r} \mathbf{d}(x)$, by (B21), we have $h_{1} \mathbf{d}(x) \omega \mathbf{d}(x)$. Since $h_{2} \omega^{l} \mathbf{r}(x)$, by the dual of (B21), we have that $\mathbf{r}(x) h_{2} \omega \mathbf{r}(x)$. By (PC1) and its dual, there exist $u \omega \mathbf{d}(x)$ and $v \omega \mathbf{r}(x)$ such that ${ }_{u}|x \rho x|_{\mathbf{r}(x) h_{2}}$ 
and $\left.x\right|_{v} \rho_{h_{1} \mathbf{d}(x)} \mid x$. From ${ }_{u}|x \rho x|_{\mathbf{r}(x) h_{2}}$, we obtain that $u \mathcal{R} h_{2}^{\prime}, \mathbf{r}\left(_{u} \mid x\right) \mathcal{L} \mathbf{r}(x) h_{2}$ and

$$
\left.\left[u, h_{2}^{\prime}\right] \cdot x\right|_{\mathbf{r}(x) h_{2}}={ }_{u} \mid x \cdot\left[\mathbf{r}\left({ }_{u} \mid x\right), \mathbf{r}(x) h_{2}\right],
$$

which implies that $\left.x\right|_{\mathbf{r}(x) h_{2}}=\left[h_{2}^{\prime}, u\right] \cdot{ }_{u} \mid x \cdot\left[\mathbf{r}\left({ }_{u} \mid x\right), \mathbf{r}(x) h_{2}\right]$. Thus,

$$
\begin{aligned}
x \diamond h_{2} & =\left.x\right|_{\mathbf{r}(x) h_{2}} \cdot\left[\mathbf{r}(x) h_{2}, h_{2}\right] \\
& =\left[h_{2}^{\prime}, u\right] \cdot{ }_{u} \mid x \cdot\left[\mathbf{r}\left({ }_{u} \mid x\right), \mathbf{r}(x) h_{2}\right] \cdot\left[\mathbf{r}(x) h_{2}, h_{2}\right] \\
& =\left[h_{2}^{\prime}, u\right] \cdot{ }_{u} \mid x \cdot\left[\mathbf{r}\left({ }_{u} \mid x\right), h_{2}\right] \quad\left(\mathbf{r}\left({ }_{u} \mid x\right) \mathcal{L} \mathbf{r}(x) h_{2} \mathcal{L} h_{2}, \text { by }(\mathrm{P} 1)\right),
\end{aligned}
$$

and so

$$
\begin{aligned}
& h *\left(x \diamond h_{2}\right) \\
& =h *\left(\left[h_{2}^{\prime}, u\right] \cdot{ }_{u} \mid x \cdot\left[\mathbf{r}\left({ }_{u} \mid x\right), h_{2}\right]\right) \\
& =\left[h, h h_{2}^{\prime}\right] \cdot{ }_{h h_{2}^{\prime}} \mid\left(\left[h_{2}^{\prime}, u\right] \cdot{ }_{u} \mid x \cdot\left[\mathbf{r}\left({ }_{u} \mid x\right), h_{2}\right]\right) \\
& =\left[h, h h_{2}^{\prime}\right] \cdot{ }_{h h_{2}^{\prime}}\left|\left[h_{2}^{\prime}, u\right] \cdot{ }_{m}\right|\left({ }_{u} \mid x\right) \cdot{ }_{n} \mid\left[\mathbf{r}\left({ }_{u} \mid x\right), h_{2}\right] \\
& \text { (by } \left.(\mathrm{P} 5), m=\mathbf{r}\left({ }_{h h_{2}^{\prime}} \mid\left[h_{2}^{\prime}, u\right]\right), n=\mathbf{r}\left({ }_{m} \mid\left({ }_{u} \mid x\right)\right)\right) \\
& =\left[h, h h_{2}^{\prime}\right] \cdot\left[h h_{2}^{\prime},\left(h h_{2}^{\prime}\right) u\right] \cdot\left[\left(h h_{2}^{\prime}\right) u,\left(\left(h h_{2}^{\prime}\right) u\right)^{\star}\right] \cdot\left(\left(h h_{2}^{\prime}\right) u\right)^{\star}\left|\left({ }_{u} \mid x\right) \cdot{ }_{n}\right|\left[\mathbf{r}\left({ }_{u} \mid x\right), h_{2}\right] \\
& \text { (by (P3), since } h \omega^{r} h_{2}^{\prime} \text {, we have } h h_{2}^{\prime} \omega h_{2}^{\prime} \mathcal{R} u, m=\left(\left(h h_{2}^{\prime}\right) u\right)^{\star} \text { ) } \\
& =\left[h, h h_{2}^{\prime}\right] \cdot\left[h h_{2}^{\prime},\left(h h_{2}^{\prime}\right) u\right] \cdot\left[\left(h h_{2}^{\prime}\right) u,\left(\left(h h_{2}^{\prime}\right) u\right)^{\star}\right] \cdot\left(\left(h h_{2}^{\prime}\right) u\right)^{\star}\left|x \cdot{ }_{n}\right|\left[\mathbf{r}\left({ }_{u} \mid x\right), h_{2}\right] \\
& \left(\left(\left(h h_{2}^{\prime}\right) u\right)^{\star} \mathcal{L}\left(h h_{2}^{\prime}\right) u \omega u \text {, by }(\mathrm{P} 4)\right) \\
& =\left[h, h h_{2}^{\prime}\right] \cdot\left[h h_{2}^{\prime}, h u\right] \cdot\left[h u,(h u)^{\star}\right] \cdot(h u)^{\star}\left|x \cdot{ }_{n}\right|\left[\mathbf{r}\left({ }_{u} \mid x\right), h_{2}\right] \\
& \text { (by (B31), since } h \omega^{r} h_{2}^{\prime} \mathcal{R} u \text {, we have } h \omega^{r} u \mathcal{R} h_{2}^{\prime} \text { ) } \\
& =\left[h, h h_{2}^{\prime}\right] \cdot\left[h h_{2}^{\prime}, h u\right] \cdot{ }_{h u}\left|x \cdot{ }_{n}\right|\left[\mathbf{r}\left({ }_{u} \mid x\right), h_{2}\right] \quad\left(h u \mathcal{L}(h u)^{\star}, \text { by }(\mathrm{P} 4)\right) \\
& =[h, h u] \cdot{ }_{h u}\left|x \cdot{ }_{n}\right|\left[\mathbf{r}\left({ }_{u} \mid x\right), h_{2}\right] \quad\left(h \mathcal{R} h h_{2}^{\prime} \mathcal{R} h u, \text { by }(\mathrm{P} 1)\right) \\
& =[h, h u] \cdot{ }_{h u} \mid x \cdot\left[\mathbf{r}\left({ }_{h u} \mid x\right),\left(\mathbf{r}\left({ }_{h u} \mid x\right)^{\star}\right)\right] \quad\left(n=\mathbf{r}\left({ }_{h u} \mid x\right) \omega^{l} \mathbf{r}\left({ }_{u} \mid x\right) \mathcal{L} h_{2},\right. \text { by (P3)). }
\end{aligned}
$$

Since $h_{2}^{\prime} \mathcal{R} u$, by Lemma 1.28, we have $S\left(h_{1}, h_{2}^{\prime}\right)=S\left(h_{1}, u\right)$. From $\left.x\right|_{v} \rho_{h_{1} \mathbf{d}(x)} \mid x$, we obtain that $v \mathcal{L} \mathbf{r}\left(h_{h(x)} \mid x\right)=h_{1}^{\prime}$, and so by Lemma 1.28, $S\left(h_{1}^{\prime}, h_{2}\right)=S\left(v, h_{2}\right)$. As $h \in S\left(h_{1}, h_{2}^{\prime}\right)$ and $\sigma_{x}: \omega(\mathbf{d}(x)) \rightarrow \omega(\mathbf{r}(x))$ is an isomorphism, it follows from Lemma 1.31 that there exists $h^{\prime} \in S\left(h_{1}^{\prime}, h_{2}\right)$ such that $(h \mathbf{d}(x)) \sigma_{x}=\mathbf{r}(x) h^{\prime}$. Since $h \omega^{r} u \omega \mathbf{d}(x)$, we have that $h \mathbf{d}(x) \mathcal{R} h \omega^{r} u \omega \mathbf{d}(x)$ by (B21), and so $(h \mathbf{d}(x)) u$ exists and $(h \mathbf{d}(x)) u \omega u \omega \mathbf{d}(x)$. In addition, by (B31), we have $h u=(h \mathbf{d}(x)) u$. 
Due to Lemma 11.4, we obtain that

$$
(h \mathbf{d}(x)) u|x \rho x|_{((h \mathbf{d}(x)) u) \sigma_{x}}
$$

Since $h^{\prime} \omega^{r} h_{2}$ and $h_{1}^{\prime}, h_{2} \omega^{l} \mathbf{r}(x)$, it follows from (B32) ${ }^{\circ}$ that

$$
\left(\mathbf{r}(x) h^{\prime}\right)\left(\mathbf{r}(x) h_{2}\right)=\mathbf{r}(x)\left(h^{\prime} h_{2}\right)
$$

Observe that $h^{\prime} h_{2} \omega h_{2} \omega^{l} \mathbf{r}(x)$. Thus $\mathbf{r}(x)\left(h^{\prime} h_{2}\right) \mathcal{L} h^{\prime} h_{2}$, and so

$$
\begin{aligned}
\mathbf{r}\left({ }_{h u} \mid x\right) & =\mathbf{r}\left({ }_{(h \mathbf{d}(x)) u} \mid x\right) \quad((h \mathbf{d}(x)) u=h u) \\
& \mathcal{L}((h \mathbf{d}(x)) u) \sigma_{x} \\
& =(h \mathbf{d}(x)) \sigma_{x} u \sigma_{x}=\left(\mathbf{r}(x) h^{\prime}\right)\left(\mathbf{r}(x) h_{2}\right)=\mathbf{r}(x)\left(h^{\prime} h_{2}\right) \mathcal{L} h^{\prime} h_{2} .
\end{aligned}
$$

Hence, $\left(\mathbf{r}\left({ }_{h u} \mid x\right)\right)^{\star} \mathcal{L} \mathbf{r}\left({ }_{h u} \mid x\right) \mathcal{L} h^{\prime} h_{2}$, that is, $\mathbf{r}\left(h *\left(x \diamond h_{2}\right)\right) \mathcal{L} h^{\prime} h_{2}$.

From ${ }_{h_{1} \mathbf{d}(x)}|x \rho x|_{v}$, we obtain that

$$
h_{1} \mathbf{d}(x)\left|x \cdot\left[h_{1}^{\prime}, v\right]=\left[h_{1} \mathbf{d}(x), \mathbf{d}\left(\left.x\right|_{v}\right)\right] \cdot x\right|_{v},
$$

which implies that ${ }_{h_{1} \mathbf{d}(x)}\left|x=\left[h_{1} \mathbf{d}(x), \mathbf{d}\left(\left.x\right|_{v}\right)\right] \cdot x\right|_{v} \cdot\left[v, h_{1}^{\prime}\right]$. Thus

$$
\begin{aligned}
& h_{1} * x \\
= & {\left[h_{1}, h_{1} \mathbf{d}(x)\right] \cdot h_{1} \mathbf{d}(x) \mid x } \\
= & {\left.\left[h_{1}, h_{1} \mathbf{d}(x)\right] \cdot\left[h_{1} \mathbf{d}(x), \mathbf{d}\left(\left.x\right|_{v}\right)\right] \cdot x\right|_{v} \cdot\left[v, h_{1}^{\prime}\right] } \\
= & {\left.\left[h_{1}, \mathbf{d}\left(\left.x\right|_{v}\right)\right] \cdot x\right|_{v} \cdot\left[v, h_{1}^{\prime}\right] \quad\left(h_{1} \mathcal{R} h_{1} \mathbf{d}(x) \mathcal{R} \mathbf{d}\left(\left.x\right|_{v}\right), \text { by }(\mathrm{P} 1)\right), }
\end{aligned}
$$

and so 


$$
\begin{aligned}
& \left(h_{1} * x\right) \diamond h^{\prime} \\
& =\left.\left(\left.\left[h_{1}, \mathbf{d}\left(\left.x\right|_{v}\right)\right] \cdot x\right|_{v} \cdot\left[v, h_{1}^{\prime}\right]\right)\right|_{h_{1}^{\prime} h^{\prime}} \cdot\left[h_{1}^{\prime} h^{\prime}, h^{\prime}\right] \\
& =\left.\left.\left.\left[h_{1}, \mathbf{d}\left(\left.x\right|_{v}\right)\right]\right|_{t} \cdot\left(\left.x\right|_{v}\right)\right|_{s} \cdot\left[v, h_{1}^{\prime}\right]\right|_{h_{1}^{\prime} h^{\prime}} \cdot\left[h_{1}^{\prime} h^{\prime}, h^{\prime}\right] \\
& \text { (by } \left.(\mathrm{P} 5)^{\circ}, s=\mathbf{d}\left(\left.\left[v, h_{1}^{\prime}\right]\right|_{h_{1}^{\prime} h^{\prime}}\right), t=\mathbf{d}\left(\left.\left(\left.x\right|_{v}\right)\right|_{s}\right)\right) \\
& =\left.\left.\left[h_{1}, \mathbf{d}\left(\left.x\right|_{v}\right)\right]\right|_{t} \cdot\left(\left.x\right|_{v}\right)\right|_{\left(v\left(h_{1}^{\prime} h^{\prime}\right)\right)^{+}} \cdot\left[\left(v\left(h_{1}^{\prime} h^{\prime}\right)\right)^{+}, v\left(h_{1}^{\prime} h^{\prime}\right)\right] \cdot\left[v\left(h_{1}^{\prime} h^{\prime}\right), h_{1}^{\prime} h^{\prime}\right] \cdot\left[h_{1}^{\prime} h^{\prime}, h^{\prime}\right] \\
& =\left.\left.\left[h_{1}, \mathbf{d}\left(\left.x\right|_{v}\right)\right]\right|_{t} \cdot\left(\left.x\right|_{v}\right)\right|_{\left(v h^{\prime}\right)^{+}} \cdot\left[\left(v h^{\prime}\right)^{+}, v h^{\prime}\right] \cdot\left[v h^{\prime}, h_{1}^{\prime} h^{\prime}\right] \cdot\left[h_{1}^{\prime} h^{\prime}, h^{\prime}\right] \\
& \left(h^{\prime} \omega^{l} h_{1}^{\prime} \mathcal{L} v \text {, by }(\mathrm{B} 31)^{\circ}, v\left(h_{1}^{\prime} h^{\prime}\right)=v h^{\prime}\right) \\
& =\left.\left.\left[h_{1}, \mathbf{d}\left(\left.x\right|_{v}\right)\right]\right|_{t} \cdot\left(\left.x\right|_{v}\right)\right|_{\left(v h^{\prime}\right)^{+}} \cdot\left[\left(v h^{\prime}\right)^{+}, v h^{\prime}\right] \cdot\left[v h^{\prime}, h^{\prime}\right] \\
& \left(v h^{\prime} \mathcal{L} h_{1}^{\prime} h^{\prime} \mathcal{L} h^{\prime} \text { by }(\mathrm{P} 1)\right) \\
& =\left.\left.\left[h_{1}, \mathbf{d}\left(\left.x\right|_{v}\right)\right]\right|_{t} \cdot x\right|_{\left(v h^{\prime}\right)^{+}} \cdot\left[\left(v h^{\prime}\right)^{+}, v h^{\prime}\right] \cdot\left[v h^{\prime}, h^{\prime}\right] \quad\left(\left(v h^{\prime}\right)^{+} \mathcal{R} v h^{\prime} \mathcal{R} v \text {, by }(\mathrm{P} 4)^{\circ}\right) \\
& =\left.\left.\left[h_{1}, \mathbf{d}\left(\left.x\right|_{v}\right)\right]\right|_{t} \cdot x\right|_{v h^{\prime}} \cdot\left[v h^{\prime}, h^{\prime}\right] \quad\left(\left(v h^{\prime}\right)^{+} \mathcal{R} v h^{\prime}, \text { by }(\mathrm{P} 4)^{\circ}\right) \\
& =\left.\left.\left[h_{1}, \mathbf{d}\left(\left.x\right|_{v}\right)\right]\right|_{\mathbf{d}\left(\left.x\right|_{v h^{\prime}}\right)} \cdot x\right|_{v h^{\prime}} \cdot\left[v h^{\prime}, h^{\prime}\right] \quad\left(t=\mathbf{d}\left(\left.x\right|_{v h^{\prime}}\right)\right) \\
& =\left.\left[\left(\mathbf{d}\left(\left.x\right|_{v h^{\prime}}\right)\right)^{+}, \mathbf{d}\left(\left.x\right|_{v h^{\prime}}\right)\right] \cdot x\right|_{v h^{\prime}} \cdot\left[v h^{\prime}, h^{\prime}\right] \\
& \left(\mathbf{d}\left(\left.x\right|_{v h^{\prime}}\right) \omega^{r} \mathbf{d}\left(\left.x\right|_{v}\right) \mathcal{R} h_{1} \text {, by }(\mathrm{P} 3)^{\circ}\right) .
\end{aligned}
$$

Since $v \mathcal{L} h_{1}^{\prime}$ and $h^{\prime} \omega^{l} h_{1}^{\prime}$, we have that $h^{\prime} \omega^{l} v \omega \mathbf{r}(x)$, and so by $(\mathrm{B} 31)^{\circ}$, $v h^{\prime}=v\left(\mathbf{r}(x) h^{\prime}\right)$. Also, by $(\mathrm{B} 21)^{\circ}, v h^{\prime} \omega v \omega \mathbf{r}(x)$. Thus, $\left(v h^{\prime}\right) \tau_{x}$ exists, and

$$
\left(v h^{\prime}\right) \tau_{x}=\left(v\left(\mathbf{r}(x) h^{\prime}\right)\right) \tau_{x}=\left(h_{1} \mathbf{d}(x)\right)(h \mathbf{d}(x))=\left(h_{1} h\right) \mathbf{d}(x)
$$

so that

$$
\begin{aligned}
\left(\mathbf{d}\left(\left.x\right|_{v h^{\prime}}\right)\right)^{+} & \mathcal{R} \mathbf{d}\left(\left.x\right|_{v h^{\prime}}\right) \\
& \mathcal{R}\left(v h^{\prime}\right) \tau_{x} \quad\left(\left.x\right|_{v h^{\prime}} \rho_{\left(v h^{\prime}\right) \tau_{x}} \mid x\right) \\
& =\left(h_{1} h\right) \mathbf{d}(x) \mathcal{R} h_{1} h,
\end{aligned}
$$

that is, $\mathbf{d}\left(\left(h_{1} * x\right) \diamond h^{\prime}\right) \mathcal{R} h_{1} h$.

Let $g=\mathbf{d}\left(\left(h_{1} * x\right) \diamond h^{\prime}\right)=\left(\mathbf{d}\left(\left.x\right|_{v h^{\prime}}\right)\right)^{+}$and $k=\mathbf{r}\left(h *\left(x \diamond h_{2}\right)\right)=\left(\mathbf{r}\left({ }_{h u} \mid x\right)\right)^{\star}$. As $g \mathcal{R} h_{1} h$ and $k \mathcal{L} h^{\prime} h_{2}$, it follows that $\left[g, h_{1} h\right]$ and $\left[h^{\prime} h_{2}, k\right]$ are well-defined. Since $h \in S\left(h_{1}, h_{2}^{\prime}\right)$ and $h_{1} \in S(e, \mathbf{d}(x))$, we have $h \omega^{l} h_{1} \omega^{r} \mathbf{d}(x)$ and $h \omega^{r} h_{2}^{\prime} \omega^{r} \mathbf{d}(x)$, and so $h \omega^{r} \mathbf{d}(x)$ and $h \mathcal{L} h_{1} h \omega h_{1} \omega^{r} \mathbf{d}(x)$ so that $\left(\begin{array}{cc}h & h \mathbf{d}(x) \\ h_{1} h & \left(h_{1} h\right) \mathbf{d}(x)\end{array}\right)$ is a 
column-singular matrix. By the comments succeeding Definition 10.1, we have

$$
\left[h_{1} h, h\right] \cdot[h, h \mathbf{d}(x)]=\left[h_{1} h,\left(h_{1} h\right) \mathbf{d}(x)\right] \cdot\left[\left(h_{1} h\right) \mathbf{d}(x), h \mathbf{d}(x)\right],
$$

which implies that

$$
\begin{aligned}
& {\left[\left(h_{1} h\right) \mathbf{d}(x), h_{1} h\right] \cdot\left[h_{1} h, h\right] \cdot[h, h \mathbf{d}(x)] } \\
= & {\left[\left(h_{1} h\right) \mathbf{d}(x), h_{1} h\right] \cdot\left[h_{1} h,\left(h_{1} h\right) \mathbf{d}(x)\right] \cdot\left[\left(h_{1} h\right) \mathbf{d}(x), h \mathbf{d}(x)\right], }
\end{aligned}
$$

that is,

$$
\begin{aligned}
& {\left[\left(h_{1} h\right) \mathbf{d}(x), h_{1} h\right] \cdot\left[h_{1} h, h\right] \cdot[h, h \mathbf{d}(x)] } \\
= & {\left[\left(h_{1} h\right) \mathbf{d}(x),\left(h_{1} h\right) \mathbf{d}(x)\right] \cdot\left[\left(h_{1} h\right) \mathbf{d}(x), h \mathbf{d}(x)\right] }
\end{aligned}
$$

by $(\mathrm{P} 1)$, that is,

$$
\left[\left(h_{1} h\right) \mathbf{d}(x), h_{1} h\right] \cdot\left[h_{1} h, h\right] \cdot[h, h \mathbf{d}(x)]=\left[\left(h_{1} h\right) \mathbf{d}(x), h \mathbf{d}(x)\right],
$$

from which it follows that

$$
\begin{aligned}
& {\left[\left(h_{1} h\right) \mathbf{d}(x), h_{1} h\right] \cdot\left[h_{1} h, h\right] \cdot[h, h \mathbf{d}(x)] \cdot[h \mathbf{d}(x), h] } \\
= & {\left[\left(h_{1} h\right) \mathbf{d}(x), h \mathbf{d}(x)\right] \cdot[h \mathbf{d}(x), h], }
\end{aligned}
$$

that is,

$$
\left[\left(h_{1} h\right) \mathbf{d}(x), h_{1} h\right] \cdot\left[h_{1} h, h\right] \cdot[h, h]=\left[\left(h_{1} h\right) \mathbf{d}(x), h \mathbf{d}(x)\right] \cdot[h \mathbf{d}(x), h],
$$

or equivalently,

$$
\left[\left(h_{1} h\right) \mathbf{d}(x), h_{1} h\right] \cdot\left[h_{1} h, h\right]=\left[\left(h_{1} h\right) \mathbf{d}(x), h \mathbf{d}(x)\right] \cdot[h \mathbf{d}(x), h] .
$$


So,

$$
\begin{aligned}
& {\left[g, h_{1} h\right] \cdot\left[h_{1} h, h\right] \cdot\left(h *\left(x \diamond h_{2}\right)\right) } \\
= & {\left[g,\left(h_{1} h\right) \mathbf{d}(x)\right] \cdot\left[\left(h_{1} h\right) \mathbf{d}(x), h_{1} h\right] \cdot\left[h_{1} h, h\right] \cdot\left(h *\left(x \diamond h_{2}\right)\right) } \\
& \left(g \mathcal{R} h_{1} h \mathcal{R}\left(h_{1} h\right) \mathbf{d}(x), \text { by }(\mathrm{P} 1)\right) \\
= & {\left[g,\left(h_{1} h\right) \mathbf{d}(x)\right] \cdot\left[\left(h_{1} h\right) \mathbf{d}(x), h \mathbf{d}(x)\right] \cdot[h \mathbf{d}(x), h] \cdot\left(h *\left(x \diamond h_{2}\right)\right) } \\
= & {\left[g,\left(h_{1} h\right) \mathbf{d}(x)\right] \cdot\left[\left(h_{1} h\right) \mathbf{d}(x), h \mathbf{d}(x)\right] \cdot[h \mathbf{d}(x), h] \cdot[h, h u] \cdot{ }_{h u} \mid x \cdot\left[\mathbf{r}\left({ }_{h u} \mid x\right), k\right] } \\
& \left(k=\left(\mathbf{r}(h u \mid x)^{\star}\right)\right) \\
= & {\left[g,\left(h_{1} h\right) \mathbf{d}(x)\right] \cdot\left[\left(h_{1} h\right) \mathbf{d}(x), h \mathbf{d}(x)\right] \cdot[h \mathbf{d}(x), h u] \cdot{ }_{h u} \mid x \cdot\left[\mathbf{r}\left({ }_{h u} \mid x\right), k\right] } \\
= & {[h \mathbf{d}(x) \mathcal{R} h \mathcal{R} h u, b y(\mathrm{P} 1)) } \\
& \left(\mathbf{r}\left(h_{1} h\right) \mathbf{d}(x)\right] \cdot\left[\left(h_{1} h\right) \mathbf{d}(x), h \mathbf{d}(x)\right] \cdot[h \mathbf{d}(x), h u] \cdot{ }_{h u} \mid x \cdot \\
\quad\left[\mathbf{r}(x)\left(h_{h u}^{\prime} h_{2}\right) \mathcal{L} h^{\prime} h_{2} \mathcal{L} k, \mathbf{r}(x)\left(h^{\prime} h_{2}\right)\right] \cdot\left[\mathbf{r}(x)\left(h^{\prime} h_{2}\right), k\right] & (\mathrm{P} 1)) .
\end{aligned}
$$

Since $h \omega^{r} u \omega \mathbf{d}(x)$, we have $h \mathbf{d}(x) \mathcal{R} h \mathcal{R} h u$ and $h \mathbf{d}(x), h u \in \omega(\mathbf{d}(x))$. Also, we have

$$
(h \mathbf{d}(x)) \sigma_{x}=\mathbf{r}(x) h^{\prime} \omega \mathbf{r}(x) \text { and }(h u) \sigma_{x}=((h \mathbf{d}(x)) u) \sigma_{x}=\mathbf{r}(x)\left(h^{\prime} h_{2}\right) \omega \mathbf{r}(x)
$$

As $\sigma_{x}$ is an isomorphism, we obtain that $\mathbf{r}(x) h^{\prime} \mathcal{R} \mathbf{r}(x)\left(h^{\prime} h_{2}\right)$. By Lemma 11.3,

$$
\begin{aligned}
& {[h \mathbf{d}(x), h u] \cdot{ }_{h u} \mid x \cdot\left[\mathbf{r}\left({ }_{h u} \mid x\right), \mathbf{r}(x)\left(h^{\prime} h_{2}\right)\right] } \\
= & { }_{h \mathbf{d}(x)} \mid x \cdot\left[\mathbf{r}\left({ }_{h \mathbf{d}(x)} \mid x\right), \mathbf{r}(x) h^{\prime}\right] \cdot\left[\mathbf{r}(x) h^{\prime}, \mathbf{r}(x)\left(h^{\prime} h_{2}\right)\right] .
\end{aligned}
$$

Thus,

$$
\begin{aligned}
& {\left[g, h_{1} h\right] \cdot\left[h_{1} h, h\right] \cdot\left(h *\left(x \diamond h_{2}\right)\right) } \\
= & {\left[g,\left(h_{1} h\right) \mathbf{d}(x)\right] \cdot\left[\left(h_{1} h\right) \mathbf{d}(x), h \mathbf{d}(x)\right] \cdot{ }_{h \mathbf{d}(x)} \mid x \cdot\left[\mathbf{r}(h \mathbf{d}(x) \mid x), \mathbf{r}(x) h^{\prime}\right] \cdot } \\
& {\left[\mathbf{r}(x) h^{\prime}, \mathbf{r}(x)\left(h^{\prime} h_{2}\right)\right] \cdot\left[\mathbf{r}(x)\left(h^{\prime} h_{2}\right), k\right] }
\end{aligned}
$$




$$
\begin{aligned}
& =\left[g,\left(h_{1} h\right) \mathbf{d}(x)\right] \cdot{ }_{\left(h_{1} h\right) \mathbf{d}(x)} \mid x \cdot\left[\mathbf{r}\left({ }_{h \mathbf{d}(x)} \mid x\right), \mathbf{r}(x) h^{\prime}\right] \cdot\left[\mathbf{r}(x) h^{\prime}, \mathbf{r}(x)\left(h^{\prime} h_{2}\right)\right] \cdot \\
& \left(\left(h_{1} h\right) \mathbf{d}(x) \mathcal{L} h \mathbf{d}(x), \text { by }(\mathrm{P} 4)\right) \\
& =\left[g,\left(h_{1} h\right) \mathbf{d}(x)\right] \cdot{ }_{\left(h_{1} h\right) \mathbf{d}(x)} \mid x \cdot\left[\mathbf{r}\left({ }_{\left(h_{1} h\right) \mathbf{d}(x)} \mid x\right), \mathbf{r}(x) h^{\prime}\right] \cdot\left[\mathbf{r}(x) h^{\prime}, \mathbf{r}(x)\left(h^{\prime} h_{2}\right)\right] \cdot \\
& {\left[\mathbf{r}(x)\left(h^{\prime} h_{2}\right), k\right]} \\
& =\left[g,\left(h_{1} h\right) \mathbf{d}(x)\right] \cdot{ }_{\left(h_{1} h\right) \mathbf{d}(x)} \mid x \cdot\left[\mathbf{r}\left({ }_{\left(h_{1} h\right) \mathbf{d}(x)} \mid x\right), v h^{\prime}\right] \cdot\left[v h^{\prime}, \mathbf{r}(x) h^{\prime}\right] \cdot \\
& {\left[\mathbf{r}(x) h^{\prime}, \mathbf{r}(x)\left(h^{\prime} h_{2}\right)\right] \cdot\left[\mathbf{r}(x)\left(h^{\prime} h_{2}\right), k\right]} \\
& \left(v h^{\prime} \mathcal{L} \mathbf{r}\left({ }_{\left(h_{1} h\right) \mathbf{d}(x)} \mid x\right) \mathcal{L} \mathbf{r}(x) h^{\prime}\right) \\
& =\left.\left[g,\left(h_{1} h\right) \mathbf{d}(x)\right] \cdot\left[\left(h_{1} h\right) \mathbf{d}(x), \mathbf{d}\left(\left.x\right|_{v h^{\prime}}\right)\right] \cdot x\right|_{v h^{\prime}} \cdot\left[v h^{\prime}, \mathbf{r}(x) h^{\prime}\right] \cdot \\
& {\left[\mathbf{r}(x) h^{\prime}, \mathbf{r}(x)\left(h^{\prime} h_{2}\right)\right] \cdot\left[\mathbf{r}(x)\left(h^{\prime} h_{2}\right), k\right]} \\
& \left({ }_{\left(h_{1} h\right) \mathbf{d}(x)}|x \rho x|_{v h^{\prime}}\right) \\
& =\left.\left[g, \mathbf{d}\left(\left.x\right|_{v h^{\prime}}\right)\right] \cdot x\right|_{v h^{\prime}} \cdot\left[v h^{\prime}, \mathbf{r}(x) h^{\prime}\right] \cdot\left[\mathbf{r}(x) h^{\prime}, \mathbf{r}(x)\left(h^{\prime} h_{2}\right)\right] \cdot\left[\mathbf{r}(x)\left(h^{\prime} h_{2}\right), k\right] \\
& \left(g \mathcal{R}\left(h_{1} h\right) \mathbf{d}(x) \mathcal{R} \mathbf{d}\left(\left.x\right|_{v h^{\prime}}\right) \text {, by }(\mathrm{P} 1)\right) \\
& =\left.\left[g, \mathbf{d}\left(\left.x\right|_{v h^{\prime}}\right)\right] \cdot x\right|_{v h^{\prime}} \cdot\left[v h^{\prime}, h^{\prime}\right] \cdot\left[h^{\prime}, \mathbf{r}(x) h^{\prime}\right] \cdot\left[\mathbf{r}(x) h^{\prime}, \mathbf{r}(x)\left(h^{\prime} h_{2}\right)\right] \cdot\left[\mathbf{r}(x)\left(h^{\prime} h_{2}\right), k\right] \\
& \left(v h^{\prime} \mathcal{L} h^{\prime} \mathcal{L} \mathbf{r}(x) h^{\prime} \text {, by }(\mathrm{P} 1)\right) \text {. }
\end{aligned}
$$

Since $h^{\prime} \in S\left(h_{1}^{\prime}, h_{2}\right)$, we have $h^{\prime} \omega^{r} h_{2}$ and $h^{\prime} \omega^{l} h_{1}^{\prime}$, and so $h^{\prime} \mathcal{R} h^{\prime} h_{2} \omega h_{2}$. As $h_{1}^{\prime}, h_{2} \omega^{l} \mathbf{r}(x)$, we have $h^{\prime}, h^{\prime} h_{2} \omega^{l} \mathbf{r}(x)$, it follows that $\left(\begin{array}{cc}h^{\prime} & h^{\prime} h_{2} \\ \mathbf{r}(x) h^{\prime} & \mathbf{r}(x)\left(h^{\prime} h_{2}\right)\end{array}\right)$ is a row-singular matrix, and so by (P6),

$$
\left[h^{\prime}, \mathbf{r}(x) h^{\prime}\right] \cdot\left[\mathbf{r}(x) h^{\prime}, \mathbf{r}(x)\left(h^{\prime} h_{2}\right)\right]=\left[h^{\prime}, h^{\prime} h_{2}\right] \cdot\left[h^{\prime} h_{2}, \mathbf{r}(x)\left(h^{\prime} h_{2}\right)\right]
$$

Thus,

$$
\begin{aligned}
& {\left[g, h_{1} h\right] \cdot\left[h_{1} h, h\right] \cdot\left(h *\left(x \diamond h_{2}\right)\right) } \\
= & {\left.\left[g, \mathbf{d}\left(\left.x\right|_{v h^{\prime}}\right)\right] \cdot x\right|_{v h^{\prime}} \cdot\left[v h^{\prime}, h^{\prime}\right] \cdot\left[h^{\prime}, h^{\prime} h_{2}\right] \cdot\left[h^{\prime} h_{2}, \mathbf{r}(x)\left(h^{\prime} h_{2}\right)\right] \cdot\left[\mathbf{r}(x)\left(h^{\prime} h_{2}\right), k\right] } \\
= & {\left.\left[g, \mathbf{d}\left(\left.x\right|_{v h^{\prime}}\right)\right] \cdot x\right|_{v h^{\prime}} \cdot\left[v h^{\prime}, h^{\prime}\right] \cdot\left[h^{\prime}, h^{\prime} h_{2}\right] \cdot\left[h^{\prime} h_{2}, k\right] } \\
& \left(h^{\prime} h_{2} \mathcal{L} \mathbf{r}(x)\left(h^{\prime} h_{2}\right) \mathcal{L} k, \text { by }(\mathrm{P} 1)\right) \\
= & {\left.\left[\left(\mathbf{d}\left(\left.x\right|_{v h^{\prime}}\right)\right)^{+}, \mathbf{d}\left(\left.x\right|_{v h^{\prime}}\right)\right] \cdot x\right|_{v h^{\prime}} \cdot\left[v h^{\prime}, h^{\prime}\right] \cdot\left[h^{\prime}, h^{\prime} h_{2}\right] \cdot\left[h^{\prime} h_{2}, k\right] } \\
& \left(g=\left(\mathbf{d}\left(\left.x\right|_{v h^{\prime}}\right)\right)^{+}\right) \\
= & \left(\left(h_{1} * x\right) \diamond h^{\prime}\right) \cdot\left[h^{\prime}, h^{\prime} h_{2}\right] \cdot\left[h^{\prime} h_{2}, k\right] .
\end{aligned}
$$


Together with,

$$
\mathbf{d}\left(\left(\left(h_{1} * x\right) \diamond h^{\prime}\right) \cdot\left[h^{\prime}, h^{\prime} h_{2}\right]\right)=g \mathcal{R} h_{1} h=\mathbf{d}\left(\left[h_{1} h, h\right] \cdot\left(h *\left(x \diamond h_{2}\right)\right)\right)
$$

and

$$
\mathbf{r}\left(\left(\left(h_{1} * x\right) \diamond h^{\prime}\right) \cdot\left[h^{\prime}, h^{\prime} h_{2}\right]\right)=h^{\prime} h_{2} \mathcal{L} k=\mathbf{r}\left(\left[h_{1} h, h\right] \cdot\left(h *\left(x \diamond h_{2}\right)\right)\right),
$$

we have that $\left(\left(h_{1} * x\right) \diamond h^{\prime}\right) \cdot\left[h^{\prime}, h^{\prime} h_{2}\right] \rho\left[h_{1} h, h\right] \cdot\left(h *\left(x \diamond h_{2}\right)\right)$.

Now, we turn our attention to concordant semigroups.

Corollary 11.6. The category of concordant semigroups and good morphisms is equivalent to the category of $I C$ - RBS categories and RBS functors.

Proof. Suppose that $S$ is a concordant semigroup with set of idempotents $E(S)$. In view of Lemma 10.32, it is sufficient to show that $S \mathbf{C}$ satisfies (PC1) and (PC2).

(PC1) If $(e, x, f) \in S \mathbf{C}$ and $h \leq e$, then $e \mathcal{R}^{*} x \mathcal{L}^{*} f$. Since $S$ satisfies (IC), it follows from the comments succeeding Lemma 2.19 that there exists a unique $k \in E(S)$ such that $k \leq f$ and $h x=x k$. In addition,

$$
{ }_{h} \mid(e, x, f)=\left(h, h x,(h x)^{\star}\right) \text { and }\left.(e, x, f)\right|_{k}=\left((x k)^{+}, x k, k\right) .
$$

As $h x=x k$, we obtain that $h \mathcal{R}(x k)^{+}$and $(h x)^{\star} \mathcal{L} k$. Further, we have

$$
\begin{aligned}
{ }_{h} \mid(e, x, f) \cdot\left[(h x)^{\star}, k\right] & =\left(h, h x,(h x)^{\star}\right) \cdot\left((h x)^{\star},(h x)^{\star}, k\right) \\
& =\left(h, h x(h x)^{\star}, k\right)=(h, h x, k)
\end{aligned}
$$

and

$$
\begin{aligned}
{\left.\left[h,(x k)^{+}\right] \cdot(e, x, f)\right|_{k} } & =\left(h,(x k)^{+},(x k)^{+}\right) \cdot\left((x k)^{+}, x k, k\right) \\
& =(h, x k, k) \\
& =(h, h x, k) \quad(x k=h x),
\end{aligned}
$$

so that ${ }_{h}|(e, x, f) \rho(e, x, f)|_{k}$.

In particular, if $h=e$, then $e_{e}(e, x, f)=\left(e, x, x^{\star}\right)$. Certainly, $x^{\star} \mathcal{L} f$ and

$$
\left(e, x, x^{\star}\right) \cdot\left(x^{\star}, x^{\star}, f\right)=\left(e, x x^{\star}, f\right)=(e, x, f),
$$


that is, ${ }_{e} \mid(e, x, f) \cdot\left[x^{\star}, f\right]=(e, x, f)$, so ${ }_{e} \mid(e, x, f) \rho(e, x, f)$.

(PC2) Let $(e, x, f) \in S \mathbf{C}$ and for $i=1,2, e_{i}, f_{i} \in U$ be such that $e_{i} \leq e$, $f_{i} \leq f$ and $e_{i}|(e, x, f) \rho(e, x, f)|_{f_{i}}$. Then

$$
e_{i} \mid(e, x, f)=\left(e_{i}, e_{i} x,\left(e_{i} x\right)^{\star}\right) \text { and }\left.(e, x, f)\right|_{f_{i}}=\left(\left(x f_{i}\right)^{+}, x f_{i}, f_{i}\right) .
$$

As $_{e_{i}}|(e, x, f) \rho(e, x, f)|_{f_{i}}$, we have that $e_{i} \mathcal{R}\left(x f_{i}\right)^{+},\left(e_{i} x\right)^{\star} \mathcal{L} f_{i}$ and

$$
{ }_{e_{i}}\left|(e, x, f) \cdot\left[\left(e_{i} x\right)^{\star}, f\right]=\left[e_{i},\left(x f_{i}\right)^{+}\right] \cdot(e, x, f)\right|_{f_{i}},
$$

that is,

$$
\left(e_{i}, e_{i} x,\left(e_{i} x\right)^{\star}\right) \cdot\left(\left(e_{i} x\right)^{\star},\left(e_{i} x\right)^{\star}, f_{i}\right)=\left(e_{i},\left(x f_{i}\right)^{+},\left(x f_{i}\right)^{+}\right) \cdot\left(\left(x f_{i}\right)^{+}, x f_{i}, f_{i}\right),
$$

that is,

$$
\left(e_{i}, e_{i} x, f_{i}\right)=\left(e_{i}, x f_{i}, f_{i}\right)
$$

and so $e_{i} x=x f_{i}$.

If $e_{1} \leq_{\mathcal{R}} e_{2}$, then

$$
e_{1} x=e_{2} e_{1} x=e_{2}\left(e_{1} x\right)=e_{2} x f_{1}=x f_{2} f_{1} .
$$

As $e_{1} x=x f_{1}$, we get that $x f_{2} f_{1}=x f_{1}$. Since $x \mathcal{L}^{*} f$, we have that $f f_{2} f_{1}=f f_{1}$, and so $f_{2} f_{1}=f_{1}$ as $f_{1}, f_{2} \leq f$. Thus, $f_{1} \leq_{\mathcal{R}} f_{2}$. Note that $e_{1} \leq_{\mathcal{R}} e_{2}$ and $f_{1} \leq_{\mathcal{R}} f_{2}$, we have that $e_{1} e_{2} \leq e_{2} \leq e$ and $f_{1} f_{2} \leq f_{2} \leq f$, so that $e_{1} e_{2} \mid(e, x, f)$ and $\left.(e, x, f)\right|_{f_{1} f_{2}}$ exist. Also, we have

$$
e_{e_{1} e_{2}} \mid(e, x, f)=\left(e_{1} e_{2}, e_{1} e_{2} x,\left(e_{1} e_{2} x\right)^{\star}\right) \text { and }\left.(e, x, f)\right|_{f_{1} f_{2}}=\left(\left(x f_{1} f_{2}\right)^{+}, x f_{1} f_{2}, f_{1} f_{2}\right) \text {. }
$$

As $e_{1} e_{2} x=e_{1} x f_{2}=x f_{1} f_{2}$, we obtain that $e_{1} e_{2} \mathcal{R}\left(x f_{1} f_{2}\right)^{+},\left(e_{1} e_{2} x\right)^{\star} \mathcal{L} f_{1} f_{2}$, and so $\left[e_{1} e_{2},\left(x f_{1} f_{2}\right)^{+}\right]$and $\left[f_{1} f_{2},\left(e_{1} e_{2} x\right)^{\star}\right]$ exist. Further, we have

$$
\begin{aligned}
{\left[\left(x f_{1} f_{2}\right)^{+}, e_{1} e_{2}\right] \cdot e_{1} e_{2} \mid(e, x, f) } & =\left(\left(x f_{1} f_{2}\right)^{+}, e_{1} e_{2}, e_{1} e_{2}\right) \cdot\left(e_{1} e_{2}, e_{1} e_{2} x,\left(e_{1} e_{2} x\right)^{\star}\right) \\
& =\left(\left(x f_{1} f_{2}\right)^{+}, e_{1} e_{2} x,\left(e_{1} e_{2} x\right)^{\star}\right)
\end{aligned}
$$


and

$$
\begin{aligned}
\left.(e, x, f)\right|_{f_{1} f_{2}} \cdot\left[f_{1} f_{2},\left(e_{1} e_{2} x\right)^{\star}\right] & =\left(\left(x f_{1} f_{2}\right)^{+}, x f_{1} f_{2}, f_{1} f_{2}\right) \cdot\left(f_{1} f_{2}, f_{1} f_{2},\left(e_{1} e_{2} x\right)^{\star}\right) \\
& =\left(\left(x f_{1} f_{2}\right)^{+}, x f_{1} f_{2},\left(e_{1} e_{2} x\right)^{\star}\right) \\
& =\left(\left(x f_{1} f_{2}\right)^{+}, e_{1} e_{2} x,\left(e_{1} e_{2} x\right)^{\star}\right) \quad\left(e_{1} e_{2} x=x f_{1} f_{2}\right) .
\end{aligned}
$$

So $e_{e_{1} e_{2}}|(e, x, f) \rho(e, x, f)|_{f_{1} f_{2}}$.

Dually, if $e_{1} \leq_{\mathcal{L}} e_{2}$, then $f_{1} \leq_{\mathcal{L}} f_{2}$ and $e_{e_{2} e_{1}}|(e, x, f) \rho(e, x, f)|_{f_{2} f_{1}}$.

Conversely, let $P$ be an IC-RBS category over $U$. In view of Lemma 11.5 and Lemma 11.2, it is sufficient to show that Condition (P8) holds and PS satisfies (IC).

(P8) Suppose that $x, y, z \in P, h \in S(\mathbf{r}(x), \mathbf{d}(y))$ and $h^{\prime} \in S(\mathbf{r}(x), \mathbf{d}(z))$ are such that $(x \otimes y)_{h} \rho(x \otimes z)_{h^{\prime}}$. Then $\mathbf{d}\left((x \otimes y)_{h}\right) \mathcal{R} \mathbf{d}\left((x \otimes z)_{h^{\prime}}\right)$, that is, $\mathbf{d}(x \diamond h) \mathcal{R} \mathbf{d}\left(x \diamond h^{\prime}\right)$, or equivalently, $\mathbf{d}\left(\left.x\right|_{\mathbf{r}(x) h}\right) \mathcal{R} \mathbf{d}\left(\left.x\right|_{\mathbf{r}(x) h^{\prime}}\right)$. As $\mathbf{r}(x) h, \mathbf{r}(x) h^{\prime} \leq$ $\mathbf{r}(x)$, by (PC1), there exist $e_{1}, e_{2} \leq \mathbf{d}(x)$ such that $e_{1}|x \rho x|_{\mathbf{r}(x) h}$ and $e_{2}|x \rho x|_{\mathbf{r}(x) h^{\prime}}$. Thus, $e_{1} \mathcal{R} \mathbf{d}\left(\left.x\right|_{\mathbf{r}(x) h}\right) \mathcal{R} \mathbf{d}\left(\left.x\right|_{\mathbf{r}(x) h^{\prime}}\right) \mathcal{R} e_{2}$. In addition, from $e_{1}|x \rho x|_{\mathbf{r}(x) h}$ and $e_{2}|x \rho x|_{\mathbf{r}(x) h^{\prime}}$, we obtain that $e_{1} \sigma_{x}=\mathbf{r}(x) h$ and $e_{2} \sigma_{x}=\mathbf{r}(x) h^{\prime}$ by Lemma 11.4. As $\sigma_{x}$ is an isomorphism and $e_{1} \mathcal{R} e_{2}$, we obtain that $\mathbf{r}(x) h \mathcal{R} \mathbf{r}(x) h^{\prime}$. Hence, Condition (P8) holds.

To show that $P \mathrm{~S}$ satisfies (IC), we assume that $\bar{x} \in P$ and $\overline{1_{e}} \in \bar{U}$ with $\overline{1_{e}} \leq \overline{1_{\mathbf{d}(x)}}$. Then by Lemma 10.24, $e \leq \mathbf{d}(x)$. By (PC1), there exists a unique $k \in U$ such that $k \leq \mathbf{r}(x)$ and ${ }_{e}|x \rho x|_{k}$, that is, $\bar{e}|x=\bar{x}|_{k}$. By Lemma 10.21 and its dual, we have that $\overline{1_{e}} \odot \bar{x}=\bar{x} \odot \overline{1_{k}}$. Dually, if $\overline{1_{f}} \in \bar{U}$ with $\overline{1_{f}} \leq \overline{1_{\mathbf{r}(x)}}$, then there exists $\overline{1_{g}} \in \bar{U}$ such that $\overline{1_{g}} \leq \overline{1_{\mathbf{d}(x)}}$ and $\overline{1_{g}} \odot \bar{x}=\bar{x} \odot \overline{1_{f}}$. Thus, PS has (IC).

Now, we aim to define new restrictions and co-restrictions on an IC-RBS category to recover the original result of Armstrong. We first show that an IC-RBS category forms an inductive ${ }_{2}$ cancellative category with respect to the restriction and co-restriction defined below.

Let $P$ be an IC-RBS category over $U$. If $k, h \in U, x \in P$ with $h \omega \mathbf{d}(x)$, $k \omega \mathbf{r}(x)$ and ${ }_{h}|x \rho x|_{k}$, then we define the restriction and co-restriction by the rule that

$$
{ }_{h}|| x={ }_{h}\left|x \cdot\left[\mathbf{r}\left({ }_{h} \mid x\right), k\right], \quad x \|_{k}=\left[h, \mathbf{d}\left(\left.x\right|_{k}\right)\right] \cdot x\right|_{k} .
$$


Notice that if $h \omega \mathbf{d}(x), k \omega \mathbf{r}(x)$ and ${ }_{h}|x \rho x|_{k}$, then by (PC1) and Lemma 11.5, we have $h \sigma_{x}=k$. In partucular, if $h=\mathbf{d}(x)$, then we must have that $k=\mathbf{r}(x)$, and so

$$
\mathbf{d}(x)|| x=\mathbf{d}(x)_{1}\left|x \cdot\left[\mathbf{r}\left(_{\mathbf{d}(x)} \mid x\right), k\right]=\mathbf{d}(x)_{1}\right| x \cdot[\mathbf{r}(\mathbf{d}(x) \mid x), \mathbf{r}(x)],
$$

that is, $\mathbf{d}(x) \| x=x$ by (P2). Dually, $x \|_{\mathbf{r}(x)}=x$.

We define a relation on $P$ by the rule that for any $x, y \in P$,

$$
x \leq y \text { if and only if } x=e_{e} \| y \quad \text { for some } e \in U .
$$

Clearly, if $x \leq y$ then there exists $e \in U$ such that $x={ }_{e} \| y$. Then $\mathbf{d}(x)=e$, and so $x=\mathbf{d}(x) \| y$. For $e \| y$ to exist, we have $e \omega \mathbf{d}(y)$, that is, $\mathbf{d}(x) \omega \mathbf{d}(y)$. By the definition of the restriction, we obtain that $\mathbf{r}\left({ }_{e} \| y\right) \omega \mathbf{r}(y)$, and so $\mathbf{r}(x) \omega \mathbf{r}(y)$.

Lemma 11.7. The relation $\leq$ is a partial order on $P$.

Proof. It is easy to see that $\leq$ is reflexive by the comments above. Suppose that $x \leq y$ and $y \leq x$. Then $\mathbf{d}(x)=\mathbf{d}(y)$, and so $x={ }_{\mathbf{d}(x)}\|y=\mathbf{d}(y)\| y=y$. To show that $\leq$ is transitive, suppose that $x \leq y$ and $y \leq z$. Then $\mathbf{d}(x) \omega \mathbf{d}(y)$ and $\mathbf{d}(y) \omega \mathbf{d}(z)$. Thus, $\mathbf{d}(x) \omega \mathbf{d}(z)$. Also, we have $y={ }_{\mathbf{d}(y)}|| z={ }_{\mathbf{d}(y)} \mid z \cdot[\mathbf{r}(\mathbf{d}(y) \mid z), \mathbf{r}(y)]$. Then

$$
\begin{aligned}
\mathbf{d}(x) \mid y & =\mathbf{d}_{\mathbf{d}(x)} \mid(\mathbf{d}(y) \mid z \cdot[\mathbf{r}(\mathbf{d}(y) \mid z), \mathbf{r}(y)]) \\
& ={ }_{\mathbf{d}(x)}\left|\left(_{\mathbf{d}(y)} \mid z\right) \cdot{ }_{g}\right|[\mathbf{r}(\mathbf{d}(y) \mid z), \mathbf{r}(y)] \quad\left(g=\mathbf{r}\left({ }_{\mathbf{d}(x)} \mid\left(_{\mathbf{d}(y)} \mid z\right)\right), \text { by }(\mathrm{P} 5)\right) \\
& ={ }_{\mathbf{d}(x)} \mid(\mathbf{d}(y) \mid z) \cdot\left[g, g^{\star}\right] \quad\left(g=\mathbf{r}(\mathbf{d}(x) \mid(\mathbf{d}(y) \mid z)) \omega^{l} \mathbf{r}(\mathbf{d}(y) \mid z) \mathcal{L} \mathbf{r}(y), \text { by }(\mathrm{P} 3)\right) \\
& ={ }_{\mathbf{d}(x)} \mid z \cdot\left[g, g^{\star}\right] \quad(\mathbf{d}(x) \omega \mathbf{d}(y), \text { by }(\mathrm{P} 4)) \\
& ={ }_{\mathbf{d}(x)} \mid z \cdot\left[\mathbf{r}(\mathbf{d}(x) \mid z),\left(\mathbf{r}\left(\mathbf{d}_{(x)} \mid z\right)\right)^{\star}\right] .
\end{aligned}
$$

From $x \leq y$, we have that

$$
\begin{array}{rll}
x & ={ }_{\mathbf{d}(x)}|| y={ }_{\mathbf{d}(x)} \mid y \cdot[\mathbf{r}(\mathbf{d}(x) \mid y), \mathbf{r}(x)] \\
& ={ }_{\mathbf{d}(x)} \mid z \cdot\left[\mathbf{r}\left({ }_{\mathbf{d}(x)} \mid z\right),(\mathbf{r}(\mathbf{d}(x) \mid z))^{\star}\right] \cdot\left[\left(\mathbf{r}(\mathbf{d}(x) \mid z)^{\star}, \mathbf{r}(x)\right] \quad\left(\mathbf{r}(\mathbf{d}(x) \mid y)=(\mathbf{r}(\mathbf{d}(x) \mid z))^{\star}\right)\right. \\
& ={ }_{\mathbf{d}(x)} \mid z \cdot[\mathbf{r}(\mathbf{d}(x) \mid z), \mathbf{r}(x)] & \left(\mathbf{r}(\mathbf{d}(x) \mid z) \mathcal{L}\left(\mathbf{r}\left(\mathbf{d}_{(x)} \mid z\right)\right)^{\star} \mathcal{L} \mathbf{r}(x), \text { by }(\mathrm{P} 1)\right) \\
& =\mathbf{d}_{\mathbf{d}(x)}|| z .
\end{array}
$$

Lemma 11.8. An IC-RBS category over a regular biordered set $U$ with the order defined above forms an ordered 2 category. 
Proof. Let $P$ be an IC-RBS category over $U$.

(OC1) If $x \leq y$, then by the comments before Lemma 11.7, $\mathbf{d}(x) \boldsymbol{\omega} \mathbf{d}(y)$ and $\mathbf{r}(x) \omega \mathbf{r}(y)$.

(OC3) Suppose that $x^{\prime} \leq x, y^{\prime} \leq y$ and $x^{\prime} \cdot y^{\prime}$ and $x \cdot y$ are defined. Then $x^{\prime}={\mathbf{d}\left(x^{\prime}\right)} \| x$ and $y^{\prime}={ }_{\mathbf{d}\left(y^{\prime}\right)} \| y$. Also,

$$
\begin{aligned}
& x^{\prime} \cdot y^{\prime}=\mathbf{d}\left(x^{\prime}\right)|| x \cdot \mathbf{d}\left(y^{\prime}\right) \| y \\
& ={ }_{\mathbf{d}\left(x^{\prime}\right)}\left|x \cdot\left[\mathbf{r}\left(\mathbf{d}\left(x^{\prime}\right) \mid x\right), \mathbf{r}\left(x^{\prime}\right)\right] \cdot \mathbf{d}\left(y^{\prime}\right)\right| y \cdot\left[\mathbf{r}\left(\mathbf{d}\left(y^{\prime}\right) \mid y\right), \mathbf{r}\left(y^{\prime}\right)\right] \\
& =\mathbf{d}\left(x^{\prime}\right)\left|x \cdot\left[\mathbf{r}\left(\mathbf{d}\left(x^{\prime}\right) \mid x\right), \mathbf{r}\left(x^{\prime}\right)\right] \cdot \mathbf{r}\left(x^{\prime}\right)\right| y \cdot\left[\mathbf{r}\left(\mathbf{r}\left(x^{\prime}\right) \mid y\right), \mathbf{r}\left(y^{\prime}\right)\right] \\
& \text { (since } x^{\prime} \cdot y^{\prime} \text { is defined, } \mathbf{r}\left(x^{\prime}\right)=\mathbf{d}\left(y^{\prime}\right) \text { ) }
\end{aligned}
$$

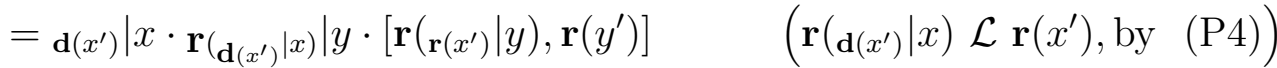

$$
\begin{aligned}
& \left.=\mathbf{d}\left(x^{\prime}\right) \mid(x \cdot y) \cdot\left[\mathbf{r}\left(\mathbf{d}\left(x^{\prime}\right) \mid(x \cdot y)\right), \mathbf{r}\left(y^{\prime}\right)\right] \quad \text { (by }(\mathrm{P} 5)\right) \\
& =\mathbf{d}\left(x^{\prime}\right) \|(x \cdot y) \text {. }
\end{aligned}
$$

Thus, $x^{\prime} \cdot y^{\prime} \leq x \cdot y$.

(OC4) For part $(i)$, if $x \in P$ and $e \in U$ are such that $e \omega \mathbf{d}(x)$, then by the definition of the order, $e_{e} \| x$ is the unique element satisfying that ${ }_{e} \| x \leq x$ and $\mathbf{d}\left({ }_{e} \| x\right)=e$. Dually, part $(i i)$ holds.

Hence, $P$ is an ordered $_{2}$ category.

Lemma 11.9. An $I C$ - $R B S$ category $P$ over $U$ with respect to the order, restrictions and co-restrictions forms an inductive ${ }_{2}$ cancellative category.

Proof. In view of Lemma 11.8, it is sufficient to show that $P$ satisfies Conditions (IC1)-(IC6) mentioned in Chapter 6.

(IC1) Clearly.

(IC2) Suppose that $e, f \in U$ are such that $e \omega f$. Then ${ }_{e}\left|1_{f}={ }_{e}\right|[f, f]=\left[e, e^{\star}\right]$ by $(\mathrm{P} 3)$. Also, by $(\mathrm{P} 3)^{\circ}$, we have $\left.1_{f}\right|_{e}=\left[e^{+}, e\right]$. By Lemma 10.2, we obtain that

$$
{ }_{e}\left|1_{f}=\left[e, e^{\star}\right] \rho 1_{e} \rho\left[e^{+}, e\right]=1_{f}\right|_{e},
$$

and so by the definition of the restriction, we have that

$$
e^{\|}\left|1_{f}={ }_{e}\right| 1_{f} \cdot\left[e^{\star}, e\right],
$$


that is,

$$
{ }_{e} \| 1_{f}=\left[e, e^{\star}\right] \cdot\left[e^{\star}, e\right]=[e, e]=1_{e},
$$

and so $1_{e} \leq 1_{f}$.

Conversely, if $1_{e} \leq 1_{f}$, by the comments before Lemma 11.7, we obtain that $e \omega f$.

(IC3) It follows from (P1).

(IC4) Suppose that $g, h, e \in U$ are such that $e \omega g \mathcal{L} h$. Then $[g, h]$ exists and $e \mathcal{L} h e=h e h \omega h$, and so $[e, h e]$ exists. By (P3), we have ${ }_{e} \mid[g, h]=\left[e, e^{\star}\right]$. Also, we have

$$
\begin{array}{rlr}
{\left.[g, h]\right|_{h e}} & =\left[(g(h e))^{+}, g(h e)\right] \cdot[g(h e), h e] \quad\left(\text { by }(\mathrm{P} 3)^{\circ}\right) \\
& =\left[e^{+}, e\right] \cdot[e, h e] \quad\left(e \omega g \mathcal{L} h, \text { by }(\mathrm{B} 31)^{\circ}, g(h e)=g e=e\right) .
\end{array}
$$

Clearly, $e \mathcal{R} e^{+}, e^{\star} \mathcal{L} e$ and

$$
\left[e, e^{\star}\right] \cdot\left[e^{\star}, h e\right]=[e, h e]=\left[e, e^{+}\right] \cdot\left[e^{+}, e\right] \cdot[e, e h],
$$

that is, $e|[g, h] \rho[g, h]|_{h e}$, and so

$$
e^{\|}\left|[g, h]=e_{e}\right|[g, h] \cdot\left[e^{\star}, h e\right]=\left[e, e^{\star}\right] \cdot\left[e^{\star}, h e\right]=[e, h e],
$$

so that $[e, h e] \leq[g, h]$.

Dually, if $g \mathcal{R} h$, then $[e, e h]$ exists and $[e, e h] \leq[g, h]$.

(IC5) It follows from Lemma 11.3.

(IC6) It follows from (P6).

Thus, $P$ is an inductive ${ }_{2}$ cancellative category.

Conversely, let $Q$ be an inductive 2 cancellative category with regular biordered set $U$. For each $\mathcal{L}$-class and $\mathcal{R}$-class, we pick out a special element as its representative. If $h, k \in U$ with $h \omega^{l} \mathbf{d}(x)$ and $k \omega^{r} \mathbf{r}(x)$, then we define

$$
{ }_{h}|x=[h, \mathbf{d}(x) h] \cdot \mathbf{d}(x) h||| x \cdot\left[\mathbf{r}(\mathbf{d}(x) h|| \mid x),(\mathbf{r}(\mathbf{d}(x) h|| \mid x))^{\star}\right]
$$

and

$$
\left.x\right|_{k}=\left[\left(\mathbf{d}\left(x \mid \|_{k \mathbf{r}(x)}\right)\right)^{+}, \mathbf{d}\left(x \mid \|_{k \mathbf{r}(x)}\right)\right] \cdot x \mid \|_{k \mathbf{r}(x)} \cdot[k \mathbf{r}(x), k],
$$


where if $e \omega \mathbf{d}(x)$ and $f \omega \mathbf{r}(x)$, then we use $e l \| x$ and $x\|\|_{f}$ to mean the restriction of $x$ to $e$ and the co-restriction of $x$ to $f$ in the sense of inductive cancellative $^{2}$ categories. So if $e=\mathbf{d}(x)$ and $f=\mathbf{r}(x)$, then $e \| x=x$ and $x\|\|_{f}=x$.

In particular, if $h, k \in U$ with $h \omega \mathbf{d}(x)$ and $k \omega \mathbf{r}(x)$, then we have that

$$
{ }_{h}\left|x={ }_{h}\right||| x \cdot\left[\mathbf{r}\left({ }_{h}|| \mid x\right),\left(\mathbf{r}\left({ }_{h}|| \mid x\right)\right)^{\star}\right]
$$

and

$$
\left.x\right|_{k}=\left[\left(\mathbf{d}\left(x \mid \|_{k}\right)\right)^{+}, \mathbf{d}\left(x \mid \|_{k}\right)\right] \cdot x \mid \|_{k} .
$$

In addition, if $h=\mathbf{d}(x)$, then

$$
{ }_{h}|x=\mathbf{d}(x)||| x \cdot\left[\mathbf{r}(\mathbf{d}(x)|| \mid x),(\mathbf{r}(\mathbf{d}(x)|| \mid x))^{\star}\right]=x \cdot\left[\mathbf{r}(x),(\mathbf{r}(x))^{\star}\right],
$$

and so $\mathbf{r}\left({ }_{h} \mid x\right)=(\mathbf{r}(x))^{\star}$ and

$$
{ }_{h} \mid x \cdot\left[(\mathbf{r}(x))^{\star}, \mathbf{r}(x)\right]=x \cdot\left[\mathbf{r}(x),(\mathbf{r}(x))^{\star}\right] \cdot\left[(\mathbf{r}(x))^{\star}, \mathbf{r}(x)\right],
$$

that is,

$$
{ }_{h} \mid x \cdot\left[(\mathbf{r}(x))^{\star}, \mathbf{r}(x)\right]=x \cdot[\mathbf{r}(x), \mathbf{r}(x)],
$$

by (IC3), or equivalently,

$$
{ }_{h} \mid x \cdot\left[(\mathbf{r}(x))^{\star}, \mathbf{r}(x)\right]=x
$$

Dually, if $k=\mathbf{r}(x)$, then $\left.\left[\mathbf{d}(x), \mathbf{d}\left(\left.x\right|_{k}\right)\right] \cdot x\right|_{k}=x$.

So, we have:

Lemma 11.10. An inductive $_{2}$ cancellative category $Q$ over $U$ forms an $I C-R B S$ category with the restriction and co-restriction defined above.

Proof. Clearly, Condition (P1) holds by (IC3).

(P2) It follows from the statement before this lemma.

(P3) If $g \omega e$ and $e \mathcal{R} f$ or $e \mathcal{L} f$, then by (IC4),

$$
{ }_{g}\left|[e, f]={ }_{g}\right|||[e, f] \cdot\left[\mathbf{r}(g|| \mid[e, f]),(\mathbf{r}(g|| \mid[e, f]))^{\star}\right]=[g, f g f] \cdot\left[f g f,(f g f)^{\star}\right] .
$$


If $g \omega^{l} e$ and $e \mathcal{L} f$, then $g \omega^{l} f$, which implies that $f g f=f g \mathcal{L} g$, and so

$$
\begin{array}{rlr}
{ }_{g} \mid[e, f] & =[g, e g] \cdot{ }_{e g} \mid \|[e, f] \cdot\left[\mathbf{r}(e g \mid \|[e, f]),(\mathbf{r}(e g \mid \|[e, f]))^{\star}\right] \\
& =[g, e g] \cdot[e g, f(e g) f] \cdot\left[f(e g) f,(f(e g) f)^{\star}\right] & (\text { by }(\mathrm{IC} 4)) \\
& =[g, e g] \cdot[e g, f g] \cdot\left[f g,(f g)^{\star}\right] \\
& =\left[g,(f g)^{\star}\right] \quad\left(g \mathcal{L} e g \mathcal{L} f g \mathcal{L}(f g)^{\star}\right) \\
& =\left[g, g^{\star}\right] & (g \mathcal{L} f g) .
\end{array}
$$

(P4) If $x \in Q$ and $e, f \in U$ with $e \omega^{l} f \omega^{l} \mathbf{d}(x)$, then

$$
{ }_{f} \mid x=[f, \mathbf{d}(x) f] \cdot(\mathbf{d}(x) f|| \mid x) \cdot\left[\mathbf{r}(\mathbf{d}(x) f|| \mid x),(\mathbf{r}(\mathbf{d}(x) f|| \mid x))^{\star}\right],
$$

and so

$$
\begin{aligned}
& e^{e}\left({ }_{f} \mid x\right)={ }_{e} \mid\left([f, \mathbf{d}(x) f] \cdot(\mathbf{d}(x) f|| \mid x) \cdot\left[\mathbf{r}(\mathbf{d}(x) f|| \mid x),\left(\mathbf{r}\left({ }_{\mathbf{d}(x) f}|| \mid x\right)\right)^{\star}\right]\right) \\
& =[e, f e] \cdot{ }_{f e} \|\left([f, \mathbf{d}(x) f] \cdot \mathbf{d}(x) f \mid \| x \cdot\left[\mathbf{r}(\mathbf{d}(x) f|| \mid x),(\mathbf{r}(\mathbf{d}(x) f|| \mid x))^{\star}\right]\right) \cdot\left[u, u^{\star}\right] \\
& \left(u=\mathbf{r}\left(f_{e} \| \mid\left([f, \mathbf{d}(x) f] \cdot \mathbf{d}(x) f \mid \| x \cdot\left[\mathbf{r}(\mathbf{d}(x) f|| \mid x),(\mathbf{r}(\mathbf{d}(x) f \mid \| x))^{\star}\right]\right)\right)\right) \\
& \left.=[e, f e] \cdot[f e, k] \cdot{ }_{k}||\left|(\mathbf{d}(x) f|| \mid x) \cdot{ }_{g}\right||| \mathbf{r}(\mathbf{d}(x) f|| \mid x),\left(\mathbf{r}\left({ }_{\mathbf{d}(x) f}|| \mid x\right)\right)^{\star}\right] \cdot\left[u, u^{\star}\right] \\
& \left(k=(\mathbf{d}(x) f)(f e)(\mathbf{d}(x) f), g=\mathbf{r}\left({ }_{k}|| \mid((\mathbf{d}(x) f|| \mid x))\right)\right. \\
& =[e, f e] \cdot[f e, k] \cdot{ }_{k}|| \mid(\mathbf{d}(x) f|| \mid x) \cdot\left[g,(\mathbf{r}(\mathbf{d}(x) f|| \mid x))^{\star} g\right] \cdot\left[u, u^{\star}\right] \\
& =[e, f e] \cdot[f e, k] \cdot{ }_{k}||(\mathbf{d}(x) f \mid \| x) \cdot\left[g, u^{\star}\right] \\
& \left(g \mathcal{L}(\mathbf{r}(\mathbf{d}(x) f|| \mid x))^{\star} g=u \mathcal{L} u^{\star}, \text { by }(\mathrm{IC} 3)\right) \\
& =[e, f e] \cdot[f e, k] \cdot{ }_{k}||\left({ }_{\mathbf{d}(x) f}|| x\right) \cdot\left[g, g^{\star}\right] \quad(g \mathcal{L} u) .
\end{aligned}
$$

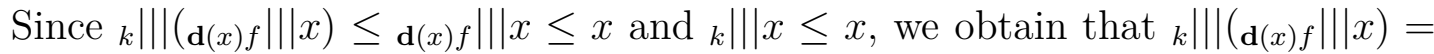
${ }_{k}|| \mid x$ by (OC4). Thus

$$
\begin{aligned}
e \mid(f \mid x) & =[e, f e] \cdot[f e, k] \cdot{ }_{k} \||| x \cdot\left[g, g^{\star}\right] \\
& =[e, f e] \cdot[f e, k] \cdot{ }_{k} \||| x \cdot\left[\mathbf{r}\left({ }_{k}|| \mid x\right),\left(\mathbf{r}\left({ }_{k}|| \mid x\right)\right)^{\star}\right] .
\end{aligned}
$$

Notice that $e \omega^{l} f \omega^{l} \mathbf{d}(x)$, by (B21) $)^{\circ}$, we have that

fe $\mathcal{L}$ e $\mathcal{L} \mathbf{d}(x) e \omega \mathbf{d}(x)$ and $f e \omega f \mathcal{L} \mathbf{d}(x) f \omega \mathbf{d}(x)$. 
Thus,

$$
k=(\mathbf{d}(x) f)(f e)(\mathbf{d}(x) f)=(\mathbf{d}(x) f)(f e) \mathcal{L} \text { fe } \mathcal{L} \text { e } \mathcal{L} \mathbf{d}(x) e
$$

and also, $k \omega \mathbf{d}(x)$. In addition, by the dual of (IC5), we have that

$$
\mathbf{r}(\mathbf{d}(x) e \mid \| x) \mathcal{L} \mathbf{r}\left({ }_{k}|| \mid x\right)
$$

and

$$
[\mathbf{d}(x) e, k(\mathbf{d}(x) e)] \cdot{ }_{k}(\mathbf{d}(x) e)\|\| x=\mathbf{d}(x) e \mid \| x \cdot\left[\mathbf{r}(\mathbf{d}(x) e\|\mid\| x), \mathbf{r}\left({ }_{k} \||| x\right) \mathbf{r}(\mathbf{d}(x) e\|\mid\| x)\right]
$$

that is,

$$
[\mathbf{d}(x) e, k] \cdot{ }_{k}\left|\|x=\mathbf{d}(x) e \mid\| x \cdot\left[\mathbf{r}(\mathbf{d}(x) e \mid \| x), \mathbf{r}\left({ }_{k} \mid \| x\right)\right],\right.
$$

and so, we have

$$
\begin{aligned}
& e^{\mid}\left({ }_{f} \mid x\right) \\
& =[e, k] \cdot\left({ }_{k}|| \mid x\right) \cdot\left[\mathbf{r}\left({ }_{k} \mid \| x\right),\left(\mathbf{r}\left({ }_{k} \mid \| x\right)\right)^{\star}\right] \quad(e \mathcal{L} \text { fe } \mathcal{L} k, \text { by }(\mathrm{IC} 3)) \\
& =[e, \mathbf{d}(x) e] \cdot[\mathbf{d}(x) e, k] \cdot{ }_{k}|| \mid x \cdot\left[\mathbf{r}\left({ }_{k}|| \mid x\right),\left(\mathbf{r}\left({ }_{k}|| \mid x\right)\right)^{\star}\right] \\
& (e \mathcal{L} \mathbf{d}(x) e \mathcal{L} k, \text { by }(\mathrm{IC} 3)) \\
& =[e, \mathbf{d}(x) e] \cdot \mathbf{d}(x) e\|\| x \cdot\left[\mathbf{r}(\mathbf{d}(x) e|| \mid x), \mathbf{r}\left({ }_{k}|| \mid x\right)\right] \cdot\left[\mathbf{r}\left({ }_{k}|| \mid x\right),\left(\mathbf{r}\left({ }_{k}|| \mid x\right)\right)^{\star}\right] \\
& =[e, \mathbf{d}(x) e] \cdot \mathbf{d}(x) e \mid \| x \cdot\left[\mathbf{r}(\mathbf{d}(x) e\|\| x),\left(\mathbf{r}\left({ }_{k} \mid \| x\right)\right)^{\star}\right] \quad(\text { by }(\mathrm{IC} 3)) \\
& =[e, \mathbf{d}(x) e] \cdot \mathbf{d}(x) e \mid \| x \cdot\left[\mathbf{r}(\mathbf{d}(x) e \mid \| x),\left(\mathbf{r}\left({ }_{\mathbf{d}(x) e} \||| x\right)\right)^{\star}\right] \quad\left(\mathbf{r}(\mathbf{d}(x) e \mid \| x) \mathcal{L} \mathbf{r}\left({ }_{k} \||| x\right)\right) \\
& ={ }_{e} \mid x \text {. }
\end{aligned}
$$

If $e \mathcal{L} f \omega^{l} \mathbf{d}(x)$, then $\mathbf{d}(x) e \mathcal{L}$ e $\mathcal{L} f \mathcal{L} \mathbf{d}(x) f$ and $\mathbf{d}(x) e, \mathbf{d}(x) f \omega \mathbf{d}(x)$. By the dual of (IC5), we have that

$[\mathbf{d}(x) e,(\mathbf{d}(x) f)(\mathbf{d}(x) e)] \cdot{ }_{(\mathbf{d}(x) f)(\mathbf{d}(x) e)}||\left|x=\mathbf{d}_{\mathbf{d}(x) e}\right| \| x \cdot[\mathbf{r}(\mathbf{d}(x) e \mid \| x), \mathbf{r}((\mathbf{d}(x) f)(\mathbf{d}(x) e)|| \mid x)]$,

that is,

$$
[\mathbf{d}(x) e, \mathbf{d}(x) f] \cdot \mathbf{d}(x) f\|\| x=\mathbf{d}(x) e\|\| x \cdot[\mathbf{r}(\mathbf{d}(x) e \mid \| x), \mathbf{r}(\mathbf{d}(x) f \mid \| x)]
$$


as $\mathbf{d}(x) e \mathcal{L} \mathbf{d}(x) f$. Then, we have

$$
\begin{aligned}
& {[e, f] \cdot\left({ }_{f} \mid x\right)} \\
& =[e, f] \cdot[f, \mathbf{d}(x) f] \cdot(\mathbf{d}(x) f \mid \| x) \cdot\left[\mathbf{r}\left(\mathbf{d}_{\mathbf{d}(x) f} \mid \| x\right),\left(\mathbf{r}\left(\mathbf{d}_{\mathbf{d}(x) f} \mid \| x\right)\right)^{\star}\right] \\
& =[e, \mathbf{d}(x) f] \cdot(\mathbf{d}(x) f|| \mid x) \cdot\left[\mathbf{r}(\mathbf{d}(x) f|| \mid x),(\mathbf{r}(\mathbf{d}(x) f|| \mid x))^{\star}\right] \\
& (\text { e } \mathcal{L} f \mathcal{L} \mathbf{d}(x) f \text {, by }(\mathrm{IC} 3)) \\
& =[e, \mathbf{d}(x) e] \cdot[\mathbf{d}(x) e, \mathbf{d}(x) f] \cdot(\mathbf{d}(x) f|| \mid x) \cdot\left[\mathbf{r}(\mathbf{d}(x) f|| \mid x),(\mathbf{r}(\mathbf{d}(x) f|| \mid x))^{\star}\right] \\
& =[e, \mathbf{d}(x) e] \cdot(\mathbf{d}(x) e \mid \| x) \cdot[\mathbf{r}(\mathbf{d}(x) e|| \mid x), \mathbf{r}(\mathbf{d}(x) f|| \mid x)] \cdot\left[\mathbf{r}(\mathbf{d}(x) f|| \mid x),(\mathbf{r}(\mathbf{d}(x) f|| \mid x))^{\star}\right] \\
& \left.=[e, \mathbf{d}(x) e] \cdot \mathbf{d}(x) e \mid \| x \cdot[\mathbf{r}(\mathbf{d}(x) e) \mid \| x),(\mathbf{r}(\mathbf{d}(x) e \mid \| x))^{\star}\right] \\
& ={ }_{e} \mid x \text {. }
\end{aligned}
$$

(P5) If $h \omega^{l} \mathbf{d}(x)$ and $x \cdot y$ is defined. Then

$$
\begin{aligned}
& { }_{h} \mid(x \cdot y) \\
& =[h, \mathbf{d}(x) h] \cdot \mathbf{d}(x) h|| \mid(x \cdot y) \cdot\left[v, v^{\star}\right] \quad(v=\mathbf{r}(h|| \mid(x \cdot y))) \\
& =[h, \mathbf{d}(x) h] \cdot \mathbf{d}(x) h\left|\left\|x \cdot{ }_{g}\right\|\right| y \cdot\left[v, v^{\star}\right] \quad(g=\mathbf{r}(\mathbf{d}(x) h \mid \| x)) \\
& =[h, \mathbf{d}(x) h] \cdot \mathbf{d}(x) h||\left|x \cdot[g, g] \cdot \mathbf{r}\left(\mathbf{d}_{(x) h}|| \mid x\right)\right||| y \cdot\left[v, v^{\star}\right] \\
& =[h, \mathbf{d}(x) h] \cdot \mathbf{d}(x) h\|\mid\| x \cdot\left[g, g^{\star}\right] \cdot\left[g^{\star}, g\right] \cdot g \| y \cdot\left[v, v^{\star}\right] \\
& ={ }_{h}\left|x \cdot\left[g^{\star}, g\right] \cdot{ }_{g}\right||| y \cdot\left[v, v^{\star}\right] \\
& ={ }_{h}\left|x \cdot\left[g^{\star}, \mathbf{d}(y) g^{\star}\right] \cdot\left[\mathbf{d}(y) g^{\star}, g\right] \cdot{ }_{g}\right||| y \cdot\left[v, v^{\star}\right] \\
& \left(g^{\star} \mathcal{L} g \omega \mathbf{d}(y) \text {, and so } g^{\star} \mathcal{L} \mathbf{d}(y) g^{\star} \omega \mathbf{d}(y)\right) .
\end{aligned}
$$

As $\mathbf{d}(y) g^{\star} \mathcal{L} g^{\star} \mathcal{L} g$ and $\mathbf{d}(y) g^{\star}, g \omega \mathbf{d}(y)$, by the dual of (IC5), we have that

$$
\mathbf{r}\left(\mathbf{d}(y) g^{*}|| \mid y\right) \mathcal{L} \mathbf{r}\left(g_{g} \| y\right)
$$

and

$$
\left[\mathbf{d}(y) g^{\star}, g\left(\mathbf{d}(y) g^{\star}\right)\right] \cdot{ }_{g\left(\mathbf{d}(y) g^{\star}\right)}||\left|y={ }_{\mathbf{d}(y) g^{\star}}\right| \| y \cdot\left[\mathbf{r}\left(\mathbf{d}_{(y) g^{\star}}|| y\right), \mathbf{r}(g \mid \| y) \mathbf{r}\left(\mathbf{d}(y) g^{\star}|| \mid y\right)\right],
$$

that is,

$$
\left[\mathbf{d}(y) g^{\star}, g\right] \cdot{ }_{g}\left\|y={ }_{\mathbf{d}(y) g^{\star}} \mid\right\| y \cdot\left[\mathbf{r}\left(\mathbf{d}(y) g^{\star}|| y\right), \mathbf{r}(g|| \mid y)\right],
$$


and so

$$
\begin{aligned}
&{ }_{h}\left|x \cdot\left[g^{\star}, \mathbf{d}(y) g^{\star}\right] \cdot\left[\mathbf{d}(y) g^{\star}, g\right] \cdot{ }_{g}\right||| y \cdot\left[v, v^{\star}\right] \\
&={ }_{h}\left|x \cdot\left[g^{\star}, \mathbf{d}(y) g^{\star}\right] \cdot \mathbf{d}(y) g^{\star}\right||| y \cdot\left[\mathbf{r}\left(\mathbf{d}(y) g^{\star}|| \mid y\right), \mathbf{r}\left(g_{g}|| \mid y\right)\right] \cdot\left[v, v^{\star}\right] \\
&={ }_{h}\left|x \cdot\left[g^{\star}, \mathbf{d}(y) g^{\star}\right] \cdot \mathbf{d}(y) g^{\star}\right||| y \cdot\left[\mathbf{r}\left(\mathbf{d}(y) g^{\star}|| \mid y\right), v^{\star}\right] \\
& \quad\left(v=\mathbf{r}\left({ }_{h}|| \mid(x \cdot y)\right)=\mathbf{r}\left({ }_{g}|| \mid y\right), \text { by }(\mathrm{IC} 3)\right) \\
&={ }_{h}\left|x \cdot\left[g^{\star}, \mathbf{d}(y) g^{\star}\right] \cdot \mathbf{d}(y) g^{\star}\right||| y \cdot\left[\mathbf{r}\left(\mathbf{d}(y) g^{\star}|| \mid y\right),\left(\mathbf{r}\left(\mathbf{d}(y) g^{\star}|| \mid y\right)\right)^{\star}\right] \\
& \quad\left(v=\mathbf{r}\left({ }_{g}|| \mid y\right) \mathcal{L} \mathbf{r}\left(\mathbf{d}(y) g^{\star}|| \mid y\right), \text { and so } v^{\star}=\left(\mathbf{r}\left(\mathbf{d}(y) g^{\star}|| \mid y\right)\right)^{\star}\right) \\
&={ }_{h}\left|x \cdot{ }_{g \star}\right| y \quad\left(g^{\star}=\mathbf{r}\left({ }_{h} \mid x\right)\right) .
\end{aligned}
$$

(P6) It follows from (IC6).

(PC1) Suppose that $x \in Q, h \in U$ and $h \omega \mathbf{d}(x)$. Then by Proposition 1.41, $\mathbf{r}\left({ }_{h} \mid \| x\right) \omega \mathbf{r}(x)$ and ${ }_{h}\|\mid x=x\|_{\mathbf{r}\left({ }_{h} \mid \| x\right)}$. Thus, $\mathbf{d}\left(x\|\|_{\mathbf{r}\left({ }_{h} \||| x\right)}\right)=h$. In addition, we have that

$$
{ }_{h}\left|x={ }_{h}\right||| x \cdot\left[\mathbf{r}\left({ }_{h}|| \mid x\right),\left(\mathbf{r}\left({ }_{h}|| \mid x\right)\right)^{\star}\right]
$$

and

$$
\begin{aligned}
\left.x\right|_{\mathbf{r}(h \||| x)} & =\left[\left(\mathbf{d}\left(x|\||_{\mathbf{r}(h|| \mid x)}\right)\right)^{+}, \mathbf{d}\left(x|\||_{\mathbf{r}(h|| x)}\right)\right] \cdot x\|\|_{\mathbf{r}(h \||| x)} \\
& =\left[h^{+}, h\right] \cdot x \|_{\mathbf{r}(h|| \mid x)} .
\end{aligned}
$$

Clearly, we have that

$$
\begin{aligned}
& { }_{h} \mid x \cdot\left[\left(\mathbf{r}\left({ }_{h}|| \mid x\right)\right)^{\star}, \mathbf{r}\left({ }_{h}|| \mid x\right)\right] \\
& ={ }_{h}|| \mid x \cdot\left[\mathbf{r}\left({ }_{h}|| \mid x\right),\left(\mathbf{r}\left({ }_{h}|| \mid x\right)\right)^{\star}\right] \cdot\left[\left(\mathbf{r}\left({ }_{h}|| \mid x\right)\right)^{\star}, \mathbf{r}\left({ }_{h}|| \mid x\right)\right] \\
& ={ }_{h} \| x \\
& =x\|\|_{\mathbf{r}}\left({ }_{h}|| x\right) \\
& =\left.\left[h,\left(\mathbf{d}\left(\left.x||\right|_{\mathbf{r}(h|| \mid x)}\right)\right)^{+}\right] \cdot x\right|_{\mathbf{r}(h|| \mid x)} \\
& \left(\left.x\right|_{\mathbf{r}(h|| \mid x)}=\left[\left(\mathbf{d}\left(x|\||_{\mathbf{r}(h|| x)}\right)\right)^{+}, h\right] \cdot x \|_{\left.\mathbf{r}_{(h}|| x\right)}\right) .
\end{aligned}
$$

Hence, ${ }_{h}|x \rho x|_{\left.\mathbf{r}_{h}|| \mid x\right)}$. 
Suppose that $k \omega \mathbf{r}(x)$ and ${ }_{h}|x \rho x|_{k}$. Then

$$
\begin{aligned}
& { }_{h}\left|x \cdot\left[\mathbf{r}\left({ }_{h} \mid x\right), k\right]=\left[h, \mathbf{d}\left(\left.x\right|_{k}\right)\right] \cdot x\right|_{k} \\
& \Rightarrow\left({ }_{h}|| \mid x\right) \cdot\left[\mathbf{r}\left({ }_{h}|| \mid x\right),\left(\mathbf{r}\left({ }_{h}|| \mid x\right)\right)^{\star}\right] \cdot\left[\left(\mathbf{r}\left({ }_{h}|| \mid x\right)\right)^{\star}, k\right] \\
& =\left[h,\left(\mathbf{d}\left(x \mid \|_{k}\right)\right)^{+}\right] \cdot\left[\left(\mathbf{d}\left(x \mid \|_{k}\right)\right)^{+}, \mathbf{d}\left(x \mid \|_{k}\right)\right] \cdot x \|_{k} \\
& \Rightarrow\left({ }_{h}|| \mid x\right) \cdot\left[\mathbf{r}\left({ }_{h} \mid \| x\right), k\right]=\left[h, \mathbf{d}\left(\left.x||\right|_{k}\right)\right] \cdot x \|_{k} \quad(\text { by (IC3)), }
\end{aligned}
$$

that is, $h\||| x \rho x\| \| k$ in $Q$, where $\rho$ is defined in Chapter 6. Since $h \omega \mathbf{d}(x)$, we have that $h \in S(h, \mathbf{d}(x))$. Similarly, $k \in S(\mathbf{r}(x), k)$. Now, we calculate in $Q / \rho$

$$
\begin{aligned}
& \overline{1_{h}} \odot \bar{x}=\overline{\left(1_{h} \diamond h\right) \cdot(h * x)} \\
& =\overline{\left(1_{h} \otimes x\right)_{h}} \\
& =\overline{1_{h}\left|\|_{h} \cdot[h, h] \cdot[h, h \mathbf{d}(x)] \cdot{ }_{h \mathbf{d}(x)}\right||| x} \\
& =\overline{1_{h}\left|\|_{h} \cdot h\right||| x} \quad(h \omega \mathbf{d}(x)) \\
& =\overline{1_{h} \cdot{ }_{h} \|} \\
& =\overline{h \| \mid x}
\end{aligned}
$$

and dually, we have $\bar{x} \odot \overline{1_{k}}=\overline{x \mid \|_{k}}$. As ${ }_{h}|\|x \rho x\|| \mid k$, we obtain that $\overline{1_{h}} \odot \bar{x}=\bar{x} \odot \overline{1_{k}}$. Since $\left.{ }_{h}\|\mid x=x\|\left\|_{\mathbf{r}(h)}\right\| x\right)$, we obtain that $\overline{1_{h}} \odot \bar{x}=\bar{x} \odot \overline{\left.1_{\mathbf{r}(h)} \| x\right)}$. Hence, $\bar{x} \odot \overline{1_{k}}=$ $\bar{x} \odot \overline{1_{\mathbf{r}(h|| \mid x)}}$. Due to $\bar{x} \mathcal{L}^{*} \overline{1_{\mathbf{r}(x)}}$ and $k, \mathbf{r}\left({ }_{h} \mid \| x\right) \in \omega(\mathbf{r}(x))$, we have that $\overline{1_{k}}=\overline{1_{\mathbf{r}\left({ }_{h} \| \mid x\right)}}$. Since $U$ is isomorphic to $\bar{U}$, it follows that $k=\mathbf{r}\left({ }_{h} \mid \| x\right)$, and consequently, the uniqueness holds.

We note that $\mathbf{d}(x)|x=\mathbf{d}(x)||| x \cdot\left[\mathbf{r}\left(\mathbf{d}_{(x)}|| \mid x\right),(\mathbf{r}(\mathbf{d}(x)|| \mid x))^{\star}\right]=x \cdot\left[\mathbf{r}(x),(\mathbf{r}(x))^{\star}\right]$. Clearly, $\mathbf{d}(x) \mid x \rho x$. Thus, (PC1) holds.

(PC2) Suppose that $x \in Q$ and for $i=1,2, e_{i}, f_{i} \in U$ are such that $e_{i} \omega \mathbf{d}(x)$, $f_{i} \omega \mathbf{r}(x)$ and $e_{i}|x \rho x|_{f_{i}}$. In view of the proof of (PC1), we have that $f_{i}=\mathbf{r}\left(e_{i}|| \mid x\right)$. If $e_{1} \omega^{r} e_{2}$, by (IC5), we have that $f_{1} \omega^{r} f_{2}$ and

$$
\left[e_{1}, e_{1} e_{2}\right] \cdot\left({ }_{e_{1} e_{2}}|| \mid x\right)=\left(e_{1}|| \mid x\right) \cdot\left[f_{1}, f_{1} f_{2}\right] .
$$

Thus, $\mathbf{r}\left(e_{1} e_{2}|| \mid x\right)=f_{1} f_{2}$. Again in view of the proof of (PC1), we have that $e_{1} e_{2}|x \rho x|_{f_{1} f_{2}}$.

Similarly, the second part holds.

To sum up, we have: 
Corollary 11.11. An IC-RBS category with respect to the restriction and corestriction defined before Lemma 11.7 forms an inductive $e_{2}$ cancellative category.

Conversely, an inductive ${ }_{2}$ cancellative category with respect to the restriction and co-restriction defined before Lemma 11.10 forms an IC-RBS category.

Also, we have:

Lemma 11.12. Let $P_{1}$ and $P_{2}$ be $I C$-RBS categories and $F: P_{1} \rightarrow P_{2}$ be an

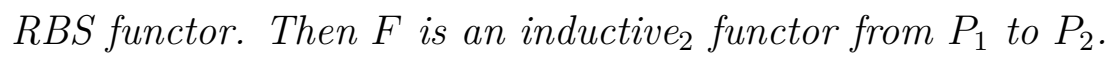

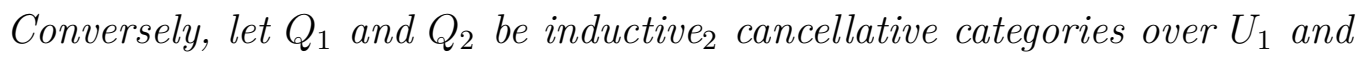

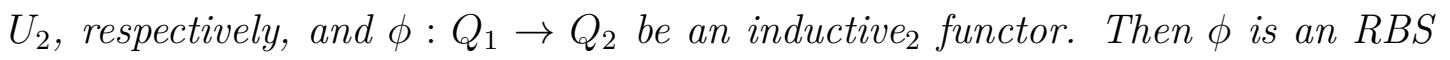
functor from $Q_{1}$ to $Q_{2}$.

Proof. Suppose that $P_{1}$ and $P_{2}$ are IC-RBS categories and $F: P_{1} \rightarrow P_{2}$ is an RBS functor. If $x \leq y$ in $P_{1}$, then there exists $e \in U$ such that $e \omega \mathbf{d}(y)$ and $x=e_{e}|| y$, that is, $x={ }_{e} \mid y \cdot[\mathbf{r}(e \mid y), k]$, where $k \in \mathbf{r}(y)$ and $e_{e}|y \rho y|_{k}$. Certainly, we have $e F \omega \mathbf{d}(y F)$ and $k F \omega \mathbf{r}(y F)$. From ${ }_{e}|y \rho y|_{k}$, we have $\left.{ }_{e} \mid y\right) F \rho\left(\left.y\right|_{k}\right) F$ by Lemma 10.8, that is, $e F|y F \rho y F|_{k F}$ by (PF3). Since $(e \mid y) F \rho_{e F} \mid y F$ and $\mathbf{d}((e \mid y) F)=e F=\mathbf{d}\left({ }_{e F} \mid y F\right)$, we have that

$$
\left({ }_{e} \mid y\right) F={ }_{e F} \mid y F \cdot\left[\mathbf{r}\left({ }_{e F} \mid y F\right), \mathbf{r}((e \mid y) F)\right]
$$

and so we have

$$
\begin{aligned}
x F & \left.={ }_{e} \mid y\right) F \cdot\left[\mathbf{r}\left({ }_{e} \mid y\right), k\right] F \\
& ={ }_{e F} \mid y F \cdot\left[\mathbf{r}\left({ }_{e F} \mid y F\right), \mathbf{r}\left(\left({ }_{e} \mid y\right) F\right)\right] \cdot\left[\mathbf{r}\left({ }_{e} \mid y\right) F, k F\right] \quad(\text { by }(\mathrm{PF} 2)) \\
& ={ }_{e F} \mid y F \cdot\left[\mathbf{r}\left({ }_{e F} \mid y F\right), \mathbf{r}\left(\left({ }_{e} \mid y\right) F\right)\right] \cdot\left[\mathbf{r}\left(\left(_{e} \mid y\right) F\right), k F\right] \\
& ={ }_{e F} \mid y F \cdot\left[\mathbf{r}\left({ }_{e F} \mid y F\right), k F\right] \quad\left(\mathbf{r}\left(_{e F} \mid y F\right) \mathcal{L} \mathbf{r}\left(\left(_{e} \mid y\right) F\right) \mathcal{L} k F\right) \\
& ={ }_{e F} \| y F
\end{aligned}
$$

so that $x F \leq y F$. Hence $F$ is order-preserving. Together with (PF1) and (PF2), $F$ is an inductive functor. $_{2}$

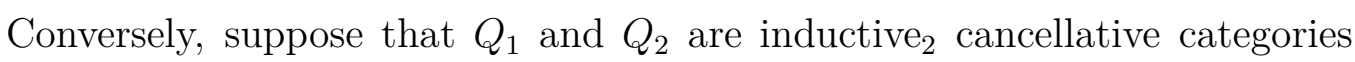

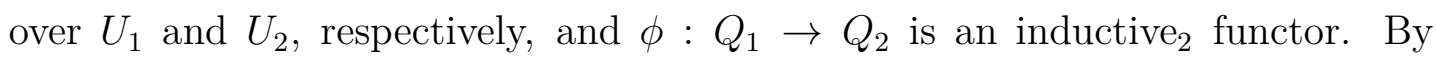
(IOF1) and (IOF2), (PF1) and (PF2) hold. We now show that Condition (PF3) 
holds. If $x \in Q_{1}$ and $h \in U_{1}$ with $h \omega^{l} \mathbf{d}(x)$, then

$$
{ }_{h}|x=[h, \mathbf{d}(x) h] \cdot \mathbf{d}(x) h||| x \cdot\left[\mathbf{r}(\mathbf{d}(x) h|| \mid x),(\mathbf{r}(\mathbf{d}(x) h|| \mid x))^{\star}\right] .
$$

Note that $\mathbf{d}(x) h \mid \| x \leq x$, and so $\mathbf{d}(x) h \mid \| x \phi \leq x \phi$ as $\phi$ is order-preserving. Since $(\mathbf{d}(x) h) \phi \omega \mathbf{d}(x) \phi,{ }_{(\mathbf{d}(x) h) \phi} \| \mid x \phi$ is defined and ${ }_{(\mathbf{d}(x) h) \phi} \| x \phi \leq x \phi$. As $\mathbf{d}((\mathbf{d}(x) h|| \mid x) \phi)=(\mathbf{d}(x) h) \phi$, we obtain that

$$
(\mathbf{d}(x) h \mid \| x) \phi={ }_{(\mathbf{d}(x) h) \phi} \||| x \phi
$$

by the uniqueness of restrictions. Then

$$
\begin{aligned}
\left(_{h} \mid x\right) \phi & =[h, \mathbf{d}(x) h] \phi \cdot(\mathbf{d}(x) h|| \mid x) \phi \cdot\left[\mathbf{r}(\mathbf{d}(x) h|| \mid x),(\mathbf{r}(\mathbf{d}(x) h|| \mid x))^{\star}\right] \phi \\
& =[h \phi,(\mathbf{d}(x) h) \phi] \cdot{ }_{(\mathbf{d}(x) h) \phi}|| \mid x \phi \cdot\left[\mathbf{r}(\mathbf{d}(x) h|| \mid x) \phi,(\mathbf{r}(\mathbf{d}(x) h|| \mid x))^{\star} \phi\right] \quad(\text { by }(\text { IOF2 })) \\
& =[h \phi, \mathbf{d}(x) \phi h \phi] \cdot \mathbf{d}(x) \phi h \phi|| \mid x \phi \cdot\left[\mathbf{r}(\mathbf{d}(x) \phi h \phi|| \mid x \phi),(\mathbf{r}(\mathbf{d}(x) h|| \mid x))^{\star} \phi\right] \\
& =[h \phi, \mathbf{d}(x) \phi h \phi] \cdot \mathbf{d}(x) \phi h \phi|| \mid x \phi \cdot\left[\mathbf{r}(\mathbf{d}(x) \phi h \phi|| \mid x \phi),(\mathbf{r}(\mathbf{d}(x) \phi h \phi|| \mid x \phi))^{\star}\right] \cdot \\
& ={ }_{h \phi} \mid x \phi \cdot\left[(\mathbf{r}(\mathbf{r}(x) \phi h \phi|| \mid x \phi))^{\star},\left(\mathbf{r}\left({ }_{\mathbf{d}(x) h) \phi h \phi}|| \mid x\right)\right)^{\star} \phi\right] \\
& \left.={ }_{h \phi} \mid x \phi \cdot\left[\mathbf{r}\left({ }_{h \phi} \mid x \phi\right), \mathbf{r}\left({ }_{h} \mid x\right) \phi\right)\right]
\end{aligned}
$$

so that $\left.{ }_{h} \mid x\right) \phi \rho_{h \phi} \mid x \phi$ and dually, if $k \omega^{r} \mathbf{r}(x)$, we have $\left.\left(\left.x\right|_{k}\right) \phi \rho x \phi\right|_{k \phi}$ so that (PF3) holds. Hence, $\phi$ is an RBS functor.

Further, by Corollary 11.6, Corollary 11.11 and Lemma 11.12, we obtain Theorem $\mathrm{C}$ mentioned in Chapter 6 as follows:

Theorem C (Armstrong [1]) The category of concordant semigroups and good morphisms is equivalent to the category of inductive ${ }_{2}$ cancellative categories and inductive $_{2}$ functors.

\subsection{The regular case}

We focus on regular semigroups in this section. An RBS category $P$ over $U$ is a regular groupoid over $U$ if Conditions ( $\mathrm{RG}),(\mathrm{PC} 1),(\mathrm{PC} 2)$ and the duals $(\mathrm{PC} 1)^{\circ}$ and $(\mathrm{PC} 2)^{\circ}$ of $(\mathrm{PC} 1)$ and $(\mathrm{PC} 2)$ hold: 
(RG) for all $x \in P$, there exists $y \in P$ with $\mathbf{d}(y)=\mathbf{r}(x)$ and $\mathbf{r}(y)=\mathbf{d}(x)$ such that $1_{\mathbf{d}(x)}=x \cdot y$ and $y \cdot x=1_{\mathbf{r}(x)}$.

Notice that in a regular groupoid $P$, for any $x \in P$, there exists $y \in P$ such that $1_{\mathbf{d}(x)}=x \cdot y$ and $y \cdot x=1_{\mathbf{r}(x)}$, and so $y$ is the inverse of $x$ so that $P$ is a groupoid. Consequently, $P$ is cancellative and so it is an IC-RBS category. Together with the comments succeeding the definition of inductive ${ }_{2}$ cancellative category in Chapter 6, we have an immediate consequence of Corollary 11.11 as follows:

Corollary 11.13. A regular groupoid with respect to the restriction and corestriction defined before Lemma 11.7 forms an inductive 2 groupoid.

Conversely, an inductive 2 groupoid with respect to the restriction and corestriction defined before Lemma 11.10 forms a regular groupoid.

Since a regular groupoid is an IC-RBS category and a regular semigroup is a special concordant semigroup, it follows from Lemma 11.6 that:

Corollary 11.14. The category of regular semigroups and morphisms is equivalent to the category of regular groupoids over regular biordered sets and $R B S$ functors.

Proof. Let $S$ be a regular semigroup with $U=E(S)$. It is sufficient to show that $S \mathbf{C}$ satisfies Condition $(\mathrm{RG})$. Suppose that $(e, x, f) \in S \mathbf{C}$. Since $\mathcal{R}=\widetilde{\mathcal{R}}_{U}$ and $\mathcal{L}=\widetilde{\mathcal{L}}_{U}$, we have that $e \mathcal{R} x \mathcal{L} f$. It follows from the fact that $S$ is regular that there exists $y \in S$ with $e \mathcal{L} y \mathcal{R} f, e=x y$ and $y x=f$. Then $(f, y, e) \in S \mathbf{C}$ and the products $(e, x, f) \cdot(f, y, e),(f, y, e) \cdot(e, x, f)$ exist in $S \mathbf{C}$. Moreover, $(e, x, f) \cdot(f, y, e)=(e, x y, e)=(e, e, e)=[e, e]=1_{e}$ and similarly, $(f, y, e) \cdot(e, x, f)=1_{f}$. Hence, Conditon $(\mathrm{RG})$ holds.

Conversely, let $P$ be a regular groupoid over $U$. We need to show that $P \mathbf{S}$ is regular. Suppose that $x \in P$. Then there exists $y \in P$ with $\mathbf{d}(y)=\mathbf{r}(x)$ and $\mathbf{r}(y)=\mathbf{d}(x)$ such that $1_{\mathbf{r}(x)}=y \cdot x$ and $1_{\mathbf{d}(x)}=x \cdot y$. So $\overline{1_{\mathbf{d}(x)}}=\overline{x \cdot y}=\bar{x} \odot \bar{y}$. Hence, $\bar{x} \odot \bar{y} \odot \bar{x}=(\bar{x} \odot \bar{y}) \odot \bar{x}=\overline{1_{\mathbf{d}(x)}} \odot \bar{x}=\bar{x}$ so that $P \mathbf{S}$ is regular.

In view of Corollary 11.13 and Corollary 11.14, we have:

Theorem B (Nambooripad [38]) The category of regular semigroups and mor-

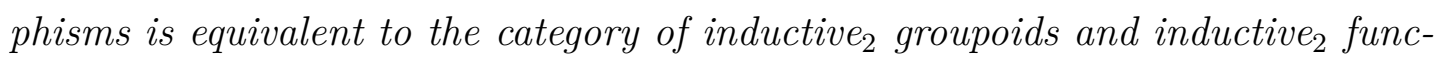
tors. 


\section{Bibliography}

[1] S.M. Armstrong, 'Structure of concordant semigroups', J. Algebra 118 (1988), 205-260.

[2] S.M. Armstrong, 'The structure of type A semigroups', Semigroup Forum 29 (1984), 319-336.

[3] D. Easdown, 'Biordered sets come from semigroups', J. Algebra 96 (1985), $581-591$.

[4] C. Ehresmann, 'Oeuvres complètes et comentées', Suppl. Chaiers Topologie Géom. Différentielle (Amiens, 1980-1984).

[5] S. Eilenberg and S. Mac Lane, 'General theory of natural equivalences', Trans. Amer. Math. Soc. 58 (1945), 231-294.

[6] A. El-Qallali, J. Fountain and V.A.R. Gould, 'Fundamental representations for classes of semigroups containing a band of idempotents', Communications in Algebra 36 (2008), 2991-3031.

[7] A. El-Qallali and J. Fountain, 'Quasi-adequate semigroups', Proc. Roy. Soc. Edinburgh Sec. A 91 (1981), 91-99.

[8] A. El-Qallali and J. Fountain, 'Idempotent-connected abundant semigroups', Proc. Roy. Soc. Edinburgh Sec. A 91 (1981), 79-90.

[9] J. Fountain, 'Adequate semigroups', Proc. Edinburgh Math. Soc. 22 (1979), $113-125$.

[10] J. Fountain, 'Abundant semigroups', Proc. London Math. Soc. 44 (1982), 103-129. 
[11] J. Fountain and G.M.S. Gomes, 'Finite abundant semigroups in which the idempotents form a subsemigroup', J. Algebra 295 (2006), 303-313.

[12] J. Fountain and V.A.R. Gould, 'Idempotent bounded C-semigroups', Monatsh. Math. 117 (1994), 237-254.

[13] J. Fountain, G.M.S. Gomes and V.A.R. Gould, 'The free ample monoid', I.J.A.C. 19 (2009), 527-554.

[14] J. Fountain, G.M.S. Gomes and V.A.R. Gould, 'Membership of $\mathcal{A} \vee \mathcal{B}$ for classes of finite weakly abundant semigroups', Periodica Mathematica Hungarica 59 (2009), 9-36.

[15] J. Fountain, G.M.S. Gomes and V.A.R. Gould, 'A Munn type representaion for a class of E-semiadequate semigroups', J. Algebra 218 (1999), 693-714.

[16] G.M.S. Gomes and V.A.R. Gould, 'Left adequate and left Ehresmann monoids II', J. Algebra, to appear.

[17] G.M.S. Gomes and V.A.R. Gould, 'Fundamental semigroups having a band of idempotents', Semigroup Forum 77 (2008), 279-299.

[18] G.M.S. Gomes and V.A.R. Gould, 'Fundamental Ehresmann semigroups', Semigroup Forum 63 (2001), 11-33.

[19] V.A.R. Gould, 'Notes on restriction semigroups and related structures', http://www-users.york.ac.uk/ varg1/restriction.pdf.

[20] V.A.R. Gould and Y.H. Wang, 'Beyond orthodox semigroups', preprint.

[21] J.A. Green, 'On the structure of semigroups' Ann. of Math. (2) 54 (1951), 163-172.

[22] X. Guo, 'F-abundant semigroups', Glasgow Math. J. 43 (2001), 153-163.

[23] T.E. Hall, 'On regular semigroups whose idempotents form a subsemigroup', Bull. Australian Math. Soc. 1 (1969), 195-208.

[24] C.D. Hollings, 'Partial actions of semigroups and monoids', PhD thesis, the University of York (2007). 
[25] J.M. Howie, Fundamentals of Semigroup Theory, Clarendon Press, Oxford (1995).

[26] J.M. Howie, An Introduction to Semigroup Theory, Academic Press (1976).

[27] J.M. Howie, 'The maximum idempotent-separating congruence on an inverse semigroup', Proc. Edinburgh Math. Soc. 14 (1964), 71-79.

[28] M. Kilp, U. Knauer and A.V. Mikhalev, Monoids, Acts and Categories: with applications to wreath products and graphs, De Gruyter Expositions in Mathematics vol. 29 (2000).

[29] M. Kambites, 'Free adequate semigroups', J. Australian Math. Soc., to appear.

[30] M. Kambites, 'Retracts of trees and free left adequate semigroups', Proc. Edinburgh Math. Soc., to appear.

[31] G. Lallement, 'Congruences et équivalences de Green sur un demi-groupe régulier', C. R. Acad. Sci. Paris, Sér. A. 262 (1966), 613-616.

[32] M.V. Lawson, 'Semigroups and ordered categories. I. the reduced case', J. Algebra 141 (1991), 422-462.

[33] M.V. Lawson, 'Rees matrix semigroups', Proc. Edinburgh Math. Soc. 33 (1990), 23-37.

[34] S. Mac Lane, Categories for the working Mathematician Graduate Texts in Mathematics 5 (2nd ed.) Springer-Verlag, ISBN 0-387-98403-8 (1998).

[35] J. Meakin, 'On the structure of inverse semigroups', Semigroup Forum 12 (1976), 6-4.

[36] J. Meakin, 'The structure mappings on a regular semigroups', Proc. Edinburgh Math. Soc. 21 (1978), 135-142.

[37] W.D. Munn, 'Uniform semilattices and bisimple inverse semigroups', Quart. J. Math. Oxford (2), 17(1966), 151-159.

[38] K.S.S. Nambooripad, 'Structure of regular semigroups', Mem. American Math. Soc. 22 (1979), No. 224. 
[39] K.S.S. Nambooripad, 'Structure of regular semigroups I: fundamental regular semigroups', Semigroup Forum 9 (1976), 354-363.

[40] K.S.S. Nambooripad, 'Structure of regular semigroups II: the general case', Semigroup Forum 9 (1975), 364-371.

[41] K.S.S. Nambooripad and R. Veeramony, 'Subdirect products of regular semigroups', Semigroup Forum 28 (1983), 265-307.

[42] J. von Neuman, 'On regular rings', Proc. Nat. Acad. Sci., U. S. A. 22 (1936), 503-554.

[43] N.R. Reilly and H.E. Scheiblich, 'Congruences on regular semigroups', Pacific J. Math. 23 (1967), 349-360.

[44] X.M. Ren, K.P. Shum and Y.Q. Guo, 'A generalized Clifford theorem of semigroups', Science in China A 53 (2010), 1097-1101.

[45] X.M. Ren, K.P. Shum and Q.Y. Yin, ' Comprehensive congruences on $U$ cyber semigroups', Int. Math. Forum 3 (2008), 685-693.

[46] X.M. Ren, Y.H. Wang and K.P. Shum, 'On U-orthodox semigroups', Science in China A 52 (2009), 329-350.

[47] B.M. Schein, 'On the theory of inverse semigroups and generalised groups', Amer. Math. Soc. Transl. Ser. 2113 (1979), 89-112.

[48] C.P. Simmons, 'Small category theory applied to semigroups and monoids', PhD thesis, the University of York (2001).

[49] M.B. Szendrei, private communication (2010).

[50] Y.H. Wang, 'Weakly B-orthodox semigroups', preprint.

[51] Y.H. Wang, 'Structure theorems for weakly B-abundant semigroups', Semigroup Forum 84 (2012), 39-58.

[52] M. Yamada, 'Regular semigroups whose idempotents satisfy permutation identities', Pacific J. Math. 21(1967), 371-392.

[53] http://en.wikipedia.org/wiki/Equivalence-of-categories. 